\title{
TRENCH SAMPLING REPORT SALMON SITE LAMAR COUNTY, MISSISSIPPI
}

\author{
Prepared for: \\ DOE Nevada Operations Office \\ Las Vegas, Nevada \\ Prepared by: \\ IT CORPORATION \\ 4330 South Valley View Boulevard, Suite 114 \\ Las Vegas, Nevada
}

Work Performed Under Contract No.:

DE-AC08-92NV10972

July 1994

MASTER

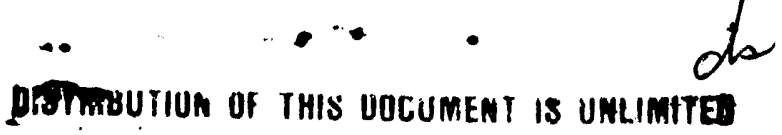




\section{Table of Contents}

List of Figures $\ldots \ldots \ldots \ldots \ldots \ldots \ldots \ldots \ldots \ldots \ldots \ldots \ldots \ldots \ldots$

List of Tables $\ldots \ldots \ldots \ldots \ldots \ldots \ldots \ldots \ldots \ldots \ldots \ldots \ldots \ldots$ iii

List of Abbreviations and Acronyms $\ldots \ldots \ldots \ldots \ldots \ldots \ldots \ldots \ldots \ldots$ iv

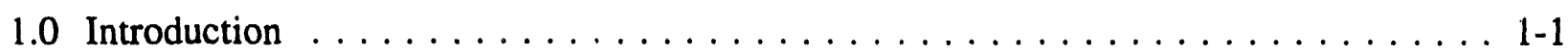

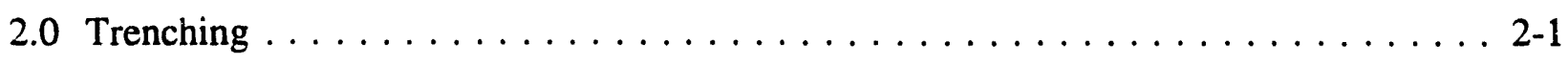

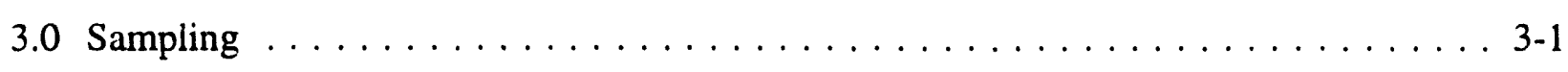

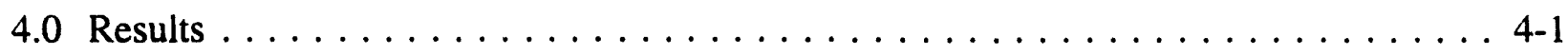

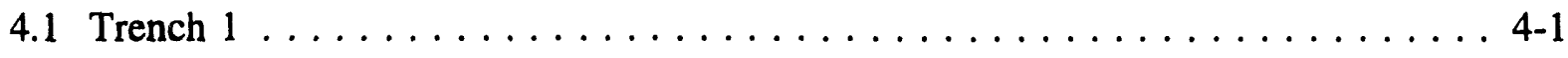

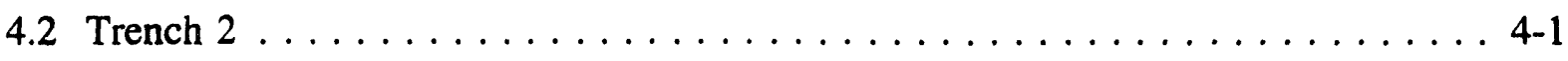

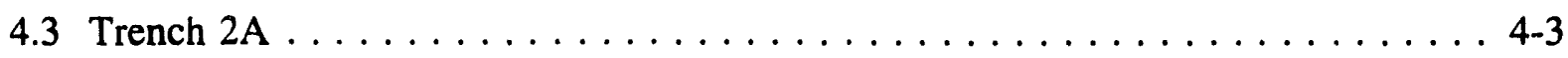

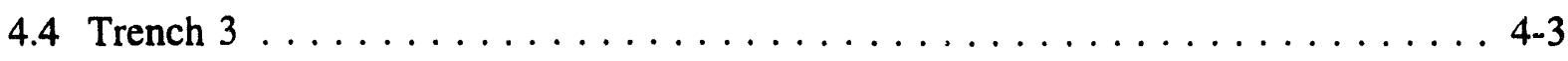

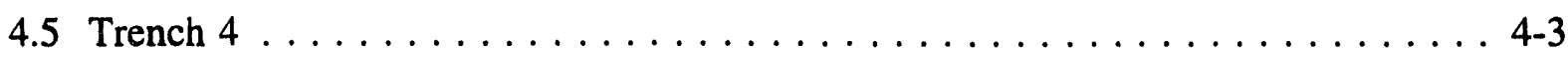

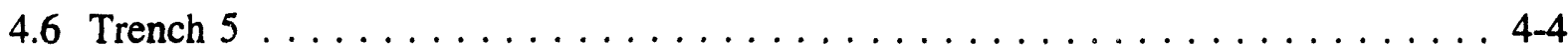

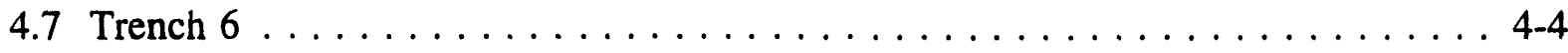

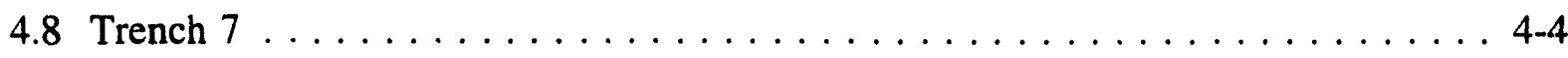

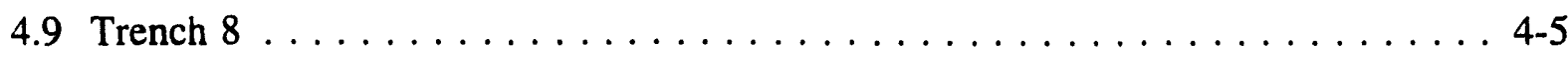

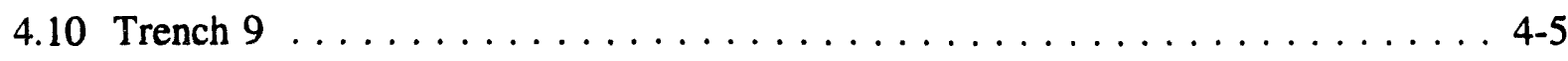

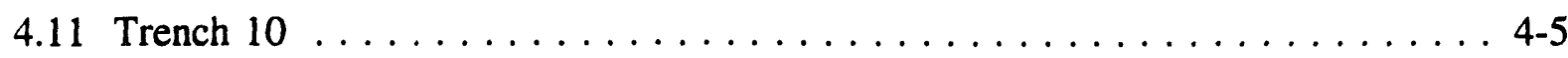

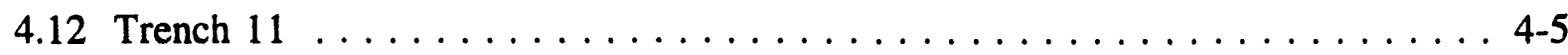

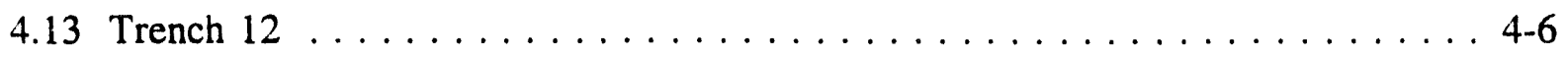

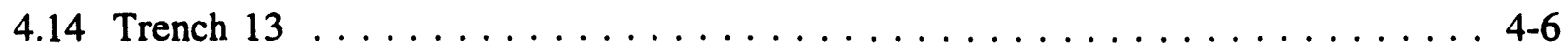

4.15 Trench $14 \ldots \ldots \ldots \ldots \ldots \ldots \ldots \ldots \ldots \ldots \ldots \ldots \ldots \ldots \ldots \ldots$

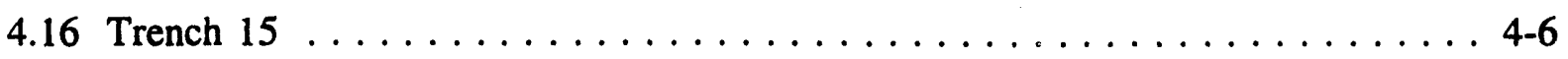

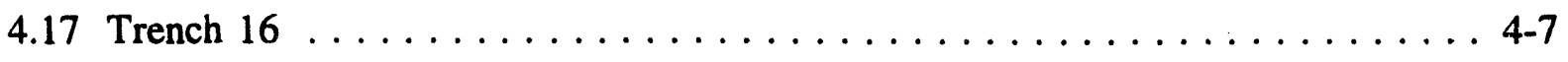

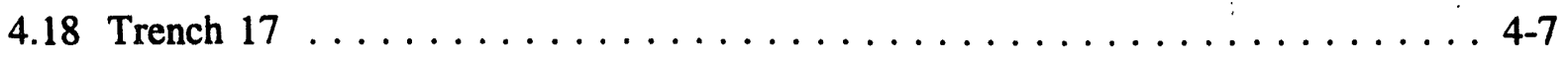

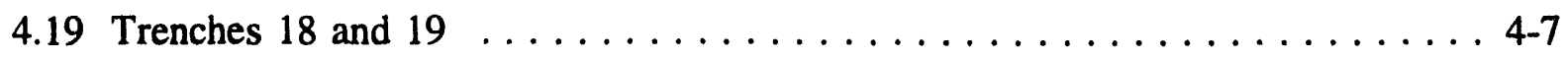




\section{Table of Contents (Continued)}

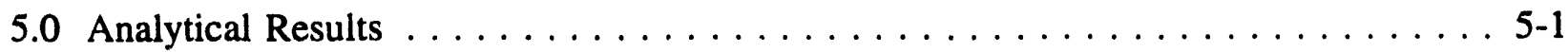

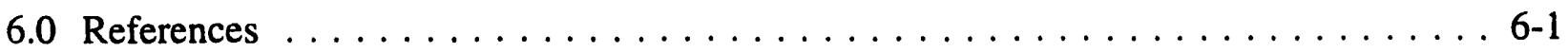

Appendix A - Corrected Sample Locations . . . . . . . . . . . . . . . . A-1

Appendix B - List of Applicable Terms, Letters, or Symbols found in

Data Analyses Tables . . . . . . . . . . . . . . . . . B-1

Appendix C - Results of Volatile Organic Compound Analyses . . . . . . . . . . . . C-1

Appendix D - Results of Semivolatile Organic Compound Analyses . . . . . . . . . . D-1

Appendix E - Results of Gross Alpha and Beta Analyses . . . . . . . . . . . . . E-1

Appendix F - Results of Gamma Spectroscopy Analyses . . . . . . . . . . . . . F-1

Appendix $\mathrm{G}$ - Results of Metals and Cyanide Analyses $\ldots \ldots \ldots \ldots \ldots \ldots$

Appendix $\mathrm{H}$ - Results of Dioxin/Furan Compound Analyses . . . . . . . . . . . . H-1

Appendix I - Results of Total Petroleum Hydrocarbon Analyses . . . . . . . . . . . . . I-1

Appendix $\mathrm{J}-$ Results of Tritium Analyses . . . . . . . . . . . . . . . . J

Appendix $\mathrm{K}$ - Sample Collection Logs . . . . . . . . . . . . . . . . . . . K-1 


\section{List of Figures}

Number

Title

Page

1-1 Salmon Site Map Showing REECo Pits Area $\ldots \ldots \ldots \ldots \ldots \ldots \ldots \ldots \ldots$

1-2 Vertical Magnetic Gradient Map of the Main REECo Pits Area $\ldots \ldots \ldots \ldots$ 1-3

1-3 Vertical Magnetic Gradient Map of Disposal Area $5 \ldots \ldots \ldots \ldots \ldots \ldots \ldots$

1-4 Vertical Magnetic Gradient Map of the Gas Station Area $\ldots \ldots \ldots \ldots \ldots \ldots$

4-1 Trench Locations, REECo Pits Area $\ldots \ldots \ldots \ldots \ldots \ldots \ldots \ldots \ldots . \ldots \ldots .2$

\section{List of Tables}

Number

Title

Page

3-1 Samples Collected From Test Pit Excavations, December 8 to 17, $1993 \ldots \ldots$. . . 3-2

5-1 Summary of Preliminary Analytical Results $\ldots \ldots \ldots \ldots \ldots \ldots . \ldots \ldots$ 5-2

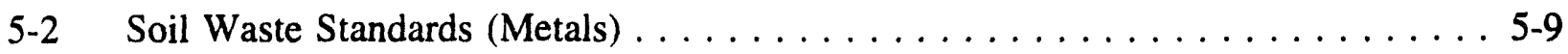

5-3 Radionuclides Included in the Gamma Spectroscopy Analysis . . . . . . . 5-11

5-4 Radionuclide Activities for Soil Sample REFC-19-SS, Salmon Site . . . . . . 5 5-12 


\section{List of Abbreviations and Acronyms}

$\begin{array}{ll}\text { bgs } & \text { below ground surface } \\ \text { BNA } & \text { base-neutral-acid extractaible compounds } \\ \text { CFR } & \text { Code of Federal Regulations } \\ \text { CLP } & \text { Contract Laboratory Program } \\ \text { cm } & \text { centimeters } \\ \text { CN } & \text { cyanide } \\ \text { CRDL } & \text { contract required detection limit } \\ \text { DIOX } & \text { dioxin/furan } \\ \text { DOE } & \text { U.S. Department of Energy } \\ \text { EERF } & \text { Eastern Environmental Radiation Facilities } \\ \text { EPA } & \text { U.S. Environmental Protection Agency } \\ \text { ft } & \text { feet } \\ { }^{3} \text { H } & \text { tritium } \\ \text { HASL } & \text { Health and Safety Laboratory } \\ \text { in. } & \text { inch(es) } \\ \text { IT } & \text { IT Corporation } \\ \text { ITAS } & \text { IT Analytical Services } \\ \ell & \text { liter } \\ \text { m } & \text { meter(s) } \\ \text { MCC } & \text { maximum contaminant concentration } \\ \text { MET } & \text { metals } \\ \text { mg/kg } & \text { milligrams per kilograms } \\ \text { MMCC } & \text { modified maximum contaminant concentration } \\ \text { MSDEQ } & \text { Mississippi State Department of Environmental Quality } \\ \text { me } & \text { milliliter } \\ \text { pCi } & \text { picocurie } \\ \text { REECo } & \text { Reynolds Electrical \& Engineering Co., Inc. } \\ \text { SM } & \text { Standard Method } \\ \text { TCLP } & \text { toxicity characteristic leaching procedure } \\ \text { TPH } & \text { total petroleum hydrocarbons } \\ \text { VOC } & \text { volatile organic compounds } \\ & \end{array}$




\subsection{Introduction}

This report describes trench excavation and sample-collection activities conducted by IT Corporation (IT) as part of the ongoing Remedial Investigation and Feasibility Study at the Salmon Site, Lamar County, Mississippi (DOE, 1992). During construction. operation, and closure of the site wastes of unknown composition were buried in pits on site. All of the potential burial sites are located in Area SA-2 in the northeastern part of the Salmon Site (Figure 1-1). Surface-geophysical field investigations were conducted intermittently between November 1992 and October 1993 to identify potential waste-burial sites and buried metallic materials. The geophysical investigations included vertical magnetic gradient, electromagnetic conductivity, electromagnetic in-phase component. and ground-penetrating radar surveys. A number of anomalies identified by the magnetic gradiometer survey in the Reynolds Electrical \& Engineering Co., Inc., (REECo) pits area indicated buried metallic objects (Figures 1-2, 1-3 and 1-4) (DOE, 1994a). All of the anomalies were field checked to determine if any were caused by surface features or debris. After field checking, 17 anomalies were still unexplained; trenching was planned to attempt to identify their sources.

Between December 8, 1993, and December 17, 1993, 15 trenches were excavated and soil samples were collected at the anomalies. Two other trenches were planned but not excavated, one because a culvert was found at the ground surface and the other because a gopher tortoise burrow was found at the center of the anomaly. After all of the trenches were excavated and sampled, permission was received from the Mississippi State Department of Environmental Quality (MSDEQ) to close the trenches. The trenches were refilled with the excavated material on December 29 and 30, 1993.

Samples were collected, placed in 250- and 500-milliliter $(\mathrm{m} \ell)$ amber glass containers, and shipped on ice to IT Analytical Services (ITAS) in St. Louis, Missouri, using standard IT chain-of-custody procedures. The samples were analyzed for various chemical and radiological parameters. Data validation has not been conducted on any of the samples.

During excavation and sampling, soil samples were also collected by IT for the MSDEQ and the Mississippi Department of Radiological Health, in accordance with their instructions, and delivered into their custody. 


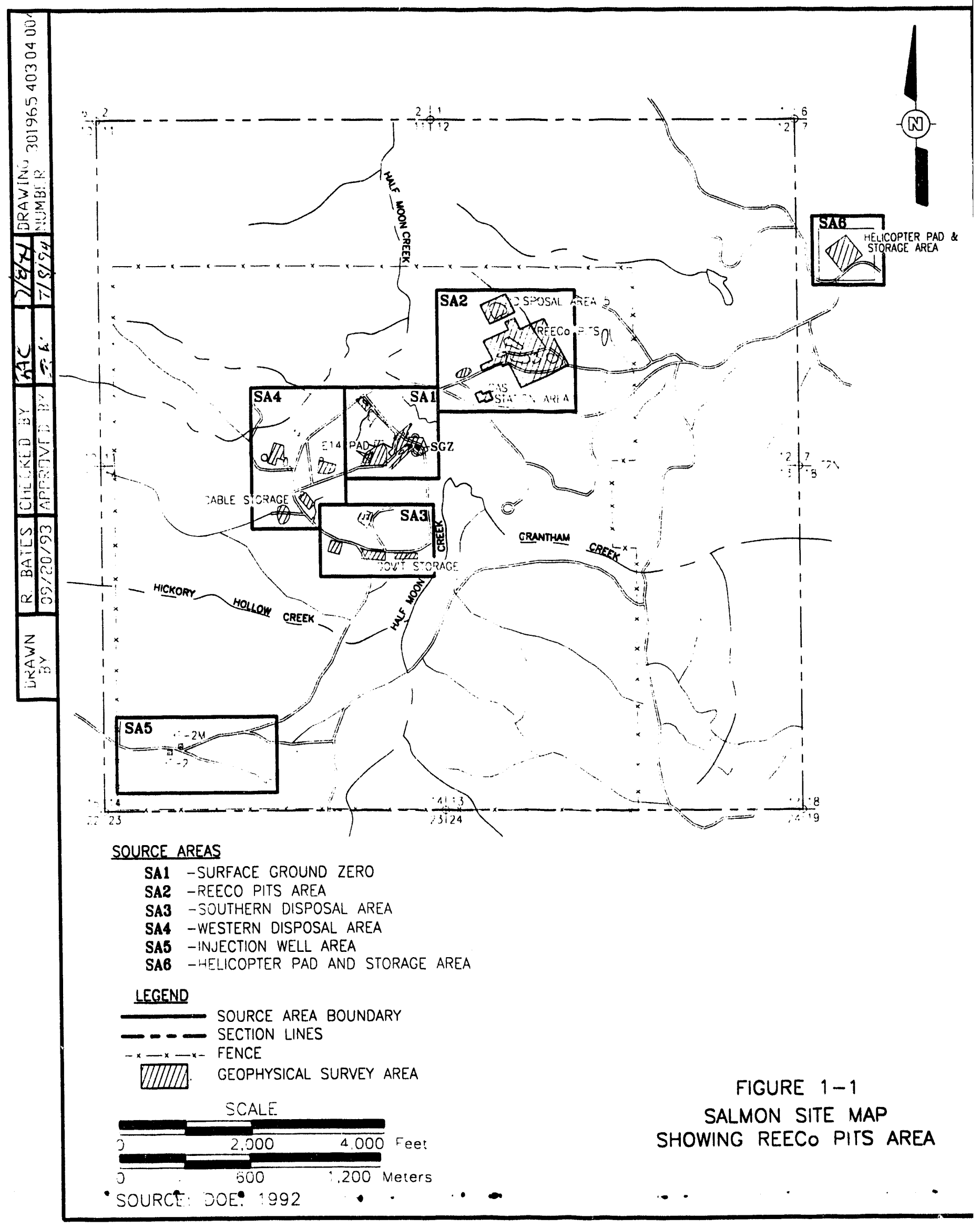




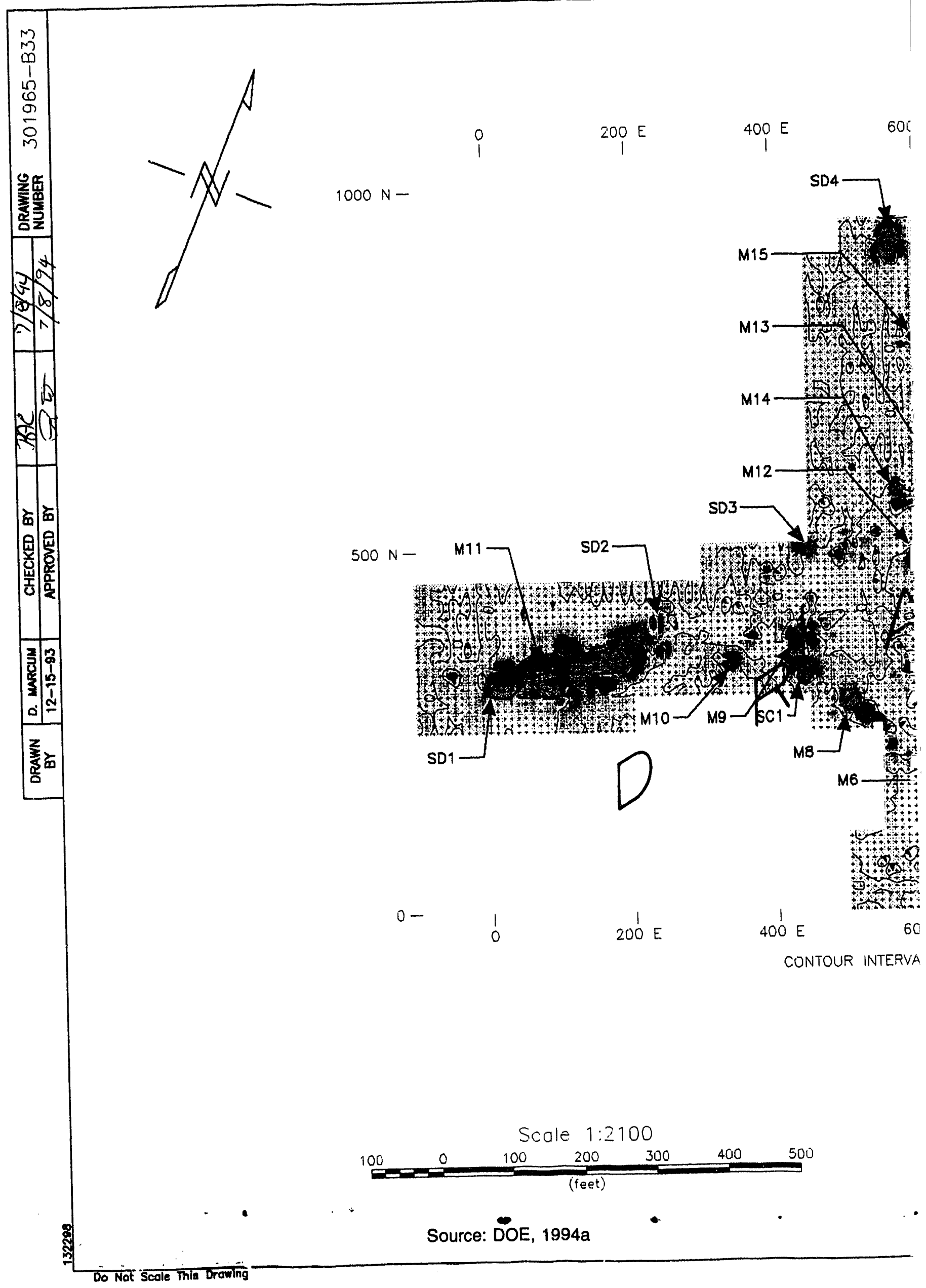




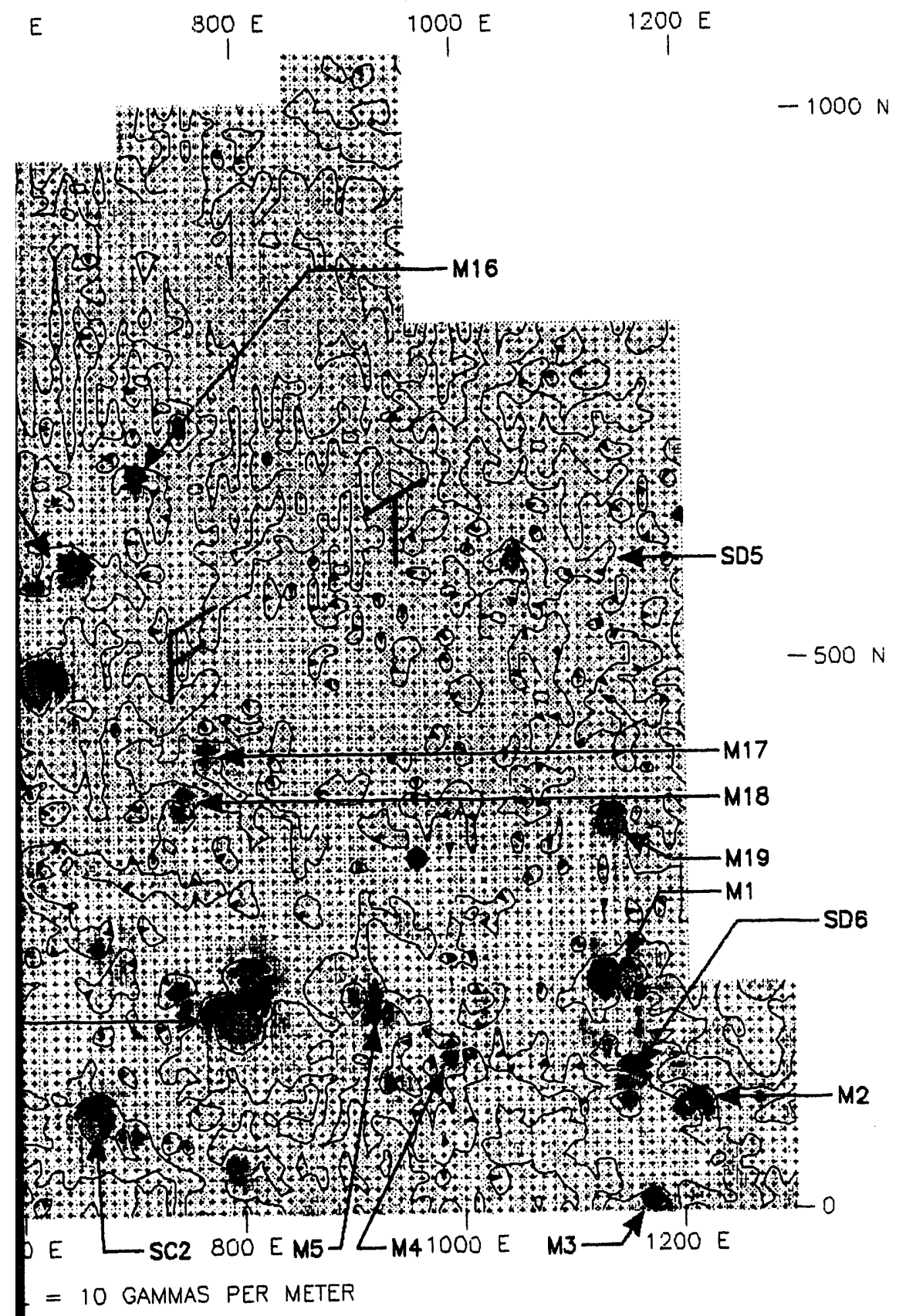

LEGEND

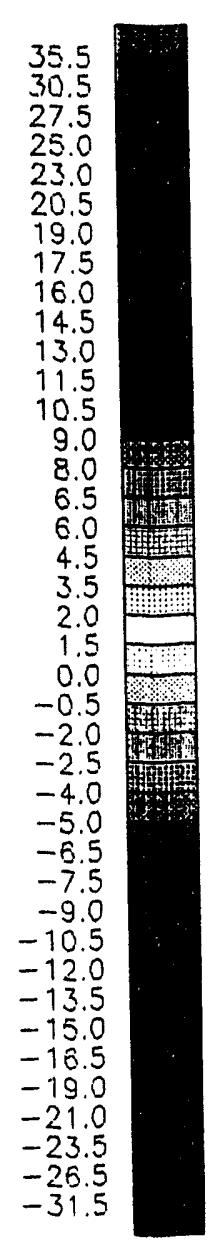

FIGURE 1-2

VERTICAL MAGNETIC GRADIENT MAP OF THE MAIN REECO PITS AREA DECEMBER 1993

SALMON SITE

LAMAR COUNTY, MISSISSIPPI

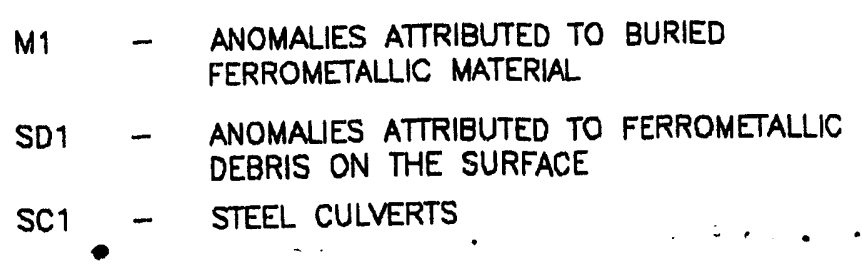




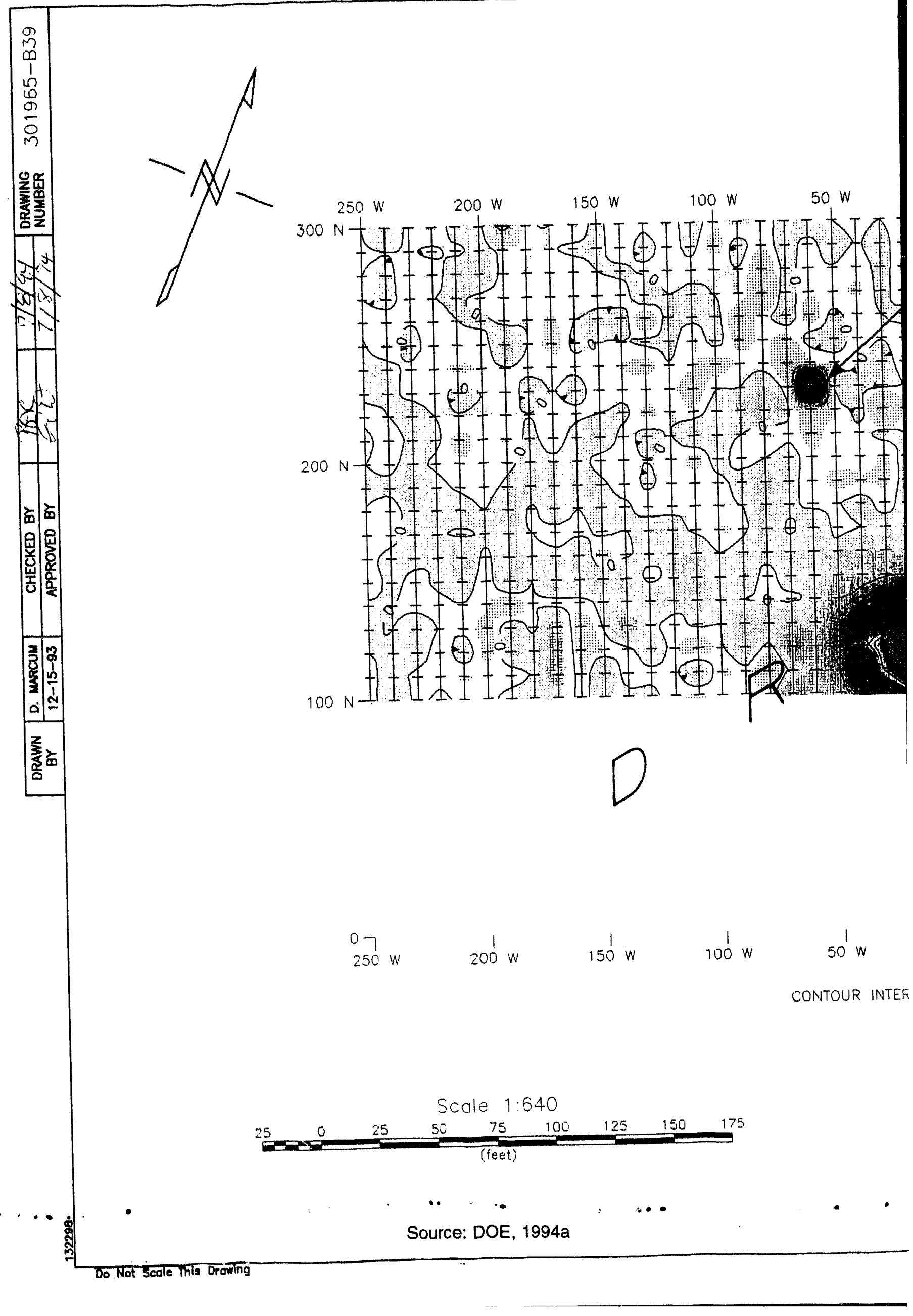




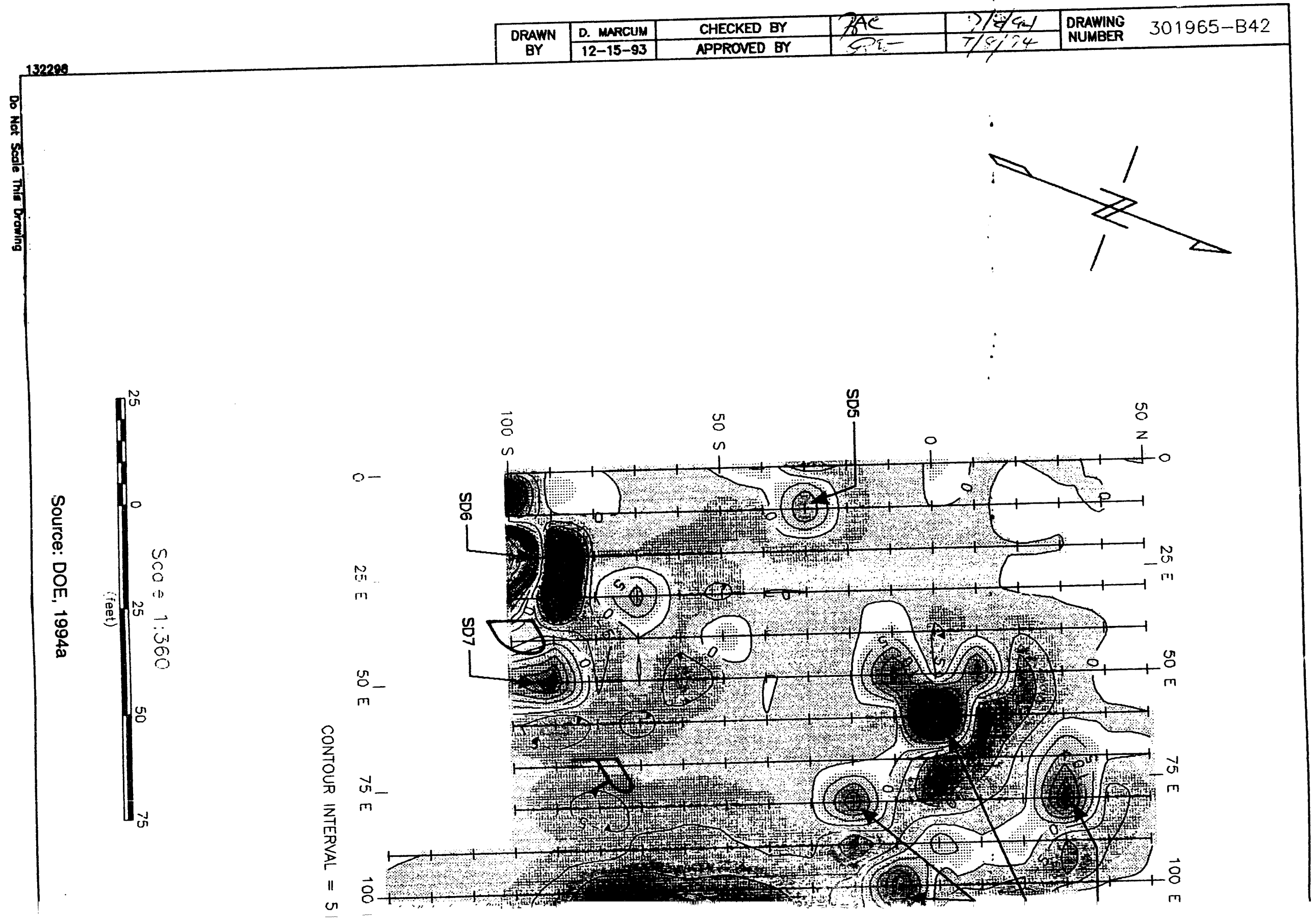




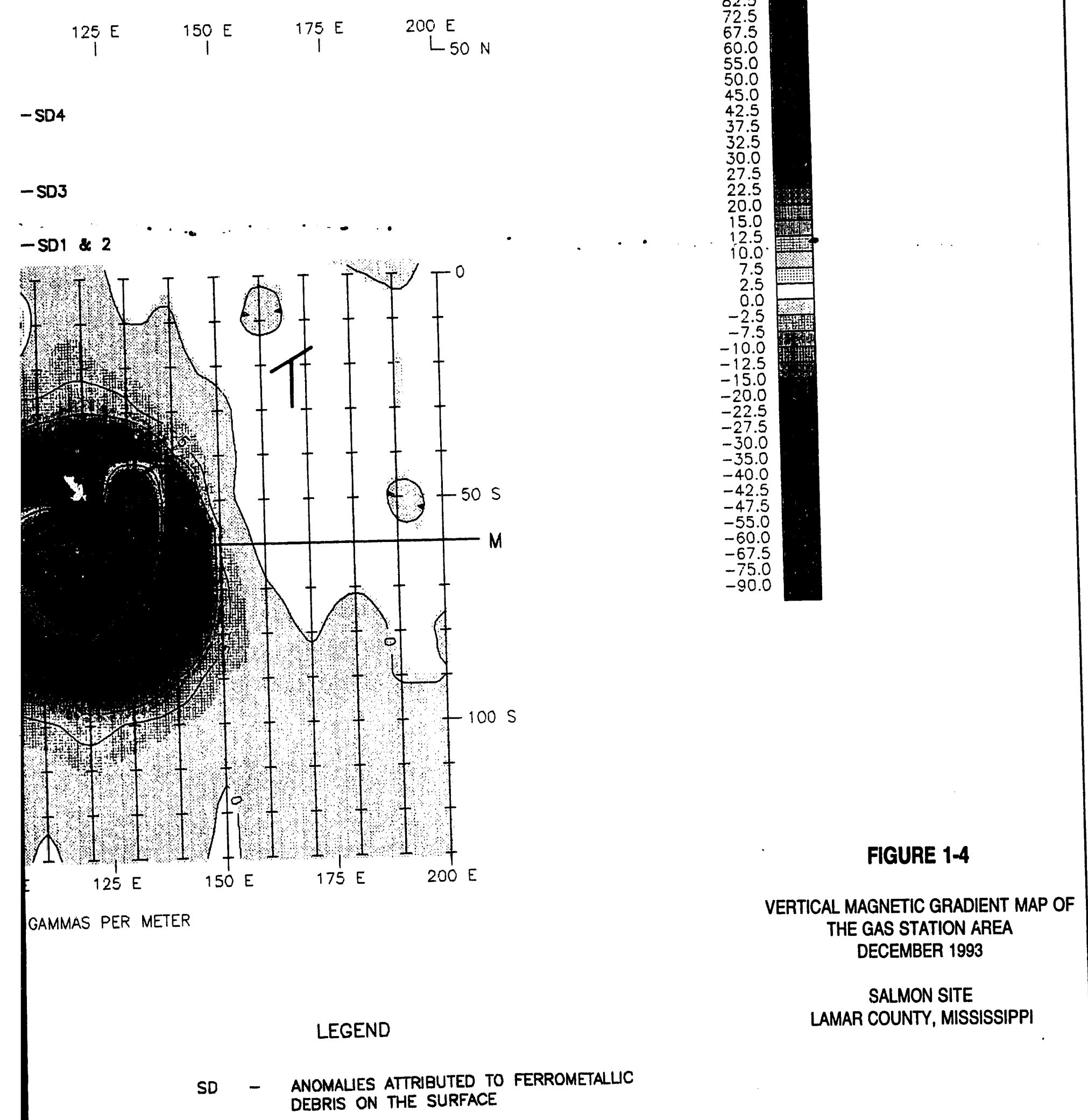




\subsection{Trenching}

All trenching activities were conducted in accordance with Field Operating Procedure 14, Test Pit/Trench Excavation and Sampling (DOE, 1992), the Salmon Site Test Pit Excavation Health and Safety Plan (DOE, 1993), and IT Policy HS307, Excavation and Trenching. Trench locations were identified using the survey grid established during the geophysical investigation. Prior to excavation, all access roads to the REECo pits area were blocked, the work area was surveyed by the site biologist for tortoise burrows, cleared of obstructions, and an exclusion zone was established. Level $\mathrm{C}$ personal protective equipment was required at the beginning of the job, this consisted of air-purifying respirators, poly-Tyvek jumpsuits; and double gloves in addition to the standard Level D requirements of safety glasses, hard hat, and hard toed shoes. After excavating the first three trenches, the site health and safety officer evaluated the real-time health and safety monitoring data and downgraded personal protective equipment requirements to Level D. At the onset of excavation for each trench, and at regular intervals thereafter, the health and safety technician surveyed the hole for organic vapors, gross radiological contamination, and noise levels. None of these parameters exceeded the predetermined health and safety limits.

Except as noted, trenches were approximately 60 centimeters $(\mathrm{cm})$ [24 inches (in.)] wide, up to 18.3 meters $(\mathrm{m})$ [60 feet $(\mathrm{ft})]$ long and ranged in depth from $1.8 \mathrm{~m}$ to $4.6 \mathrm{~m}(6 \mathrm{ft}$ to $15 \mathrm{ft}$ ). Excavation was controlled by the site supervisor, who maintained visual contact with the backhoe operator. Excavated soil was placed on visquine.

After completion of a trench, the backhoe bucket, shovels, and other equipment that came into contact with $\mathrm{e}^{-}$- avated soil were decontaminated using a high-pressure steam cleaner to wash soil back intc . trench. The decontaminated equipment was then surveyed for gross contamination. The spoils pile was covered with visquine and the trench was fenced with construction fencing as an additional safety measure and to prevent entry by gopher tortoises or small mammals.

Following the completion of sampling, the test pits were closed by backfilling the trenches with the excavated soil and grading the surface. 


\subsection{Sampling}

During excavation of the anomalies, one to three soil samples were collected from each trench. The intent was to collect a soil sample from immediately below the material that caused the anomaly or from obviously stained areas in the trenches. Thirty-three samples were collected and analyzed for volatile organic compounds (VOC), base-neutral-aidid (BNA) extractable compounds (semivolatile organic compounds), gross alpha $(\alpha)$ and beta $(\beta)$ radiaticn, gamma spectroscopy $(\gamma)$, metals $(\mathrm{MET})$, and cyanide $(\mathrm{CN})$. Additional analytes were added either because of what the trench was excavated to determine or because of what was found once the trench was excavated. Trenches 18 and 19 were conducted to try and locate the source of the tritium $\left({ }^{3} \mathrm{H}\right)$ found in the stream below Disposal Area 5 , trench 2 uncovered a burn pit so the four samples from that trench were also analyzed for dioxin/furan (DIOX), and 26 of the trenches uncovered diesel based drilling mud. so they were analyzed for total petroleum hydrocarbons (TPA). The thirty-three soil samples were collected and shipped to the ITAS laboratory in St. Louis for analysis. A list of the samples and the requested analyses is provided in Table 3-1.

Sample numbers follow the general form TRX-ES-XXXN-YYYE-ZZZ, where:

- TRX is the trench number

- ES is the excavated soil

- XXXN is the geophysical grid coordinate north

- YYYE is the geophysical grid coordinate east

- $\mathrm{ZZZ}$ is the depth, in $\mathrm{ft}$, of sample below the surface.

After the first six samples were collected, the sample identification was simplified by dropping the "N" in "XXXN," the "E" in "YYYE," and two "Zs" in "ZZZ." It is understood that the northing is stated first, followed by the easting and the depth. Sample identification numbers with "MS," "MSD," or "Dup" at the end are quality control samples. Two samples, one each from Trenches 18 and 19, are exceptions to this format. Because surface conditions prevented the actual excavation of the trenches, samples TR18-BS-90-150A-E and TR19-BS0-150A-E were composited from five auger holes at each site.

Several samples were mislabeled as to the geophysical survey grid coordinates. In order to prevent confusion, the sample numbers were not changed, however, the actual collection coordinates are provided in Appendix A. 


\section{Table 3-1}

\section{Samples Collected from Test Pit Excavations December 8 to 17, 1993}

\begin{tabular}{|c|c|c|c|}
\hline Trench & Date & Sample & Analysis \\
\hline TA1 & $12 / 8 / 93$ & TR1-ES-001 & VOC $^{a}, \mathrm{BNA}^{\mathrm{b}}, \alpha^{c}, \beta^{d}, \gamma^{\rho}, \mathrm{MET}^{\prime}, \mathrm{CN}^{\mathrm{g}}$ \\
\hline TR2 & $12 / 9 / 93$ & TR2-ES-355N-175E-5Z & VOC, BNA, $\alpha, \beta, \gamma$, MET, CN, DIOX \\
\hline TR2 & $12 / 9 / 93$ & TA2-ES-356N-175E-8Z & VOC, BNA, $\alpha, \beta, \gamma$, MET, CN, DIOX \\
\hline TR2A & $12 / 9 / 93$ & TR2A-ES-365N-200E-3.5Z & VOC, BNA, $\alpha, \beta, \gamma$, MET, CN, DIOX \\
\hline TR2A & $12 / 9 / 93$ & TR2A-ES-360N-200E-8Z & VOC, BNA, $\alpha, \beta, \gamma$, MET, CN, DIOX \\
\hline TR3 & $12 / 9 / 93$ & TR3-ES-450N-400E-3Z" & VOC. BNA, TPH', MET, $\alpha, \beta, \gamma, C N$ \\
\hline This. & $12 / 9 / 93$ & TR3-ES-430N-400E-15Z* & VOC, BNA, TPH, MET, $\alpha, \beta, \gamma, \mathrm{CN}$ \\
\hline TR: & $12 / 10 / 93$ & TR4-ES-2:60-490-8 & VOC, BNA, 'TPH, MET, $\alpha, \beta, \gamma, \mathrm{CN}$ \\
\hline 蚆 & $12 / 13 / 93$ & TR5-ES.590-570-12 & VOC. BNA, TPH, MET, $\alpha, \beta, \gamma, \mathrm{CN}$ \\
\hline TR6 & $12 / 14 / 93$ & TR6-ES-495-585-12* & VOC, BNA, TPH, MET, $\alpha, \beta, \gamma, C N$ \\
\hline TR6 & $12 / 14 / 93$ & TR6-ES-485-590-6* & VOC, BNA. TPH, MET, $\alpha, \beta, \gamma, \mathrm{CN}$ \\
\hline TR7 & $12 / 13 / 93$ & TR7-ES-558-650-5 & VOC. BNA, TPH, MET, $\alpha, \beta, \gamma, C N$ \\
\hline TR7 & $12 / 13 / 93$ & TR7-ES-560-650-14 & VOC, BNA,TPH, MET, $\alpha, \beta, \gamma, C N$ \\
\hline TR8 & $12 / 11 / 93$ & TR8-ES-780-600-3 & VOC, BNA, TPH, MET, $\alpha, \beta, \gamma$, CN \\
\hline TR8 & $12 / 13 / 93$ & TA8-ES-780-600-3-MS & VOC, BNA, TPH, MET, $\alpha, \beta, \gamma$, CN \\
\hline TR8 & $12 / 13 / 93$ & TR8-ES-780-600-3-MSD & VOC, BNA, TPH, MET, $\alpha, \beta, \gamma, \mathrm{CN}$ \\
\hline TR8 & $12 / 13 / 93$ & TR8-ES-780-600-3-DUP & VOC, BNA, TPH, MET, $\alpha, \beta, \gamma, \mathrm{CN}$ \\
\hline TR10 & $12 / 10 / 93$ & TR10-ES-180-790-15 & VOC, BNA, TPH, MET, $\alpha, \beta, \gamma, \mathrm{CN}$ \\
\hline TR10 & $12 / 10 / 93$ & TR10-ES-190-790-8 & VOC. BNA. TPH, MET, $\alpha, \beta, \gamma, \mathrm{CN}$ \\
\hline TR11 & $12 / 15 / 93$ & TR11-ES-180-930-3 & VOC. BNA. TPH, MET, $\alpha, \beta, \gamma, \mathrm{CN}$ \\
\hline TR13 & $12 / 14 / 93$ & TR13-ES-210-1110-6 & VOC, BNA, TPH, MET, $\alpha, \beta, \gamma$, CN \\
\hline TR13 & $12 / 14 / 93$ & TR13-ES-212-1150-6 & VOC, BNA, TPH, MET, $\alpha, \beta, \gamma, \mathrm{CN}$ \\
\hline TR14 & $12 / 14 / 93$ & TR14-ES-80-1189-5 & VOC, BNA, TPH, MET, $\alpha, \beta, \gamma$, CN \\
\hline TR14 & $12 / 14 / 93$ & TR14-ES-90-1200-3 & VOC. BNA, TPH, MET, $\alpha, \beta, \gamma$, CN \\
\hline TR15 & $12 / 15 / 93$ & TR15-ES-20-1180-..5 & VOC, BNA, TPH, MET, $\alpha, \beta, \gamma, \mathrm{CN}$ \\
\hline TR15 & $12 / 15 / 93$ & TR15-ES-20-1180-.5-MS* & VOC, BNA, TPH, MET, $\alpha, \beta, \gamma, \mathrm{CN}$ \\
\hline TR15 & $12 / 15 / 93$ & TR15-ES-20-1180-.5-MSD* & VOC, BNA, TPH, MET, $\alpha, \beta, \gamma, \mathrm{CN}$ \\
\hline TR15 & $12 / 15 / 93$ & TR15-ES-20-1180-.5-DUP* & BNA, TPH, MET, $\alpha, \beta, \gamma$, CN \\
\hline TR16 & $12 / 15 / 93$ & TR16-ES-200-80-1.5 & VOC, BNA, TPH, MET, $\alpha, \beta, \gamma, C N$ \\
\hline TR17 & $12 / 16 / 93$ & TR17-ES-30-120-5 & VOC, BNA, TPH, MET, $\alpha, \beta, \gamma$, CN \\
\hline TR17 & $12 / 16 / 93$ & TR17-ES-30-125-8 & VOC, BNA, TPH, MET, $\alpha, \beta, \gamma, \mathrm{CN}$ \\
\hline TR18 & $12 / 17 / 93$ & TR18-BS-90-150A-E & VOC, BNA, MET, $\alpha, \beta, \gamma, C N,{ }^{3} H$ \\
\hline TR19 & $12 / 17 / 93$ & TR19-BS-0-150A-E* & VOC, BNA, TPH, MET, $\alpha, \beta, \gamma, C N,{ }^{3} H$ \\
\hline
\end{tabular}

"See Appendix A for notes regarding sample identfication.

- Volatile organic compounds

- Base-neutral/acid extractable compounds

c Gross alpha radiation

- Beta radiation

- Gamma spectroscopy

\footnotetext{
'Metals

- Cyanide

"Dioxinfturan compounds

' Total petroleum hydrocarbons

1 Tritium
} 


\subsection{Results}

The sections below describe the excavation and sampling activities and the results of the excavation activities. Analytical results from the soi: sampling are discussed in Section 5.0 and the results are summarized in Table 5-2. Sample collection logs are included in Appendix K.

\subsection{Trench 1}

Trench 1 was excavated in a north-south direction across the west end of a large, linear anomaly on tive west side of the REECQ pits grid (Figures 1-2 and 4-1): The excavated trench was approxisuately $18.3 \mathrm{~m}(60 \mathrm{ft})$ long and $3.6 \mathrm{~m}(12 \mathrm{ft})$ deep at its deepest point. Portions of field fencing, barbed wire, and fence posts were encountered at a depth of approximately $1 \mathrm{~m}(3 \mathrm{ft})$ at grid location 330 north $(\mathrm{N}), 10$ east $(\mathrm{E})$. Excavation continued to the south, where additional fencing materials, cinder blocks, and steel cable were unearthed. The bulk of the cable was braided and unsheathed, although several lengths of sheathed electrical-type cable were also unearthed. Sample TR1-ES-001 was collected from a depth of 1 to $1.2 \mathrm{~m}$ ( 3 to $4 \mathrm{ft}$ ), immediately below the main mass of cable. This sample was analyzed for VOC, BNA, MET, CN, $\alpha, \beta$, and $\gamma$.

\subsection{Trench 2}

Trench 2 was excavated in a north-south direction across the eastern portion of the same anomaly as Trench 1 (Figure 4-1). Trench excavation was from north to south. The trench was approximately $15.2 \mathrm{~m}(50 \mathrm{ft})$ long and $3 \mathrm{~m}(10 \mathrm{ft})$ deep. Immediately below the surface [less than $0.3 \mathrm{~m}(1 \mathrm{ft})$ ], small pieces of wire, sheet metal, and fencing were encountered. Discolored soil was encountered at a depth of approximately $1.5 \mathrm{~m}(5 \mathrm{ft})$. This soil was dark brown to black, while native soil is red to red orange. Sample TR2-ES-355N-175E-5Z was collected at this location. As work continued, the excavated soil became more discolored and the litter included pieces of wire, broken glass, and assorted charred debris. The excavation revealed a buried burn pit approximately $7.6 \mathrm{~m}(25 \mathrm{ft})$ wide. The base of the pit ranges from

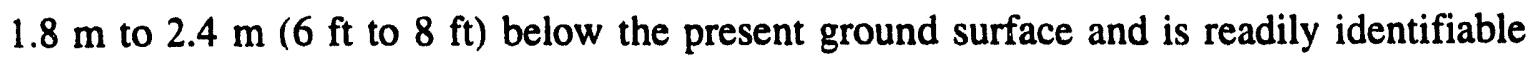
because of the layer of burned material. Sample TR2-ES-356N-175E-8Z was collected for analysis from the more discolored soils. Requested analyses were for VOC, BNA, MET, CN, $\alpha, \beta$, and $\gamma$. Due to the presence of burned cables and wire, additional analyses for DIOX were also requested. 


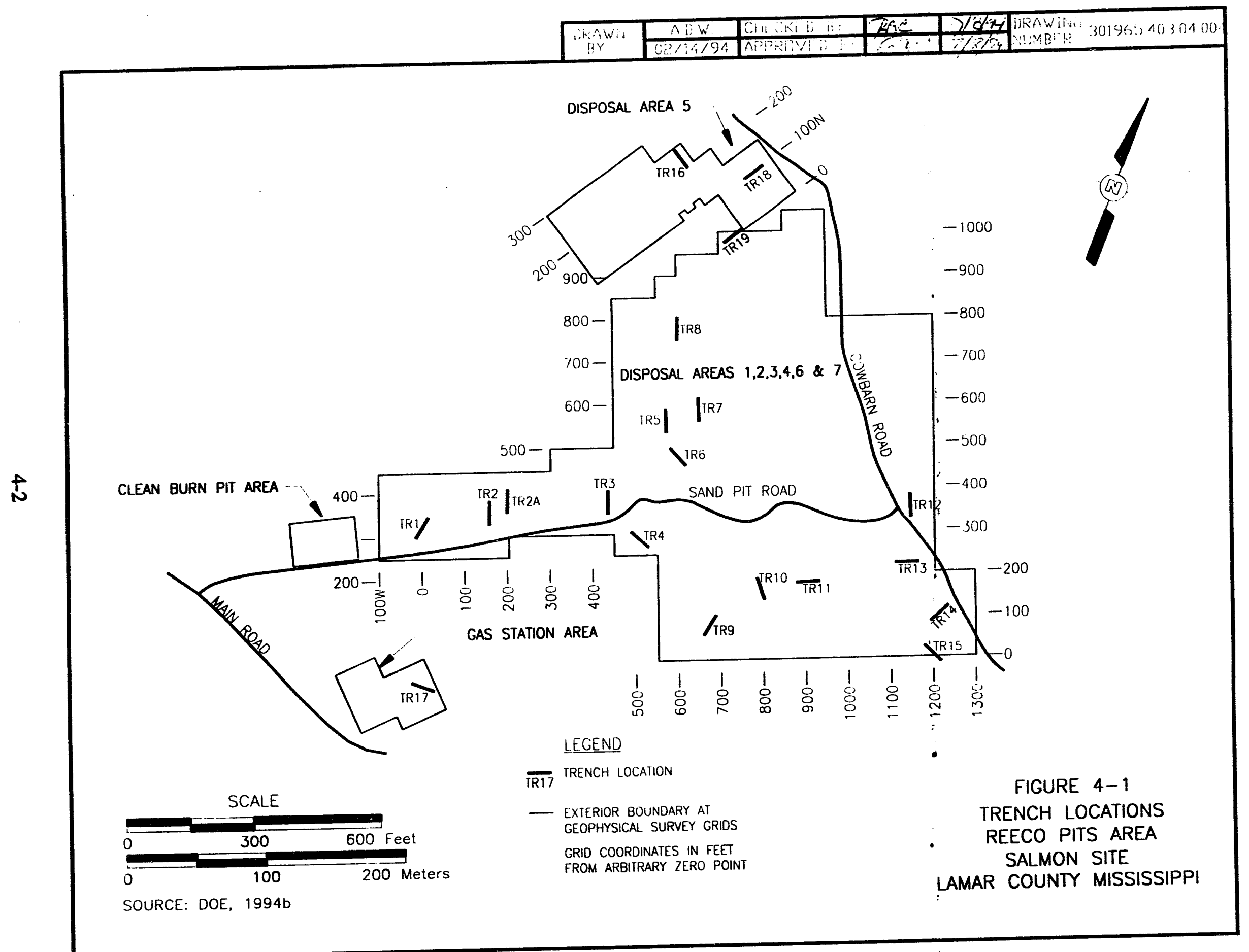




\subsection{Trench $2 A$}

Trench 2A was excavated to the east of, and parallel to. Trench 2 in an effort to ascertain the lateral extent of the burn pit (Figure 4-1). Trench $2 \mathrm{~A}$ was approximately $12.2 \mathrm{~m}(40 \mathrm{ft}$ ) long and $3 \mathrm{~m}(10 \mathrm{ft})$ deep. Discolored soil was first found at a depth of approximately $1 \mathrm{~m}(3 \mathrm{ft})$ below ground surface (bgs). The base of the burn pit in this trench was identified at approximately $1.8 \mathrm{~m}(6 \mathrm{ft}) \mathrm{bgs}$ by strongly discolored soil and a layer of small pieces of wire, broken glass, and metallic material. Two samples, TR2A-ES-365N-200E-3.5Z and TR2A$360 \mathrm{~N}-200 \mathrm{E}-8 \mathrm{Z}$, were collected from this trench. Requested analyses were the same as those requested for Trench 2 .

\subsection{Trench 3}

Trench 3 was excavated in a north-south direction across an anomaly on the west side of the REECo pits grid (Figure 4-1). The trench was approximately $12.2 \mathrm{~m} \mathrm{(40} \mathrm{ft)} \mathrm{long} \mathrm{and} 4.6 \mathrm{~m}$

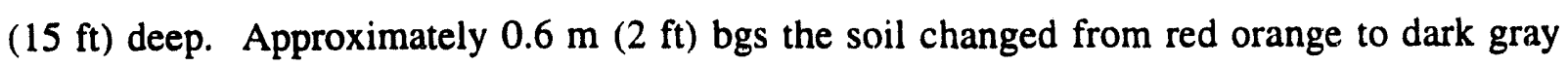
to black, and had a strong diesel odor. Excavated material had a submetallic luster when wet and there was a dark-black fibrous material, presumed to be lignite, interspersed throughout. The ground was saturated and the walls sloughed nearly as fast as the trench could be excavated. The condition of the trench prevented certain identification of the base of the

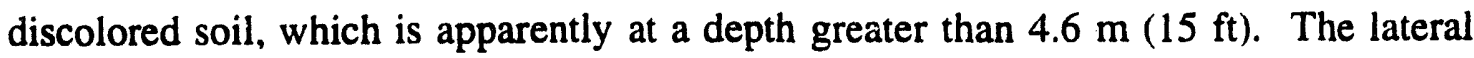
discoloration appears to continue north beyond grid location $400 \mathrm{~N}$ and end at approximately $360 \mathrm{~N}$ at its south end. The excavated discolored material is presumed to be drilling mud. Although several pieces of cement block were uncovered, no readily identifiable metallic objects vere uncovered that could account for the magnetic gradient anomaly. Samples TR3ES-450N-400E-3Z and TR3-ES-430N-400E-15Z were collected from this trench. Requested analyses included VOC, BNA, TPH, MET, CN, $\alpha, \beta$, and $\gamma$.

\subsection{Trench 4}

Trench 4 was excavated in a southeast-northwest direction immediately to the south of Sand Pit Road along the western edge of a large portion of the geophysical survey grid

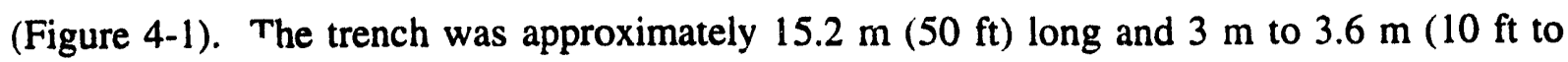

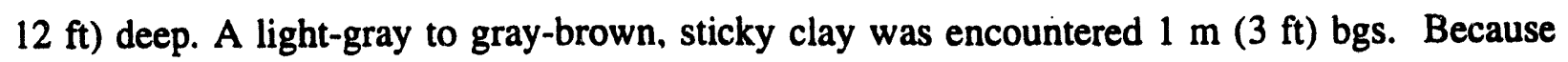
of a local high water table, water flowed into the excavation almost immediately upon removal of the second or third backhoe bucket. As excavation continued, the soil became blacker and more unstable. The material excavated at this trench also appeared to be drilling 
mud. Braided cable was encountered at a depth of $2.4 \mathrm{~m}(8 \mathrm{ft})$ near the northwest end of the trench; Sample TR4-ES-260N-490E-8Z was taken from this depth. The analyses requested for Trench 3 were repeated for this trench.

\subsection{Trench 5}

Trench 5 was excavated in a north-south direction in the central portion of the REECo pits

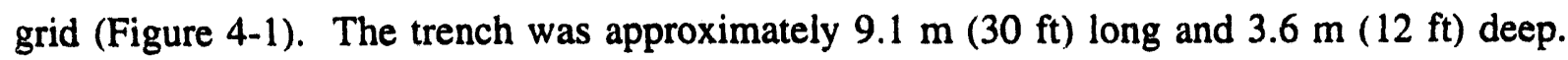

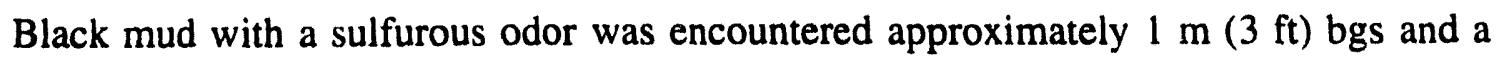
wooden fence post was encountered at $1.5 \mathrm{~m}(5 \mathrm{ft})$. A short section of braided cable found immediately below the ground surface toward the south end of the trench is presumed to have caused the anomaly. Sample TR5-ES-590-570-12 was collected at the base of the discolored soil. Requested analyses were the same as those for Trench 3.

\subsection{Trench 6}

Trench 6 was excavated in a southeasterly direction beginning at a point approximately $21.3 \mathrm{~m} \mathrm{(70} \mathrm{ft)} \mathrm{southeast} \mathrm{of} \mathrm{the} \mathrm{south} \mathrm{end} \mathrm{of} \mathrm{Trench} 5$ (Figure 4-1). The total length of the trench was approximately $15.2 \mathrm{~m}(50 \mathrm{ft})$ and $3.6 \mathrm{~m}$ to $4.6 \mathrm{~m}(12 \mathrm{ft}$ to $15 \mathrm{ft})$ deep. At a depth

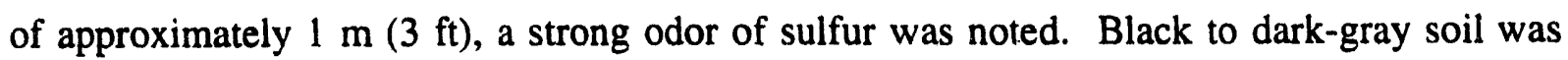
excavated, along with abundant lignite. Sample TR6-ES-495-585-12 was collected $3.6 \mathrm{~m}$ (12 ft) from the bottom of the discolored zone. A gray, unsolidified, concrete-like material was excavated and sampled (TR6-ES-485-590-6). Requested analyses were the same as those for Trench 3.

Because no metallic material was encountered, concern was expressed that a ferric component to the drilling mud had possibly caused the anomaly. Because no magnetic anomaly was indicated to the east of Trench 6, a test hole was dug to determine whether the drilling mud had caused the anomaly; if so, none should be encountered at the "step out" (designated 6A in the field notes and not shown on Figure 4-1) from Trench 6. Drilling mud was encountered

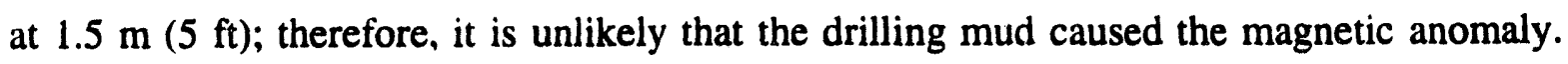
No sample was collected from Trench $6 \mathrm{~A}$.

\subsection{Trench 7}

Trench 7 was excavated in a north-south direction in the central portion of the REECo pits area (Figure 4-1). The total length of the trench was approximately $9.1 \mathrm{~m}$ long (30 ft) and 
$4.6 \mathrm{~m}$ ( $15 \mathrm{ft}$ ) deep. Drilling mud was encountered at a depth of approximately $1 \mathrm{~m}(3 \mathrm{ft})$ bgs. The ground at this location was saturated and the trench would not stay open. The excavated material had a "ropey" texture and a dull metallic luster. No metallic materials were encountered that could explain the geophysical anomaly. Two samples, TR7-ES-558-650-5 and TR7-ES-560-650-14, were collected from this trench. The second sample (TR7-ES-560650-14) was collected from the base of the presumed drilling mud. Both samples were analyzed for the same constituents as those requested for Trench 3.

\subsection{Trench 8}

Trench 8 was excavated in the northwest portion of the main disposal area (Figure 4-1). The trench trended north to south and was approximately $15.2 \mathrm{~m}(50 \mathrm{ft})$ long and $3.6 \mathrm{~m} \mathrm{(12} \mathrm{ft)}$ deep. Digging was difficult because soil in the area was apparently undisturbed. A piece of 3/4-in. braided cable was located approximately $8 \mathrm{~cm}$ (3 in.) below the surface. Samples TR8-ES-780-600-3, TR8-ES-780-600-3-MS, and TR8-ES-780-600-3-MSD, TR8-ES-780-6003-DUP were collected from this location and analyzed for the same constituents as those requested for Trench 3 .

\subsection{Trench 9}

Trench 9 was not excavated. The magnetic anomaly was explained after a reevaluation of the proposed location revealed the presence of a steel culvert.

\subsection{Trench 10}

Trench 10 was located in the south-central part of the main REECo disposal pits area (Figure 4-1) and was excavated in a northwest to southeast direction. The trench was approximately $18.3 \mathrm{~m}(60 \mathrm{ft})$ long and $4.6 \mathrm{~m}(15 \mathrm{ft})$ deep. Drilling mud was encountered

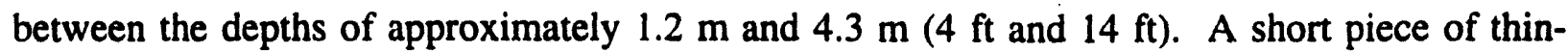
walled steel pipe, approximately $5 \mathrm{~cm}$ ( 2 in.) in diameter, was uncovered at the north end of the trench at a depth of $1.9 \mathrm{~m}$ (6 ft). Sample TR10-ES-180-790-15 was collected from below the drilling mud. A second sample, TR10-ES-190-790-8, which was taken from mid-depth, appeared to be 100 percent drilling mud. Requested analyses were the same as those requested for Trench 3.

\subsection{Trench 11}

Trench 11 was excavated in a west-east direction in the southern portion of the burial pits (Figure 4-1). The trench was approximately $12.2 \mathrm{~m} \mathrm{(40} \mathrm{ft)} \mathrm{long} \mathrm{and} 3.6 \mathrm{~m} \mathrm{(12} \mathrm{ft)} \mathrm{deep.} \mathrm{This}$ 
trench uncovered several wooden fence posts, short pieces of field fencing, drilling mud, and a small piece of 5/8-in. braided cable. Trench 11 appeared to cross the eastern edge of a mud disposal pit. Sample TR11-ES-180-930-3 was collected and sent to the laboratory for the same analyses as those requestid for Trench 3.

\subsection{Trench 12}

Trench 12 was not excavated because of its proximity to a tortoise burrow.

\subsection{Trench 13}

Treñch 13 was' excavated on the east edge of the burial pits; west of Cowbarn Road

(Figure 4-1). The west end of the trench began at grid line 1,110E and was excavated in an easterly direction. At a depth of approximately $0.6 \mathrm{~m}(2 \mathrm{ft}) \mathrm{bgs}$, a hard, fine-grained, gray, cement-like layer was encountered. Immediately below this layer, black soil and lignite with a strong sulfur odor were encountered. Sample TR13-ES-210-1110-6 was collected at this point. Additional gray material was unearthed as trench excavation continued to the east. The trench was extended approximately $15.2 \mathrm{~m}(50 \mathrm{ft})$ and widened to approximately $6.1 \mathrm{~m}$ $(20 \mathrm{ft})$ because insufficient metallic materials were encountered to explain the anomaly. $\mathrm{A}$ small cable, a large coiled spring, and the head of a grease gun were uncovered. A second sample (TR13-ES-212-1150-6) was collected. Both samples were analyzed for the same constituents as those requested for Trench 3.

\subsection{Trench 14}

Trench 14 was excavated in the extreme southeastern corner of the REECo pits area southwest of Cowbarn Road (Figure 4-1). The trench trended northeast to southwest and was

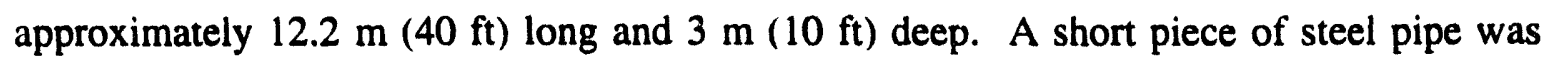
uncovered at approximately $0.6 \mathrm{~m}(2 \mathrm{ft})$, and drilling mud was encountered at a depth of

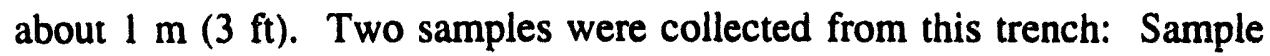
TR14-ES-90-1200-3 from beneath the pipe toward the southwest end of the trench and Sample TR14-ES-80-1189-5 from mid-depth at the southwestern end of the trench. The second sample contained more clay and sand and was much redder than previous samples. Both samples were analyzed for the same constituents as those requested for Trench 3.

\subsection{Trench 15}

Trench 15 was excavated in the extreme southeastern end of the REECo disposal pits (Figure 4-1). The trench trended northwest to southeast. The total length of the trench was 


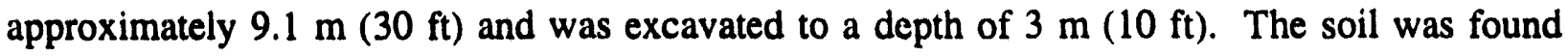
to be hard, red, and stratified and, therefore, presumed undisturbec. A short piece of cable was unearthed at a depth of $.15 \mathrm{~m}$ (6 in.) bgs, and the following samples were collected from immediately below the cable: TR15-ES-20-1180-.5, TR15-ES-20-1180-.5-MS, TR15-ES-201180-.5-MSD, and TR15-ES-20-1180-.5-DUP. Requested analyses were the same as those requested for Trench 3 .

\subsection{Trench 16}

Trench 16 was excavated on the small geophysical grid in Disposal Area 5 to the north of the main grid (Figure 4-1). A north-south trench was excavated to intersect a strong east-west trending anomaly. The trench was approximately $9.1 \mathrm{~m}(30 \mathrm{ft})$ long and $1.5 \mathrm{~m}(5 \mathrm{ft})$ deep. A large section of steel pipe [less than $30 \mathrm{~cm}$ (12 in.) in diameter] was uncovered and sample TR16-ES-200-80-1.5 was collected. No attempt was made to establish the lateral extent of the pipe. Requested analyses were the same as those requested for Trench 3.

\subsection{Trench 17}

Trench 17 was located in the "Gas Pump" area southwest of the main grid (Figure 4-1) and north of the main road. The strong anomaly for this trench was thought to indicate an underground storage tank (Figure 1-3). A trench was excavated adjacent to the suspected tank location to collect samples and information with minimal disturbance to the tank. A

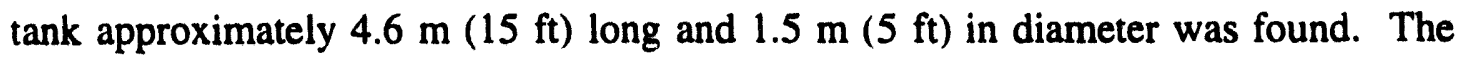
excavation revealed that a large hole had been cut in its top and it was full of liquid and sediment. Stacks of landing mats were unearthed alongside the tank. Sample TR17-ES-30120-5 was taken from the ground beside the tank and Sample TR17-ES-30-125-8 from the bottom of the trench at a depth of $0.6 \mathrm{~m}(2 \mathrm{ft})$ below the base of the tank. No sample was collected from the tank contents because the tank appeared to be filled with coarse gravel. Both samples were analyzed for the same constituents as those requested for Trench 3 .

\subsection{Trenches 18 and 19}

Magnetic anomalies were not identified at either Trench 18 or Trench 19, but both were located at strategic points on the small grid to the north of the main disposal area (Figure 4-1). Trench 18 was installed at the base of an outcrop of the Citronelle Formation and Trench 19 was placed south of the headward erosion of the main drainage through the REECo pits area. The trenches were planned to attempt to locate the source of elevated 
tritium levels found during U.S. Environmental Protection Agency sampling (Trench 18) and to check for the presence of drilling mud (Trench 19). However, the soft ground surface prevented access by a backhoe so five shallow auger holes were drilled on approximately 3-m $(10-\mathrm{ft})$ centers at each location. Each hole was $1.2 \mathrm{~m}$ to $1.5 \mathrm{~m}(4 \mathrm{ft}$ to $5 \mathrm{ft})$ deep and the last $30 \mathrm{~cm}$ (12 in.) of each hole was collected. The five samples from each trench were then composited to make samples TR18-BS-90-150A-E and TR19-BS-40A-E. Requested analyses were the same as those requested for Trench 3 with the addition of ${ }^{3} \mathrm{H}$. 


\subsection{Analytical Results}

Results of analyses from the trench soil samples indicate the presence of chemical, metal, dioxin/furans, and radiological constituents at levels that exceed the contract required detection limit (CRDL). These results are summarized in Table 5-1. The results for each sample are detailed in Appendices $\mathrm{C}$ through $\mathrm{J}$. One sample delivery group, consisting of 14 samples (42\%), was validated. Analytical results that exceeded the CRDL or the minimum detectable activity are highlighted by shading in the appendices. The requested analytical protocols and the methods used were:

- Semivolatile

- Volatile

- Dioxin

- Metals

- Cyanide

- TPH

- Alpha/beta

- Gamma scan

- Tritium
Contract Laboratory Program (CLP) 88 CLP 88

U.S. Environmental Protection Agency (EPA) 8280

CLP 90

CLP 90

EPA 418.1

Standard Method (SM) 7110

Health and Safety Laboratory (HASL) 300.0

Eastern Environmental Radiation Facilities (EERF) H.01

Findings included the detection of chromium and lead concentrations above the modified maximum contaminate concentration (MMCC). The metal concentrations in the analyzed samples were compared to requirements in Title 40. Code of Federal Regulations (CFR), Part 261.26 (DOE, 1990) toxicity characteristics for classifying hazardous wastes. The CFR specifies the maximum contaminate concentration (MCC) in milligrams per liter. Because a leachate is analyzed and not the soil sample, the results are not directly comparable. The toxicity characteristic leaching procedure (TCLP) specifies that $2,000 \mathrm{~m} \ell$ of liquid are to be used to extract the metals from 100 grams of soil. This leachate is then analyzed for the metal concentration. If 100 percent of the metal leaches, the concentration in the leachate will be diluted by a factor of 20 compared to the original concentration in the soil. In order to compare the TCLP MCC to the soil values expressed in milligrams per kilogram (mg/kg), the MCC should be multiplied by 20 . This new value is then called the MMCC. Table 5-2 lists the metals, the MCC, and the MMCC.

TPH in excess of $100 \mathrm{mg} / \mathrm{kg}$ was detected in 10 of the samples. The majority of the chemical constituents identified, except the furans, appear to be related to the drilling mud. 
Table 5-1

\section{Summary of Analytical Results}

(Page 1 of 7 )

\begin{tabular}{|c|c|c|c|c|c|}
\hline $\begin{array}{l}\text { Trench } \\
\text { Number }\end{array}$ & $\begin{array}{l}\text { Number of } \\
\text { Samples }\end{array}$ & Metals & Organics & Radionuclides" & Dioxins/Furans \\
\hline 1 & 1 & $\begin{array}{l}\mathrm{Hg}, \mathrm{Al}, \mathrm{Gr}, \mathrm{Cu}, \mathrm{Fe}, \mathrm{Mn}, \mathrm{Zn}, \text { and } \mathrm{Pb} \\
\text { exceeded } \mathrm{CRDL}^{-} \text {in Sample TR-ES- } \\
001 .\end{array}$ & $\begin{array}{l}\text { Volatiles: No analytes exceeded the } \\
\text { CRDL. } \\
\text { Semivolatiles: No analytes exceeded } \\
\text { the CRDL. } \\
\frac{\mathrm{TPH}^{\mathrm{b}} \text { : (Total Petroleum hydrocarbons) }}{\mathrm{ND}^{\mathrm{c}}}\end{array}$ & $\begin{array}{l}\text { Gross alpha: The activity was } \\
\text { below the reference sample value. } \\
\text { Gross beta: The activity was below } \\
\text { the reference sample value. } \\
\text { Gamma scan: Pb-212 exceeded } \\
\text { the reference sample value. }\end{array}$ & No analysis done. \\
\hline 2 & 2 & $\begin{array}{l}\mathrm{As}, \mathrm{Hg}, \mathrm{Al}, \mathrm{Ba}, \mathrm{Cd}, \mathrm{Ca}, \mathrm{Cr}, \mathrm{Cu}, \mathrm{Fe}, \\
\text { Mn, } \mathrm{Mg}, \mathrm{Ni}, \mathrm{Ag}, \mathrm{Va}, \mathrm{Zn} \text {, and } \mathrm{Pb} \\
\text { exceeded the CRDL in both TR2-ES- } \\
355 \mathrm{~N}-175 \mathrm{E}-5 \mathrm{Z} \text { and TR2-ES-356N- } \\
\text { 175E-8Z. Co exceeded CRDL in } \\
\text { TR2-ES-356N-175E-8Z. }\end{array}$ & $\begin{array}{l}\text { Volatiles:- Toluene was indicated in } \\
\text { Sample TR2-ES-356N-175E-8Z. Two } \\
\text { unknown compounds were identified } \\
\text { in Sample TR2-ES-355N-175E-5Z and } \\
\text { four in Sample TR2-ES-356N-175E- } \\
8 Z \text {. } \\
\text { Semivolatiles: There were no } \\
\text { detections for either of the samples. } \\
\text { TPH: ND }\end{array}$ & $\begin{array}{l}\text { Gross alpha: The activities were } \\
\text { below the reference sample value. } \\
\text { Gross beta: Activities were slightly } \\
\text { above the reference sample value. } \\
\text { Gamma scan: Pb-212 and Ra-226 } \\
\text { exceeded the values for the } \\
\text { reference sample in both samples } \\
\text { and Cs-137 in TR2A-ES-356-175E- } \\
8 Z \text {. }\end{array}$ & $\begin{array}{l}\text { No dioxins or furans } \\
\text { were detected i Sample } \\
\text { TR2-ES-355N-175E-5Z. } \\
\text { TCDF, HxCDF', and } \\
\text { HpCDF' were detected } \\
\text { above CADL in TR2-ES- } \\
\text { 356N-175E-8Z. }\end{array}$ \\
\hline $2 A$ & 2 & $\begin{array}{l}\mathrm{Cr} \text { and } \mathrm{Pb} \text { exceeded the } \mathrm{MCC}^{\circ} \text { in } \\
\text { Sample TR2A-ES-360N-200E-3Z. Cr } \\
\text { and Pb were detected above the } \\
\text { CRDL in TR2A-Es-360N-200E-8Z. }\end{array}$ & $\begin{array}{l}\text { Volatiles: Sample TR2A-ES-360N- } \\
200 E-3 \text { had one unknown; TR2A-ES- } \\
360 N-200 E-8 Z \text { had toluene below the } \\
\text { CRDL and three unknown } \\
\text { compounds. } \\
\text { Semivolatiles: There were no } \\
\text { exceedances of the MCC or } \\
\text { detections above the CRDL. } \\
\text { TPH: ND }\end{array}$ & $\begin{array}{l}\text { Gross alpha: The activities were } \\
\text { slightly above the reference sample. } \\
\text { Gross beta: The activities for both } \\
\text { samples exceeded the reference } \\
\text { sample value. } \\
\text { Gamma scan: Cs-137 was } \\
\text { detected in sample } 3.5 \mathrm{Z} \text { at a level } \\
\text { above that of the reference sample. } \\
\text { Pb-212 and Ra-226 were detected } \\
\text { in both samples at levels above } \\
\text { those of the reference sample. }\end{array}$ & ND \\
\hline
\end{tabular}

See Footnotes at end of table. 
Table 5-1

Summary of Analytical Results

(Page 2 of 7 )

\begin{tabular}{|c|c|c|c|c|c|}
\hline $\begin{array}{l}\text { Trench } \\
\text { Number }\end{array}$ & $\begin{array}{l}\text { Number of } \\
\text { Samples }\end{array}$ & Metais & Organics & Radionuclides & Dioxins/Furans \\
\hline 3 & 2 & $\begin{array}{l}\text { There were no exceedances above } \\
\text { the MMCC' for either sample. }\end{array}$ & $\begin{array}{l}\text { Volatiles: There were no } \\
\text { exceedances of the CRDL. Toluene } \\
\text { and Xylene were detected below the } \\
\text { CRDL in Sample TR3-ES-430N-400E- } \\
\text { 15Z. } \\
\text { Semivolatiles: } \\
\text { 2-Methylnaphthalene and Bis } \\
\text { (2-Ethylhexyl)phthalate exceeded } \\
\text { the CRDL in Sample TR3-ES-450N- } \\
\text { 400E-3Z. } \\
\text { 2- Methylnaphthalene exceeded the } \\
\text { CRDL and Naphthalene was } \\
\text { detected below the CRDL in Sample } \\
\text { TR3-ES-430-400E-15Z. } \\
\text { TPH: Both samples had values that } \\
\text { exceeded the CRDL. }\end{array}$ & $\begin{array}{l}\text { Gross Alpha: The activity for one } \\
\text { sample was slightly below and one } \\
\text { slightly above the reference sample } \\
\text { value. } \\
\text { Gross beta: The activity for one } \\
\text { sample was slightly below and one } \\
\text { slightly above the reference sample } \\
\text { value. } \\
\text { Gamma scan: TI-208 was detected } \\
\text { at slightly elevated levels in both } \\
\text { samples. Pb-212 was detected at a } \\
\text { level above that of the reference } \\
\text { sample in TR3-ES-430N-400E-15Z. }\end{array}$ & No analysis done. \\
\hline 4 & 1 & $\begin{array}{l}\text { There were no exceedances above } \\
\text { the MMCC. }\end{array}$ & $\begin{array}{l}\text { Volatiles: Carbon Disulfide, } \\
\text { Toluene, and Xylene were detected } \\
\text { at below the CRDL in Sample TR4- } \\
\text { ES-260-490-8. } \\
\text { Semivolatiles: Fluorene exceeded } \\
\text { the CRDL. Naphthalene and Bis(2- } \\
\text { Ethylhexyl)phthalate were detected } \\
\text { below the CRDL. } \\
\text { TPH: Exceeded the CRDL. }\end{array}$ & $\begin{array}{l}\text { Gross alpha: The activities was } \\
\text { slightly above the reference sample } \\
\text { value. } \\
\text { Gross beta: Activities for this } \\
\text { sample were approximately twice } \\
\text { reference value. } \\
\text { Gamma scan: Tl-208 } \mathrm{Pb}-210, \mathrm{~Pb}- \\
212, \mathrm{Ra}-226, \text { and Th-234 were } \\
\text { detected at levels slightly above the } \\
\text { reference sample. }\end{array}$ & No analysis done. \\
\hline 5 & 1 & $\begin{array}{l}\text { There were no exceedances above } \\
\text { the MMCC. }\end{array}$ & $\begin{array}{l}\text { Volatiles: There were no } \\
\text { exceedances of the CRDL. } \\
\text { Semivolatiles: } \\
\text { 2-Wethylnaphthalene exceeded the } \\
\text { CRDL. Naphthalene and Fluorene } \\
\text { were detected but below CRDL. } \\
\text { TPH: Exceeded the CRDL. }\end{array}$ & $\begin{array}{l}\text { Gross alpha: Activities exceeded } \\
\text { that of the reference value. } \\
\text { Gross beta: Activities exceeded that } \\
\text { of the reference value. } \\
\text { Gamma scan: Pb212 exceeded the } \\
\text { values for the reference sample. }\end{array}$ & No analysis done. \\
\hline
\end{tabular}

See footnotes at end of table. 
Table 5-1

\section{Summary of Analytical Results \\ (Page 3 of 7)}

\begin{tabular}{|c|c|c|c|c|c|}
\hline $\begin{array}{l}\text { Trench } \\
\text { Number }\end{array}$ & $\begin{array}{c}\text { Number of } \\
\text { Samples }\end{array}$ & Metals & Organics & Radionuclides & Dioxins/Furans \\
\hline 6 & 2 & $\begin{array}{l}\text { There were no exceedances above } \\
\text { the MMCC for either sample. Cr was } \\
\text { above the CRDL in both samples. }\end{array}$ & $\begin{array}{l}\text { Volatiles: There were no exceedances } \\
\text { in either sample. Toluene was } \\
\text { detected at below the CRDL in } \\
\text { Sample TR6-ES-485-590-6. } \\
\text { Semivolatiles: Fluorene was detected } \\
\text { below the CADL in Sample TR6-ES- } \\
495-585-12 \text {. There were } 22 \\
\text { unknowns. No compounds exceeded } \\
\text { the CRDL in Sample TR6-ES-485- } \\
590-6 \text { but there were four unknowns. } \\
\text { TPH: Both samples exceeded the } \\
\text { CRDL. }\end{array}$ & $\begin{array}{l}\text { Gross alpha: Activity for both } \\
\text { samples exceeded the reference } \\
\text { sample value. } \\
\text { Gross beta: Activities for both } \\
\text { samples were below the reference } \\
\text { sample value. } \\
\text { Gamma scan: T1-208, Pb-212, Ra- } \\
\text { 226, Ra-228 were detected at levels } \\
\text { which exceeded the reference } \\
\text { sample value in TR6-ES-495-585- } \\
\text { 12. Pb-212 exceeded the reference } \\
\text { sample value in TR6-ES-485-590-6. }\end{array}$ & No analysis done. \\
\hline 7 & 2 & $\begin{array}{l}\text { There were no exceedances of the } \\
\text { MMCC for gither sample. As, Cr, and } \\
\text { Pb exceeded the CRDL. }\end{array}$ & $\begin{array}{l}\text { Volatiles: Xylenes were detected at } \\
\text { above the CRDL in Sample TR7-ES- } \\
560-650-14 \text {. Ethyl Benzene was } \\
\text { detected but at below the CRDL. } \\
\text { Semivolatiles: } \\
\text { 2-Methylnaphthalene exceeded the } \\
\text { CRDL in Sample TR7-ES-558-650-5. } \\
\text { Fluorene and Phenanthrene were } \\
\text { detected below the CRDL. Bis(2- } \\
\text { Ethylhexyl)phthalate exceeded the } \\
\text { CRDL in Sample TR7-ES-560-650-14. } \\
\text { TPH: Both samples exceeded the } \\
\text { CRDL. }\end{array}$ & $\begin{array}{l}\text { Gross alpha: Activities for both } \\
\text { samples exceeded the reference } \\
\text { sample value. } \\
\text { Gross beta: The activity for TR7- } \\
\text { ES-558-650-5 exceeded the } \\
\text { reference sample; activity for TR7- } \\
\text { ES-560-650-14 exceeded the } \\
\text { reference sample value by a factor } \\
\text { of three. } \\
\text { Gamma scan: T1-208, Pb-212, Ra- } \\
226 \text { were detected at levels that } \\
\text { exceeded the reference sample } \\
\text { value in both samples. }\end{array}$ & No analysis done. \\
\hline 8 & 1 & $\begin{array}{l}\text { There were no exceedances of the } \\
\mathrm{MMCC} \text { for either sample. } \mathrm{As}, \mathrm{Cr} \text {, and } \\
\mathrm{Pb} \text { exceeded the CRDL. }\end{array}$ & $\begin{array}{l}\text { Volatiles: There were no } \\
\text { exceedances of the CRDL. } \\
\text { Semivolatiles: There were no } \\
\text { exceedances of the CRDL. Benzoic } \\
\text { acid was detected below the CRDL. } \\
\text { TPH: Did not exceed the CRDL. }\end{array}$ & $\begin{array}{l}\text { Gross alpha: The activity for both } \\
\text { samples was approximately the } \\
\text { same as in the reference sample. } \\
\text { Gross beta: The activities for both } \\
\text { samples were approximately the } \\
\text { same as in the reference sample. } \\
\text { Gamma scan: Pb-212 exceeded } \\
\text { the reference sample value. }\end{array}$ & No analysis done. \\
\hline 9 & $0^{j}$ & & & & \\
\hline
\end{tabular}

\footnotetext{
See footnotes at end of table.
} 
Table 5-1

Summary of Analytical Results

(Page 4 of 7)

\begin{tabular}{|c|c|c|c|c|c|}
\hline $\begin{array}{l}\text { Trench } \\
\text { Number }\end{array}$ & $\begin{array}{c}\text { Number of } \\
\text { Samples }\end{array}$ & Metals & Organics & Radionuclides & Dioxins/Furnas \\
\hline 10 & 2 & $\begin{array}{l}\text { Cr exceeded the MMCC in Sample } \\
\text { TR10-ES-190-790-8. As, Cr, and Pb } \\
\text { exceeded the CRDL in both samples. }\end{array}$ & $\begin{array}{l}\text { Volatiles: Xylene was detected above } \\
\text { the CRDL in Sample TR10-ES-180- } \\
790-15 \text {. Both samples had numerous } \\
\text { unknowns. } \\
\text { Semivolatiles: There were no } \\
\text { compounds that exceeded the CRDL } \\
\text { in either sample. Sample TR10-ES- } \\
180-930-2 \text { had eight uniknowns. } \\
\text { TPH: Both samples exceeded the } \\
\text { CRDL. }\end{array}$ & $\begin{array}{l}\text { Gross alpha: The activities for both } \\
\text { samples were below the reference } \\
\text { sample value. } \\
\text { Gross beta: The activities for both } \\
\text { samples were below the reference } \\
\text { sample value. } \\
\text { Gamma scan: TI-208 and Pb-212 } \\
\text { exceeded the reference sample } \\
\text { value in TR10-ES-190-790-8. }\end{array}$ & No analysis done. \\
\hline 11 & 1 & $\begin{array}{l}\text { There were no exceedances of the } \\
\text { MMCC. Cr and Pb exceeded the } \\
\text { CRDL. }\end{array}$ & $\begin{array}{l}\text { Volatiles: There were no } \\
\text { exceedances of the CRDL. } \\
\text { Semivolatiles: There were no } \\
\text { compounds that exceeded the CRDL } \\
\text { in this sample. } \\
\text { TPH: Exceeded the CRDL. }\end{array}$ & $\begin{array}{l}\text { Gross alpha: The activity exceeded } \\
\text { the reference sample value. } \\
\text { Gross beta: The activity was below } \\
\text { the reference sample value. } \\
\text { Gamma scan: T1-208 and } \mathrm{Pb}-212 \\
\text { exceeded the values for the } \\
\text { reference samples. }\end{array}$ & No analysis done. \\
\hline 12 & $0^{1}$ & & & & \\
\hline
\end{tabular}

See footnotes at end of table. 
Table 5-1

Summary of Analytical Results

(Page 5 of 7)

\begin{tabular}{|c|c|c|c|c|c|}
\hline $\begin{array}{l}\text { Trench } \\
\text { Number }\end{array}$ & $\begin{array}{c}\text { Number of } \\
\text { Samples }\end{array}$ & Metals & Organics & Radionuclides & Dioxins/Furans \\
\hline 13 & 2 & $\begin{array}{l}\text { Cr exceeded the MMCC in Sample } \\
\text { TR13-ES-212-1150-6. Cr and Pb } \\
\text { were detected above the CRDL in } \\
\text { both samples. }\end{array}$ & $\begin{array}{l}\text { Volatiles } \\
\text { Tetrachloroethane was identified } \\
\text { above the CRDL in both samples. } \\
\text { Trichloroethane, 1,1,2,2,- } \\
\text { Tetrachloroethane, Ethyl Benzene } \\
\text { and Xylene were deteried in Sample } \\
\text { TR13-ES-212-1150-6. Toluene was } \\
\text { detected below the CRDL. } \\
\text { Semivolatiles: } \\
\text { 2-methylneghthalene was detected } \\
\text { at levels above the CRDL in both } \\
\text { samples. Naphthalene was detected } \\
\text { in both samples at below the CRDL. } \\
\text { Fluorene and Phenanthrene were } \\
\text { detected at below the CRDL in } \\
\text { Sample TR13-ES-212-1150-6. There } \\
\text { were } 22 \text { unknown or associated } \\
\text { compounds in this sample. } \\
\text { TPH: Both samples exceeded the } \\
\text { CRDL. }\end{array}$ & $\begin{array}{l}\text { Gross alpha: The activity for } \\
\text { sample TR13-ES-210-1110-6 was } \\
\text { below the reference sample value. } \\
\text { The activity for TR13-ES-212-1150- } \\
6 \text { exceeded the reference sample } \\
\text { value. } \\
\text { Gross beta: The activities for both } \\
\text { samples were below the reference } \\
\text { sample value. } \\
\text { Gamma scan: Pb-212 exceeded } \\
\text { the values for the reference } \\
\text { samples in both samples. }\end{array}$ & No analysis done. \\
\hline 14 & 2 & $\begin{array}{l}\text { There were no values in excess of the } \\
\text { MMCC for either sample. } \mathrm{Cr} \text { and } \mathrm{Pb} \\
\text { were detected above the CRDL in } \\
\text { both samples. }\end{array}$ & $\begin{array}{l}\text { Volatiles: There were no } \\
\text { exceedances of the CRDL in either } \\
\text { sample. } \\
\text { Semivolatiles: } \\
\text { 2-Methylnaphthalene was detected } \\
\text { but below CRDL. in Sample TR14-ES- } \\
80-1189-5 \text { along with } 12 \text { unknowns. } \\
\text { Bis(2-Ethylhexyl)phthalate was } \\
\text { detected below the CRDL in Sample } \\
\text { TR14-ES-90-1200-3. } \\
\text { TPH: Neither sample exceeded } \\
\text { CRDL. }\end{array}$ & $\begin{array}{l}\text { Gross alpha: The activity for } \\
\text { sample TR14-ES-80-1189-5 } \\
\text { exceeded the reference sample } \\
\text { value. Activity for sample TA14-ES- } \\
90-1200-3 \text { was below the value for } \\
\text { the reference sample. } \\
\text { Gross beta: The activity for sample } \\
\text { TR14-ES-80-1189-5 exceeded the } \\
\text { reference sample value. Activity for } \\
\text { sample TR14-ES-90-1200-3 was } \\
\text { below the reference sample value. } \\
\text { Gamma scan: Pb212 exceeded the } \\
\text { values for the reference sample. } \\
\text { TR14-ES-80-1189-5 was the only } \\
\text { sample to have a detection of } U^{35} \text {. }\end{array}$ & No analysis done. \\
\hline
\end{tabular}

See footnotes at end of table. 
Table 5-1

Summary of Analytical Results

(Page 6 of 7)

\begin{tabular}{|c|c|c|c|c|c|}
\hline $\begin{array}{l}\text { Trench } \\
\text { Number }\end{array}$ & $\begin{array}{c}\text { Number of } \\
\text { Samples }\end{array}$ & Metals & Organics & Radionuclides & Dioxinafurans \\
\hline$\overline{15}$ & $\overline{2}$ & $\begin{array}{l}\text { There were no values above the } \\
\text { MMCC for either sample. As, } \mathrm{Cr} \text {, and } \\
\text { Pb exceeded the CRDL for both } \\
\text { samples. }\end{array}$ & $\begin{array}{l}\text { Volatiles: There were no } \\
\text { exceedances of the CRDL in either } \\
\text { sample. } \\
\text { Semivolatiles: There were no } \\
\text { exceedances of the CRDL. There } \\
\text { were } 22 \text { unknowns. } \\
\text { TPH: Sample exceeded CRDL. }\end{array}$ & $\begin{array}{l}\text { Gross alpha: The activities for both } \\
\text { samples exceeded the reference } \\
\text { sample value. } \\
\text { Gross beta: The activities for both } \\
\text { samples were below the reference } \\
\text { sample value. } \\
\text { Gamma scan: Th-208, Ra-226 } \\
\text { were detected above the roference } \\
\text { sample values in TR15-ES-20- } \\
1180-.5 \text { and Pt-212 was detected } \\
\text { above the reference sample value in } \\
\text { both. }\end{array}$ & No analysis done. \\
\hline 16 & 1 & $\begin{array}{l}\text { There were no values that exceeded } \\
\text { the MMCC. CR and Pb exceeded } \\
\text { the CRDL. }\end{array}$ & $\begin{array}{l}\text { Volatiles: There were no } \\
\text { exceedances of the CRDL. } \\
\text { Semivolatiles: There were no } \\
\text { exceedances of the CRDL. } \\
\text { Phenanthrene, Fluoranthene, } \\
\text { Pyrene, and bis } \\
\text { (2-Ethylhexyl)phthalate were } \\
\text { detected below the CRDL. } \\
\text { TPH: Below CRDL. }\end{array}$ & $\begin{array}{l}\text { Gross alpha: The activity for this } \\
\text { sample exceeded the reference } \\
\text { sample value. } \\
\text { Gross beta: The activity for this } \\
\text { sample was below the reference } \\
\text { sample value. } \\
\text { Gamma scan: Pb-212 exceeded } \\
\text { the reference sample value. }\end{array}$ & No analysis done. \\
\hline 17 & 2 & $\begin{array}{l}\text { There were no exceedances of the } \\
\text { MMCC. As, Cr, and Pb exceeded the } \\
\text { CRDL. } \\
\text { The concentration of } \mathrm{Pb} \text { in Sample } \\
\text { TR17-ES-30-125-8 was the highest of } \\
\text { any sample. }\end{array}$ & $\begin{array}{l}\text { Volatiles: There were no } \\
\text { exceedances of the CRDL in either } \\
\text { sample. } \\
\text { Semivolatiles: There were no } \\
\text { exceedances of the CRDL in either } \\
\text { sample. Bis } \\
\text { (2-Ethylhexyl)phthalate was detected } \\
\text { below the CHDL in Sample TR17-ES- } \\
\text { 30-125-8. } \\
\text { TPH: Did not exceed the CRDL. }\end{array}$ & $\begin{array}{l}\text { Gross alpha: The activities for both } \\
\text { samples exceeded the reference } \\
\text { sample value. } \\
\text { Gross beta: The activity for sample } \\
\text { TR17-ES-30-120-5 was below the } \\
\text { reference sample and TR17-ES-30- } \\
125-8 \text { was above the reference } \\
\text { sample value. } \\
\text { Gamma scan: T1-208 and Pb-212 } \\
\text { exceeded the reference sample } \\
\text { values in both samples. Ra-226 } \\
\text { and Th-234 were detected above } \\
\text { the reference value in } \\
\text { TR17-ES-30-125-8. }\end{array}$ & No analysis done. \\
\hline
\end{tabular}


Table 5-1

\section{Summary of Analytical Results \\ (Page 7 of 7)}

\begin{tabular}{|c|c|c|c|c|c|}
\hline $\begin{array}{l}\text { Trench } \\
\text { Number }\end{array}$ & $\begin{array}{c}\text { Number of } \\
\text { Samples }\end{array}$ & Metals & Organics & Radionuclides & Dioxins/Furans \\
\hline 18 & $\overline{1}$ & $\begin{array}{l}\text { There were no exceedances of the } \\
\text { MMCC. Cr and } \mathrm{Pb} \text { exceeded the } \\
\text { CRDL. }\end{array}$ & $\begin{array}{l}\text { Volatiles: There were no } \\
\text { exceedances of the CRDL. } \\
\text { Semivolatiles: There were no } \\
\text { exceedances of the CRDL. } \\
\text { TPH: ND }\end{array}$ & $\begin{array}{l}\text { Gross alpha: Activities for sample } \\
\text { Th18-BS-90-150-A-E was above } \\
\text { the reference sample value. } \\
\text { Gross beta: Activities were below } \\
\text { the reference sample value. } \\
\text { Gamma scan: Th-208, Pb-210, Pb- } \\
\text { 212, and Ra-228 were detected } \\
\text { above the reference sample values. } \\
\text { Tritium: Activities were well below } \\
\text { the MDA for this sample. }\end{array}$ & No analysis done. \\
\hline 19 & 1 & $\begin{array}{l}\text { There were no exceedances of the } \\
\text { MMCC. As, Cr, and Pb exceeded the } \\
\text { CRDL. }\end{array}$ & $\begin{array}{l}\text { Volatiles: There were no } \\
\text { exceedances of the CRDL. } \\
\text { Semivolatiles: There were no } \\
\text { exceedances. } \\
\text { TPH: Exceeded the CRDL. }\end{array}$ & $\begin{array}{l}\text { Gross alpha: Activities for Sample } \\
\text { TR19-BS-0-150-A-E was above the } \\
\text { reference sample value. } \\
\text { Gross beta: Activities were below } \\
\text { the reference sample value. } \\
\text { Gamma scan: Pb-212 and Ra-226 } \\
\text { exceeded the reference sample } \\
\text { values. } \\
\text { Tritium: Activities were well below } \\
\text { the MDA for this sample. }\end{array}$ & No analysis done. \\
\hline
\end{tabular}

- CRDL Contract required detection limits

- Note: Reference sample REFC-19-SS collected March 1993 (DOE, 1994a)

- TPH Total petroleum hydrocarbons

- ND Not detected

- TCDF Terrachlorodibenzofuran

- HXCDF Hexachlorodibenzofuran

HPCDF Heptachlorocibenzoluran

- MCC Maxirrum contaminate concentration

"MMCC Modified maximum contaminant concentration

' Trench was not excavated - blank cells indicate no data

$\begin{array}{lll}\mathrm{Ag}=\text { silver } & \mathrm{Cr}=\text { chronium } & \mathrm{Pb}=\text { lead } \\ \mathrm{Al}=\text { aluminum } & \mathrm{Cv}=\text { copper } & \mathrm{TI}=\text { thallium } \\ \mathrm{As}=\text { arsenic } & \mathrm{Fe}=\text { iron } & \mathrm{Th}=\text { thorium } \\ \mathrm{Ba}=\text { barium } & \mathrm{Hg}=\text { mercury } & \mathrm{Ra}=\text { radium } \\ \mathrm{Ca}=\text { calcium } & \mathrm{Mn}=\text { manganese } & \mathrm{U}=\text { uranium } \\ \mathrm{Cd}=\text { cadmium } & \mathrm{Mg}=\text { magnesium } & \mathrm{Va}=\text { vanadium } \\ \mathrm{Cn}=\text { cyanide } & \mathrm{Na}=\text { sodium } & \mathrm{Zn}=\text { zinc } \\ \mathrm{Co}=\text { cobalt } & \mathrm{Ni}=\text { nickle } & \end{array}$


Table 5-2

Soll Waste Standards (Metals)

\begin{tabular}{||l|c|c||}
\hline \multicolumn{1}{|c|}{ Element } & $\begin{array}{c}\text { Maximum Contaminant } \\
\text { Concentration (mg/l) }\end{array}$ & $\begin{array}{c}\text { Modified Maximum } \\
\text { Contaminant Concentration } \\
\text { (mg/l) }\end{array}$ \\
\hline \hline Arsenic (As) & 5.0 & 100.0 \\
\hline Barium (Ba) & 100.0 & 2000.0 \\
\hline Cadmium (Cd) & 1.0 & 20.0 \\
\hline Chromium (Cr) & 5.0 & 100.0 \\
\hline Lead $(\mathrm{Pb})$ & 5.0 & 100.0 \\
\hline Mercury $(\mathrm{Hg})$ & 0.2 & 4.0 \\
\hline Selenium $(\mathrm{Se})$ & 1.0 & 20.0 \\
\hline Silver $(\mathrm{Ag})$ & 5.0 & 100.0 \\
\hline
\end{tabular}

Drilling mud consists of four basic components: (1) a liquid phase; (2) colloid phase; (3) inert solid phase; and (4) a chemical phase. The liquid phase is normally water (i.e., fresh, brackish, or saline, or less commonly oil or a polymer/emulsion). The colloid phase is principally clay, most commonly bentonite, and is used to increase the viscosity of the liquid. The inert solid phase is used to increase the density of the drilling fluid and/or limit the loss of fluid into cracks, crevices, or pores in the rocks while drilling. Chemicals are added to increase or decrease the viscosity, change the rheologic properties, improve lubrication, or limit corrosion of drilling equipment. The chemicals generally work by altering ionic exchanges, with typical examples being soda ash, sodium hydroxide, or sodium bicarbonate. Organic thinners typically are phosphates, lignites or heavy metal lignosulfonates (ferrochrome lignosulfonate) used to control flocculation of solids and viscosity. Often diesel oil is added to the drilling mud to improve lubrication. limit flocculation of the colloids, and prevent hydration of shale and clay formations. In regions like Tatum Dome, where salt beds or domes are penetrated, the diesel oil limits the dissolution of the salt by the drilling mud.

The volatile and semivolatile organic constituents identified are either extracts from the lignite (coal) in the drilling mud or solvents. The only exception is the bis (2-Ethylhexyl) phthalate used in vacuum pumps and is probably a laboratory contaminant. 
A complete list of those radionuclides included in the gamma spectroscopy analysis is given in Table 5-3. The results of the gamma spectroscopy were compared to those of a reference soil sample collected in conjunction with previous activities at the Salmon Site (DOE, 1994b). The reference sample was collected from an off-site location near the Salmon Site. which is believed to not have been affected by the testing activities at Salmon Site. The values for each radionuclide detected in the reference sample are listed in Table 5-4. Trench samples whose radionuclide activities exceeded those of the reference sample are listed in the "radionuclides" column of Table 5-2. Gross alpha and beta activities for the reference sample were 8.34 and $12.79 \mathrm{pCi} / \mathrm{g}$, respectively. 


\section{Table 5-3 \\ Radionuclides Included in the Gamma Spectroscopy Analysisa}

\begin{tabular}{|c|c|c|c|c|c|}
\hline Radlonuclldo & Symbol & Mase & Radlonuolide & Symbol & Mases \\
\hline Beryllium 7 & Be-7 & 7 & Barium 131 & Ba-131 & 131 \\
\hline Sodium 22 & $\mathrm{Na}-22$ & 22 & Barium 133 & $\mathrm{Ba}-133$ & 133 \\
\hline Sodium 24 & $\mathrm{Na}-24$ & 24 & Barium 140 & $B a-14 C$ & 140 \\
\hline Potassium 40 & $K-40$ & 40 & Lanthanum 140 & La-140 & 140 \\
\hline Scandium 46 & Sc-46 & 46 & Cerium 139 & Ce-139 & 139 \\
\hline Vanadium 48 & $V-48$ & 48 & Cerium 141 & $\mathrm{Ce}-141$ & 141 \\
\hline Chromium 51 & $\mathrm{Cr}-51$ & 51 & Cerium 144 & $\mathrm{C}_{\theta-144}$ & 144 \\
\hline Manganese 54 & $M n \cdot 54$ & 54 & Europium 152 & Eu-152 & 152 \\
\hline Iron 59 & Fe-59 & 59 & Europium 154 & Eu-154 & 154 \\
\hline Cobalt 56 & Co-56 & 56 & Europium 155 & Eu-155 & 155 \\
\hline Cobalt 57 & Co-57 & 57 & Gadolinium 153 & Gd-153 & 153 \\
\hline Cobalt 58 & Co.58 & 58 & Iridium 192 & Ir-192 & 192 \\
\hline Cobalt 60 & Co-60 & 60 & Mercury 203 & $\mathrm{Hg}-203$ & 203 \\
\hline Nickel 65 & $\mathrm{Ni}-65$ & 65 & Thallium 208 & $\mathrm{TI}-208$ & 208 \\
\hline Zinc 65 & Zn-65 & 65 & Lead 210 & $\mathrm{~Pb}-210$ & 210 \\
\hline Selenium 75 & Se-75 & 75 & Lead 211 & $\mathrm{~Pb}-211$ & 211 \\
\hline Strontium 85 & Sr-85 & 85 & Lead 212 & $\mathrm{~Pb}-212$ & 212 \\
\hline Yttrium 88 & $Y-88$ & 88 & Bismuth 207 & Bi-207 & 207 \\
\hline Zirconium 95 & Zr-95 & 95 & Bismuth 212 & $\mathrm{Bi}-212$ & 212 \\
\hline Niobium 95 & $\mathrm{Nb}-95$ & 95 & Bismuth 214 & Bi-214 & 214 \\
\hline Technetium 99M & Tc-99M & 99 & Radium 223 & Ra-223 & 223 \\
\hline Ruthenium 103 & Ru-103 & 103 & Radium 224 & Ra-224 & 224 \\
\hline Ruthenium 106 & $\mathrm{Ru}-106$ & 106 & Radium 226 & $\mathrm{Ra}-226$ & 226 \\
\hline Silver $110 \mathrm{M}$ & $\mathrm{Ag}-110 \mathrm{M}$ & 110 & Radium 228 & $\mathrm{Ra}-228$ & 228 \\
\hline Cadmium 109 & Cd-109 & 109 & Actinium 227 & Ac-227 & 227 \\
\hline $\operatorname{Tin} 113$ & Sn-113 & 113 & Thorium 228 & Th-228 & 228 \\
\hline Antimony 124 & Sb-124 & 124 & Thorium 230 & Th-230 & 230 \\
\hline Antimony 125 & Sb-125 & 125 & Thorium 234 & Th-234 & 234 \\
\hline lodine 129 & $1-129$ & 129 & Proactinium 231 & Pa-231 & 231 \\
\hline lodine 131 & $1-131$ & 131 & |Proactinium 234 & Pa-234 & 234 \\
\hline lodine 133 & $1-133$ & 133 & Uranium 234 & U.234 & 234 \\
\hline Cesium 134 & Cs-134 & 134 & Uranium 235 & U-235 & 234 \\
\hline Cesium 137 & Cs-137 & 137 & Americium 241 & Am-241 & 241 \\
\hline
\end{tabular}

a IT Corporation, St. Louis, 1993, SL13030 
Table 5-4

\section{Radionuclide Activities for Soll Sample REFC-19-SS", Salmon Site}

\begin{tabular}{|l|c||l|c|}
\hline \multicolumn{1}{|c|}{ Radionuclide } & Aotivity (pCVg) & Radionuclide & Activity (pCVg) \\
\hline Cesium 137 & 0.24 & Uranium 234 & ND \\
\hline Potassium 40 & 2.70 & Uranium 235 & ND \\
\hline Lead 212 & 0.31 & Blamuth 214 & ND \\
\hline Radium 226 & 0.46 & Protactinium 234 & ND \\
\hline Radium 228 & 0.47 & Thorium 234 & ND \\
\hline Thallium 208 & 0.13 & Cd-109 & ND \\
\hline Uranium 238 & 0.60 & Strontium 85 & ND \\
\hline Lead 210 & 1.23 & Yttrium 88 & ND \\
\hline Radium 224 & 0.43 & Cerium 144 & ND \\
\hline Radium 223 & 0.16 & Thorium 230 & ND \\
\hline Thorium 228 & ND & Bismuth 212 & 0.32 \\
\hline Protactinium 231 & ND & lodine 131 & ND \\
\hline Tin 113 & ND & Beryllium 7 & ND \\
\hline Mercury 203 & ND & & \\
\hline
\end{tabular}

" Sample collected March 1993 (DOE, 1994a)

${ }^{\circ} \mathrm{ND}=$ nondetected 


\subsection{Reforences}

Code of Federal Regulations. 1990, Title 40, "Protection of Environment." U.S. Government Printing Office, Washington, DC.

DOE, See U.S. Department of Energy

U.S. Department of Energy, 1992, Remedial Investigation and Feasibility Study of the Tatum Dome Test Site, Lamar County, Mississippi, Final Work Plan, Washington, DC.

U.S. Department of Energy, 1994a, Draft Geophysical Investigation, Salmon Site, Lamar County, Mississippi, Washington. DC.

U.S. Department of Energy, 1994b, Draft Preliminary Data Report of Investigations Conducted at the Salmon Site, Lamar County, Mississippi, Washington, DC.

U.S. Department of Energy, Nevada Operations Office. 1993. Salmon Site Test Pit Excavation Site-Specific Health and Safety Plan, Las Vegas, NV. 
Appendix A

Corrected Sample Locations 


\section{Corrected Sample Locations}

\begin{tabular}{|c|l|l||}
\hline \multicolumn{1}{|c|}{ Trench } & \multicolumn{1}{|c|}{ Sample } & \multicolumn{1}{c|}{ Actual Location" } \\
\hline 3 & TR3-ES-450N-400E-3Z & $395 \mathrm{~N}-430 \mathrm{E}-32$ \\
\hline 3 & TR3-ES-430N-400E-15Z & $380 \mathrm{~N}-430 \mathrm{E}-15 \mathrm{Z}$ \\
\hline 6 & TR6-ES-495-585-12 & $495 \mathrm{~N}-595 \mathrm{E}-12$ \\
\hline 6 & TR6-ES-485-590-6 & $485 \mathrm{~N}-605 \mathrm{E}-6$ \\
\hline 15 & $\begin{array}{l}\text { TR15-ES-20-1180-.5 } \\
\text { TR15-ES-20-1180-.5MS } \\
\text { TR15-ES-20-1180-.5MSD } \\
\text { TR15-ES-20-1180-.5DUP }\end{array}$ & $\begin{array}{l}\text { TR15-ES-0-1180-.5 } \\
\text { TR15-ES-0-1180-.5MS } \\
\text { TR15-ES-0-1180-.5MSD } \\
\text { TR15-ES-0-1180-.5DUP }\end{array}$ \\
\hline 19 & TR19-BS-0-150A-E & ON-40EA-E \\
\hline
\end{tabular}

- Coordinates are in feet and reference the geophysical survey grid established in November 1992 and April 1993. 


\section{Appendix B \\ List of Applicable Terms, Letters, or Symbols Found in Data Analyses Tables}




\section{Appendix B \\ Explanation of Terms Used in Data Tables}

Terms (letters or symbols) found in the Data Tables are explained below.

CRDL Contract required detection limit.

MDA Minimum detectable activity.

RE Following the sample number indicates a laboratory re-extraction.

DIL Following the sample number indicates a laboratory dilution.

UG/KG Micrograms per kilogram.

pCi picocurie.

\section{Organic Compounds}

B Analyte was found in associated blank as well as in sample.

IATA QUAL Data qualifier.

Dup Indicates sample is a duplicate.

E Concentrations exceed the calibration range of the Gas

Chromatograph/Multispectral Scanner (GC/MS).

$\mathrm{J} \quad$ Reported result is quantitatively estimated.

MS Indicates that the sample is a matrix spike sample.

MSD Indicates that the sample is a matrix spike duplicate.

NJ There is presumptive evidence that a Tentatively Identified Compound (TIC) is present at an estimated concentration.

$\mathrm{U} \quad$ Compound was analyzed for but not detected.

UJ Reported quantitation limit is qualified as non-detect.

$\mathrm{X} \quad$ Data entered manually.

"- Shaded entry indicates that the reported value exceeds the Contract Required

Detection Limit (CRDL).

Inorganic Compounds

B The reported value is below the CRDL but above the Instrument Detection Limit (IDL).

DATA QUAL Data qualifier.

Dup Indicates sample is a duplicate.

E ICP dilution \%D is out of control limits.

J Reported result is quantitatively estimated.

MS Indicates that the sample is a matrix spike. 
MSD Indicates that the sample is a matrix spike duplicate.

N Spiked sample recovery not within control limits.

NJ There is presumptive evidence that a TIC is present at an estimated concentration.

$\mathbf{R} \quad$ Reject, data is qualified as unusable.

S The reported value was determined by Method of Standard Addition.

U Compound/element was analyzed for but not detected.

UJ Reported non-detect result is qualified as estimated.

W Post digestion spike for furnace Atomic Absorption Analysis is out of control limit.

$X \quad$ Data entered manually.

"1"

Shaded entry indicates that the reported value exceeds the CRDL.

\section{Radiochemical Compounds}

Dup Indicates sample is a duplicate.

J Reported result is quantitatively estimated.

MDA Minimum detectable activity.

ND Recorded as non-detect since the result was below the Minimum Detectable Activity (MDA).

"-" $\quad$ Single dash indicate not applicable.

U Compound was not detected at specified detection limit.

UJ Reported non-detect result is qualified as estimated.

" $\square$ Shaded entry indicates that the reported value exceeds the reference sample. 


\section{Appendix C}

Results of Volatile Organic Compound Analyses 


\section{Appendix C: Results of Volatile Organic Compound Analyses, Salmon Site}

\begin{tabular}{|c|c|c|c|c|c|c|c|}
\hline COMPOUND & RESULTS & UNITS & $\begin{array}{l}\text { DATA } \\
\text { QUAL. }\end{array}$ & $\begin{array}{l}\text { DATA } \\
\text { VALID. }\end{array}$ & CRDL & UNITS & DILUTION \\
\hline \multicolumn{8}{|l|}{ TR1-ES-001 } \\
\hline Chloromethane & 10 & UG/KG & $u$ & $-\cdots$ & 10 & UG/KG & 1 \\
\hline Bromomethane & 10 & UG/KG & $u$ & -- & 10 & UG/KG & 1 \\
\hline Vinyl Chloride & 10 & UG/KG & $u$ & -- & 10 & UG/KG & 1 \\
\hline Chloroethane & 10 & UG/KG & $u$ & $\cdots$ & 10 & UG/KG & 1 \\
\hline Methylene Chloride & 21 & UG/KG & B & $u$ & 5 & UG/KG & 1 \\
\hline Acetone & 35 & UG/KG & & UJ & 10 & UG/KG & 1 \\
\hline Carbon Disulfide & 5 & UG/KG & $u$ & $\cdots$ & 5 & UG/KG & 1 \\
\hline 1,1-Dichloroethene & 5 & UG/KG & $u$ & -.- & 5 & UG/KG & 1 \\
\hline 1.1-Dichloroethane & 5 & UG/KG & $u$ & $\cdots$ & 5 & UG/KG & 1 \\
\hline 1,2-Dichloroethene (total) & 5 & UG/KG & $u$ & $\cdots$ & 5 & UG/KG & 1 \\
\hline Chloroform & 5 & UG/KG & $u$ & $\cdots$ & 5 & UG/KG & 1 \\
\hline 1.2-Dichloroethane & 5 & UG/KG & $u$ & -- & 5 & UG/KG & 1 \\
\hline 2-Butanone & 10 & UG/KG & $u$ & UJ & 10 & UG/KG & 1 \\
\hline 1,1,1-Trichloroethane & 5 & UG/KG & $\mathbf{u}$ & $\cdots$ & 5 & UG/KG & 1 \\
\hline Carbon Tetrachloride & 5 & UG/KG & $U$ & $\cdots$ & 5 & UG/KG & 1 \\
\hline Vinyl Acetate & 10 & UG/KG & $u$ & $\cdots$ & 10 & UG/KG & 1 \\
\hline Bromodichloromethane & 5 & UG/KG & $\mathbf{U}$ & $\cdots$ & 5 & UG/KG & 1 \\
\hline 1,2-Dichloropropane & 5 & UG/KG & $U$ & $\cdots$ & 5 & UG/KG & 1 \\
\hline cis-1,3-Dichloropropene & 5 & UG/KG & $U$ & $\cdots$ & 5 & UG/KG & 1 \\
\hline Trichloroethene & 5 & UG/KG & $u$ & $\cdots$ & 5 & UG/KG & 1 \\
\hline Dibromochloromethane & 5 & UG/KG & $u$ & $\cdots$ & 5 & UG/KG & 1 \\
\hline 1,1,2-Trichloroethane & 5 & UG/KG & $U$ & $\cdots$ & 5 & UG/KG & 1 \\
\hline Benzene & 5 & UG/KG & $u$ & $\cdots$ & 5 & UG/KG & 1 \\
\hline trans-1,3-Dichloropropene & 5 & UG/KG & $u$ & $\cdots$ & 5 & UG/KG & 1 \\
\hline Bromoform & 5 & UG/KG & $u$ & $\cdots$ & 5 & UG/KG & 1 \\
\hline 4-Methyl-2-Pentanone & 10 & UG/KG & $u$ & $\cdots$ & 10 & UG/KG & 1 \\
\hline 2-Hexanone & 10 & UG/KG & $u$ & $\cdots$ & 10 & UG/KG & 1 \\
\hline Tetrachloroethene & 5 & UG/KG & $U$ & $\cdots$ & 5 & UG/KG & 1 \\
\hline 1,1,2,2-Tetrachloroethane & 5 & UG/KG & $u$ & $-\cdots$ & 5 & UG/KG & 1 \\
\hline Toluene & 2 & UG/KG & $J$ & $\cdots$ & 5 & UG/KG & 1 \\
\hline Chlorobenzene & 5 & UG/KG & $U$ & $\cdots$ & 5 & UG/KG & 1 \\
\hline Ethylbenzene & 5 & UG/KG & 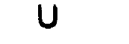 & $\cdots$ & 5 & UG/KG & 1 \\
\hline Styrene & 5 & UG/KG & $U$ & $\cdots$ & 5 & UG/KG & 1 \\
\hline Xylenes (total) & 5 & UG/KG & $u$ & $\ldots$ & 5 & UG/KG & 1 \\
\hline Unknown & 5 & UG/KG & $\mathrm{J}$ & $\cdots$ & & UG/KG & 1 \\
\hline Toluene-d8 (SURR) & 102 & $\%$ & & & & $\%$ & 1 \\
\hline Bromofluorobenzene (SURR) & 95 & $\%$ & & & & $\%$ & 1 \\
\hline 1,2-Dichloroethane-d4 (SURR) & 100 & $\%$ & & & & $\%$ & 1 \\
\hline
\end{tabular}




\section{Appendix C: Results of Volatile Organic Compound Analyses, Salmon Site}

\begin{tabular}{|c|c|c|c|c|c|c|c|}
\hline COMPOUND & RESULTS & UNITS & $\begin{array}{l}\text { DATA } \\
\text { QUAL. }\end{array}$ & $\begin{array}{l}\text { DATA } \\
\text { VAUD. }\end{array}$ & CRDL & UNITS & DILUTION \\
\hline \multicolumn{8}{|l|}{ TR2-ES-355N-175E-5Z } \\
\hline Chloromethane & 20 & UG/KG & $u$ & -- & 20 & UG/KG & 2 \\
\hline Bromomethane & 20 & UG/KG & $u$ & $\cdots$ & 20 & UG/KG & 2 \\
\hline Vinyl Chloride & 20 & UG/KG & $u$ & -- & 20 & UG/KG & 2 \\
\hline Chloroethane & 20 & UG/KG & $u$ & $\cdots$ & 20 & UG/KG & 2 \\
\hline Methylene Chloride & 33 & UG/KG & B & $u$ & 10 & UG/KG & 2 \\
\hline Acetone & 210 & UGKG & & UJ & 20 & UG/KG & 2 \\
\hline Carbon Disulfide & 10 & UG/KG & U & $\cdots$ & 10 & UG/KG & 2 \\
\hline 1,1-Dichloroethene & 10 & UG/KG & U & $\cdots$ & 10 & UG/KG & 2 \\
\hline 1,1-Dichloroethane & 10 & UG/KG & U & -.. & 10 & UG/KG & 2 \\
\hline 1,2-Dichloroethene (total) & 10 & UG/KG & $u$ & $\cdots$ & 10 & UG/KG & 2 \\
\hline Chloroform & 10 & UG/KG & $u$ & -- & 10 & UG/KG & 2 \\
\hline 1,2-Dichloroethane & 10 & UG/KG & $U$ & $\cdots$ & 10 & UG/KG & 2 \\
\hline 2-Butanone & 20 & UG/KG & $U$ & $\cdots$ & 20 & UG/KG & 2 \\
\hline 1.1.1-Trichloroethane & 10 & UG/KG & $\mathrm{U}$ & -.. & 10 & UG/KG & 2 \\
\hline Carbon Tetrachloride & 10 & UG/KG & $u$ & $\cdots$ & 10 & UG/KG & 2 \\
\hline Vinyl Acetate & 20 & UG/KG & $u$ & UJ & 20 & UG/KG & 2 \\
\hline Bromodichloromethane & 10 & UG/KG & $u$ & $\cdots$ & 10 & UG/KG & 2 \\
\hline 1,2-Dichloropropane & 10 & UG/KG & $\mathbf{u}$ & $\cdots$ & 10 & UG/KG & 2 \\
\hline cis-1,3-Dichloropropene & 10 & UG/KG & $u$ & $\cdots$ & 10 & UG/KG & 2 \\
\hline Trichloroethene & 10 & UG/KG & $U$ & $\cdots$ & 10 & UG/KG & 2 \\
\hline Dibromochloromethane & 10 & UG/KG & $u$ & $\cdots$ & 10 & UG/KG & 2 \\
\hline 1,1,2-Trichloroethane & 10 & UG/KG & U & $\cdots$ & 10 & UG/KG & 2 \\
\hline Benzene & 10 & UG/KG & $u$ & -- & 10 & UG/KG & 2 \\
\hline trans-1,3-Dichloropropene & 10 & UG/KG & $u$ & $\cdots$ & 10 & UG/KG & 2 \\
\hline Bromoform & 10 & UG/KG & U & $\cdots$ & 10 & UG/KG & 2 \\
\hline 4-Methyl-2-Pentanone & 20 & UG/KG & $U$ & -- & 20 & UG/KG & 2 \\
\hline 2-Hexanone & 20 & UG/KG & U & $\cdots$ & 20 & UG/KG & 2 \\
\hline Tetrachloroethene & 10 & UG/KG & $\mathrm{U}$ & $\cdots$ & 10 & UG/KG & 2 \\
\hline 1,1,2,2-Tetrachloroethane & 10 & UG/KG & $\mathbf{U}$ & $\cdots$ & 10 & UG/KG & 2 \\
\hline Toluene & 5 & UGKG & J & $\cdots$ & 10 & UG/KG & 2 \\
\hline Chlorobenzene & 10 & UG/KG & U & -- & 10 & UG/KG & 2 \\
\hline Ethylbenzene & 10 & UG/KG & U & $\cdots$ & 10 & UG/KG & 2 \\
\hline Styrene & 10 & UG/KG & U & $\cdots$ & 10 & UG/KG & 2 \\
\hline Xylenes (total) & 10 & UG/KG & U & $\cdots$ & 10 & UG/KG & 2 \\
\hline Unknown-1 & 15 & UG/KG & $\mathrm{J}$ & $\cdots$ & & UG/KG & 2 \\
\hline Unknown-2 & 31 & UG/KG & BJ & $\mathbf{R}$ & & UG/KG & 2 \\
\hline Toluene-d8 (SURR) & 109 & $\%$ & & & & $\%$ & 2 \\
\hline Bromofluorobenzene (SURR) & 92 & $\%$ & & & & $\%$ & 2 \\
\hline 1,2-Dichloroethane-d4 (SURR) & 99 & $\%$ & & & & $\%$ & 2 \\
\hline
\end{tabular}


Appendix C: Results of Volatile Organic Compound Analyses, Salmon Site

\begin{tabular}{|c|c|c|c|c|c|c|c|}
\hline COMPOUND & RESULTS & UNITS & $\begin{array}{l}\text { DATA } \\
\text { QUAL. } \\
\end{array}$ & $\begin{array}{l}\text { DATA } \\
\text { VALID. }\end{array}$ & CRDL & UNITS & DILUTION \\
\hline \multicolumn{8}{|l|}{$\begin{array}{l}\text { COMPOUND } \\
\text { TR2-ES-356N-175E-82 }\end{array}$} \\
\hline Chloromethane & 20 & UG/KG & U & $\cdots$ & 20 & UG/KG & 2 \\
\hline Bromomethane & 20 & UG/KG & $u$ & 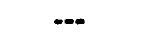 & 20 & UG/KG & 2 \\
\hline Vinyl Chloride & 20 & UG/KG & $U$ & $\cdots$ & 20 & UG/KG & 2 \\
\hline Chloroethane & 20 & UG/KG & $u$ & $\cdots$ & 20 & UG/KG & 2 \\
\hline Methylene Chloride & 10 & UG/KG & $u$ & --- & 10 & UG/KG & 2 \\
\hline Acetone & 350 & UG/KG & B & J & 20 & UG/KG & 2 \\
\hline Carbon Disulfide & 10 & UG/KG & $U$ & $\cdots$ & 10 & UG/KG & 2 \\
\hline 1,1-Dichloroethene & 10 & UG/KG & $U$ & $\cdots$ & 10 & UG/KG & 2 \\
\hline 1.1-Dichloroethane & 10 & UG/KG & $U$ & $\cdots$ & 10 & UG/KG & 2 \\
\hline 1,2-Dichloroethene (total) & 10 & UG/KG & $U$ & $\cdots$ & 10 & UG/KG & 2 \\
\hline Chloroform & 10 & UG/KG & $U$ & $\cdots$ & 10 & UG/KG & 2 \\
\hline 1,2-Dichloroethane & 10 & UG/KG & $U$ & $\cdots$ & 10 & UG/KG & 2 \\
\hline 2-Butanone & 20 & UG/KG & $U$ & --- & 20 & UG/KG & 2 \\
\hline $1,1,1$-Trichloroethane & 10 & UG/KG & $u$ & $\cdots$ & 10 & UG/KG & 2 \\
\hline Carbon Tetrachloride & 10 & UG/KG & $U$ & $\ldots$ & 10 & UG/KG & 2 \\
\hline Vinyl Acetate & 20 & UG/KG & $U$ & $-\cdot$ & 20 & UG/KG & 2 \\
\hline Bromodichloromethane & 10 & UG/KG & $U$ & $\ldots$ & 10 & UG/KG & 2 \\
\hline 1.2-Dichloropropane & 10 & UG/KG & $u$ & $\cdots$ & 10 & UG/KG & 2 \\
\hline cis-1,3-Dichloropropene & 10 & UG/KG & $U$ & --- & 10 & UG/KG & 2 \\
\hline Trichloroethene & 10 & UG/KG & $U$ & --- & 10 & UG/KG & 2 \\
\hline Dibromochloromethane & 10 & UG/KG & $u$ & -- & 10 & UG/KG & 2 \\
\hline 1,1,2-Trichloroethane & 10 & UG/KG & $U$ & $\cdots$ & 10 & UG/KG & 2 \\
\hline Benzene & 10 & UG/KG & $U$ & $\ldots$ & 10 & UG/KG & 2 \\
\hline trans-1,3-Dichloropropene & 10 & UG/KG & $u$ & $\cdots$ & 10 & UG/KG & 2 \\
\hline Bromoform & 10 & UG/KG & $U$ & $\cdots$ & 10 & UG/KG & 2 \\
\hline 4-Methyl-2-Pentanone & 20 & UG/KG & U & $\ldots$ & 20 & UG/KG & 2 \\
\hline 2-Hexanone & 20 & UG/KG & $u$ & $\cdots$ & 20 & UG/KG & 2 \\
\hline Tetrachloroethene & 10 & UG/KG & $U$ & $\ldots$ & 10 & UG/KG & 2 \\
\hline 1,1,2,2-Tetrachloroethane & 10 & UG/KG & U & $\cdots$ & 10 & UG/KG & 2 \\
\hline Toluene & 16 & UG/KG & & $\ldots$ & 10 & UG/KG & 2 \\
\hline Chlorobenzene & 10 & UG/KG & $U$ & $\ldots$ & 10 & UG/KG & 2 \\
\hline Ethylbenzene & 10 & UG/KG & $U$ & $\ldots$ & 10 & UG/KG & 2 \\
\hline Styrene & 10 & UG/KG & $u$ & $\cdots$ & 10 & UG/KG & 2 \\
\hline Xylenes (total) & 10 & UG/KG & $U$ & ... & 10 & UG/KG & 2 \\
\hline Unknown-1 & 66 & UG/KG & J & --- & & UG/KG & 2 \\
\hline Unknown-2 & 12 & UG/KG & J & -- & & UG/KG & 2 \\
\hline Unknown-3 & 26 & UG/KG & J & --. & & UG/KG & 2 \\
\hline Unknown-4 & 20 & UG/KG & $J$ & $\ldots$ & & UG/KG & 2 \\
\hline Toluene-d8 (SURR) & 111 & $\%$ & & & & $\%$ & 2 \\
\hline Bromofluorobenzene (SURR) & 87 & $\%$ & & & & $\%$ & 2 \\
\hline 1,2-Dichloroethane-d4 (SURR) & 101 & $\%$ & & & & $\%$ & 2 \\
\hline
\end{tabular}




\section{Appendix C: Results of Volatile Organic Compound Analyses, Salmon Site}

\begin{tabular}{|c|c|c|c|c|c|c|c|}
\hline COMPOUND & RESULTS & UNITS & $\begin{array}{l}\text { DATA } \\
\text { QUAL. }\end{array}$ & $\begin{array}{l}\text { DATA } \\
\text { VALID. }\end{array}$ & CRDL & UNITS & DILUTION \\
\hline \multicolumn{8}{|l|}{ TR2A-ES-365N-200E-3.5Z } \\
\hline Chloromethane & 10 & UG/KG & $u$ & $\cdots$ & 10 & UG/KG & 1 \\
\hline Bromomethane & 10 & UG/KG & $u$ & $\cdots$ & 10 & UG/KG & 1 \\
\hline Vinyl Chloride & 10 & UG/KG & $u$ & $\cdots$ & 10 & UG/KG & 1 \\
\hline Chloroethane & 10 & UG/KG & $u$ & -- & 10 & UG/KG & 1 \\
\hline Methylene Chloride & 7 & UG/KG & B & $u$ & 5 & UG/KG & 1 \\
\hline Acetone & 64 & UG/KG & B & UJ & 10 & UG/KG & 1 \\
\hline Carbon Disulfide & 5 & UG/KG & $u$ & $-\cdots$ & 5 & UG/KG & 1 \\
\hline 1,1-Dichloroethene & 5 & UG/KG & $u$ & -.. & 5 & UG/KG & 1 \\
\hline 1.1-Dichloroethane & 5 & UG/KG & U & $\cdots$ & 5 & UG/KG & 1 \\
\hline 1,2-Dichloroethene (total) & 5 & UG/KG & $u$ & $\ldots$ & 5 & UG/KG & 1 \\
\hline Chloroform & 5 & UG/KG & $u$ & $\ldots$ & 5 & UG/KG & 1 \\
\hline 1,2-Dichloroethane & 5 & UG/KG & $u$ & $\cdots$ & 5 & UG/KG & 1 \\
\hline 2-Butanone & 10 & UG/KG & $u$ & $\cdots$ & 10 & UG/KG & 1 \\
\hline 1.1.1-Trichloroethane & 5 & UG/KG & $u$ & $\ldots$ & 5 & UG/KG & 1 \\
\hline Carbon Tetrachloride & 5 & UG/KG & $u$ & -.- & 5 & UG/KG & 1 \\
\hline Vinyl Acetate & 10 & UG/KG & $u$ & --- & 10 & UG/KG & 1 \\
\hline Bromodichloromethane & 5 & UG/KG & $u$ & --- & 5 & UG/KG & 1 \\
\hline 1,2-Dichloropropane & 5 & UG/KG & $u$ & $\cdots$ & 5 & UG/KG & 1 \\
\hline cis-1,3-Dichloropropene & 5 & UG/KG & $u$ & $\cdots$ & 5 & UG/KG & 1 \\
\hline Trichloroethene & 5 & UG/KG & $u$ & $\cdots$ & 5 & UG/KG & 1 \\
\hline Dibromochloromethane & 5 & UG/KG & $u$ & $\cdots$ & 5 & UG/KG & 1 \\
\hline 1,1,2-Trichloroethane & 5 & UG/KG & $u$ & $\cdots$ & 5 & UG/KG & 1 \\
\hline Benzene & 5 & UG/KG & $u$ & $\cdots$ & 5 & UG/KG & 1 \\
\hline trans-1,3-Dichloropropene & 5 & UG/KG & $u$ & $\cdots$ & 5 & UG/KG & 1 \\
\hline Bromoform & 5 & UG/KG & $u$ & $\cdots$ & 5 & $U G / K G$ & 1 \\
\hline 4-Methyl-2-Pentanone & 10 & UG/KG & $u$ & $\cdots$ & 10 & UG/KG & 1 \\
\hline 2-Hexanone & 10 & UG/KG & $u$ & $\ldots$ & 10 & UG/KG & 1 \\
\hline Tetrachloroethene & 5 & UG/KG & $u$ & --- & 5 & UG/KG & 1 \\
\hline 1,1,2,2-Tetrachloroethane & 5 & UG/KG & $u$ & $\ldots$ & 5 & UG/KG & 1 \\
\hline Toluene & 5 & UG/KG & $u$ & --- & 5 & UG/KG & 1 \\
\hline Chlorobenzene & 5 & UG/KG & $u$ & $\cdots$ & 5 & UG/KG & 1 \\
\hline Ethylbenzene & 5 & UG/KG & $u$ & -.- & 5 & UG/KG & 1 \\
\hline Styrene & 5 & UG/KG & $u$ & -.- & 5 & UG/KG & 1 \\
\hline Xylenes (total) & 5 & UG/KG & $u$ & $--\cdot$ & 5 & UG/KG & 1 \\
\hline Toluene-d8 (SURR) & 110 & $\%$ & & & & $\%$ & 1 \\
\hline Bromofluorobenzene (SURR) & 95 & $\%$ & & & & $\%$ & 1 \\
\hline 1,2-Dichloroethane-d4 (SURR) & 105 & $\%$ & & & & $\%$ & 1 \\
\hline
\end{tabular}




\section{Appendix C: Results of Volatile Organic Compound Analyses, Salmon Site}

\begin{tabular}{|c|c|c|c|c|c|c|c|}
\hline COMPOUND & RESULTS & UNITS & $\begin{array}{l}\text { DATA } \\
\text { QUAL. }\end{array}$ & $\begin{array}{l}\text { DATA } \\
\text { VALID. }\end{array}$ & CRDL & UNITS & DILUTION \\
\hline \multicolumn{8}{|l|}{ TR2A-ES-360N-200E-8Z } \\
\hline Chloromethane & 10 & UG/KG & $u$ & $\cdots$ & 10 & UG/KG & 1 \\
\hline Bromomethane & 10 & UG/KG & $u$ & $\cdots$ & 10 & UG/KG & 1 \\
\hline Vinyl Chloride & 10 & UG/KG & U & $\cdots$ & 10 & UG/KG & 1 \\
\hline Chloroethane & 10 & UG/KG & $u$ & $\cdots$ & 10 & UG/KG & 1 \\
\hline Methylene Chloride & 13 & UG/KG & B & $u$ & 5 & UG/KG & 1 \\
\hline Acetone & 130 & UG/KG & & UJ & 10 & UG/KG & 1 \\
\hline Carbon Disulfide & 5 & UG/KG & $u$ & -.. & 5 & UG/KG & 1 \\
\hline 1,1-Dichloroethene & 5 & UG/KG & $u$ & $\cdots$ & 5 & UG/KG & 1 \\
\hline 1,1-Dichloroethane & 5 & UG/KG & $u$ & -. & 5 & UG/KG & 1 \\
\hline 1,2-Dichloroethene (total) & 5 & UG/KG & $u$ & -.- & 5 & UG/KG & 1 \\
\hline Chloroform & 5 & UG/KG & $U$ & -.. & 5 & UG/KG & 1 \\
\hline 1,2-Dichloroethane & 5 & UG/KG & $u$ & ..- & 5 & UG/KG & 1 \\
\hline 2-Butanone & 10 & UG/KG & $U$ & UJ & 10 & UG/KG & 1 \\
\hline 1,1,1-Trichloroethane & 5 & UG/KG & $\mathrm{U}$ & UJ & 5 & UG/KG & 1 \\
\hline Carbon Tetrachloride & 5 & UG/KG & $u$ & UJ & 5 & UG/KG & 1 \\
\hline Vinyl Acetate & 10 & UG/KG & $U$ & UJ & 10 & UG/KG & 1 \\
\hline Bromodichloromethane & 5 & UG/KG & $u$ & UJ & 5 & UG/KG & 1 \\
\hline 1,2-Dichloropropane & 5 & UG/KG & $u$ & UJ & 5 & UG/KG & 1 \\
\hline cis-1,3-Dichloropropene & 5 & UG/KG & $u$ & UJ & 5 & UG/KG & 1 \\
\hline Trichloroethene & 5 & UG/KG & $u$ & UJ & 5 & UG/KG & 1 \\
\hline Dibromochloromethane & 5 & UG/KG & $u$ & UJ & 5 & UG/KG & 1 \\
\hline 1,1,2-Trichloroethane & 5 & UG/KG & U & UJ & 5 & UG/KG & 1 \\
\hline Benzene & 5 & UG/KG & $U$ & UJ & 5 & UG/KG & 1 \\
\hline trans-1,3-Dichloropropene & 5 & UG/KG & $\mathrm{U}$ & UJ & 5 & UG/KG & 1 \\
\hline Bromoform & 5 & UG/KG & $U$ & UJ & 5 & UG/KG & 1 \\
\hline 4-Methyl-2-Pentanone & 10 & UG/KG & $U$ & UJ & 10 & UG/KG & 1 \\
\hline 2-Hexanone & 10 & UG/KG & U & UJ & 10 & UG/KG & 1 \\
\hline Tetrachloroethene & 5 & UG/KG & $U$ & UJ & 5 & UG/KG & 1 \\
\hline 1,1,2,2-Tetrachloroethane & 5 & UG/KG & $u$ & UJ & 5 & UG/KG & 1 \\
\hline Toluene & 5 & UG/KG & $u$ & UJ & 5 & UG/KG & 1 \\
\hline Chlorobenzene & 5 & UG/KG & $\mathrm{U}$ & UJ & 5 & UG/KG & 1 \\
\hline Ethylbenzene & 5 & UG/KG & $\mathrm{U}$ & UJ & 5 & UG/KG & 1 \\
\hline Styrene & 5 & UG/KG & 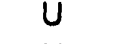 & UJ & 5 & UG/KG & 1 \\
\hline Xylenes (total) & 5 & UG/KG & $\mathrm{U}$ & UJ & 5 & UG/KG & 1 \\
\hline Unknown & 6 & UG/KG & $J$ & $\cdots$ & & UG/KG & 1 \\
\hline Toluene-d8 (SURA) & 100 & $\%$ & & & & $\%$ & 1 \\
\hline Bromofluorobenzene (SURA) & 104 & $\%$ & & & & $\%$ & 1 \\
\hline 1,2-Dichloroethane-d4 (SURR) & 96 & $\%$ & & & & $\%$ & 1 \\
\hline
\end{tabular}


Appendix C: Results of Volatile Organic Compound Analyses, Salmon Site

\begin{tabular}{|c|c|c|c|c|c|c|c|}
\hline COMPOUND & RESULTS & UNITS & $\begin{array}{l}\text { DATA } \\
\text { QUAL. }\end{array}$ & $\begin{array}{l}\text { DATA } \\
\text { VALID. }\end{array}$ & CRDL & UNITS & DILUTION \\
\hline \multicolumn{8}{|l|}{ TR2A-ES-360N-200E-6ZRE } \\
\hline Chloromethane & 10 & Ua/KG & $u$ & $\mathbf{R}$ & 10 & Ua/KO & 1 \\
\hline Bromomethane & 10 & UG/KG & $\mathbf{U}$ & P & 10 & UQ/KG & 1 \\
\hline Vinyi Chloride & 10 & UG/KG & $u$ & $\mathbf{R}$ & 10 & Ua/KG & 1 \\
\hline Chloroethane & 10 & UG/KG & $u$ & R & 10 & UG/KG & 1 \\
\hline Mothylene Chloride & 8 & UG/KG & B & $\mathbf{R}$ & 5 & UG/KG & 1 \\
\hline Acetone & 190 & UG/KG & B & $\mathbf{R}$ & 10 & UCKKG & 1 \\
\hline Carbon Disulfide & 5 & UG/KG & $u$ & $\mathbf{R}$ & 5 & UG/KG & 1 \\
\hline 1,1-Dichloroethene & 5 & UG/KG & $U$ & $\mathbf{R}$ & 5 & UG/KG & 1 \\
\hline 1,1-Dichloroethane & 5 & UG/KG & $u$ & $\mathbf{R}$ & 5 & UG/KG & 1 \\
\hline 1,2-Dichloroethene (total) & 5 & UG/KG & $u$ & $\mathbf{R}$ & 5 & UG/KG & 1 \\
\hline Chloroform & 5 & UG/KG & $u$ & $\mathbf{R}$ & 5 & UG/KG & 1 \\
\hline 1,2-Dichloroethane & 5 & UG/KG & $u$ & $\mathbf{R}$ & 5 & UG/KG & 1 \\
\hline 2-Butanone & 10 & UG/KG & $u$ & $\mathbf{R}$ & 10 & UG/KG & 1 \\
\hline 1,1,1-Trichloroethane & 5 & UG/KG & $u$ & $\mathbf{R}$ & 5 & UG/KG & 1 \\
\hline Carbon Tetrachloride & 5 & UG/KG & $U$ & R & 5 & UG/KG & 1 \\
\hline Vinyl Acetate & 10 & UG/KG & $U$ & R & 10 & UG/KG & 1 \\
\hline Bromodichloromethane & 5 & UG/KG & $U$ & $\mathbf{R}$ & 5 & UG/KG & 1 \\
\hline 1,2-Dichloropropane & 5 & UG/KG & U & $R$ & 5 & UGKG & 1 \\
\hline cis-1,3-Dichloropropene & 5 & UG/KG & $u$ & $\mathbf{R}$ & 5 & UG/KG & 1 \\
\hline Trichloroethene & 5 & LG/KG & $u$ & A & 5 & UG/KG & 1 \\
\hline Dibromochloromethane & 5 & UG/KG & $u$ & $\mathbf{R}$ & 5 & UG/KG & 1 \\
\hline 1,1,2-Trichloroethane & 5 & UG/KG & $U$ & $\mathbf{R}$ & 5 & UG/KG & 1 \\
\hline Benzene & 5 & UG/KG & $U$ & $\mathbf{R}$ & 5 & UG/KG & 1 \\
\hline trans-1,3-Dichloropropene & 5 & UG/KG & $U$ & $\mathbf{R}$ & 5 & UG/KG & 1 \\
\hline Bromoform & 5 & UG/KG & $U$ & R & 5 & UG/KG & 1 \\
\hline 4-Methyl-2-Pentanone & 10 & UG/KG & $U$ & $\mathbf{R}$ & 10 & UG/KG & 1 \\
\hline 2-Hexanone & 10 & UG/KG & $U$ & $\mathbf{R}$ & 10 & UG/KG & 1 \\
\hline Tetrachloroethene & 5 & UG/KG & $u$ & R & 5 & UG/KG & 1 \\
\hline 1,1,2,2-Tetrachloroethane & 5 & UG/KG & $u$ & R & 5 & UG/KG & 1 \\
\hline Toluene & 1 & UG/KG & $J$ & R & 5 & UG/KG & 1 \\
\hline Chlorobenzene & 5 & UG/KG & $u$ & $\mathbf{R}$ & 5 & UG/KG & 1 \\
\hline Ethylbenzene & 5 & UG/KG & $U$ & $\mathbf{R}$ & 5 & UG/KG & 1 \\
\hline Styrene & 5 & UG/KG & $u$ & $\mathbf{R}$ & 5 & UG/KG & 1 \\
\hline Xylenes (total) & 5 & UG/KG & U & $\mathbf{R}$ & 5 & UG/KG & 1 \\
\hline Unknown-1 & 9 & UG/KG & $J$ & $\mathbf{R}$ & & UG/KG & 1 \\
\hline Unknown-2 & 12 & UG/KG & $J$ & R & & UG/KG & 1 \\
\hline Unknown-3 & 6 & UG/KG & $J$ & $\mathbf{R}$ & & UG/KG & 1 \\
\hline Toluene-d8 (SURR) & 96 & $\%$ & & & & $\%$ & 1 \\
\hline Bromofluorobenzene (SURR) & 103 & $\%$ & & & & $\%$ & 1 \\
\hline 1,2-Dichloroethane-d4 (SURR) & 105 & $\%$ & & & & $\%$ & 1 \\
\hline
\end{tabular}




\section{Appendix C: Results of Volatlle Organic Compound Analyses, Salmon Site}

\begin{tabular}{|c|c|c|c|c|c|c|c|}
\hline COMPOUND & RESULTS & UNITS & $\begin{array}{l}\text { DATA } \\
\text { QUAL. }\end{array}$ & $\begin{array}{l}\text { DATA } \\
\text { VALID. }\end{array}$ & CRDL & UNITS & DILUTION \\
\hline \multicolumn{8}{|l|}{ TR3-ES-450N-400E-32 } \\
\hline Chloromethane & 10 & UG/KG & U & $\ldots$ & 10 & UG/KG & 1 \\
\hline Bromomethane & 10 & UG/KG & $U$ & $\cdots$ & 10 & UG/KG & 1 \\
\hline Vinyl Chloride & 10 & UG/KG & $u$ & $\cdots$ & 10 & Ua/KG & 1 \\
\hline Chloroethane & 10 & UG/KG & $u$ & $\cdots$ & 10 & UG/KG & 1 \\
\hline Methylene Chloride & 13 & UG/KG & B & $\mathbf{U}$ & 5 & UGKG & 1 \\
\hline Acetone & 62 & UG/KG & & $w$ & 10 & UaKG & 1 \\
\hline Carbon Disulfide & 5 & UG/KG & $u$ & $\cdots$ & 5 & UG/KG & 1 \\
\hline 1,1-Dichioroethene & 5 & UG/KG & $u$ & $\cdots$ & 5 & UGKG & 1 \\
\hline 1,1-Dichloroethane & 5 & UG/KG & $u$ & $\cdots$ & 5 & UG/KG & 1 \\
\hline 1,2-Dichloroethene (total) & 5 & UG/KG & $u$ & $\cdots$ & 5 & UGKG & 1 \\
\hline Chioroform & 5 & UGKK & $u$ & -- & 5 & UGKG & 1 \\
\hline 1,2-Dichloroethane & 5 & UG/KG & $u$ & $\cdots$ & 5 & UG/KG & 1 \\
\hline 2-Butanone & 10 & UG/KG & $u$ & UJ & 10 & UG/KG & 1 \\
\hline 1,1,1-Trichloroethane & 5 & UG/KG & $u$ & $\cdots$ & 5 & UG/KG & 1 \\
\hline Carbon Tetrachloride & 5 & UG/KG & $u$ & $\cdots$ & 5 & UG/KG & 1 \\
\hline Vinyl Acetate & 10 & UG/KG & $u$ & UJ & 10 & UG/KG & 1 \\
\hline Bromodichloromethane & 5 & UG/KG & $u$ & $\cdots$ & 5 & UG/KG & 1 \\
\hline 1,2-Dichloropropane & 5 & UG/KG & $u$ & $\cdots$ & 5 & UG/KG & 1 \\
\hline cis-1,3-Dichloropropene & 5 & UG/KG & $u$ & $\cdots$ & 5 & UG/KG & 1 \\
\hline Trichloroethene & 5 & UG/KG & $u$ & $\cdots$ & 5 & UG/KG & 1 \\
\hline Dibromochloromethane & 5 & UG/KG & $u$ & $-\cdots$ & 5 & UG/KG & 1 \\
\hline 1,1,2-Trichloroethane & 5 & UG/KG & $u$ & $\cdots$ & 5 & UG/KG & 1 \\
\hline Benzene & 5 & UG/KG & $u$ & $\cdots$ & 5 & UG/KG & 1 \\
\hline trans-1.3-Dichloropropene & 5 & UG/KG & $u$ & $\cdots$ & 5 & UG/KG & 1 \\
\hline Bromoform & 5 & UG/KG & $u$ & $\cdots$ & 5 & UG/KG & 1 \\
\hline 4-Methyl-2-Pentanone & 10 & UG/KG & $u$ & $\cdots$ & 10 & UG/KG & 1 \\
\hline 2-Hexanone & 10 & UG/KG & $u$ & $\cdots$ & 10 & UG/KG & 1 \\
\hline Tetrachloroethene & 5 & UG/KG & $u$ & $\cdots$ & 5 & UG/KG & 1 \\
\hline 1,1,2,2-Tetrachloroethane & 5 & UG/KG & $u$ & $\cdots$ & 5 & UG/KG & 1 \\
\hline Toluene & 5 & UG/KG & $u$ & $\cdots$ & 5 & UG/KG & 1 \\
\hline Chlorobenzene & 5 & UG/KG & $U$ & $\cdots$ & 5 & UG/KG & 1 \\
\hline Ethylbenzene & 5 & UG/KG & $U$ & $\cdots$ & 5 & UG/KG & 1 \\
\hline Styrene & 5 & UG/KG & $u$ & $\cdots$ & 5 & UG/KG & 1 \\
\hline Xylenes (total) & 5 & UG/KG & $u$ & $\cdots$ & 5 & UG/KG & 1 \\
\hline Unknown-1 & 24 & UG/KG & J & $\cdots$ & & UG/KG & 1 \\
\hline Unknown $\mathrm{C} 11 \mathrm{H} 14-1$ & 15 & UG/KG & $J$ & $\cdots$ & & UG/KG & 1 \\
\hline Unkric : in C11H14-2 & 16 & UG/KG & $J$ & $\cdots$ & & UG/KG & 1 \\
\hline Naphthalene & 14 & UG/KG & J & $\cdots$ & & UG/KG & 1 \\
\hline Unknown C11H14-3 & 73 & UG/KG & J & $\cdots$ & & UG/KG & 1 \\
\hline Unknown-2 & 57 & UG/KG & J & $\cdots$ & & UG/KG & 1 \\
\hline Unknown C11H14-4 & 39 & UG/KG & J & $\cdots$ & & UG/KG & 1 \\
\hline Unknown-3 & 210 & UG/KG & $J$ & $\cdots$ & & UG/KG & 1 \\
\hline Unknown-4 & 14 & UGKG & J & $\cdots$ & & UG/KG & 1 \\
\hline Unknown-5 & 31 & UG/KG & $J$ & $\cdots$ & & UG/KG & 1 \\
\hline Toluene-d8 (SURR) & 101 & $\%$ & & & & $\%$ & 1 \\
\hline Bromofluorobenzene (SURR) & 102 & $\%$ & & & & $\%$ & 1 \\
\hline 1,2-Dichloroethano-d4 (SURA) & 100 & $\%$ & & & & $\%$ & 1 \\
\hline
\end{tabular}


Appendlx C: Results of Volatlle Organic Compound Analyses, Salmon Site

\begin{tabular}{|c|c|c|c|c|c|c|c|}
\hline COMPOUND & RESULTS & UNITS & $\begin{array}{l}\text { DATA } \\
\text { QUAL. }\end{array}$ & $\begin{array}{l}\text { DATA } \\
\text { VAUD. }\end{array}$ & CROL & UNITS & DILUTION \\
\hline \multicolumn{8}{|l|}{ TAB-E8-430N-400-15Z } \\
\hline Chloromothane & 10 & UaKo & $u$ & $\cdots$ & 10 & vaKa & 1 \\
\hline Bromomethane & 10 & UaKa & $u$ & $\cdots$ & 10 & Ua/KG & 1 \\
\hline Vinyl Chloride & 10 & UaKo & $u$ & - & 10 & Ua/Ko & 1 \\
\hline Chloroethane & 10 & UaKG & u & $\cdots$ & 10 & VG/KG & 1 \\
\hline Methylene Chloride & 7 & VaKO & $\mathbf{B}$ & $u$ & 5 & UGKO & 1 \\
\hline Acotone & 42 & UaKG & $\mathbf{B}$ & $\omega$ & 10 & UaKa & 1 \\
\hline Carbon Disulflide & 5 & UG/KG & U & $\ldots$ & 5 & UG/KG & 1 \\
\hline 1.1-Dlchloroethene & 5 & UaKG & $u$ & ... & 5 & Ua/KG & 1 \\
\hline 1,1-Dichloroethane & 5 & Ua/KO & $u$ & $\cdots$ & 5 & Ua/KG & 1 \\
\hline 1,2-Dlchloroethene (total) & 5 & UGKG & $u$ & $\cdots$ & 5 & UQKG & 1 \\
\hline Chloroform & $\mathbf{5}$ & UaKG & $u$ & $\cdots$ & 5 & vaKG & 1 \\
\hline 1,2-Dichloroethane & 5 & UG/KG & $U$ & $\cdots$ & 5 & Ua/KG & 1 \\
\hline 2-Butanone & 10 & Ua/KG & $u$ & -.. & 10 & UG/KG & 1 \\
\hline 1,1,1-Trichloroethane & 5 & UGKG & $\mathbf{u}$ & $\cdots$ & 5 & UQKKG & 1 \\
\hline Carbon Tetrachloride & 5 & UG/KG & $u$ & $\ldots$ & 5 & UG/KG & 1 \\
\hline Vinyl Acetate & 10 & UG/KG & $u$ & $\ldots$ & 10 & UG/KG & 1 \\
\hline Bromodichloromethane & 5 & Ua/KG & $u$ & $\cdots$ & 5 & UGKG & 1 \\
\hline 1,2-Dichloropropane & 5 & UGKG & $u$ & $\cdots$ & 5 & UGKG & 1 \\
\hline cis-1,3-Dichloropropene & 5 & UG/KG & $u$ & $\cdots$ & 5 & UG/KG & 1 \\
\hline Trichloroethene & 5 & UG/KG & $u$ & $\cdots$ & 5 & UG/KG & 1 \\
\hline Dibromochloromethane & 5 & UG/KG & $u$ & ... & 5 & UG/KG & 1 \\
\hline 1,1,2-Trichloroethane & 5 & UG/KG & $u$ & $\cdots$ & 5 & UG/KG & 1 \\
\hline Benzene & 5 & UQKKG & $u$ & $\cdots$ & 5 & UG/KG & 1 \\
\hline trans-1,3-Dichloropropene & 5 & UG/KG & $u$ & $\cdots$ & 5 & UG/KG & 1 \\
\hline Bromoform & 5 & UG/KG & $u$ & $\cdots$ & 5 & UG/KG & 1 \\
\hline 4-Methyl-2-Pentanone & 10 & UG/KG & $u$ & $\cdots$ & 10 & UG/KG & 1 \\
\hline 2-Hexanone & 10 & UG/KG & $u$ & $\cdots$ & 10 & UG/KG & 1 \\
\hline Tetrachloroethene & 5 & UG/KG & $u$ & $\cdots$ & 5 & UG/KG & 1 \\
\hline 1,1,2,2-Tetrachloroethane & 5 & UG/KG & $u$ & $\cdots$ & 5 & UG/KG & 1 \\
\hline Toluene & 2 & UG/KG & $\mathrm{J}$ & $\cdots$ & 5 & UG/KG & 1 \\
\hline Chlorobenzene & 5 & UG/KG & $u$ & $\cdots$ & 5 & UG/KG & 1 \\
\hline Ethylbenzene & 5 & UG/KG & $u$ & ... & 5 & UG/KG & 1 \\
\hline Styrene & 5 & UG/KG & $u$ & $\cdots$ & 5 & UG/KG & 1 \\
\hline Xylenes (total) & 2 & UGKG & J & $\cdots$ & 5 & UG/KG & 1 \\
\hline Unknown Alkane-1 & 83 & UG/KG & $\mathrm{J}$ & $\cdots$ & & UG/KG & 1 \\
\hline Unknown-1 & 45 & UG/KG & $\mathrm{J}$ & $\cdots$ & & UGKG & 1 \\
\hline Unknown Alkane-2 & 150 & UG/KG & $\mathrm{J}$ & $\cdots$ & & UG/KG & 1 \\
\hline Unknown-2 & 100 & UG/KG & $\mathrm{J}$ & ..- & & UG/KG & 1 \\
\hline Unknown-3 & 49 & UG/KG & $\mathrm{J}$ & $\cdots$ & & UGKG & 1 \\
\hline Naphthalene & 50 & UG/KG & $\mathrm{J}$ & NJ & & UG/KG & 1 \\
\hline Unknown C11H14 & 180 & UG/KG & $\mathrm{J}$ & $\cdots$ & & UGKG & 1 \\
\hline Unknown-4 & 170 & UGKG & $\mathrm{J}$ & $\cdots$ & & UG/KG & 1 \\
\hline Unknown-5 & 130 & UG/KG & $J$ & $\cdots$ & & UGKG & 1 \\
\hline Unknown-6 & 71 & UG/KG & $J$ & $\cdots$ & & UG/KG & 1 \\
\hline Toluene-d8 (SURR) & 99 & $\%$ & & & & $\%$ & 1 \\
\hline Bromofluorobenzene (SURA) & 99 & $\%$ & & & & $\%$ & 1 \\
\hline 1,2-Dichloroethane-d4 (SURR) & 102 & $\%$ & & & & $\%$ & 1 \\
\hline
\end{tabular}




\begin{tabular}{|c|c|c|c|c|c|c|c|}
\hline COMPOUND & RESULTS & UNITS & $\begin{array}{l}\text { DATA } \\
\text { QUAL. }\end{array}$ & $\begin{array}{l}\text { DATA } \\
\text { VALD. }\end{array}$ & CRDL & UNITS & DILUTION \\
\hline \multicolumn{8}{|l|}{ TA4-E8-280-490-8 } \\
\hline Chloromethane & 10 & UG/KG & $u$ & $\cdots$ & 10 & UG/KG & 1 \\
\hline Bromomethane & 10 & UG/KO & $u$ & $\cdots$ & 10 & UG/KG & 1 \\
\hline Vinyl Chloride & 10 & Ua/KG & $u$ & ... & 10 & UG/KG & 1 \\
\hline Chloroethane & 10 & Ua/KG & $u$ & $\cdots$ & 10 & UG/KG & 1 \\
\hline Methylene Chloride & $\mathbf{5}$ & UG/KG & $u$ & $\cdots$ & 5 & UGKG & 1 \\
\hline Acetone & 41 & Ua/KG & B & UJ & 10 & Ua/KG & 1 \\
\hline Carbon Disulfide & 2 & Ua/KG & $J$ & $\cdots$ & 5 & va/Ko & 1 \\
\hline 1,1-Dichloroethene & 5 & UG/KG & U & $\cdots$ & 5 & UG/KG & 1 \\
\hline 1,1-Dichloroethane & 5 & Ua/KG & $u$ & $\cdots$ & 5 & UG/KG & 1 \\
\hline 1,2-Dichloroethene (total) & 5 & UaKG & $u$ & $\cdots$ & 5 & UG/KG & 1 \\
\hline Chloroform & 5 & UG/KG & $u$ & $\cdots$ & 5 & UG/KG & 1 \\
\hline 1,2-Dichloroethane & 5 & UG/KG & $u$ & $\cdots$ & 5 & UG/KG & 1 \\
\hline 2-Butanone & 10 & UG/KG & $u$ & $\cdots$ & 10 & UG/KG & 1 \\
\hline 1,1,1-Trichloroethane & 5 & UG/KG & $u$ & UJ & 5 & UG/KG & 1 \\
\hline Carbon Tetrachloride & 5 & UG/KG & $u$ & UJ & 5 & UG/KG & 1 \\
\hline Vinyl Acetate & 10 & UG/KG & $u$ & UJ & 10 & UG/KG & 1 \\
\hline Bromodichloromethane & 5 & UG/KG & u & UJ & 5 & UG/KG & 1 \\
\hline 1,2-Dichloropropane & 5 & UG/KG & $u$ & UJ & 5 & UG/KG & 1 \\
\hline cis-1,3-Dichloropropene & 5 & UG/KG & u & UJ & 5 & UG/KG & 1 \\
\hline Trichloroethene & 5 & Ua/KG & $u$ & UJ & 5 & UG/KG & 1 \\
\hline Dibromochloromethane & 5 & UG/KG & $\mathbf{U}$ & UJ & 5 & UG/KG & 1 \\
\hline 1,1,2-Trichloroethane & 5 & UG/KG & $u$ & UJ & 5 & UG/KG & 1 \\
\hline Benzene & 5 & UG/KG & $u$ & UJ & 5 & UG/KG & 1 \\
\hline trans-1,3-Dichloropropene & 5 & UG/KG & u & UJ & 5 & UG/KG & 1 \\
\hline Bromoform & 5 & UG/KG & $u$ & UJ & 5 & UG/KG & 1 \\
\hline 4-Methyl-2-Pentanone & 10 & UG/KG & u & UJ & 10 & UG/KG & 1 \\
\hline 2-Hexanone & 10 & UG/KG & U & UJ & 10 & UG/KG & 1 \\
\hline Tetrachloroethene & 5 & UG/KG & u & UJ & 5 & UG/KG & 1 \\
\hline $1,1,2,2$-Tetrachloroethane & 5 & UG/KG & $\mathrm{U}$ & UJ & 5 & UG/KG & 1 \\
\hline Toluene & 3 & UG/KG & $\mathrm{J}$ & $\cdots$ & 5 & UG/KG & 1 \\
\hline Chlorobenzene & 5 & UG/KG & $u$ & UJ & 5 & UG/KG & 1 \\
\hline Ethylbenzene & 5 & UG/KG & & $\mathrm{J}$ & 5 & UG/KG & 1 \\
\hline Styrene & 5 & UG/KG & $u$ & UJ & 5 & UG/KG & 1 \\
\hline Xylenes (total) & 2 & UG/KG & J & $\cdots$ & 5 & UG/KG & 1 \\
\hline Unknown Alkane-1 & 400 & UG/KG & $J$ & $\cdots$ & & UG/KG & 1 \\
\hline Unknown Alkane-2 & 470 & UG/KG & $J$ & $\cdots$ & & UG/KG & 1 \\
\hline Unknown Alkane-3 & 340 & UG/KG & J & $\cdots$ & & UG/KG & 1 \\
\hline Unknown Alkane-4 & 480 & UG/KG & $\mathrm{J}$ & $\cdots$ & & UG/KG & 1 \\
\hline Unknown Alkane-5 & 1100 & UG/KG & $J$ & $\cdots$ & & UG/KG & 1 \\
\hline Unknown Alkane-6 & 700 & UG/KG & $J$ & $\cdots$ & & UG/KG & 1 \\
\hline Unknown Alkane-7 & 480 & UG/KG & $J$ & $\cdots$ & & UG/KG & 1 \\
\hline Unknown-1 & 860 & UG/KG & $J$ & $\cdots$ & & UG/KG & 1 \\
\hline Unknown-2 & 400 & UG/KG & $J$ & $\cdots$ & & UG/KG & 1 \\
\hline Unknown Alkane-8 & 990 & UG/KG & $\mathrm{J}$ & $\cdots$ & & UGKG & 1 \\
\hline Toluene-d8 (SUAR) & 105 & $\%$ & & & & $\%$ & 1 \\
\hline Bromofluorobenzene (SURA) & 96 & $\%$ & & & & $\%$ & 1 \\
\hline 1,2-Dichloroethane-d4 (SURR) & 103 & $\%$ & & & & $\%$ & 1 \\
\hline
\end{tabular}


Appendix C: Results of Volatile Organic Compound Analyses, Salmon Site

\begin{tabular}{|c|c|c|c|c|c|c|c|}
\hline COMPOUND & RESULTS & UNITS & $\begin{array}{l}\text { DATA } \\
\text { QUAL. }\end{array}$ & $\begin{array}{l}\text { DATA } \\
\text { VALID. }\end{array}$ & CRDL & UNITS & DILUTION \\
\hline \multicolumn{8}{|l|}{ TRA-ES-260-400-8RE } \\
\hline Chloromethane & 10 & va/kg & $u$ & A & 10 & UaKG & 1 \\
\hline Bromomethane & 10 & UGKG & $U$ & $\mathbf{R}$ & 10 & UG/KG & 1 \\
\hline Vinyl Chloride & 10 & UG/KG & $u$ & A & 10 & UG/KG & 1 \\
\hline Chloroethane & 10 & UG/KG & $U$ & R & 10 & UG/KG & 1 \\
\hline Methylene Chloride & 20 & UaKG & B & $\mathbf{R}$ & 5 & UaKG & 1 \\
\hline Acetone & 37 & UG/KG & & A & 10 & UaKG & 1 \\
\hline Carbon Disulfide & 5 & UGKG & $U$ & A & 5 & UGKG & 1 \\
\hline 1,1-Dichloroethene & 5 & UG/KG & $U$ & A & 5 & Ua/KG & 1 \\
\hline 1,1-Dichloroethane & 5 & UG/KG & $\mathbf{U}$ & R & 5 & UG/KG & 1 \\
\hline 1,2-Dichloroethene (total) & 5 & UG/KG & $u$ & A & 5 & UG/KG & 1 \\
\hline Chloroform & 5 & UG/KG & $u$ & R & 5 & UG/KG & 1 \\
\hline 1,2-Dichloroethane & 5 & UG/KG & $u$ & R & 5 & UGKG & 1 \\
\hline 2-Butanone & 10 & UG/KG & $u$ & $\mathbf{R}$ & 10 & UG/KG & 1 \\
\hline 1,1,1-Trichloroethane & 5 & UG/KG & U & $\mathbf{R}$ & 5 & VG/KG & 1 \\
\hline Carbon Tetrachloride & 5 & UG/KG & $u$ & R & 5 & UG/KG & 1 \\
\hline Vinyl Acetate & 10 & UG/KG & $U$ & R & 10 & UG/KG & 1 \\
\hline Bromodichloromethane & 5 & UG/KG & $u$ & A & 5 & UG/KG & 1 \\
\hline 1,2-Dichloropropane & 5 & UG/KG & $u$ & $\mathbf{R}$ & 5 & UGKG & 1 \\
\hline cis-1,3-Dichloropropene & 5 & UG/KG & U & $\boldsymbol{A}$ & 5 & UGKG & 1 \\
\hline Trichloroethene & 5 & UG/KG & $\mathbf{u}$ & $\mathbf{R}$ & 5 & UG/KG & 1 \\
\hline Dibromochloromethane & 5 & UG/KG & $u$ & $\mathbf{R}$ & 5 & UG/KG & 1 \\
\hline 1,1,2-Trichloroethane & 5 & UGKG & $u$ & $\mathbf{R}$ & 5 & UG/KG & 1 \\
\hline Benzene & 5 & UG/KG & $u$ & $\mathbf{R}$ & 5 & UG/KG & 1 \\
\hline trans-1,3-Dichloropropene & 5 & UG/KG & $u$ & R & 5 & UG/KG & 1 \\
\hline Bromotorm & 5 & UG/KG & $u$ & A & 5 & UG/KG & 1 \\
\hline 4-Methyl-2-Pentanone & 10 & UG/KG & $u$ & R & 10 & UG/KG & 1 \\
\hline 2-Hexanone & 10 & UG/KG & $u$ & R & 10 & UG/KG & 1 \\
\hline Tetrachloroethene & 5 & UG/KG & $u$ & $\mathbf{R}$ & 5 & UG/KG & 1 \\
\hline 1,1,2,2-Tetrachloroethane & 5 & UG/KG & $u$ & R & 5 & UG/KG & 1 \\
\hline Toluene & 5 & UG/KG & $u$ & $\mathbf{R}$ & 5 & UGKG & 1 \\
\hline Chlorobenzene & 5 & UG/KG & $U$ & $\mathbf{R}$ & 5 & UG/KG & 1 \\
\hline Ethylbenzene & 5 & UG/KG & $u$ & $\mathbf{A}$ & 5 & UG/KG & 1 \\
\hline Styrene & 5 & UG/KG & $u$ & $\mathbf{R}$ & 5 & UG/KG & 1 \\
\hline Xylenes (total) & 5 & UG/KG & $u$ & $\mathbf{A}$ & 5 & UG/KG & 1 \\
\hline Unknown Alkane-1 & 130 & UG/KG & J & $\mathbf{A}$ & & Ua/KG & 1 \\
\hline Unknown-1 & 120 & UG/KG & $J$ & A & & UG/KG & 1 \\
\hline Unknown Alkane-2 & 350 & UG/KG & $J$ & $\mathbf{A}$ & & UG/KG & 1 \\
\hline Unknown Alkane-3 & 170 & UG/KG & $\mathrm{J}$ & $\mathbf{R}$ & & UG/KG & 1 \\
\hline Unknown Alkane-4 & 160 & UG/KG & J & $\mathbf{R}$ & & UG/KG & 1 \\
\hline Unknown-2 & 350 & UG/KG & $J$ & $\mathbf{R}$ & & UG/KG & 1 \\
\hline Unknown-3 & 160 & UG/KG & J & $\mathbf{R}$ & & UG/KG & 1 \\
\hline Unknown Alkane-5 & 470 & UG/KG & J & A & & UG/KG & 1 \\
\hline Unknown-4 & 350 & UG/KG & $J$ & $\mathbf{R}$ & & UG/KG & 1 \\
\hline Unknown-5 & 78 & UG/KG & $J$ & $\mathbf{R}$ & & UG/KG & 1 \\
\hline Toluene-d8 (SURR) & 105 & $\%$ & & & & $\%$ & 1 \\
\hline Bromofluorobenzene (SURA) & 98 & $\%$ & & & & $\%$ & 1 \\
\hline 1,2-Dichloroethane-d4 (SURR) & 99 & $\%$ & & & & $\%$ & 1 \\
\hline
\end{tabular}




\section{Appendix C: Results of Volatile Organic Compound Analyses, Salmon Site}

\begin{tabular}{|c|c|c|c|c|c|c|c|}
\hline COMPOUND & RESULTS & UNITS & $\begin{array}{l}\text { DATA } \\
\text { QUAL. }\end{array}$ & $\begin{array}{l}\text { DATA } \\
\text { VALID. }\end{array}$ & CRDL & UNITS & DILUTION \\
\hline \multicolumn{8}{|l|}{ TR5-ES-590-570-12 } \\
\hline Chloromethane & 10 & UG/KG & $u$ & $\cdots$ & 10 & UG/KG & 1 \\
\hline Bromomethane & 10 & UG/KG & $u$ & $\cdots$ & 10 & UG/KG & 1 \\
\hline Vinyl Chloride & 10 & UG/KG & $u$ & $\cdots$ & 10 & UG/KG & 1 \\
\hline Chloroethane & 10 & UG/KG & $u$ & $\cdots$ & 10 & UG/KG & 1 \\
\hline Methylene Chioride & 21 & UG/KG & B & $u$ & 5 & UG/KG & 1 \\
\hline Acetone & 48 & UG/KG & B & UJ & 10 & UG/KG & 1 \\
\hline Carbon Disulfide & 5 & UG/KG & u & $\cdots$ & 5 & UG/KG & 1 \\
\hline 1,1-Dichloroethene & 5 & UG/KG & $u$ & $\cdots$ & 5 & UG/KG & 1 \\
\hline 1.1-Dichloroethane & 5 & UG/KG & $u$ & $\cdots$ & 5 & UG/KG & 1 \\
\hline 1,2-Dichloroethene (total) & 5 & UG/KG & $u$ & $\cdots$ & 5 & UG/KG & 1 \\
\hline Chloroform & 5 & UG/KG & $u$ & $\cdots$ & 5 & UG/KG & 1 \\
\hline 1.2-Dichloroethane & 5 & UG/KG & $u$ & $\cdots$ & 5 & UG/KG & 1 \\
\hline 2-Butanone & 10 & UG/KG & $u$ & UJ & 10 & UG/KG & 1 \\
\hline 1,1,1-Trichloroethane & 5 & UG/KG & $u$ & $\cdots$ & 5 & UG/KG & 1 \\
\hline Carbon Tetrachloride & 5 & UG/KG & $u$ & $\cdots$ & 5 & UG/KG & 1 \\
\hline Vinyl Acetate & 10 & UG/KG & $u$ & $\cdots$ & 10 & UG/KG & 1 \\
\hline Bromodichloromethane & 5 & UG/KG & $u$ & $\cdots$ & 5 & UG/KG & 1 \\
\hline 1,2-Dichloropropane & 5 & UG/KG & $u$ & $\cdots$ & 5 & UG/KG & 1 \\
\hline cis-1,3-Dichloropropene & 5 & UG/KG & $u$ & --- & 5 & UG/KG & 1 \\
\hline Trichloroethene & 5 & UG/KG & $u$ & $\cdots$ & 5 & UG/KG & 1 \\
\hline Dibromochloromethane & 5 & UG/KG & $u$ & $\cdots$ & 5 & UG/KG & 1 \\
\hline 1,1,2-Trichloroethane & 5 & UG/KG & $u$ & $\cdots$ & 5 & UG/KG & 1 \\
\hline Benzene & 5 & UG/KG & $u$ & $\cdots$ & 5 & UG/KG & 1 \\
\hline trans-1,3-Dichloropropene & 5 & UG/KG & $u$ & $\cdots$ & 5 & UG/KG & 1 \\
\hline Bromoform & 5 & UG/KG & $u$ & $\cdots$ & 5 & UG/KG & 1 \\
\hline 4-Methyl-2-Pentanone & 10 & UG/KG & $u$ & -- & 10 & UG/KG & 1 \\
\hline 2-Hexanone & 10 & UG/KG & $u$ & $\cdots$ & 10 & UG/KG & 1 \\
\hline Tetrachloroethene & 5 & UG/KG & $u$ & $\cdots$ & 5 & UG/KG & 1 \\
\hline 1,1,2,2-Tetrachloroethane & 5 & UG/KG & $u$ & $\cdots$ & 5 & UG/KG & 1 \\
\hline Toluene & 5 & UG/KG & $u$ & $\cdots$ & 5 & UG/KG & 1 \\
\hline Chlorobenzene & 5 & UG/KG & $u$ & $\cdots$ & 5 & UG/KG & 1 \\
\hline Etrylbenzene & 5 & UG/KG & $u$ & -.- & 5 & UG/KG & 1 \\
\hline Styrene & 5 & UG/KG & $U$ & $\cdots$ & 5 & UG/KG & 1 \\
\hline Xylenes (total) & 5 & UG/KG & $u$ & $\cdots$ & 5 & UG/KG & 1 \\
\hline Unknown-1 & 36 & UG/KG & J & $\cdots$ & & UG/KG & 1 \\
\hline Unknown-2 & 64 & UG/KG & BJ & $J$ & & UG/KG & 1 \\
\hline Unknown Alkane-1 & 38 & UG/KG & BJ & $J$ & & UG/KG & 1 \\
\hline Unknown-3 & 42 & UG/KG & j & -.- & & UG/KG & 1 \\
\hline Unknown Alkane-2 & 49 & UG/KG & J & $\cdots$ & & UG/KG & 1 \\
\hline Unknown C10H8 & 60 & UG/KG & $J$ & -- & & UG/KG & 1 \\
\hline Unknown-4 & 120 & UG/KG & $J$ & $\cdots$ & & UG/KG & 1 \\
\hline Unknown-5 & 65 & UG/KG & J & $\cdots$ & & UG/KG & 1 \\
\hline Unknown-6 & 75 & UG/KG & J & $\cdots$ & & UG/KG & 1 \\
\hline Unknown-7 & 220 & UG/KG & $\mathbf{J}$ & $\cdots$ & & UG/KG & 1 \\
\hline Unknown-8 & 21 & UG/KG & $\mathrm{J}$ & $\cdots$ & & UG/KG & 1 \\
\hline Toluene-d8 (SURA) & 106 & $\%$ & & & & $\%$ & 1 \\
\hline Bromofluorobenzene (SURR) & 101 & $\%$ & & & & $\%$ & 1 \\
\hline 1,2-Dichloroethane-d4 (SURR) & 102 & $\%$ & & & & $\%$ & 1 \\
\hline
\end{tabular}


Appendix C: Results of Volatile Organic Compound Analyses, Salmon Site

\begin{tabular}{|c|c|c|c|c|c|c|c|}
\hline COMPOUND & RESULTS & UNITS & $\begin{array}{l}\text { DATA } \\
\text { QUAL. }\end{array}$ & $\begin{array}{l}\text { DATA } \\
\text { VAUD. }\end{array}$ & CRDL & UNITS & DILUTION \\
\hline \multicolumn{8}{|l|}{ TRG-ES-495-585-12 } \\
\hline Chloromethane & 10 & UG/KG & $u$ & NP & 10 & UG/KG & 1 \\
\hline Bromomethane & 10 & UG/KG & $u$ & NP & 10 & UG/KG & 1 \\
\hline Vinyl Chloride & 10 & UGKG & $u$ & NP & 10 & UG/KG & 1 \\
\hline Chloroethane & 10 & UG/KG & $u$ & NP & 10 & UG/KG & 1 \\
\hline Methylene Chloride & 5 & UG/KG & $u$ & NP & 5 & UG/KG & 1 \\
\hline Acetone & 10 & UG/KG & $u$ & NP & 10 & UG/KG & 1 \\
\hline Carbon Disulfide & 5 & UG/KG & $u$ & NP & 5 & UG/KG & 1 \\
\hline 1.1-Dichloroethene & 5 & UG/KG & $U$ & NP & 5 & UG/KG & 1 \\
\hline 1.1-Dichloroethane & 5 & UG/KG & $U$ & NP & 5 & UG/KG & 1 \\
\hline 1,2-Dichloroethiene (total) & 5 & UG/KG & $U$ & NP & 5 & UG/KG & 1 \\
\hline Chloroform & 5 & UG/KG & $U$ & NP & 5 & UG/KG & 1 \\
\hline 1,2-Dichloroethane & 5 & UG/KG & $u$ & NP & 5 & UG/KG & 1 \\
\hline 2-Butanone & 10 & UG/KG & $u$ & NP & 10 & UG/KG & 1 \\
\hline 1,1,1-Trichloroethane & 5 & UG/KG & $u$ & NP & 5 & UG/KG & 1 \\
\hline Carbon Tetrachloride & 5 & UG/KG & $u$ & NP & 5 & UG/KG & 1 \\
\hline Vinyl Acetate & 10 & UG/KG & $u$ & NP & 10 & UG/KG & 1 \\
\hline Bromodichloromethane & 5 & UG/KG & $u$ & NP & 5 & UG/KG & 1 \\
\hline 1,2-Dichloropropane & 5 & UG/KG & $u$ & NP & 5 & UG/KG & 1 \\
\hline cis-1.3-Dichloropropene & 5 & UG/KG & $U$ & NP & 5 & UG/KG & 1 \\
\hline Trichloroethene & 5 & UG/KG & $U$ & NP & 5 & UG/KG & 1 \\
\hline Dibromochloromethane & 5 & UG/KG & $U$ & NP & 5 & UG/KG & 1 \\
\hline 1,1,2-Trichloroethane & 5 & UG/KG & $u$ & NP & 5 & UG/KG & 1 \\
\hline Benzene & 5 & UG/KG & $u$ & NP & 5 & UG/KG & 1 \\
\hline trans-1,3-Dichloropropene & 5 & UG/KG & $u$ & NP & 5 & UG/KG & 1 \\
\hline Bromoform & 5 & UG/KG & $u$ & NP & 5 & UG/KG & 1 \\
\hline 4-Methyl-2-Pentanone & 10 & UG/KG & $u$ & NP & 10 & UG/KG & 1 \\
\hline 2-Hexanone & 10 & UG/KG & $u$ & NP & 10 & UG/KG & 1 \\
\hline Tetrachioroethene & 5 & UG/KG & $u$ & NP & 5 & UG/KG & 1 \\
\hline 1,1,2,2-Tetrachloroethane & 5 & UG/KG & $U$ & NP & 5 & UG/KG & 1 \\
\hline Toluene & 5 & UG/KG & $u$ & NP & 5 & UG/KG & 1 \\
\hline Chlorobenzene & 5 & UG/KG & $u$ & NP & 5 & UG/KG & 1 \\
\hline Ethylbenzene & 5 & UG/KG & $u$ & NP & 5 & UG/KG & 1 \\
\hline Styrene & 5 & UG/KG & $u$ & NP & 5 & UG/KG & 1 \\
\hline Xylenes (total) & 5 & UG/KG & $U$ & NP & 5 & UG/KG & 1 \\
\hline Unknown-1 & 150 & UG/KG & $J$ & NP & & UG/KG & 1 \\
\hline Unknown Alkane-1 & 240 & UG/KG & $J$ & NP & & UG/KG & 1 \\
\hline Unknown Alkane-2 & 140 & UG/KG & $J$ & NP & & UG/KG & 1 \\
\hline Unknuwn-2 & 120 & UG/KG & J & NP & & UG/KG & 1 \\
\hline Unknown Alkane-3 & 380 & UG/KG & J & NP & & UG/KG & 1 \\
\hline Unknown-3 & 180 & UG/KG & $J$ & NP & & UG/KG & 1 \\
\hline Unknown-4 & 130 & UGKKG & J & NP & & UG/KG & 1 \\
\hline Unknown-5 & 280 & UGKG & $J$ & NP & & UG/KG & 1 \\
\hline Unknown-6 & 330 & UG/KG & J & NP & & UG/KG & 1 \\
\hline Unknown-7 & 140 & UG/KG & $J$ & NP & & UG/KG & 1 \\
\hline Toluene-d8 (SURA) & 106 & $\%$ & & & & $\%$ & 1 \\
\hline Bromofluorobenzene (SURR) & 99 & $\%$ & & & & $\%$ & 1 \\
\hline 1,2-Dichloroethane-d4 (SURR) & 110 & $\%$ & & & & $\%$ & 1 \\
\hline
\end{tabular}


Appendix C: Results of Volatile Organic Compound Analyses, Salmon Site

\begin{tabular}{|c|c|c|c|c|c|c|c|}
\hline COMPOUND & RESULTS & UNITS & $\begin{array}{l}\text { DATA } \\
\text { QUAL. }\end{array}$ & $\begin{array}{l}\text { DATA } \\
\text { VALID. }\end{array}$ & CRDL & UNITS & DILUTION \\
\hline \multicolumn{8}{|l|}{ TR6-ES-485-590-6 } \\
\hline Chloromethane & 10 & UG/KG & $u$ & NP & 10 & UG/KG & 1 \\
\hline Bromomethane & 10 & UG/KG & $u$ & NP & 10 & UG/KG & 1 \\
\hline Vinyl Chloride & 10 & UG/KG & $u$ & NP & 10 & UG/KG & 1 \\
\hline Chloroethane & 10 & UG/KG & $u$ & NP & 10 & UG/KG & 1 \\
\hline Methylene Chloride & 5 & UG/KG & $u$ & NP & 5 & UG/KG & 1 \\
\hline Acetone & 10 & UG/KG & $u$ & NP & 10 & UG/KG & 1 \\
\hline Carbon Disulfide & 5 & UG/KG & $u$ & NP & 5 & UG/KG & 1 \\
\hline 1,1-Dichloroethene & 5 & UG/KG & $u$ & NP & 5 & UG/KG & 1 \\
\hline 1,1-Dichloroethane & 5 & UG/KG & $u$ & NP & 5 & UG/KG & 1 \\
\hline 1,2-Dichloroethene (total) & 5 & UG/KG & $u$ & NP & 5 & UG/KG & 1 \\
\hline Chloroform & 5 & UG/KG & u & NP & 5 & UG/KG & 1 \\
\hline 1,2-Dichloroethane & 5 & UG/KG & $u$ & NP & 5 & UG/KG & 1 \\
\hline 2-Butanone & 10 & UG/KG & $u$ & NP & 10 & UG/KG & 1 \\
\hline 1,1,1-Trichloroethane & 5 & UG/KG & $\mathrm{u}$ & NP & 5 & UG/KG & 1 \\
\hline Carbon Tetrachloride & 5 & UG/KG & $u$ & NP & 5 & UG/KG & 1 \\
\hline Vinyl Acetate & 10 & UG/KG & $u$ & NP & 10 & UG/KG & 1 \\
\hline Bromodichloromethane & 5 & UG/KG & U & NP & 5 & UG/KG & 1 \\
\hline 1,2-Dichloropropane & 5 & UG/KG & $u$ & NP & 5 & UG/KG & 1 \\
\hline cis-1,3-Dichloropropene & 5 & UG/KG & U & NP & 5 & UG/KG & 1 \\
\hline Trichloroethene & 5 & UG/KG & $U$ & NP & 5 & UG/KG & 1 \\
\hline Dibromochloromethane & 5 & UG/KG & $\mathrm{U}$ & NP & 5 & UG/KG & 1 \\
\hline 1,1,2-Trichloroethan $\theta$ & 5 & UG/KG & $u$ & NP & 5 & UG/KG & 1 \\
\hline Benzene & 5 & UG/KG & $u$ & NP & 5 & UG/KG & 1 \\
\hline trans-1,3-Dichloropropene & 5 & UG/KG & $U$ & NP & 5 & UG/KG & 1 \\
\hline Bromoform & 5 & UG/KG & $\mathrm{U}$ & NP & 5 & UG/KG & 1 \\
\hline 4-Methyl-2-Pentanone & 10 & UG/KG & $U$ & NP & 10 & UG/KG & 1 \\
\hline 2-Hexanone & 10 & UG/KG & $U$ & NP & 10 & UG/KG & 1 \\
\hline Tetrachloroethene & 5 & UG/KG & U & NP & 5 & UG/KG & 1 \\
\hline 1,1,2,2-Tetrachloroethane & 5 & UG/KG & $\mathrm{U}$ & NP & 5 & UG/KG & 1 \\
\hline Toluene & 2 & UG/KG & $\mathrm{J}$ & NP & 5 & UG/KG & 1 \\
\hline Chlorobenzene & 5 & UG/KG & $\mathrm{U}$ & NP & 5 & UG/KG & 1 \\
\hline Ethylbenzene & 5 & UG/KG & U & NP & 5 & UG/KG & 1 \\
\hline Styrene & 5 & UG/KG & $u$ & NP & 5 & UG/KG & 1 \\
\hline Xylenes (total) & 5 & UG/KG & $\mathrm{U}$ & NP & 5 & UG/KG & 1 \\
\hline Toluene-d8 (SURA) & 108 & $\%$ & & & & $\%$ & 1 \\
\hline Bromofluorobenzene (SURR) & 99 & $\%$ & & & & $\%$ & 1 \\
\hline 1,2-Dichloroethane-d4 (SURR) & 112 & $\%$ & & & & $\%$ & 1 \\
\hline
\end{tabular}


Appendix C: Results of Volatile Organic Compound Analyses, Salmon Site

\begin{tabular}{|c|c|c|c|c|c|c|c|}
\hline COMPOUND & RESULTS & UNITS & $\begin{array}{l}\text { DATA } \\
\text { QUAL. }\end{array}$ & $\begin{array}{l}\text { DATA } \\
\text { VALD. } \\
\end{array}$ & CRDL & UNITS & DILUTION \\
\hline \multicolumn{8}{|l|}{ TR7-ES-558-650-5 } \\
\hline Chloromethane & 10 & UG/KG & $U$ & -- & 10 & UG/KG & 1 \\
\hline Bromomethane & 10 & UG/KG & $U$ & --- & 10 & UG/KG & 1 \\
\hline Vinyl Chloride & 10 & UG/KG & $u$ & $-\cdots$ & 10 & UG/KG & 1 \\
\hline Chloroethane & 10 & UG/KG & $U$ & $\ldots$ & 10 & UG/KG & 1 \\
\hline Methylene Chloride & 26 & UG/KG & B & $u$ & 5 & UG/KG & 1 \\
\hline Acetone & 71 & UGKG & & US & 10 & UG/KG & 1 \\
\hline Carbon Disulfide & 5 & UG/KG & $u$ & $\cdots$ & 5 & UG/KG & 1 \\
\hline 1,1-Dichloroethene & 5 & UG/KG & $u$ & $\cdots$ & 5 & UG/KG & 1 \\
\hline 1.1-Dichloroethane & 5 & UG/KG & $u$ & $-\cdots$ & 5 & UG/KG & 1 \\
\hline 1.2-Dichloroethene (total) & 5 & UG/KG & $u$ & $\cdots$ & 5 & UG/KG & 1 \\
\hline Chloroform & 5 & UG/KǴ & $u$ & $-\cdots$ & 5 & UG/KG & 1 \\
\hline 1,2-Dichloroethane & 5 & UG/KG & $u$ & $\cdots$ & 5 & UG/KG & 1 \\
\hline 2-Butanone & 10 & UG/KG & $u$ & Us & 10 & UG/KG & 1 \\
\hline 1,1,1-Trichloroethane & 5 & UG/KG & $u$ & ..- & 5 & UG/KG & 1 \\
\hline Carbon Tetrachloride & 5 & UG/KG & $u$ & -.- & 5 & UG/KG & 1 \\
\hline Vinyl Acetate & 10 & UG/KG & $U$ & $\cdots$ & 10 & UG/KG & 1 \\
\hline Bromodichloromethane & 5 & UG/KG & $u$ & $\cdots$ & 5 & UG/KG & 1 \\
\hline 1,2-Dichloropropane & 5 & UG/KG & $u$ & $\cdots$ & 5 & UG/KG & 1 \\
\hline cis-1.3-Dichloropropene & 5 & UG/KG & $u$ & $\cdots$ & 5 & UG/KG & 1 \\
\hline Trichloroethene & 5 & UG/KG & $u$ & $\cdots$ & 5 & UG/KG & 1 \\
\hline Dibromochloromethane & 5 & UǴ/KG & $u$ & $-\cdots$ & 5 & UG/KG & 1 \\
\hline 1,1,2-Trichloroethane & 5 & UG/KG & $u$ & -- & 5 & UG/KG & 1 \\
\hline Benzene & 5 & UG/KG & $u$ & -- & 5 & UG/KG & 1 \\
\hline trans-1,3-Dichloropropene & 5 & UG/KG & $u$ & $\ldots$ & 5 & UG/KG & -1 \\
\hline Bromoform & 5 & UG/KG & $u$ & -.- & 5 & $U G / K G$ & 1 \\
\hline 4-Methyl-2-Pentanone & 10 & UG/KG & $u$ & -.. & 10 & UGKG & 1 \\
\hline 2.Hexanone & 10 & UG/KG & $u$ & $-\cdots$ & 10 & UG/KG & 1 \\
\hline Tetrachloroethene & 5 & UG/KG & $u$ & $\cdots$ & 5 & UG/KG & 1 \\
\hline 1,1,2,2-Tetrachloroethane & 5 & UG/KG & $u$ & --- & 5 & UG/KG & 1 \\
\hline Toluene & 5 & UG/KG & $u$ & -.. & 5 & UG/KG & 1 \\
\hline Chlorobenzene & 5 & UG/KG. & $u$ & -.. & 5 & UG/KG & 1 \\
\hline Ethylbenzene & 5 & UG/KG & $u$ & $\cdots$ & 5 & UG/KG & 1 \\
\hline Styrene & 5 & UG/KG & u & -.. & 5 & UG/KG & 1 \\
\hline Xylenes (total) & 5 & UG/KG & $u$ & $\ldots$ & 5 & UG/KG & 1 \\
\hline Unknown Alkane-1 & 180 & UG/KG & $J$ & $\cdots$ & & UG/KG & 1 \\
\hline Unknown-1 & 130 & UG/KG & $J$ & $\ldots$ & & UG/KG & 1 \\
\hline Unknown Alkane-2 & 350 & UG/KG & J & $\ldots$ & & UG/KG & 1 \\
\hline Unknown Alkane-3 & 260 & UG/KG & J & $\cdots$ & & UG/KG & 1 \\
\hline Unknown Alkane-4 & 170 & UG/KG & $\mathrm{j}$ & $\cdots$ & & UG/KG & 1 \\
\hline Unknown-2 & 440 & UG/KG & J & $\cdots$ & & UG/KG & 1 \\
\hline Unknown-3 & 230 & UG/KG & J & $\cdots$ & & UG/KG & 1 \\
\hline Unknown-4 & 180 & UG/KG & J & $\cdots$ & & UG/KG & 1 \\
\hline Unknown-5 & 230 & UG/KG & $J$ & $\cdots$ & & UG/KG & 1 \\
\hline Unknown-6 & 590 & UG/KG & J & $\ldots$ & & UG/KG & 1 \\
\hline Toluene-d8 (SURR) & 105 & $\%$ & & & & $\%$ & 1 \\
\hline Bromofluorobenzene (SURR) & 97 & $\%$ & & & & $\%$ & 1 \\
\hline 1,2-Dichloroethane-d4 (SURR) & 97 & $\%$ & & & & $\%$ & 1 \\
\hline
\end{tabular}


Appendix C: Results of Volatile Organic Compound Analyses. Salmon Site

\begin{tabular}{|c|c|c|c|c|c|c|c|}
\hline COMPOUND & RESULTS & UNITS & $\begin{array}{l}\text { DATA } \\
\text { QUAL. }\end{array}$ & $\begin{array}{l}\text { DATA } \\
\text { VALID. }\end{array}$ & CRDL & UNITS & DILUTION \\
\hline \multicolumn{8}{|l|}{ TR7-ES-560-650-14 } \\
\hline Chloromethane & 10 & UG/KG & $u$ & $\ldots$ & 10 & UG/KG & 1 \\
\hline Bromomethane & 10 & UG/KG & $u$ & $\ldots$ & 10 & UG/KG & 1 \\
\hline Vinyl Chloride & 10 & UG/KG & $u$ & -- & 10 & UG/KG & 1 \\
\hline Chloroethane & 10 & UG/KG & $u$ & $\ldots$ & 10 & UG/KG & 1 \\
\hline Methylene Chloride & 5 & UG/KG & B & $U$ & 5 & UG/KG & 1 \\
\hline Acetone & 24 & UG/KG & $\mathbf{B}$ & WJ & 10 & UG/KG & 1 \\
\hline Carbon Disulfide & 5 & UG/KG & $u$ & $--\cdot$ & 5 & UG/KG & 1 \\
\hline 1.1-Dichloroethene & 5 & UG/KG & $u$ & -- & 5 & UG/KG & 1 \\
\hline 1,1-Dichloroethane & 5 & UG/KG & $u$ & --- & 5 & UG/KG & 1 \\
\hline 1.2-Dichloroethene (total) & 5 & UG/KG & $u$ & $\ldots$ & 5 & UG/KG & 1 \\
\hline Chloroform & 5 & UG/KG & $u$ & -- & 5 & UG/KG & 1 \\
\hline 1.2-Dichloroethane & 5 & UG/KG & $u$ & $\ldots$ & 5 & UG/KG & 1 \\
\hline 2-Butanone & 10 & UG/KG & $u$ & UJ & 10 & UG/KG & 1 \\
\hline 1,1,1-Trichloroethane & 5 & UG/KG & $u$ & -- & 5 & UG/KG & 1 \\
\hline Carbon Tetrachloride & 5 & UG/KG & $u$ & $\cdots$ & 5 & UG/KG & 1 \\
\hline Vinyl Acetate & 10 & UG/KG & $u$ & $\cdots$ & 10 & UG/KG & 1 \\
\hline Bromodichloromethane & 5 & UG/KG & $u$ & --- & 5 & UG/KG & 1. \\
\hline 1,2-Dichloropropane & 5 & UG/KG & $u$ & $\cdots$ & 5 & UG/KG & 1 \\
\hline cis-1,3-Dichloropropene & 5 & UG/KG & $u$ & $\cdots$ & 5 & UG/KG & 1 \\
\hline Trichloroethene & 5 & UG/KG & $u$ & $-\cdots$ & 5 & UG/KG & 1 \\
\hline Dibromochloromethane & 5 & UG/KG & $u$ & $\cdots$ & 5 & UG/KG & 1 \\
\hline 1,1,2-Trichloroethane & 5 & UG/KG & $u$ & $\cdots$ & 5 & UG/KG & 1 \\
\hline Benzene & 5 & UG/KG & $u$ & $\cdots$ & 5 & UG/KG & 1 \\
\hline trans-1,3-Dichloropropene & 5 & UG/KG & $u$ & -- & 5 & UG/KG & 1 \\
\hline Bromoform & 5 & UG/KG & $U$ & $\cdots$ & 5 & UG/KG & 1 \\
\hline 4-Methyl-2-Pentanone & 10 & UG/KG & $U$ & $\cdots$ & 10 & UG/KG & 1 \\
\hline 2-Hexanone & 10 & UG/KG & U & $\cdots$ & 10 & UG/KG & 1 \\
\hline Tetrachloroethene & 5 & UG/KG & $u$ & -- & 5 & UG/KG & 1 \\
\hline 1,1,2,2-Tetrachloroethane & 5 & UG/KG & u & $\cdots$ & 5 & UG/KG & 1 \\
\hline Toluene & 5 & UG/KG & $u$ & $\ldots$ & 5 & UG/KG & 1 \\
\hline Chlorobenzene & 5 & UG/KG & $u$ & $\cdots$ & 5 & UG/KG & 1 \\
\hline Ethylbenzene & 1 & UG/KG & $J$ & $\cdots$ & 5 & UG/KG & 1 \\
\hline Styrene & 5 & UG/KG & $U$ & $\cdots$ & 5 & UG/KG & 1 \\
\hline Xylenes (total) & 13 & UG/KG & & $\cdots$ & 5 & UG/KG & 1 \\
\hline Unknown Alkane-1 & 780 & UG/KG & J & $-\cdots$ & & UG/KG & 1 \\
\hline Unknown Alkane-2 & 2000 & UG/KG & BJ & J & & UG/KG & 1 \\
\hline Unknown-1 & 650 & UG/KG & J & -- & & UG/KG & 1 \\
\hline Unknown Alkane-3 & 710 & UG/KG & J & --. & & UG/KG & 1 \\
\hline Unknown Alkane-4 & 2700 & UG/KG & BJ & $J$ & & UG/KG & 1 \\
\hline Unknown Alkane-5 & 1600 & UG/KG & $J$ & -- & & UG/KG & 1 \\
\hline Unknown-2 & 760 & UG/KG & $J$ & $\cdots$ & & UG/KG & 1 \\
\hline Unknown Alkane-6 & 1300 & UG/KG & $J$ & $\cdots$ & & UG/KG & 1 \\
\hline Unknown Alkane-7 & 2800 & UG/KG & BJ & $J$ & & UG/KG & 1 \\
\hline Unknown-3 & 710 & UG/KG & $J$ & $\cdots$ & & UG/KG & 1 \\
\hline Toluene-d8 (SURR) & 103 & $\%$ & & & & $\%$ & 1 \\
\hline Bromofluorobenzene (SURR) & 101 & $\%$ & & & & $\%$ & 1 \\
\hline 1,2-Dichloroethane-d4 (SURR) & 97 & $\%$ & & & & $\%$ & 1 \\
\hline
\end{tabular}


Appendix C: Results of Volatile Organic Compound Analyses, Salmon Site

\begin{tabular}{|c|c|c|c|c|c|c|c|}
\hline COMPOUND & RESULTS & UNITS & $\begin{array}{l}\text { DATA } \\
\text { QUAL. }\end{array}$ & $\begin{array}{l}\text { DATA } \\
\text { VALD. }\end{array}$ & CRDL & UNITS & DILUTION \\
\hline \multicolumn{8}{|l|}{ TR8-ES-780-600-3 } \\
\hline Chloromethane & 10 & UG/KG & $u$ & $\cdots$ & 10 & UG/KG & 1 \\
\hline Bromomethane & 10 & UG/KG & $u$ & -- & 10 & UG/KG & 1 \\
\hline Vinyl Chloride & 10 & UG/KG & $\mathbf{U}$ & $\cdots$ & 10 & UG/KG & 1 \\
\hline Chloroethane & 10 & UG/KG & $\mathbf{U}$ & $\cdots$ & 10 & UG/KG & 1 \\
\hline Methylene Chloride & 5 & UG/KG & $u$ & UJ & 5 & UG/KG & 1 \\
\hline Acetone & 10 & UG/KG & $U$ & UJ & 10 & UG/KG & 1 \\
\hline Carbon Disulfide & 5 & UG/KG & $u$ & UJ & 5 & UG/KG & 1 \\
\hline 1,1-Dichloroethene & 5 & UG/KG & $u$ & UJ & 5 & UG/KG & 1 \\
\hline 1,1-Dlchloroethane & 5 & UG/KG & $u$ & UJ & 5 & UG/KG & 1 \\
\hline 1,2-Dichloroethene (total) & 5 & UG/KG & $U$ & UJ & 5 & UG/KG & 1 \\
\hline Chloroform & 5 & UG/KG & $U$ & UJ & 5 & UG/KG & 1 \\
\hline 1,2-Dichloroethane & 5 & UG/KG & $U$ & UJ & 5 & UG/KG & 1 \\
\hline 2-Butanone & 10 & UG/KG & $U$ & UJ & 10 & UG/KG & 1 \\
\hline 1,1,1-Trichloroethane & 5 & UG/KG & $u$ & UJ & 5 & UG/KG & 1 \\
\hline Carbon Tetrachloride & 5 & UG/KG & $u$ & UJ & 5 & UG/KG & 1 \\
\hline Vinyl Acetate & 10 & UG/KG & $u$ & UJ & 10 & UG/KG & 1 \\
\hline Bromodichloromethane & 5 & UG/KG & $u$ & UJ & 5 & UG/KG & 1 \\
\hline 1,2-Dichloropropane & 5 & UG/KG & U & UJ & 5 & UG/KG & 1 \\
\hline cis-1,3-Dichloropropene & 5 & UG/KG & $u$ & UJ & 5 & UG/KG & 1 \\
\hline Trichloroethene & 5 & UG/KG & $u$ & UJ & 5 & UG/KG & 1 \\
\hline Dibromochloromethane & 5 & UG/KG & $u$ & U.J & 5 & UG/KG & 1 \\
\hline 1,1,2-Trichloroethane & 5 & UG/KG & U & UJ & 5 & UG/KG & 1 \\
\hline Benzene & 5 & UG/KG & $u$ & UJ & 5 & UG/KG & 1 \\
\hline trans-1,3-Dichloropropene & 5 & UG/KG & $u$ & UJ & 5 & UG/KG & 1 \\
\hline Bromoform & 5 & UG/KG & $u$ & UJ & 5 & UG/KG & 1 \\
\hline 4-Methyl-2-Pentanone & 10 & UG/KG & u & UJ & 10 & UG/KG & 1 \\
\hline 2-Hexanone & 10 & UG/KG & $u$ & UJ & 10 & UG/KG & 1 \\
\hline Tetrachloroethene & 5 & UG/KG & $u$ & UJ & 5 & UG/KG & 1 \\
\hline 1,1,2,2-Tetrachloroethane & 5 & UG/KG & u & UJ & 5 & UG/KG & 1 \\
\hline Toluene & 5 & UG/KG & u & UJ & 5 & UG/KG & 1 \\
\hline Chlorobenzene & 5 & UG/KG & U & US & 5 & UG/KG & 1 \\
\hline Ethylbenzene & 5 & UG/KG & U & UJ & 5 & UG/KG & 1 \\
\hline Styrene & 5 & UG/KG & $u$ & US & 5 & UG/KG & 1 \\
\hline Xylenes (total) & 5 & UG/KG & U & US & 5 & UG/KG & 1 \\
\hline Unknown & 5 & UG/KG & $\mathrm{J}$ & $\cdots$ & & UG/KG & 1 \\
\hline Toluene-d8 (SURA) & 98 & $\%$ & & & & $\%$ & 1 \\
\hline Bromofluorobenzene (SURR) & 102 & $\%$ & & & & $\%$ & 1 \\
\hline 1,2-Dichloroethane-d4 (SURR) & 102 & $\%$ & & & & $\%$ & 1 \\
\hline
\end{tabular}


Appendix C: Results of Volatile Organic Compound Analyses, Salmon Site

\begin{tabular}{|c|c|c|c|c|c|c|c|}
\hline COMPOUND & RESULTS & UNITS & $\begin{array}{l}\text { DATA } \\
\text { QUAL. }\end{array}$ & $\begin{array}{l}\text { DATA } \\
\text { VALID. }\end{array}$ & CRDL & UNITS & DILUTION \\
\hline \multicolumn{8}{|l|}{ TR8-ES-780-600-3-MS } \\
\hline 1.1-Dichloroethene & 114 & $\%$ & & NP & & $\%$ & \\
\hline Trichloroethene & 114 & $\%$ & & NP & & $\%$ & 1 \\
\hline Benzene & 116 & $\%$ & & NP & & $\%$ & 1 \\
\hline Toluene & 120 & $\%$ & & NP & & $\%$ & 1 \\
\hline Chlorobenzene & 119 & $\%$ & & NP & & $\%$ & 1 \\
\hline Toluene-d8 (SURA) & 99 & $\%$ & & NP & & $\%$ & 1 \\
\hline Bromofluorobenzene (SURR) & 100 & $\%$ & & NP & & $\%$ & 1 \\
\hline 1,2-Dichloroethane-d4 (SURR) & 90 & $\%$ & & NP & & $\%$ & 1 \\
\hline \multicolumn{8}{|l|}{ TR8-ES-780-600-3-MSD } \\
\hline 1,1-Dichloroethene & 109 & $\%$ & & NP & & $\%$ & \\
\hline Trichloroethene & 104 & $\%$ & & NP & & $\%$ & 1 \\
\hline Benzene & 113 & $\%$ & & NP & & $\%$ & 1 \\
\hline Toluene & 109 & $\%$ & & NP & & $\%$ & 1 \\
\hline Chlorobenzene & 111 & $\%$ & & NP & & $\%$ & 1 \\
\hline Toluene-d8 (SURR) & 97 & $\%$ & & NP & & $\%$ & 1 \\
\hline Bromofluorobenzene (SURA) & 99 & $\%$ & & NP & & $\%$ & 1 \\
\hline 1.2-Dichloroethane-d4 (SURR) & 98 & $\%$ & & NP & & $\%$ & 1 \\
\hline
\end{tabular}


Appendix C: Results of Volatile Organic Compound Analyses, Salmon Site

\begin{tabular}{|c|c|c|c|c|c|c|c|}
\hline COMPOUND & RESULTS & UNITS & $\begin{array}{l}\text { DATA } \\
\text { QUAL. }\end{array}$ & $\begin{array}{l}\text { DATA } \\
\text { VALID. }\end{array}$ & CRDL & UNITS & DILUTION \\
\hline \multicolumn{8}{|l|}{ TA10-ES-180-780-15 } \\
\hline Chloromethane & 10 & UG/KG & $u$ & $\ldots$ & 10 & UG/KG & 1 \\
\hline Bromomethane & 10 & UG/KG & U & $\ldots$ & 10 & UG/KG & 1 \\
\hline Vinyl Chloride & 10 & UG/KG & U & -. & 10 & UG/KG & 1 \\
\hline Chloroethane & 10 & UG/KG & $u$ & $\cdots$ & 10 & UG/KG & 1 \\
\hline Methylene Chloride & 22 & UG/KG & B & $u$ & 5 & UG/KG & 1 \\
\hline Acetone & 42 & UG/KG & & UJ & 10 & UG/KG & 1 \\
\hline Carbon Disulfide & 5 & UG/KG & $u$ & $\cdots$ & 5 & UG/KG & 1 \\
\hline 1,1-Dichloroethene & 5 & UG/KG & $\mathrm{U}$ & $\cdots$ & 5 & UG/KG & 1 \\
\hline 1,1-Dichloroethane & 5 & UG/KG & $U$ & $\cdots$ & 5 & UG/KG & 1 \\
\hline 1,2-Dichloroethene (total) & 5 & UG/KG & 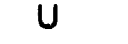 & $\cdots$ & 5 & UG/KG & 1 \\
\hline Chloroform & 5 & UG/KG & $u$ & $\cdots$ & 5 & UG/KG & 1 \\
\hline 1,2-Dichloroethane & 5 & UG/KG & $u$ & $\cdots$ & 5 & UG/KG & 1 \\
\hline 2-Butanone & 10 & UG/KG & $u$ & UJ & 10 & UG/KG & 1 \\
\hline 1.1,1-Trichloroethane & 5 & UG/KG & $u$ & $\cdots$ & 5 & UG/KG & 1 \\
\hline Carbon Tetrachioride & 5 & UG/KG & $u$ & $\cdots$ & 5 & UG/KG & 1 \\
\hline Vinyl Acetate & 10 & UG/KG & $u$ & $\cdots$ & 10 & $U G / K G$ & 1 \\
\hline Bromodichloromethane & 5 & UG/KG & $u$ & $\cdots$ & 5 & UG/KG & 1 \\
\hline 1,2-Dichloropropane & 5 & UG/KG & $u$ & $\cdots$ & 5 & UG/KG & 1 \\
\hline cis-1,3-Dichloropropene & 5 & UG/KG & $u$ & $\cdots$ & 5 & $U$ UG/KG & 1 \\
\hline Trichloroethene & 5 & UG/KG & $u$ & $\cdots$ & 5 & UG/KG & 1 \\
\hline Dibromochloromethane & 5 & UG/KG & $u$ & $\cdots$ & 5 & UG/KG & 1 \\
\hline 1,1,2-Trichloroethane & 5 & UG/KG & U & $\cdots$ & 5 & UG/KG & 1 \\
\hline Benzene & 5 & UG/KG & $U$ & $\cdots$ & 5 & UG/KG & 1 \\
\hline trans-1,3-Dichloropropene & 5 & UG/KG & U & $\cdots$ & 5 & UG/KG & 1 \\
\hline Bromoform & 5 & UG/KG & $u$ & $\cdots$ & 5 & UG/KG & 1 \\
\hline 4-Methyl-2-Pentanone & 10 & UG/KG & $u$ & $\cdots$ & 10 & UG/KG & 1 \\
\hline 2-Hexanone & 10 & UG/KG & U & $\cdots$ & 10 & UG/KG & 1 \\
\hline Tetrachloroethene & 5 & UG/KG & 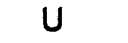 & $\cdots$ & 5 & UG/KG & 1 \\
\hline 1,1,2,2-Tetrachloroethane & 5 & UG/KG & $u$ & $\cdots$ & 5 & UG/KG & 1 \\
\hline Toluene & 3 & UG/KG & $J$ & $\cdots$ & 5 & UG/KG & 1 \\
\hline Chlorobenzene & 5 & UG/KG & U & $\cdots$ & 5 & UG/KG & 1 \\
\hline Ethylbenzene & 1 & UG/KG & $\mathrm{J}$ & $\cdots$ & 5 & UG/KG & 1 \\
\hline Styrene & 5 & UG/KG & $u$ & $\cdots$ & 5 & UG/KG & 1 \\
\hline Xylenes (total) & 6 & UG/KG & & $\cdots$ & 5 & UG/KG & 1 \\
\hline Unknown Alkane-1 & 2100 & UG/KG & $J$ & $\cdots$ & & UG/KG & 1 \\
\hline Unknown Alkane-2 & 5400 & UG/KG & $\mathrm{J}$ & $\cdots$ & & UG/KG & 1 \\
\hline Unknown Alkane-3 & 3600 & UG/KG & $\mathrm{J}$ & $\cdots$ & & UG/KG & 1 \\
\hline Unknown-1 & 3500 & UG/KG & $\mathrm{J}$ & $\cdots$ & & UG/KG & 1 \\
\hline Unknown Alkane-4 & 2500 & UG/KG & $\mathrm{J}$ & $\cdots$ & & UG/KG & 1 \\
\hline Unknown Alkane-5 & 2300 & UG/KG & $\mathrm{J}$ & $\cdots$ & & UG/KG & 1 \\
\hline Unknown Alkane-6 & 4800 & UG/KG & $\mathrm{J}$ & $\cdots$ & & UG/KG & 1 \\
\hline Unknown Alkane-7 & 4900 & UG/KG & $J$ & -- & & UG/KG & 1 \\
\hline Unknown-2 & 2600 & UG/KG & $\mathrm{J}$ & $\cdots$ & & UG/KG & 1 \\
\hline Unknown-3 & 2100 & UG/KG & J & ... & & UG/KG & 1 \\
\hline Toluene-d8 (SURA) & 110 & $\%$ & & & & $\%$ & 1 \\
\hline Bromofluorobenzene (SURR) & 79 & $\%$ & & & & $\%$ & 1 \\
\hline 1,2-Dichloroethane-d4 (SURR) & 99 & $\%$ & & & & $\%$ & 1 \\
\hline
\end{tabular}




\section{Appendix C: Results of Volatile Organic Compound Analyses, Salmon Site}

\begin{tabular}{|c|c|c|c|c|c|c|c|}
\hline COMPOUND & RESULTS & UNITS & $\begin{array}{l}\text { DATA } \\
\text { QUAL. }\end{array}$ & $\begin{array}{l}\text { DATA } \\
\text { VALD. }\end{array}$ & CRDL & UNITS & DILUTION \\
\hline \multicolumn{8}{|l|}{ TR10-ES-190-790-8 } \\
\hline Chloromethane & 10 & UG/KG & $u$ & --- & 10 & UG/KG & 1 \\
\hline Bromomethane & 10 & UG/KG & $U$ & $\cdots$ & 10 & UG/KG & 1 \\
\hline Vinyl Chloride & 10 & UG/KG & $u$ & $-\cdots$ & 10 & UG/KG & 1 \\
\hline Chloroethane & 10 & UG/KG & $u$ & $\cdots$ & 10 & UG/KG & 1 \\
\hline Methylene Chloride & 26 & UG/KG & B & $u$ & 5 & UG/KG & 1 \\
\hline Acetone & 59 & UG/KG & & UJ & 10 & UG/KG & 1 \\
\hline Carbon Disulfide & 5 & UG/KG & $u$ & $\ldots$ & 5 & UG/KG & 1 \\
\hline 1,1-Dichloroethene & 5 & UG/KG & $u$ & $\cdots$ & 5 & UG/KG & 1 \\
\hline 1,1-Dichloroethane & 5 & UG/KG & $U$ & $\cdots$ & 5 & UG/KG & 1 \\
\hline 1,2-Dichloroethene (total) & 5 & UG/KG & $U$ & $\cdots$ & 5 & UG/KG & 1 \\
\hline Chlorotorm & 5 & UG/KG & $U$ & $\cdots$ & 5 & UG/KG & 1 \\
\hline 1,2-Dichloroethane & 5 & UG/KG & $u$ & $-\cdots$ & 5 & UG/KG & 1 \\
\hline 2-Butanone & 10 & UG/KG & $U$ & UJ & 10 & UG/KG & 1 \\
\hline 1,1,1-Trichloroethane & 5 & UG/KG & $u$ & $\cdots$ & 5 & UG/KG & 1 \\
\hline Carbon Tetrachloride & 5 & UG/KG & $u$ & $\cdots$ & 5 & UG/KG & 1 \\
\hline Vinyl Acetate & 10 & UG/KG & $u$ & $\cdots$ & 10 & UG/KG & 1 \\
\hline Bromodichloromethane & 5 & UG/KG & $u$ & $\cdots$ & 5 & UG/KG & 1 \\
\hline 1,2-Dichloropropane & 5 & UG/KG & $u$ & $\cdots$ & 5 & $U G / K G$ & 1 \\
\hline cis-1,3-Dichloropropene & 5 & UG/KG & $u$ & $\cdots$ & 5 & UG/KG & 1 \\
\hline Trichloroethene & 5 & UG/KG & $u$ & $\cdots$ & 5 & UG/KG & 1 \\
\hline Dibromochloromethane & 5 & UG/KG & $u$ & -.. & 5 & UG/KG & 1 \\
\hline 1,1,2-Trichloroethane & 5 & UG/KG & $u$ & $\cdots$ & 5 & UG/KG & 1 \\
\hline Benzene & 5 & UG/KG & U & $\cdots$ & 5 & UG/KG & 1 \\
\hline trans-1,3-Dichloropropene & 5 & UG/KG & $u$ & $\cdots$ & 5 & UG/KG & 1 \\
\hline Bromoform & 5 & UG/KG & $u$ & $\cdots$ & 5 & UG/KG & 1 \\
\hline 4-Methyl-2-Pentanone & 10 & UG/KG & $u$ & -- & 10 & UG/KG & 1 \\
\hline 2-Hexanone & 10 & UG/KG & $u$ & --- & 10 & UG/KG & 1 \\
\hline Tetrachloroethene & 5 & UG/KG & $u$ & --- & 5 & UG/KG & 1 \\
\hline 1,1,2,2-Tetrachloroethane & 5 & UG/KG & $u$ & --- & 5 & UG/KG & 1 \\
\hline Toluene & 5 & UG/KG & $u$ & -- & 5 & UG/KG & 1 \\
\hline Chlorobenzene & 5 & UG/KG & $u$ & $\cdots$ & 5 & UG/KG & 1 \\
\hline Ethylbenzene & 5 & UG/KG & $u$ & $\cdots$ & 5 & UG/KG & 1 \\
\hline Styrene & 5 & UG/KG & $u$ & $\cdots$ & 5 & UG/KG & 1 \\
\hline Xylenes (total) & 5 & UG/KG & $u$ & $\cdots$ & 5 & UG/KG & 1 \\
\hline Unknown-1 & 14 & UG/KG & $J$ & $\cdots$ & & UG/KG & 1 \\
\hline Unknown Alkane-1 & 15 & UG/KG & $J$ & --- & & UG/KG & 1 \\
\hline Unknown Alkane-2 & 32 & UG/KG & $J$ & $\cdots$ & & UG/KG & 1 \\
\hline Unknown Alkane-3 & 13 & UG/KG & J & $\cdots$ & & UG/KG & 1 \\
\hline Unknown-2 & 11 & UG/KG & $J$ & $\cdots$ & & UG/KG & 1 \\
\hline Unknown Alkane-4 & 14 & UG/KG & J & $\cdots$ & & UG/KG & 1 \\
\hline Unknown-3 & 22 & UG/KG & $J$ & $\cdots$ & & UG/KG & 1 \\
\hline Unknown-4 & 16 & UG/KG & J & $\cdots$ & & UG/KG & 1 \\
\hline Unknown Alkane-5 & 17 & UG/KG & J & $\cdots$ & & UG/KG & 1 \\
\hline Unknown-5 & 20 & UG/KG & J & $\cdots$ & & UG/KG & 1 \\
\hline Toluene-d8 (SURR) & 106 & $\%$ & & & & $\%$ & 1 \\
\hline Bromofluorobenzene (SURR) & 97 & $\%$ & & & & $\%$ & 1 \\
\hline 1,2-Dichloroethane-d4 (SURR) & 100 & $\%$ & & & & $\%$ & 1 \\
\hline
\end{tabular}




\section{Appendix C: Results of Volatile Organic Compound Analyses, Salmon Site}

\begin{tabular}{|c|c|c|c|c|c|c|c|}
\hline COMPOUND & RESULTS & UNITS & $\begin{array}{l}\text { DATA } \\
\text { QUAL. }\end{array}$ & $\begin{array}{l}\text { DATA } \\
\text { VALID. }\end{array}$ & CRDL & UNITS & DILUTION \\
\hline \multicolumn{8}{|l|}{ TA11-ES-180-930-3 } \\
\hline Chloromethane & 10 & UG/KG & $u$ & NP & 10 & UG/KG & 1 \\
\hline Bromomethane & 10 & UG/KG & u & NP & 10 & UG/KG & 1 \\
\hline Vinyl Chloride & 10 & UG/KG & $u$ & NP & 10 & UG/KG & 1 \\
\hline Chloroethane & 10 & UG/KG & $\mathrm{U}$ & NP & 10 & UG/KG & 1 \\
\hline Methylene Chloride & 5 & UG/KG & $U$ & NP & 5 & UG/KG & 1 \\
\hline Acetone & 10 & UG/KG & $\mathbf{u}$ & NP & 10 & UG/KG & 1 \\
\hline Carbon Disulfide & 5 & UG/KG & $u$ & NP & 5 & UG/KG & 1 \\
\hline 1,1-Dichloroethene & 5 & UG/KG & $u$ & NP & 5 & UG/KG & 1 \\
\hline 1.1-Dichloroethane & 5 & UG/KG & $u$ & NP & 5 & UG/KG & 1 \\
\hline 1,2-Dichloroethene (total) & 5 & UG/KG & $u$ & NP & 5 & UG/KG & 1 \\
\hline Chluroform & 5 & UG/KG & $u$ & NP & 5 & UG/KG & 1 \\
\hline 1,2-Dichloroethane & 5 & UG/KG & $\mathrm{u}$ & NP & 5 & UG/KG & 1 \\
\hline 2-Butanone & 10 & UG/KG & $\mathbf{u}$ & NP & 10 & UG/KG & 1 \\
\hline 1,1,1-Trichloroethane & 5 & UG/KG & $\mathbf{u}$ & NP & 5 & UG/KG & 1 \\
\hline Carbon Tetrachloride & 5 & UG/KG & $u$ & NP & 5 & UG/KG & 1 \\
\hline Vinyl Acetate & 10 & UG/KG & $u$ & NP & 10 & UG/KG & 1 \\
\hline Bromodichloromethane & 5 & UG/KG & $\mathbf{u}$ & NP & 5 & UG/KG & 1 \\
\hline 1,2-Dichloropropane & 5 & UG/KG & $u$ & NP & 5 & UG/KG & 1 \\
\hline cis-1,3-Dichloropropene & 5 & UG/KG & $u$ & NP & 5 & UG/KG & 1 \\
\hline Trichloroethene & 5 & UG/KG & $u$ & NP & 5 & UG/KG & 1 \\
\hline Dibromochloromethane & 5 & UG/KG & $u$ & NP & 5 & UG/KG & 1 \\
\hline 1,1,2-Trichloroethane & 5 & UG/KG & $\mathbf{u}$ & NP & 5 & UG/KG & 1 \\
\hline Benzene & 5 & UG/KG & $U$ & NP & 5 & UG/KG & 1 \\
\hline trans-1,3-Dichloropropene & 5 & UG/KG & $u$ & NP & 5 & UG/KG & 1 \\
\hline Bromoform & 5 & UG/KG & U & NP & 5 & UG/KG & 1 \\
\hline 4-Methyl-2-Pentanone & 10 & UG/KG & $\mathbf{U}$ & NP & 10 & UG/KG & 1 \\
\hline 2-Hexanone & 10 & UG/KG & U & NP & 10 & UG/KG & 1 \\
\hline Tetrachloroethene & 5 & UG/KG & $\mathbf{U}$ & NP & 5 & UG/KG & 1 \\
\hline 1,1,2,2-Tetrachloroethane & 5 & UG/KG & U & NP & 5 & UG/KG & 1 \\
\hline Toluene & 5 & UG/KG & U & NP & 5 & UGKG & 1 \\
\hline Chlorobenzene & 5 & UG/KG & $\mathrm{U}$ & NP & 5 & UG/KG & 1 \\
\hline Ethylbenzene & 5 & UG/KG & $U$ & NP & 5 & UG/KG & 1 \\
\hline Styrene & 5 & UG/KG & U & NP & 5 & UG/KG & 1 \\
\hline Xylenes (total) & 5 & UG/KG & U & NP & 5 & UG/KG & 1 \\
\hline Unknown & 84 & UG/KG & $\mathrm{J}$ & NP & & UG/KG & 1 \\
\hline Toluene-d8 (SURR) & 109 & $\%$ & & & & $\%$ & 1 \\
\hline Bromofluorobenzene (SURR) & 95 & $\%$ & & & & $\%$ & 1 \\
\hline 1,2-Dichloroethane-d4 (SURR) & 100 & $\%$ & & & & $\%$ & 1 \\
\hline
\end{tabular}


Appendix C: Results of Volatile Organic Compound Analyses, Salmon Site

\begin{tabular}{|c|c|c|c|c|c|c|c|}
\hline COMPOUND & RESULTS & UNITS & $\begin{array}{l}\text { DATA } \\
\text { QUAL. }\end{array}$ & $\begin{array}{l}\text { DATA } \\
\text { VALID. }\end{array}$ & CRDL & UNITS & DILUTION \\
\hline \multicolumn{8}{|l|}{ TA11-ES-180-930-2AE } \\
\hline Chloromethane & 10 & UG/KG & $u$ & NP & 10 & UC/KG & 1 \\
\hline Bromomethane & 10 & Ua/KG & $u$ & NP & 10 & UaKK & 1 \\
\hline Vinyl Chloride & 10 & UG/KG & $u$ & NP & 10 & UGKG & 1 \\
\hline Chloroethane & 10 & UG/KG & $u$ & NP & 10 & UG/KG & 1 \\
\hline Methylene Chloride & 5 & UGKG & $u$ & NP & 5 & UG/KG & 1 \\
\hline Acetone & 10 & UG/KG & $u$ & NP & 10 & UaKKG & 1 \\
\hline Carbon Disulfide & 5 & UG/KG & $U$ & NP & 5 & UG/KG & 1 \\
\hline 1,1-Dichloroethene & 5 & UQ/KG & $u$ & NP & 5 & UG/KG & 1 \\
\hline 1,1-Dichloroethane & 5 & UG/KG & $u$ & NP & 5 & UG/KG & 1 \\
\hline 1,2-Dichloroethene (total) & 5 & UG/KG & $U$ & NP & 5 & UG/KG & 1 \\
\hline Chlorotorm & 5 & UG/KG & $u$ & NP & 5 & UG/KG & $\mathrm{L}$ \\
\hline 1,2-Dichloroethane & 5 & UG/KG & $U$ & NP & 5 & UG/KG & 1 \\
\hline 2-Butanone & 10 & UG/KG & $u$ & NP & 10 & UG/KG & 1 \\
\hline 1,1,1-Trichloroethane & 5 & UG/KG & $u$ & NP & 5 & UG/KG & 1 \\
\hline Carbon Tetrachlonde & 5 & UG/KG & $u$ & NP & 5 & UG/KG & 1 \\
\hline Vinyl Acetate & 10 & UG/KG & $u$ & NP & 10 & UG/KG & 1 \\
\hline Bromodichloromethane & 5 & UG/KG & $u$ & NP & 5 & UG/KG & 1 \\
\hline 1,2-Dichloropropane & 5 & UG/KG & $u$ & NP & 5 & UG/KG & 1 \\
\hline cis-1,3-Dichloropropene & 5 & UG/KG & $U$ & NP & 5 & UG/KG & 1 \\
\hline Trichloroethene & 5 & UG/KG & $u$ & NP & 5 & UG/KG & 1 \\
\hline Dibromochloromethane & 5 & UG/KG & $u$ & NP & 5 & UG/KG & 1 \\
\hline 1,1,2-Trichloroethane & 5 & UG/KG & U & NP & 5 & UG/KG & 1 \\
\hline Benzene & 5 & UG/KG & $u$ & NP & 5 & UG/KG & 1 \\
\hline trans-1,3-Dichloropropene & 5 & UG/KG & $u$ & NP & 5 & UG/KG & 1 \\
\hline Bromoform & 5 & UG/KG & $u$ & NP & 5 & UG/KG & 1 \\
\hline 4-Methyl-2-Pentanone & 10 & UG/KG & $u$ & NP & 10 & UG/KG & 1 \\
\hline 2-Hexanone & 10 & UG/KG & $U$ & NP & 10 & UG/KG & 1 \\
\hline Tetrachloroethene & 5 & UG/KG & $u$ & NP & 5 & UG/KG & 1 \\
\hline 1,1,2,2-Tetrachloroethan $\theta$ & 5 & UG/KG & $u$ & NP & 5 & UG/KG & 1 \\
\hline Toluene & 5 & UG/KG & $u$ & NP & 5 & UG/KG & 1 \\
\hline Chlorobenzene & 5 & UG/KG & $U$ & NP & 5 & UG/KG & 1 \\
\hline Ethylbenzene & 5 & UG/KG & $u$ & NP & 5 & UG/KG & 1 \\
\hline Styrene & 5 & UG/KG & $U$ & NP & 5 & UG/KG & 1 \\
\hline Xylenes (total) & 5 & UG/KG & $U$ & NP & 5 & UG/KG & 1 \\
\hline Toluene-d8 (SURA) & 102 & $\%$ & & & & $\%$ & 1 \\
\hline Bromofluorobenzene (SURR) & 98 & $\%$ & & & & $\%$ & 1 \\
\hline 1,2-Dichloroethane-d4 (SURA) & 104 & $\%$ & & & & $\%$ & 1 \\
\hline
\end{tabular}


Appendix C: Results of Volatile Organic Compound Analyses, Salmon Site

\begin{tabular}{|c|c|c|c|c|c|c|c|}
\hline COMPOUND & RESULTS & UNITS & $\begin{array}{l}\text { DATA } \\
\text { QUAL. }\end{array}$ & $\begin{array}{l}\text { DATA } \\
\text { VALD. }\end{array}$ & CRDL & UNITS & DILUTION \\
\hline \multicolumn{8}{|l|}{ TA13-E8-210-1110-8 } \\
\hline Chloromethane & 10 & UG/KG & $U$ & NP & 10 & UGKG & 1 \\
\hline Bromomethane & 10 & UG/KG & U & NP & 10 & UGKG & 1 \\
\hline Vinyl Chlonde & 10 & UG/KG & $u$ & NP & 10 & UG/KG & 1 \\
\hline Chloroethane & 10 & UG/KG & U & NP & 10 & UG/KG & 1 \\
\hline Methylene Chloride & 5 & UCKK & $U$ & NP & 5 & UC/KG & 1 \\
\hline Acotone & 10 & UGKG & $u$ & NP & 10 & UG/KG & 1 \\
\hline Carbon Disulfide & 5 & UaKG & $u$ & NP & 5 & UaKG & 1 \\
\hline 1.1-Olchloroethene & 5 & UG/KG & $u$ & NP & 5 & UG/KG & 1 \\
\hline 1,1-Dichloroethane & 5 & UG/KG & $u$ & NP & 5 & UGKG & 1 \\
\hline 1.2-Dichloroethene (total) & 5 & UG/KG & $u$ & NP & 5 & UG/KG & 1 \\
\hline Chloroform & 5 & UG/KG & U & NP & 5 & UGKG & 1 \\
\hline 1.2-Dichloroethane & 5 & UG/KG & $U$ & NP & 5 & UG/KG & 1 \\
\hline 2-Butanone & 10 & UG/KG & $U$ & NP & 10 & UGKG & 1 \\
\hline 1,1,1-Trichloroethane & 5 & UG/KG & $U$ & NP & 5 & Ua/KG & 1 \\
\hline Carbon Tetrachloride & 5 & UG/KG & $U$ & NP & 5 & UG/KG & 1 \\
\hline Vinyl Acetate & 10 & UG/KG & U & NP & 10 & UG/KG & 1 \\
\hline Bromodichloromethane & 5 & UG/KG & $U$ & NP & 5 & UG/KG & 1 \\
\hline 1,2-Dichloropropane & 5 & UG/KG & $u$ & NP & 5 & UG/KG & 1 \\
\hline cis-1,3-Dichloropropene & 5 & UG/KG & $u$ & NP & 5 & UG/KG & 1 \\
\hline Trichloroethene & 5 & UG/KG & $u$ & NP & 5 & UG/KG & 1 \\
\hline Dibromochloromethane & 5 & UG/KG & $u$ & NP & 5 & UG/KG & 1 \\
\hline 1,1,2-Trichloroethane & 5 & UGKG & $U$ & NP & 5 & UGKG & 1 \\
\hline Benzene & 5 & UG/KG & $u$ & NP & 5 & UG/KG & 1 \\
\hline trans-1,3-Dichloropropene & 5 & UG/KG & $U$ & NP & 5 & UG/KG & 1 \\
\hline Bromoform & 5 & UG/KG & $U$ & NP & 5 & UG/KG & 1 \\
\hline 4-Methyl-2-Pentanone & 10 & UG/KG & $U$ & NP & 10 & UG/KG & 1 \\
\hline 2-Hexanone & 10 & UG/KG & U & NP & 10 & UGKG & 1 \\
\hline Tetrachloroethene & 28 & UG/KG & & NP & 5 & UG/KG & 1 \\
\hline 1,1,2,2-Tetrachloroethane & 5 & UG/KG & $U$ & NP & 5 & UG/KG & 1 \\
\hline Toluene & 5 & UG/KG & $U$ & NP & 5 & UG/KG & 1 \\
\hline Chlorobenzene & 5 & UG/KG & $U$ & NP & 5 & UG/KG & 1 \\
\hline Ethylbenzene & 5 & UG/KG & U & NP & 5 & UG/KG & 1 \\
\hline Styrene & 5 & UG/KG & $U$ & NP & 5 & UG/KG & 1 \\
\hline Xylenes (total) & 5 & UG/KG & $u$ & NP & 5 & UG/KG & 1 \\
\hline Unknown-1 & 100 & UG/KG & $\mathrm{J}$ & NP & & UG/KG & 1 \\
\hline Unknown-2 & 230 & UG/KG & $J$ & NP & & UG/KG & 1 \\
\hline Unknown-3 & 130 & UG/KG & $J$ & NP & & UG/KG & 1 \\
\hline Unknown C11H14-1 & 150 & UG/KG & $J$ & NP & & UG/KG & 1 \\
\hline Naphthalene & 170 & UGKG & $J$ & NP & & UG/KG & 1 \\
\hline Unknown-4 & 430 & UG/KG & $J$ & NP & & UG/KG & 1 \\
\hline Unknown C11H14-2 & 300 & UG/KG & $J$ & NP & & UG/KG & 1 \\
\hline Unknown C11H14-3 & 280 & UG/KG & J & NP & & UaKG & 1 \\
\hline Unknown C11H14-4 & 800 & UG/KG & $J$ & NP & & UG/KG & 1 \\
\hline Unknown-5 & 130 & UG/KG & $J$ & NP & & UG/KG & 1 \\
\hline Toluene-d8 (SL'RR) & 103 & $\%$ & & & & $\%$ & 1 \\
\hline Bromofluorobenzene (SURR) & 100 & $\%$ & & & & $\%$ & 1 \\
\hline 1,2-Dichloroethane-d4 (SURA) & 97 & $\%$ & & & & $\%$ & 1 \\
\hline
\end{tabular}




\section{Appendlx C: Results of Volatile Organic Compound Analyses, Salmon Site}

\begin{tabular}{|c|c|c|c|c|c|c|c|}
\hline COMPOUND & RESULTS & UNITS & $\begin{array}{l}\text { DATA } \\
\text { QUAL. }\end{array}$ & $\begin{array}{l}\text { DATA } \\
\text { VALID. }\end{array}$ & CRDL & UNITS & DILUTION \\
\hline \multicolumn{8}{|l|}{ TA13-E8-212-1150-6 } \\
\hline Chloromethane & 10 & Ua/KG & $u$ & NP & 10 & UG/KG & 1 \\
\hline Bromomethane & 10 & UaKG & $u$ & NP & 10 & UGKG & 1 \\
\hline Vinyl Chloride & 10 & UGKG & $u$ & NP & 10 & UaKa & 1 \\
\hline Chloroethane & 10 & UG/KG & $u$ & NP & 10 & UG/KG & 1 \\
\hline Methylene Chloride & 5 & UG/KG & $u$ & NP & 5 & UG/KG & 1 \\
\hline Acetone & 10 & UG/KG & $u$ & NP & 10 & UGKG & 1 \\
\hline Carbon Disulfide & 5 & Ua/KG & $U$ & NP & 5 & UG/KG & 1 \\
\hline 1,1-Dichloroethene & 5 & UG/KG & U & NP & 5 & UGKG & 1 \\
\hline 1,1-Dichloroethane & 5 & UG/KG & $u$ & NP & 5 & UG/KG & 1 \\
\hline 1,2-Dichloroethene (total) & 5 & UG/KG & $u$ & NP & 5 & UG/KG & 1 \\
\hline Chloroform & 5 & UG/KG & $u$ & NP & 5 & UG/KG & 1 \\
\hline 1,2-Dichloroethane & $\mathbf{5}$ & Ua/KG & $u$ & NP & 5 & UG/KG & 1 \\
\hline 2-Butanone & 10 & UG/KG & 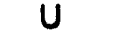 & NP & 10 & Ua/KG & 1 \\
\hline 1,1,1-Trichloroethane & 5 & UG/KG & $u$ & NP & 5 & UG/KG & 1 \\
\hline Carbon Tetrachioride & 5 & UG/KG & $u$ & NP & 5 & UG/KG & 1 \\
\hline Vinyl Acetate & 10 & UG/KG & $u$ & NP & 10 & UG/KG & 1 \\
\hline Bromodichloromethane & 5 & UG/KG & $u$ & NP & 5 & UG/KG & 1 \\
\hline 1,2-Dichloropropane & 5 & UG/KG & U & NP & 5 & UG/KG & 1 \\
\hline cis-1,3-Dichloropropene & 5 & UGKG & U & NP & 5 & UG/KG & 1 \\
\hline Trichloroethene & 19 & UG/KG & & NP & 5 & UG/KG & 1 \\
\hline Dibromochloromethane & 5 & UG/KG & $u$ & NP & 5 & UG/KG & 1 \\
\hline 1,1,2-Trichloroethane & 5 & UG/KG & $u$ & NP & 5 & UG/KG & 1 \\
\hline Benzene & 5 & UG/KG & $u$ & NP & 5 & UG/KG & 1 \\
\hline trans-1,3-Dichloropropene & 5 & UG/KG & $u$ & NP & 5 & UG/KG & 1 \\
\hline Bromoform & 5 & UG/KG & $u$ & NP & 5 & UG/KG & 1 \\
\hline 4-Methyl-2-Pentanone & 10 & UG/KG & $\mathbf{u}$ & NP & 10 & Ua/KG & 1 \\
\hline 2-Hexanone & 10 & UGKG & U & NP & 10 & UG/KG & 1 \\
\hline Tetrachloroethene & 18 & UG/KG & & NP & 5 & UG/KG & 1 \\
\hline 1,1,2,2-Tetrachloroethane & 5 & UG/KG & & NP & 5 & UG/KG & 1 \\
\hline Toluene & 3 & UG/KG & J & NP & 5 & UG/KG & 1 \\
\hline Chlorobenzene & 5 & UG/KG & U & NP & 5 & UG/KG & 1 \\
\hline Etnylbenzene & 6 & UG/KG & & NP & 5 & UG/KG & 1 \\
\hline Styrene & 5 & UG/KG & U & NP & 5 & UG/KG & 1 \\
\hline Xylenes (total) & 12 & UG/KG & & NP & 5 & UG/KG & 1 \\
\hline Unknown-1 & 450 & UG/KG & $J$ & NP & & UG/KG & 1 \\
\hline Unknown-2 & 680 & UG/KG & $J$ & NP & & UG/KG & 1 \\
\hline Unknown Alkane-1 & 1000 & UG/KG & $\mathrm{J}$ & NP & & UG/KG & 1 \\
\hline Unknown Alkane-2 & 1300 & UG/KG & $J$ & NP & & UG/KG & 1 \\
\hline Unknown Alkane-3 & 760 & UG/KG & $J$ & NP & & UG/KG & 1 \\
\hline Naphthalene & 1400 & UG/KG & $\mathrm{J}$ & NP & & UG/KG & 1 \\
\hline Unknown-3 & 1300 & UG/KG & $J$ & NP & & UG/KG & 1 \\
\hline Unknown-4 & 1000 & UG/KG & $J$ & NP & & UG/KG & 1 \\
\hline Unknown-5 & 1300 & UG/KG & $\mathrm{J}$ & NP & & UG/KG & 1 \\
\hline Unknown-6 & 2400 & UG/KG & $J$ & NP & & UG/KG & 1. \\
\hline Toluene-d8 (SURR) & 103 & $\%$ & & & & $\%$ & 1 \\
\hline Bromofluorobenzene (S & 104 & $\%$ & & & & $\%$ & 1 \\
\hline 1,2-Dichloroetha & 110 & $\%$ & & & & $\%$ & 1 \\
\hline
\end{tabular}


Appendix C: Results of Volatile Organic Compound Analyses, Salmon Site

\begin{tabular}{|c|c|c|c|c|c|c|c|}
\hline COMPOUND & RESULTS & UNITS & $\begin{array}{l}\text { DATA } \\
\text { QLAL. }\end{array}$ & $\begin{array}{l}\text { DATA } \\
\text { VALID. }\end{array}$ & CRDL & UNITS & DILUTION \\
\hline \multicolumn{8}{|l|}{ TR14-ES00-1180.6 } \\
\hline Chloromethane & 10 & UG/KG & $U$ & NP & 10 & UG/KG & 1 \\
\hline Bromomethane & 10 & UG/KG & $u$ & NP & 10 & UG/KG & 1 \\
\hline Vinyl Chloride & 10 & UG/KG & $u$ & NP & 10 & UG/KG & 1 \\
\hline Chloroethane & 10 & UG/KG & $u$ & NP & 10 & UG/KG & 1 \\
\hline Methylene Chloride & 5 & UG/KG & $u$ & NP & 5 & UG/KG & 1 \\
\hline Acetone & 10 & UG/KG & $U$ & NP & 10 & UG/KG & 1 \\
\hline Carbon Disulfide & 5 & UG/KG & $U$ & NP & 5 & UG/KG & 1 \\
\hline 1,1-Dichloroethene & 5 & UG/KG & $U$ & NP & 5 & UG/KG & 1 \\
\hline 1.1-Dichloroethane & 5 & UG/KG & $U$ & NP & 5 & Ua/KG & 1 \\
\hline 1,2-Dichloroethene (total) & 5 & UG/KG & $U$ & NP & 5 & UG/KG & 1 \\
\hline Chloroform & 5 & UG/KG & $u$ & NP & 5 & UG/KG & 1 \\
\hline 1,2-Dichloroethane & 5 & UG/KG & $u$ & NP & 5 & UG/KG & 1 \\
\hline 2-Butanone & 10 & UG/KG & $u$ & NP & 10 & Ua/Ka & 1 \\
\hline 1,1,1-Trichloroethane & 5 & UG/KG & $U$ & NP & 5 & UG/KG & 1 \\
\hline Carbon Tetrachloride & 5 & UG/KG & $U$ & NP & 5 & UG/KG & 1 \\
\hline Vinyl Acetate & 10 & UG/KG & $U$ & NP & 10 & UG/KG & 1 \\
\hline Bromodichloromethane & 5 & UG/KG & $\mathbf{U}$ & NP & 5 & UG/KG & 1 \\
\hline 1,2-Dlchloropropane & 5 & UG/KG & U & NP & 5 & UG/KG & 1 \\
\hline cis-1,3-Dichloropropene & 5 & UG/KG & $u$ & NP & 5 & UQISG & 1 \\
\hline Trichloroethene & 5 & UG/KG & $u$ & NP & 5 & UG/KG & 1 \\
\hline Dibromochloromethane & 5 & UG/KG & $u$ & NP & 5 & UG/KG & 1 \\
\hline 1,1,2-Trichloroethane & 5 & UG/KG & $u$ & NP & 5 & UG/KG & 1 \\
\hline Benzene & 5 & UG/KG & $U$ & NP & 5 & UG/KG & 1 \\
\hline trans-1,3-Dichloropropene & 5 & UG/KG & $\mathbf{U}$ & NP & 5 & UG/KG & 1 \\
\hline Bromoform & 5 & UG/KG & $U$ & NP & 5 & UG/KG & 1 \\
\hline 4-Methyl-2-Pentanone & 10 & UG/KG & U & NP & 10 & UGKG & 1 \\
\hline 2-Hexanone & 10 & UG/KG & $\mathbf{U}$ & NP & 10 & UG/KG & 1 \\
\hline Tetrachloroethene & 5 & UG/KG & $\mathbf{U}$ & NP & 5 & Ua/KG & 1 \\
\hline 1,1,2,2-Tetrachloroethane & 5 & UG/KG & $\mathbf{U}$ & NP & 5 & UG/KG & 1 \\
\hline Toluene & 5 & UG/KG & U & NP & 5 & UG/KG & 1 \\
\hline Chlorobenzene & 5 & UG/KG & U & NP & 5 & UG/KG & 1 \\
\hline Ethylbenzene & 5 & UG/KG & U & NP & 5 & UG/KG & 1 \\
\hline Styrene & 5 & UG/KG & $U$ & NP & 5 & UG/KG & 1 \\
\hline Xylenes (total) & 5 & UG/KG & U & NP & 5 & UG/KG & 1 \\
\hline Unknown-1 & 5 & UG/KG & $J$ & NP & & UG/KG & 1 \\
\hline Unknown-2 & 6 & UG/KG & J & NP & & UG/KG & 1 \\
\hline Unknown-3 & 8 & UG/KG & $J$ & NP & & UG/KG & 1 \\
\hline Unknown-4 & 10 & UG/KG & $J$ & NP & & UG/KG & 1 \\
\hline Unknown-5 & 7 & UG/KG & $J$ & NP & & UG/KG & 1 \\
\hline Unknown-6 & 7 & UG/KG & J & NP & & UG/KG & 1 \\
\hline Unknown-7 & 28 & UG/KG & $J$ & NP & & UGKG & 1 \\
\hline Toluene-d8 (SURR) & 98 & $\%$ & & & & $\%$ & 1 \\
\hline Bromofluorobenzene (SURR) & 106 & $\%$ & & & & $\%$ & 1 \\
\hline 1,2-Dichloroethane-d4 (SURR) & 109 & $\%$ & & & & $\%$ & 1 \\
\hline
\end{tabular}




\section{Appendix C: Results of Volatile Organic Compound Analyses, Salmon Site}

\begin{tabular}{|c|c|c|c|c|c|c|c|}
\hline COMPOUND & RESULTS & UNITS & $\begin{array}{l}\text { DATA } \\
\text { QUAL. }\end{array}$ & $\begin{array}{l}\text { DATA } \\
\text { VALID. }\end{array}$ & CRDL & UNITS & DILUTION \\
\hline \multicolumn{8}{|l|}{ TR14-ES-90-1200-3 } \\
\hline Chloromethane & 10 & UG/KG & $u$ & NP & 10 & UG/KG & 1 \\
\hline Bromomethane & 10 & UG/KG & $u$ & NP & 10 & UG/KG & 1 \\
\hline Vinyl Chloride & 10 & UG/KG & $u$ & NP & 10 & UG/KG & 1 \\
\hline Chloroethane & 10 & UG/KG & $u$ & NP & 10 & UG/KG & 1 \\
\hline Methylene Chloride & 5 & UG/KG & $U$ & NP & 5 & UG/KG & 1 \\
\hline Acetone & 10 & UG/KG & u & NP & 10 & UG/KG & 1 \\
\hline Carbon Disulfide & 5 & UG/KG & $u$ & NP & 5 & UG/KG & 1 \\
\hline 1,1-Dichloroethene & 5 & UG/KG & $\mathbf{u}$ & NP & 5 & UG/KG & 1 \\
\hline 1,1-Dichloroethane & 5 & UG/KG & $u$ & NP & 5 & UG/KG & 1 \\
\hline 1,2-Dichloroethene (total) & 5 & UG/KG & $u$ & NP & 5 & UG/KG & 1 \\
\hline Chloroform & 5 & UG/KG & $u$ & NP & 5 & UG/KG & 1 \\
\hline 1,2-Dichloroethane & 5 & UG/KG & $u$ & NP & 5 & UG/KG & 1 \\
\hline 2-Butanone & 10 & UG/KG & $u$ & NP & 10 & UG/KG & 1 \\
\hline 1,1,1-Trichloroethane & 5 & UG/KG & $u$ & NP & 5 & UG/KG & 1 \\
\hline Carbon Tetrachloride & 5 & UG/KG & $u$ & NP & 5 & UG/KG & 1 \\
\hline Vinyl Acetate & 10 & UG/KG & $u$ & NP & 10 & UG/KG & 1 \\
\hline Bromodichloromethane & 5 & UG/KG & $u$ & NP & 5 & UG/KG & 1 \\
\hline 1,2-Dichloropropane & 5 & UG/KG & $u$ & NP & 5 & UG/KG & 1 \\
\hline cis-1.3-Dichloropropene & 5 & UG/KG & $u$ & NP & 5 & UG/KG & 1 \\
\hline Trichloroethene & 5 & UG/KG & $u$ & NP & 5 & UG/KG & 1 \\
\hline Dibromochloromethane & 5 & UG/KG & $u$ & NP & 5 & UG/KG & 1 \\
\hline 1,1,2-Trichloroethane & 5 & UG/KG & $u$ & NP & 5 & UG/KG & 1 \\
\hline Benzene & 5 & UG/KG & $u$ & NP & 5 & UG/KG & 1 \\
\hline trans-1,3-Dichloropropene & 5 & UG/KG & $\mathrm{U}$ & NP & 5 & UG/KG & 1 \\
\hline Bromoform & 5 & UG/KG & $u$ & NP & 5 & UG/KG & 1 \\
\hline 4-Methyl-2-Pentanone & 10 & UG/KG & $\mathrm{U}$ & NP & 10 & UG/KG & 1 \\
\hline 2-Hexanone & 10 & UG/KG & U & NP & 10 & UG/KG & 1 \\
\hline Tetrachloroethene & 5 & UG/KG & $\mathrm{u}$ & NP & 5 & UG/KG & 1 \\
\hline 1,1,2,2-Tetrachloroethane & 5 & UG/KG & u & NP & 5 & UG/KG & 1 \\
\hline Toluene & 5 & UG/KG & u & NP & 5 & UG/KG & 1 \\
\hline Chlorobenzene & 5 & UG/KG & $u$ & NP & 5 & UG/KG & 1 \\
\hline Ethylbenzene & 5 & UG/KG & $u$ & NP & 5 & UG/KG & 1 \\
\hline Styrene & 5 & UG/KG & $u$ & NP & 5 & UG/KG & 1 \\
\hline Xylenes (total) & 5 & UG/KG & $U$ & NP & 5 & UG/KG & 1 \\
\hline Unknown-1 & 13 & UG/KG & J & NP & & UG/KG & 1 \\
\hline Unknown Alkane-1 & 36 & UG/KG & $J$ & NP & & UG/KG & 1 \\
\hline Unknown-2 & 13 & UG/KG & J & NP & & UG/KG & 1 \\
\hline Unknown-3 & 18 & UG/KG & $J$ & NP & & UG/KG & 1 \\
\hline Unknown-4 & 32 & UG/KG & J & NP & & UG/KG & 1 \\
\hline Unknown-5 & 17 & UG/KG & J & NP & & UG/KG & 1 \\
\hline Unknown Alkane-2 & 57 & UG/KG & $J$ & NP & & UG/KG & 1 \\
\hline Unknown-6 & 79 & UG/KG & $J$ & NP & & UG/KG & 1 \\
\hline Unknown-7 & 9 & UG/KG & J & NP & & UG/KG & 1 \\
\hline Unknown-8 & 16 & UG/KG & $\mathbf{J}$ & NP & & UG/KG & 1 \\
\hline Toluene-d8 (SURR) & 98 & $\%$ & & & & $\%$ & 1 \\
\hline Bromofluorobenzene (SURR) & 106 & $\%$ & & & & $\%$ & 1 \\
\hline 1,2-Dichloroethane-d4 (SURR) & 111 & $\%$ & & & & $\%$ & 1 \\
\hline
\end{tabular}




\section{Appendix C: Results of Volatile Organic Compound Analyses, Salmon Site}

\begin{tabular}{|c|c|c|c|c|c|c|c|}
\hline COMPOUND & RESULTS & UNITS & $\begin{array}{l}\text { DATA } \\
\text { QUAL. }\end{array}$ & $\begin{array}{l}\text { DATA } \\
\text { VALID. }\end{array}$ & CRDL & UNITS & DILUTION \\
\hline \multicolumn{8}{|l|}{ TR-15-ES-20-1180-.5 } \\
\hline Chloromethane & 10 & UG/KG & $u$ & NP & 10 & UG/KG & 1 \\
\hline Bromomethane & 10 & UG/KG & $u$ & NP & 10 & UG/KG & 1 \\
\hline Vinyl Chloride & 10 & UG/KG & $u$ & NP & 10 & UG/KG & 1 \\
\hline Chloroethane & 10 & UG/KG & $u$ & NP & 10 & UG/KG & 1 \\
\hline Methylene Chloride & 5 & UG/KG & $u$ & NP & 5 & UG/KG & 1 \\
\hline Acetone & 10 & UG/KG & $u$ & NP & 10 & UG/KG & 1 \\
\hline Carbon Disulfide & 5 & UG/KG & $u$ & NP & 5 & UG/KG & 1 \\
\hline 1,1-Dichloroethene & 5 & UG/KG & $u$ & NP & 5 & UG/KG & 1 \\
\hline 1,1-Dichloroethane & 5 & UG/KG & $u$ & NP & 5 & UG/KG & 1 \\
\hline 1,2-Dichloroethene (total) & 5 & UG/KG & $u$ & NP & 5 & UG/KG & 1 \\
\hline Chloroform & 5 & UG/KG & $u$ & NP & 5 & UG/KG & 1 \\
\hline 1,2-Dichloroethane & 5 & UG/KG & $u$ & NP & 5 & UG/KG & 1 \\
\hline 2-Butanone & 10 & UG/KG & U & NP & 10 & UG/KG & 1 \\
\hline 1,1,1-Trichloroethane & 5 & UG/KG & u & NP & 5 & UG/KG & 1 \\
\hline Carbon Tetrachloride & 5 & UG/KG & $U$ & NP & 5 & UG/KG & 1 \\
\hline Vinyl Acetate & 10 & UG/KG & $U$ & NP & 10 & UG/KG & 1 \\
\hline Bromodichloromethane & 5 & UG/KG & $u$ & NP & 5 & UG/KG & 1 \\
\hline 1,2-Dichloropropane & 5 & UG/KG & $U$ & NP & 5 & UG/KG & 1 \\
\hline cis-1,3-Dichloropropene & 5 & UG/KG & $u$ & NP & 5 & UG/KG & 1 \\
\hline Trichloroethene & 5 & UG/KG & $u$ & NP & 5 & UG/KG & 1 \\
\hline Dibromochloromethane & 5 & UG/KG & $u$ & NP & 5 & UG/KG & 1 \\
\hline 1,1,2-Trichloroethane & 5 & UG/KG & $u$ & NP & 5 & UG/KG & 1 \\
\hline Benzene & 5 & UG/KG & $u$ & NP & 5 & UG/KG & 1 \\
\hline trans-1,3-Dichloropropene & 5 & UG/KG & $U$ & NP & 5 & UG/KG & 1 \\
\hline Bromoform & 5 & UG/KG & $u$ & NP & 5 & UG/KG & 1 \\
\hline 4-Methyl-2-Pentanone & 10 & UG/KG & $u$ & NP & 10 & UG/KG & 1 \\
\hline 2-Hexanone & 10 & UG/KG & $u$ & NP & 10 & UG/KG & 1 \\
\hline Tetrachloroethene & 5 & UG/KG & $U$ & NP & 5 & UG/KG & 1 \\
\hline 1,1,2,2-Tetrachloroethan $\theta$ & 5 & UG/KG & $U$ & NP & 5 & UG/KG & 1 \\
\hline Toluene & 5 & UG/KG & $u$ & NP & 5 & UG/KG & 1 \\
\hline Chlorobenzene & 5 & UG/KG & $u$ & NP & 5 & UG/KG & 1 \\
\hline Ethylbenzene & 5 & UG/KG & U & NP & 5 & UG/KG & 1 \\
\hline Styrene & 5 & UG/KG & $u$ & NP & 5 & UG/KG & 1 \\
\hline Xylenes (total) & 5 & UG/KG & $u$ & NP & 5 & UG/KG & 1 \\
\hline Unknown & 100 & UG/KG & $J$ & NP & & UG/KG & 1 \\
\hline Toluene-d8 (SURR) & 105 & $\%$ & & & & $\%$ & 1 \\
\hline Bromofluorobenzene (SURR) & 98 & $\%$ & & & & $\%$ & 1 \\
\hline 1,2-Dichloroethane-d4 (SURR) & 114 & $\%$ & & & & $\%$ & 1 \\
\hline
\end{tabular}


Appendix C: Results of Volatile Organic Compound Analyses, Salmon Site

\begin{tabular}{|c|c|c|c|c|c|c|c|}
\hline COMPOUND & RESULTS & UNITS & $\begin{array}{l}\text { DATA } \\
\text { QUAL. }\end{array}$ & $\begin{array}{l}\text { DATA } \\
\text { VALD. }\end{array}$ & CRDL & UNITS & DILUTION \\
\hline \multicolumn{8}{|l|}{ TR-15-ES-20-1180-.5 MS } \\
\hline 1,1-Dichloroethene & 137 & $\%$ & & NP & & $\%$ & 1 \\
\hline Trichloroethene & 131 & $\%$ & & NP & & $\%$ & 1 \\
\hline Benzene & 138 & $\%$ & & NP & & $\%$ & 1 \\
\hline Toluene & 138 & $\%$ & & NP & & $\%$ & 1 \\
\hline Chlorobenzene & 139 & $\%$ & * & NP & & $\%$ & 1 \\
\hline Toluene-d8 (SURA) & 106 & $\%$ & & NP & & $\%$ & 1 \\
\hline Bromofluorobenzene (SURR) & 102 & $\%$ & & NP & & $\%$ & 1 \\
\hline 1,2-Dichloroethane-d4 (SURR) & 104 & $\%$ & & NP & & $\%$ & 1 \\
\hline \multicolumn{8}{|l|}{ TR-15-ES-20-1180-.5 MSD } \\
\hline 1.1-Dichloroethene & 141 & $\%$ & & NP & & $\%$ & 1 \\
\hline Trichloroethene & 135 & $\%$ & & NP & & $\%$ & 1 \\
\hline Benzene & 146 & $\%$ & * & NP & & $\%$ & 1 \\
\hline Toluene & 145 & $\%$ & * & NP & & $\%$ & 1 \\
\hline Chlorobenzene & 141 & $\%$ & * & NP & & $\%$ & 1 \\
\hline Toluene-d8 (SURR) & 105 & $\%$ & & NP & & $\%$ & 1 \\
\hline Bromofluorobenzene (SURR) & 96 & $\%$ & & NP & & $\%$ & 1 \\
\hline 1,2-Dichloroethane-d4 (SURR) & 103 & $\%$ & & NP & & $\%$ & 1 \\
\hline
\end{tabular}


Appendix C: Results of Volatile Organic Compound Analyses, Salmon Site

\begin{tabular}{|c|c|c|c|c|c|c|c|}
\hline COMPOUND & RESULTS & UNITS & $\begin{array}{l}\text { DATA } \\
\text { QUAL. }\end{array}$ & $\begin{array}{l}\text { DATA } \\
\text { VALID. }\end{array}$ & CRDL & UNITS & DILUTION \\
\hline \multicolumn{8}{|l|}{ TR16-ES-200-80-1.5 } \\
\hline Chloromethane & 10 & UG/KG & $u$ & NP & 10 & UG/KG & 1 \\
\hline Bromomethane & 10 & UG/KG & $u$ & NP & 10 & $U G / K G$ & 1 \\
\hline Vinyl Chloride & 10 & UG/KG & $u$ & NP & 10 & UG/KG & 1 \\
\hline Chloroethane & 10 & UG/KG & $U$ & NP & 10 & UG/KG & 1 \\
\hline Methylene Chloride & 5 & UG/KG & $u$ & NP & 5 & UG/KG & 1 \\
\hline Acetone & 17 & UG/KG & & NP & 10 & UG/KG & 1 \\
\hline Carbon Disulfide & 5 & UG/KG & $U$ & NP & 5 & UG/KG & 1 \\
\hline 1.1-Dichloroethene & 5 & UG/KG & $U$ & NP & 5 & UG/KG & 1 \\
\hline 1,1-Dichloroethane & 5 & UG/KG & $U$ & NP & 5 & UG/KG & 1 \\
\hline 1,2-Dichloroethene (total) & 5 & UG/KG & $U$ & NP & 5 & UG/KG & 1 \\
\hline Chlorotorm & 5 & UG/KG & $u$ & NP & 5 & UG/KG & 1 \\
\hline 1,2-Dichloroethane & 5 & UG/KG & $u$ & NP & 5 & UG/KG & 1 \\
\hline 2-Butanone & 10 & UG/KG & U & NP & 10 & UG/KG & 1 \\
\hline 1,1,1-Trichloroethane & 5 & UG/KG & $u$ & NP & 5 & UG/KG & 1 \\
\hline Carbon Tetrachloride & 5 & $U G / K G$ & $u$ & NP & 5 & UG/KG & 1 \\
\hline Vinyl Acetate & 10 & UG/KG & $u$ & NP & 10 & UG/KG & 1 \\
\hline Bromodichloromethane & 5 & UG/KG & $u$ & NP & 5 & UG/KG & 1 \\
\hline 1,2-Dichloropropane & 5 & UG/KG & $u$ & NP & 5 & UG/KG & 1 \\
\hline cis-1,3-Dichloropropene & 5 & UG/KG & $U$ & NP & 5 & UG/KG & 1 \\
\hline Trichloroethene & 5 & UG/KG & $U$ & NP & 5 & UG/KG & 1 \\
\hline Dibromochloromethane & 5 & UG/KG & $u$ & NP & 5 & UG/KG & 1 \\
\hline 1,1,2-Trichloroethane & 5 & UG/KG & $u$ & NP & 5 & UG/KG & 1 \\
\hline Benzene & 5 & UG/KG & $u$ & NP & 5 & UG/KG & 1 \\
\hline trans-1,3-Dichloropropene & 5 & UG/KG & U & NP & 5 & UG/KG & 1 \\
\hline Bromoform & 5 & UG/KG & U & NP & 5 & UG/KG & 1 \\
\hline 4-Methyl-2-Pentanone & 10 & UG/KG & $u$ & NP & 10 & UG/KG & 1 \\
\hline 2-Hexanone & 10 & UG/KG & $u$ & NP & 10 & UG/KG & 1 \\
\hline Tetrachloroethene & 5 & UG/KG & $u$ & NP & 5 & UG/KG & 1 \\
\hline 1,1,2,2-Tetrachloroethane & 5 & UG/KG & $u$ & NP & 5 & UG/KG & 1 \\
\hline Toluene & 5 & UG/KG & $u$ & NP & 5 & UG/KG & 1 \\
\hline Chlorobenzene & 5 & UG/KG & U & NP & 5 & UG/KG & 1 \\
\hline Ethylbenzene & 5 & UG/KG & $u$ & NP & 5 & UG/KG & 1 \\
\hline Styrene & 5 & UG/KG & $u$ & NP & 5 & UG/KG & 1 \\
\hline Xylenes (total) & 5 & UG/KG & U & NP & 5 & UG/KG & 1 \\
\hline Toluene-d8 (SURR) & 101 & $\%$ & & & & $\%$ & 1 \\
\hline Bromofluorobenzene (SURR) & 102 & $\%$ & & & & $\%$ & 1 \\
\hline 1,2-Dichloroethane-d4 (SURR) & 104 & $\%$ & & & & $\%$ & 1 \\
\hline
\end{tabular}




\section{Appendix C: Results of Volatile Organic Compound Analyses, Salmon Site}

\begin{tabular}{|c|c|c|c|c|c|c|c|}
\hline COMPOUND & RESULTS & UNITS & $\begin{array}{l}\text { DATA } \\
\text { QUAL. }\end{array}$ & $\begin{array}{l}\text { DATA } \\
\text { VALD. }\end{array}$ & CRDL & UNITS & DILUTION \\
\hline \multicolumn{8}{|l|}{ TR17-ES-30-120-5 } \\
\hline Chloromethane & 10 & UG/KG & $u$ & NP & 10 & UG/KG & 1 \\
\hline Bromomethane & 10 & UG/KG & $u$ & NP & 10 & UG/KG & 1 \\
\hline Vinyl Chloride & 10 & $U G / K G$ & $u$ & NP & 10 & UG/KG & 1 \\
\hline Chloroethane & 10 & UG/KG & $u$ & NP & 10 & UG/KG & 1 \\
\hline Methylene Chloride & 5 & UG/KG & $u$ & NP & 5 & UG/KG & 1 \\
\hline Acetone & 10 & UG/KG & $u$ & NP & 10 & UG/KG & 1 \\
\hline Carbon Disulfide & 5 & UG/KG & $u$ & NP & 5 & UG/KG & 1 \\
\hline 1,1-Dichloroethene & 5 & UG/KG & $u$ & NP & 5 & UG/KG & 1 \\
\hline 1,1-Dichloroethane & 5 & UG/KG & $u$ & NP & 5 & UG/KG & 1 \\
\hline 1,2-Dichloroethene (total) & 5 & UG/KG & $u$ & NP & 5 & UG/KG & 1 \\
\hline Chloroform & 5 & UG/KG & $u$ & NP & 5 & UG/KG & 1 \\
\hline 1,2-Dichloroethane & 5 & UG/KG & $u$ & NP & 5 & UG/KG & 1 \\
\hline 2-Butanone & 10 & UG/KG & $u$ & NP & 10 & UG/KG & 1 \\
\hline 1,1,1-Trichloroethane & 5 & UG/KG & $u$ & NP & 5 & UG/KG & 1 \\
\hline Carbon Tetrachloride & 5 & $U G / K G$ & $u$ & NP & 5 & UG/KG & 1 \\
\hline Vinyl Acetate & 10 & UG/KG & $u$ & NP & 10 & UG/KG & 1 \\
\hline Bromodichloromethane & 5 & UG/KG & $u$ & NP & 5 & UG/KG & 1 \\
\hline 1,2-Dichloropropane & 5 & UG/KG & $u$ & NP & 5 & UG/KG & 1 \\
\hline cis-1,3-Dichloropropene & 5 & UG/KG & $u$ & NP & 5 & UG/KG & 1 \\
\hline Trichloroethene & 5 & UG/KG & $u$ & NP & 5 & UG/KG & 1 \\
\hline Dibromochloromethane & 5 & UG/KG & $u$ & NP & 5 & UG/KG & 1 \\
\hline 1,1,2-Trichloroethane & 5 & UG/KG & $U$ & NP & 5 & UG/KG & 1 \\
\hline Benzene & 5 & UG/KG & $u$ & NP & 5 & UG/KG & 1 \\
\hline trans-1,3-Dichloropropene & 5 & UG/KG & $u$ & NP & 5 & UG/KG & 1 \\
\hline Bromoform & 5 & UG/KG & $u$ & NP & 5 & UG/KG & 1 \\
\hline 4-Methyl-2-Pentanone & 10 & UG/KG & $u$ & NP & 10 & UG/KG & 1 \\
\hline 2-Hexanone & 10 & UG/KG & $u$ & NP & 10 & UG/KG & 1 \\
\hline Tetrachloroethene & 5 & UG/KG & $u$ & NP & 5 & UG/KG & 1 \\
\hline 1,1,2,2-Tetrachloroethane & 5 & UG/KG & $u$ & NP & 5 & UG/KG & 1 \\
\hline Toluene & 5 & UG/KG & $u$ & NP & 5 & UG/KG & 1 \\
\hline Chlorobenzene & 5 & UG/KG & $u$ & NP & 5 & $U G / K G$ & 1 \\
\hline Ethylbenzene & 5 & UG/KG & $u$ & NP & 5 & UG/KG & 1 \\
\hline Styrene & 5 & UG/KG & $u$ & NP & 5 & UG/KG & 1 \\
\hline Xylenes (total) & 5 & UG/KG & $u$ & NP & 5 & UG/KG & 1 \\
\hline Toluene-d8 (SURA) & 105 & $\%$ & & & & $\%$ & 1 \\
\hline Bromofluorobenzene (SURR) & 98 & $\%$ & & & & $\%$ & 1 \\
\hline 1,2-Dichloroethane-d4 (SURR) & 106 & $\%$ & & & & $\%$ & 1 \\
\hline
\end{tabular}


Appendix C: Results of Volatile Organic Compound Analyses, Salmon Site

\begin{tabular}{|c|c|c|c|c|c|c|c|}
\hline COMPOUND & RESULTS & UNITS & $\begin{array}{l}\text { DATA } \\
\text { QUAL. }\end{array}$ & $\begin{array}{l}\text { DATA } \\
\text { VALID. }\end{array}$ & CRDL & UNITS & DILUTION \\
\hline \multicolumn{8}{|l|}{ TR17-ES-30-125-8 } \\
\hline Chloromethane & 10 & UG/KG & $U$ & NP & 10 & UG/KG & 1 \\
\hline Bromomethane & 10 & UG/KG & $U$ & NP & 10 & UG/KG & 1 \\
\hline Vinyl Chloride & 10 & UG/KG & $U$ & NP & 10 & UG/KG & 1 \\
\hline Chloroethane & 10 & UG/KG & $U$ & NP & 10 & UG/KG & 1 \\
\hline Methylene Chloride & 5 & UG/KG & $U$ & NP & 5 & UG/KG & 1 \\
\hline Acetone & 10 & UG/KG & $u$ & NP & 10 & UG/KG & 1 \\
\hline Carbon Disulfide & 5 & UG/KG & $u$ & NP & 5 & UG/KG & 1 \\
\hline 1,1-Dichloroethene & 5 & UG/KG & $u$ & NP & 5 & UG/KG & 1 \\
\hline 1,1-Dichloroethane & 5 & UG/KG & $u$ & NP & 5 & UG/KG & 1 \\
\hline 1,2-Dichloroethene (total) & 5 & UG/KG & $u$ & NP & 5 & UG/KG & 1 \\
\hline Chloroform & 5 & UG/KG & $U$ & NP & 5 & UG/KG & 1 \\
\hline 1.2-Dichloroethane & 5 & UG/KG & U & NP & 5 & UG/KG & 1 \\
\hline 2-Butanone & 10 & UG/KG & $U$ & NP & 10 & UG/KG & 1 \\
\hline 1,1,1-Trichloroethane & 5 & UG/KG & $u$ & NP & 5 & UG/KG & 1 \\
\hline Carbon Tetracnloride & 5 & UG/KG & $u$ & NP & 5 & UG/KG & 1 \\
\hline Vinyl Acetate & 10 & UG/KG & $u$ & NP & 10 & UG/KG & 1 \\
\hline Bromodichloromethane & 5 & UG/KG & $u$ & NP & 5 & UG/KG & 1 \\
\hline 1,2-Dichloropropane & 5 & UG/KG & $u$ & NP & 5 & UG/KG & 1 \\
\hline cis-1,3-Dichloropropene & 5 & UG/KG & $U$ & NP & 5 & UG/KG & 1 \\
\hline Trichloroethene & 5 & UG/KG & $U$ & NP & 5 & UG/KG & 1 \\
\hline Dibromochloromethane & 5 & UG/KG & $u$ & NP & 5 & UG/KG & 1 \\
\hline 1,1,2-Trichloroethane & 5 & UG/KG & $U$ & NP & 5 & UG/KG & 1 \\
\hline Benzene & 5 & UG/KG & $u$ & NP & 5 & UG/KG & 1 \\
\hline trans-1,3-Dichloropropene & 5 & UG/KG & $u$ & NP & 5 & UG/KG & 1 \\
\hline Bromoform & 5 & UG/KG & $u$ & NP & 5 & UG/KG & 1 \\
\hline 4-Methyl-2-Pentanone & 10 & UG/KG & $u$ & NP & 10 & UG/KG & 1 \\
\hline 2-Hexanone & 10 & UG/KG & $u$ & NP & 10 & UG/KG & 1 \\
\hline Tetrachloroethene & 5 & UG/KG & $U$ & NP & 5 & UG/KG & 1 \\
\hline 1,1,2,2-Tetrachloroethane & 5 & UG/KG & $U$ & NP & 5 & UG/KG & 1 \\
\hline Toluene & 5 & UG/KG & $u$ & NP & 5 & UG/KG & 1 \\
\hline Chlorobenzene & 5 & UG/KG & $U$ & NP & 5 & UG/KG & 1 \\
\hline Ethylbenzene & 5 & UG/KG & $U$ & NP & 5 & UG/KG & 1 \\
\hline Styrene & 5 & UG/KG & $U$ & NP & 5 & UG/KG & 1 \\
\hline Xylenes (total) & 5 & UG/KG & $u$ & NP & 5 & UG/KG & 1 \\
\hline Toluene-d8 (SURR) & 110 & $\%$ & & & & $\%$ & 1 \\
\hline Bromofluorobenzene (SURR) & 94 & $\%$ & & & & $\%$ & 1 \\
\hline 1,2-Dichloroethane-d4 (SURR) & 101 & $\%$ & & & & $\%$ & 1 \\
\hline
\end{tabular}




\section{Appendix C: Results of Volatile Organic Compound Analyses, Salmon Site}

\begin{tabular}{|c|c|c|c|c|c|c|c|}
\hline COMPOUND & RESULTS & UNITS & $\begin{array}{l}\text { DATA } \\
\text { QUAL. }\end{array}$ & $\begin{array}{l}\text { DATA } \\
\text { VALD. }\end{array}$ & CRDL & UNITS & DILUTION \\
\hline \multicolumn{8}{|l|}{ TR18-BS-80-150A-E } \\
\hline Chloromethane & 10 & UG/KG & $u$ & NP & 10 & UG/KG & 1 \\
\hline Bromomethane & 10 & UG/KG & $u$ & NP & 10 & UG/KG & 1 \\
\hline Vinyl Chloride & 10 & UG/KG & $u$ & NP & 10 & UG/KG & 1 \\
\hline Chloroethane & 10 & UG/KG & $u$ & NP & 10 & UG/KG & 1 \\
\hline Methylene Chloride & 1 & UG/KG & $J$ & NP & 5 & $U G / K G$ & 1 \\
\hline Acetone & 10 & UG/KG & $u$ & NP & 10 & $U G / K G$ & 1 \\
\hline Carbon Disulfide & 5 & UG/KG & $u$ & NP & 5 & UG/KG & 1 \\
\hline 1,1-Dichloroethene & 5 & UG/KG & $u$ & NP & 5 & UG/KG & 1 \\
\hline 1,1-Dichloroethane & 5 & UG/KG & $u$ & NP & 5 & UG/KG & 1 \\
\hline 1,2-Dichloroethene (total) & 5 & UG/KG & $u$ & NP & 5 & $U G / K G$ & 1 \\
\hline Chloroform & 5 & UG/KG & $u$ & NP & 5 & UG/KG & 1 \\
\hline 1,2-Dichloroethane & 5 & UG/KG & $u$ & NP & 5 & UG/KG & 1 \\
\hline 2-Butanone & 10 & UG/KG & $u$ & NP & 10 & UG/KG & 1 \\
\hline 1,1,1-Trichloroethane & 5 & UG/KG & $u$ & NP & 5 & UG/KG & 1 \\
\hline Carbon Tetrachloride & 5 & UG/KG & $u$ & NP & 5 & UG/KG & 1 \\
\hline Vinyl Acetate & 10 & UG/KG & $u$ & NP & 10 & UG/KG & 1 \\
\hline Bromodichloromethane & 5 & UG/KG & $u$ & NP & 5 & UG/KG & 1 \\
\hline 1,2-Dichloropropane & 5 & UG/KG & $u$ & NP & 5 & UG/KG & 1 \\
\hline cis-1,3-Dichloropropene & 5 & UG/KG & $u$ & NP & 5 & UG/KG & 1 \\
\hline Trichloroethene & 5 & UG/KG & $u$ & NP & 5 & UG/KG & 1 \\
\hline Dibromochloromethane & 5 & UG/KG & $u$ & NP & 5 & UG/KG & 1 \\
\hline 1,1,2-Trichloroethane & 5 & UG/KG & $U$ & NP & 5 & UG/KG & 1 \\
\hline Benzene & 5 & UG/KG & $u$ & NP & 5 & UG/KG & 1 \\
\hline trans-1,3-Dichloropropene & 5 & UG/KG & $u$ & NP & 5 & UG/KG & 1 \\
\hline Bromoform & 5 & UG/KG & $u$ & NP & 5 & UG/KG & 1 \\
\hline 4-Methyl-2-Pentanone & 10 & UG/KG & U & NP & 10 & UG/KG & 1 \\
\hline 2-Hexanone & 10 & UG/KG & $U$ & NP & 10 & UG/KG & 1 \\
\hline Tetrachloroethene & 5 & UG/KG & U & NP & 5 & UG/KG & 1 \\
\hline 1,1,2,2-Tetrachloroethane & 5 & UG/KG & $U$ & NP & 5 & UG/KG & 1 \\
\hline Toluene & 5 & UG/KG & $u$ & NP & 5 & UG/KG & 1 \\
\hline Chlorobenzene & 5 & UG/KG & $u$ & NP & 5 & UG/KG & 1 \\
\hline Ethylbenzene & 5 & UG/KG & $u$ & NP & 5 & UG/KG & 1 \\
\hline Styrene & 5 & UG/KG & $u$ & NP & 5 & UG/KG & 1 \\
\hline Xylenes (total) & 5 & UGKG & $u$ & NP & 5 & UG/KG & 1 \\
\hline Toluene-d8 (SURR) & 102 & $\%$ & & & & $\%$ & 1 \\
\hline Bromofluorobenzene (SURR) & 101 & $\%$ & & & & $\%$ & 1 \\
\hline 1,2-Dichloroethane-d4 (SURR) & 104 & $\%$ & & & & $\%$ & 1 \\
\hline
\end{tabular}


Appendix C: Results of Volatlle Organic Compound Analyses, Salmon Site

\begin{tabular}{|c|c|c|c|c|c|c|c|}
\hline COMPOUND & RESULTS & UNITS & $\begin{array}{l}\text { DATA } \\
\text { QUAL. }\end{array}$ & $\begin{array}{l}\text { DATA } \\
\text { VALID. }\end{array}$ & CRDL & UNITS & DILUTION \\
\hline \multicolumn{8}{|l|}{ TR19-BS-0-150A-E } \\
\hline Chloromethane & 10 & UG/KG & $u$ & NP & 10 & UG/KG & 1 \\
\hline Bromomethane & 10 & UG/KG & $u$ & NP & 10 & UG/KG & 1 \\
\hline Vinyl Chloride & 10 & UG/KG & $u$ & NP & 10 & UG/KG & 1 \\
\hline Chloroethane & 10 & UG/KG & $U$ & NP & 10 & UG/KG & 1 \\
\hline Methylene Chloride & 5 & UG/KG & $u$ & NP & 5 & UG/KG & 1 \\
\hline Acetone & 31 & UG/KG & & NP & 10 & UG/KG & 1 \\
\hline Carbon Disulfide & 5 & UG/KG & $u$ & NP & 5 & UG/KG & 1 \\
\hline 1,1-Dichloroethene & 5 & UG/KG & $u$ & NP & 5 & UG/KG & 1 \\
\hline 1,1-Dichloroethane & 5 & UG/KG & $u$ & NP & 5 & UG/KG & 1 \\
\hline 1,2-Dichloroethene (total) & 5 & UG/KG & $u$ & NP & 5 & UG/KG & 1 \\
\hline Chloroform & 5 & UG/KG & $u$ & NP & 5 & UG/KG & 1 \\
\hline 1.2-Dichloroethane & 5 & UG/KG & $u$ & NP & 5 & UG/KG & 1 \\
\hline 2-Butanone & 10 & UG/KG & $u$ & NP & 10 & UG/KG & 1 \\
\hline 1,1,1-Trichloroethane & 5 & UG/KG & $u$ & NP & 5 & UG/KG & 1 \\
\hline Carbon Tetrachloride & 5 & UG/KG & $u$ & NP & 5 & UG/KG & 1 \\
\hline Vinyl Acetate & 10 & UG/KG & $u$ & NP & 10 & UG/KG & 1 \\
\hline Bromodichloromethane & 5 & UG/KG & $u$ & NP & 5 & UG/KG & 1 \\
\hline 1,2-Dichloropropane & 5 & UG/KG & $u$ & NP & 5 & UG/KG & 1 \\
\hline cis-1,3-Dichloropropene & 5 & UG/KG & $u$ & NP & 5 & UG/KG & 1 \\
\hline Trichloroethene & 5 & UG/KG & $u$ & NP & 5 & UG/KG & 1 \\
\hline Dibromochloromethane & 5 & UG/KG & $u$ & NP & 5 & UG/KG & 1 \\
\hline 1,1,2-Trichloroethane & 5 & UG/KG & $u$ & NP & 5 & UG/KG & 1 \\
\hline Benzene & 5 & UG/KG & $U$ & NP & 5 & UG/KG & 1 \\
\hline trans-1,3-Dichloropropene & 5 & UG/KG & $U$ & NP & 5 & UG/KG & 1 \\
\hline Bromoform & 5 & UG/KG & $U$ & NP & 5 & UG/KG & 1 \\
\hline 4-Methyl-2-Pentanone & 10 & UG/KG & $u$ & NP & 10 & UG/KG & 1 \\
\hline 2-Hexanone & 10 & UG/KG & $u$ & NP & 10 & UG/KG & 1 \\
\hline Tetrachloroethene & 5 & UG/KG & $u$ & NP & 5 & UG/KG & 1 \\
\hline 1,1,2,2-Tetrachloroethane & 5 & UG/KG & $u$ & NP & 5 & UG/KG & 1 \\
\hline Tolvene & 5 & UG/KG & $U$ & NP & 5 & UG/KG & 1 \\
\hline Chlorobenzene & 5 & UG/KG & $U$ & NP & 5 & UG/KG & 1 \\
\hline Ethylbenzene & 5 & UG/KG & U & NP & 5 & UG/KG & 1 \\
\hline Styrene & 5 & UG/KG & $U$ & NP & 5 & UG/KG & 1 \\
\hline Xylenes (total) & 5 & UG/KG & $U$ & NP & 5 & UG/KG & 1 \\
\hline Unknown Alkane-1 & 32 & UG/KG & J & NP & & UG/KG & 1 \\
\hline Unknown Alkane-2 & 97 & UG/KG & J & NP & & UG/KG & 1 \\
\hline Unknown C1OH12-1 & 67 & UG/KG & J & NP & & UG/KG & 1 \\
\hline Naphthalene & 66 & UG/KG & J & NP & & UG/KG & 1 \\
\hline Unknown-1 & 92 & UG/KG & $J$ & NP & & UG/KG & 1 \\
\hline Unknown-2 & 76 & UG/KG & J & NP & & UG/KG & 1 \\
\hline Unknown Alkane-3 & 54 & UG/KG & $J$ & NP & & UG/KG & 1 \\
\hline Unknown C1 1H14-2 & 53 & UG/KG & J & NP & & UG/KG & 1 \\
\hline Unknown C11H14-3 & 250 & UG/KG & J & NP & & UG/KG & 1 \\
\hline Unknown-3 & 45 & UG/KG & $J$ & NP & & UG/KG & 1 \\
\hline Toluene-d8 (SURR) & 101 & $\%$ & & & & $\%$ & 1 \\
\hline Bromofluorobenzene (SURA) & 100 & $\%$ & & & & $\%$ & 1 \\
\hline 1,2-Dichloroethane-d4 (SURR) & 100 & $\%$ & & & & $\%$ & 1 \\
\hline
\end{tabular}




\section{Appendix C: Results of Volatile Organic Compound Analyses, Salmon Site}

\begin{tabular}{|c|c|c|c|c|c|c|c|}
\hline COMPOUND & RESULTS & UNITS & $\begin{array}{l}\text { DATA } \\
\text { QUAL. }\end{array}$ & $\begin{array}{l}\text { DATA } \\
\text { VALID. }\end{array}$ & CRDL & UNITS & DILUTION \\
\hline \multicolumn{8}{|l|}{ TRIP BLANK } \\
\hline Chloromethane & 10 & UG/L & $u$ & $\ldots$ & 10 & UG/L & 1 \\
\hline Bromomethane & 10 & UG/L & $u$ & $\cdots$ & 10 & UG/L & 1 \\
\hline Vinyl Chloride & 10 & UG/L & $u$ & $\cdots$ & 10 & UG/L & 1 \\
\hline Chloroethane & 10 & $U G / L$ & $u$ & -.- & 10 & UG/L & 1 \\
\hline Methylene Chloride & 5 & UG/L & $u$ & $\cdots$ & 5 & UG/L & 1 \\
\hline Acetone & 41 & $U G / L$ & B & US & 10 & UG/L & 1 \\
\hline Carbon Disulfide & 5 & UG/L & $u$ & $\cdots$ & 5 & UG/L & 1 \\
\hline 1,1-Dichloroethene & 5 & UG/L & $u$ & $\ldots$ & 5 & UG/L & 1 \\
\hline 1.1-Dichloroethane & 5 & UG/L & $u$ & $\cdots$ & 5 & UG/L & 1 \\
\hline 1,2-Dichloroethene (total) & 5 & UG/L & $u$ &.-- & 5 & UG/L & 1 \\
\hline Chloroform & 5 & UG/L & $u$ & -- & 5 & UG/L & 1 \\
\hline 1,2-Dichloroethane & 5 & UG/L & $u$ & -- & 5 & UG/L & 1 \\
\hline 2-Butanone & 10 & $U G / L$ & $u$ & ... & 10 & UG/L & 1 \\
\hline 1,1,1-Trichloroethane & 5 & UG/L & $u$ & ..- & 5 & $U G / L$ & 1 \\
\hline Carbon Tetrachloride & 5 & UG/L & $u$ & $\ldots$ & 5 & UG/L & 1 \\
\hline Vinyl Acetate & 10 & UG/L & $U$ & $\ldots$ & 10 & UG/L & 1 \\
\hline Bromodichloromethane & 5 & UG/L & $u$ & $\ldots$ & 5 & UG/L & 1 \\
\hline 1,2-Dichloropropane & 5 & UG/L & $u$ & $\cdots$ & 5 & UG/L & 1 \\
\hline cis-1,3-Dichloropropene & 5 & UG/L & $u$ & $\ldots$ & 5 & UG/L & 1 \\
\hline Trichloroethene & 5 & UG/L & $u$ & $\ldots$ & 5 & UG/L & 1 \\
\hline Dibromochloromethane & 5 & UG/L & $u$ & $\ldots$ & 5 & $U G / L$ & 1 \\
\hline 1,1,2-Trichloroethane & 5 & UG/L & $u$ & $\cdots$ & 5 & $U G / L$ & 1 \\
\hline Benzene & 5 & UG/L & $u$ & -- & 5 & UG/L & 1 \\
\hline trans-1,3-Dichloropropene & 5 & UG/L & $u$ & $\cdots$ & 5 & UG/L & 1 \\
\hline Bromoform & 5 & UG/L & $u$ & $\cdots$ & 5 & UG/L & 1 \\
\hline 4-Methyl-2-Pentanone & 10 & UG/L & $u$ & -- & 10 & UG/L & 1 \\
\hline 2-Hexanone & 10 & $U G / L$ & $u$ & -- & 10 & UG/L & 1 \\
\hline Tetrachloroethene & 5 & UG/L & U & $-\cdots$ & 5 & UG/L & 1 \\
\hline 1,1,2,2-Tetrachloroethane & 5 & $U G / L$ & $U$ & $-\cdots$ & 5 & UG/L & 1 \\
\hline Toluene & 5 & UG/L & $U$ & -- & 5 & UG/L & 1 \\
\hline Chlorobenzene & 5 & UG/L & $U$ & $\cdots$ & 5 & UG/L & 1 \\
\hline Ethylbenzene & 5 & $U G / L$ & $u$ & -- & 5 & UG/L & 1 \\
\hline Styrene & 5 & UG/L & $u$ & $\cdots$ & 5 & UG/L & 1 \\
\hline Xylenes (total) & 5 & $U G / L$ & $u$ & -- & 5 & UG/L & 1 \\
\hline Toluene-d8 (SURR) & 100 & $\%$ & & & & $\%$ & 1 \\
\hline Bromofluorobenzene (SURA) & 101 & $\%$ & & & & $\%$ & 1 \\
\hline 1,2-Dichloroethane-d4 (SURR) & 108 & $\%$ & & & & $\%$ & 1 \\
\hline
\end{tabular}




\section{Appendix D \\ Results of Semivolatile Organic Compound Analyses}




\begin{tabular}{|c|c|c|c|c|c|c|c|}
\hline COMPOUND & RESULTS & UNITS & $\begin{array}{l}\text { DATA } \\
\text { QUAL }\end{array}$ & $\begin{array}{l}\text { DATA } \\
\text { VALID. }\end{array}$ & NOTES & CADL UNITS & DILUTION \\
\hline \multicolumn{8}{|l|}{ TRI-ES-001 } \\
\hline Phenol & 330 & UG/KG & $u$ & $\ldots$ & & 330 Ua/Ka & 1 \\
\hline bis(2-Chloroethyl) ether & 330 & UG/Ka & $u$ & ... & & 330 Ua/Ka & 1 \\
\hline 2-Chlorophenol & 330 & VG/KG & $u$ & ... & & 330 Ua/KG & 1 \\
\hline 1, 3-Dichlorobenzene & 330 & VG/KG & $u$ & ... & & 330 Ua/KG & 1 \\
\hline 1, 4-Dichlorobenzene & 330 & UG/KG & $u$ & .. & & 330 Ua/KG & 1 \\
\hline Benzyl Alcohol & 330 & UG/KG & $u$ & $\cdots$ & & 330 UG/KG & 1 \\
\hline 1, 2-Dichlorobenzene & 330 & UG/KG & $u$ & $\ldots$ & & 330 UG/KG & 1 \\
\hline 2-Methylphenol & 330 & Ua/Ka & $u$ & ... & & 330 UG/KO & 1 \\
\hline bis(2-Chloroisopropyl) ether & 330 & UG/Ka & $u$ & ... & & 330 Ua/Ka & 1 \\
\hline 4-Methylphenol & 330 & UG/Ka & $u$ & ... & & 330 UQ/KG & 1 \\
\hline N-Nitroso-dl-nPropylamine & 330 & UG/KG & $u$ & $\ldots$ & & 330 Ua/KG & 1 \\
\hline Hexachloroethane & 330 & UG/KG & $u$ & ..- & & 330 Ua/Ka & 1 \\
\hline Nitrobenzene & 330 & UG/KG & $u$ & ... & & 330 Ua/KG & 1 \\
\hline Isophorone & 330 & UG/KG & $u$ & ... & & 330 Ua/Ka & 1 \\
\hline 2-Nitrophenol & 330 & $U G / K G$ & $u$ & ... & & 330 Ua/ka & 1 \\
\hline 2, 4-Dimethylphenol & 330 & UG/KG & $u$ & ... & & 330 UG/KG & 1 \\
\hline Benzoic Acid & 1600 & UG/KG & $u$ & ... & & 1600 UG/KG & 1 \\
\hline bis(2Chloroethoxy)methane & 330 & UG/KG & $u$ & $\ldots$ & & 330 UG/KG & 1 \\
\hline 2, 4-Dichlorophenol & 330 & UG/KG & $u$ & ... & & 330 UG/KG & 1 \\
\hline 1, 2, 4-Trichlorobenzene & 330 & $U G / K G$ & $U$ & $\ldots$ & & 330 UG/KG & 1 \\
\hline Naphthalene & 330 & UG/KG & $u$ & $\ldots$ & & 330 UG/KG & 1 \\
\hline 4-Chloroaniline & 330 & UG/KG & $u$ & ... & & 330 UG/KG & 1 \\
\hline Hexachlorobutadiene & 330 & UG/KG & U & $\ldots$ & & 330 UG/KG & 1 \\
\hline 4-Chloro-3-Methylphenol & 330 & $U G / K G$ & $u$ & ... & & 330 Ua/KG & 1 \\
\hline 2-Methylnaphthalene & 330 & UG/KG & $u$ & ... & & 330 UG/KG & 1 \\
\hline Hexachlorocyclopentadiene & 330 & UG/KG & $u$ & ... & & 330 Ua/KG & 1 \\
\hline 2, 4, 6-Trichlorophenol & 330 & UG/KG & $u$ & ... & & 330 UG/KG & 1 \\
\hline 2, 4, 5-Trichlorophenol & 1600 & $U G / K G$ & U & ... & & 1600 UG/KG & 1 \\
\hline 2-Chloronaphthalene & 330 & UG/KG & $u$ & ... & & 330 UG/KG & 1 \\
\hline 2-Nitroaniline & 1600 & UG/KG & U & $\ldots$ & & 1600 UG/KG & 1 \\
\hline Dimethyl Phthalate & 330 & UG/KG & $u$ & ... & & 330 UG/KG & 1 \\
\hline Acenaphthylene & 330 & $U G / K G$ & U & ... & & 330 UG/KG & 1 \\
\hline 2, 6-Dinitrotoluene & 330 & UG/KG & $u$ & ... & & 330 UG/KG & 1 \\
\hline 3-Nitroaniline & 1600 & UG/KG & $u$ & ... & & 1600 UG/KG & 1 \\
\hline Acenaphthene & 330 & UG/KG & U & ... & & 330 UG/KG & 1 \\
\hline 2, 4-Dinitrophenol & 1600 & UG/KG & U & .. & & 1600 UG/KG & 1 \\
\hline 4-Nitrophenol & 1600 & UG/KG & U & $\ldots$ & & 1600 UG/KG & 1 \\
\hline Dibenzofuran & 330 & UG/KG & u & ... & & 330 Ua/KG & 1 \\
\hline 2, 4-Dinitrotoluene & 330 & UG/KG & u & ... & & 330 Ua/KG & 1 \\
\hline Diethylphthalate & 330 & UG/KG & u & .. & & 330 UG/KG & 1 \\
\hline 4-Chlorophenyl phenyl ether & 330 & UG/KG & u & ... & & 330 UG/KG & 1 \\
\hline Fluorene & 330 & UG/KG & u & -. & & 330 UG/KG & 1 \\
\hline 4-Nitroaniline & 1600 & UG/KG & u & ... & & 1600 Ua/KG & 1 \\
\hline 4, 6-Dinitro-2-Methylphenol & 1600 & UG/KG & u & ... & & 1600 UG/KG & 1 \\
\hline N-Nitrosodiphenylamine & 330 & UG/KG & $u$ & ... & & 330 Ua/KG & 1 \\
\hline 4-Bromophenyl phenyl ether & 330 & UG/KG & $U$ & ... & & 330 UG/KG & 1 \\
\hline Hexachlorobenzene & 330 & UG/KG & $u$ & -. & & 330 UG/KG & 1 \\
\hline Pentachlorophenol & 1600 & UG/KG & $u$ & ... & & 1600 UG/KG & 1 \\
\hline Phenanthrene & 330 & UG/KG & $u$ &.- & & 330 UG/KG & 1 \\
\hline
\end{tabular}


Appendix D: Results of Semivolatile Organic Compound Analyses, Salmon Site

\begin{tabular}{|c|c|c|c|c|c|c|c|c|}
\hline COMPOUND & RESULTS & UNITS & $\begin{array}{l}\text { DATA } \\
\text { QUAL }\end{array}$ & $\begin{array}{l}\text { DATA } \\
\text { VALID. }\end{array}$ & NOTES & CADL & UNITS & DILUTION \\
\hline \multicolumn{9}{|l|}{ TAI-ES-001 (CONT) } \\
\hline Anthracene & 330 & Ua/KG & $u$ & $\ldots$ & & & Ua/Ka & 1 \\
\hline DI-N-Butylphthalate & 330 & Ua/Ka & $u$ & $\ldots$ & & & UQ/KO & 1 \\
\hline Fluoranthene & 330 & Ua/Ka & $u$ & ... & & & Ua/Ko & 1 \\
\hline Pyrene & 330 & Ua/Ko & $u$ & $\ldots$ & & & UG/Ko & 1 \\
\hline Butyl Benzyl Phthalate & 330 & Va/KG & $u$ & $\ldots$ & & & UG/KG & 1 \\
\hline 3, 3'-Dichlorobenzidine & 660 & $U Q / K G$ & $u$ & $\ldots$ & & & UG/KG & 1 \\
\hline Benzo(a)Anthracene & 330 & Ua/KG & $u$ & $\ldots$ & & & Ua/KG & 1 \\
\hline Chrysene & 330 & Ua/KG & $u$ & $\ldots$ & & & UG/KG & 1 \\
\hline bis(2-Ethylhexyl)phthalate & 330 & Ua/Ka & $u$ & $\ldots$ & & & UG/KG & 1 \\
\hline dl-N-Octyl Phthalate & 330 & UQ/KG & $u$ & ... & & & UG/KG & 1 \\
\hline Benzo(b)Fluoranthene & 330 & Ua/Ko & $u$ & $\ldots$ & & & UG/KG & 1 \\
\hline Benzo(k)Fluoranthene & 330 & Ua/Ka & $u$ & $\ldots$ & & & UG/KG & 1 \\
\hline Benzo(a)Pyrene & 330 & UQ/KG & $u$ & $\ldots$ & & & UG/KG & 1 \\
\hline Indeno(1,2,3-CD)Pyrene & 330 & $U G / K G$ & $u$ & $\ldots$ & & & UG/KG & 1 \\
\hline Dibenzo(a,h)Anthracene & 330 & UG/KG & $u$ & $\ldots$ & & & UG/KG & 1 \\
\hline Benzo(g,h,i)Perylene & 330 & UG/KG & $u$ & $\ldots$ & & & UG/KG & 1 \\
\hline Nitrobenzene-d5 & 63 & $\%$ & & & & & $\%$ & 1 \\
\hline 2-Fluorobiphenyl & 59 & $\%$ & & & & & $\%$ & 1 \\
\hline Terphenyl-d14 & 71 & $\%$ & & & & & $\%$ & 1 \\
\hline Phenol-d5 & 80 & $\%$ & & & & & $\%$ & 1 \\
\hline 2-Fluorophenol & 70 & $\%$ & & & & & $\%$ & 1 \\
\hline 2, 4, 6-Tribromophenol & 58 & $\%$ & & & & & $\%$ & 1 \\
\hline
\end{tabular}


Appendix D: Results of Semivolatile Organic Compound Analyses, Salmon Site

\begin{tabular}{|c|c|c|c|c|c|c|c|}
\hline COMPOUND & REBULTS & UNITS & $\begin{array}{l}\text { DATA } \\
\text { QUAL }\end{array}$ & $\begin{array}{l}\text { DATA } \\
\text { VALID. }\end{array}$ & NOTES & CRDL UNITS & DILUTION \\
\hline TH2-ES-355N-173E.62 & & & & & & & \\
\hline Phenol & 330 & UQKG & U & $\ldots$ & & 330 Ua/Ka & 1 \\
\hline bis(2-Chloroethyl) ether & 330 & vaka & u & $\ldots$ & & 330 vaka & 1 \\
\hline 2-Chlorophenol & 330 & veNko & u & - & & 330 va/ka & 1 \\
\hline 1, 3-Dichlorobenzene & 330 & Ua/Ko & $u$ & $\ldots$ & & 330 Va/Ka & 1 \\
\hline 1, 4-Dichlorobenzene & 330 & UG/Ka & U & - & & 330 verke & 1 \\
\hline Benzyl Alcohol & 330 & Ua/Ko & $u$ & - & & 330 UaKKO & 1 \\
\hline 1. 2-Dichlorobenzene & 330 & vaKa & $u$ & ... & & 330 Ua/Ka & 1 \\
\hline 2.Methyiphenol & 330 & va/Ko & $u$ & $\ldots$ & & 330 Ua/KO & 1 \\
\hline bis(2-Chloroisopropyl) ether & 330 & UG/Ka & $u$ & ... & & 330 Ua/Ka & 1 \\
\hline 4-Methyiphenol & 330 & va/Ka & $u$ & - & & 330 Ua/KG & 1 \\
\hline N-Nitroso-di-nPropylamine & 330 & UG/KG & $u$ & $\ldots$ & & 330 Ua/KG & 1 \\
\hline Hexachloroethane & 330 & vaKa & u & $\ldots$ & & 330 va/ka & 1 \\
\hline Nitrobenzene & 330 & UG/KO & U & - & & 330 Ua/KG & 1 \\
\hline Isophorone & 330 & UG/KO & $u$ & - & & 330 Va/Ka & 1 \\
\hline 2-Nitrophenol & 330 & UG/KG & $u$ & ... & & 330 Ua/Ko & 1 \\
\hline 2, 4-Dimethylphenol & 330 & UG/Ka & $u$ & .- & & 330 Ua/KG & 1 \\
\hline Benzoic Acld & 1600 & Ua/Ka & $u$ & ... & & 1600 va/Ka & 1 \\
\hline bis(2Chloroethoxy)methane & 330 & UG/KO & $u$ & $\cdots$ & & 330 UG/KG & 1 \\
\hline 2. 4-Dichlorophenol & 330 & Ua/KG & $u$ & ... & & 330 UQ/KG & 1 \\
\hline 1, 2, 4-Trichlorobenzene & 330 & UG/KG & $u$ & ... & & 330 Ua/Ka & 1 \\
\hline Naphthalene & 330 & UG/KG & $u$ & $\ldots$ & & 330 UQ/KO & 1 \\
\hline 4-Chloroaniline & 330 & Ua/KG & $u$ & $\cdots$ & & 330 Ua/Ka & 1 \\
\hline Hexachlorobutadiene & 330 & Ua/KG & $u$ & $\cdots$ & & 330 Ua/KG & 1 \\
\hline 4-Chloro-3-Methylphenol & 330 & UG/KG & $u$ & ... & & 330 UG/KG & 1 \\
\hline 2-Methylnaphthalene & 330 & UG/KO & $u$ & $\cdots$ & & 330 UG/KG & 1 \\
\hline Hexachlorocyclopentadiene & 330 & UG/KG & $u$ & ... & & 330 UG/KG & 1 \\
\hline 2, 4, 6-Trichlorophenol & 330 & UG/KG & $u$ & ... & & 330 Ua/KG & 1 \\
\hline 2, 4,5-Trichlorophenol & 1600 & Ua/KG & $u$ & ... & & 1600 UG/KG & 1 \\
\hline 2-Chloronaphthalene & 330 & UQ/KG & $u$ & ... & & 330 UG/KG & 1 \\
\hline 2-Nitroaniline & 1600 & UG/KG & $u$ & ... & & 1600 Ua/KG & 1 \\
\hline Dimethyl Phthalate & 330 & Ua/KG & $u$ & ... & & 330 UG/KG & 1 \\
\hline Acenaphthylene & 330 & Ua/KG & $u$ & ... & & 330 UG/KG & 1 \\
\hline 2, 6-Dinitrotoivene & 330 & UG/KG & $u$ & ... & & 330 Ua/KG & 1 \\
\hline 3-Nitroaniline & 1600 & Ua/KG & $u$ & -. & & 1600 UG/KG & 1 \\
\hline Acenaphthene & 330 & Ua/KG & $u$ & ... & & 330 UG/KG & 1 \\
\hline 2, 4-Dinitrophenol & 1600 & Ua/KG & $u$ & ... & & 1600 UG/Ka & 1 \\
\hline 4-Nitrophenol & 1600 & Ua/Ka & $u$ & ... & & 1600 va/Ka & 1 \\
\hline Dibenzofuran & 330 & UG/KG & $u$ & ... & & 330 UG/KG & 1 \\
\hline 2, 4-Dinitrotoluene & 330 & UaKG & $u$ & $\cdots$ & & 330 UG/KG & $i$ \\
\hline Diethylphthalate & 330 & Ua/Ka & u & $\ldots$ & & 330 Ua/Ka & 1 \\
\hline 4-Chlorophenyl phenyl ether & 330 & UGKG & u & $\ldots$ & & 330 UG/KG & 1 \\
\hline Fluorene & 330 & UG/KG & $u$ & $\ldots$ & & 330 Va/Ka & 1 \\
\hline 4-Nitroaniline & 1600 & Ua/KG & $u$ & .. & & 1600 va/KG & 1 \\
\hline 4, 6-Dinitro-2-Methylphenol & 1600 & UGKG & u & - & & 1600 Va/KG & 1 \\
\hline N-Nitrosodiphenylamine & 330 & Ua/Ko & u & $\cdots$ & & 330 Ua/KG & 1 \\
\hline 4-Bromophenyl phenyl ether & 330 & Ua/Ka & u & ... & & 330 va/Ka & 1 \\
\hline Hexachlorobenzene & 330 & Ua/KG & $u$ & -.. & & 330 va/Ka & 1 \\
\hline Pentachlorophenol & 1600 & Ua/Ka & u & -. & & 1600 UG/KG & 1 \\
\hline Phenanthrene & 330 & Va/KG & $\mathrm{u}$ & ... & & 330 UG/KG & 1 \\
\hline
\end{tabular}


Appendix D: Results of Semivolatile Organic Compound Analyses, Salmon Site

\begin{tabular}{|c|c|c|c|c|c|c|c|c|}
\hline COMPOUND & RESULTS & UNITS & $\begin{array}{l}\text { DATA } \\
\text { QUAL }\end{array}$ & $\begin{array}{l}\text { DATA } \\
\text { VALID. }\end{array}$ & NOTES & CRDL & UNITS & DILUTION \\
\hline \multicolumn{9}{|l|}{ 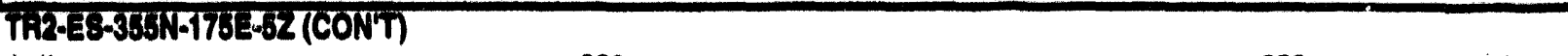 } \\
\hline Anthracene & 330 & Vako & $u$ & $\ldots$ & & 33 & UaKo & 1 \\
\hline Di-N-Butyiphthalate & 330 & UQ/KO & $u$ & $\ldots$ & & 33 & UG/KO & 1 \\
\hline Fluoranthene & 330 & UQKG & $u$ & - & & & Ua/Ko & 1 \\
\hline Pyrene & 330 & UQ/KO & $u$ & $\ldots$ & & 33 & Ua/Ka & 1 \\
\hline Butyl Benzyl Phthalate & 330 & Uaka & $u$ & - & & & Uaka & 1 \\
\hline 3. 3.Dichlorobenzidine & 650 & VaKa & $u$ & - & & & Uako & 1 \\
\hline Benzo(a)Anthracene & 330 & Ua/KG & $u$ & $\ldots$ & & & Ua/Ka & 1 \\
\hline Chrysene & 330 & UG/KG & $u$ & $\ldots$ & & & va/ko & 1 \\
\hline bis(2-Ethylhexyl)phthalate & 330 & VaKa & $u$ & - & & & Va/KG & 1 \\
\hline di-N-Octyl Phthalate & 330 & va/Ka & $u$ & $\ldots$ & & & Ua/KO & 1 \\
\hline Benzo(b)Fluoranthene & 330 & va/Ko & $u$ & $\ldots$ & & & va/ko & 1 \\
\hline Benzo(k)Fluoranthene & 330 & Ua/kG & $u$ & $\ldots$ & & & va/Ka & 1 \\
\hline Benzo(a)Pyrene & 330 & Ua/Ko & $u$ & ... & & & Va/Ko & 1 \\
\hline Indeno(1,2,3-CD)Pyrene & 330 & UGKO & $u$ & ... & & & UG/KO & 1 \\
\hline Dibenzo(a,h)Anthracene & 330 & Ua/KG & $u$ & ... & & & va/Ko & 1 \\
\hline Benzo $(g, h, i)$ Perylene & 330 & va/ka & $u$ & ... & & & UG/KO & 1 \\
\hline Nitrobenzene-d5 & 68 & $\%$ & & & & & $\%$ & 1 \\
\hline 2-Fluorobiphenyl & 62 & $\%$ & & & & & $\%$ & 1 \\
\hline Terphenyl-d14 & 72 & $\%$ & & & & & $\%$ & 1 \\
\hline Phenol-d5 & 78 & $\%$ & & & & & $\%$ & 1 \\
\hline 2-Fluorophenol & 67 & $\%$ & & & & & $\%$ & 1 \\
\hline 2, 4, 6-Tribromophenol & 61 & $\%$ & & & & & $\%$ & 1 \\
\hline
\end{tabular}


Appendix D: Results of Semivolatile Organic Compound Analyses, Salmon Site

\begin{tabular}{|c|c|c|c|c|c|c|c|}
\hline COMPOUND & RESULTS & UNITS & $\begin{array}{l}\text { DATA } \\
\text { QUAL } \\
\end{array}$ & $\begin{array}{l}\text { DATA } \\
\text { VALID. }\end{array}$ & NOTES & CRDL UNITS & DILUTION \\
\hline \multicolumn{8}{|l|}{ TR2-ES-356N-175E-8Z } \\
\hline Phenol & 330 & UG/KG & $u$ & -. & & 330 UG/KG & 1 \\
\hline bis(2-Chloroethyl) ether & 330 & UG/KG & $u$ & - & & 330 UG/KG & 1 \\
\hline 2-Chlorophenol & 330 & UGKG & $u$ & - & & 330 UG/KG & 1 \\
\hline 1, 3-Dichlorobenzene & 330 & UG/KG & $u$ & $\ldots$ & & 330 UG/KG & 1 \\
\hline 1, 4-Dichlorobenzene & 330 & UGKG & $u$ & .. & & 330 UG/KG & 1 \\
\hline Benzyl Alcohol & 330 & UG/KG & $u$ & - & & 330 UG/KG & 1 \\
\hline 1. 2-Dichlorobenzene & 330 & UG/KG & $u$ & - & & 330 UG/KG & 1 \\
\hline 2-Methylphenol & 330 & UGKG & $u$ & - & & 330 UG/KG & 1 \\
\hline bis(2-Chloroisopropyl) ether & 330 & UG/KG & $u$ & - & & 330 UG/KG & 1 \\
\hline 4-Methylphenol & 330 & UG/KG & $u$ & - & & 330 UG/KG & 1 \\
\hline N-Nitroso-di-nPropylamine & 330 & UG/KG & $u$ & - & & 330 UG/KG & 1 \\
\hline Hexachloroethane & 330 & UG/KG & $u$ & - & & 330 UG/KG & 1 \\
\hline Nitrobenzene & 330 & UG/KG & $u$ & -. & & 330 UG/KG & 1 \\
\hline Isophorone & 330 & UG/KG & $u$ & $\ldots$ & & 330 UG/KG & 1 \\
\hline 2-Nitrophenol & 330 & UG/KG & $u$ & $\ldots$ & & 330 UG/KG & 1 \\
\hline 2. 4-Dimethylphenol & 330 & UG/KG & $u$ & $\cdots$ & & 330 UG/KG & 1 \\
\hline Benzoic Acid & 1600 & UG/KG & $u$ & - & & 1600 UG/KG & 1 \\
\hline bis(2Chloroethoxy)methane & 330 & UG/KG & $u$ & - & & 330 UG/KG & 1 \\
\hline 2, 4-Dichlorophenol & 330 & UG/KG & $u$ & $\cdots$ & & 330 UG/KG & 1 \\
\hline 1,2, 4-Trichlorobenzene & 330 & UG/KG & $u$ & $\ldots$ & & 330 UG/KG & 1 \\
\hline Naphthalene & 330 & UG/KG & $u$ & - & & 330 UG/KG & 1 \\
\hline 4-Chloroaniline & 330 & UG/KG & $u$ & - & & 330 UG/KG & 1 \\
\hline Hexachlorobutadiene & 330 & UG/KG & $u$ & $\ldots$ & & 330 UG/KG & 1 \\
\hline 4-Chloro-3-Methylphenol & 330 & UGKG & $u$ & .- & & 330 UG/KG & 1 \\
\hline 2-Methyinaphthalene & 330 & UG/KG & $u$ & ... & & 330 UG/KG & 1 \\
\hline Hexachlorocyclopentadiene & 330 & UG/KG & $u$ & .. & & 330 UG/KG & 1 \\
\hline 2. 4, 6-Trichlorophenol & 330 & UGKG & $u$ & -- & & 330 UG/KG & 1 \\
\hline 2, 4,5-Trichlorophenol & 1600 & UGKG & $u$ & ... & & 1600 UG/KG & 1 \\
\hline 2-Chloronaphthalene & 330 & UG/KG & $u$ & .. & & 330 UG/KG & 1 \\
\hline 2-Nitroaniline & 1600 & UG/KG & $u$ & $\ldots$ & & 1600 UG/KG & 1 \\
\hline Dimethyl Phthalate & 330 & UGKG & $u$ & $\ldots$ & & 330 UG/KG & 1 \\
\hline Acenaphthylene & 330 & UG/KG & $u$ & -- & & 330 UGKG & 1 \\
\hline 2. 6-Dinitrotoluene & 330 & UG/KG & $u$ & ... & & 330 UG/KG & 1 \\
\hline 3-Nitroaniline & 1600 & UG/KG & $u$ & -- & & 1600 UG/KG & 1 \\
\hline Acenaphthene & 330 & UG/KG & $u$ & -. & & 330 UG/KG & 1 \\
\hline 2, 4-Dinitrophenol & 1600 & UG/KG & $u$ & -. & & 1600 UG/KG & 1 \\
\hline 4-Nitrophenol & 1600 & UGKG & $u$ & - & & 1600 UG/KG & 1 \\
\hline Dibenzofuran & 330 & UG/KG & $u$ & - & & 330 UG/KG & 1 \\
\hline 2, 4-Dinitrotoluene & 330 & UG/KG & $u$ & - & & 330 UG/KG & 1 \\
\hline Diethylphthalate & 330 & UG/KG & $u$ & $\ldots$ & & 330 UG/KG & 1 \\
\hline 4-Chlorophenyl phenyl ether & 330 & UG/KG & $u$ & - & & 330 UG/KG & 1 \\
\hline Fluorene & 330 & UGKG & $u$ & - & & 330 UG/KG & 1 \\
\hline 4-Nitroaniline & 1600 & UG/KG & $u$ & - & & 1600 UG/KG & 1 \\
\hline 4, 6-Dinitro-2-Methylphenol & 1600 & UG/KG & $u$ & - & & 1600 UG/KG & 1 \\
\hline N-Nitrosodiphenylamine & 330 & UGKG & $u$ & - & & 330 UG/KG & 1 \\
\hline 4-Bromophenyl phenyl ether & 330 & UGKG & $u$ & - & & 330 UGKG & 1 \\
\hline Hexachlorobenzene & 330 & UGKG & $u$ & - & & 330 UG/KG & 1 \\
\hline Pentacinlorophenol & 1600 & UGKG & $u$ & - & & 1600 UGKG & 1 \\
\hline Phenanthrene & 330 & UG/KG & $u$ & -. & & 330 UG/KG & 1 \\
\hline
\end{tabular}


Appendix D: Results of Semivolatile Organic Compound Analyses, Salmon Site

\begin{tabular}{|c|c|c|c|c|c|c|c|c|}
\hline COMPOUND & RESULTS & UNITS & $\begin{array}{l}\text { DATA } \\
\text { QUAL }\end{array}$ & $\begin{array}{l}\text { DATA } \\
\text { VALID. }\end{array}$ & NOTES & CADL & UNITS & DILUTION \\
\hline \multicolumn{9}{|c|}{ TR2-ES-356N-175E-8Z(CON'T) } \\
\hline Anthracene & 330 & UG/KG & $u$ & - & & & UG/KG & 1 \\
\hline Di-N-Butyiphthaiate & 330 & UG/KG & $u$ & - & & & UG/KG & 1 \\
\hline Fluoranthene & 330 & UG/KG & $u$ & - & & & UG/KG & 1 \\
\hline Pyrene & 330 & UG/KG & $u$ & - & & & UG/KG & 1 \\
\hline Butyl Benzyl Phthalate & 330 & UG/KG & $u$ & - & & & UG/KG & 1 \\
\hline 3. 3'-Dichlorobenzidine & 650 & UG/KG & $u$ & - & & & UG/KG & 1 \\
\hline Benzo(a)Anthracene & 330 & UG/KG & $u$ & - & & & UG/KG & 1 \\
\hline Chrysene & 330 & UG/KG & $u$ & - & & & UG/KG & 1 \\
\hline bis(2-Ethylhexyl)phthalate & 330 & UG/KG & $u$ & ... & & & UG/KG & 1 \\
\hline di-N-Octyl Phthalate & 330 & UG/KG & $u$ & ..- & & & UG/KG & 1 \\
\hline Benzo(b)Fluoranthene & 330 & UG/KG & $u$ & - & & & O UG/KG & 1 \\
\hline Benzo(k)Fluoranthene & 330 & UG/KG & U & - & & & O UG/KG & 1 \\
\hline Benzo(a)Pyrene & 330 & UG/KG & $u$ & -. & & & O UG/KG & 1 \\
\hline Indeno(1,2,3-CD)Pyrene & 330 & UG/KG & U & $\cdots$ & . & & O UG/KG & 1 \\
\hline Dibenzo(a,h)Anthracene & 330 & UG/KG & $u$ & ..- & & & O UG/KG & 1 \\
\hline Benzo(g,h,i)Perylene & 330 & $U G / K G$ & $u$ & $\ldots$ & & & O UG/KG & 1 \\
\hline Nitrobenzene-d5 & 67 & $\%$ & & & & & $\%$ & 1 \\
\hline 2-Fluorobiphenyl & 61 & $\%$ & & & & & $\%$ & 1 \\
\hline Terphenyl-d14 & 75 & $\%$ & & & & & $\%$ & 1 \\
\hline Phenol-d5 & 72 & $\%$ & & & & & $\%$ & 1 \\
\hline 2-Fluorophenol & 64 & $\%$ & & & & & $\%$ & 1 \\
\hline 2, 4, 6-Tribromophenol & 61 & $\%$ & & & & & $\%$ & 1 \\
\hline
\end{tabular}


Appendix D: Results of Semivolatile Organic Compound Analyses, Salmon Site

\begin{tabular}{|c|c|c|c|c|c|c|c|}
\hline COMPOUND & RESULTS & UNITS & $\begin{array}{l}\text { DATA } \\
\text { QUAL } \\
\end{array}$ & $\begin{array}{l}\text { DATA } \\
\text { VALID. }\end{array}$ & NOTES & CRDL UNITS & DILUTION \\
\hline \multicolumn{8}{|l|}{ TR2A-ES-365N-200E-3.52 } \\
\hline Phenol & 330 & UG/KG & $u$ & $\cdots$ & & 330 UG/KG & 1 \\
\hline bis(2-Chloroethyl) ether & 330 & UG/KG & $u$ & $\ldots$ & & 330 UG/KG & 1 \\
\hline 2-Chlorophenol & 330 & UG/KG & $u$ & -. & & 330 UG/KG & 1 \\
\hline 1, 3-Dichlorobenzene & 330 & UG/KG & $u$ & $\ldots$ & & 330 UG/KG & 1 \\
\hline 1, 4-Dichlorobenzene & 330 & UG/KG & u & - & & 330 UG/KG & 1 \\
\hline Benzyl Alcohol & 330 & UG/KG & $u$ & -. & & 330 UG/KG & 1 \\
\hline 1, 2-Dichlorobenzene & 330 & UG/KG & $u$ & .- & & 330 UG/KG & 1 \\
\hline 2-Methylphenol & 330 & UG/KG & $u$ & -. & & 330 UG/KG & 1 \\
\hline bis(2-Chloroisopropyl) ether & 330 & UG/KG & $u$ & ... & & 330 UG/KG & 1 \\
\hline 4-Methyiphenol & 330 & UG/KG & u & -. & & 330 UG/KG & 1 \\
\hline N-Nitroso-di-nPropylamine & 330 & UG/KG & $u$ & -.. & & 330 UG/KG & 1 \\
\hline Hexachloroethane & 330 & UG/KG & $u$ & ..- & & 330 UG/KG & 1 \\
\hline Nitrobenzene & 330 & UG/KG & $u$ & $\ldots$ & & 330 UG/KG & 1 \\
\hline Isophorone & 330 & UG/KG & $u$ & $\ldots$ & & 330 UG/KG & 1 \\
\hline 2-Nitrophenol & 330 & UG/KG & $u$ & $\ldots$ & & 330 UG/KG & 1 \\
\hline 2, 4-Dimethylphenol & 330 & UG/KG & $u$ & $\ldots$ & & 330 UG/KG & 1 \\
\hline Benzoic Acid & 1600 & UG/KG & $u$ & $\ldots$ & & 1600 UG/KG & 1 \\
\hline bis(2Chloroethoxy)methane & 330 & UG/KG & $u$ & ... & & 330 UG/KG & 1 \\
\hline 2, 4-Dichlorophenol & 330 & UG/KG & $u$ & -. & & 330 UG/KG & 1 \\
\hline 1,2, 4-Trichlorobenzene & 330 & UG/KG & $u$ & -. & & 330 UG/KG & 1 \\
\hline Naphthalene & 330 & UG/KG & $u$ & - & & 330 UG/KG & 1 \\
\hline 4-Chloroaniline & 330 & UG/KG & $u$ & -. & & 330 UG/KG & 1 \\
\hline Hexachlorobutadiene & 330 & UG/KG & $u$ & - & & 330 UG/KG & 1 \\
\hline 4-Chloro-3-Methylphenol & 330 & UG/KG & $u$ & ... & & 330 UG/KG & 1 \\
\hline 2-Methylnaphthalene & 330 & UG/KG & $u$ & $\ldots$ & & 330 UG/KG & 1 \\
\hline Hexachlorocyclopentadiene & 330 & UG/KG & $u$ & ... & & 330 UG/KG & 1 \\
\hline 2, 4, 6-Trichlorophenol & 330 & UG/KG & u & ... & & 330 UG/KG & 1 \\
\hline 2, 4, 5-Trichlorophenol & 1600 & UG/KG & $u$ & $\cdots$ & & 1600 UG/KG & 1 \\
\hline 2-Chloronaphthalene & 330 & UG/KG & u & -. & & 330 UG/KG & 1 \\
\hline 2-Nitroaniline & 1600 & UG/KG & u & $\cdots$ & & 1600 UG/KG & 1 \\
\hline Dimethyl Phthalate & 330 & UG/KG & u & .. & & 330 UG/KG & 1 \\
\hline Acenaphthylene & 330 & UG/KG & $u$ & -. & & 330 UG/KG & 1 \\
\hline 2, 6-Dinitrotoluene & 330 & UG/KG & U & ... & & 330 UG/KG & 1 \\
\hline 3-Nitroaniline & 1600 & UG/KG & u & $\ldots$ & & 1600 UG/KG & 1 \\
\hline Acenaphthene & 330 & UG/KG & $u$ & ... & & 330 UG/KG & 1 \\
\hline 2, 4-Dinitrophenol & 1600 & UG/KG & U & $\ldots$ & & 1600 UG/KG & 1 \\
\hline 4-Nitrophenol & 330 & UG/KG & $u$ & -. & & 1600 UG/KG & 1 \\
\hline Dibenzofuran & 330 & UG/KG & $u$ & ... & & 330 UG/KG & 1 \\
\hline 2, 4-Dinitrotoluene & 330 & UG/KG & u & -. & & 330 UG/KG & 1 \\
\hline Diethylphthalate & 330 & UGKG & U & - & & 330 UG/KG & 1 \\
\hline 4-Chlorophenyl phenyl ether & 330 & UG/KG & u & -. & & 330 UG/KG & 1 \\
\hline Fluorene & 330 & UG/KG & U & - & & 330 UG/KG & 1 \\
\hline 4-Nitroaniline & 1600 & UG/KG & u & - & & 1600 UG/KG & 1 \\
\hline 4, 6-Dinitro-2-Methylphenol & 1600 & UG/KG & U & - & & 1600 UG/KG & 1 \\
\hline N-Nitrosodiphenylamine & 330 & UG/KG & $u$ & - & & 330 UG/KG & 1 \\
\hline 4-Bromophenyl phenyl ether & 330 & UG/KG & u & - & & 330 UG/KG & 1 \\
\hline Hexachlorobenzene & 330 & UG/KG & U & - & & 330 UG/KG & 1 \\
\hline Pentachlorophenol & 1600 & UG/KG & u & - & & 1600 UG/KG & 1 \\
\hline Phenanthrene & 330 & UG/KG & U & -. & & 330 UG/KG & 1 \\
\hline
\end{tabular}


Appendix D: Results of Semivolatile Organic Compound Analyses, Salmon Site

\begin{tabular}{|c|c|c|c|c|c|c|c|c|}
\hline COMPOUND & RESULTS & UNITS & $\begin{array}{l}\text { DATA } \\
\text { QUAL }\end{array}$ & $\begin{array}{l}\text { DATA } \\
\text { VALID. }\end{array}$ & NOTES & CRDL & UNITS & DILUTION \\
\hline \multicolumn{9}{|c|}{ TR2A-ES-365N-200E-3.52(CON'T) } \\
\hline Anthracene & 330 & UG/KG & $u$ & .- & & & O UG/KG & 1 \\
\hline Di-N-Butyiphthalate & 330 & UG/KG & $u$ & - & & & O UG/KG & 1 \\
\hline Fluoranthene & 330 & UG/KG & $u$ & - & & & O UG/KG & 1 \\
\hline Pyrene & 330 & UG/KG & $u$ & $\ldots$ & & & O UG/KG & 1 \\
\hline Butyl Benzyl Phthalate & 330 & UG/KG & $u$ & ... & & & O UG/KG & 1 \\
\hline 3, 3'-Dichlorobenzidine & 660 & UG/KG & $u$ & - & & & O UG/KG & 1 \\
\hline Benzo(a)Anthracene & 330 & UG/KG & $u$ & - & & & O UG/KG & 1 \\
\hline Chrysene & 330 & UG/KG & $u$ & ... & & & O UG/KG & 1 \\
\hline bis(2-Ethylhexyl)phthalate & 330 & UG/KG & $u$ & ... & & & O UG/KG & 1 \\
\hline di-N-Octyl Phthalate & 330 & UG/KG & $u$ & ..- & & & $0 \mathrm{U}$ UG/KG & 1 \\
\hline Benzo(b)Fluoranthene & 330 & $U G / K G$ & $u$ & $\ldots$ & & & O UG/KG & 1 \\
\hline Benzo(k)Fluoranthene & 330 & UG/KG & $u$ & $\ldots$ & & & $30 \mathrm{UG} / \mathrm{KG}$ & 1 \\
\hline Benzo(a)Pyrene & 330 & UG/KG & $u$ & $\ldots$ & & & 30 UG/KG & 1 \\
\hline Indeno(1,2,3-CD)Pyrene & 330 & UG/KG & $u$ & $\ldots$ & & & 30 UG/KG & 1 \\
\hline Dibenzo(a,h)Anthracene & 330 & $U G / K G$ & $u$ & $\ldots$ & & & 30 UG/KG & 1 \\
\hline Benzo(g,h,i)Perylene & 330 & UG/KG & $u$ & $\cdots$ & & & 30 UG/KG & 1 \\
\hline Nitrobenzene-d5 & 66 & $\%$ & & & & & $\%$ & 1 \\
\hline 2-Fluorobiphenyl & 59 & $\%$ & & & & & $\%$ & 1 \\
\hline Terphenyl-d14 & 70 & $\%$ & & & & & $\%$ & 1 \\
\hline Phenol-d5 & 75 & $\%$ & & & & & $\%$ & 1 \\
\hline 2-Fluorophenol & 64 & $\%$ & & & & & $\%$ & 1 \\
\hline 2, 4, 6-Tribromophenol & 57 & $\%$ & & & & & $\%$ & 1 \\
\hline
\end{tabular}




\begin{tabular}{|c|c|c|c|c|c|c|c|}
\hline COMPOUND & RESULTS & UNITS & $\begin{array}{l}\text { DATA } \\
\text { QUAL }\end{array}$ & $\begin{array}{l}\text { DATA } \\
\text { VALID. }\end{array}$ & NOTES & CRDL UNITS & DILUTION \\
\hline \multicolumn{8}{|l|}{ TR2A-ES-360N-200E-8Z } \\
\hline Phenol & 330 & UG/KG & $u$ & ... & & 330 UG/KG & 1 \\
\hline bis(2-Chloroethyl) ether & 330 & UG/KG & $u$ & ... & & 330 UG/KG & 1 \\
\hline 2-Chlorophenol & 330 & UG/KG & $u$ & ... & & 330 UG/KG & 1 \\
\hline 1. 3-Dichlorobenzene & 330 & $U G / K G$ & $u$ & $\ldots$ & & 330 UG/KG & 1 \\
\hline 1, 4-Dichlorobenzene & 330 & UG/KG & $u$ & $\ldots$ & & 330 UG/KG & 1 \\
\hline Benzyl Alcohol & 330 & UG/KG & $u$ & -. & & 330 UG/KG & 1 \\
\hline 1, 2-Dichlorobenzene & 330 & UG/KG & $u$ & .. & & 330 UG/KG & 1 \\
\hline 2-Methylphenol & 330 & UG/KG & $u$ & $\ldots$ & & 330 UG/KG & 1 \\
\hline bis(2-Chloroisopropyi) ether & 330 & UG/KG & $u$ & $\ldots$ & & 330 UG/KG & 1 \\
\hline 4-Methylphenol & 330 & UG/KG & $u$ & $\ldots$ & & 330 UG/KG & 1 \\
\hline N-Nitroso-di-nPropylamine & 330 & UG/KG & $u$ & $\ldots$ & & 330 UG/KG & 1 \\
\hline Hexachloroethane & 330 & UG/KG & u & $\ldots$ & & 330 UG/KG & 1 \\
\hline Nitrobenzene & 330 & UG/KG & $u$ & ... & & 330 UG/KG & 1 \\
\hline Isophorone & 330 & UG/KG & u & ... & & 330 UG/KG & 1 \\
\hline 2-Nitrophenol & 330 & UG/KG & $u$ & ... & & 330 UG/KG & 1 \\
\hline 2, 4-Dimethylphenol & 330 & UG/KG & $u$ & $\ldots$ & & 330 UG/KG & 1 \\
\hline Benzoic Acid & 1600 & $U G / K G$ & $u$ & ... & & 1600 UG/KG & 1 \\
\hline bis(2Chloroethoxy)methane & 330 & UG/KG & $u$ & ... & & 330 UG/KG & 1 \\
\hline 2, 4-Dichlorophenol & 330 & UG/KG & $u$ & ... & & 330 UG/KG & 1 \\
\hline 1, 2, 4-Trichlorobenzene & 330 & UG/KG & $u$ & ... & & 330 UG/KG & 1 \\
\hline Naphthalene & 330 & UG/KG & $u$ & ... & & 330 UG/KG & 1 \\
\hline 4-Chloroaniline & 330 & UG/KG & $u$ & ... & & 330 UG/KG & 1 \\
\hline Hexachlorobutadiene & 330 & UG/KG & $u$ & $\ldots$ & & 330 UG/KG & 1 \\
\hline 4-Chloro-3-Methylphenol & 330 & UG/KG & $u$ & ... & & 330 UG/KG & 1 \\
\hline 2-Methylnaphthalene & 330 & UG/KG & $u$ & $\ldots$ & & 330 UG/KG & 1 \\
\hline Hexachlorocyclopentadiene & 330 & UG/KG & $u$ & ... & & 330 UG/KG & 1 \\
\hline 2, 4, 6-Trichlorophenol & 330 & UG/KG & u & $\cdots$ & & 330 UG/KG & 1 \\
\hline 2, 4, 5-Trichlorophenol & 1600 & UG/KG & $u$ & $\cdots$ & & 1600 UG/KG & 1 \\
\hline 2-Chloronaphthalene & 330 & UG/KG & u & ... & & 330 UG/KG & 1 \\
\hline 2-Nitroaniline & 1600 & UG/KG & $u$ & ... & & 1600 UG/KG & 1 \\
\hline Dimethyl Phthalate & 330 & UG/KG & u & .. & & 330 UG/KG & 1 \\
\hline Acenaphthylene & 330 & UG/KG & u & ... & & 330 UG/KG & 1 \\
\hline 2, 6-Dinitrotoluene & 330 & UG/KG & $u$ & ... & & 330 UG/KG & 1 \\
\hline 3-Nitroaniline & 1600 & UG/KG & $u$ & -.. & & 1600 UG/KG & 1 \\
\hline Acenaphthene & 330 & UG/KG & $u$ & $\cdots$ & & 330 UG/KG & 1 \\
\hline 2, 4-Dinitrophenol & 1600 & UG/KG & u & $\cdots$ & & 1600 UG/KG & 1 \\
\hline 4-Nitrophenol & 1600 & $U G / K G$ & $u$ & $\ldots$ & & 1600 UG/KG & 1 \\
\hline Dibenzofuran & 330 & $U G / K G$ & $u$ & ... & & 330 UG/KG & 1 \\
\hline 2, 4-Dinitrotoluene & 330 & UG/KG & $u$ & ... & & 330 UG/KG & 1 \\
\hline Diethylphthalate & 330 & UG/KG & $u$ & ... & & 330 UG/KG & 1 \\
\hline 4-Chlorophenyl phenyl ether & 330 & $U G / K G$ & u & ... & & 330 UG/KG & 1 \\
\hline Fluorene & 330 & UG/KG & $u$ & ... & & 330 UG/KG & 1 \\
\hline 4-Nitroaniline & 1600 & UG/KG & $u$ & -. & & 1600 UG/KO & 1 \\
\hline 4, 6-Dinitro-2-Methylphenol & 1600 & UG/KG & $u$ & ... & & 1600 UG/KG & 1 \\
\hline N-Nitrosodiphenylamine & 330 & UG/KG & $u$ & $\ldots$ & & 330 UG/KG & 1 \\
\hline 4-Bromophenyl phenyl ether & 330 & UG/KG & $u$ & $\ldots$ & & 330 UG/KG & 1 \\
\hline Hexachlorobenzene & 330 & UG/KG & $u$ & ... & & 330 UG/KG & 1 \\
\hline Pentachlorophenol & 1600 & UG/KG & $u$ & ... & & 1600 UG/KG & 1 \\
\hline Phenanthrene & 330 & UG/KG & $u$ & $\ldots$ & & 330 UG/KG & 1 \\
\hline
\end{tabular}


Appendix D: Results of Semivolatile Organic Compound Analyses, Salmon Site

\begin{tabular}{|c|c|c|c|c|c|c|c|c|}
\hline COMPOUND & RESULTS & UNITS & $\begin{array}{l}\text { DATA } \\
\text { QUAL }\end{array}$ & $\begin{array}{l}\text { DATA } \\
\text { VALID. }\end{array}$ & NOTES & CRDL & UNITS & DILUTION \\
\hline \multicolumn{9}{|l|}{ TA2A-ES-360N-200E-8Z(CONT) } \\
\hline Anthracene & 330 & UG/KG & $u$ & -. & & & UG/KG & 1 \\
\hline Di-N-Butylphthalate & 330 & UG/KG & $u$ & ... & & & UG/KG & 1 \\
\hline Fluoranthene & 330 & UG/KG & $u$ & ... & & & UG/KG & 1 \\
\hline Pyrene & 330 & UG/KG & $u$ & $\ldots$ & & & UG/KG & 1 \\
\hline Butyl Benzyl Phthalate & 330 & UG/KG & $u$ & $\ldots$ & & & UG/KG & 1 \\
\hline 3, 3'-Dichlorobenzidine & 660 & UG/KG & $u$ & - & & & UG/KG & 1 \\
\hline Benzo(a)Anthracene & 330 & UG/KG & $u$ & ... & & & Ua/Ka & 1 \\
\hline Chrysene & 330 & UG/KG & $u$ & $\ldots$ & & & UG/KG & 1 \\
\hline bis(2-Ethylhexyl)phthalate & 330 & UG/KG & $u$ & $\ldots$ & & & UG/KG & 1 \\
\hline di-N-Octyl Phthalate & 330 & UG/KG & $u$ & $\ldots$ & & & Ua/KG & 1 \\
\hline Benzo(b)Fluoranthene & 330 & $U G / K G$ & $u$ & $\ldots$ & & & UG/KG & 1 \\
\hline Benzo(k)Fluoranthene & 330 & UG/KG & $u$ & $\ldots$ & & & UG/KG & 1 \\
\hline Benzo(a)Pyrene & 330 & UG/KG & $u$ & $\ldots$ & & & UG/KG & 1 \\
\hline Indeno(1,2,3-CD)Pyrene & 330 & $U G / K G$ & u & $\ldots$ & & & UG/KG & 1 \\
\hline Dibenzo(a.h)Anthracene & 330 & UG/KG & $u$ & $\ldots$ & & & UG/KG & 1 \\
\hline Benzo(g,h,i)Perylene & 330 & UG/KG & $u$ & $\ldots$ & & & UG/KG & 1 \\
\hline Nitrobenzene-d5 & 64 & $\%$ & & & & & $\%$ & 1 \\
\hline 2-Fluorobiphenyl & 55 & $\%$ & & & & & $\%$ & 1 \\
\hline Terphenyi-d14 & 67 & $\%$ & & & & & $\%$ & 1 \\
\hline Phenol-d5 & 67 & $\%$ & & & & & $\%$ & 1 \\
\hline 2-Fluorophenol & 57 & $\%$ & & & & & $\%$ & 1 \\
\hline 2, 4, 6-Tribromophenol & 52 & $\%$ & & & & & $\%$ & 1 \\
\hline
\end{tabular}


Appendix D: Results of Semivolatile Organic Compound Analyses, Salmon Site

\begin{tabular}{|c|c|c|c|c|c|c|c|}
\hline COMPOUND & RESULTS & UNITS & $\begin{array}{l}\text { DATA } \\
\text { QUAL }\end{array}$ & $\begin{array}{l}\text { DATA } \\
\text { VALID. }\end{array}$ & NOTES & CADL UNITS & DILUTION \\
\hline \multicolumn{8}{|l|}{ TA3-ES-450N-400E-3Z } \\
\hline Phenol & 330 & UG/KG & $u$ & ... & & 330 UG/KG & 1 \\
\hline bis(2-Chloroethyl) ether & 330 & UG/KG & $u$ & $\ldots$ & & 330 Ua/KG & 1 \\
\hline 2-Chlorophenol & 330 & UG/KG & $u$ & ... & & 330 Ua/KG & 1 \\
\hline 1, 3-Dichlorobenzene & 330 & UG/KG & $u$ & $\ldots$ & & 330 UG/KG & 1 \\
\hline 1, 4-Dichlorobenzene & 330 & UG/KG & $u$ & $\ldots$ & & 330 UG/KG & 1 \\
\hline Benzyl Alcohol & 330 & UG/KG & $U$ & ... & & 330 UG/KG & 1 \\
\hline 1, 2-Dichlorobenzene & 330 & UG/KG & $u$ & $\ldots$ & & 330 UG/KG & 1 \\
\hline 2-Methylphenol & 330 & UG/KG & $u$ & .. & & $330 \mathrm{UG} / \mathrm{KG}$ & 1 \\
\hline bis(2-Chloroisopropyl) ether & 330 & UG/KG & $u$ & ... & & 330 UG/KG & 1 \\
\hline 4-Methylphenol & 330 & UG/KG & $u$ & $\ldots$ & & 330 UG/KG & 1 \\
\hline N-Nitroso-di-nPropylamine & 330 & UG/KG & u & ... & & 330 UG/KG & 1 \\
\hline Hexachloroethane & 330 & UG/KG & $u$ & ... & & 330 UG/KG & 1 \\
\hline Nitrobenzene & 330 & UG/KG & $u$ & $\ldots$ & & 330 UG/KG & 1 \\
\hline Isophorone & 330 & UG/KG & $u$ & $\ldots$ & & 330 UG/KG & 1 \\
\hline 2-Nitrophenol & 330 & UG/KG & $\mathrm{U}$ & $\ldots$ & & 330 UG/KG & 1 \\
\hline 2, 4-Dimethylphenol & 330 & UG/KG & $u$ & ... & & 330 UG/KG & 1 \\
\hline Benzolc Acid & 1600 & UG/KG & $u$ & ... & & 1600 UG/KG & 1 \\
\hline bis(2Chloroethoxy)methane & 330 & UG/KG & $u$ & $\ldots$ & & 330 UG/KG & 1 \\
\hline 2, 4-Dichlorophenol & 330 & UG/KG & $u$ & $\ldots$ & & 330 UG/KG & 1 \\
\hline 1, 2, 4-Trichlorobenzene & 330 & UG/KG & $u$ & ... & & 330 UG/KG & 1 \\
\hline Naphthalene & 330 & UG/KO & u & ... & & 330 Ua/KG & 1 \\
\hline 4-Chloroaniline & 330 & UG/KG & u & ... & & 330 UG/KG & 1 \\
\hline Hexachlorobutadiene & 330 & UG/KG & u & $\cdots$ & & 330 UG/KG & 1 \\
\hline 4-Chloro-3-Methylphenol & 330 & UG/KG & $u$ & $\ldots$ & & 330 UG/KG & 1 \\
\hline 2-Methylnaphthalene & 4200 & UG/KG & $E$ & R & A & 330 UG/KG & 1 \\
\hline Hexachlorocyclopentadiene & 330 & UG/KG & $u$ & A & A & 330 UG/KG & 1 \\
\hline 2, 4, 6-Trichlorophenol & 330 & UG/KG & $u$ & R & A & 330 UG/KG & 1 \\
\hline 2, 4, 5-Trichlorophenol & 1600 & UG/KG & $u$ & R & A & 1600 UG/KG & 1 \\
\hline 2-Chloronaphthalene & 330 & UG/KG & $U$ & R & A & 330 UG/KG & 1 \\
\hline 2-Nitroaniline & 1600 & UG/KG & $U$ & A & A & 1600 UG/KG & 1 \\
\hline Dimethyl Phthalate & 330 & UG/KG & $U$ & A & A & 330 UG/KG & 1 \\
\hline Acenaphthylene & 330 & UG/KG & $u$ & A & A & 330 UG/KG & 1 \\
\hline 2, 6-Dinitrotoluene & 330 & UG/KG & U & $R$ & A & 330 UG/KG & 1 \\
\hline 3-Nitroaniline & 1600 & UG/KG & $u$ & A & & 1600 UG/KG & 1 \\
\hline Acenaphthene & 330 & UG/KG & u & R & & 330 UG/KG & 1 \\
\hline 2. 4-Dinitrophenol & 1600 & UG/KG & u & ค & & 1600 UG/KG & 1 \\
\hline 4-Nitrophenol & 1600 & UG/KG & u & R & & 1600 UG/KG & 1 \\
\hline Dibenzofuran & 330 & UG/KG & $u$ & R & & 330 UG/KG & 1 \\
\hline 2, 4-Dinitrotoluene & 330 & UG/KG & $u$ & R & & 330 UG/KG & 1 \\
\hline Diethylphthalate & 330 & UG/KG & $u$ & A & & 330 UG/KG & 1 \\
\hline 4-Chlorophenyl phenyl ether & 330 & UG/KG & u & A & & 330 UG/KG & 1 \\
\hline Fluorene & 330 & UG/KG & u & A & & 330 UG/KG & 1 \\
\hline 4-Nitroaniline & 1600 & VGKG & u & -. & & 1600 UG/KG & 1 \\
\hline 4, 6-Dinitro-2-Methylphenol & 1600 & UG/KG & u & -.. & & 1600 UG/KG & 1 \\
\hline N-Nitrosodiphenylamine & 330 & VGKG & $u$ & -. & & 330 Ua/rG & 1 \\
\hline 4-Bromophenyl phenyl ether & 330 & VG/KG & u & -.. & & 330 UG/KG & 1 \\
\hline Hexachlorobenzene & 330 & UG/KG & $u$ & ... & & 330 UG/KG & 1 \\
\hline Pentachlorophenol & 1600 & VG/KG & $u$ & - & & 1600 UG/KG & 1 \\
\hline Phenanthrene & 330 & UG/KG & $u$ & $\ldots$ & & 330 UG/KG & 1 \\
\hline
\end{tabular}


Appendix D: Results of Semivolatile Organic Compound Analyses, Salmon Site

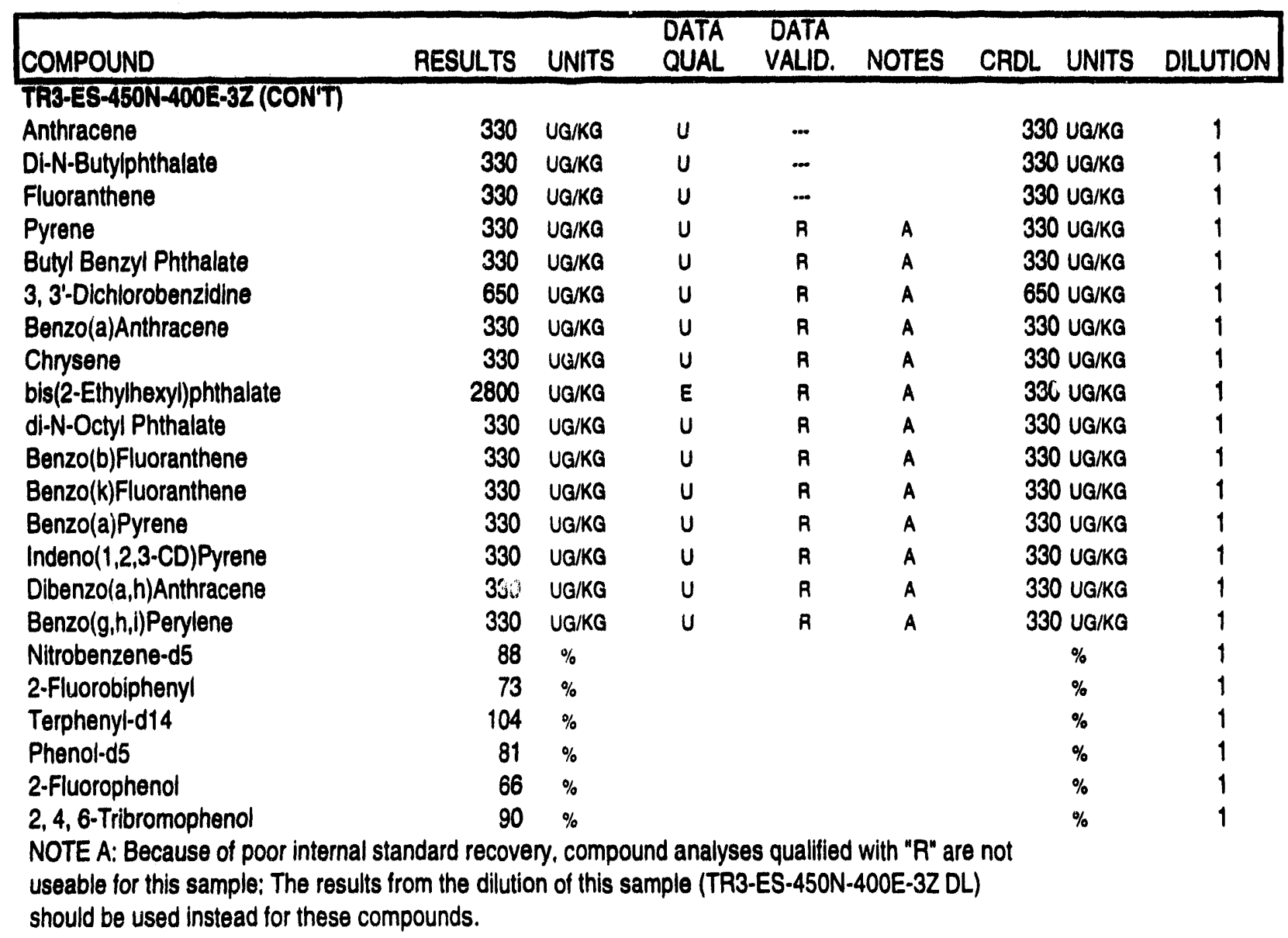




\begin{tabular}{|c|c|c|c|c|c|c|c|}
\hline COMPOUND & RESULTS & UNITS & $\begin{array}{l}\text { DATA } \\
\text { QUAL }\end{array}$ & $\begin{array}{l}\text { DATA } \\
\text { VALID. }\end{array}$ & NOTES & CADL UNITS & DILUTION \\
\hline \multicolumn{8}{|l|}{ TA3-ES-450N-400E-32DL } \\
\hline Phenol & 3300 & UG/KG & $u$ & $\mathbf{R}$ & & 3300 UG/KG & 10 \\
\hline bis(2-Chloroethyl) ether & 3300 & UG/KG & u & ค & & 3300 UG/KG & 10 \\
\hline 2-Chlorophenol & 3300 & VG/Ka & $u$ & R & & 3300 UG/KG & 10 \\
\hline 1, 3-Dichlorobenzene & 3300 & UG/KG & $u$ & R & & 3300 UG/KG & 10 \\
\hline 1, 4-Dichlorobenzene & 3300 & UG/KG & $u$ & R & & 3300 UG/KG & 10 \\
\hline Benzyl Alcohol & 3300 & UG/KG & $u$ & R & & 3300 UG/KG & 10 \\
\hline 1. 2-Dichlorobenzene & 3300 & UG/KG & $u$ & A & & 3300 UG/KG & 10 \\
\hline 2-Methylphenol & 3300 & Ua/Ka & $u$ & A & & 3300 UG/KG & 10 \\
\hline bis(2-Chloroisopropyl) ether & 3300 & UG/KG & $u$ & R & & 3300 UG/KG & 10 \\
\hline 4-Methylphenol & 3300 & Ua/Ko & $u$ & R & & 3300 UG/KG & 10 \\
\hline N-Nitroso-di-nPropylamine & 3300 & Va/KG & $u$ & R & & 3300 UG/KG & 10 \\
\hline Hexachloroethane & 3300 & Ua/KG & u & R & & 3300 UG/KG & 10 \\
\hline Nitrobenzene & 3300 & UG/KG & $u$ & R & & 3300 VG/KG & 10 \\
\hline Isophorone & 3300 & UG/KG & $u$ & A & & 3300 Ua/KG & 10 \\
\hline 2-Nitrophenol & 3300 & UG/KG & $u$ & R & & 3300 UG/KG & 10 \\
\hline 2. 4-Dimethyiphenol & 3300 & $U G / K G$ & $u$ & R & & 3300 UG/KG & 10 \\
\hline Benzoic Acid & 16000 & $U G / K G$ & $u$ & R & & 16000 UG/KG & 10 \\
\hline bis(2Chloroethoxy)methane & 3300 & UG/KG & $u$ & R & & 3300 UG/KG & 10 \\
\hline 2, 4-Dichlorophenol & 3300 & UG/KG & $u$ & R & & 3300 UQ/KG & 10 \\
\hline 1, 2, 4-Trichlorobenzene & 3300 & UQ/KG & $u$ & R & & 3300 UG/KG & 10 \\
\hline Naphthalene & 3300 & UG/KG & $u$ & A & & 3300 UG/KG & 10 \\
\hline 4-Chloroaniline & 3300 & UQ/KG & u & R & & 3300 UG/KG & 10 \\
\hline Hexachlorobutadiene & 3300 & UG/KG & $u$ & $\mathbf{R}$ & & 3300 UG/KG & 10 \\
\hline 4-Chloro-3-Methylphenol & 3300 & $\mathrm{UG} / \mathrm{KG}$ & $u$ & R & & 3300 UG/KG & 10 \\
\hline 2-Methylnaphthalene & 11000 & UG/KG & D & ... & & 3300 UG/KG & 10 \\
\hline Hexachlorocyclopentadiene & 3300 & UG/KG & $u$ & ... & & 3300 UG/KG & 10 \\
\hline 2, 4, 6-Trichlorophenol & 3300 & UG/KG & $u$ & ... & & 3300 Ua/KG & 10 \\
\hline 2, 4,5-Trichlorophenol & 16000 & UG/KG & $u$ & ... & & 16000 UG/KG & 10 \\
\hline 2-Chloronaphthalene & 3300 & UG/KG & U & ... & & 3300 UG/KG & 10 \\
\hline 2-Nitroaniline & 16000 & UG/KG & U & $\ldots$ & & 16000 UG/KG & 10 \\
\hline Dimethyl Phthalate & 3300 & UG/KG & $u$ & $\ldots$ & & 3300 UG/KG & 10 \\
\hline Acenaphthylene & 3300 & UG/KG & $u$ & $\cdots$ & & 3300 UG/KG & 10 \\
\hline 2, 6-Dinitrotoluene & 3300 & UG/KG & $u$ & ... & & 3300 UG/KG & 10 \\
\hline 3-Nitroaniline & 16000 & UG/KG & $u$ & ... & & 16000 UG/KG & 10 \\
\hline Acenaphthene & 3300 & UG/KG & $u$ & ... & & 3300 UG/KG & 10 \\
\hline 2, 4-Dinitrophenol & 16000 & UG/KG & $u$ & $\ldots$ & & 16000 UG/KG & 10 \\
\hline 4-Nitrophenol & 16000 & UG/KG & $u$ & ... & & $16000 \mathrm{UG} / \mathrm{KG}$ & 10 \\
\hline Dibenzofuran & 3300 & UG/KG & $u$ & $\ldots$ & & 3300 UG/KG & 10 \\
\hline 2, 4-Dinitrotoluene & 3300 & UG/KG & $u$ & $\ldots$ & & 3300 UG/KG & 10 \\
\hline Diethylphthalate & 3300 & $U G / K G$ & $u$ & ... & & 3300 UG/KG & 10 \\
\hline 4-Chlorophenyl phenyl ether & 3300 & UG/KG & U & $\ldots$ & & 3300 UG/KG & 10 \\
\hline Fluorene & 1100 & UG/KG & DJ & $\mathrm{J}$ & & 3300 UG/KG & 10 \\
\hline 4-Nitroaniline & 16000 & UG/KG & u & R & B & $16000 \mathrm{Ua} / \mathrm{Ka}$ & 10 \\
\hline 4, 6-Dinitro-2-Methylphenol & 16000 & UG/KG & $u$ & R & B & 16000 UG/KG & 10 \\
\hline N-Nitrosodiphenylamine & 3300 & UG/KG & u & ค & B & $3300 \mathrm{Va} / \mathrm{Ka}$ & 10 \\
\hline 4-Bromophenyl phenyl ether & 3300 & UG/KG & u & R & B & 3300 UG/KG & 10 \\
\hline Hexachlorobenzene & 3300 & UG/KG & u & $\mathbf{R}$ & B & 3300 UG/KG & 10 \\
\hline Pentachlorophenol & 16000 & UG/KG & u & A & $B$ & 16000 UG/KG & 10 \\
\hline Phenanthrene & 3300 & UG/KG & $u$ & R & B & 3300 UG/KG & 10 \\
\hline
\end{tabular}


Appendix D: Results of Semivolatile Organic Compound Analyses, Salmon Site

\begin{tabular}{|c|c|c|c|c|c|c|c|c|}
\hline COMPOUND & RESULTS & UNITS & $\begin{array}{l}\text { DATA } \\
\text { QUAL }\end{array}$ & $\begin{array}{l}\text { DATA } \\
\text { VALID. }\end{array}$ & NOTES & CRDL & UNITS & DILUTION \\
\hline \multicolumn{9}{|c|}{ TR3-ES-450N-400E-32 DL (CON'T) } \\
\hline Anthracene & 3300 & Va/KG & $u$ & R & B & 3300 & UG/KG & 10 \\
\hline DI-N-Butylphthalate & 3300 & UQ/KG & $u$ & $\mathbf{R}$ & B & 3300 & UG/KG & 10 \\
\hline Fluoranthene & 3300 & UG/KG & $u$ & R & B & 3300 & UG/KG & 10 \\
\hline Pyrene & 3300 & UG/KG & $u$ & $\ldots$ & & 3300 & UG/KG & 10 \\
\hline Butyl Benzyl Phthalate & 3300 & UG/KG & $u$ & $\ldots$ & & 3300 & UG/KG & 10 \\
\hline 3, 3'-Dichlorobenzidine & 6500 & UG/KG & $u$ & $\ldots$ & & 6500 & UG/Ka & 10 \\
\hline Benzo(a)Anthracene & 3300 & UG/KG & $u$ & ... & & 3300 & UG/KG & 10 \\
\hline Chrysene & 3300 & UG/KG & $u$ & .. & & 3300 & UG/KG & 10 \\
\hline bis(2-Ethylhexyl)phthalate & 2800 & UG/KG & DJ & J & & 3300 & UG/KG & 10 \\
\hline di-N-Octyl Phthalate & 3300 & UG/KG & $u$ & ... & & 3300 & UG/KG & 10 \\
\hline Benzo(b)Fluoranthene & 3300 & $U G / K G$ & $u$ & $\ldots$ & & 3300 & VG/KG & 10 \\
\hline Benzo(k)Fluoranthene & 3300 & UQ/KG & $u$ & ... & & 3300 & UG/KG & 10 \\
\hline Benzo(a)Pyrene & 3300 & UG/KG & $u$ & $\ldots$ & & 3300 & UG/KG & 10 \\
\hline Indeno(1,2,3-CD)Pyrene & 3300 & UG/KG & U & ... & & 3300 & UG/KG & 10 \\
\hline Dibenzo(a,h)Anthracene & 3300 & UG/KG & $U$ & ... & & 3300 & UG/KG & 10 \\
\hline Benzo(g,h,i)Perylene & 3300 & UG/KG & $u$ & $\ldots$ & & 3300 & UG/KG & 10 \\
\hline Nitrobenzene-d5 & 71 & $\%$ & & & & & $\%$ & 10 \\
\hline 2-Fluorobiphenyl & 75 & $\%$ & & & & & $\%$ & 10 \\
\hline Terphenyl-d14 & 70 & $\%$ & & & & & $\%$ & 10 \\
\hline Phenol-d5 & 79 & $\%$ & & & & & $\%$ & 10 \\
\hline 2-Fluorophenol & 71 & $\%$ & & & & & $\%$ & 10 \\
\hline 2, 4,6-Tribromophenol & 73 & $\%$ & & & & & $\%$ & 10 \\
\hline
\end{tabular}




\begin{tabular}{|c|c|c|c|c|c|c|c|}
\hline COMPOUND & RESULTS & UNITS & $\begin{array}{l}\text { DATA } \\
\text { QUAL }\end{array}$ & $\begin{array}{l}\text { DATA } \\
\text { VALID. }\end{array}$ & NOTES & CRDL UNITS & DILUTION \\
\hline \multicolumn{8}{|l|}{ TR3-ES-450N-400E-3Z AE } \\
\hline Phenol & 330 & UQ/Ka & $u$ & A & & 330 UG/KG & 1 \\
\hline bis(2-Chloroethyl) ether & 330 & UG/KG & $u$ & A & & 330 UG/KG & 1 \\
\hline 2-Chlorophenol & 330 & UQ/KG & $u$ & A & & 330 UG/Ka & 1 \\
\hline 1, 3-Dichlorobenzene & 330 & UG/KG & $u$ & R & & 330 UG/KG & 1 \\
\hline 1, 4-Dichlorobenzene & 330 & UG/KG & $u$ & R & & 330 UG/KG & 1 \\
\hline Benzyl Alcohol & 330 & UG/KG & $u$ & R & & 330 UG/KG & 1 \\
\hline 1, 2-Dichlorobenzene & 330 & $U G / K G$ & $u$ & A & & 330 UG/KG & 1 \\
\hline 2-Methylphenol & 330 & $U G / K G$ & $u$ & A & & 330 UG/KG & 1 \\
\hline bis(2-Chloroisopropyl) ether & 330 & UG/KG & $u$ & ค & & 330 UG/KG & 1 \\
\hline 4-Methylphenol & 330 & UG/KG & $u$ & A & & 330 UG/KG & 1 \\
\hline N-Nitroso-di-nPropylamine & 330 & UQ/KG & $u$ & A & & 330 UG/KG & 1 \\
\hline Hexachloroethane & 330 & UQ/KG & $u$ & A & & 330 UG/KG & 1 \\
\hline Nitrobenzene & 330 & UG/KG & $u$ & R & & 330 UG/KG & 1 \\
\hline Isophorone & 330 & UG/KG & $u$ & R & & 330 UG/KG & 1 \\
\hline 2-Nitrophenol & 330 & UG/KG & $u$ & R & & 330 UG/KG & 1 \\
\hline 2. 4-Dimethyiphenol & 330 & UG/KG & $u$ & A & & 330 UG/KG & 1 \\
\hline Benzolc Acid & 1600 & UG/KG & $u$ & ค & & 1600 UG/KG & 1 \\
\hline bis(2Chloroethoxy)methane & 330 & UG/KG & $u$ & R & & 330 UG/KG & 1 \\
\hline 2, 4-Dichlorophenol & 330 & UG/KG & $u$ & R & & 330 UG/KG & 1 \\
\hline 1,2, 4-Trichlorobenzene & 330 & UG/KG & $u$ & $R$ & & 330 UG/KG & 1 \\
\hline Naphthalene & 330 & UG/KG & $u$ & R & & 330 UG/KG & 1 \\
\hline 4-Chloroaniline & 330 & UG/KG & $u$ & A & & 330 UG/KG & 1 \\
\hline Hexachlorobutadiene & 330 & UG/KG & $u$ & R & & 330 UG/KG & 1 \\
\hline 4-Chloro-3-Methylphenol & 330 & UG/KG & $u$ & R & & 330 UG/KG & 1 \\
\hline 2-Methylnaphthalene & 4700 & UG/KG & E & R & & 330 UG/KG & 1 \\
\hline Hexachlorocyclopentadiene & 330 & $U G / K G$ & $u$ & R & & 330 UG/KG & 1 \\
\hline 2, 4, 6-Trichlorophenol & 330 & UG/KG & $u$ & A & & 330 UG/KG & 1 \\
\hline 2, 4, 5-Trichlorophenol & 1600 & UG/KG & $u$ & R & & 1600 UG/KG & 1 \\
\hline 2-Chloronaphthalene & 330 & UG/KG & U & A & & 330 UG/KG & 1 \\
\hline 2-Nitroaniline & 1600 & UG/KG & $\mathrm{U}$ & A & & 1600 UG/KG & 1 \\
\hline Dimethyl Phthalate & 330 & UG/KG & $u$ & R & & 330 UG/KG & 1 \\
\hline Acenaphthylene & 330 & UG/KG & u & ค & & 330 UG/Ka & 1 \\
\hline 2, 6-Dinitrotoluene & 330 & UG/KG & $u$ & R & & 330 UG/KG & 1 \\
\hline 3-Nitroaniline & 1600 & UG/KG & $u$ & A & & 1600 UG/KG & 1 \\
\hline Acenaphthene & 330 & UG/KG & $u$ & R & & 330 UG/KG & 1 \\
\hline 2, 4-Dinitrophenol & 1600 & UG/KG & $u$ & R & & 1600 UG/KG & 1 \\
\hline 4-Nitrophenol & 1600 & UG/KG & $u$ & A & & 1600 UG/KG & 1 \\
\hline Dibenzofuran & 330 & UG/KG & $u$ & R & & 330 UG/KG & 1 \\
\hline 2, 4-Dinitrotoluene & 330 & UG/KG & $u$ & R & & 330 UG/KG & 1 \\
\hline Diethylphthalate & 330 & UG/KG & $u$ & R & & 330 UG/KG & 1 \\
\hline 4-Chlorophenyl phenyl ether & 330 & $U G / K G$ & $u$ & R & & 330 UG/KG & 1 \\
\hline Fluorene & 330 & UG/KG & $u$ & R & & 330 UG/KG & 1 \\
\hline 4-Nitroaniline & 1600 & UG/KG & $u$ & R & & 1600 UG/KG & 1 \\
\hline 4,6-Dinitro-2-Methylphenol & 1600 & UG/KG & $u$ & R & & 1600 UG/KG & 1 \\
\hline N-Nitrosodiphenylamine & 330 & UG/KG & $u$ & R & & 330 UG/KG & 1 \\
\hline 4-Bromophenyl phenyl ether & 330 & UG/KG & $u$ & $\mathbf{R}$ & & 330 UG/KG & 1 \\
\hline Hexachlorobenzene & 330 & $U G / K G$ & $u$ & A & & 330 UG/KG & 1 \\
\hline Pentachlorophenol & 1600 & $U G / K G$ & $u$ & A & & 1600 UG/KG & 1 \\
\hline Phenanthrene & 330 & UG/KG & $u$ & R & & 330 UG/KG & 1 \\
\hline
\end{tabular}


Appendix D: Results of Semivolatile Organic Compound Analyses, Salmon Site

\begin{tabular}{|c|c|c|c|c|c|c|c|}
\hline COMPOUND & RESULTS & UNITS & $\begin{array}{l}\text { DATA } \\
\text { QUAL }\end{array}$ & $\begin{array}{l}\text { DATA } \\
\text { VALID. }\end{array}$ & NOTES & CRDL UNITS & DILUTION \\
\hline \multicolumn{8}{|l|}{ TA3-ES-45ON-4OOE-3Z RE } \\
\hline Anthracene & 330 & Ua/Ka & $u$ & A & & 330 UG/KG & 1 \\
\hline DI-N-Butylphthalate & 330 & vaka & $u$ & A & & 330 UG/KG & 1 \\
\hline Fluoranthene & 330 & UG/Ka & $u$ & P & & 330 Ua/KG & 1 \\
\hline Pyrene & 330 & UG/KG & $u$ & R & & 330 Ua/KG & 1 \\
\hline Butyl Benzyl Phthalate & 330 & UQ/KO & $u$ & A & & 330 Ua/KG & 1 \\
\hline 3. 3'-Dichlorobenzidine & 650 & Ua/KG & $u$ & ค & & 650 Ua/Ka & 1 \\
\hline Benzo(a)Anthracene & 330 & UG/KO & $u$ & A & & 330 UG/KG & 1 \\
\hline Chrysene & 330 & UG/KG & $u$ & A & & 330 UG/KG & 1 \\
\hline bis(2-Ethylhexyl)phthalate & 2700 & UG/Ka & $\mathbf{E}$ & R & & 330 UG/KG & 1 \\
\hline di-N-Octyl Phthalate & 330 & UG/KG & $u$ & R & & 330 UG/KG & 1 \\
\hline Benzo(b)Fluoranthene & 330 & UG/KO & $u$ & R & & 330 UG/KG & 1 \\
\hline Benzo(k)Fluoranthene & 330 & UG/KO & $u$ & $\mathbf{R}$ & & 330 UG/KG & 1 \\
\hline Benzo(a)Pyrene & 330 & UG/KG & $u$ & A & & 330 UG/KG & 1 \\
\hline Indeno(1,2,3-CD)Pyrene & 330 & UG/KG & u & A & & 330 UG/KG & 1 \\
\hline Dibenzo(a,h)Anthracene & 330 & UG/KG & $u$ & R & & 330 UG/KG & 1 \\
\hline Benzo(g,h,i)Perylene & 330 & UG/KG & $u$ & $R$ & & 330 Ua/KG & 1 \\
\hline Nitrobenzene-d5 & 73 & $\%$ & & & & $\%$ & 1 \\
\hline 2-Fluorobiphenyl & 101 & $\%$ & & & & $\%$ & 1 \\
\hline Terphenyl-d14 & 128 & $\%$ & & & & $\%$ & 1 \\
\hline Phenol-d5 & 66 & $\%$ & & & & $\%$ & 1 \\
\hline 2-Fluorophenol & 64 & $\%$ & & & & $\%$ & 1 \\
\hline 2, 4, 6-Tribromophenol & 94 & $\%$ & & & & $\%$ & 1 \\
\hline
\end{tabular}




\begin{tabular}{|c|c|c|c|c|c|c|c|}
\hline COMPOUND & RESULTS & UNITS & $\begin{array}{l}\text { DATA } \\
\text { QUAL }\end{array}$ & $\begin{array}{l}\text { DATA } \\
\text { VALID. }\end{array}$ & NOTES & CRDL UNITS & DILUTION \\
\hline \multicolumn{8}{|c|}{ 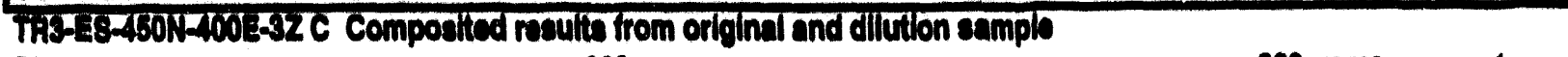 } \\
\hline Phenol & 330 & vaka & $u$ & $\ldots$ & & 330 vake & 1 \\
\hline bis(2-Chloroethyl) ether & 330 & vako & $u$ & - & & 330 vaka & 1 \\
\hline 2-Chlorophenol & 330 & VaKa & $u$ & $\cdots$ & & 330 vaka & 1 \\
\hline 1, 3-Dichlorobenzene & 330 & UaKo & $u$ & - & & 330 VaRe & 1 \\
\hline 1, 4-Dichlorobenzene & 330 & VOKO & $u$ & - & & 330 vaka & 1 \\
\hline Benzyl Alcohol & 330 & VaKa & $u$ & - & & 330 UaKa & 1 \\
\hline 1, 2-Dichlorobenzene & 330 & UaKG & u & $\cdots$ & & 330 UaKO & 1 \\
\hline 2-Mothylphenol & 330 & Ua/Ka & u & -. & & 330 VaKa & 1 \\
\hline bis(2-Chloroisopropyl) ether & 330 & UaKo & u & $\cdots$ & & 330 vaKa & 1 \\
\hline 4-Methylphenol & 330 & Ua/Ka & $u$ & ... & & 330 UGKG & 1 \\
\hline N-Nitroso-di-nPropylamine & 330 & va/Ka & $u$ & $\cdots$ & & 330 va/Ka & 1 \\
\hline Hexachloroethane & 330 & UEKO & u & ... & & 330 Ua/Ka & 1 \\
\hline Nitrobenzene & 330 & VAKG & $u$ & .. & & 330 Ua/KG & 1 \\
\hline Isophorone & 330 & VAKG & $u$ & - & & 330 Ua/KO & 1 \\
\hline 2-Nitrophenol & 330 & UQKG & $u$ & ... & & 330 Ua/KG & 1 \\
\hline 2, 4-Dimethyiphenol & 330 & UaKG & $u$ & -. & & 330 Ua/ka & 1 \\
\hline Benzoic Acid & 1600 & VaKa & $u$ & - & & 1600 veKK & 1 \\
\hline bis(2Chloroethoxy)/methane & 330 & Ua/KG & u & ... & & 330 UG/KO & 1 \\
\hline 2, 4-Dichlorophenol & 330 & UaKo & $u$ & - & & 330 UaKa & 1 \\
\hline 1, 2, 4-Trichlorobenzene & 330 & UGKO & $u$ & - & & 330 vaka & 1 \\
\hline Naphthalene & 330 & UGKG & $u$ & - & & 330 UGKK & 1 \\
\hline 4-Chloroaniline & 330 & vaka & $u$ & -. & & 330 UaKa & 1 \\
\hline Hexachlorobutadiene & 330 & UaKe & $u$ & - & & 330 UaKa & 1 \\
\hline 4-Chloro-3-Methylphenol & 330 & UaKa & $u$ & -. & & 330 vaka & 1 \\
\hline 2-Methylnaphthalene & 11000 & UGKG & $u$ & ... & & 3300 UGKO & 10 \\
\hline Hexachlorocyclopentadiene & 3300 & vaKa & $u$ & - & $\cdots$ & 3300 Ua/KG & 10 \\
\hline 2, 4, 6-Trichlorophenol & 3300 & VaKa & $u$ & - & & 3300 UGKG & 10 \\
\hline 2, 4,5-Trichlorophenol & 16000 & vaka & $u$ & -.. & & 16000 UaKa & 10 \\
\hline 2-Chloronaphthalene & 3300 & UG/KG & $u$ & - & & 3300 Va/Ka & 10 \\
\hline 2-Nitroaniline & 16000 & UGKG & $u$ & -. & & 16000 UG/KG & 10 \\
\hline Dimethyl Phthalate & 3300 & UGKG & $u$ & ... & & 3300 UGKa & 10 \\
\hline Acenaphthylene & 3300 & UGKG & $u$ & -. & & 3300 Ua/Ka & 10 \\
\hline 2, 6-Dinitrotoluene & 3300 & UGKG & $u$ & $\ldots$ & & 3300 Ua/Ka & 10 \\
\hline 3-Nitroaniline & 16000 & UQKO & $u$ & .. & & 16000 :Ja/Ka & 10 \\
\hline Acenaphthene & 3300 & UG/KG & $u$ & -. & & 3300 Ua/Ka & 10 \\
\hline 2, 4-Dinitrophenol & 16000 & UQ/KG & $u$ & $\cdots$ & & 16000 UG/KG & 10 \\
\hline 4-Nitrophenol & 16000 & va/Ka & $u$ & - & & 16000 vakg & 10 \\
\hline Dibenzofuran & 3300 & UaKG & $u$ & - & & 3300 UaKKG & 10 \\
\hline 2, 4-Dinitrotoluene & 3300 & UGKG & $u$ & -- & & 3300 UaKKO & 10 \\
\hline Diethylphthalate & 3300 & VaKa & $u$ & - & & 3300 UGKG & 10 \\
\hline 4-Chlorophenyl phenyl ether & 3300 & UaKo & $u$ & .. & & 3300 UG/KG & 10 \\
\hline Fluorene & 1100 & vaka & DJ & J & & 3300 Ua/Ko & 10 \\
\hline 4-Nitroaniline & 1600 & VaKa & $u$ & .. & & 1600 VaKG & 1 \\
\hline 4, 6-Dinitro-2-Methyiphenol & 1600 & UaKa & $u$ & - & & 1600 UaKG & 1 \\
\hline N-Nitrosodiphenylamine & 330 & vako & $u$ & - & & 330 บaKa & 1 \\
\hline 4-Bromophenyl phenyl ether & 330 & UGKG & $u$ & - & & 330 UaKG & 1 \\
\hline Hexachlorobenzene & 330 & Vaka & $u$ & - & & 330 UaKG & 1 \\
\hline Pentachlorophenol & 1600 & UGKG & $u$ & - & & 1600 UGKG & 1 \\
\hline Phenanthrene & 330 & VaKa & U & - & & 330 UaKa & 1 \\
\hline
\end{tabular}


Appendix D: Results of Semivolatile Organic Compound Analyses, Salmon Site

\begin{tabular}{|c|c|c|c|c|c|c|c|c|}
\hline COMPOUND & AESULTS & UNITS & $\begin{array}{l}\text { DATA } \\
\text { QUAL }\end{array}$ & $\begin{array}{l}\text { DATA } \\
\text { VALID. }\end{array}$ & NOTES & CADL & UNITS & DILUTION \\
\hline \multicolumn{9}{|l|}{ TH3-ES-460N400E-32C (CONT) } \\
\hline Anthracene & 330 & UQ/KO & $u$ & ... & & & va/ka & 1 \\
\hline DI-N-Butylphthalate & 330 & Ua/Ka & $u$ & ... & & & Va/Ka & 1 \\
\hline Fluoranthene & 330 & UG/KG & $u$ & ... & & & VGKG & 1 \\
\hline Pyrene & 3300 & $U G / K a$ & $u$ & $\ldots$ & & 3300 & VQ/KG & 10 \\
\hline Butyl Benzyl Phthalate & 3300 & UQ/KG & $u$ & ... & & 3300 & O Va/Ka & 10 \\
\hline 3, 3'-Dichlorobenzidine & 6500 & UG/KG & $u$ & ... & & 6500 & D VQ/KG & 10 \\
\hline Benzo(a)Anthracene & 3300 & va/ka & $u$ & ... & & 3300 & o va/Ka & 10 \\
\hline Chrysene & 3300 & Ua/Ko & $u$ & .. & & 3300 & O va/Ka & 10 \\
\hline bis(2-Ethylhexyl)phthalate & 2800 & Ua/Ko & DJ & J & & 3300 & O Ua/KG & 10 \\
\hline di-N-Octyl Phthalate & 3300 & VG/KO & $u$ & $\ldots$ & & 3300 & O VQ/KG & 10 \\
\hline Benzo(b)Fluoranthene & 3300 & UG/KG & $u$ & $\cdots$ & & 3300 & 0 UG/KG & 10 \\
\hline Benzo(k)Fluoranthene & 3300 & UG/KO & $u$ & $\cdots$ & & 3300 & O UG/KG & 10 \\
\hline Benzo(a)Pyrene & 3300 & UG/KG & $u$ & $\cdots$ & & 3300 & O Va/Ko & 10 \\
\hline Indeno(1,2,3-CD)Pyrene & 3300 & UG/KG & $u$ & $\cdots$ & & 3300 & O UG/KG & 10 \\
\hline Dibenzo(a.h)Anthracene & 3300 & UG/KG & $u$ & $\ldots$ & & 3300 & 0 Ua/KG & 10 \\
\hline Benzo $(g, n, i)$ Perylene & 3300 & Ua/KG & $u$ & ... & & 3300 & 0 va/ka & 10 \\
\hline Nitrobenzene-d5 & 71 & $\%$ & & & & & $\%$ & 10 \\
\hline 2-Fluorobiphenyl & 75 & $\%$ & & & & & $\%$ & 10 \\
\hline Terphenyl-d14 & 70 & $\%$ & & & & & $\%$ & 10 \\
\hline Phenol-d5 & 79 & $\%$ & & & & & $\%$ & 10 \\
\hline 2.Fluorophenol & 71 & $\%$ & & & & & $\%$ & 10 \\
\hline 2, 4, 6-Tribromophenol & 73 & $\%$ & & & & & $\%$ & 10 \\
\hline
\end{tabular}


Appendix D: Results of Semivolatile Organic Compound Analyses. Salmon Site

\begin{tabular}{|c|c|c|c|c|c|c|c|}
\hline COMPOUND & RESULTS & UNITS & $\begin{array}{l}\text { DATA } \\
\text { QUAL }\end{array}$ & $\begin{array}{l}\text { DATA } \\
\text { VALID. }\end{array}$ & NOTES & CRDL UNITS & DILUTION \\
\hline \multicolumn{8}{|l|}{ TR3-ES-430N-400E-15Z } \\
\hline Phenol & 330 & UG/KG & $u$ & .. & & $330 \cup G / K G$ & 1 \\
\hline bis(2-Chloroethyl) ether & 330 & UG/KG & $u$ & ... & & 330 UG/KG & 1 \\
\hline 2-Chlorophenol & 330 & UG/KG & $u$ & .. & & 330 UG/KG & 1 \\
\hline 1, 3-Dichlorobenzene & 330 & UG/KG & u & ... & & 330 UG/KG & 1 \\
\hline 1. 4-Dichlorobenzene & 330 & UG/KG & $u$ & ... & & $330 \mathrm{UG} / \mathrm{KG}$ & 1 \\
\hline Benzyl Alcohol & 330 & UG/KG & $u$ & ... & & 330 UG/KG & 1 \\
\hline 1,2-Dichlorobenzene & 330 & $U G / K G$ & $u$ & -- & & 330 UG/KG & 1 \\
\hline 2-Methylphenol & 330 & $U G / K G$ & $u$ & -.. & & 330 UG/KG & 1 \\
\hline bis(2-Chloroisopropyl) ether & 330 & $U G / K G$ & $u$ & -. & & 330 UG/KG & 1 \\
\hline 4-Methylphenoi & 330 & $U G / K G$ & $u$ & ... & & 330 UG/KG & 1 \\
\hline N-Nitroso-di-nPropylamine & 330 & $U G / K G$ & $u$ & $\ldots$ & & 330 UG/KG & 1 \\
\hline Hexachloroethane & 330 & UG/KG & $u$ & $\ldots$ & & 330 UG/KG & 1 \\
\hline Nitrobenzene & 330 & UG/KG & $u$ & ... & & 330 UG/KG & 1 \\
\hline Isophorone & 330 & UG/KG & $u$ & ... & & 330 UG/KG & 1 \\
\hline 2-Nitrophenol & 330 & UG/KG & $u$ & ... & & 330 UG/KG & 1 \\
\hline 2, 4-Dimethylphenol & 330 & $U G / K G$ & $u$ & ... & & 330 UG/KG & 1 \\
\hline Benzoic Acid & 1600 & $U G / K G$ & $u$ & ... & & 1600 UG/KG & 1 \\
\hline bis(2Chloroethoxy)methane & 330 & UG/KG & $u$ & ... & & 330 UG/KG & 1 \\
\hline 2, 4-Dichlorophenol & 330 & UG/KG & $u$ & $\cdots$ & & 330 UG/KG & 1 \\
\hline 1, 2, 4-Trichlorobenzene & 330 & UG/KG & u & - & & 330 UG/KG & 1 \\
\hline Naphthalene & 250 & UG/KG & $\mathrm{J}$ & .. & & 330 UG/KG & 1 \\
\hline 4-Chloroaniline & 330 & UG/KG & $u$ & ... & & 330 UG/KG & 1 \\
\hline Hexachlorobutadiene & 330 & UG/KG & $u$ & -.. & & 330 UG/KG & 1 \\
\hline 4-Chloro-3-Methylphenol & 330 & UG/KG & $u$ & -.. & & 330 UG/KG & 1 \\
\hline 2-MethyInaphthalene & 2900 & UG/KG & E & R & A & 330 UG/KG & 1 \\
\hline Hexachlorocyclopentadiene & 330 & UG/KG & $u$ & $\cdots$ & & 330 UG/KG & 1 \\
\hline 2, 4, 6-Trichlorophenol & 330 & UG/KG & $u$ & ... & & 330 UG/KG & 1 \\
\hline 2, 4, 5-Trichlorophenol & 1600 & UG/KG & $u$ & ... & & 1600 UG/KG & 1 \\
\hline 2-Chloronaphthalene & 330 & UG/KG & $u$ & ... & & 330 UG/KG & 1 \\
\hline 2-Nitroaniline & 1600 & UG/KG & $u$ & -.. & & 1600 UG/KG & 1 \\
\hline Dimethyl Phthalate & 330 & UG/KG & $u$ & ... & & 330 UG/KG & 1 \\
\hline Acenaphthylene & 330 & UG/KG & $u$ & $\ldots$ & & 330 UG/KG & 1 \\
\hline 2, 6-Dinitrotoluene & 330 & UG/KG & $u$ & -.. & & 330 UG/KG & 1 \\
\hline 3-Nitroaniline & 1600 & UG/KG & $u$ & -. & & 1600 UG/KG & 1 \\
\hline Acenaphthene & 330 & UG/KG & $u$ & $\ldots$ & & 330 UG/KG & 1 \\
\hline 2, 4-Dinitrophenol & 1600 & UG/KG & $u$ & ... & & 1600 UG/KG & 1 \\
\hline 4-Nitrophenol & 1600 & UG/KG & $u$ & -- & & 1600 UG/KG & 1 \\
\hline Dibenzofuran & 330 & UG/KG & $u$ & ... & & 330 UG/KG & 1 \\
\hline 2, 4-Dinitrotoluene & 330 & UG/KG & $u$ & -. & & 330 UG/KG & 1 \\
\hline Diethylphthalate & 330 & UG/KG & $u$ & ..- & & 330 UG/KG & 1 \\
\hline 4-Chlorophenyl phenyl ether & 330 & UG/KG & $u$ & ... & & 330 UG/KG & 1 \\
\hline Fluorene & 330 & UG/KG & u & -. & & 330 UG/KG & 1 \\
\hline 4-Nitroaniline & 1600 & UG/KG & $u$ & -- & & 1600 UG/KG & 1 \\
\hline 4, 6-Dinitro-2-Methylphenol & 1600 & UG/KG & $u$ & -. & & 1600 UG/KG & 1 \\
\hline N-Nitrosodiphenylamine & 330 & UG/KG & U & $\cdots$ & & 330 UG/KG & 1 \\
\hline 4-Bromophenyl phenyl ether & 330 & UG/KG & $u$ & -. & & 330 UG/KG & 1 \\
\hline Hexachlorobenzene & 330 & UG/KG & $u$ & - & & 330 UG/KG & 1 \\
\hline Pentachiorophenol & 1600 & UG/KG & $u$ & $\cdots$ & & 1600 UG/KG & 1 \\
\hline Phenanthrene & 330 & UG/KG & $u$ & $\cdots$ & & 330 UG/KG & 1 \\
\hline
\end{tabular}


Appendix D: Results of Semivolatile Organic Compound Analyses, Salmon Site

\begin{tabular}{|c|c|c|c|c|c|c|c|c|}
\hline COMPOUND & RESULTS & UNITS & $\begin{array}{l}\text { DATA } \\
\text { QUAL }\end{array}$ & $\begin{array}{l}\text { DATA } \\
\text { VALID. }\end{array}$ & NOTES & CRDL & UNITS & DILUTION \\
\hline \multicolumn{9}{|c|}{ TR3-ES-430N-400E-15Z (CON'T) } \\
\hline Anthracene & 330 & $U G / K G$ & $u$ & $\ldots$ & & & UG/KG & 1 \\
\hline Di-N-Butylphthalate & 330 & UG/KG & U & -- & & 330 & UG/KG & 1 \\
\hline Fluoranthene & 330 & UG/KG & $u$ & - & & & UG/KG & 1 \\
\hline Pyrene & 330 & UG/KG & $u$ & $\ldots$ & & & UG/KG & 1 \\
\hline Butyl Benzyl Phthalate & 330 & UG/KG & $u$ & ... & & & UG/KG & 1 \\
\hline 3, 3'-Dichlorobenzidine & 660 & UG/KG & $u$ & ... & & & UG/KG & 1 \\
\hline Benzo(a)Anthracene & 330 & UG/KG & $u$ & $\ldots$ & & & UG/KG & 1 \\
\hline Chrysene & 330 & UG/KG & $u$ & $\ldots$ & & & UG/KG & 1 \\
\hline bis(2-Elhylhexyl)phthalate & 330 & $U G / K G$ & $u$ & ... & & & UG/KG & 1 \\
\hline di-N-Octyl Phthalate & 330 & $U G / K G$ & $u$ & $\ldots$ & & & UG/KG & 1 \\
\hline Benzo(b)Fluoranthene & 330 & $U G / K G$ & $u$ & $\cdots$ & & & UG/KG & 1 \\
\hline Benzo(k)Fluoranthene & 330 & $U G / K G$ & $u$ & $\cdots$ & & & UG/KG & 1 \\
\hline Benzo(a)Pyrene & 330 & $U G / K G$ & $u$ & $\cdots$ & & & UG/KG & 1 \\
\hline Indeno(1,2,3-CD)Pyrene & 330 & $U G / K G$ & $u$ & $\cdots$ & & & UG/KG & 1 \\
\hline Dibenzo(a,h)Anthracene & 330 & $U G / K G$ & $u$ & $\cdots$ & & & UG/KG & 1 \\
\hline Benzo(g,h,i)Perylene & 330 & UG/KG & $u$ & $\cdots$ & & & UG/KG & 1 \\
\hline Nitrobenzene-d5 & 72 & $\%$ & & & & & $\%$ & 1 \\
\hline 2-Fluorobiphenyl & 64 & $\%$ & & & & & $\%$ & 1 \\
\hline Terphenyl-d14 & 68 & $\%$ & & & & & $\%$ & 1 \\
\hline Phenol-d5 & 78 & $\%$ & & & & & $\%$ & 1 \\
\hline 2-Fluorophenol & 62 & $\%$ & & & & & $\%$ & 1 \\
\hline $\begin{array}{l}\text { 2, 4, 6-Tribromophenol } \\
\text { NOTE A: Use results from }\end{array}$ & 69 & $\%$ & & & & & $\%$ & 1 \\
\hline
\end{tabular}




\section{Appendix D: Results of Semivolatile Organic Compound Analyses, Salmon Site}

\begin{tabular}{|c|c|c|c|c|c|c|c|}
\hline COMPOUND & RESULTS & UNITS & $\begin{array}{l}\text { DATA } \\
\text { QUAL }\end{array}$ & $\begin{array}{l}\text { DATA } \\
\text { VALID. }\end{array}$ & NOTES & CRDL UNITS & DILUTION \\
\hline \multicolumn{8}{|l|}{ TR3-ES-430N-400E-15Z CL } \\
\hline Phenol & 660 & UG/KG & $u$ & R & & 660 UG/KG & 2 \\
\hline bis(2-Chloroethyl) ether & 660 & UG/KG & $u$ & $R$ & & 660 UG/KG & 2 \\
\hline 2-Chlorophenol & 660 & UG/KG & $u$ & $R$ & & 660 UG/KG & 2 \\
\hline 1, 3-Dichlorobenzene & 660 & UG/KG & U & $R$ & & 660 UG/KG & 2 \\
\hline 1, 4-Dichlorobenzene & 660 & UG/KG & $u$ & R & & 660 UG/KG & 2 \\
\hline Benzyl Alcohol & 660 & $U G / K G$ & $u$ & R & & 660 UG/KG & 2 \\
\hline 1, 2-Dichlorobenzene & 660 & UG/KG & $u$ & A & & 660 UG/KG & 2 \\
\hline 2-Methylphenol & 660 & UG/KG & u & A & & 660 UG/KG & 2 \\
\hline bis(2-Chloroisopropyl) ether & 660 & UG/KG & u & A & & 660 UG/KG & 2 \\
\hline 4-Methyiphenol & 660 & UG/KG & $u$ & R & & 660 UG/KG & 2 \\
\hline N-Nitroso-di-nPropylamine & 660 & UG/KG & $u$ & R & & 660 UG/KG & 2 \\
\hline Hexachloroethane & 660 & UG/KG & $u$ & R & & 660 UG/KG & 2 \\
\hline Nitrobenzene & 660 & UG/KG & $u$ & R & & 660 UG/KG & 2 \\
\hline Isophorone & 660 & UG/KG & $u$ & R & & 660 UG/KG & 2 \\
\hline 2-Nitrophenol & 660 & UG/KG & $u$ & R & & 660 UG/KG & 2 \\
\hline 2, 4-Dimethylphenol & 660 & UG/KG & $u$ & R & & 660 UG/KG & 2 \\
\hline Benzoic Acid & 3200 & UG/KG & $u$ & R & & 3200 UG/KG & 2 \\
\hline bis(2Chloroethoxy)methane & 660 & UG/KG & u & A & & 660 UG/KG & 2 \\
\hline 2, 4-Dichlorophenol & 660 & UG/KG & $u$ & R & & 660 UG/KG & 2 \\
\hline 1,2, 4-Trichlorobenzene & 660 & UG/KG & $u$ & R & & 660 UG/KG & 2 \\
\hline Naphthalene & 660 & UG/KG & U & $\mathbf{R}$ & & 660 UG/KG & 2 \\
\hline 4-Chloroaniline & 660 & UGKG & u & R & & 660 UG/KG & 2 \\
\hline Hexachlorobutadiene & 660 & UG/KG & U & R & & 660 UG/KG & 2 \\
\hline 4-Chloro-3-Methylphenol & 660 & UG/KG & U & $\mathbf{R}$ & & 660 UG/KG & 2 \\
\hline 2-Methylnaphthalene & 3200 & UG/KG & D & - & $B$ & 660 UG/KG & 2 \\
\hline Hexachlorocyclopentadiene & 660 & UG/KG & $u$ & R & & 660 UG/KG & 2 \\
\hline 2, 4, 6-Trichlorophenol & 660 & UG/KG & U & $\mathbf{R}$ & & 660 UG/KG & 2 \\
\hline 2, 4, 5-Trichlorophenol & 3200 & UG/KG & U & R & & 3200 UG/KG & 2 \\
\hline 2-Chloronaphthalene & 660 & UG/KG & $u$ & R & & $660 \mathrm{UG} / \mathrm{KG}$ & 2 \\
\hline 2-Nitroaniline & 3200 & UG/KG & $u$ & R & & 3200 UG/KG & 2 \\
\hline Dimethyl Phthalate & 660 & UG/KG & U & $\mathbf{R}$ & & 660 UG/KG & 2 \\
\hline Acenaphthylene & 660 & UG/KG & $u$ & R & & 660 UG/KG & 2 \\
\hline 2, 6-Dinitrotoluene & 660 & $U G / K G$ & $u$ & R & & 660 UG/KG & 2 \\
\hline 3-Nitroaniline & 3200 & UG/KG & $u$ & R & & 3200 UG/KG & 2 \\
\hline Acenaphthene & 660 & UG/KG & u & R & & 660 UG/KG & 2 \\
\hline 2, 4-Dinitrophenol & 3200 & UG/KG & U & A & & 3200 UG/KG & 2 \\
\hline 4-Nitrophenol & 3200 & UG/KG & $u$ & A & & 3200 UG/KG & 2 \\
\hline Dibenzofuran & 660 & UG/KG & u & ค & & 660 UG/KG & 2 \\
\hline 2, 4-Dinitrotoluene & 660 & UG/KG & u & A & & 660 UG/KG & 2 \\
\hline Diethylphthalate & 660 & UG/KG & u & ค & & 660 UG/KG & 2 \\
\hline 4-Chlorophenyl phenyl ether & 660 & UG/KG & $u$ & R & & 660 UG/KG & 2 \\
\hline Fluorene & 660 & UGKG & $u$ & A & & 660 UG/KG & 2 \\
\hline 4-Nitroaniline & 3200 & UG/KG & $u$ & R & & 3200 UG/KG & 2 \\
\hline 4,6-Dinitro-2-Methylphenol & 3200 & UGKG & u & R & & 3200 UG/KG & 2 \\
\hline N-Nitrosodiphenylamine & 660 & UGKG & u & R & & 660 UG/KG & 2 \\
\hline 4-Bromophenyl phenyl ether & 660 & UG/KG & $u$ & R & & 660 UG/KG & 2 \\
\hline Hexachlorobenzene & 660 & UGKG & u & R & & 660 UG/KG & 2 \\
\hline Pentachlorophenol & 3200 & UG/KG & u & R & & 3200 UG/KG & 2 \\
\hline Phenanthrene & 660 & UG/KG & $u$ & A & & 660 UG/KG & 2 \\
\hline
\end{tabular}


Appendix D: Results of Semivolatile Organic Compound Analyses, Salmon Site

\begin{tabular}{|c|c|c|c|c|c|c|c|c|}
\hline COMPOUND & RESULTS & UNITS & $\begin{array}{l}\text { DATA } \\
\text { QUAL }\end{array}$ & $\begin{array}{l}\text { DATA } \\
\text { VALID. }\end{array}$ & NOTES & CRDL & UNITS & DILUTION \\
\hline \multicolumn{9}{|l|}{ TR3-ES-430N-400E-15Z DL } \\
\hline Anthracene & 660 & UG/KG & U & R & & & UG/KG & 2 \\
\hline Di-N-Butylphthalate & 660 & UG/KG & $u$ & R & & 660 & UG/KG & 2 \\
\hline Fluoranthene & 660 & UG/KG & $u$ & R & & & UG/KG & 2 \\
\hline Pyrene & 660 & UG/KG & $u$ & R & & & UG/KG & 2 \\
\hline Butyl Benzyl Phthalate & 660 & $U G / K G$ & $u$ & A & & & UG/KG & 2 \\
\hline 3, 3'-Dichlorobenzidine & 1300 & UG/KG & $u$ & ค & & 1300 & $U G / K G$ & 2 \\
\hline Benzo(a)Anthracene & 660 & UG/KG & $u$ & R & & & UG/KG & 2 \\
\hline Chrysene & 660 & UG/KG & $u$ & R & & & UG/KG & 2 \\
\hline bis(2-Ethylhexyl)phthalate & 660 & UG/KG & $u$ & R & & & UG/KG & 2 \\
\hline di-N-Octyl Phthalate & 660 & UG/KG & $u$ & A & & & UG/KG & 2 \\
\hline Benzo(b)Fluoranthene & 660 & UG/KG & $u$ & R & & & UG/KG & 2 \\
\hline Benzo(k)Fluoranthene & 660 & UG/KG & $u$ & R & & & UG/KG & 2 \\
\hline Benzo(a)Pyrene & 660 & UG/KG & $u$ & $R$ & & & UG/KG & 2 \\
\hline Indeno(1,2,3-CD)Pyrene & 660 & UG/KG & $u$ & A & & & UG/KG & 2 \\
\hline Dibenzo(a,h)Anthracene & 660 & UG/KG & $u$ & A & & & $U G / K G$ & 2 \\
\hline Benzo(g,h,i)Perylene & 660 & UG/KG & $u$ & R & & & UG/KG & 2 \\
\hline Nitrobenzene-d5 & 60 & $\%$ & & & & & $\%$ & 2 \\
\hline 2-Fluorobiphenyl & 69 & $\%$ & & & & & $\%$ & 2 \\
\hline Terphenyl-d14 & 63 & $\%$ & & & & & $\%$ & 2 \\
\hline Phenol-d5 & 64 & $\%$ & & & & & $\%$ & 2 \\
\hline 2-Fluorophenol & 65 & $\%$ & & & & & $\%$ & 2 \\
\hline 2, 4, 6-Tribromophenol & 81 & $\%$ & & & & & $\%$ & 2 \\
\hline
\end{tabular}




\begin{tabular}{|c|c|c|c|c|c|c|c|}
\hline COMPOUND & RESULTS & UNITS & $\begin{array}{l}\text { DATA } \\
\text { QUAL }\end{array}$ & $\begin{array}{l}\text { DATA } \\
\text { VALID. }\end{array}$ & NOTES & CRDL UNITS & DILUTION \\
\hline \multicolumn{8}{|l|}{ TR4-ES-260-490-8 } \\
\hline Phenol & 1300 & UG/KG & $u$ & ... & & 1300 UG/KG & 4 \\
\hline bis(2-Chloroethyl) ether & 1300 & UG/KG & $u$ & -. & & $1300 \mathrm{UG} / \mathrm{KG}$ & 4 \\
\hline 2-Chiorophenol & 1300 & UG/KG & $u$ & - & & 1300 UG/KG & 4 \\
\hline 1. 3-Dichlorobenzene & 1300 & UG/KG & $u$ & $\ldots$ & & 1300 UG/KG & 4 \\
\hline 1, 4-Dichlorobenzene & 1300 & $U G / K G$ & $u$ & $\cdots$ & & 1300 UG/KG & 4 \\
\hline Benzyl Alcohol & 1300 & UG/KG & $u$ & ... & & 1300 UG/KG & 4 \\
\hline 1, 2-Dichlorobenzene & 1300 & UG/KG & $u$ & $\ldots$ & & 1300 UG/KG & 4 \\
\hline 2-Methyiphenol & 1300 & UG/KG & $u$ & .. & & 1300 UG/KG & 4 \\
\hline bis(2-Chloroisopropyl) ether & 1300 & UG/KG & $u$ & $\ldots$ & & 1300 UG/KG & 4 \\
\hline 4-Methylphenol & 1300 & UG/KG & $u$ & $\ldots$ & & 1300 UG/KG & 4 \\
\hline N-Nitroso-di-nPropylamine & 1300 & UG/KG & $u$ & $\cdots$ & & 1300 UG/KG & 4 \\
\hline Hexachloroethane & 1300 & UG/KG & $u$ & $\ldots$ & & 1300 UG/KG & 4 \\
\hline Nitrobenzene & 1300 & $U G / K G$ & $u$ & $\ldots$ & & 1300 UG/KG & 4 \\
\hline Isophorone & 1300 & UG/KG & $u$ & $\cdots$ & & 1300 UG/KG & 4 \\
\hline 2-Nitrophenol & 1300 & UG/KG & $u$ & $\cdots$ & & 1300 UG/KG & 4 \\
\hline 2, 4-Dimethylphenol & 1300 & UG/KG & $u$ & ... & & 1300 UG/KG & 4 \\
\hline Benzoic Acid & 6400 & UG/KG & $u$ & $\ldots$ & & 6400 UG/KG & 4 \\
\hline bis(2Chloroethoxy)methane & 1300 & $U G / K G$ & $u$ & $\cdots$ & & 1300 UG/KG & 4 \\
\hline 2, 4-Dichlorophenol & 1300 & UG/KG & $u$ & $\cdots$ & & 1300 UG/KG & 4 \\
\hline 1, 2, 4-Trichlorobenzene & 1300 & UG/KG & $u$ & $\cdots$ & & 1300 UG/KG & 4 \\
\hline Naphthalene & 480 & UG/KG & J & $\ldots$ & & $1300 \mathrm{UG} / \mathrm{KG}$ & 4 \\
\hline 4-Chloroaniline & 1300 & UG/KG & $u$ & $\ldots$ & & 1300 UG/KG & 4 \\
\hline Hexachlorobutadiene & 1300 & UG/KG & $u$ & $\cdots$ & & 1300 UG/KG & 4 \\
\hline 4-Chloro-3-Methylphenol & 1300 & UG/KG & $u$ & $\ldots$ & & 1300 UG/KG & 4 \\
\hline 2-Methylnaphthalene & 1300 & UG/KG & $u$ & $\cdots$ & & 1300 UG/KG & 4 \\
\hline Hexachlorocyclopentadiene & 1300 & UG/KG & $u$ & $\cdots$ & & 1300 UG/KG & 4 \\
\hline 2, 4, 6-Trichlorophenol & 1300 & UG/KG & $u$ & -- & & 1300 UG/KG & 4 \\
\hline 2, 4, 5-Trichlorophenol & 6400 & UG/KG & $u$ & $\ldots$ & & 6400 UG/KG & 4 \\
\hline 2-Chloronaphthalene & 1300 & UG/KG & $u$ & $\ldots$ & & 1300 UG/KG & 4 \\
\hline 2-Nitroaniline & 6400 & UG/KG & $u$ & $\ldots$ & & 6400 UG/KG & 4 \\
\hline Dimethyl Phthalate & 1300 & UG/KG & $u$ & $\ldots$ & & 1300 UG/KG & 4 \\
\hline Acenaphthylene & 1300 & $U G / K G$ & $u$ & $\ldots$ & & 1300 UG/KG & 4 \\
\hline 2, 6-Dinitrotoluene & 1300 & $U G / K G$ & $u$ & $\ldots$ & & 1300 UG/KG & 4 \\
\hline 3-Nitroaniline & 6400 & UG/KG & $u$ & $\cdots$ & & 6400 UG/KG & 4 \\
\hline Acenaphthene & 1300 & UG/KG & $u$ & $\ldots$ & & 1300 UG/KG & 4 \\
\hline 2, 4-Dinitrophenol & 6400 & UG/KG & $u$ & $\ldots$ & & 6400 UG/KG & 4 \\
\hline 4-Nitrophenol & 6400 & UG/KG & $u$ & $\ldots$ & & $6400 \mathrm{UIZ/KG}$ & 4 \\
\hline Dibenzofuran & 1300 & UG/KG & $u$ & $\ldots$ & & $1300 \mathrm{UG} / \mathrm{KG}$ & 4 \\
\hline 2, 4-Dinitrotoluene & 1300 & UG/KG & $U$ & - & & 1300 UG/KG & 4 \\
\hline Diethylphthalate & 1300 & UG/KG & $u$ & -- & & $1300 \mathrm{UG} / \mathrm{KG}$ & 4 \\
\hline 4-Chlorophenyl phenyl ether & 1300 & UG/KG & $u$ & $\ldots$ & & 1300 UG/KG & 4 \\
\hline Fluorene & 2400 & UGKG & & $\cdots$ & & 1300 UG/KG & 4 \\
\hline 4-Nitroaniline & 6400 & UGKG & $u$ & $\ldots$ & & 6400 UG/KG & 4 \\
\hline 4, 6-Dinitro-2-Methylphenol & 6400 & UG/KG & $u$ & $\cdots$ & & 6400 UG/KG & 4 \\
\hline N-Nitrosodiphenylamine & 1300 & UG/KG & $u$ & $\ldots$ & & 1300 UG/KG & 4 \\
\hline 4-Bromophenyl phenyl ether & 1300 & UG/KG & $u$ & - & & 1300 UG/KG & 4 \\
\hline Hexachlorobenzene & 1300 & UG/KG & $u$ & $\ldots$ & & 1300 UG/KG & 4 \\
\hline Pentachlorophenol & 6400 & UG/KG & $u$ & .- & & 6400 UG/KG & 4 \\
\hline Phenanthrene & 1300 & UG/KG & $u$ & $\ldots$ & & 1300 UG/KG & 4 \\
\hline
\end{tabular}


Appendix D: Results of Semivolatile Organic Compound Analyses, Salmon Site

\begin{tabular}{|c|c|c|c|c|c|c|c|c|}
\hline COMPOUND & RESULTS & UNITS & $\begin{array}{l}\text { DATA } \\
\text { QUAL }\end{array}$ & $\begin{array}{l}\text { DATA } \\
\text { VALID. }\end{array}$ & NOTES & CRDL & UNITS & DILUTION \\
\hline \multicolumn{9}{|l|}{ TR4-ES-260-490-8 (CON'T) } \\
\hline Anthracene & 1300 & UG/KG & u & $\ldots$ & & 130 & UG/KG & 4 \\
\hline Di-N-Butylphthalate & 1300 & UG/KG & $u$ & $\ldots$ & & 130 & UG/KG & 4 \\
\hline Fluoranthene & 1300 & UG/KG & u & $\ldots$ & & 130 & UG/KG & 4 \\
\hline Pyrene & 1300 & UG/KG & u & $\cdots$ & & 130 & UG/KG & 4 \\
\hline Butyl Benzyl Phthalate & 1300 & UG/KG & u & $\ldots$ & & 130 & UG/KG & 4 \\
\hline 3. 3'-Dichlorobenzidine & 2600 & UG/KG & $u$ & ... & & 260 & UG/KG & 4 \\
\hline Benzo(a)Anthracene & 1300 & $\mathrm{U} / \mathrm{KG}$ & U & $\ldots$ & & 130 & UG/KG & 4 \\
\hline Chrysene & 1300 & UG/KG & U & ... & & 130 & UG/KG & 4 \\
\hline bis(2-Ethylhexyl)phthalate & 250 & UG/KG & J & $\ldots$ & & 130 & UG/KG & 4 \\
\hline di-N-Octyl Phthalate & 1300 & UG/KG & U & ... & & 130 & UG/KG & 4 \\
\hline Benzo(b)Fluoranthene & 1300 & UG/KG & U & $\ldots$ & & 130 & UG/KG & 4 \\
\hline Benzo(k)Fluoranthene & 1300 & ¡UG/KG & U & $\ldots$ & & 130 & UG/KG & 4 \\
\hline Benzo(a)Pyrene & 1300 & $U G / K G$ & $u$ & $\ldots$ & & 130 & UG/KG & 4 \\
\hline Indeno(1,2,3-CD)Pyrene & 1300 & $U G / K G$ & $u$ & $\ldots$ & & 130 & UG/KG & 4 \\
\hline Dibenzo(a,h)Anthracene & 1300 & $U G / K G$ & $u$ & $\ldots$ & & 130 & UG/KG & 4 \\
\hline Benzo(g,h,i)Perylene & 1300 & $U G / K G$ & $u$ & $\ldots$ & & 130 & UG/KG & 4 \\
\hline Nitrobenzene-d5 & 73 & $\%$ & & & & & $\%$ & 4 \\
\hline 2-Fluorobiphenyl & 87 & $\%$ & & & & & $\%$ & 4 \\
\hline Terphenyl-d14 & 72 & $\%$ & & & & & $\%$ & 4 \\
\hline Phenol-d5 & 67 & $\%$ & & & & & $\%$ & 4 \\
\hline 2-Fluorophenol & 69 & $\%$ & & & & & $\%$ & 4 \\
\hline 2, 4, 6-Tribromophenol & 95 & $\%$ & & & & & $\%$ & 4 \\
\hline
\end{tabular}




\begin{tabular}{|c|c|c|c|c|c|c|c|}
\hline COMPOUND & RESULTS & UNITS & $\begin{array}{l}\text { DATA } \\
\text { QUAL }\end{array}$ & $\begin{array}{l}\text { DATA } \\
\text { VALID. }\end{array}$ & NOTES & CADL UNITS & DILUTION \\
\hline \multicolumn{8}{|l|}{ TR4-ES-260-490-8 RE } \\
\hline Phenol & 1300 & UG/KG & $u$ & NP & & 1300 UG/KG & 4 \\
\hline bis(2-Chloroethyl) ether & 1300 & UG/KG & $u$ & NP & & 1300 UG/KG & 4 \\
\hline 2-Chlorophenol & 1300 & UG/KG & $u$ & NP & & 1300 UG/KG & 4 \\
\hline 1, 3-Dichlorobenzene & 1300 & UG/KG & $u$ & NP & & 1300 UG/KG & 4 \\
\hline 1, 4-Dichlorobenzene & 1300 & UG/KG & $u$ & NP & & 1300 UG/KG & 4 \\
\hline Benzyl Alcohol & 1300 & UG/KG & $u$ & NP & & 1300 UG/KG & 4 \\
\hline 1. 2-Dichlorobenzene & 1300 & UG/KG & U & NP & & 1300 UG/KG & 4 \\
\hline 2-Methylphenol & 1300 & UG/KG & U & NP & & 1300 UG/KG & 4 \\
\hline bis(2-Chloroisopropyl) ether & 1300 & UG/KG & U & NP & & 1300 UG/KG & 4 \\
\hline 4-Methylphenol & 1300 & UG/KG & $u$ & NP & & 1300 UG/KG & 4 \\
\hline N-Nitroso-di-nPropylamine & 1300 & UG/KG & $u$ & NP & & 1300 UG/KG & 4 \\
\hline Hexachloroethane & 1300 & UG/KG & $u$ & NP & & 1300 UG/KG & 4 \\
\hline Nitrobenzene & 1300 & UG/KG & $u$ & NP & & 1300 UG/KG & 4 \\
\hline Isophorone & 1300 & UG/KG & $u$ & NP & & 1300 UG/KG & 4 \\
\hline 2-Nitrophenol & 1300 & UG/KG & U & NP & & 1300 UG/KG & 4 \\
\hline 2, 4-Dimethylphenol & 1300 & UG/KG & $U$ & NP & & 1300 UG/KG & 4 \\
\hline Benzoic Acid & 6400 & UG/KG & U & NP & & 6400 UG/KG & 4 \\
\hline bis(2Chloroethoxy)methane & 1300 & UG/KG & $u$ & NP & & 1300 UG/KG & 4 \\
\hline 2, 4-Dichlorophenol & 1300 & UG/KG & $u$ & NP & & 1300 UG/KG & 4 \\
\hline 1, 2, 4-Trichlorobenzene & 1300 & UG/KG & $u$ & NP & & 1300 UG/KG & 4 \\
\hline Naphthalene & 480 & UG/KG & $\mathrm{J}$ & NP & & 1300 UG/KG & 4 \\
\hline 4-Chloroaniline & 1300 & UG/KG & u & NP & & $1300 \mathrm{UG} / \mathrm{KG}$ & 4 \\
\hline Hexacnlorobutadiene & 1300 & $U G / K G$ & $u$ & NP & & 1300 UG/KG & 4 \\
\hline 4-Chloro-3-Methylphenol & 1300 & UG/KG & $u$ & NP & & 1300 UG/KG & 4 \\
\hline 2-MethyInaphthalene & 1300 & $U G / K G$ & $u$ & NP & & $1300 \mathrm{UG} / \mathrm{KG}$ & 4 \\
\hline Hexachlorocyclopentadiene & 1300 & UG/KG & u & NP & & 1300 UG/KG & 4 \\
\hline 2, 4,6-Trichlorophenol & 1300 & UG/KG & u & NP & & 1300 UG/KG & 4 \\
\hline 2, 4, 5-Trichlorophenol & 6400 & UG/KG & u & NP & & 6400 UG/KG & 4 \\
\hline 2-Chloronaphthalene & 1300 & $U G / K G$ & $u$ & NP & & 1300 UG/KG & 4 \\
\hline 2-Nitroaniline & 6400 & UG/KG & $u$ & NP & & 6400 UG/KG & 4 \\
\hline Dimethyl Phthalate & 1300 & UG/KG & u & NP & & 1300 UG/KG & 4 \\
\hline Acenaphthylene & 1300 & UG/KG & $u$ & NP & & 1300 UG/KG & 4 \\
\hline 2, 6-Dinitrotoluene & 1300 & UG/KG & $u$ & NP & & 1300 UG/KG & 4 \\
\hline 3-Nitroaniline & 6400 & Ua/KG & $u$ & NP & & $6400 \mathrm{JG} / \mathrm{KG}$ & 4 \\
\hline Acenaphthene & 1300 & UG/KG & $u$ & NP & & 1300 UG/KG & 4 \\
\hline 2, 4-Dinitrophenol & 6400 & $U G / K G$ & $u$ & NP & & 6400 UG/KG & 4 \\
\hline 4-Nitrophenol & 6400 & UG/KG & $u$ & NP & & 6400 UG/KG & 4 \\
\hline Dibenzofuran & 1300 & UG/KG & $u$ & NP & & 1300 UG/KG & 4 \\
\hline 2, 4-Dinitrotoluene & 1300 & UG/KG & $u$ & NP & & 1300 UG/KG & 4 \\
\hline Diethylphthalate & 1300 & UG/KG & $u$ & NP & & 1300 UG/KG & 4 \\
\hline 4-Chlorophenyl phenyl ether & 1300 & UG/KG & $u$ & $\mathrm{NP}$ & & 1300 UG/KG & 4 \\
\hline Fluorene & 2300 & UGKG & & NP & & $: 300$ UQ/KG & 4 \\
\hline 4-Nitroaniline & 6400 & UGKG & $u$ & NP & & 6400 UG/KG & 4 \\
\hline 4, 6-Dinitro-2-Methylphenol & 6400 & UG/KG & $u$ & NP & & 6400 UG/KG & 4 \\
\hline N-Nitrosodiphenylamine & 1300 & UG/KG & $u$ & NP & & 1300 UG/KG & 4 \\
\hline 4-Bromophenyl phenyl ether & 1300 & UG/KG & $u$ & NP & & 1300 UG/KG & 4 \\
\hline Hexachlorobenzene & 1300 & UG/KG & $u$ & NP & & 1300 UG/KG & 4 \\
\hline Pentachlorophenol & 6400 & UG/KG & $u$ & NP & & 6400 UG/KG & 4 \\
\hline Phenanthrene & 1300 & UG/KG & $u$ & NP & & 1300 UG/KG & 4 \\
\hline
\end{tabular}


Appendix D: Results of Semivolatile Organic Compound Analyses, Salmon Site

\begin{tabular}{|c|c|c|c|c|c|c|c|c|}
\hline COMPOUND & RESULTS & UNITS & $\begin{array}{l}\text { DATA } \\
\text { QUAL } \\
\end{array}$ & $\begin{array}{l}\text { DATA } \\
\text { VALID. }\end{array}$ & NOTES & CRDL & UNITS & DILUTION \\
\hline \multicolumn{9}{|c|}{ TR4-ES-260-490-8RE (CON'T) } \\
\hline Anthracene & 1300 & UG/KG & u & NP & & 1300 & $U G / K G$ & 4 \\
\hline Di-N-Butylphthalate & 1300 & $U G / K G$ & $u$ & $N P$ & & 1300 & UG/KG & 4 \\
\hline Fluoranthene & 1300 & UG/KG & $u$ & NP & & 1300 & UG/KG & 4 \\
\hline Pyrene & 1300 & UG/KG & $u$ & NP & & 1300 & UG/KG & 4 \\
\hline Butyl Benzyl Phthalate & 1300 & $U G / K G$ & $u$ & NP & & 1300 & UG/KG & 4 \\
\hline 3, 3'-Dichlorobenzidine & 2600 & UG/KG & $u$ & NP & & 2600 & UG/KG & 4 \\
\hline Benzo(a)Anthracene & 1300 & UG/KG & $u$ & NP & & 1300 & UG/KG & 4 \\
\hline Chrysene & 1300 & UG/KG & $u$ & NP & & 1300 & UG/KG & 4 \\
\hline bis(2-Ethylhexyl)phthalate & 250 & UG/KG & J & NP & & 1300 & UG/KG & 4 \\
\hline di-N-Octyl Phthalate & 1300 & UG/KG & $u$ & NP & & 1300 & UG/KG & 4 \\
\hline Benzo(b)Fluoranthene & 1300 & JJ/KG & $u$ & NP & & 1300 & UG/KG & 4 \\
\hline Benzo(k)Fluoranthene & 1300 & UG/KG & $u$ & NP & & 1300 & UG/KG & 4 \\
\hline Benzo(a)Pyrene & 1300 & UG/KG & $u$ & NP & & 130 & O UG/KG & 4 \\
\hline Indeno $(1,2,3-C D)$ Pyrene & 1300 & UG/KG & $u$ & NP & & 130 & O UG/KG & 4 \\
\hline Dibenzo(a,h)Anthracene & 1300 & UG/KG & $u$ & NP & & 130 & O UG/KG & 4 \\
\hline Benzo(g,h,i)Perylene & 1300 & UG/KG & $u$ & NP & & 130 & O UG/KG & 4 \\
\hline Nitrobenzene-d5 & 75 & $\%$ & & & & & $\%$ & 4 \\
\hline 2-Fluorobiphenyl & 72 & $\%$ & & & & & $\%$ & 4 \\
\hline Terphenyl-d14 & 62 & $\%$ & & & & & $\%$ & 4 \\
\hline Phenol-d5 & 71 & $\%$ & & & & & $\%$ & 4 \\
\hline 2-Fluorophenol & 65 & $\%$ & & & & & $\%$ & 4 \\
\hline 2, 4, 6-Tribromophenol & 83 & $\%$ & & & & & $\%$ & 4 \\
\hline
\end{tabular}




\begin{tabular}{|c|c|c|c|c|c|c|c|}
\hline COMPOUND & RESULTS & UNITS & $\begin{array}{l}\text { DATA } \\
\text { QUAL }\end{array}$ & $\begin{array}{l}\text { DATA } \\
\text { VALID. }\end{array}$ & NOTES & CRDL UNITS & DILUTIO \\
\hline \multicolumn{8}{|l|}{ TR5-ES-590-570-12 } \\
\hline Phenol & 330 & UG/KG & $u$ & ... & & 330 UG/KG & 1 \\
\hline bis(2-Chloroethyl) ether & 330 & $U G / K G$ & $u$ & $\ldots$ & & 330 UG/KG & 1 \\
\hline 2-Chlorophenol & 330 & UG/KG & $u$ & $\ldots$ & & 330 UG/KG & 1 \\
\hline 1, 3-Dichlorobenzene & 330 & $U G / K G$ & $u$ & $\ldots$ & & 330 UG/KG & 1 \\
\hline 1, 4-Dichlorobenzene & 330 & UG/KG & $u$ & ... & & 330 UG/KG & 1 \\
\hline Benzyl Alcohol & 330 & UG/KG & $u$ & ... & & 330 UG/KG & 1 \\
\hline 1. 2-Dichlorobenzene & 330 & UG/KG & $u$ & $\ldots$ & & 330 UG/KG & 1 \\
\hline 2-Methylphenol & 330 & UG/KG & $u$ & ... & & 330 UG/KG & 1 \\
\hline bis(2-Chloroisopropyl) ether & 330 & UG/KG & $u$ & $\ldots$ & & 330 UG/KG & 1 \\
\hline 4-Methylphenol & 330 & UG/KG & $u$ & ... & & 330 UG/KG & 1 \\
\hline N-Nitroso-di-nPropylamine & 330 & UG/KG & $u$ & $\ldots$ & & 330 UG/KG & 1 \\
\hline Hexachloroethane & 330 & UG/KG & $u$ & $\ldots$ & & 330 UG/KG & 1 \\
\hline Nitrobenzene & 330 & UG/KG & $u$ & ... & & 330 UG/KG & 1 \\
\hline Isophorone & 330 & UG/KG & $u$ & ... & & 330 UG/KG & 1 \\
\hline 2-Nitrophenol & 330 & $U G / K G$ & $u$ & $\ldots$ & & 330 UG/KG & 1 \\
\hline 2. 4-Dimethylphenol & 330 & UG/KG & $u$ & ... & & 330 UG/KG & 1 \\
\hline Benzoic Acid & 1600 & UG/KG & $u$ & $\ldots$ & & 1600 UG/KG & 1 \\
\hline bis(2Chloroethoxy)methane & 330 & UG/KG & $u$ & $\ldots$ & & 330 UG/KG & 1 \\
\hline 2, 4-Dichlorophenol & 330 & UG/KG & $u$ & ... & & 330 UG/KG & 1 \\
\hline 1, 2, 4-Trichlorobenzene & 330 & UG/KG & $u$ & -.- & & 330 UG/KG & 1 \\
\hline Naphthalene & 100 & UG/KG & J & -- & & 330 UG/KG & 1 \\
\hline 4-Chloroaniline & 330 & UG/KG & $u$ & - & & 330 UG/KG & 1 \\
\hline Hexachlorobutadiene & 330 & UG/KG & $u$ & ... & & 330 UG/KG & 1 \\
\hline 4-Chloro-3-Methylphenol & 330 & UG/KG & $u$ & -. & & 330 UG/KG & 1 \\
\hline 2-Methyinaphthalene & 1500 & UG/KG & & ... & . & 330 UG/KG & 1 \\
\hline Hexachlorocyclopentadiene & 330 & UG/KG & $u$ & $\ldots$ & & 330 UG/KG & 1 \\
\hline 2, 4, 6-Trichlorophenol & 330 & UG/KG & $u$ & ... & & 330 UG/KG & 1 \\
\hline 2, 4,5-Trichlorophenol & 1600 & UG/KG & $u$ & -. & & 1600 UG/KG & 1 \\
\hline 2-Chloronaphthalene & 330 & UG/KG & $u$ & $\ldots$ & & 330 UG/KG & 1 \\
\hline 2-Nitroaniline & 1600 & UG/KG & $u$ & $\ldots$ & & 1600 UG/KG & 1 \\
\hline Dimethyl Phthalate & 330 & $U G / K G$ & $u$ & $\ldots$ & & 330 UG/KG & 1 \\
\hline Acenaphthylene & 330 & $U G / K G$ & $u$ & $\ldots$ & & 330 UG/KG & 1 \\
\hline 2. 6-Dinitrotoluene & 330 & $U G / K G$ & $u$ & ... & & 330 UG/KG & 1 \\
\hline 3-Nitroaniline & 1600 & UG/KG & $u$ & $\ldots$ & & 1600 UG/KG & 1 \\
\hline Acenaphthene & 330 & UG/KG & $u$ & $\ldots$ & & 330 UG/KG & 1 \\
\hline 2, 4-Dinitrophenol & 1600 & UG/KG & $u$ & $\ldots$ & & 1600 UG/KG & 1 \\
\hline 4-Nitrophenol & 1600 & UG/KG & $u$ & - & & 1600 UG/KG & 1 \\
\hline Dibenzofuran & 330 & UG/KG & $u$ & -.. & & 330 UG/KG & 1 \\
\hline 2, 4-Dinitrotoluene & 330 & UG/KG & $u$ & -- & & 330 UG/KG & 1 \\
\hline Diethylphthalate & 330 & UG/KG & $u$ & $\ldots$ & & 330 UG/KG & 1 \\
\hline 4-Chlorophenyl phenyl ether & 330 & UG/KG & $u$ & $\ldots$ & & 330 UG/KG & 1 \\
\hline Fluorene & 170 & UG/KG & J & $\ldots$ & & 330 UG/KG & 1 \\
\hline 4-Nitroaniline & 1600 & UG/KG & $u$ & $\ldots$ & & 1600 UG/KG & 1 \\
\hline 4, 6-Dinitro-2-Methylphenol & 1600 & UG/KG & $u$ & $\cdots$ & & 1600 UG/KG & 1 \\
\hline N-Nitrosodiphenylamine & 330 & UG/KG & $u$ & - & & 330 UG/KG & 1 \\
\hline 4-Bromophenyl phenyl ether & 330 & UG/KG & $u$ & $\ldots$ & & 330 UG/KG & 1 \\
\hline Hexachlorobenzene & 330 & UG/KG & $u$ & - & & 330 UG/KG & 1 \\
\hline Pentachlorophenol & 1600 & UG/KG & $u$ & -. & & 1600 UG/KG & 1 \\
\hline Phenanthrene & 330 & UG/KG & $u$ & $\cdots$ & & 330 UG/KG & 1 \\
\hline
\end{tabular}


Appendix D: Results of Semivolatile Organic Compound Analyses, Salmon Site

\begin{tabular}{|c|c|c|c|c|c|c|c|c|}
\hline COMPOUND & RESULTS & UNITS & $\begin{array}{l}\text { DATA } \\
\text { QUAL }\end{array}$ & $\begin{array}{l}\text { DATA } \\
\text { VALID. }\end{array}$ & NOTES & CRDL & UNITS & DILUTION \\
\hline \multicolumn{9}{|l|}{ TR5-ES-590-570-12 (CON'T) } \\
\hline Anthracene & 330 & $U G / K G$ & u & ... & & & O UG/KG & 1 \\
\hline Di-N-Butylphthalate & 330 & UG/KG & u & $\ldots$ & & & U UG/KG & 1 \\
\hline Fluoranthene & 330 & UG/KG & $u$ & ... & & & $0 \cup G / K G$ & 1 \\
\hline Pyrene & 330 & $U G / K G$ & $u$ & ... & & & $0 \cup G / K G$ & 1 \\
\hline Butyl Benzyl Phthalate & 330 & UG/KG & $u$ & ... & & & O UG/KG & 1 \\
\hline 3, 3'-Dichlorobenzidine & 650 & UG/KG & $u$ & ... & & & O UG/KG & 1 \\
\hline Benzo(a)Anthracene & 330 & UG/KG & $u$ & $\ldots$ & & & 0 UG/KG & 1 \\
\hline Chrysene & 330 & UG/KG & $u$ & ... & & & 0 UG/KG & 1 \\
\hline bis(2-Ethylhexyl)phthalate & 330 & $U G / K G$ & $u$ & $\ldots$ & & & 0 UG/KG & 1 \\
\hline di-N-Octyl Phthaiate & 330 & UG/KG & $u$ & $\ldots$ & & & 0 UG/KG & 1 \\
\hline Benzo(b)Fluoranthene & 330 & UG/KG & $u$ & $\ldots$ & & & 0 UG/KG & 1 \\
\hline Benzo(k)Fluoranthene & 330 & UG/KG & $u$ & ... & & & 0 UG/KG & 1 \\
\hline Benzo(a)Pyrene & 330 & UG/KG & $u$ & $\ldots$ & & & 0 UG/KG & 1 \\
\hline Indeno $(1,2,3-C D)$ Pyrene & 330 & $U G / K G$ & $u$ & $\ldots$ & & & O UG/KG & 1 \\
\hline Dibenzo(a,h)Anthracene & 330 & UG/KG & $u$ & $\ldots$ & & & 0 UG/KG & 1 \\
\hline Benzo(g,h,i)Perylene & 330 & UG/KG & $u$ & $\ldots$ & & & 0 UG/KG & 1 \\
\hline Nitrobenzene-d5 & 53 & $\%$ & & & & & $\%$ & 1 \\
\hline 2-Fluorobiphenyl & 62 & $\%$ & & & & & $\%$ & 1 \\
\hline Terphenyl-d14 & 58 & $\%$ & & & & & $\%$ & 1 \\
\hline Phenol-d5 & 59 & $\%$ & & & & & $\%$ & 1 \\
\hline 2-Fluoropheriol & 55 & $\%$ & & & & & $\%$ & 1 \\
\hline 2, 4, 6-Tribromophenol & 71 & $\%$ & & & & & $\%$ & 1 \\
\hline
\end{tabular}




\begin{tabular}{|c|c|c|c|c|c|c|c|}
\hline COMPOUND & RESULTS & UNITS & $\begin{array}{l}\text { DATA } \\
\text { QUAL }\end{array}$ & $\begin{array}{l}\text { DATA } \\
\text { VALID. }\end{array}$ & NOTES & CRDL UNITS & DILUTION \\
\hline \multicolumn{8}{|l|}{ TR6-ES-495-585-12 } \\
\hline Phenol & 330 & UG/KG & $u$ & NP & & 330 UG/KG & 1 \\
\hline bis(2-Chloroethyl) ether & 330 & $U G / K G$ & $u$ & NP & & 330 UG/KG & 1 \\
\hline 2-Chlorophenol & 330 & $U G / K G$ & $u$ & NP & & 330 UG/KG & 1 \\
\hline 1. 3-Dichlorobenzene & 330 & UG/KG & $u$ & NP & & 330 UG/KG & 1 \\
\hline 1, 4-Dichlorobenzene & 330 & UG/KG & $u$ & NP & & 330 UG/KG & 1 \\
\hline Benzyl Alcohol & 330 & UG/KG & $u$ & NP & & 330 UG/KG & 1 \\
\hline 1, 2-Dichlorobenzene & 330 & UG/KG & $u$ & NP & & 330 UG/KG & 1 \\
\hline 2-Methylphenol & 330 & UG/KG & U & NP & & 330 UG/KG & 1 \\
\hline bis(2-Chloroisopropyl) ether & 330 & UG/KG & U & NP & & 330 UG/KG & 1 \\
\hline 4-Methylphenol & 330 & UG/KG & U & NP & & 330 UG/KG & 1 \\
\hline N-Nitroso-di-nPropylamine & 330 & UG/KG & $u$ & NP & & 330 UG/KG & 1 \\
\hline Hexachloroethane & 330 & UG/KG & $u$ & NP & & 330 UG/KG & 1 \\
\hline Nitrobenzene & 330 & UG/KG & $u$ & NP & & 330 UG/KG & 1 \\
\hline Isophorone & 330 & UG/KG & u & NP & & 330 UG/KG & 1 \\
\hline 2-Nitrophenol & 330 & UG/KG & $u$ & NP & & 330 UG/KG & 1 \\
\hline 2. 4-Dimethylphenol & 330 & UG/KG & u & NP & & 330 UG/KG & 1 \\
\hline Benzoic Acid & 1600 & UG/KG & $u$ & NP & & 1600 UG/KG & 1 \\
\hline bis(2Chloroethoxy)methane & 330 & UG/KG & $u$ & NP & & 330 UG/KG & 1 \\
\hline 2, 4-Dichlorophenol & 330 & UG/KG & $u$ & NP & & 330 UG/KG & 1 \\
\hline 1, 2, 4-Trichlorobenzene & 330 & UG/KG & $u$ & NP & & 330 UG/KG & 1 \\
\hline Naphthalene & 330 & UG/KG & u & NP & & 330 UG/KG & 1 \\
\hline 4-Chloroaniline & 330 & UG/KG & $u$ & NP & & 330 UG/KG & 1 \\
\hline Hexachlorobutadiene & 330 & UG/KG & U & NP & & 330 UG/KG & 1 \\
\hline 4-Chloro-3-Methylphenol & 330 & UG/KG & $u$ & NP & & 330 UG/KG & 1 \\
\hline 2-Methylnaphthalene & 330 & UG/KG & $u$ & NP & & 330 UG/KG & 1 \\
\hline Hexachlorocyclopentadiene & 330 & UG/KG & $u$ & NP & & 330 UG/KG & 1 \\
\hline 2, 4, 6-Trichlorophenol & 330 & UG/KG & u & NP & & 330 UG/KG & 1 \\
\hline 2, 4, 5-Trichlorophenol & 1600 & UG/KG & $u$ & NP & & 1600 UG/KG & 1 \\
\hline 2-Chloronaphthalene & 330 & UG/KG & $u$ & NP & & 330 UG/KG & 1 \\
\hline 2-Nitroaniline & 1600 & UG/KG & u & NP & & 1600 UG/KG & 1 \\
\hline Dimethyl Phthalate & 330 & UG/KG & u & NP & & 330 UG/KG & 1 \\
\hline Acenaphthylene & 330 & UG/KG & u & NP & & 330 UG/KG & 1 \\
\hline 2, 6-Dinitrotoluene & 330 & UG/KG & $u$ & NP & & 330 UG/KG & 1 \\
\hline 3-Nitroaniline & 1600 & UG/KG & $u$ & NP & & 1600 UG/KG & 1 \\
\hline Acenaphthene & 330 & UG/KG & $u$ & NP & & 330 UG/KG & 1 \\
\hline 2. 4-Dinitrophenol & 1600 & UG/KG & $u$ & NP & & $1600 \mathrm{UG} / \mathrm{KG}$ & 1 \\
\hline 4-Nitrophenol & 1600 & $U G / K G$ & $u$ & NP & & 1600 UG/KG & 1 \\
\hline Dibenzofuran & 330 & $U G / K G$ & u & NP & & 330 UG/KG & 1 \\
\hline 2, 4-Dinitrotoluene & 330 & UG/KG & u & NP & & 330 UG/KG & 1 \\
\hline Diethylphthalate & 330 & UG/KG & $u$ & NP & & 330 UG/KG & 1 \\
\hline 4-Chlorophenyl phenyl ether & 330 & UG/KG & $u$ & NP & & 330 UG/KG & 1 \\
\hline Fluorene & 70 & UGKG & $J$ & NP & & 330 UG/KG & 1 \\
\hline 4-Nitroaniline & 1600 & UG/KG & $u$ & NP & & 1600 UG/KG & 1 \\
\hline 4, 6-Dinitro-2-Methyiphenol & 1600 & UG/KG & $u$ & NP & & 1600 UG/KG & 1 \\
\hline N-Nitrosodiphenylamine & 330 & UG/KG & $u$ & NP & & 330 UG/KG & 1 \\
\hline 4-Bromophenyl phenyl ether & 330 & UG/KG & $u$ & NP & & 330 UG/KG & 1 \\
\hline Hexachlorobenzene & 330 & UGKG & $u$ & NP & & 330 UG/KG & 1 \\
\hline Pentachlorophenol & 1600 & UG/KG & $u$ & NP & & 1600 UG/KG & 1 \\
\hline Phenanthrene & 330 & UG/KG & u & NP & & 330 UG/KG & 1 \\
\hline
\end{tabular}


Appendix D: Results of Semivolatile Organic Compound Analyses, Salmon Site

\begin{tabular}{|c|c|c|c|c|c|c|c|}
\hline COMPOUND & RESULTS & UNITS & $\begin{array}{l}\text { DATA } \\
\text { QUAL }\end{array}$ & $\begin{array}{l}\text { DATA } \\
\text { VALID. }\end{array}$ & NOTES & CRDL UNITS & DILUTION \\
\hline \multicolumn{8}{|l|}{ TR6-ES-495-585-12 (CON'T) } \\
\hline Anthracene & 330 & UG/KG & $u$ & NP & & 330 UG/KG & 1 \\
\hline Di-N-Butylphthalate & 330 & $U G / K G$ & $u$ & NP & & 330 UG/KG & 1 \\
\hline Fluoranthene & 330 & UG/KG & $u$ & NP & & 330 UG/KG & 1 \\
\hline Pyrene & 330 & UG/KG & $u$ & NP & & 330 UG/KG & 1 \\
\hline Butyl Benzyl Phthalate & 330 & UG/KG & $u$ & NP & & 330 UG/KG & 1 \\
\hline 3, 3'-Dichlorobenzidine & 660 & $U G / K G$ & $u$ & NP & & 660 UG/KG & 1 \\
\hline Benzo(a)Anthracene & 330 & UG/KG & $u$ & NP & & 330 UG/KG & 1 \\
\hline Chrysene & 330 & UG/KG & $u$ & NP & & 330 UG/KG & 1 \\
\hline bis(2-Ethylhexyl)phthalate & 330 & $U G / K G$ & $u$ & NP & & 330 UG/KG & 1 \\
\hline di-N-Octyl Phthalate & 330 & $U G / K G$ & $u$ & NP & & 330 UG/KG & 1 \\
\hline Benzo(b)Fluoranthine & 330 & UG/KGi & $u$ & NP & & 330 UG/KG & 1 \\
\hline Benzo(k)Fluoranthene & 330 & $U G / K G i$ & $u$ & NP & & 330 UG/KG & 1 \\
\hline Benzo(a)Pyrene & 330 & UG/KG & $u$ & $\mathrm{NP}$ & & 330 UG/KG & 1 \\
\hline Indeno(1.2,3-CD)Pyrene & 330 & UG/KG & $u$ & NP & & 330 UG/KG & 1 \\
\hline Dibenzo(a.h)Anthracene & 330 & UG/KG & $u$ & NP & & 330 UG/KG & 1 \\
\hline Benzo(g,h,i)Perylene & 330 & UG/KG & $u$ & NP & & 330 UG/KG & 1 \\
\hline Unknown-1 & 200 & $U G / K G$ & J & NP & & UGKG & 1 \\
\hline Unknown-2 & 190 & $U G / K G$ & J & NP & & UG/KG & 1 \\
\hline Unknown-3 & 150 & UG/KG & J & NP & & UG/KG & 1 \\
\hline Unknown-4 & 210 & UG/KG & J & NP & & UG/KG & 1 \\
\hline Unknown-5 & 280 & $U G / K G$ & J & NP & & UG/KG & 1 \\
\hline Unknown-6 & 160 & $U G / K G$ & J & NP & & $U G / K G$ & 1 \\
\hline Unknown-7 & 150 & UG/KG & J & NP & & $U G / K G$ & 1 \\
\hline Unknown-8 & 360 & UG/KG & $\downarrow$ & NP & & UG/KG & 1 \\
\hline Unknown-9 & 200 & UG/KG & J & NP & & UG/KG & 1 \\
\hline Unknown-10 & 160 & UG/KG & J & NP & & UG/KG & 1 \\
\hline Unknown-11 & 270 & UG/KG & J & NP & & UG/KG & 1 \\
\hline Unknown-12 & 290 & UG/KG & J & NP & & UG/KG & 1 \\
\hline Unknown-13 & 210 & $U G / K G$ & J & NP & & UG/KG & 1 \\
\hline Unknown-14 & 250 & UG/KG & J & NP & & UG/KG & 1 \\
\hline Unknown-15 & 340 & UG/KG & J & NP & & UG/KG & 1 \\
\hline Unknown-16 & 180 & UG/KG & J & NP & & UG/KG & 1 \\
\hline Unknown-17 & 210 & $U G / K G$ & J & NP & & UG/KG & 1 \\
\hline Unknown-18 & 280 & UG/KG & J & NP & & UG/KG & 1 \\
\hline Unknown-19 & 370 & UG/KG & J & NP & & UG/KG & 1 \\
\hline Unknown-20 & 190 & UG/KG & J & NP & & UG/KG & 1 \\
\hline Unknown-21 & 220 & UG/KG & J & NP & & UG/KG & 1 \\
\hline Unknown-22 & 270 & $U G / K G$ & BS & NP & & UG/KG & 1 \\
\hline Nitrobenzene-d5 & 78 & $\%$ & & & & $\%$ & 1 \\
\hline 2-Fluorobiphenyl & 71 & $\%$ & & & & $\%$ & 1 \\
\hline Terphenyl-d14 & 58 & $\%$ & & & & $\%$ & 1 \\
\hline Phenol-d5 & 81 & $\%$ & & & & $\%$ & 1 \\
\hline 2-Fluorophenol & 75 & $\%$ & & & & $\%$ & 1 \\
\hline 2, 4, 6-Tribromophenol & 80 & $\%$ & & & & $4 \%$ & 1 \\
\hline
\end{tabular}


Appendix D: Results of Semivolatile Organic Compound Analyses, Salmon Site

\begin{tabular}{|c|c|c|c|c|c|c|c|}
\hline COMPOUND & RESULTS & UNITS & $\begin{array}{l}\text { DATA } \\
\text { QUAL }\end{array}$ & $\begin{array}{l}\text { DATA } \\
\text { VALID. }\end{array}$ & NOTES & CRDL UNITS & DILUTION \\
\hline \multicolumn{8}{|l|}{ TR6-ES-485-590-6 } \\
\hline Phenol & 330 & UG/KG & $u$ & NP & & $330 \mathrm{UG} / \mathrm{KG}$ & 1 \\
\hline bis(2-Chloroethyl) ether & 330 & UG/KG & $u$ & NP & & 330 UG/KG & 1 \\
\hline 2-Chlorophenol & 330 & UG/KG & $u$ & NP & & 330 UG/KG & 1 \\
\hline 1, 3-Dichlorobenzene & 330 & UG/KG & $u$ & NP & & 330 UG/KG & 1 \\
\hline 1, 4-Dichlorobenzene & 330 & UG/KG & $u$ & NP & & 330 UG/RG & 1 \\
\hline Benzyl Alcohol & 330 & UG/KG & $u$ & NP & & 330 UG/KG & 1 \\
\hline 1, 2-Dichlorobenzene & 330 & UGKG & $u$ & NP & & 330 UG/KG & 1 \\
\hline 2-Methylphenol & 330 & UGKG & $u$ & NP & & 330 UG/KG & 1 \\
\hline bis(2-Chloroisopropyl) ether & 330 & UGKG & $u$ & NP & & 330 UG/KG & 1 \\
\hline 4-Methylphenol & 330 & UG/KG & $u$ & NP & & 330 UG/KG & 1 \\
\hline N-Nitroso-di-nPropylamine & 330 & UG/KG & $u$ & NP & & 330 UG/KG & 1 \\
\hline Hexachloroethane & 330 & Ua/KG & $u$ & NP & & 330 UG/KG & 1 \\
\hline Nitrobenzene & 330 & UGKG & $u$ & NP & & 330 UG/KG & 1 \\
\hline Isophorone & 330 & UG/KG & $u$ & NP & & 330 UG/KG & 1 \\
\hline 2-Nitrophenol & 330 & UG/KG & $u$ & NP & & 330 UG/KG & 1 \\
\hline 2, 4-Dimethylphenol & 330 & UG/KG & $u$ & NP & & 330 UG/KG & 1 \\
\hline Benzoic Acid & 1600 & UGKG & $u$ & NP & & 1600 VG/KG & 1 \\
\hline bis(2Chloroethoxy)methane & 330 & UG/KG & $u$ & NP & & 330 UG/KG & 1 \\
\hline 2, 4-Dichlorophenol & 330 & UG/KG & $u$ & NP & & 330 UG/KG & 1 \\
\hline 1, 2, 4-Trichlorobenzene & 330 & UG/KG & $u$ & NP & & 330 UG/KG & 1 \\
\hline Naphthalene & 330 & VGKG & $u$ & NP & & 330 UGKG & 1 \\
\hline 4-Chloroaniline & 330 & UG/KG & $u$ & NP & & 330 UG/KG & 1 \\
\hline Hexachlorobutadiene & 330 & UG/KG & $u$ & NP & & 330 UG/KG & 1 \\
\hline 4-Chloro-3-Methylphenol & 330 & UG/KG & $u$ & NP & & 330 UG/KG & 1 \\
\hline 2-Methylnaphthalene & 330 & UGKG & $u$ & NP & & 330 UG/KG & 1 \\
\hline Hexachlorocyclopentadiene & 330 & UG/KG & $u$ & NP & & 330 UG/KG & 1 \\
\hline 2, 4, 6-Trichlorophenol & 330 & UGKG & $u$ & NP & & 330 UG/KG & 1 \\
\hline 2, 4, 5-Trichlorophenol & 1600 & UG/KG & $u$ & NP & & 1600 UG/KG & 1 \\
\hline 2-Chloronaphthalene & 330 & VGKG & $u$ & NP & & 330 UG/KG & 1 \\
\hline 2-Nitroaniline & 1600 & UG/KG & $u$ & NP & & 1600 UG/KG & 1 \\
\hline Dimethyl Phthalate & 330 & UG/KG & $u$ & NP & & 330 UG/KG & 1 \\
\hline Acenaphthylene & 330 & UGKG & $u$ & NP & & 330 UGKG & 1 \\
\hline 2, 6-Dinitrotoluene & 330 & UGKG & $u$ & NP & & 330 UG/KG & 1 \\
\hline 3-Nitroaniline & 1600 & UGKG & $u$ & NP & & 1600 UG/KG & 1 \\
\hline Acenaphthene & 330 & UGKG & $u$ & NP & & 330 UG/KG & 1 \\
\hline 2, 4-Dinitrophenol & 1600 & UG/KG & $u$ & NP & & 1600 UG/KG & 1 \\
\hline 4-Nitrophenol & 1600 & UG/KG & $u$ & NP & & 1600 UG/KG & 1 \\
\hline Dibenzofuran & 330 & UGKG & $u$ & NP & & 330 UG/KG & 1 \\
\hline 2, 4-Dinitrotoluene & 330 & VGKG & $u$ & NP & & 330 UG/KG & 1 \\
\hline Diethylphthalate & 330 & UGKG & $u$ & NP & & 330 UG/KG & 1 \\
\hline 4-Chlorophenyl phenyl ether & 330 & UGKG & $u$ & NP & & 330 UG/KG & 1 \\
\hline Fluorene & 330 & UGKG & $u$ & NP & & 330 UGKG & 1 \\
\hline 4-Nitroaniline & 1600 & UGKG & $u$ & NP & & 1600 UGKG & 1 \\
\hline 4, 6-Dinitro-2-Methylphenol & 1600 & URER & u & NP & & 1600 UG/KG & 1 \\
\hline N-Nitrosodiphenylamine & 330 & UGKG & $u$ & NP & & 330 UG/KG & 1 \\
\hline 4-Bromophenyl phenyl ether & 330 & UGKG & $u$ & NP & & 330 UGKG & 1 \\
\hline Hexachlorobenzene & 330 & UGKG & $u$ & NP & & 330 UG/KG & 1 \\
\hline Pentachiorophenol & 1600 & UGKG & $u$ & NP & & 1600 UaKG & 1 \\
\hline Phenanthrene & 330 & UG/KG & $u$ & NP & & 330 UG/KG & 1 \\
\hline
\end{tabular}


Appendix D: Results of Semivolatile Organic Compound Analyses, Salmon Site

\begin{tabular}{|c|c|c|c|c|c|c|c|c|}
\hline COMPOUND & RESULTS & UNITS & $\begin{array}{l}\text { DATA } \\
\text { QUAL }\end{array}$ & $\begin{array}{l}\text { DATA } \\
\text { VALID. }\end{array}$ & NOTES & CADL & UNITS & DILUTION \\
\hline \multicolumn{9}{|l|}{ TR6-ES-485-590-6 } \\
\hline Anthracene & 330 & UG/KG & $u$ & NP & & & UG/KG & 1 \\
\hline Di-N-Butylphthalate & 330 & UG/KG & $u$ & NP & & & UG/KG & 1 \\
\hline Fluoranthene & 330 & UG/KG & $u$ & NP & & & UG/KG & 1 \\
\hline Pyrene & 330 & UG/KG & $u$ & NP & & & U UG/KG & 1 \\
\hline Butyl Benzyl Phthalate & 330 & UG/KG & $u$ & NP & & & O UG/KG & 1 \\
\hline 3, 3'-Dichlorobenzidine & 660 & UG/KG & $u$ & NP & & & O UG/KG & 1 \\
\hline Benzo(a)Anthracene & 330 & UG/KG & $u$ & NP & & & UG/KG & 1 \\
\hline Chrysene & 330 & UG/KG & $u$ & NP & & & UG/KG & 1 \\
\hline bis(2-Ethylhexyl)phthalate & 330 & UG/KG & $u$ & NP & & & 0 UG/KG & 1 \\
\hline di-N-Octyl Phthalate & 330 & UG/KG & $u$ & NP & & & 0 UG/KG & 1 \\
\hline Benzo(b)Fluoranthene & 330 & UG/KG & $u$ & NP & & & O UG/KG & 1 \\
\hline Benzo(k)Fluoranthene & 330 & UG/KG & $u$ & NP & & & 0 UG/KG & 1 \\
\hline Benzo(a)Pyrene & 330 & UG/KG & $u$ & NP & & & UG/KG & 1 \\
\hline Indeno(1,2,3-CD)Pyrene & 330 & UG/KG & $u$ & NP & & & UG/KG & 1 \\
\hline Dibenzo(a.h)Anthracene & 330 & UG/KG & $u$ & NP & & & UG/KG & $?$ \\
\hline Benzo(g,h,1)Perylene & 330 & UG/KG & $u$ & NP & & & UG/KG & 1 \\
\hline Unknown-1 & 120 & $U G / K G$ & J & NP & & & UG/KG & 1 \\
\hline Unknown-2 & 84 & UG/KG & J & NP & & & UG/KG & 1 \\
\hline Unknown-3 & 71 & UG/KG & J & NP & & & UG/KG & 1 \\
\hline Unknown-4 & 86 & $U G / K G$ & BS & NP & & & UG/KG & 1 \\
\hline Nitrobenzene-d5 & 79 & $\%$ & & & & & $\%$ & 1 \\
\hline 2-Fluorobiphenyl & 71 & $\%$ & & & & & $\%$ & 1 \\
\hline Terphenyl-d14 & 61 & $\%$ & & & & & $\%$ & 1 \\
\hline Phenol-d5 & 71 & $\%$ & & & & & $\%$ & 1 \\
\hline 2-Fluorophenol & 67 & $\%$ & & & & & $\%$ & 1 \\
\hline 2, 4, 6-Tribromophenol & 85 & $\%$ & & & & & $\%$ & 1 \\
\hline
\end{tabular}


Appendix D: Results of Semivolatile Organic Compound Analyses, Salmon Site

\begin{tabular}{|c|c|c|c|c|c|c|c|}
\hline COMPOUND & RESULTS & UNITS & $\begin{array}{l}\text { DATA } \\
\text { QLAL }\end{array}$ & $\begin{array}{l}\text { DATA } \\
\text { VALID. }\end{array}$ & NOTES & CRDL UNITS & DILUTION \\
\hline \multicolumn{8}{|l|}{ TR7-ES-558-650-5 } \\
\hline Phenol & 330 & UG/KG & $u$ & - & & 330 UG/KG & 1 \\
\hline bis(2-Chloroethyl) ether & 330 & UG/KG & $u$ & - & & 330 UG/KG & 1 \\
\hline 2-Chlorophenol & 330 & UG/KG & $u$ & - & & 330 UG/KG & 1 \\
\hline 1, 3-Dichlorobenzene & 330 & UG/KG & $u$ & -. & & 330 UG/KG & 1 \\
\hline 1, 4-Dichlorobenzene & 330 & UG/KG & $u$ & - & & 330 UG/KG & 1 \\
\hline Benzyl Alcohol & 330 & UG/KG & $u$ & - & & 330 UG/KG & 1 \\
\hline 1, 2-Dichlorobenzene & 330 & UG/KG & $u$ & - & & 330 UG/KG & 1 \\
\hline 2-Methylphenol & 330 & UGKG & $u$ & - & & 330 UG/KG & 1 \\
\hline bis(2-Chloroisopropyl) ether & 330 & UGKG & $u$ & - & & 330 UG/KG & 1 \\
\hline$\therefore$ Methylphenol & 330 & UG/KG & $u$ & - & & 330 UG/KG & 1 \\
\hline N-Nitroso-di-nPropylamine & 330 & UGKG & $u$ & ... & & 330 UG/KG & 1 \\
\hline Hexachloroethane & 330 & UG/KG & $u$ & - & & 330 UG/KG & 1 \\
\hline Nitrobenzene & 330 & UG/KG & $u$ & ... & & 330 UG/KG & 1 \\
\hline Isophorone & 330 & UG/KG & $u$ & -. & & 330 UG/KG & 1 \\
\hline 2-Nitrophenol & 330 & UG/KG & $u$ & -. & & 330 UG/KG & 1 \\
\hline 2, 4-Dimethylphenol & 330 & UG/KG & $u$ & - & & 330 UG/KG & 1 \\
\hline Benzoic Acid & 1600 & UGKG & $u$ & - & & 1600 UG/KG & 1 \\
\hline bis(2Chloroethoxy)methane & 330 & UG/KG & $u$ & - & & 330 UG/KG & 1 \\
\hline 2, 4-Dichlorophenol & 330 & UGKG & $u$ & - & & 330 UG/KG & 1 \\
\hline 1, 2, 4-Trichlorobenzene & 330 & UG/KG & $u$ & - & & 330 UG/KG & 1 \\
\hline Naphthalene & 330 & UG/KG & $u$ & - & & 330 UG/KG & 1 \\
\hline 4-Chloroaniline & 330 & UGKG & $u$ & - & & 330 UG/KG & 1 \\
\hline Hexachlorobutadiene & 330 & UG/KG & u & - & & 330 UG/KG & 1 \\
\hline 4-Chloro-3-Methylphenol & 330 & UG/KG & $u$ & - & & 330 UG/KG & 1 \\
\hline 2-Methylnaphthalene & 940 & UG/KG & & $\ldots$ & & 330 UG/KG & 1 \\
\hline Hexachlorocyclopentadiene & 330 & UG/KG & $u$ & - & & 330 UG/KG & 1 \\
\hline 2, 4, 6-Trichlorophenol & 330 & UG/KG & $u$ & - & & 330 UG/KG & 1 \\
\hline 2, 4, 5-Trichlorophenol & 1600 & UGKG & $u$ & - & & 1600 UG/KG & 1 \\
\hline 2-Chloronaphthalene & 330 & UG/KG & $u$ & -- & & 330 UG/KG & 1 \\
\hline 2-Nitroaniline & 1600 & UGKG & $u$ & - & & 1600 UG/KG & 1 \\
\hline Dimethyl Phthalate & 330 & UG/KG & u & -- & & 330 UG/KG & 1 \\
\hline Acenaphthylene & 330 & UGKG & u & - & & 330 UG/KG & 1 \\
\hline 2, 6-Dinitrotoluene & 330 & UG/KG & $u$ & -. & & 330 UG/KG & 1 \\
\hline 3-Nitroaniline & 1600 & UG/KG & $u$ & - & & 1600 UG/KG & 1 \\
\hline Acenaphthene & 330 & UG/KG & u & -.. & & 330 UG/KG & 1 \\
\hline 2, 4-Dinitrophenol & 1600 & UGKG & $u$ & - & & 1600 UG/KG & 1 \\
\hline 4-Nitrophenol & 1600 & UG/KG & u & - & & 1600 UG/KG & 1 \\
\hline Dibenzofuran & 330 & UG/KG & u & -- & & 330 UG/KG & 1 \\
\hline 2, 4-Dinitrotoluene & 330 & UGKG & u & - & & 330 UG/KG & 1 \\
\hline Diethylphthalate & 330 & UGKG & u & - & & 330 UG/KG & 1 \\
\hline 4-Chlorophenyl phenyl ether & 330 & UGKG & $u$ & .. & & 330 UG/KG & 1 \\
\hline Fluorene & 230 & UGKG & $\mathrm{J}$ & - & & 330 UG/KG & 1 \\
\hline 4-Nitroaniline & 1600 & UGKG & u & - & & 1600 UGKG & 1 \\
\hline 4, 6-Dinitro-2-Methylphenol & 1600 & UGKG & u & - & & 1600 UG/KG & 1 \\
\hline N-Nitrosodiphenylamine & 330 & UGKG & $u$ & - & & 330 UG/KG & 1 \\
\hline 4-Bromophenyl phenyl ether & 330 & UGKG & U & - & & 330 UG/KG & 1 \\
\hline Hexachlorobenzene & 330 & UGKG & U & - & & 330 UGKG & 1 \\
\hline Pentachlorophenol & 1600 & UGKG & u & - & & 1500 UGKG & 1 \\
\hline Phenanthrene & 130 & UG/KG & $\mathrm{J}$ & - & & 330 UG/KG & 1 \\
\hline
\end{tabular}


Appendix D: Results of Semivolatile Organic Compound Analyses, Salmon Site

\begin{tabular}{|c|c|c|c|c|c|c|c|c|}
\hline COMPOUND & RESULTS & UNITS & $\begin{array}{l}\text { DATA } \\
\text { QUAL }\end{array}$ & $\begin{array}{l}\text { DATA } \\
\text { VALID. }\end{array}$ & NOTES & CRDL & UNITS & DILUTION \\
\hline \multicolumn{9}{|l|}{ TR7-ES-556-650-5 (CON'T) } \\
\hline Anthracene & 330 & UG/KG & $u$ & ... & & & UG/KG & 1 \\
\hline Di-N-Butylphthalate & 330 & UG/KG & $u$ & ... & & & UG/KG & 1 \\
\hline Fluoranthene & 330 & UG/KG & $u$ & ..- & & & UG/KG & 1 \\
\hline Pyrene & 330 & UG/KG & $u$ & $\ldots$ & & & UG/KG & 1 \\
\hline Butyl Benzyl Phthalate & 330 & UG/KG & U & ... & & & UG/KG & 1 \\
\hline 3, 3'-Dichlorobenzidine & 660 & UG/KG & $u$ & $\ldots$ & & & UG/KG & 1 \\
\hline Benzo(a)Anthracene & 330 & UG/KG & $u$ & $\ldots$ & & & UG/KG & 1 \\
\hline Chrysene & 330 & UG/KG & $u$ & $\ldots$ & & & UG/KG & 1 \\
\hline bis(2-Ethylhexyl)phthalate & 330 & UG/KG & $u$ & $\ldots$ & & & UG/KG & 1 \\
\hline di-N-Cctyl Phthalate & 330 & UG/KG & $u$ & $\ldots$ & & & UG/KG & 1 \\
\hline Benzo(b)Fluoranthene & 330 & $U G / K G$ & $u$ & $\ldots$ & & & UG/KG & 1 \\
\hline Benzo(k)Fluoranthene & 330 & UG/KG & $u$ & -- & & & UG/KG & 1 \\
\hline Benzo(a)Pyrene & 330 & UG/KG & $u$ & $\ldots$ & & & UG/KG & 1 \\
\hline Indeno(1,2,3-CD)Pyrene & 330 & UG/KG & $u$ & $\ldots$ & & & UG/KG & 1 \\
\hline Dibenzo(a.h)An'hracene & 330 & UG/KG & $u$ & $\ldots$ & & & UG/KG & 1 \\
\hline Benzo(g,h,i)Perylene & 330 & UG/KG & $u$ & $\ldots$ & & & UG/KG & 1 \\
\hline Nitrobenzene-d5 & 52 & $\%$ & & & & & $\%$ & 1 \\
\hline 2-Fluorobiphenyl & 56 & $\%$ & & & & & $\%$ & 1 \\
\hline Terphenyl-d14 & 55 & $\%$ & & & & & $\%$ & 1 \\
\hline Phenol-d5 & 56 & $\%$ & & & & & $\%$ & 1 \\
\hline 2-Fluorophenol & 55 & $\%$ & & & & & $\%$ & 1 \\
\hline 2, 4, 6-Tribromophenol & 62 & $\%$ & & & & & $\%$ & 1 \\
\hline
\end{tabular}


Appendix D: Results of Semivolatile Organic Compound Analyses, Salmon Site

\begin{tabular}{|c|c|c|c|c|c|c|c|}
\hline COMPOUND & RESULTS & UNITS & $\begin{array}{l}\text { DATA } \\
\text { QUAL }\end{array}$ & $\begin{array}{l}\text { DATA } \\
\text { VALID. }\end{array}$ & NOTES & CRDL UNITS & DILUTION \\
\hline \multicolumn{8}{|l|}{ TR7-ES-560-650-14 } \\
\hline Phenol & 330 & UG/KG & $u$ & - & & 330 UG/KG & 1 \\
\hline bis(2-Chloroethyl) ether & 330 & UG/KG & $u$ & -. & & 330 UG/KG & 1 \\
\hline 2-Chlorophenol & 330 & UGKG & $u$ & $\ldots$ & & 330 UG/KG & 1 \\
\hline 1, 3-Dichlorobenzene & 330 & UG/KG & $u$ & ... & & 330 UG/KG & 1 \\
\hline 1, 4-Dichlorobenzene & 330 & UG/KG & $u$ & ... & & 330 UG/KG & 1 \\
\hline Benzyl Alcohol & 330 & UG/KG & $u$ & .. & & 330 UG/KG & 1 \\
\hline 1, 2-Dichlorobenzene & 330 & UG/KG & $u$ & ... & & 330 UG/KG & 1 \\
\hline 2-Methylphenol & 330 & UG/KG & $u$ & $\ldots$ & & 330 UG/KG & 1 \\
\hline bis(2-Chloroisopropyl) ether & 330 & UG/KG & $u$ & .- & & $330 \mathrm{UG} / \mathrm{KG}$ & 1 \\
\hline 4-Methylphenol & 330 & UG/KG & $u$ & .- & & 330 UG/KG & 1 \\
\hline N-Nitroso-di-nPropylamine & 330 & UG/KG & $u$ & $\ldots$ & & 330 UG/KG & 1 \\
\hline Hexachloroethane & 330 & UG/KG & $u$ & $\ldots$ & & 330 UG/KG & 1 \\
\hline Nitrobenzene & 330 & UG/KG & $u$ & -. & & 330 UG/KG & 1 \\
\hline Isophorone & 330 & UG/KG & $u$ & $\ldots$ & & 330 UG/KG & 1 \\
\hline 2-Nitrophenol & 330 & UG/KG & $u$ & ... & & 330 UG/KG & 1 \\
\hline 2, 4-Dimethylphenol & 330 & UG/KG & $u$ & $\ldots$ & & 330 UG/KG & 1 \\
\hline Benzoic Acid & 1600 & UG/KG & $u$ & ... & & 1600 UG/KG & 1 \\
\hline bis(2Chloroethoxy)methane & 330 & UG/KG & $u$ & ... & & $330 \mathrm{UG} / \mathrm{KG}$ & 1 \\
\hline 2, 4-Dichlorophenol & 330 & UG/KG & $u$ & -. & & 330 UG/KG & 1 \\
\hline 1, 2, 4-Trichlorobenzene & 330 & UG/KG & $u$ & -. & & 330 UG/KG & 1 \\
\hline Napinthalene & 330 & UG/KG & $u$ & -. & & 330 UG/KG & 1 \\
\hline 4-Chloroaniline & 330 & UG/KG & $u$ & $\ldots$ & & 330 UG/KG & 1 \\
\hline Hexachlorobutadiene & 330 & $U G / K G$ & $u$ & -- & & 330 UG/KG & 1 \\
\hline 4-Chloro-3-Methylphenol & 330 & UG/KG & $u$ & - & & 330 UG/KG & 1 \\
\hline 2-Methylnaphthalene & 330 & UG/KG & $u$ & $\ldots$ & & $330 \mathrm{UG} / \mathrm{KG}$ & 1 \\
\hline Hexachlorocyclopentadiene & 330 & $U G / K G$ & $u$ & $\ldots$ & & 330 UG/KG & 1 \\
\hline 2, 4, 6-Trichlorophenol & 330 & UG/KG & $u$ & -. & & 330 UG/KG & 1 \\
\hline 2, 4, 5-Trichlorophenol & 1600 & UG/KG & $u$ & $\ldots$ & & 1600 UG/KG & 1 \\
\hline 2-Chloronaphthalene & 330 & $U G / K G$ & $u$ & $\ldots$ & & 330 UG/KG & 1 \\
\hline 2-Nitroaniline & 1600 & UG/KG & $u$ & ... & & 1600 UG/KG & 1 \\
\hline Dimethyl Phthalate & 330 & UG/KG & $u$ & $\ldots$ & & 330 UG/KG & 1 \\
\hline Acenaphthylene & 330 & UGKG & $u$ & $\ldots$ & & 330 UG/KG & 1 \\
\hline 2, 6-Dinitrotoluene & 330 & UG/KG & $u$ & $\ldots$ & & $330 \mathrm{UG} / \mathrm{KG}$ & 1 \\
\hline 3-Nitroaniline & 1600 & UG/KG & $u$ & $\ldots$ & & 1600 UG/KG & 1 \\
\hline Acenaphthene & 330 & UG/KG & $u$ & $\ldots$ & & 330 UG/KG & 1 \\
\hline 2, 4-Dinitrophenol & 1600 & UG/KG & $u$ & -- & & 1600 UG/KG & 1 \\
\hline 4-Nitrophenol & 1600 & UGKG & $u$ & $\ldots$ & & 1600 UG/KG & 1 \\
\hline Dibenzofuran & 330 & UG/KG & $u$ & -.. & & 330 UG/KG & 1 \\
\hline 2, 4-Dinitrotoluene & 330 & UG/KG & $u$ & .. & & 330 UG/KG & 1 \\
\hline Diethylphthalate & 330 & UG/KG & $u$ & -. & & 330 UG/KG & 1 \\
\hline 4-Chlorophenyl phenyl ether & 330 & UG/KG & $u$ & .- & & 330 UG/KG & 1 \\
\hline Fluorene & 330 & UGKG & $u$ & - & & 330 UG/KG & 1 \\
\hline 4-Nitroaniline & 1600 & UGKG & $u$ & - & & 1600 UG/KG & 1 \\
\hline 4, 6-Dinitro-2-Methylphenol & 1600 & UG/KG & $u$ & R & A & 1600 UG/KG & 1 \\
\hline N-Nitrosodiphenylamine & 330 & UG/KG & $u$ & $\mathbf{R}$ & A & 330 UG/KG & 1 \\
\hline 4-Bromophenyl phenyl ether & 330 & UGKG & $u$ & R & A & 330 UG/KG & 1 \\
\hline Hexachlorobenzene & 330 & UGKG & $u$ & A & A & 330 UG/KG & 1 \\
\hline Pentachlcrophenol & 1600 & UGKG & $u$ & R & A & 1600 UG/KG & 1 \\
\hline Phenanthrene & 330 & UG/KG & $u$ & $\mathbf{R}$ & A & 330 UG/KG & 1 \\
\hline
\end{tabular}


Appendix D: Results of Semivolatile Organic Compound Analyses. Salmon Site

\begin{tabular}{|c|c|c|c|c|c|c|c|c|}
\hline COMPOUND & RESULTS & UNITS & $\begin{array}{l}\text { DATA } \\
\text { QUAL }\end{array}$ & $\begin{array}{l}\text { DA } T A \\
\text { VALID. }\end{array}$ & NOTES & CRDL & UNITS & DILUTION \\
\hline \multicolumn{9}{|l|}{ TR7-ES-560-650-14 (CON'T) } \\
\hline Anthracene & 330 & UG/KG & u & R & A & & $U G / K G$ & 1 \\
\hline Di-N-Butylphthalate & 330 & UG/KG & $u$ & A & A & & $\cup G / K G$ & 1 \\
\hline Fluoranthene & 330 & UG/KG & $u$ & R & A & & UG/KG & 1 \\
\hline Pyrene & 330 & UG/KG & $u$ & A & A & & UG/KG & 1 \\
\hline Butyl Benzyl Phthalate & 330 & UG/KG & $u$ & $R$ & A & & UG/KG & 1 \\
\hline 3, 3'-Dichlorobenzidine & 660 & UG/KG & $u$ & $\mathbf{R}$ & A & & UG/KG & 1 \\
\hline Benzo(a)Anthracene & 330 & UG/KG & $u$ & R & $A$ & & UG/KG & 1 \\
\hline Chrysene & 330 & UG/KG & $u$ & R & A & & UG/KG & 1 \\
\hline bis(2-Ethylhexyl)phthalate & 2100 & UG/KG & & R & A & & UG/KG & 1 \\
\hline di-N-Octyl Phthaiate & 330 & UG/KG & $u$ & R & & & $O U G / K G$ & 1 \\
\hline Benzo(b)Fluoranthene & 330 & UG/KG & $u$ & R & & & $O U G / K G$ & 1 \\
\hline Benzo(k)Fluoranthene & 330 & UG/KG & $u$ & R & & & O UG/KG & 1 \\
\hline Benzo(a)Pyrene & 330 & UG/KG & $u$ & R & & & $O$ UG/KG & 1 \\
\hline Indeno(1,2,3-CD)Pyrene & 330 & UG/KG & $u$ & A & & & O UG/KG & 1 \\
\hline Dibenzo(a.h)Anthracene & 330 & $U G / K G$ & $u$ & R & & & O UG/KG & 1 \\
\hline Benzo(g,h,i)Perylene & 330 & $U G / K G$ & $u$ & $R$ & & & O UG/KG & 1 \\
\hline Nitrobenzene-d5 & 67 & $\%$ & & & & & $\%$ & 1 \\
\hline 2-Fluorobiphenyl & 99 & $\%$ & & & & & $\%$ & 1 \\
\hline Terphenyl-d14 & 75 & $\%$ & & & & & $\%$ & 1 \\
\hline Phenol-d5 & 56 & $\%$ & & & & & $\%$ & 1 \\
\hline 2-Fluorophenol & 57 & $\%$ & & & & & $\%$ & 1 \\
\hline 2, 4, 6-Tribromophenol & 84 & $\%$ & & & & & $\%$ & 1 \\
\hline
\end{tabular}

NOTE A: Results of analyses for these compounds are unuseable: Use the results from the re-run sample (TR7-ES-560-650-14 RE) 


\begin{tabular}{|c|c|c|c|c|c|c|c|}
\hline COMPOUND & RESULTS & UNITS & $\begin{array}{l}\text { DATA } \\
\text { QUAL } \\
\end{array}$ & $\begin{array}{l}\text { DATA } \\
\text { VALID. }\end{array}$ & NOTES & CRDL UNITS & DILUTION \\
\hline \multicolumn{8}{|l|}{ TA7-ES-560-650-14 RE } \\
\hline Phenol & 330 & UG/KG & $u$ & R & & 330 UG/KG & 1 \\
\hline bis(2-Chloroethyl) ether & 330 & UG/KG & $u$ & R & & 330 UG/KG & 1 \\
\hline 2-Chlorophenol & 330 & UG/KG & $u$ & R & & 330 UG/KG & 1 \\
\hline 1, 3-Dichlorobenzene & 330 & UG/KG & $u$ & R & & 330 UG/KG & 1 \\
\hline 1, 4-Dichlorobenzene & 330 & $U G / K G$ & $u$ & R & & 330 UG/KG & 1 \\
\hline Benzyl Alcohol & 330 & UG/KG & $u$ & $\mathbf{R}$ & & 330 UG/KG & 1 \\
\hline 1, 2-Dichlorobenzene & 330 & UG/KG & $u$ & R & & 330 UG/KG & 1 \\
\hline 2-Methylphenol & 330 & UG/KG & $u$ & R & & 330 UG/KG & 1 \\
\hline bis(2-Chloroisopropyl) ether & 330 & UG/KG & $u$ & R & & 330 UG/KG & 1 \\
\hline 4-Methylphenol & 330 & UG/KG & $u$ & R & & 330 UG/KG & 1 \\
\hline N-Nitroso-di-nPropylamine & 330 & UG/KG & $u$ & R & & 330 UG/KG & 1 \\
\hline Hexachloroethane & 330 & UG/KG & u & R & & 330 UG/KG & 1 \\
\hline Nitrobenzene & 330 & UG/KG & $u$ & A & & 330 UG/KG & 1 \\
\hline Isophorone & 330 & UG/KG & $u$ & R & & 330 UG/KG & 1 \\
\hline 2-Nitrophenol & 330 & UG/KG & $u$ & R & & 330 UG/KG & 1 \\
\hline 2, 4-Dimethylphenol & 330 & UG/KG & $u$ & R & & 330 UG/KG & 1 \\
\hline Benzoic Acid & 1600 & UG/KG & ن & R & & 1600 UG/KG & 1 \\
\hline bis(2Chloroethoxy)methane & 330 & UG/KG & $u$ & R & & 330 UG/KG & 1 \\
\hline 2, 4-Dichlorophenol & 330 & UG/KG & $u$ & ค & & 330 UG/KG & 1 \\
\hline 1, 2, 4-Trichiorobenzene & 330 & UG/KG & $u$ & R & & 330 UG/KG & 1 \\
\hline Naphthalene & 330 & UG/KG & $u$ & R & & 330 UG/KG & 1 \\
\hline 4-Chloroaniline & 330 & UG/KG & $u$ & R & & 330 UG/KG & 1 \\
\hline Hexachlorobutadiene & 330 & UG/KG & $u$ & R & & 330 UG/KG & 1 \\
\hline 4-Chloro-3-Methylphenol & 330 & UG/KG & $u$ & R & & 330 UG/KG & 1 \\
\hline 2-Methyinaphthalene & 330 & UG/KG & $u$ & R & & 330 UG/KG & 1 \\
\hline Hexachlorocyclopentadiene & 330 & $U G / K G$ & $u$ & R & & 330 UG/KG & 1 \\
\hline 2, 4, 6-Trichlorophenol & 330 & UG/KG & $u$ & $\mathbf{R}$ & & 330 UG/KG & 1 \\
\hline 2, 4, 5-Trichlorophenol & 1600 & UG/KG & $u$ & R & & 1600 UG/KG & 1 \\
\hline 2-Chloronaphthalene & 330 & UG/KG & $u$ & R & & 330 UG/KG & 1 \\
\hline 2-Nitroaniline & 1600 & UG/KG & $u$ & $\mathbf{R}$ & & 1600 UG/KG & 1 \\
\hline Dimethyl Phthalate & 330 & UG/KG & $u$ & R & & 330 UG/KG & 1 \\
\hline Acenar nthylene & 330 & UG/KG & $u$ & R & & 330 UG/KG & 1 \\
\hline 2, 6-Dinitrotoluene & 330 & UG/KG & $u$ & R & & 330 UG/KG & 1 \\
\hline 3-Nitroaniline & 1600 & UG/KG & $u$ & $\mathbf{R}$ & & 1600 UG/KG & 1 \\
\hline Acenaphthene & 330 & UG/KG & $u$ & A & & 330 UG/KG & 1 \\
\hline 2, 4-Dinitrophenol & 1600 & UG/KG & $u$ & $\mathbf{R}$ & & 1600 UG/KG & 1 \\
\hline 4-Nitrophenol & 1600 & UG/KG & $u$ & R & & 1600 UG/KG & 1 \\
\hline Dibenzofuran & 330 & UG/KG & $u$ & A & & 330 UG/KG & 1 \\
\hline 2, 4-Dinitrotoluene & 330 & UGKG & $u$ & ค & & 330 UG/KG & 1 \\
\hline Diethylphthalate & 330 & UG/KG & $u$ & R & & 330 UG/KG & 1 \\
\hline 4-Chlorophenyl phenyl ether & 330 & UG/KG & $u$ & R & & 330 UG/KG & 1 \\
\hline Fluorene & 330 & UG/KG & $u$ & A & & 330 UG/KG & 1 \\
\hline 4-Nitroanii.. 9 & 1600 & UGKG & $u$ & A & & 1600 UG/KG & 1 \\
\hline 4, 6-Dinitro-2-Methylphenol & 1600 & UGKG & $u$ & $\ldots$ & B & 1600 UG/KG & 1 \\
\hline N-Nitrosodiphenylamine & 330 & UG/KG & $u$ & - & B & 330 UG/KG & 1 \\
\hline 4-Bromophenyl phenyl ether & 330 & UG/KG & $u$ & $\ldots$ & B & 330 UG/KG & 1 \\
\hline Hexachlorobenzene & 330 & UGKG & $u$ & - & B & 330 UG/KG & 1 \\
\hline Pentachlorophenol & 1600 & UGKG & $u$ & - & 8 & 1600 UG/KG & 1 \\
\hline Phenanthrene & 330 & UG/KG & $u$ & $\ldots$ & $B$ & 330 UG/KG & 1 \\
\hline
\end{tabular}


Appendix D: Results of Semivolatile Organic Compound Analyses, Salmon Site

\begin{tabular}{|c|c|c|c|c|c|c|c|c|}
\hline COMPOUND & RESULTS & UNITS & $\begin{array}{l}\text { DATA } \\
\text { QUAL }\end{array}$ & $\begin{array}{l}\text { DATA } \\
\text { VALID. }\end{array}$ & NOTES & CRDL & UNITS & DILUTIOA \\
\hline \multicolumn{9}{|c|}{ TR7-ES-560-650-14 RE (CON'T) } \\
\hline Anthracene & 330 & UG/KG & $u$ & $\ldots$ & $B$ & & UG/KG & 1 \\
\hline Di-N.Butylphthalate & 330 & UG/KG & $u$ & ... & $B$ & & UG/KG & 1 \\
\hline Fluoranthene & 330 & UG/KG & $u$ & ... & B & & UG/KG & 1 \\
\hline Pyrene & 330 & UG/KG & $u$ & $\ldots$ & B & & UG/KG & 1 \\
\hline Butyl Benzyl Phthalate & 330 & UG/KG & $u$ & ... & $B$ & & UG/KG & 1 \\
\hline 3. 3'-Dichlorobenzidine & 660 & UG/KG & $u$ & $\ldots$ & $B$ & & UG/KG & 1 \\
\hline Benzo(a)Anthracene & 330 & UG/KG & $u$ & ... & B & & UG/KG & 1 \\
\hline Chrysene & 330 & UG/KG & $u$ & $\ldots$ & $B$ & & O UG/KG & 1 \\
\hline bis(2-Ethylhexyl)phthalate & 2000 & UG/KG & & .. & $B$ & & UG/KG & 1 \\
\hline di-N-Octyl Phthalate & 330 & UG/KG & $u$ & R & & & O UG/KG & 1 \\
\hline Benzo(b)Fluoranthene & 330 & UG/KG & $u$ & R & & & O UG/KG & 1 \\
\hline Benzo(k)Fluoranthene & 330 & UG/KG & $u$ & A & & & O UG/KG & 1 \\
\hline Benzo(a)Pyrene & 330 & UG/KG & $u$ & $R$ & & & O UG/KG & 1 \\
\hline Indeno(1,2,3-CD)Pyrene & 330 & UG/KG & $u$ & A & & & O UG/KG & 1 \\
\hline Dibenzo(a.h)Anthracene & 330 & UG/KG & $u$ & R & & & 0 UG/KG & 1 \\
\hline Benzo(g,h,i)Perylene & 330 & UG/KG & $u$ & A & & & O UG/KG & 1 \\
\hline Nitrobenzene-d5 & 65 & $\%$ & & & & & $\%$ & 1 \\
\hline 2-Fluorobiphenyl & 82 & $\%$ & & & & & $\%$ & 1 \\
\hline Terphenyl-d14 & 70 & $\%$ & & & & & $\%$ & 1 \\
\hline Phenol-d5 & 64 & $\%$ & & & & & $\%$ & 1 \\
\hline 2-Fluorophenol & 54 & $\%$ & & & & & $\%$ & 1 \\
\hline 2, 4, 6-Tribromophenol & 73 & $\%$ & & & & & $\%$ & 1 \\
\hline
\end{tabular}

NOTE A: Results of analyses for these compounds are unuseable: Use the results from the original sample (TR7-ES-560-650-14) 
Appendix D: Results of Semivolatile Organic Compound Analyses. Salmon Site

\begin{tabular}{|c|c|c|c|c|c|c|c|}
\hline COMPOUND & RESULTS & UNITS & $\begin{array}{l}\text { DATA } \\
\text { QUAL }\end{array}$ & $\begin{array}{l}\text { DATA } \\
\text { VALID. }\end{array}$ & NOTES & CRDL UNITS & DILUTION \\
\hline \multicolumn{8}{|l|}{ TR8-ES-780-600-3 } \\
\hline Phenol & 330 & UG/KG & $u$ & ... & & 330 UG/KG & 1 \\
\hline bis(2-Chloroethyl) ether & 330 & UG/KG & $u$ & ... & & 330 UG/KG & 1 \\
\hline 2-Chlorophenol & 330 & UG/KG & $u$ & ... & & 330 UG/KG & 1 \\
\hline 1. 3-Dichlorobenzene & 330 & UG/KG & $u$ & $\ldots$ & & 330 UG/KG & 1 \\
\hline 1. 4-Dichlorobenzene & 330 & UG/KG & $u$ & $\ldots$ & & 330 UG/KG & 1 \\
\hline Benzyl Alcohol & 330 & $U G / K G$ & $u$ & $\ldots$ & & 330 UG/KG & 1 \\
\hline 1.2-Dichlorobenzene & 330 & UG/KG & $u$ & $\ldots$ & & $330 \mathrm{UG} / \mathrm{KG}$ & 1 \\
\hline 2-Methylphenol & 330 & $U G / K G$ & $u$ & ... & & 330 UG/KG & 1 \\
\hline bis(2-Chloroisopropyl) ether & 330 & UG/KG & $u$ & $\ldots$ & & 330 UG/KG & 1 \\
\hline 4-Methylphenol & 330 & UG/KG & $u$ & $\ldots$ & & 330 UG/KG & 1 \\
\hline N-Nitroso-di-nPropylamine & 330 & $U G / K G$ & $u$ & $\ldots$ & & 330 UG/KG & 1 \\
\hline Hexachloroethane & 330 & UGKG & $u$ & $\ldots$ & & 330 UG/KG & 1 \\
\hline Nitrobenzene & 330 & UG/KG & $u$ & $\ldots$ & & 330 UG/KG & 1 \\
\hline Isophorone & 330 & UG/KG & $u$ & $\ldots$ & & 330 UG/KG & 1 \\
\hline 2-Nitrophenol & 330 & UG/KG & $v$ & $\ldots$ & & 330 UG/KG & 1 \\
\hline 2. 4-Dimethylphenol & 330 & $U G / K G$ & $u$ & $\ldots$ & & 330 UG/KG & 1 \\
\hline Benzoic Acid & 73 & UG/KG & J & $\ldots$ & & 1600 UG/KG & 1 \\
\hline bis(2Chloroethoxy)methane & 330 & UG/KG & $u$ & $\ldots$ & & 330 UG/KG & 1 \\
\hline 2, 4-Dichlorophenol & 330 & UG/KG & 11 & $\ldots$ & & 330 UG/KG & 1 \\
\hline 1, 2, 4-Trichlorobenzene & 330 & UG/KG & $u$ & $\ldots$ & & 330 UG/KG & 1 \\
\hline Naphthalene & 330 & UG/KG & $u$ & $\ldots$ & & 330 UG/KG & 1 \\
\hline 4-Chloroaniline & 330 & UG/KG & $u$ & $\ldots$ & & 330 UG/KG & 1 \\
\hline Hexachlorobutadiene & 330 & UG/KG & $u$ & $\cdots$ & & 330 UG/KG & 1 \\
\hline 4-Chloro-3-Methylphenol & 330 & UG/KG & $u$ & $\cdots$ & & 330 UG/KG & 1 \\
\hline 2-Methylnaphthalene & 330 & $U G / K G$ & $u$ & $\ldots$ & & 330 UG/KG & 1 \\
\hline Hexachlorocyclopentadiene & 330 & UG/KG & $u$ & $\ldots$ & & 330 UG/KG & 1 \\
\hline 2, 4, 6-Trichlorophenol & 330 & UG/KG & $u$ & $\ldots$ & & 330 UG/KG & 1 \\
\hline 2. 4,5-Trichlorophenol & 1600 & UG/KG & $u$ & $\ldots$ & & 1600 UG/KG & 1 \\
\hline 2-Chloronaphthalene & 330 & UG/KG & $u$ & $\ldots$ & & 330 UG/KG & 1 \\
\hline 2-Nitroaniline & 1600 & UGKG & $u$ & ... & & 1600 UG/KG & 1 \\
\hline Dimethyl Phthalate & 330 & $U G / K G$ & $u$ & $\ldots$ & & 330 UG/KG & 1 \\
\hline Acenaphthylene & 330 & $U G / K G$ & $u$ & $\ldots$ & & 330 UG/KG & 1 \\
\hline 2, 6-Dinitrotoluene & 330 & UG/KG & $u$ & $\ldots$ & & $330 \mathrm{UG} / \mathrm{KG}$ & 1 \\
\hline 3-Nitroaniline & 1600 & UG/KG & $u$ & $\ldots$ & & 1600 UG/KG & 1 \\
\hline Acenaphthene & 330 & UG/KG & $u$ & $\ldots$ & & 330 UG/KG & 1 \\
\hline 2, 4-Dinitrophenol & 1600 & UG/KG & $u$ & $\ldots$ & & $1600 \cup G / K G$ & 1 \\
\hline 4-Nitrophenol & 1600 & UG/KG & $u$ & $\ldots$ & & 1600 UG/KG & 1 \\
\hline Dibenzofuran & 330 & UG/KG & $u$ & ... & & 330 UG/KG & 1 \\
\hline 2, 4-Dinitrotoluene & 330 & UG/KG & $u$ & $\ldots$ & & $330 \mathrm{UG} / \mathrm{KG}$ & 1 \\
\hline Diethylphthalate & 330 & UG/KG & $u$ & $\ldots$ & & $330 \mathrm{UG} / \mathrm{KG}$ & 1 \\
\hline 4-Chlorophenyl phenyl ether & 330 & $U G / K G$ & $u$ & $\ldots$ & & 330 UG/KG & 1 \\
\hline Fluorene & 330 & UG/KG & $u$ & $\ldots$ & & 330 UG/KG & 1 \\
\hline 4-Nitroaniline & 1600 & UG/KG & $u$ & $\ldots$ & & 1600 UG/KG & 1 \\
\hline 4, 6-Dinitro-2-Methylphenol & 1600 & UG/KG & $u$ & ... & & 1600 UG/KG & 1 \\
\hline N-Nitrosodiphenylamine & 330 & UG/KG & $u$ & $\ldots$ & & 330 UG/KG & 1 \\
\hline 4-Bromophenyl phenyl ether & 330 & UG/KG & $u$ & $\ldots$ & & 330 UG/KG & 1 \\
\hline Hexachlorobenzene & 330 & UG/KG & $u$ & $\ldots$ & & 330 UG/KG & 1 \\
\hline Pentachlorophenol & 1600 & UG/KG & $u$ & $\ldots$ & & 1600 UG/KG & 1 \\
\hline Phenanthrene & 330 & UG/KG & $u$ & $\ldots$ & & 330 UG/KG & 1 \\
\hline
\end{tabular}


Appendix D: Results of Semivolatile Organic Compound Analyses, Salmon Site

\begin{tabular}{|c|c|c|c|c|c|c|c|c|}
\hline COMPOUND & RESULTS & UNITS & $\begin{array}{l}\text { DATA } \\
\text { QUAL }\end{array}$ & $\begin{array}{l}\text { DATA } \\
\text { VALID. }\end{array}$ & NOTES & CRDL & UNITS & DILUTION \\
\hline \multicolumn{9}{|l|}{ TR8-ES-780-600-3 (CON'T) } \\
\hline Anthracene & 330 & UG/KG & $u$ & $\ldots$ & & & UG/KG & 1 \\
\hline Di-N-Butviphthalate & 330 & UG/KG & $u$ & $\ldots$ & & & UG/KG & 1 \\
\hline Fluoranthene & 330 & UG/KG & $u$ & ... & & & UG/KG & 1 \\
\hline Pyrene & 330 & UG/KG & $u$ & $\ldots$ & & & UG/KG & 1 \\
\hline Butyl Benzyl Phthalate & 330 & $U G / K G$ & $u$ & $\ldots$ & & & UG/KG & 1 \\
\hline 3. 3'-Dichlorobenzidine & 660 & UG/KG & $u$ & $\ldots$ & & & UG/KG & 1 \\
\hline Benzo(a)Anthracene & 330 & UG/KG & $u$ & $\ldots$ & & & UG/KG & 1 \\
\hline Chrysene & 330 & $U G / K G$ & $u$ & $\ldots$ & & & UG/KG & 1 \\
\hline bis(2-Ethylhexyl)phthalate & 330 & $U G / K G$ & $u$ & ... & & & UG/KG & 1 \\
\hline di-N-Octyl Phthalate & 330 & UG/KG & $u$ & .. & & & UG/KG & 1 \\
\hline Benzo(b)Fluoranthene & 330 & $U G / K G$ & $u$ & ... & & & UG/KG & 1 \\
\hline Benzo(k)Fluoranthene & 330 & $U G / K G$ & $u$ & - & & & UG/KG & 1 \\
\hline Benzo(a)Pyrene & 330 & UG/KG & $u$ & ... & & & UG/KG & 1 \\
\hline li ideno(1,2,3-CD)Pyrene & 330 & UG/KG & $u$ & ... & & & UG/KG & 1 \\
\hline Dibenzola.h)Anthracene & 330 & UG/KG & $u$ & ... & & & UR/KG & 1 \\
\hline Benzo(g,h,i)Perylene & 330 & UG/KG & $u$ & ... & & & UG/KG & 1 \\
\hline Nitrobenzene-d5 & 58 & $\%$ & & & & & $\%$ & 1 \\
\hline 2-Fluorobiphenyl & 57 & $\%$ & & & & & $\%$ & 1 \\
\hline Terphenyl-d14 & 57 & $\%$ & & & & & $\%$ & 1 \\
\hline Phenol-d5 & 59 & $\%$ & & & & & $\%$ & 1 \\
\hline 2-Fluorophenol & 57 & $\%$ & & & & & $\%$ & 1 \\
\hline 2, 4, 6-Tribromophenol & 66 & $\%$ & & & & & $\%$ & 1 \\
\hline
\end{tabular}


Appendix D: Results of Semivolatile Organic Compound Analyses. Salmon Site

\begin{tabular}{|c|c|c|c|c|c|c|c|c|}
\hline COMPOUND & RESULTS & UNITS & $\begin{array}{l}\text { DATA } \\
\text { QUAL }\end{array}$ & $\begin{array}{l}\text { DATA } \\
\text { VALID. }\end{array}$ & NOTES & CRDL & UNITS & DILUTION \\
\hline \multicolumn{9}{|l|}{ TR8-ES-780-600-3-MS } \\
\hline Phenol & 55 & $\%$ & & NP & & & $\%$ & 1 \\
\hline 2-Chlorophenol & 70 & $\%$ & & NP & & & $\%$ & 1 \\
\hline 1. 4-Dichlorobenzene & 62 & $\%$ & & NP & & & $\%$ & 1 \\
\hline N-Nitroso-di-nPropylamıne & 62 & $\%$ & & NP & & & $\%$ & 1 \\
\hline 1, 2, 4-Trichlorobenzene & 67 & $\%$ & & NP & & & $\%$ & 1 \\
\hline 4-Chloro-3-Methylphenol & 65 & $\%$ & & NP & & & $\%$ & 1 \\
\hline Acenaphthene & 64 & $\%$ & & NP & & & $\%$ & 1 \\
\hline 4-Nitrophenol & 85 & $\%$ & & NP & & & $\%$ & 1 \\
\hline 2. 4-Dinitrotoluene & 76 & $\%$ & & NP & & & $\%$ & 1 \\
\hline Pentachlorophenol & 82 & $\%$ & & NP & & & $\%$ & 1 \\
\hline Pyrene & 65 & $\%$ & & NP & & & $\%$ & 1 \\
\hline Nitrobenzene-d5 & 68 & $\%$ & & NP & & & $\%$ & 1 \\
\hline 2.Fluorobiphenyl & 67 & $\%$ & & NP & & & $\%$ & 1 \\
\hline Terphenyl-d14 & 64 & $\%$ & & NP & & & $\%$ & 1 \\
\hline Phenol-d5 & 67 & $\%$ & & NP & & & $\%$ & 1 \\
\hline 2-Fluorophenol & 69 & $\%$ & & NP & & & $\%$ & 1 \\
\hline 2, 4, 6-Tribromophenol & 76 & $\%$ & & NP & & & $\%$ & 1 \\
\hline \multicolumn{9}{|l|}{ TR8-ES-780-600-3-MSD } \\
\hline Phenol & 48 & $\%$ & & NP & & & $\%$ & 1 \\
\hline 2-Chlorophenol & 59 & $\%$ & & NP & & & $\%$ & 1 \\
\hline 1, 4-Dichlorobenzene & 53 & $\%$ & & NP & & & $\%$ & 1 \\
\hline N-Nitroso-di-nPropylamine & 51 & $\%$ & & NP & & & $\%$ & 1 \\
\hline 1, 2, 4-Trichlorobenzene & 59 & $\%$ & & NP & & & $\%$ & 1 \\
\hline 4-Chloro-3-Methylphenol & 57 & $\%$ & & NP & & & $\%$ & 1 \\
\hline Acenaphthene & 55 & $\%$ & & NP & & & $\%$ & 1 \\
\hline 4-Nitrophenol & 72 & $\%$ & & NP & & & $\%$ & 1 \\
\hline 2, 4-Dinitrotoluene & 66 & $\%$ & & NP & & & $\%$ & 1 \\
\hline Pentachlorophenol & 74 & $\%$ & & NP & & & $\%$ & 1 \\
\hline Pyrene & 56 & $\%$ & & NP & & & $\%$ & 1 \\
\hline Nitrobenzene-d5 & 59 & $\%$ & & NP & & & $\%$ & 1 \\
\hline 2-Fluorobiphenyl & 59 & $\%$ & & NP & & & $\%$ & 1 \\
\hline Terphenyl-d14 & 56 & $\%$ & & $\mathrm{NP}$ & & & $\%$ & 1 \\
\hline Phenol-d5 & 57 & $\%$ & & NP & & & $\%$ & 1 \\
\hline 2-Fluorophenol & 58 & $\%$ & & NP & & & $\%$ & 1 \\
\hline 2, 4,6-Tribromophenol & 67 & $\%$ & & NP & & & $\%$ & 1 \\
\hline
\end{tabular}


Appendix D: Results of Semivolatile Organic Compound Analyses, Salmon Site

\begin{tabular}{|c|c|c|c|c|c|c|c|}
\hline COMPOUND & RESULTS & UNITS & $\begin{array}{l}\text { DATA } \\
\text { QUAL }\end{array}$ & $\begin{array}{l}\text { DATA } \\
\text { VALID. }\end{array}$ & NOTES & CRDL UNITS & DILUTION \\
\hline \multicolumn{8}{|l|}{ TR10-ES-180-790-15 } \\
\hline Phenol & 330 & $J G / K G$ & $u$ & ... & & 330 UG/KG & 1 \\
\hline bis(2-Chloroethyl) ether & 330 & JG/KG & $u$ & .. & & 330 UG/KG & 1 \\
\hline 2-Chlorophenol & 330 & $J G / K G$ & $u$ & $\ldots$ & & 330 UG/KG & 1 \\
\hline 1. 3-Dichlorobenze & 330 & JG/KG & $u$ & ... & & 330 UG/KG & 1 \\
\hline 1, 4-Dichlorobenze & 330 & JG/KG & $u$ & ... & & 330 UG/KG & 1 \\
\hline Berizyl Alcohol & 330 & JG/KG & $u$ & ... & & 330 UG/KG & 1 \\
\hline 1,2-Dichlorobenzene & 330 & JG/KG & $u$ & $\ldots$ & & 330 UG/KG & 1 \\
\hline 2-Methylphenol & 330 & JG/KG & $u$ & $\ldots$ & & 330 UG/KG & 1 \\
\hline bis(2-Chloroisopropyl) ether & 330 & JG/KG & $u$ & $\ldots$ & & 330 UG/KG & 1 \\
\hline 4-Methylphenol & 330 & JG/KG & $u$ & $\ldots$ & & 330 UG/KG & 1 \\
\hline N-Nitroso-Di-npropylamine & 330 & UG/KG & $u$ & ... & & 330 UG/KG & 1 \\
\hline Hexachloroethane & 330 & JG/KG & $u$ & .. & & 330 UG/KG & 1 \\
\hline Nitrobenzene & 330 & UG/KG & $u$ & $\ldots$ & & 330 UG/KG & 1 \\
\hline Isophorone & 330 & UG/KG & $u$ & $\ldots$ & & 330 UG/KG & 1 \\
\hline 2-Nitrophenol & 330 & UG/KG & $u$ & $\ldots$ & & 330 UG/KG & 1 \\
\hline 2. 4-Dimethylphenol & 330 & UG/KG & $u$ & $\ldots$ & & 330 UG/KG & 1 \\
\hline Benzoic Acid & 1600 & UG/KG & $u$ & $\ldots$ & & 1600 UG/KG & 1 \\
\hline bis(2Chloroethoxy)methane & 330 & UG/KG & $u$ & $\ldots$ & & 330 UG/KG & 1 \\
\hline 2, 4-Dichlorophenol & 330 & UG/KG & $u$ & $\ldots$ & & 330 UG/KG & 1 \\
\hline 1,2, 4-Trichlorobenzene & 330 & UG/KG & $u$ & $\ldots$ & & 330 UG/KG & 1 \\
\hline Naphthalene & 330 & UG/KG & $u$ & $\ldots$ & & 330 UG/KG & 1 \\
\hline 4-Chloroaniline & 330 & UG/KG & $u$ & $\ldots$ & & 330 UG/KG & 1 \\
\hline Hexachlorobutadiene & 330 & UG/KG & $u$ & ... & & 330 UG/KG & 1 \\
\hline 4-Chloro-3-Methylphenol & 330 & UG/KG & $u$ & $\ldots$ & & 330 UG/KG & 1 \\
\hline 2-Methylnaphthalene & & UG/KG & $\mathrm{J}$ & $\ldots$ & & 330 UG/KG & 1 \\
\hline Hexachlorocyclopentadiene & 330 & UG/KG & u & $\ldots$ & & 330 UG/KG & 1 \\
\hline 2, 4, 6-Trichlorophenol & 330 & UG/KG & u & $\ldots$ & & 330 UGiKG & 1 \\
\hline 2, 4, 5-Trichlorophenol & 1600 & UG/KG & $u$ & .. & & 1600 UG/KG & 1 \\
\hline 2-Chloronaphthalene & 330 & UG/KG & u & $\ldots$ & & 330 UG/KG & 1 \\
\hline 2-Nitroaniline & 1600 & UG/KG & $u$ & $\ldots$ & & 1600 UG/KG & 1 \\
\hline Dimethyl Phthalate & 330 & UG/KG & $u$ & $\ldots$ & & $330 \mathrm{UG} / \mathrm{KG}$ & 1 \\
\hline Acenaphthylene & 330 & UG/KG & $u$ & $\ldots$ & & 330 UG/KG & 1 \\
\hline 2, 6, Dinitrotoluene & 330 & UG/KG & $u$ & ... & & 330 UG/KG & 1 \\
\hline 3-Nitroaniline & 1600 & UG/KG & $u$ & $\ldots$ & & 1600 UG/KG & 1 \\
\hline Acenaphthene & 330 & UG/KG & $u$ & $\ldots$ & & 330 UG/KG & 1 \\
\hline 2, 4-Dinitrophenol & 1600 & UG/KG & $u$ & $\ldots$ & & 1600 UG/KG & 1 \\
\hline 4-Nitrophenol & 1600 & UG/KG & $u$ & $\ldots$ & & 1600 UG/KG & 1 \\
\hline Dibenzofuran & & UG/KG & $u$ & $\ldots$ & & 330 UG/KG & 1 \\
\hline 2, 4-Dinitrotoluene & 330 & UG/KG & $u$ & $\ldots$ & & 330 UG/KG & 1 \\
\hline Diethylphthalate & 330 & UGKG & $u$ & $\ldots$ & & 330 UG/KG & 1 \\
\hline 4-Chlorophenyl phenyl ether & 330 & UG/KG & $u$ & $\ldots$ & & 330 UG/KG & 1 \\
\hline Fluorene & 330 & UGKG & u & .. & & 330 UG/KG & 1 \\
\hline 4-Nitroaniline & 1600 & UG/KG & u & $\ldots$ & & 1600 UG/KG & 1 \\
\hline 4, 6-Dinitro-2-Methylphenol & 1600 & UGKG & $u$ & $\ldots$ & & 1600 UG/KG & 1 \\
\hline N-Nitrosodiphenylamine & & UGKG & $u$ & - & & 330 UG/KG & 1 \\
\hline 4-Bromophenyl phenyl ether & 330 & UG/KG & $u$ & .- & & 330 UG/KG & 1 \\
\hline Hexachlorobenzene & 330 & UGKG & $u$ & .. & & 330 UG/KG & 1 \\
\hline Pentachlorophenol & 1600 & UGKG & $u$ & -- & & 1600 UG/KG & 1 \\
\hline Phenanthrene & 330 & UGKG & $u$ & $\ldots$ & & 330 UG/KG & 1 \\
\hline
\end{tabular}


Appendix D: Results of Semivolatile Organic Compound Analyses. Salmon Site

\begin{tabular}{|c|c|c|c|c|c|c|}
\hline COMPOUND & RESULTS UNITS & $\begin{array}{l}\text { DATA } \\
\text { QUAL }\end{array}$ & $\begin{array}{l}\text { DATA } \\
\text { VALID. }\end{array}$ & NOTES & CRDL L'NITS & DILUTIO \\
\hline \multicolumn{7}{|l|}{ TR10-ES-180-790-15 (CON'T) } \\
\hline Anthracene & 330 UG/KG & $u$ & $\ldots$ & & 330 UG/KG & 1 \\
\hline Di-N-Butylphthalate & 330 UG/KG & $u$ & ... & & 330 UG/KG & 1 \\
\hline Fluoranthene & 330 UG/KG & $u$ & -.. & & 330 UG/KG & 1 \\
\hline Pyrene & 330 UG/KG & $u$ & $\ldots$ & & 330 UG/KG & 1 \\
\hline Butyl Benzyl Phthalate & 330 UG/KG & $u$ & ... & & 330 UG/KG & 1 \\
\hline 3. 3'-Dichlorobenzidine & 660 UG/KG & $u$ & $\ldots$ & & 660 UG/KG & 1 \\
\hline Benzo(a)Anthracene & 330 UG/KG & $u$ & ... & & 330 UG/KG & 1 \\
\hline Chrysene & 330 UG/KG & $u$ & ... & & 330 UG/KG & 1 \\
\hline bis(2-Ethylhexyl)phthalate & 330 UG/KG & $u$ & $\ldots$ & & 330 UG/KG & 1 \\
\hline di-N-Octyl Phthalate & 330 UG/KG & $u$ & $\ldots$ & & 330 UG/KG & 1 \\
\hline Benzo(b)Fluoranthene & 330 UG/KG & $u$ & $\ldots$ & & 330 UG/KG & 1 \\
\hline Benzo(k)Fluoranthene & 330 UG/KG & $u$ & $\ldots$ & & 330 UG/KG & 1 \\
\hline Benzo(a)Pyrene & 330 UG/KG & $u$ & $\ldots$ & & 330 UG/KG & 1 \\
\hline Indeno $(1,2,3-C D)$ Pyrene & 330 UG/KG & $u$ & ... & & 330 UG/KG & 1 \\
\hline Dibenzo(a,h)Anthracene & 330 UG/KG & $u$ & $\ldots$ & & 330 UG/KG & 1 \\
\hline Benzo(g,h,i)Perylene & 330 UG/KG & $u$ & $\ldots$ & & 330 UG/KG & 1 \\
\hline Nitrobenzene-d5 & $74 \%$ & & & & $\%$ & 1 \\
\hline 2-Fluorobiphenyl & $66 \%$ & & & & $\%$ & 1 \\
\hline Terphenyl-d14 & $74 \%$ & & & & $\%$ & 1 \\
\hline Phenol-d5 & $83 \%$ & & & & $\%$ & 1 \\
\hline 2-Fluorophenol & $67 \%$ & & & & $\%$ & 1 \\
\hline 2, 4,6-Tribromophenol & $63 \%$ & & & & $\%$ & 1 \\
\hline
\end{tabular}


Appendix D: Results of Semivolatile Organic Compound Analyses, Salmon Site

\begin{tabular}{|c|c|c|c|c|c|c|c|}
\hline COMPOUND & RESULTS & UNITS & $\begin{array}{l}\text { DATA } \\
\text { QUAL }\end{array}$ & $\begin{array}{l}\text { DATA } \\
\text { VALID. }\end{array}$ & NOTES & CRDL UNITS & DILUTION \\
\hline \multicolumn{8}{|l|}{ TR10-ES-190-790-8 } \\
\hline Phenol & 330 & UG/KG & $u$ & $\ldots$ & & 330 UG/KG & 1 \\
\hline bis(2-Chloroethyl) ether & 330 & UGKKG & u & $\ldots$ & & 330 UG/KG & 1 \\
\hline 2-Chiorophenol & 330 & UG/KG & $u$ & -- & & 330 UG/KG & 1 \\
\hline 1, 3-Dichlorobenzene & 330 & UG/KG & $u$ & $\ldots$ & & 330 UG/KG & 1 \\
\hline 1, 4-Dichlorobenzene & 330 & UG/KG & $u$ & $\ldots$ & & 330 UG/KG & 1 \\
\hline Benzyl Alcohol & 330 & UG/KG & $u$ & - & & 330 UG/KG & 1 \\
\hline 1, 2-Dichlorobenzene & 330 & UG/KG & $u$ & $\ldots$ & & 330 UG/KG & 1 \\
\hline 2-Methylphenol & 330 & UG/KG & $u$ & ... & & 330 UG/KG & 1 \\
\hline bis(2-Chloroisopropyl) ether & 330 & UG/KG & $u$ & $\ldots$ & & 330 UG/KG & 1 \\
\hline 4-Methylphenol & 330 & UG/KG & $u$ & ... & & 330 UG/KG & 1 \\
\hline N-Nitroso-di-nPropylamine & 330 & UGKG & $u$ & -- & & 330 UG/KG & 1 \\
\hline Hexachloroethane & 330 & UG,KG & $u$ & - & & 330 UG/KG & 1 \\
\hline Nitrobenzene & 330 & UG/KG & $u$ & -. & & 330 UG/KG & 1 \\
\hline Isophorone & 330 & UG/KG & $u$ & ... & & 330 UG/KG & 1 \\
\hline 2-Nitrophenol & 330 & UG/KG & $u$ & $\ldots$ & & 330 UG/KG & 1 \\
\hline 2, 4-Dimethylphenol & 330 & UG/KG & $u$ & $\ldots$ & & 330 UG/KG & 1 \\
\hline Benzolc Acid & 1600 & UG/KG & $u$ & $\ldots$ & & 1600 UG/KG & 1 \\
\hline bis(2Chloroethoxy)methane & 330 & UG/KG & $u$ & $\ldots$ & & 330 UG/KG & 1 \\
\hline 2. 4-Dichlorophenol & 330 & UG/KG & $u$ & $\ldots$ & & 330 UG/KG & 1 \\
\hline 1, 2, 4-Trichlorobenzene & 330 & UG/KG & $u$ & $\ldots$ & & 330 UG/KG & 1 \\
\hline Naphthalene & 330 & UG/KG & $u$ & $\ldots$ & & 330 UG/KG & 1 \\
\hline 4-Chloroaniline & 330 & UG/KG & $u$ & $\ldots$ & & 330 UG/KG & 1 \\
\hline Hexachlorobutadiene & 330 & UG/KG & $u$ & $\ldots$ & & 330 UG/KG & 1 \\
\hline 4-Chloro-3-Methylphenol & 330 & UG/KG & $u$ & $\ldots$ & & 330 UG/KG & 1 \\
\hline 2-Methyinaphthalene & 330 & UG/KG & $u$ & $\ldots$ & & 330 UG/KG & 1 \\
\hline Hexachlorocyclopentadiene & 330 & UG/KG & $u$ & R & & 330 UG/KG & 1 \\
\hline 2, 4, 6-Trichlorophenol & 330 & UG/KG & $u$ & R & & 330 UG/KG & 1 \\
\hline 2, 4,5-Trichlorophenol & 1600 & UG/KG & $u$ & A & & 1600 UG/KG & 1 \\
\hline 2-Chloronaphthalene & 330 & UG/KG & u & R & & 330 UG/KG & 1 \\
\hline 2-Nitroaniline & 1600 & UG/KG & u & R & & 1600 UG/KG & 1 \\
\hline Dimethyl Phthalate & 330 & UG/KG & $u$ & R & & 330 UG/KG & 1 \\
\hline Acenaphthylene & 330 & UG/KG & $u$ & R & & 330 UG/KG & 1 \\
\hline 2. 6-Dinitrotoluene & 330 & UGKG & $u$ & R & & 330 UG/KG & 1 \\
\hline 3-Nitroaniline & 1600 & UGKG & $u$ & R & & 1600 UG/KG & 1 \\
\hline Acenaphthene & 330 & UG/KG & $u$ & R & & 330 UG/KG & 1 \\
\hline 2, 4-Dinitrophenol & 1600 & UGKG & $u$ & R & & $1600 \mathrm{UG} / \mathrm{KG}$ & 1 \\
\hline 4-Nitrophenol & 1600 & UG/KG & $u$ & A & & 1600 UG/KG & 1 \\
\hline Dibenzofuran & 330 & UG/KG & $u$ & A & & 330 UG/KG & 1 \\
\hline 2, 4-Dinitrotoluene & 330 & UG/KG & $u$ & R & & 330 UG/KG & 1 \\
\hline Diethylphthalate & 330 & UG/KG & $u$ & R & & 330 UG/KG & 1 \\
\hline 4-Chlorophenyl phenyl ether & 330 & UG/KG & $u$ & R & & 330 UG/KG & 1 \\
\hline Fluorene & 330 & UG/KG & $u$ & R & & 330 UG/KG & 1 \\
\hline 4-Nitroaniline & 1600 & UG/KG & $u$ & A & & 1600 UG/KG & 1 \\
\hline 4, 6-Dinitro-2-Methylphenol & 1600 & UG/KG & $u$ & - & & 1600 UG/KG & 1 \\
\hline N-Nitrosodiphenylamine & 330 & UG/KG & $u$ & - & & 330 UG/KG & 1 \\
\hline 4-Bromophenyl phenyl ether & 330 & UG/KG & $u$ & - & & 330 UGKG & 1 \\
\hline Hexachlorobenzene & 330 & UG/KG & $u$ & - & & 330 UG/KG & 1 \\
\hline Pentachlorophenol & 1600 & UG/KG & $u$ & - & & 1600 UG/KG & 1 \\
\hline Phenanthrene & 330 & UG/KG & $u$ & - & & 330 UG/KG & 1 \\
\hline
\end{tabular}


Appendix D: Results of Semivolatile Organic Compound Analyses, Salmon Site

\begin{tabular}{|c|c|c|c|c|c|c|c|c|}
\hline COMPOUND & RESULTS & UNITS & $\begin{array}{l}\text { DATA } \\
\text { QUAL }\end{array}$ & $\begin{array}{l}\text { DATA } \\
\text { VALID. }\end{array}$ & NOTES & CRDL & UNITS & DILUTION \\
\hline \multicolumn{9}{|l|}{ TR10-ES-190-790-8 (CON'T) } \\
\hline Anthracene & 330 & UG/KG & $u$ & $\cdots$ & & & UG/KG & 1 \\
\hline Di-N-Butylphthalate & 330 & UG/KG & $u$ & -. & & & UG/KG & 1 \\
\hline Fluoranthene & 330 & UG/KG & $u$ & $\ldots$ & & 33 & UG/KG & 1 \\
\hline Pyrene & 330 & UG/KG & $u$ & ... & & 33 & UG/KG & 1 \\
\hline Butyl Benzyl Phthalate & 330 & UG/KG & $u$ & $\ldots$ & & & UG/KG & 1 \\
\hline 3, 3'-Dichlorobenzidine & 660 & UG/KG & $u$ & $\ldots$ & & 66 & UG/KG & 1 \\
\hline Benzo(a)Anthracene & 330 & UG/KG & $u$ & ... & & 33 & UG/KG & 1 \\
\hline Chrysene & 330 & UG/KG & $u$ & ... & & 33 & UG/KG & 1 \\
\hline bis(2-Ethylhexyl)phthalate & 330 & UG/KG & $u$ & ... & & 33 & UG/KG & 1 \\
\hline di-N-Octyl Phthalate & 330 & $U G / K G$ & $u$ & ... & & 33 & UG/KG & 1 \\
\hline Benzo(b)Fluoranthene & 330 & UG/KG & $u$ & ... & & 33 & UG/KG & 1 \\
\hline Benzo(k)Fluoranthene & 330 & UG/KG & $u$ & ... & & 33 & UG/KG & 1 \\
\hline Benzo(a)Pyrene & 330 & UG/KG & $u$ & ... & & 33 & UG/KG & 1 \\
\hline Indeno(1.2,3-CD)Pyrene & 330 & UG/KG & $u$ & ... & & 33 & UG/KG & 1 \\
\hline Dibenzo(a.h)Anthracene & 330 & UG/KG & $u$ & $\ldots$ & & & UG/KG & 1 \\
\hline Benzo(g,h,j)Perylene & 330 & $U G / K G$ & $u$ & ... & & & UG/KG & 1 \\
\hline Nitrobenzene-d5 & 76 & $\%$ & & & & & $\%$ & 1 \\
\hline 2-Fluorobiphenyl & 75 & $\%$ & & & & & $\%$ & 1 \\
\hline Terphenyl-d14 & 63 & $\%$ & & & & & $\%$ & 1 \\
\hline Phenol-d5 & 73 & $\%$ & & & & & $\%$ & 1 \\
\hline 2-Fluorophenol & 63 & $\%$ & & & & & $\%$ & 1 \\
\hline 2, 4,6-Tribromophenol & 78 & $\%$ & & & & & $\%$ & 1 \\
\hline
\end{tabular}




\begin{tabular}{|c|c|c|c|c|c|c|c|}
\hline COMPOUND & RESULTS & UNITS & $\begin{array}{l}\text { DATA } \\
\text { QUAL }\end{array}$ & $\begin{array}{l}\text { DATA } \\
\text { VALID. }\end{array}$ & NOTES & CRDL UNITS & DILUTION \\
\hline \multicolumn{8}{|l|}{ TR11-ES-180-930-2 } \\
\hline Phenol & 330 & UG/KG & $u$ & NP & & $330 \mathrm{UG} / \mathrm{KG}$ & 1 \\
\hline bis(2-Chloroethyl) ether & 330 & UG/KG & $u$ & NP & & $330 \mathrm{UG} / \mathrm{KG}$ & 1 \\
\hline 2-Chlorophenol & 330 & UG/KG & $u$ & NP & & 330 UG/KG & 1 \\
\hline 1, 3-Dichlorobenzene & 330 & UG/KG & $u$ & NP & & 330 UG/KG & 1 \\
\hline 1, 4-Dichlorobenzene & 330 & UG/KG & $u$ & NP & & 330 UG/KG & 1 \\
\hline Benzyl Alcohol & 330 & UG/KG & $u$ & NP & & 330 UG/KG & 1 \\
\hline 1, 2-Dichlorobenzene & 330 & UG/KG & $u$ & NP & & 330 UG/KG & 1 \\
\hline 2-Methylphenol & 330 & UG/KG & $u$ & NP & & 330 UG/KG & 1 \\
\hline bis(2-Chloroisopropyl) ether & 330 & UG/KG & $u$ & NP & & 330 UG/KG & 1 \\
\hline 4-Methylphenol & 330 & UG/KG & $u$ & NP & & 330 UG/KG & 1 \\
\hline N-Nitroso-di-nPropylamine & 330 & UG/KG & $u$ & NP & & $330 \mathrm{UG} / \mathrm{KG}$ & 1 \\
\hline Hexachloroethane & 330 & UG/KG & $u$ & NP & & 330 UG/KG & 1 \\
\hline Nitrobenzene & 330 & UG/KG & $u$ & NP & & 330 UG/KG & 1 \\
\hline Isophorone & 330 & UG/KG & $u$ & NP & & 330 UG/KG & 1 \\
\hline 2-Nitrophenol & 330 & UG/KG & $u$ & NP & & 330 UG/KG & 1 \\
\hline 2. 4-Dimethylphenol & 330 & UG/KG & $u$ & NP & & 330 UG/KG & 1 \\
\hline Benzolc Acid & 1600 & UG/KG & $u$ & NP & & 1600 UG/KG & 1 \\
\hline bis(2Chloroethoxy)methane & 330 & UG/KG & $u$ & NP & & 330 UG/KG & 1 \\
\hline 2, 4-Dichlorophenol & 330 & UGKG & $u$ & NP & & 330 UG/KG & 1 \\
\hline 1, 2, 4-Trichlorobenzene & 330 & UG/KG & $u$ & NP & & 330 UG/KG & 1 \\
\hline Naphthalene & 330 & UG/KG & $u$ & NP & & 330 UG/KG & 1 \\
\hline 4-Chloroaniline & 330 & UG/KG & $u$ & NP & & 330 UG/KG & 1 \\
\hline Hexachlorobutadiene & 330 & UG/KG & $u$ & NP & & 330 UG/KG & 1 \\
\hline 4-Chloro-3-Methyiphenol & 330 & $U G / K G$ & $u$ & NP & & 330 UG/KG & 1 \\
\hline 2-Methyinaphthalene & 330 & UG/KG & $u$ & NP & & 330 UG/KG & 1 \\
\hline Hexachlorocyclopentadiene & 330 & UG/KG & $u$ & NP & & 330 UG/KG & 1 \\
\hline 2, 4, 6-Trichlorophenol & 330 & UG/KG & $u$ & NP & & 330 UG/KG & 1 \\
\hline 2, 4, 5-Trichlorophenol & 1600 & UG/KG & $u$ & NP & & 1600 UG/KG & 1 \\
\hline 2-Chloronaphthalene & 330 & UGKG & $u$ & NP & & 330 UG/KG & 1 \\
\hline 2-Nitroaniline & 1600 & UGKG & $u$ & NP & & 1600 UG/KG & 1 \\
\hline Dimethyl Phthalate & 330 & UG/KG & $u$ & NP & & 330 UG/KG & 1 \\
\hline Acenaphthylene & 330 & UG/KG & $u$ & NP & & 330 UG/KG & 1 \\
\hline 2, 6-Dinitrotoluene & 330 & UG/KG & $u$ & NP & & 330 UG/KG & 1 \\
\hline 3-Nitroaniline & 1600 & UG/KG & $u$ & NP & & 1600 UG/KG & 1 \\
\hline Acenaphthene & 330 & UG/KG & $u$ & NP & & 330 UG/KG & 1 \\
\hline 2, 4-Dinitrophenol & 1600 & UGKG & $u$ & NP & & 1600 UG/KG & 1 \\
\hline 4-Nitrophenol & 1600 & UGKKG & $u$ & NP & & 1600 UG/KG & 1 \\
\hline Dibenzofuran & 330 & UG/KG & $u$ & NP & & 330 UG/KG & 1 \\
\hline 2, 4-Dinitrotoluene & 330 & UG/KG & $u$ & NP & & 330 UG/KG & 1 \\
\hline Diethylphthalate & 330 & UGKG & $u$ & NP & & 330 UG/KG & 1 \\
\hline 4-Chlorophenyl phenyl ether & 330 & UG/KG & $u$ & NP & & 330 UG/KG & 1 \\
\hline Fluorene & 330 & UGKG & $u$ & NP & & 330 UG/KG & 1 \\
\hline 4-Nitroaniline & 1600 & UGKG & $u$ & NP & & 1600 UG/KG & 1 \\
\hline 4, 6-Dinitro-2-Methylphenol & 1600 & UG/KG & $u$ & NP & & 1600 UG/KG & 1 \\
\hline N-Nitrosodiphenylamine & 330 & UG/KG & $u$ & NP & & 330 UG/KG & 1 \\
\hline 4-Bromophenyl phenyl ether & 330 & UG/KG & u & NP & & 330 UG/KG & 1 \\
\hline Hexachlorobenzene & 330 & UG/KG & $u$ & NP & & 330 UG/KG & 1 \\
\hline Pentachlorophenol & 1600 & UG/KG & $u$ & NP & & 1600 UG/KG & 1 \\
\hline Phenanthrene & 330 & UG/KG & $u$ & NP & & 330 UG/KG & 1 \\
\hline
\end{tabular}


Appendix D: Results of Semivolatile Organic Compound Analyses, Salmon Site

\begin{tabular}{|c|c|c|c|c|c|c|c|c|}
\hline COMPOUND & RESULTS & UNITS & $\begin{array}{l}\text { DATA } \\
\text { QUAL }\end{array}$ & $\begin{array}{l}\text { DATA } \\
\text { VALID. }\end{array}$ & NOTES & CRDL & UNITS & DILUTION \\
\hline \multicolumn{9}{|l|}{ TR11-ES-180-930-2 (CON'T) } \\
\hline Anthracene & 330 & UG/KG & $u$ & NP & & & 0 UG/KG & 1 \\
\hline Di-N-Butylphthalate & 330 & UG/KG & $u$ & NP & & & 0 UG/KG & 1 \\
\hline Fluoranthene & 330 & UG/KG & $u$ & NP & & & 0 UG/KG & 1 \\
\hline Pyrane & 330 & UG/KG & $u$ & NP & & & 0 UG/KG & 1 \\
\hline Butyl Benzyl Phthalate & 330 & UG/KG & $u$ & NP & & & 0 UG/KG & 1 \\
\hline 3. 3'-Dichlorobenzidine & 660 & UG/KG & $u$ & NP & & & 0 UG/KG & 1 \\
\hline Benzo(a)Anthracene & 330 & UG/KG & $u$ & NP & & & 0 UG/KG & 1 \\
\hline Chrysene & 330 & UG/KG & $u$ & NP & & & O UG/KG & 1 \\
\hline bis(2-Ethylhexyl)phthalate & 330 & UG/KG & $u$ & NP & & & 0 UG/KG & 1 \\
\hline di-N-Octyl Phthalate & 330 & UG/KG & $u$ & NP & & & 0 UG/KG & 1 \\
\hline Benzo(b)Fluoranthene & 330 & UG/KG & $u$ & NP & & & 0 UG/KG & 1 \\
\hline Benzo(k)Fluoranthene & 330 & UG/KG & $u$ & NP & & & 0 UG/KG & 1 \\
\hline Benzo(a)Pyrene & 330 & UG/KG & $u$ & NP & & & 0 UG/KG & 1 \\
\hline Indeno(1,2,3-CD)Pyrene & 330 & UG/KG & $u$ & NP & & & 0 UG/KG & 1 \\
\hline Dibenzo(a.h)Anthracene & 330 & $U G / K G$ & $u$ & NP & & & O UG/KG & 1 \\
\hline Benzo(g,h.i)Perylene & 330 & UG/KG & $u$ & NP & & & 0 UG/KG & 1 \\
\hline Unknown-1 & 150 & UG/KG & J & NP & & & UG/KG & 1 \\
\hline Unknown-2 & 84 & UG/KG & J & NP & & & UG/KG & 1 \\
\hline Unknown-3 & 81 & UG/KG & 小 & NP & & & UG/KG & 1 \\
\hline Unknown-4 & 83 & UG/KG & BJ & NP & & & UGKG & 1 \\
\hline Unknown-5 & 99 & UG/KG & J & NP & & & UG/KG & 1 \\
\hline Unknown-6 & 77 & UG/KG & J & NP & & & UG/KG & 1 \\
\hline Unknown-7 & 280 & UG/KG & J & NP & & & $U G / K G$ & 1 \\
\hline Unknown-8 & 130 & UG/KG & J & NP & & & UG/KG & 1 \\
\hline Nitrobenzene-d5 & 81 & $\%$ & & & & & $\%$ & 1 \\
\hline 2-Fluorobiphenyl & 78 & $\%$ & & & & & $\%$ & 1 \\
\hline Terphenyl-d14 & 70 & $\%$ & & & & & $\%$ & 1 \\
\hline Phenol-d5 & 82 & $\%$ & & & & & $\%$ & 1 \\
\hline 2-Fluorophenol & 77 & $\%$ & & & & & $\%$ & 1 \\
\hline 2, 4, 6-Tribromophenol & 80 & $\%$ & & & & & $\%$ & 1 \\
\hline
\end{tabular}




\begin{tabular}{|c|c|c|c|c|c|c|c|}
\hline COMPOUND & RESULTS & UNITS & $\begin{array}{l}\text { DATA } \\
\text { QUAL }\end{array}$ & $\begin{array}{l}\text { DATA } \\
\text { VALID. }\end{array}$ & NOTES & CRDL UNITS & DILUTION \\
\hline \multicolumn{8}{|l|}{ TR13-ES-210-1110-6 } \\
\hline Phenol & 16000 & UG/KG & $u$ & NP & & $160 D 0$ UG/KG & 50 \\
\hline bis(2-Chloroethyl) ether & 16000 & UG/KG & $u$ & NP & & 16000 UG/KG & 50 \\
\hline 2-Chlorophenol & 16000 & UG/KG & $u$ & NP & & 16000 UG/KG & $5 C$ \\
\hline 1. 3-Dichlorobenzene & 16000 & UG/KG & $u$ & NP & & 16000 UG/KG & 50 \\
\hline 1. 4-Dichlorobenzene & 16000 & UG/KG & $u$ & NP & & 16000 UG/KG & 50 \\
\hline Benzyl Alcohol & 16000 & UG/KG & $u$ & NP & & 16000 UG/KG & 50 \\
\hline 1, 2-Dichlorobenzene & 16000 & UG/KG & $u$ & NP & & $16000 \mathrm{UG} / \mathrm{KG}$ & 50 \\
\hline 2-Methyiphenol & 16000 & UG/KG & $u$ & NP & & 16000 UG/KG & 50 \\
\hline bis(2-Chloroisopropyl) ether & 16000 & UG/KG & $u$ & NP & & 16000 UG/KG & 50 \\
\hline 4-Methylphenol & 16000 & $U G / K G$ & $u$ & NP & & 16000 UG/KG & 50 \\
\hline N-Nitroso-di-nPropylamine & 16000 & UG/KG & $u$ & NP & & 16000 UG/KG & 50 \\
\hline Hexachloroethane & 16000 & UG/KG & $u$ & NP & & 16000 UG/KG & 50 \\
\hline Nitrobenzene & 16000 & UG/KG & $u$ & NP & & 16000 UG/KG & 50 \\
\hline Isophorone & 16000 & UG/KG & $u$ & NP & & 16000 UG/KG & 50 \\
\hline 2-Nitrophenol & 16000 & UG/KG & $u$ & NP & & 16000 UG/KG & 50 \\
\hline 2, 4-Dimethylphenol & 16000 & UG/KG & $u$ & NP & & 16000 UG/KG & 50 \\
\hline Benzoic Acid & 80000 & UG/KG & $u$ & NP & & 80000 UG/KG & 50 \\
\hline bis(2Chloroethoxy)methane & 16000 & UG/KG & $u$ & NP & & 16000 UG/KG & 50 \\
\hline 2. 4-Dichlorophenol & 16000 & UG/KG & $u$ & NP & & 16000 UG/KG & 50 \\
\hline 1, 2, 4-Trichlorobenzene & 16000 & UG/KG & $u$ & NP & & 16000 UG/KG & 50 \\
\hline Naphthalene & 5400 & UG/KG & DJ & NP & & 16000 UG/KG & 50 \\
\hline 4. Chloroaniline & 16000 & UG/KG & $u$ & NP & & 16000 UG/KG & 50 \\
\hline Hexachlorchutadiene & 16000 & UG/KG & $u$ & NP & & 16000 UG/KG & 50 \\
\hline 4-Chloro-3-Muthyiphenol & 16000 & UG/KG & $u$ & NP & & 16000 UG/KG & 50 \\
\hline 2-Methyinaph halene & 79000 & UG/KG & 0 & NP & & 16000 UG/KG & 50 \\
\hline Hexachlorocyclopentadiene & 16000 & UG/KG & $u$ & NP & & 16000 UG/KG & 50 \\
\hline 2, 4, 6-Trichlorophenol & 16000 & UG/KG & $u$ & NP & & 16000 UG/KG & 50 \\
\hline 2, 4, 5-Trichlorophenol & 80000 & UG/KG & $u$ & NP & & 80000 UG/KG & 50 \\
\hline 2-Chloronaphthalene & 16000 & UG/KG & $u$ & NP & & 16000 UG/KG & 50 \\
\hline 2-Nitroaniline & 80000 & UG/KG & $u$ & NP & & 80000 UG/KG & 50 \\
\hline Dimethyl Phthalate & 16000 & UG/KG & $u$ & NP & & 16000 UG/KG & 50 \\
\hline Acenaphthylene & 16000 & UG/KG & $u$ & NP & & 16000 UG/KG & 50 \\
\hline 2, 6-Dinitrotoluene & 16000 & UG/KG & $u$ & NP & & 16000 UG/KG & 50 \\
\hline 3-Nitroaniline & 80000 & UG/KG & $u$ & NP & & 80000 UG/KG & 50 \\
\hline Acenaphthene & 16000 & UG/KG & $u$ & NP & & 16000 UG/KG & 50 \\
\hline 2, 4-Dinitrophenol & 80000 & UG/KG & u & NP & & 80000 UG/KG & 50 \\
\hline 4-Nitrophenol & 80000 & UG/KG & $u$ & NP & & 80000 UG/KG & 50 \\
\hline Dibenzofuran & 16000 & $U G / K G$ & $u$ & NP & & 16000 UG/KG & 50 \\
\hline 2, 4-Dinitrotoluene & 16000 & UG/KG & $u$ & NP & & 16000 UG/KG & 50 \\
\hline Diethylphthalate & 16000 & UG/KG & $u$ & NP & & 16000 UG/KG & 50 \\
\hline 4-Chlorophenyl phenyl ether & 16000 & UG/KG & $u$ & NP & & 16000 UG/KG & 50 \\
\hline Fluorene & 16000 & UG/KG & $u$ & NP & & 16000 UG/KG & 50 \\
\hline 4-Nitroaniline & 80000 & UGKG & $u$ & NP & & 80000 UGKG & 50 \\
\hline 4, 6-Dinitro-2-Methylphenol & 80000 & UG/KG & $u$ & NP & & 80000 UG/KG & 50 \\
\hline N-Nitrosodiphenylamine & 16000 & UG/KG & $u$ & NP & & 16000 UGKG & 50 \\
\hline 4-Bromophenyl phenyl ether & 16000 & UG/KG & $u$ & NP & & 16000 UG/KG & 50 \\
\hline Hexachlorobenzene & 16000 & UGKG & $u$ & NP & & 16000 UG/KG & 50 \\
\hline Pentachlorophenol & 80000 & UGKG & $u$ & NP & & 80000 UG/KG & 50 \\
\hline Phenanthrene & 16000 & UG/KG & $u$ & NP & & 16000 UG/KG & 50 \\
\hline
\end{tabular}


Appendix D: Results of Semivolatile Organic Compound Analyses, Salmon Site

\begin{tabular}{|c|c|c|c|c|c|c|c|}
\hline COMPOUND & RESULTS & UNITS & $\begin{array}{l}\text { DATA } \\
\text { QUAL }\end{array}$ & $\begin{array}{l}\text { DATA } \\
\text { VALID. }\end{array}$ & NOTES & CRDL UNITS & DILUTION \\
\hline \multicolumn{8}{|l|}{ TR13-ES-210-1110-6 (CON'T) } \\
\hline Anthracene & 16000 & UG/KG & $u$ & NP & & 16000 UG/KG & 50 \\
\hline Di-N-Butylphthalate & 16000 & UG/KG & $u$ & NP & & 16000 UG/KG & 50 \\
\hline Fluoranthene & 16000 & UG/KG & $u$ & NP & & 16000 UG/KG & 50 \\
\hline Pyrene & 16000 & UG/KG & $u$ & NP & & 16000 UG/KG & 50 \\
\hline Butyl Benzyl Phthalate & 16000 & UG/KG & $u$ & NP & & 16000 UG/KG & 50 \\
\hline 3, 3'-Dichlorobenzidine & 33000 & UG/KG & $u$ & NP & & 33000 UG/KG & 50 \\
\hline Benzo(a)Anthracene & 16000 & UG/KG & $u$ & NP & & 16000 UG/KG & 50 \\
\hline Chrysene & 16000 & UG/KG & $u$ & NP & & 16000 UG/KG & 50 \\
\hline bis(2-Ethylhexyl)phthalate & $1600 C$ & UG/KG & $u$ & NP & & 16000 UG/KG & 50 \\
\hline di-N-Octyl Phthalate & 16000 & UG/KG & $u$ & NP & & 16000 UG/KG & 50 \\
\hline Benzo(b)Fluoranthene & 16000 & UG/KG & $u$ & NP & & 16000 UG/KG & 50 \\
\hline Benzo(k)Fluoranthene & 16000 & UG/KG & $u$ & NP & & 16000 UG/KG & 50 \\
\hline Benzo(a)Pyrene & 16000 & UG/KG & $u$ & NP & & 16000 UG/KG & 50 \\
\hline Indeno(1,2,3-CD)Pyrene & 16000 & UG/KG & $u$ & NP & & 16000 UG/KG & 50 \\
\hline Dibenzo(a,h)Anthracene & 16000 & UG/KG & $u$ & NP & & 16000 UG/KG & 50 \\
\hline Benzo(g,h,i)Perylene & 16000 & UG/KG & $u$ & NP & & 16000 UG/KG & 50 \\
\hline Dodecane & 26000 & UG/KG & J & NP & & UG/KG & 50 \\
\hline Naphthalene, 1,2,3,4-tetrahy & 16000 & UG/KG & $\mathrm{J}$ & NP & & $1 \mathrm{UG} / \mathrm{KG}$ & 50 \\
\hline Unknown $\mathrm{C} 11 \mathrm{H} 14$ & 24000 & UG/KG & $\mathrm{J}$ & NP & & UG/KG & 50 \\
\hline Unknown-1 & 45000 & UG/KG & J & NP & & UG/KG & 50 \\
\hline Unknown-2 & 26000 & $U G / K G$ & $\mathrm{~J}$ & NP & & UG/KG & 50 \\
\hline Unknown $\mathrm{C} 11 \mathrm{H} 10$ & 57000 & UG/KG & J & NP & & UG/KG & 50 \\
\hline Unknown-3 & 32000 & $U G / K G$ & $J$ & NP & & UG/KG & 50 \\
\hline Unknown-4 & 15000 & UG/KG & J & NP & & UG/KG & 50 \\
\hline Unknown-5 & 18000 & UG/KG & $j$ & NP & & UG/KG & 50 \\
\hline Unknown-6 & 15000 & UG/KG & J & NP & & UG/KG & 50 \\
\hline Unknown-7 & 51000 & UG/KG & J & NP & & UG/KG & 50 \\
\hline Unknown $\mathrm{C} 12 \mathrm{H} 12-1$ & 44000 & UG/KG & J & NP & & UG/KG & 50 \\
\hline Unknown $\mathrm{C} 12 \mathrm{H} 12-2$ & 47000 & UG/KG & J & NP & & UG/KG & 50 \\
\hline Unknown $\mathrm{C} 12 \mathrm{H} 12-3$ & 27000 & UG/KG & j & NP & & UG/KG & 50 \\
\hline Unknown $\mathrm{C} 12 \mathrm{H} 12-4$ & 33000 & UG/KG & J & NP & & UG/KG & 50 \\
\hline Unknown-8 & 16000 & UG/KG & J & NP & & UG/KG & 50 \\
\hline Pentadecane & 33000 & UG/KG & J & NP & & UG/KG & 50 \\
\hline Unknown $\mathrm{C} 13 \mathrm{H} 14$ & 17000 & UG/KG & J & NP & & UG/KG & 50 \\
\hline Hexadecane & 16000 & $U G / K G$ & J & NP & & UG/KG & 50 \\
\hline Heptadecane & 17000 & UG/KG & $\mathrm{J}$ & NP & & UG/KG & 50 \\
\hline Unknown-9 & 27000 & UG/KG & J & NP & & UG/KG & 50 \\
\hline Unknown-10 & 65000 & UG/KG & $\mathrm{J}$ & NP & & UG/KG & 50 \\
\hline Nitrobenzene-d5 & 71 & $\%$ & & & & $\%$ & 50 \\
\hline 2-Fluorobiphenyl & 78 & $\%$ & & & & $\%$ & 50 \\
\hline Terphenyl-d14 & 72 & $\%$ & & & & $\%$ & 50 \\
\hline Phenol-d5 & 69 & $\%$ & & & & $\%$ & 50 \\
\hline 2-Fluorophenol & 64 & $\%$ & & & & $\%$ & 50 \\
\hline 2, 4,6-Tribromophenol & 62 & $\%$ & & & & $\%$ & 50 \\
\hline
\end{tabular}




\begin{tabular}{|c|c|c|c|c|c|c|c|c|}
\hline COMPOUND & RESULTS & UNITS & $\begin{array}{l}\text { DATA } \\
\text { QUAL }\end{array}$ & $\begin{array}{l}\text { DATA } \\
\text { VALID. }\end{array}$ & NOTES & CRDL & UNITS & DILUTION \\
\hline \multicolumn{9}{|l|}{ TR13-ES-212-1150-6 } \\
\hline Phenol & 33000 & UG/KG & $u$ & NP & & 33000 & UG/KG & 100 \\
\hline bis(2-Chloroethyl) ether & 33000 & UG/KG & $u$ & NP & & 33000 & UG/KG & 100 \\
\hline 2-Chlorophenol & 33000 & UG/KG & $u$ & NP & & 33000 & UG/KG & 100 \\
\hline 1. 3-Dichlorobenzene & 33000 & UG/KG & $u$ & NP & & 33000 & UG/KG & 100 \\
\hline 1, 4-Dichlorobenzene & 33000 & UG/KG & $u$ & NP & & 33000 & UG/KG & 100 \\
\hline Benzyl Alcohol & 33000 & UG/KG & $u$ & NP & & 33000 & UG/KG & 100 \\
\hline 1. 2-Dichlorobenzene & 33000 & UG/KG & $u$ & NP & & 33000 & UG/KG & 100 \\
\hline 2-Methylphenol & 33000 & $U G / K G$ & $u$ & NP & & 33000 & UG/KG & 100 \\
\hline bis(2-Chloroisopropyl) ether & 33000 & UG/KG & $u$ & NP & & 33000 & UG/KG & 100 \\
\hline 4-Methylphenol & 33000 & UG/KG & $u$ & NP & & 33000 & UG/KG & 100 \\
\hline N-Nitroso-di-nPropylamine & 33000 & UG/KG & $u$ & NP & & 33000 & UG/KG & 100 \\
\hline Hexachioroethane & 33000 & UG/KG & $u$ & NP & & 33000 & O UG/KG & 100 \\
\hline Nitrobenzene & 33000 & UG/KG & $u$ & NP & & 33000 & UG/KG & 100 \\
\hline Isophorone & 33000 & $U G / K G$ & $u$ & NP & & 33000 & O UG/KG & 100 \\
\hline 2-Nitrophenol & 33000 & UG/KG & $u$ & NP & & 33000 & O UG/KG & 100 \\
\hline 2, 4-Dimethylphenol & 33000 & UG/KG & $u$ & NP & & 33000 & O UG/KG & 100 \\
\hline Benzolc Acid & 160000 & UG/KG & $u$ & NP & & 160000 & O UG/KG & 100 \\
\hline bis(2Chloroethoxy)methane & 33000 & UG/KG & $u$ & NP & & 33000 & O UG/KG & 100 \\
\hline 2, 4-Dichlorophenol & 33000 & UG/KG & $u$ & NP & & 33000 & 0 UG/KG & 100 \\
\hline 1, 2, 4-Trichlorobenzene & 33000 & UG/KG & $u$ & NP & & 33000 & 0 UG/KG & 100 \\
\hline Naphthalene & 15000 & UG/KG & DJ & NP & & 3300 & 0 UG/KG & 100 \\
\hline 4-Chloroaniline & 33000 & UG/KG & $u$ & NP & & 3300 & 0 UG/KG & 100 \\
\hline Hexachlorobutadiene & 33000 & UG/KG & $u$ & NP & & 3300 & 0 UG/KG & 100 \\
\hline 4-Chloro-3-Methylphenol & 33000 & UG/KG & $u$ & NP & & 3300 & $0 \cup G / K G$ & 100 \\
\hline 2-Methylnaphthalene & 210000 & UG/KG & $D$ & NP & & 3300 & 0 UG/KG & 100 \\
\hline Hexachlorocyclopentadiene & 33000 & UG/KG & $u$ & NP & & 3300 & 0 UG/KG & 100 \\
\hline 2, 4, 6-Trichlorophenol & 33000 & UG/KG & $u$ & NP & & 3300 & 0 UG/KG & 100 \\
\hline 2, 4, 5-Trichlorophenol & 160000 & UG/KG & $u$ & NP & & 3300 & 0 UG/KG & 100 \\
\hline 2-Chloronaphthalene & 33000 & UG/KG & $u$ & NP & & 3300 & 0 UG/KG & 100 \\
\hline 2-Nitroaniline & 160000 & UG/KG & $u$ & NP & & 16000 & O UG/KG & 100 \\
\hline Dimethyl Phthalate & 33000 & UG/KG & $u$ & NP & & 3300 & 0 UG/KG & 100 \\
\hline Acenaphthylene & 33000 & UG/KG & $u$ & NP & & 3300 & 0 UG/KG & 100 \\
\hline 2, 6-Dinitrotoluene & 33000 & UGKKG & u & NP & & 3300 & 0 UG/KG & 100 \\
\hline 3-Nitroaniline & 160000 & UGKG & $u$ & NP & & 16000 & O UG/KG & 100 \\
\hline Acenaphthene & 33000 & UG/KG & $u$ & NP & & 3300 & O UG/KG & 100 \\
\hline 2, 4-Dinitrophenol & 160000 & UG/KG & $u$ & NP & & 16000 & O UG/KG & 100 \\
\hline 4-Nitrophenol & 160000 & UG/KG & $u$ & NP & & 16000 & O UG/KG & 100 \\
\hline Dibenzofuran & 33000 & UG/KG & $u$ & NP & & 3300 & O UG/KG & 100 \\
\hline 2, 4-Dinitrotoluene & 33000 & UG/KG & $u$ & NP & & 3300 & O UG/KG & 100 \\
\hline Diethylphthalate & 33000 & UG/KG & $u$ & NP & & 3300 & O UG/KG & 100 \\
\hline 4-Chlorophenyl phenyl ether & 33000 & UG/KG & $u$ & NP & & 3300 & O UG/KG & 100 \\
\hline Fluorene & 9500 & UGKG & DJ & NP & & 3300 & D UG/KG & 100 \\
\hline 4-Nitroaniline & 160000 & UGKG & $u$ & NP & & 16000 & O UG/KG & 100 \\
\hline 4, 6-Dinitro-2-Methylphenol & 160000 & UG/Ka & $u$ & NP & & 16000 & IO UG/KG & 100 \\
\hline N-Nitrosodiphenylamine & 33000 & UG/KG & $u$ & NP & & 3300 & D UG/KG & 100 \\
\hline 4-Bromophenyl phenyl ether & 33000 & UG/KG & $u$ & NP & & 3300 & 0 UG/KG & 100 \\
\hline Hexachlorobenzene & 33000 & UG/KG & $u$ & NP & & 3300 & D UG/Ka & 100 \\
\hline Pentachlorophenol & 160000 & UGKG & $u$ & NP & & 16000 & 0 UG/KG & 100 \\
\hline Phenanthrene & 5100 & UG/KG & DJ & NP & & 3300 & 0 UG/KG & 100 \\
\hline
\end{tabular}


- Appendix D: Results of Semivolatile Organic Compound Analyses, Salmon Site

\begin{tabular}{|c|c|c|c|c|c|c|c|c|}
\hline COMPOUND & RESULTS & UNITS & $\begin{array}{l}\text { DATA } \\
\text { QUAL }\end{array}$ & $\begin{array}{l}\text { DATA } \\
\text { VALID. }\end{array}$ & NOTES & CRDL & UNITS & DILUTION \\
\hline \multicolumn{9}{|l|}{ TR13-ES-212-1150-6 (CON'T) } \\
\hline Anthracene & 33000 & UG/KG & $u$ & NP & & 33000 & UG/KG & 100 \\
\hline Di-N-Butylphthalate & 33000 & IJG/KG & $u$ & NP & & 33000 & UG/KG & 100 \\
\hline Fluoranthene & 33000 & UG/KG & $u$ & NP & & 33000 & UG/KG & 100 \\
\hline Pyrene & 33000 & UG/KG & $u$ & $N P$ & & 3300 & UG/KG & 100 \\
\hline Butyl Benzyl Phthalate & 33000 & UG/KG & $u$ & NP & & 3300 & UG/KG & 100 \\
\hline 3, 3'-Dichlorobenzidine & 66000 & UG/KG & $u$ & NP & & 6600 & UG/KG & 100 \\
\hline Benzo(a)Anthracene & 33000 & UG/KG & $u$ & NP & & 3300 & UG/KG & 100 \\
\hline Chrysene & 33000 & UG/KG & $u$ & NP & & 3300 & UG/KG & 100 \\
\hline bis(2-Ethylhexyl)phthalate & 33000 & UG/KG & $u$ & NP & & 33000 & UG/KG & 100 \\
\hline di-N-Octyl Phthalate & 33000 & UG/KG & $u$ & NP & & 33000 & UG/KG & 100 \\
\hline Benzo(b)Fluoranthene & 33000 & UG/KG & $u$ & NP & & 3300 & UG/KG & 100 \\
\hline Benzo(k)Fluoranthene & 33000 & UG/KG & $u$ & NP & & 3300 & UG/KG & 100 \\
\hline Benzo(a)Pyrene & 33000 & UG/KG & $u$ & NP & & 3300 & UG/KG & 100 \\
\hline Indeno(1,2,3-CD)Pyrene & 33000 & UG/KG & $u$ & NP & & 3300 & UG/KG & 100 \\
\hline Dibenzo(a.h)Anthracene & 33000 & UG/KG & $u$ & NP & & 3300 & UG/KG & 100 \\
\hline Benzo(g,h,i)Perylene & 33000 & UG/KG & $u$ & NP & & 3300 & UG/KG & 100 \\
\hline Dodecane & 62000 & UG/KG & J & NP & & & UG/KG & 100 \\
\hline Unknown-1 & 53000 & UG/KG & J & NP & & & $U G / K G$ & 100 \\
\hline Unknown C11H14-1 & 86000 & UG/KG & J & NP & & & UG/KG & 100 \\
\hline Unknown $\mathrm{C} 11 \mathrm{H} 14-2$ & 46000 & UG/KG & J & NP & & & $U G / K G$ & 100 \\
\hline Unknown $\mathrm{C} 11 \mathrm{H} 10$ & 120000 & UG/KG & J & NP & & & $U G / K G$ & 100 \\
\hline Unknown $\mathrm{C} 12 \mathrm{H} 16$ & 66000 & $U G / K G$ & J & NP & & & UG/KG & 100 \\
\hline Unknown-2 & 28000 & UG/KG & J & NP & & & UG/KG & 100 \\
\hline Unknown-3 & 27000 & UG/KG & J & NP & & & $U G / K G$ & 100 \\
\hline Unknown-4 & 32000 & UG/KG & j & NP & & & UG/KG & 100 \\
\hline Unknown-5 & 31000 & UG/KG & J & NP & & & UG/KG & 100 \\
\hline Unknown-6 & 23000 & UG/KG & J & NP & & & UG/KG & 100 \\
\hline Unknown-7 & 120000 & UG/KG & J & NP & & & UG/KG & 100 \\
\hline Unknown $\mathrm{C} 12 \mathrm{H} 12-1$ & 110000 & UG/KG & J & $N P$ & & & UG/KG & 100 \\
\hline Unknown $\mathrm{C} 12 \mathrm{H} 12-2$ & 100000 & UG/KG & J & NP & & & UG/KG & 100 \\
\hline Unknown $\mathrm{C} 12 \mathrm{H} 12-3$ & 59000 & UG/KG & J & NP & & & UG/KG & 100 \\
\hline Unknown $\mathrm{C} 12 \mathrm{H} 12-4$ & 75000 & UG/KG & 小 & NP & & & UG/KG & 100 \\
\hline Unknown-8 & 33000 & UG/KG & $\mathrm{J}$ & NP & & & UG/KG & 100 \\
\hline Unknown-9 & 21000 & UG/KG & J & NP & & & UG/KG & 100 \\
\hline Pentadecane & 84000 & UG/KG & J & NP & & & UG/KG & 100 \\
\hline Unknown $\mathrm{C} 13 \mathrm{H} 14-1$ & 37000 & UG/KG & $\mathrm{J}$ & NP & & & $U G / K G$ & 100 \\
\hline Unknown $\mathrm{C} 13 \mathrm{H} 14-2$ & 27000 & UG/KG & j & $N P$ & & & UG/KG & 100 \\
\hline Heptadecane & 41000 & UG/KG & J & NP & & & $U G / K G$ & 100 \\
\hline Nitrobenzene-d5 & 0 & $\%$ & D & & & & $\%$ & 100 \\
\hline 2-Fluorobiphenyl & 0 & $\%$ & $D$ & & & & $\%$ & 100 \\
\hline Terphenyl-d14 & 0 & $\%$ & D & & & & $\%$ & 100 \\
\hline Phenol-d5 & 0 & $\%$ & D & & & & $\%$ & 100 \\
\hline 2-Fluorophenol & 0 & $\%$ & D & & & & $\%$ & 100 \\
\hline 2, 4, 6-Tribromophenol & 0 & $\%$ & D & & & & $\%$ & 100 \\
\hline
\end{tabular}




\begin{tabular}{|c|c|c|c|c|c|c|c|}
\hline COMPOUND & RESULTS & UNITS & $\begin{array}{l}\text { DATA } \\
\text { QUAL }\end{array}$ & $\begin{array}{l}\text { DATA } \\
\text { VALID. }\end{array}$ & NOTES & CROL UNITS & DILUTION \\
\hline \multicolumn{8}{|l|}{ TR14-ES-80-1189-5 } \\
\hline Phenol & 330 & UG/KG & $u$ & NP & & 330 UG/KG & 1 \\
\hline bis(2-Chloroethyl) ether & 330 & UG/KG & $u$ & NP & & 330 UG/KG & 1 \\
\hline 2-Chlorophenol & 330 & UG/KG & $u$ & MP & & 330 UG/KG & 1 \\
\hline 1, 3-Dichlorobenzene & 330 & Ua/Ka & $u$ & NP & & 330 UG/KG & 1 \\
\hline 1, 4-Dichlorobenzene & 330 & Ua/KG & $u$ & NP & & 330 UG/KG & 1 \\
\hline Benzyl Alcohol & 330 & UG/KG & $u$ & NP & & 330 UG/KG & 1 \\
\hline 1, 2-Dichlorobenzene & 330 & UG/KG & $u$ & NP & & 330 UG/KG & 1 \\
\hline 2-Methylphenol & 330 & UGKG & $u$ & NP & & 330 UG/KG & 1 \\
\hline bis(2-Chloroisopropyl) ether & 330 & UG/KG & $u$ & NP & & 330 UG/KG & 1 \\
\hline 4-Methylphenol & 330 & UG/KG & $u$ & NP & & $330 \mathrm{UG} / \mathrm{KG}$ & 1 \\
\hline N-Nitroso-di-nPropylamine & 330 & UG/KG & $u$ & NP & & $330 \mathrm{UG} / \mathrm{KG}$ & 1 \\
\hline Hexachloroethane & 330 & UG/KG & $u$ & NP & & $330 \mathrm{UG} / \mathrm{KG}$ & 1 \\
\hline Nitrobenzene & 330 & UG/KG & $u$ & NP & & 330 UG/KG & 1 \\
\hline Isophorone & 330 & UG/KG & $u$ & NP & & 330 UG/KG & 1 \\
\hline 2-Nitrophenol & 330 & UG/KG & $u$ & NP & & 330 UG/KG & 1 \\
\hline 2. 4-Dimethylphenol & 330 & UG/KG & $u$ & NP & & 330 UG/KG & 1 \\
\hline Benzoic Acid & 1600 & UGKG & $u$ & NP & & 1600 UG/KG & 1 \\
\hline bis(2Chloroethoxy)methane & 330 & UG/KG & $u$ & NP & & 330 UG/KG & 1 \\
\hline 2, 4-Dichlorophenol & 330 & UGKG & $u$ & NP & & 330 UG/KG & 1 \\
\hline 1,2, 4-Trichlorobenzene & 330 & UG/KG & $u$ & NP & & 330 UG/KG & 1 \\
\hline Naphthalene & 330 & UG/KG & $u$ & NP & & 330 UG/KG & 1 \\
\hline 4-Chloroaniline & 330 & UG/KG & $u$ & NP & & 330 UG/KG & 1 \\
\hline Hexachlorobutadiene & 330 & UGKG & $u$ & NP & & 330 UG/KG & 1 \\
\hline 4-Chloro-3-Methyiphenol & 330 & UG/KG & $u$ & NP & & 330 UG/KG & 1 \\
\hline 2-Methyinaphthalene & 190 & UG/KG & J & NP & & 330 UG/KG & 1 \\
\hline Hexachlorocyclopentadiene & 330 & UGKG & $u$ & NP & & 330 UG/KG & 1 \\
\hline 2, 4, 6-Trichiorophenol & 330 & UG/KG & $u$ & NP & & 330 UG/KG & 1 \\
\hline 2, 4, 5-Trichlorophenol & 1600 & UG/KG & $u$ & NP & & 1600 UG/KG & 1 \\
\hline 2-Chloronaphthalene & 330 & UaKG & $u$ & NP & & 330 UG/KG & 1 \\
\hline 2-Nitroaniline & 1600 & UG/KG & $u$ & NP & & 1600 Ua/KG & 1 \\
\hline Dimethyl Phthalate & 330 & VGKG & $u$ & NP & & 330 UG/KG & 1 \\
\hline Acenaphthylerie & 330 & UGKG & $u$ & NP & & 330 UG/KG & 1 \\
\hline 2, 6-Dinitrotoluene & 330 & UGKG & $u$ & NP & & 330 UG/KG & 1 \\
\hline 3-Nitroaniline & 1600 & UGKG & u & NP & & 1600 UG/KG & 1 \\
\hline Acenaphthene & 330 & UG/KG & $u$ & NP & & 330 UG/KG & 1 \\
\hline 2, 4-Dinitrophenol & 1600 & UaKG & u & NP & & 1600 UG/KG & 1 \\
\hline 4-Nitrophenol & 1600 & UG/KG & $u$ & NP & & 1600 UG/KG & 1 \\
\hline Dibenzofuran & 330 & UGKG & $u$ & NP & & 330 UG/KG & 1 \\
\hline 2, 4-Dinitrotoluene & 330 & UGKG & $u$ & NP & & 330 UG/KG & 1 \\
\hline Diethylphthalate & 330 & UG/KG & $u$ & NP & & 330 UG/KG & 1 \\
\hline 4-Chlorophenyl phenyl ether & 330 & UGKG & $u$ & NP & & 330 UG/KG & 1 \\
\hline Fluorene & 330 & UGKG & $u$ & NP & & 330 UG/KG & 1 \\
\hline 4-Nitroaniline & 1600 & VGKG & u & NP & & 1600 Ua/KG & 1 \\
\hline 4, 6-Dinitro-2-Methylphenol & 1600 & UGKG & $u$ & NP & & 1600 UG/KG & 1 \\
\hline N-Nitrosodiphenylamine & 330 & UGKG & $u$ & NP & & 330 UG/KG & 1 \\
\hline 4-Bromophenyl phenyl ether & 330 & UGKG & $u$ & NP & & 330 UG/KG & 1 \\
\hline Hexachlorobenzene & 330 & UaKG & $u$ & NP & & 330 UG/KG & 1 \\
\hline Pentachlorophenol & 1600 & UGKG & $u$ & NP & & 1600 UG/KG & 1 \\
\hline Phenanthrene & 330 & UGKG & $U$ & NP & & 330 UG/KG & 1 \\
\hline
\end{tabular}


Appendix D: Results of Semivolatile Organic Compound Analyses, Salmon Site

\begin{tabular}{|c|c|c|c|c|c|c|c|c|}
\hline COMPOUND & RESULTS & UNITS & $\begin{array}{l}\text { DATA } \\
\text { QUAL }\end{array}$ & $\begin{array}{l}\text { DATA } \\
\text { VALID. }\end{array}$ & NOTES & CRDL & UNITS & DILUTION \\
\hline \multicolumn{9}{|l|}{ TR14-ES-80-1189-5 (CON'T) } \\
\hline Anthracene & 330 & UG/KG & $u$ & NP & & & D Ua/Ko & 1 \\
\hline Di-N-Butylphthalate & 330 & UG/KG & $u$ & NP & & 330 & O Va/Ka & 1 \\
\hline Fluoranthene & 330 & UG/KG & $u$ & NP & & & O UG/KG & 1 \\
\hline Pyrene & 330 & UG/KG & $u$ & NP & & 33 & O UG/KG & 1 \\
\hline Butyl Benzyl Phthalate & 330 & UG/KG & $u$ & NP & & 33 & O UG/KG & 1 \\
\hline 3, 3'-Dichlorobenzidine & 660 & Va/KG & $u$ & NP & & 660 & O UG/KG & 1 \\
\hline Benzo(a)Anthracene & 330 & UGKG & $u$ & NP & & 33 & UG/KG & 1 \\
\hline Chrysene & 330 & UG/KG & $u$ & NP & & 33 & UG/KG & 1 \\
\hline bis(2-Ethylhexyl)phthalate & 330 & VaKa & $u$ & NP & & 33 & O VQ/KG & 1 \\
\hline di-N-Octyl Phthalate & 330 & UG/KO & $u$ & NP & & 33 & O UG/Ko & 1 \\
\hline Benzo(b)Fluoranthene & 330 & UG/KG & $u$ & NP & & 33 & O UG/KG & 1 \\
\hline Benzo(k)Fluoranthene & 330 & UQ/KG & $u$ & NP & & & O UGKG & 1 \\
\hline Benzo(a)Pyrene & 330 & UG/Ka & $u$ & NP & & & O UG/KG & 1 \\
\hline Indeno(1,2,3-CD)Pyrene & 330 & UG/KG & $u$ & NP & & & O UG/KG & 1 \\
\hline Dibenzola.h)Anthracene & 330 & UG/KG & $u$ & NP & & & O UG/KG & 1 \\
\hline Benzo(g,h,i)Perylene & 330 & UG/KO & $u$ & NP & & & O UG/KG & 1 \\
\hline Unknown-1 & 140 & UG/KG & J & NP & & & UG/KG & 1 \\
\hline Unknown-2 & 82 & UGKG & J & NP & & & UGKG & 1 \\
\hline Unknown $\mathrm{C} 11 \mathrm{H} 10$ & 120 & UG/KO & J & NP & & & VG/KG & 1 \\
\hline Unknown-3 & 170 & UaKG & J & NP & & & UGKG & 1 \\
\hline Unknown $\mathrm{C} 12 \mathrm{H} 12-1$ & 83 & VaKKa & J & NP & & & UG/KG & 1 \\
\hline Unknown $\mathrm{C} 12 \mathrm{H} 12-2$ & 84 & UG/KG & J & NP & & & UG/KG & 1 \\
\hline Unknown C15H32 & 100 & UGKG & J & NP & & & UG/Ka & 1 \\
\hline Unknown-4 & 89 & UG/KG & J & NP & & & UGKG & 1 \\
\hline Unknown-5 & 93 & $U G / K G$ & j & NP & & & UG/KG & 1 \\
\hline Unknown-6 & 68 & UG/KG & J & NP & & & UG/KG & 1 \\
\hline Unknown-7 & 74 & UG/KG & J & NP & & & UG/KG & 1 \\
\hline Nitrobenzene-d5 & 71 & $\%$ & & & & & $\%$ & 1 \\
\hline 2-Fluorobiphenyl & 67 & $\%$ & & & & & $\%$ & 1 \\
\hline Terphenyl-d14 & 60 & $\%$ & & & & & $\%$ & 1 \\
\hline Phenol-d5 & 68 & $\%$ & & & & & $\%$ & 1 \\
\hline 2-Fluorophenol & 34 & $\%$ & & & & & $\%$ & 1 \\
\hline 2, 4, 6-Tribromophenol & 35 & $\%$ & & & & & $\%$ & 1 \\
\hline
\end{tabular}


Appendix D: Results of Semivolatile Organic Compound Analyses, Salmon Site

\begin{tabular}{|c|c|c|c|c|c|c|c|}
\hline COMPOUND & RESULTS & UNITS & $\begin{array}{l}\text { DATA } \\
\text { QUAL }\end{array}$ & $\begin{array}{l}\text { DATA } \\
\text { VALID. }\end{array}$ & NOTES & CRDL UNITS & DILUTION \\
\hline \multicolumn{8}{|l|}{ THI4-ES-90-1200-3 } \\
\hline Phenol & 330 & UG/KG & $u$ & NP & & 330 UG/KG & 1 \\
\hline bis(2-Chloroethyl) ether & 330 & UQKG & $u$ & NP & & 330 UG/KG & 1 \\
\hline 2.Chlorophenol & 330 & UG/KO & $u$ & NP & & 330 UG/KG & 1 \\
\hline 1. 3-Dichlorobenzene & 330 & UG/KO & $u$ & NP & & 330 UG/KG & 1 \\
\hline 1. 4-Dichlorobenzene & 330 & UG/KG & $u$ & NP & & 330 va/Ka & 1 \\
\hline Benzyl Alcohol & 330 & vako & $u$ & NP & & 330 va/ka & 1 \\
\hline 1.2-Dichlorobenzene & 330 & vako & $u$ & NP & & 330 Ua/KG & 1 \\
\hline 2-Methylphenol & 330 & UG/KO & $u$ & NP & & 330 vaKG & 1 \\
\hline bis(2-Chloroisopropyl) ether & 330 & UGKG & $u$ & NP & & 330 UG/KO & 1 \\
\hline 4-Mothylphenol & 330 & UG/KO & $u$ & NP & & 330 UG/KO & 1 \\
\hline N-Nitroso-di-nPropylamine & 330 & UGKO & $u$ & NP & & $330 \cup G / K a$ & 1 \\
\hline Hexachloroethane & 330 & va/Ka & $u$ & NP & & 330 UG/KG & 1 \\
\hline Nitrobenzene & 330 & va/Ka & $u$ & NP & & 330 UG/KO & 1 \\
\hline Isophorone & 330 & UaKo & $u$ & NP & & 330 Ua/Ko & 1 \\
\hline 2-Nitrophenol & 330 & UG/KG & $u$ & NP & & 330 UG/KG & 1 \\
\hline 2. 4-Dimethylphenol & 330 & vako & $u$ & NP & & 330 UG/KG & 1 \\
\hline Benzoic Acid & 1600 & UGKO & $u$ & NP & & 1600 UaKG & 1 \\
\hline bis(2Chloroethoxy)methane & 330 & VOKG & $u$ & NP & & 330 UG/KG & 1 \\
\hline 2, 4-Dichlorophenol & 330 & VGKo & u & NP & & 330 vaKo & 1 \\
\hline 1. 2, 4-Trichlorobenzene & 330 & VGKa & $u$ & NP & & 330 UaKG & 1 \\
\hline Naphthalene & 330 & vaKa & $u$ & NP & & 330 Ua/KG & 1 \\
\hline 4-Chloroaniline & 330 & VGKG & $u$ & NP & & 330 va/KG & 1 \\
\hline Hexachlorobutadiene & 330 & UaKG & $u$ & NP & & 330 UGKO & 1 \\
\hline 4-Chloro-3-Methylphenol & 330 & UG/KG & $u$ & NP & & 330 UG/Ka & 1 \\
\hline 2-Methyinaphthalene & 330 & UG/KG & $u$ & NP & & 330 UG/KG & 1 \\
\hline Hexachlorocyclopentadiene & 330 & vaKo & $u$ & NP & & 330 UG/KG & 1 \\
\hline 2. 4, 6-Trichlorophenol & 330 & VaKa & $u$ & NP & & 330 VG/KG & 1 \\
\hline 2, 4, 5-Trichlorophenol & 1600 & vaKo & $u$ & NP & & 1600 vaKa & 1 \\
\hline 2-Chloronaphthalene & 330 & UGKG & $u$ & NP & & 330 Uaka & 1 \\
\hline 2-Nitroaniline & 1600 & vaKa & $u$ & NP & & 1600 Ua/KO & 1 \\
\hline Dimethyl Phthalate & 330 & UGKG & $u$ & NP & & 330 UG/KG & 1 \\
\hline Acenaphthylene & 330 & UGKG & $u$ & NP & & 330 UGKO & 1 \\
\hline 2, 6-Dinitrotoluene & 330 & UG/KG & $u$ & NP & & 330 UG/KO & 1 \\
\hline 3-Nitroaniline & 1600 & UEKG & $u$ & NP & & 1600 Ua/KG & 1 \\
\hline Acenaphthene & 330 & UG/Ko & $u$ & NP & & 330 UG/KG & 1 \\
\hline 2, 4-Dinitrophenol & 1600 & UGKO & $u$ & NP & & 1600 UG/KG & 1 \\
\hline 4-Nitrophenol & 1600 & UG/KQ & $u$ & NP & & 1600 UG/KG & 1 \\
\hline Dibenzofuran & 330 & UG/KG & $u$ & NP & & 330 UG/KG & 1 \\
\hline 2, 4-Dinitrotoluene & 330 & UG/Ka & $u$ & NP & & 330 UG/KG & 1 \\
\hline Diethylphthalate & 330 & UGKG & $u$ & NP & & 330 UGKG & 1 \\
\hline 4-Chlorophenyl phenyl ether & 330 & UaKa & $u$ & NP & & 330 Ua/KG & 1 \\
\hline Fluorene & 330 & vaKa & $u$ & NP & & 330 UG/Ka & 1 \\
\hline 4-Nitroaniline & 1600 & UaKo & $u$ & NP & & 1600 va/Ko & 1 \\
\hline 4, 6-Dinitro-2-Methylphenol & 1600 & VaKo & $u$ & NP & & 1600 va/Ko & 1 \\
\hline N-Nitrosodiphenylamine & 330 & UaKa & $u$ & NP & & 330 Ua/Ko & 1 \\
\hline 4-Bromophenyl phenyl ether & 330 & VaKo & u & NP & & 330 va/Ka & 1 \\
\hline Hexachlorobenzene & 330 & UaKo & $u$ & NP & & 330 UG/KG & 1 \\
\hline Pentachlorophenol & 1600 & VGKG & $u$ & NP & & 1600 UG/KG & 1 \\
\hline Phenanthrene & 330 & UaKG & $U$ & NP & & 330 UG/KG & 1 \\
\hline
\end{tabular}


Appendix D: Results of Semivolatile Organic Compound Analyses. Salmon Site

\begin{tabular}{|c|c|c|c|c|c|c|c|c|}
\hline COMPOUND & RESULTS & UNITS & $\begin{array}{l}\text { DATA } \\
\text { QUAL. }\end{array}$ & $\begin{array}{l}\text { DATA } \\
\text { VALID. }\end{array}$ & NOTES & CADL & UNITS & DILUTION \\
\hline \multicolumn{9}{|l|}{ TR14-ES-90-1200-3 (CON'T) } \\
\hline Anthracene & 330 & UG/KG & $u$ & NP & & & UG/KG & 1 \\
\hline Di-N-Butylphthalate & 330 & UaKa & $u$ & NP & & & UG/KG & 1 \\
\hline Fluoranthene & 330 & Ua/KO & $u$ & NP & & & va/ka & 1 \\
\hline Pyrene & 330 & va/ko & $u$ & NP & & & Ua/Ko & 1 \\
\hline Butyl Benzyl Phthalate & 330 & UG/Ka & $u$ & NP & & & UG/KG & 1 \\
\hline 3. 3'-Dichlorobenzidine & 660 & Ua/KO & $u$ & NP & & & UG/KG & 1 \\
\hline Benzo(a)Anthracene & 330 & Ua/KO & $u$ & NP & & & Va/KG & 1 \\
\hline Chrysene & 330 & va/KG & $u$ & NP & & & UG/KG & 1 \\
\hline bis(2-Ethylhexyl)phthalate & 38 & Ua/KG & J & NP & & & UG/KG & 1 \\
\hline di-N-Octyl Phthalate & 330 & UG/KG & $u$ & NP & & & UG/KG & 1 \\
\hline Benzo(b)Fluoranthene & 330 & UG/KG & $u$ & NP & & & UG/KG & 1 \\
\hline Benzo(k)Fluoranthene & 330 & VGKO & $u$ & NP & & & O VG/Ka & 1 \\
\hline Benzo(a)Pyrene & 330 & UQ/KG & $u$ & NP & & & O UG/KO & 1 \\
\hline Indeno(1,2,3-CD)Pyrene & 330 & UG/KO & $u$ & NP & & & 0 Ua/KG & 1 \\
\hline Dibenzo(a.h)Anthracene & 330 & Ua/KG & $u$ & NP & & & O UG/KG & 1 \\
\hline Benzo(g,h,i)Perylent & 330 & UG/KG & $u$ & NP & & & O UG/KG & 1 \\
\hline Nitrobenzene-d5 & 71 & $\%$ & & & & & $\%$ & 1 \\
\hline 2-Fluorobiphenyl & 68 & $\because$ & & & & & $\%$ & 1 \\
\hline Terphenyl-d14 & 58 & 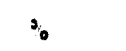 & & & & & $\%$ & 1 \\
\hline Phenol-d5 & 68 & $\%$ & & & & & $\%$ & 1 \\
\hline 2-Fluorophenol & 58 & $\%$ & & & & & $\%$ & 1 \\
\hline 2, 4, 6-Tribromophenol & 40 & $\because$ & & & & & $\%$ & 1 \\
\hline
\end{tabular}


Appendix D: Results of Semivolatile Organic Compound Analyses, Salmon Site

\begin{tabular}{|c|c|c|c|c|c|c|c|}
\hline COMPOUND & RESULTS & UNITS & $\begin{array}{l}\text { DATA } \\
\text { QUAL }\end{array}$ & $\begin{array}{l}\text { DATA } \\
\text { VALID. }\end{array}$ & NOTES & CRDL UNITS & DILUTION \\
\hline \multicolumn{8}{|l|}{ TR-15-ES-20-1180-.5 } \\
\hline Phenol & 330 & VG/KG & $u$ & NP & & 330 UG/KG & 1 \\
\hline bis(2-Chloroethyl) ether & 330 & VGKG & $u$ & NP & & 330 UG/KG & 1 \\
\hline 2-Chlorophenol & 330 & UG/Ko & $u$ & NP & & 330 UG/KG & 1 \\
\hline 1. 3-Dichlorobenzene & 330 & UG/KG & $u$ & NP & & 330 UG/KG & 1 \\
\hline 1. 4-Dichlorobenzene & 330 & UG/KG & $u$ & NP & & 330 UG/KG & 1 \\
\hline Benzyl Alcohol & 330 & UG/KG & $u$ & NP & & 330 UG/KG & 1 \\
\hline 1. 2-Dichlorobenzene & 330 & UG/KG & $u$ & NP & & 330 UG/KG & 1 \\
\hline 2-Methylphenol & 330 & UGKG & $u$ & NP & & 330 UG/KG & 1 \\
\hline bis(2-Chloroisopropyl) ether & 330 & UG/KG & $u$ & NP & & 330 UG/KG & 1 \\
\hline 4-Methylphenol & 330 & UG/KG & $u$ & NP & & 330 UG/KG & 1 \\
\hline N-Nitroso-dl-nPropylamine & 330 & UG/KG & $u$ & NP & & 330 UG/KG & 1 \\
\hline Hexachloroethane & 330 & UG/KG & $u$ & NP & & 330 Ua/KG & 1 \\
\hline Nitrobenzıne & 330 & UGKG & $u$ & NP & & 330 UG/KG & 1 \\
\hline Isophorone & 330 & UaKG & $u$ & NP & & 330 UG/KG & 1 \\
\hline 2-Nitrophenol & 330 & UG/KG & $u$ & NP & & 330 UG/KG & 1 \\
\hline 2. 4-Dimethylphenol & 330 & UGKG & $u$ & NP & & 330 UG/KG & 1 \\
\hline Benzoic Acid & 1600 & UG/KG & $u$ & NP & & 1600 UG/KG & 1 \\
\hline bis(2Chloroethoxy)methane & 330 & UGKG & $u$ & NP & & 330 UG/KG & 1 \\
\hline 2, 4-Dichlorophenol & 330 & UQKG & $u$ & NP & & 330 UG/KG & 1 \\
\hline 1, 2, 4-Trichlorobenzene & 330 & UG/KG & $u$ & NP & & 330 UG/KG & 1 \\
\hline Naphthalene & 330 & UG/KG & $u$ & NP & & 330 UG/KG & 1 \\
\hline 4-Chloroaniline & 330 & UG/KG & $u$ & NP & & 330 UG/KG & 1 \\
\hline Hexachlorobutadiene & 330 & UG/KG & $u$ & NP & & 330 UG/KG & 1 \\
\hline 4-Chloro-3-Methylphenol & 330 & UG/KG & u & NP & & $330 \cup G / K G$ & 1 \\
\hline 2-Methyinaphthalene & 330 & VG/KG & $u$ & NP & & 330 UG/KG & 1 \\
\hline Hexachlorocyclopentadiene & 330 & Ua/KG & $u$ & NP & & 330 UG/KG & 1 \\
\hline 2, 4, 6-Trichlorophenol & 330 & UG/KG & $u$ & NP & & 330 UG/KG & 1 \\
\hline 2, 4, 5-Trichlorophenol & 1600 & UG/KG & $u$ & NP & & 1600 UG/KG & 1 \\
\hline 2-Chloronaphthalene & 330 & UG/KG & $u$ & NP & & 330 UG/KG & 1 \\
\hline 2-Nitroaniline & 1600 & UGKG & $u$ & NP & & 1600 UG/KG & 1 \\
\hline Dimethyl Phthalate & 330 & UGKG & $u$ & NP & & 330 UG/KG & 1 \\
\hline Acenaphthylene & 330 & UGKG & $u$ & NP & & 330 UG/KG & 1 \\
\hline 2, 6-Dinitrotoluene & 330 & UGKG & u & NP & & 330 UG/KG & 1 \\
\hline 3-Nitroaniline & 1600 & UGKG & $u$ & NP & & 1600 Ua/KG & 1 \\
\hline Acenaphthene & 330 & UG/KG & $u$ & NP & & 330 UG/KG & 1 \\
\hline 2. 4-Dinitrophenol & 1600 & UGKG & $u$ & NP & & 1600 Ua/KG & 1 \\
\hline 4-Nitrophenol & 1600 & UG/KG & $u$ & NP & & 1600 Ua/KG & 1 \\
\hline Dibenzoturan & 330 & UG/KG & $u$ & NP & & 330 UG/KG & 1 \\
\hline 2, 4-Dinitrotoluene & 330 & UGKG & $u$ & NP & & 330 Ua/KG & 1 \\
\hline Diethylphthalate & 330 & UG/KG & $u$ & NP & & 330 UG/KG & 1 \\
\hline 4-Chlorophenyl phenyl ether & 330 & UGKG & $u$ & NP & & 330 UG/KG & 1 \\
\hline Fluorene & 330 & Ua/KG & $u$ & NP & & 330 UG/KG & 1 \\
\hline 4-Nitroaniline & 1600 & UGKa & $u$ & NP & & 1600 UG/KG & 1 \\
\hline 4, 6-Dinitro-2-Methylphenol & 1600 & UGKG & $u$ & NP & & 1600 UG/KG & 1 \\
\hline N-Nitrosodiphenylamine & 330 & UGKG & $u$ & NP & & 330 Ua/KG & 1 \\
\hline 4-Bromophenyl phenyl ether & 330 & UGKG & $u$ & NP & & 330 UG/KG & 1 \\
\hline Hexachlorobenzene & 330 & UG/KG & $u$ & NP & & 330 UG/KG & 1 \\
\hline Pentachlorophenol & 1600 & UGKG & $u$ & NP & & 1600 UG/KG & 1 \\
\hline Phenanthrene & 330 & UGKG & $u$ & NP & & 330 UG/KG & 1 \\
\hline
\end{tabular}




\begin{tabular}{|c|c|c|c|c|c|c|c|c|}
\hline COMPOUND & RESULTS & UNITS & $\begin{array}{l}\text { DATA } \\
\text { QUAL }\end{array}$ & $\begin{array}{l}\text { DATA } \\
\text { VALID. }\end{array}$ & NOTES & CROL & UNITS & DILUTION \\
\hline \multicolumn{9}{|l|}{ TR-15-ES-20-1180-.5 (CON'T) } \\
\hline Anthracene & 330 & UG/KG & $u$ & NP & & & UG/KG & 1 \\
\hline DI-N-Butylphthalate & 330 & UG/KG & $u$ & NP & & & UG/KG & 1 \\
\hline Fluoranthene & 330 & UG/KG & u & NP & & & UG/KG & 1 \\
\hline Pyrene & 330 & UG/KG & u & NP & & & UG/KG & 1 \\
\hline Butyl Benzyl Phthalate & 330 & $U G / K G$ & $u$ & NP & & & U U/KG & 1 \\
\hline 3. 3'-Dichlorobenzidine & 660 & UG/KG & $u$ & NP & & & D UG/KG & 1 \\
\hline Benzo(a)Anthracene & 330 & Ua/KG & $u$ & NP & & & O UG/KG & 1 \\
\hline Chrysene & 330 & UG/KG & $u$ & NP & & & O UG/KG & 1 \\
\hline bis(2-Ethylhexyl)phthalate & 330 & UG/KG & $u$ & NP & & & O UG/KG & 1 \\
\hline di-N-Octyl Phthalate & 330 & UG/KG & $u$ & NP & & & UG/KG & 1 \\
\hline Benzo(b)Fluoranthene & 330 & UG/KG & $u$ & NP & & & VG/KG & 1 \\
\hline Benzo(k)Fluoranthene & 330 & UG/KG & $u$ & NP & & & UG/KG & 1 \\
\hline Benzo(a)Pyrene & 330 & UG/KG & $u$ & NP & & & U UG/KG & 1 \\
\hline Indeno(1,2,3-CD)Pyrene & 330 & VG/KG & $u$ & NP & & & O UG/KG & 1 \\
\hline Dibenzo(a.h)Anthracene & 330 & UG/KG & $u$ & NP & & & O UG/KG & 1 \\
\hline Benzo(g,h,i)Perylene & 330 & UG/KG & $u$ & NP & & & O UG/KG & 1 \\
\hline Pentadecane & 220 & UG/KG & J & NP & & & UG/KG & 1 \\
\hline Unknown-1 & 140 & UGiKG & J & NP & & & UG/KG & 1 \\
\hline Unknown-2 & 190 & UG/KG & J & NP & & & UG/KG & 1 \\
\hline Hexadecane & 390 & UG/KG & J & NP & & & UG/KG & 1 \\
\hline Pentadecane, 2,6,10,14-tetrame & 1500 & UG/KG & J & NP & & & UG/KG & 1 \\
\hline Unknown-3 & 160 & UG/KG & J & NP & & & UG/KG & 1 \\
\hline Hexadecane,2,6,10,14,-tetrame & 840 & UG/KG & J & NP & & & UG/KG & 1 \\
\hline Unknown-4 & 210 & UG/KG & J & NP & & & UG/KG & 1 \\
\hline Unknown-5 & 110 & UG/KG & J & NP & & & UG/KG & 1 \\
\hline Unknown-6 & 270 & UG/KG & J & NP & & & UG/KG & 1 \\
\hline Nonadecane & 580 & UG/KG & J & NP & & & UG/KG & 1 \\
\hline Unknown-7 & 160 & UG/KG & J & NP & & & UG/KG & 1 \\
\hline Unknown-8 & 190 & UG/KG & J & NP & & & UG/KG & 1 \\
\hline Unknown-9 & 120 & UG/KG & Ј & NP & & & UG/KG & 1 \\
\hline Eicosane & 380 & UG/KG & j & NP & & & UG/KG & 1 \\
\hline Heneicosane & 150 & UG/KG & J & NP & & & UG/KG & 1 \\
\hline Unknown-10 & 110 & UG/KG & J & NP & & & UG/KG & 1 \\
\hline Unknown-11 & 220 & UG/KG & BJ & NP & & & UG/KG & 1 \\
\hline Unknown-12 & 140 & UG/KG & J & NP & & & UG/KG & 1 \\
\hline Unknown-13 & 1300 & UG/KG & J & NP & & & UG/KG & 1 \\
\hline Unknown-14 & 110 & UG/KG & J & NP & & & UG/KG & 1 \\
\hline Unknown-15 & 130 & UG/KG & $\mathrm{J}$ & NP & & & UG/KG & 1 \\
\hline Nitrobenzene-d5 & 82 & $\%$ & & & & & $\%$ & 1 \\
\hline 2-Fluorobiphenyl & 75 & $\%$ & & & & & $\%$ & 1 \\
\hline Terphenyl-d14 & 68 & $\%$ & & & & & $\%$ & 1 \\
\hline Phenol-d5 & 78 & $\%$ & & & & & $\%$ & 1 \\
\hline 2-Fluorophenol & 75 & $\%$ & & & & & $\%$ & 1 \\
\hline 2, 4, 6-Tribromophenol & 91 & $\%$ & & & & & $\%$ & 1 \\
\hline
\end{tabular}


Appendix D: Results of Semivolatile Organic Compound Analyses, Salmon Site

\begin{tabular}{|c|c|c|c|c|c|c|c|c|}
\hline COMPOUND & RESULTS & UNITS & $\begin{array}{l}\text { DATA } \\
\text { QUAL }\end{array}$ & $\begin{array}{l}\text { DATA } \\
\text { VALID. }\end{array}$ & NOTES & CRDL & UNITS & DILUTION \\
\hline \multicolumn{9}{|l|}{ TR15-ES-20-1180-.5 MS } \\
\hline Phenol & 66 & $\%$ & & NP & & & $\%$ & 1 \\
\hline 2-Chlorophenol & 70 & $\%$ & & NP & & & $\%$ & 1 \\
\hline 1, 4-Dichiorobenzene & 65 & $\%$ & & NP & & & $\%$ & 1 \\
\hline N-Nitroso-di-nPropylamine & 74 & $\%$ & & NP & & & $\%$ & 1 \\
\hline 1, 2, 4-Trichlorobenzene & 66 & $\%$ & & NP & & & $\%$ & 1 \\
\hline 4-Chloro-3-Methylphenol & 75 & $\%$ & & NP & & & $\%$ & 1 \\
\hline Acenaphthene & 63 & $\%$ & & NP & & & $\%$ & 1 \\
\hline 4-Nitrophenol & 90 & $\%$ & & NP & & & $\%$ & 1 \\
\hline 2. 4-Dinitrotoluene & 73 & $\%$ & & NP & & & $\%$ & 1 \\
\hline Pentachlorophenol & 42 & $\%$ & & NP & & & $\%$ & 1 \\
\hline Pyrene & 62 & $\%$ & & NP & & & $\%$ & 1 \\
\hline Nitrobenzene-d5 & 75 & $\%$ & & NP & & & $\%$ & 1 \\
\hline 2-Fluorobiphenyl & 71 & $\%$ & & NP & & & $\%$ & 1 \\
\hline Terpinenyl-d14 & 64 & $\%$ & & NP & & & $\%$ & 1 \\
\hline Phenol-d5 & 74 & $\%$ & & NP & & & $\%$ & 1 \\
\hline 2-Fluorophenol & 70 & $\%$ & & NP & & & $\%$ & 1 \\
\hline 2. 4,6-Tribromophenol & 82 & $\%$ & & NP & & & $\%$ & 1 \\
\hline \multicolumn{9}{|l|}{ TR15-ES-20-1180-.5 MSD } \\
\hline Phenol & 68 & $\%$ & & NP & & & $\%$ & 1 \\
\hline 2-Chlorophenol & 68 & $\%$ & & NP & & & $\%$ & 1 \\
\hline 1, 4-Dichlorobenzene & 61 & $\%$ & & NP & & & $\%$ & 1 \\
\hline N-Nitroso-di-nPropylamine & 71 & $\%$ & & NP & & & $\%$ & 1 \\
\hline 1, 2, 4-Trichlorobenzene & 66 & $\%$ & & NP & · & & $\%$ & 1 \\
\hline 4-Chloro-3-Methylphenol & 73 & $\%$ & & NP & & & $\%$ & 1 \\
\hline Acenaphthene & 66 & $\%$ & & NP & & & $\%$ & 1 \\
\hline 4-Nitrophenol & 116 & $\%$ & • & NP & & & $\%$ & 1 \\
\hline 2, 4-Dinitrotoluene & 72 & $\%$ & & NP & & & $\%$ & 1 \\
\hline Pentachlorophenol & 51 & $\%$ & & NP & & & $\%$ & 1 \\
\hline Pyrene & 62 & $\%$ & & NP & & & $\%$ & 1 \\
\hline Nitrobenzene-d5 & 74 & $\%$ & & NP & & & $\%$ & 1 \\
\hline 2-Fluorobiphenyi & 71 & $\%$ & & NP & & & $\%$ & 1 \\
\hline Terphenyl-d14 & 64 & $\%$ & & NP & & & $\%$ & 1 \\
\hline Phenol-d5 & 74 & $\%$ & & NP & & & $\%$ & 1 \\
\hline 2-Fluorophenol & 68 & $\%$ & & NP & & & $\%$ & 1 \\
\hline 2, 4, 6-Tribromophenol & 80 & $\%$ & & NP & & & $\%$ & 1 \\
\hline
\end{tabular}


Appendix D: Results of Semivolatile Organic Compound Analyses, Salmon Site

\begin{tabular}{|c|c|c|c|c|c|c|c|}
\hline COMPOUND & RESULTS & UNITS & $\begin{array}{l}\text { DATA } \\
\text { QUAL }\end{array}$ & $\begin{array}{l}\text { DATA } \\
\text { VALID. }\end{array}$ & NOTES & CRDL UNITS & DILUTION \\
\hline \multicolumn{8}{|l|}{ TR16-ES-200-80-1.5 } \\
\hline Phenol & 330 & UG/KG & $u$ & NP & & 330 UG/KG & 1 \\
\hline bis(2-Chloroethyl) ether & 330 & UG/KG & $u$ & NP & & 330 UG/KG & 1 \\
\hline 2-Chlorophenol & 330 & UG/KG & $u$ & NP & & 330 UG/KG & 1 \\
\hline 1. 3-Dichlorobenzene & 330 & UG/KG & $u$ & NP & & 330 UG/KG & 1 \\
\hline 1, 4-Dichlorobenzene & 330 & UG/KG & $u$ & NP & & 330 UG/KG & 1 \\
\hline Benzyl Alcohol & 330 & UG/KG & $u$ & NP & & 330 UG/KG & 1 \\
\hline 1, 2-Dichlorobenzene & 330 & UG/KG & $u$ & NP & & 330 UG/KG & 1 \\
\hline 2-Methylphenol & 330 & UG/KG & $u$ & NP & & 330 UG/KG & 1 \\
\hline bis(2-Chloroisopropyl) ether & 330 & UG/KG & $u$ & NP & & 330 UG/KG & 1 \\
\hline 4-Methyiphenol & 330 & UG/KG & $u$ & NP & & 330 UG/KG & 1 \\
\hline N-Nitroso-di-nPropylamine & 330 & UG/KG & $u$ & NP & & 330 UG/KG & 1 \\
\hline Hexachloroethane & 330 & UG/KG & $u$ & NP & & 330 UG/KG & 1 \\
\hline Nitrobenzene & 330 & UG/KG & $u$ & NP & & 330 UG/KG & 1 \\
\hline Isophorone & 330 & UG/KG & $u$ & NP & & 330 UG/KG & 1 \\
\hline 2-Nitrophenol & 330 & UG/KG & $u$ & NP & & 330 UG/KG & 1 \\
\hline 2, 4-Dimethylphenol & 330 & UG/KG & $u$ & NP & & 330 UG/KG & 1 \\
\hline Benzoic Acid & 1600 & UG/KG & $u$ & NP & & 1600 UG/KG & 1 \\
\hline bis(2Chloroethoxy)methane & 330 & UG/KG & $u$ & NP & & 330 UG/KG & 1 \\
\hline 2, 4-Dichlorophenol & 330 & UG/KG & $u$ & NP & & 330 UG/KG & 1 \\
\hline 1, 2. 4-Trichlorobenzene & 330 & UG/KG & $u$ & NP & & 330 UG/KG & 1 \\
\hline Naphthalene & 330 & UG/KG & $u$ & NP & & 330 UG/KG & 1 \\
\hline 4-Chloroaniline & 330 & UG/KG & $u$ & NP & & 330 UG/KG & 1 \\
\hline Hexachlorobutadiene & 330 & UG/KG & $u$ & NP & & 330 UG/KG & 1 \\
\hline 4-Chloro-3-Methyiphenol & 330 & UG/KG & $u$ & NP & & 330 UG/KG & 1 \\
\hline 2-Methyinaphthalene & 330 & UG/KG & $u$ & NP & & 330 UG/KG & 1 \\
\hline Hexachlorocyclopentadiene & 330 & UG/KG & $u$ & NP & & 330 UG/KG & 1 \\
\hline 2, 4, 6-Trichlorophenol & 330 & UG/KG & $u$ & NP & & 330 UG/KG & 1 \\
\hline 2, 4, 5-Trichlorophenol & 1600 & UG/KG & $u$ & NP & & 1600 UG/KG & 1 \\
\hline 2-Chloronaphthalene & 330 & UG/KG & $u$ & NP & & 330 UG/KG & 1 \\
\hline 2-Nitroaniline & 1600 & UG/KG & $u$ & NP & & 1600 UG/KG & 1 \\
\hline Dimethyl Phthalate & 330 & UG/KG & $u$ & NP & & 330 UG/KG & 1 \\
\hline Acenaphthylene & 330 & UG/KG & $u$ & NP & & 330 UG/KG & 1 \\
\hline 2, 6-Dinitrotoluene & 330 & UGKG & $u$ & NP & & 330 UG/KG & 1 \\
\hline 3-Nitroaniline & 1600 & UGKG & $u$ & NP & & 1600 UG/KG & 1 \\
\hline Acenaphthene & 330 & UG/KG & $u$ & NP & & 330 UG/KG & 1 \\
\hline 2, 4-Dinitrophenol & 1600 & UG/KG & $u$ & NP & & 1600 UG/KG & 1 \\
\hline 4-Nitrophenol & 1600 & UG/KG & $u$ & NP & & 1600 UG/KG & 1 \\
\hline Dibenzofuran & 330 & UG/KG & $u$ & NP & & 330 UG/KG & 1 \\
\hline 2, 4-Dinitrotoluene & 330 & UGKG & $u$ & NP & & 330 UG/KG & 1 \\
\hline Diethylphthalate & 330 & UGKG & $u$ & NP & & 330 UG/KG & 1 \\
\hline 4-Chlorophenyl phenyl ether & 330 & UG/KG & $u$ & NP & & 330 UG/KG & 1 \\
\hline Fluorene & 330 & UG/KG & $u$ & NP & & 330 UG/KG & 1 \\
\hline 4-Nitroaniline & 1600 & UGKG & $u$ & NP & & 1600 UG/KG & 1 \\
\hline 4, 6-Dinitro-2-Methylphenol & 1600 & UGKG & $u$ & NP & & 1600 UG/KG & 1 \\
\hline N-Nitrosodiphenylamine & 330 & UG/KG & $u$ & NP & & 330 UG/KG & 1 \\
\hline 4-Bromophenyl phenyl ether & 330 & UG/KG & $u$ & NP & & 330 UG/KG & 1 \\
\hline Hexachlorobenzene & 330 & UG/KG & $u$ & NP & & 330 UG/KG & 1 \\
\hline Pentachlorophenol & 1600 & UG/KG & $u$ & NP & & 1600 UG/KG & 1 \\
\hline Phenanthrene & 76 & UG/KG & J & NP & & 330 UG/KG & 1 \\
\hline
\end{tabular}


Appendix D: Results of Semivolatile Organic Compound Analyses, Salmon Site

\begin{tabular}{|c|c|c|c|c|c|c|c|c|}
\hline COMPOUND & RESULTS & UNITS & $\begin{array}{l}\text { DATA } \\
\text { QUAL }\end{array}$ & $\begin{array}{l}\text { DATA } \\
\text { VALID. }\end{array}$ & NOTES & CRDL & UNITS & DILUTION \\
\hline \multicolumn{9}{|c|}{ TR16-ES-200-80-1.5 (CON'T) } \\
\hline Anthracene & 330 & UG/KG & $u$ & NP & & 330 & $O U G / K G$ & 1 \\
\hline Di-N-Butylphthalate & 330 & UG/KG & $u$ & NP & & 330 & O UG/KG & 1 \\
\hline Fluoranthene & 74 & UG/KG & j & NP & & 330 & O UG/KG & 1 \\
\hline Pyrene & 46 & UG/KG & J & NP & & 330 & 0 UG/KG & 1 \\
\hline Butyl Benzyl Phthalate & 330 & UG/KG & $u$ & $\mathrm{NP}$ & & 330 & O UG/KG & 1 \\
\hline 3, 3'-Dichlorobenzidine & 660 & UG/KG & $u$ & NP & & 660 & O UG/KG & 1 \\
\hline Benzo(a)Anthracene & 330 & UG/KG & $u$ & NP & & 330 & UG/KG & 1 \\
\hline Chrysene & 330 & UG/KG & $u$ & NP & & 330 & UG/KG & 1 \\
\hline bis(2-Ethylhexyl)phthalate & 77 & UG/KG & $\mathrm{J}$ & NP & & 330 & UG/KG & 1 \\
\hline di-N-Octyl Phthalate & 330 & UG/KG & $u$ & NP & & 330 & UG/KG & 1 \\
\hline Benzo(b)Fluoranthene & 330 & UG/KG & $u$ & NP & & 330 & O UG/KG & 1 \\
\hline Benzo(k)Fluoranthene & 330 & UG/KG & $u$ & NP & & 330 & O UG/KG & 1 \\
\hline Benzo(a)Pyrene & 330 & UG/KG & $u$ & NP & & 330 & $O$ UG/KG & 1 \\
\hline Indeno(1,2,3-CD)Pyrene & 330 & UG/KG & $u$ & NP & & 330 & UG/KG & 1 \\
\hline Dibenzo(a.h)Anthracene & 330 & UG/KG & $u$ & NP & & & 0 UG/KG & 1 \\
\hline Benzo(g,h,i)Perylene & 330 & UG/KG & $u$ & NP & & & 0 UG/KG & 1 \\
\hline Unknown-1 & 250 & UG/KG & $\mathrm{J}$ & NP & & & UG/KG & 1 \\
\hline Unknown-2 & 220 & UG/KG & J & NP & & & UG/KG & 1 \\
\hline Unknown-3 & 280 & UG/KG & J & NP & & & UG/KG & 1 \\
\hline Unknown-4 & 75 & UG/KG & J & NP & & & UG/KG & 1 \\
\hline Unknown-5 & 310 & UG/KG & $\mathrm{J}$ & NP & & & UG/KG & 1 \\
\hline Unknown-6 & 200 & UG/KG & J & NP & & & UG/KG & 1 \\
\hline Unknown-7 & 180 & UG/KG & J & NP & & & UG/KG & 1 \\
\hline Unknown-8 & 70 & UG/KG & BJ & NP & & & UG/KG & 1 \\
\hline Unknown-9 & 75 & UG/KG & $\mathrm{J}$ & NP & & & UG/KG & 1 \\
\hline Nitrobenzene-d5 & 78 & $\%$ & & & & & $\%$ & 1 \\
\hline 2-Fluorobiphenyl & 73 & $\%$ & & & & & $\%$ & 1 \\
\hline Terphenyl-d14 & 64 & $\%$ & & & & & $\%$ & 1 \\
\hline Phenol-d5 & 79 & $\%$ & & & & & $\%$ & 1 \\
\hline 2-Fluorophenol & 71 & $\%$ & & & & & $\%$ & 1 \\
\hline 2. 4, 6-Tribromophenol & 73 & $\%$ & & & & & $\%$ & 1 \\
\hline
\end{tabular}


Appendix D: Results of Semivolatile Organic Compound Analyses, Salmon Site

\begin{tabular}{|c|c|c|c|c|c|c|c|}
\hline COMPOUND & RESULTS & UNITS & $\begin{array}{l}\text { DATA } \\
\text { QUAL }\end{array}$ & $\begin{array}{l}\text { DATA } \\
\text { VALID. }\end{array}$ & NOTES & CRDL UNITS & DILUTION \\
\hline \multicolumn{8}{|l|}{ TA17-ES-30-120-5 } \\
\hline Phenol & 330 & UG/KG & $u$ & NP & & 330 UG/KG & 1 \\
\hline bis(2-Chloroethyl) ether & 330 & UG/KG & $u$ & NP & & 330 UG/KG & 1 \\
\hline 2-Chlorophenol & 330 & UG/KG & $u$ & NP & & 330 UG/KG & 1 \\
\hline 1, 3-Dichlorobenzene & 330 & UG/KG & $u$ & NP & & 330 UG/KG & 1 \\
\hline 1, 4-Dichlorobenzene & 330 & UG/KG & $u$ & NP & & 330 UG/KG & 1 \\
\hline Benzyl Alcohol & 330 & UG/KG & $u$ & NP & & 330 UG/KG & 1 \\
\hline 1, 2-Dichlorobenzene & 330 & UG/KG & $u$ & NP & & 330 UG/KG & 1 \\
\hline 2-Methylphenol & 330 & UG/KG & $u$ & NP & & 330 UG/KG & 1 \\
\hline bis(2-Chloroisopropyl) ether & 330 & UG/KG & $u$ & NP & & 330 UG/KG & 1 \\
\hline 4-Methylphenol & 330 & UG/KG & $u$ & NP & & 330 UG/KG & 1 \\
\hline N-Nitroso-di-nPropylamine & 330 & UG/KG & $u$ & NP & & 330 UG/KG & 1 \\
\hline Hexachloroethane & 330 & VG/KG & $u$ & NP & & 330 UG/KG & 1 \\
\hline Nitrobenzene & 330 & UG/KG & $u$ & NP & & 330 UG/KG & 1 \\
\hline Isophorone & 330 & UG/KG & $u$ & NP & & 330 UG/KG & 1 \\
\hline 2-Nitrophenol & 330 & UG/KG & $u$ & NP & & 330 UG/KG & 1 \\
\hline 2. 4-Dimethylphenol & 330 & UG/KG & $u$ & NP & & 330 UG/KG & 1 \\
\hline Benzoic Acid & $160 \mathrm{C}$ & UG/KG & $u$ & NP & & 1600 UG/KG & 1 \\
\hline bis(2Chloroethoxy)methane & 330 & UG/KG & $u$ & NP & & 330 UG/KG & 1 \\
\hline 2, 4-Dichloropnenol & 330 & UG/KG & $u$ & NP & & 330 UG/KG & 1 \\
\hline 1, 2, 4-Trichlorobenzene & 330 & UG/KG & $u$ & NP & & 330 UG/KG & 1 \\
\hline Naphthalene & 330 & UG/KG & $u$ & NP & & 330 UG/KG & 1 \\
\hline 4-Chloroaniline & 330 & UG/KG & $u$ & NP & & 330 UG/KG & 1 \\
\hline Hexachlorobutadiene & 330 & UG/KG & $u$ & NP & & 330 UG/KG & 1 \\
\hline 4-Chloro-3-Methylphenol & 330 & UG/KG & $u$ & NP & & 330 UG/KG & 1 \\
\hline 2-Methyinaphthalene & 330 & UG/KG & $u$ & NP & & 330 UG/KG & 1 \\
\hline Hexachlorocyclopentadiene & 330 & UG/KG & $u$ & NP & & 330 UG/KG & 1 \\
\hline 2, 4, 6-Trichlorophenol & 330 & UG/KG & $u$ & NP & & 330 UG/KG & 1 \\
\hline 2, 4, 5-Trichlorophenol & 1600 & UG/KG & $u$ & NP & & 1600 UG/KG & 1 \\
\hline 2-Chloronaphthalene & 330 & UG/KG & $u$ & NP & & 330 UG/KG & 1 \\
\hline 2-Nitroaniline & 1600 & UG/KG & $u$ & NP & & 1600 UG/KG & 1 \\
\hline Dimethyl Phthalate & 330 & UG/KG & $u$ & NP & & 330 UG/KG & 1 \\
\hline Acenaphthylene & 330 & UG/KG & $u$ & NP & & 330 UG/KG & 1 \\
\hline 2, 6-Dinitrotoluene & 330 & UG/KG & $u$ & NP & & 330 UG/KG & 1 \\
\hline 3-Nitroaniline & 1600 & UGKG & $u$ & NP & & 1600 UG/KG & 1 \\
\hline Acenaphthene & 330 & UG/KG & $u$ & NP & & 330 UG/KG & 1 \\
\hline 2, 4-Dinitrophenol & 1600 & UG/KG & $u$ & NP & & 1600 UG/KG & 1 \\
\hline 4-Nitrophenol & 1600 & UGKG & $u$ & NP & & 1600 UG/KG & 1 \\
\hline Dibenzoifuran & 330 & UG/KG & $u$ & NP & & 330 UG/KG & 1 \\
\hline 2, 4-Dinitrotoluene & 330 & UG/KG & $u$ & NP & & 330 UG/KG & 1 \\
\hline Diethylphthalate & 330 & UG/KG & $u$ & NP & & 330 UG/KG & 1 \\
\hline 4-Chlorophenyl phenyl ether & 330 & UG/KG & $u$ & NP & & 330 UG/KG & 1 \\
\hline Fluorene & 330 & UGKG & $u$ & NP & & 330 UG/KG & 1 \\
\hline 4-Nitroaniline & 1600 & UG/KG & $u$ & NP & & 1600 UG/KG & 1 \\
\hline 4, 6-Dinitro-2-Methylphenol & 1600 & UGKG & $u$ & NP & & 1600 UG/KG & 1 \\
\hline N-Nitrosodiphenylamine & 330 & UGKG & $u$ & NP & & 330 UG/KG & 1 \\
\hline 4-Bromophenyl phenyl ether & 330 & UG/KG & $u$ & NP & & 330 UG/KG & 1 \\
\hline Hexachlorobenzene & 330 & UG/KG & $u$ & NP & & 330 UG/KG & 1 \\
\hline Pentachlorophenol & 1600 & UGKG & $u$ & NP & & 1600 UG/KG & 1 \\
\hline Phenanthrene & 330 & UG/KG & $u$ & NP & & 330 UG/KG & 1 \\
\hline
\end{tabular}


Appendix D: Results of Semivolatile Organic Compound Analyses, Salmon Site

\begin{tabular}{|c|c|c|c|c|c|c|c|c|}
\hline COMPOUND & RESULTS & UNITS & $\begin{array}{l}\text { DATA } \\
\text { QUAL }\end{array}$ & $\begin{array}{l}\text { DATA } \\
\text { VALID. }\end{array}$ & NOTES & CRDL & UNITS & DILUTION \\
\hline \multicolumn{9}{|l|}{ TA17-ES-30-120-5 (CON'T) } \\
\hline Anthracene & 330 & UG/KG & $u$ & NP & & & UG/KG & 1 \\
\hline Di-N-Butylphthalate & 330 & UG/KG & $u$ & NP & & & UG/KG & 1 \\
\hline Fluoranthene & 330 & UG/KG & $u$ & NP & & & UG/KG & 1 \\
\hline Pyrene & 330 & $U G / K G$ & $u$ & NP & & & UG/KG & 1 \\
\hline Butyl Benzyl Phthalate & 330 & UG/KG & $u$ & NP & & & O UG/KG & 1 \\
\hline 3, 3'-Dichlorobenzidine & 660 & UG/KG & u & NP & & & O UG/KG & 1 \\
\hline Benzo(a)Anthracene & 330 & UG/KG & $u$ & NP & & & O UG/KG & 1 \\
\hline Chrysene & 330 & UG/KG & $u$ & NP & & & $O U G / K G$ & 1 \\
\hline bis(2-Ethylhexyl)phthalate & 330 & UG/KG & $u$ & NP & & & O UG/KG & 1 \\
\hline di-N-Octyl Phthalate & 330 & UG/KG & $u$ & NP & & & $O$ UG/KG & 1 \\
\hline Benzo(b)Fluoranthene & 330 & UG/KG & $u$ & NP & & & $O$ UG/KG & 1 \\
\hline Benzo(k)Fluoranthene & 330 & UG/KG & $u$ & NP & & & $O U G / K G$ & 1 \\
\hline Benzo(a)Pyrene & 330 & UG/KG & $u$ & NP & & & $O$ UG/KG & 1 \\
\hline Indeno(1,2,3-CD)Pyrene & 330 & UG/KG & $u$ & NP & & & $O$ UG/KG & 1 \\
\hline Dibenzo(a,h)Anthracene & 330 & UG/KG & $u$ & NP & & & 0 UG/KG & 1 \\
\hline Benzo(g,h,i)Perylene & 330 & UG/KG & $u$ & NP & & & 0 UG/KG & 1 \\
\hline Unknown-1 & 1100 & UG/KG & J & NP & & & UG/KG & 1 \\
\hline Unknown-2 & 170 & UG/KG & J & NP & & & UG/KG & 1 \\
\hline Unknown-3 & 1700 & UG/KG & J & NP & & & UG/KG & 1 \\
\hline Unknown-4 & 170 & UG/KG & J & NP & & & UG/KG & 1 \\
\hline Nitrobenzene-d5 & 83 & $\%$ & & & & & $\%$ & 1 \\
\hline 2-Fluorobiphenyl & 77 & $\%$ & & & & & $\%$ & 1 \\
\hline Terphenyl-d14 & 69 & $\%$ & & & & & $\%$ & 1 \\
\hline Phenol-d5 & 77 & $\%$ & & & & & $\%$ & 1 \\
\hline 2-Fluorophenol & 71 & $\%$ & & & & & $\%$ & 1 \\
\hline 2, 4,6-Tribromophenol & 80 & $\%$ & & & & & $\%$ & 1 \\
\hline
\end{tabular}




\begin{tabular}{|c|c|c|c|c|c|c|c|}
\hline COMPOUND & RESULTS & UNITS & $\begin{array}{l}\text { DATA } \\
\text { QUAL }\end{array}$ & $\begin{array}{l}\text { DATA } \\
\text { VALID. }\end{array}$ & NOTES & CRDL UNITS & DILUTION \\
\hline \multicolumn{8}{|l|}{ TR17-ES-30-125-8 } \\
\hline Phenol & 330 & UG/KG & $u$ & NP & & 330 UG/KG & 1 \\
\hline bis(2-Chloroethyl) ether & 330 & UG/KG & $u$ & NP & & 330 UG/KG & 1 \\
\hline 2-Chlorophenol & 330 & UG/KG & $u$ & NP & & 330 UG/KG & 1 \\
\hline 1, 3-Dichlorobenzene & 330 & UGKG & $u$ & NP & & 330 UG/KG & 1 \\
\hline 1, 4-Dichlorobenzene & 330 & UG/KG & $u$ & NP & & $330 \mathrm{UG} / \mathrm{KG}$ & 1 \\
\hline Benzyl Alcohol & 330 & UG/KG & $u$ & NP & & 330 UG/KG & 1 \\
\hline 1, 2-Dichlorobenzene & 330 & UGKG & $u$ & NP & & 330 UG/KG & 1 \\
\hline 2-Methylphenol & 330 & UGKG & $u$ & NP & & 330 UG/KG & 1 \\
\hline bis(2-Chloroisopropyl) ether & 330 & UG/KG & $u$ & NP & & 330 UG/KG & 1 \\
\hline 4-Methylphenol & 330 & UG/KG & $u$ & NP & & 330 UG/KG & 1 \\
\hline N-Nitroso-di-nPropylamine & 330 & UGKG & $u$ & NP & & 330 UG/KG & 1 \\
\hline Hexachloroethane & 330 & VG/KG & $u$ & NP & & 330 UG/KG & 1 \\
\hline Nitrobenzene & 330 & UG/KG & $u$ & NP & & 330 UG/KG & 1 \\
\hline Isophorone & 330 & UGKG & $u$ & NP & & 330 UG/KG & 1 \\
\hline 2-Nitrophenol & 330 & UG/KG & $u$ & NP & & 330 UG/KG & 1 \\
\hline 2, 4-Dimethylphenol & 330 & UG/KG & $u$ & NP & & 330 UG/KG & 1 \\
\hline Benzoic Acid & 1600 & Ua/KG & $u$ & NP & & 1600 UG/KG & 1 \\
\hline bis(2Chloroethoxy)methane & 330 & UGKG & $u$ & NP & & 330 UG/KG & 1 \\
\hline 2, 4-Dichlorophenol & 330 & UG/KG & $u$ & NP & & 330 UG/KG & 1 \\
\hline 1, 2, 4-Trichlorobenzene & 330 & UG/KG & $u$ & NP & & 330 UG/KG & 1 \\
\hline Naphthalene & 330 & UGKG & $u$ & NP & & 330 UG/KG & 1 \\
\hline 4-Chloroaniline & 330 & UGKG & $u$ & NP & & 330 UG/KG & 1 \\
\hline Hexachlorobutadiene & 330 & UG/KG & $u$ & NP & & 330 UG/KG & 1 \\
\hline 4-Chloro-3-Methylphenol & 330 & UG/KG & $u$ & NP & & 330 UG/KG & 1 \\
\hline 2-Methylnaphthalene & 330 & UG/KG & $u$ & NP & & $330 \mathrm{UG} / \mathrm{KG}$ & 1 \\
\hline Hexachlorocyclopentadiene & 330 & UG/KG & $u$ & NP & & 330 UG/KG & 1 \\
\hline 2, 4,6-Trichlorophenol & 330 & UG/KG & $u$ & NP & & 330 UG/KG & 1 \\
\hline 2, 4, 5-Trichiorophenol & 1600 & UG/KG & $u$ & NP & & 1600 UG/KG & 1 \\
\hline 2-Chloronaphthalene & 330 & UG/KG & $u$ & NP & & 330 UG/KG & 1 \\
\hline 2-Nitroaniline & 1600 & UG/KG & $u$ & NP & & 1600 UG/KG & 1 \\
\hline Dimethyl Phthalate & 330 & UG/KG & $u$ & NP & & 330 UG/KG & 1 \\
\hline Acenaphthylene & 330 & UG/KG & $u$ & NP & & 330 UG/KG & 1 \\
\hline 2.6-Dinitrotoluene & 330 & UG/KG & $u$ & NP & & 330 UG/KG & 1 \\
\hline 3-Nitroaniline & 1600 & UG/KG & $u$ & NP & & 1600 UG/KG & 1 \\
\hline Acenaphthene & 330 & UG/KG & $u$ & NP & & 330 UG/KG & 1 \\
\hline 2, 4-Dinitrophenol & 1600 & UGKG & $u$ & NP & & 1600 UG/KG & 1 \\
\hline 4-Nitrophenol & 1600 & UGKG & $u$ & NP & & $1600 \mathrm{UG} / \mathrm{KG}$ & 1 \\
\hline Dibenzofuran & 330 & UG/KG & $u$ & NP & & $330 \mathrm{UG} / \mathrm{KG}$ & 1 \\
\hline 2, 4-Dinitrotoluene & 330 & UGKG & $u$ & NP & & 330 UG/KG & 1 \\
\hline Diethylphthalate & 330 & UG/KG & $u$ & NP & & 330 UG/KG & 1 \\
\hline 4-Chlorophenyl phenyl ether & 330 & UG/KG & $u$ & NP & & $330 \mathrm{UG} / \mathrm{KG}$ & 1 \\
\hline Fluorene & 330 & UGKG & $u$ & NP & & 330 UG/KG & 1 \\
\hline 4-Nitroaniline & 1600 & UGKG & $u$ & NP & & 1600 UG/KG & 1 \\
\hline 4, 6-Dinitro-2-Methylphenol & 1600 & UG/KG & $u$ & NP & & 1600 UG/KG & 1 \\
\hline N-Nitrosodiphenylamine & 330 & UGKG & $u$ & NP & & 330 UG/KG & 1 \\
\hline 4-Bromophenyl phenyl ether & 330 & UGKG & $u$ & NP & & 330 UG/KG & 1 \\
\hline Hexachlorobenzene & 330 & UGKG & $u$ & NP & & 330 UG/KG & 1 \\
\hline Pentachlorophenol & 1600 & UGKG & $u$ & NP & & 1600 UG/KG & 1 \\
\hline Phenanthrene & 330 & UG/KG & $u$ & NP & & 330 UG/KG & 1 \\
\hline
\end{tabular}


Appendix D: Results of Semivolatile Organic Compound Analyses, Salmon Site

\begin{tabular}{|c|c|c|c|c|c|c|c|c|}
\hline COMPOUND & RESULTS & UNITS & $\begin{array}{l}\text { DATA } \\
\text { QUAL }\end{array}$ & $\begin{array}{l}\text { DATA } \\
\text { VALID. }\end{array}$ & NOTES & CRDL & UNITS & DILUTION \\
\hline \multicolumn{9}{|l|}{ TR17-ES-30-125-8 (CON'T) } \\
\hline Anthracene & 330 & UG/KG & $u$ & NP & & 330 & UG/KG & 1 \\
\hline Di-N-Butylphthalate & 330 & $U G / K G$ & $u$ & NP & & 330 & UG/KG & 1 \\
\hline Fluoranthene & 330 & UG/KG & $u$ & NP & & 330 & UG/KG & 1 \\
\hline Pyrene & 330 & UG/KG & $u$ & NP & & 330 & UG/KG & 1 \\
\hline Butyl Benzyl Phthalate & 330 & UG/KG & $u$ & NP & & 330 & UG/KG & 1 \\
\hline 3. 3'-Dichlorobenzidine & 660 & UG/KG & $u$ & NP & & 660 & UG/KG & 1 \\
\hline Benzo(a)Anthracene & 330 & UG/KG & $u$ & NP & & 330 & UG/KG & 1 \\
\hline Chrysene & 330 & UG/KG & $u$ & NP & & 330 & UG/KG & 1 \\
\hline bis(2-Ethylhexyl)phthalate & 33 & UG/KG & $\mathrm{J}$ & NP & & 330 & UG/KG & 1 \\
\hline di-N-Octyl Phthalate & 330 & UG/KG & $u$ & NP & & 330 & UG/KG & 1 \\
\hline Benzo(b)Fluoranthene & 330 & UG/KG & $u$ & NP & & 330 & UG/KG & 1 \\
\hline Benzo(k)Fluoranthene & 330 & UG/KG & $u$ & NP & & 330 & UG/KG & 1 \\
\hline Benzo(a)Pyrene & 330 & UG/KG & $u$ & NP & & 330 & UG/KG & 1 \\
\hline Indeno(1,2,3-CD)Pyrene & 330 & UG/KG & $u$ & NP & & 330 & UG/KG & 1 \\
\hline Dibenzo(a,h)Anthracene & 330 & UG/KG & $u$ & NP & & 330 & UG/KG & 1 \\
\hline Benzo(g,h,i)Perylene & 330 & UG/KG & $u$ & NP & & 330 & UG/KG & 1 \\
\hline Unknown C10H16-1 & 950 & $U G / K G$ & $\mathrm{~J}$ & NP & & & UG/KG & 1 \\
\hline Unknown $\mathrm{C} 10 \mathrm{H} 16-2$ & 200 & UG/KG & J & NP & & & UG/KG & 1 \\
\hline Unknown-1 & 78 & UG/KG & J & NP & & & UG/KG & 1 \\
\hline Unknown-2 & 250 & UG/KG & J & NP & & & UG/KG & 1 \\
\hline Unknown-3 & 240 & UG/KG & $\mathrm{J}$ & NP & & & UG/KG & 1 \\
\hline Nitrobenzene-d5 & 82 & $\%$ & & & & & $\%$ & 1 \\
\hline 2-Fluorobiphenyl & 80 & $\%$ & & & & & $\%$ & 1 \\
\hline Terphenyl-d14 & 70 & $\%$ & & & & & $\%$ & 1 \\
\hline Phenol-d5 & 87 & $\%$ & & & & & $\%$ & 1 \\
\hline 2-Fluorophenol & 82 & $\%$ & & & & & $\%$ & 1 \\
\hline 2, 4, 6-Tribromophenol & 82 & $\%$ & & & & & $\%$ & 1 \\
\hline
\end{tabular}


Appendix D: Results of Semivolatile Organic Compound Analyses, Salmon Site

\begin{tabular}{|c|c|c|c|c|c|c|c|}
\hline COMPOUND & RESULTS & UNITS & $\begin{array}{l}\text { DATA } \\
\text { QUAL }\end{array}$ & $\begin{array}{l}\text { DATA } \\
\text { VALID. }\end{array}$ & NOTES & CRDL UNITS & DILUTION \\
\hline \multicolumn{8}{|l|}{ TR18-BS-90-150A-E } \\
\hline Phenol & 330 & UG/KG & $u$ & NP & & $330 \mathrm{UG} / \mathrm{KG}$ & 1 \\
\hline bis(2-Chloroethyl) ether & 330 & UG/KG & $u$ & NP & & 330 UG/KG & 1 \\
\hline 2-Chlorophenol & 330 & UG/KG & $u$ & NP & & 330 UG/KG & 1 \\
\hline 1, 3-Dichlorobenzene & 330 & UG/KG & $u$ & NP & & 330 UG/KG & 1 \\
\hline 1, 4-Dichlorobenzene & 330 & UG/KG & $u$ & NP & & 330 UG/KG & 1 \\
\hline Benzyl Alcohol & 330 & UG/KG & $u$ & NP & & 330 UG/KG & 1 \\
\hline 1, 2-Dichlorobenzene & 330 & UG/KG & $u$ & NP & & 330 UG/KG & 1 \\
\hline 2-Methylphenol & 330 & UG/KG & $u$ & NP & & 330 UG/KG & 1 \\
\hline bis(2-Chloroisopropyl) ether & 330 & Ua/KG & $u$ & NP & & 330 UG/KG & 1 \\
\hline 4-Methyiphenol & 330 & UG/KG & $u$ & NP & & 330 UG/KG & 1 \\
\hline N-Nitroso-di-nPropylamine & 330 & UG/KG & $u$ & NP & & 330 UG/KG & 1 \\
\hline Hexachloroethane & 330 & VaKG & $u$ & NP & & 330 UG/KG & 1 \\
\hline Nitrobenzene & 330 & UG/KG & $u$ & NP & & 330 UG/KG & 1 \\
\hline Isophorone & 330 & UG/KG & $u$ & NP & & 330 UG/KG & 1 \\
\hline 2-Nitrophenol & 330 & UG/KG & $u$ & NP & & 330 UG/KG & 1 \\
\hline 2, 4-Dimethylphenol & 330 & UGKG & $u$ & NP & & 330 UG/KG & 1 \\
\hline Benzoic Acid & 1600 & UG/KG & $u$ & NP & & 1600 UG/KG & 1 \\
\hline bis(2Chloroethoxy)methane & 330 & UG/KG & $u$ & NP & & 330 UG/KG & 1 \\
\hline 2, 4-Dichlorophenol & 330 & UG/KG & $u$ & NP & & 330 UG/KG & 1 \\
\hline 1, 2, 4-Trichlorobenzene & 330 & UG/KG & $u$ & NP & & 330 UG/KG & 1 \\
\hline Naphthalene & 330 & Ua/KG & $u$ & NP & & 330 UG/KG & 1 \\
\hline 4-Chloroaniline & 330 & UG/KG & $u$ & NP & & 330 UG/KG & 1 \\
\hline Hexachlorobutadiene & 330 & UG/KG & $u$ & NP & & 330 UG/KG & 1 \\
\hline 4-Chloro-3-Methylphenol & 330 & UGKG & $u$ & NP & & 330 UG/KG & 1 \\
\hline 2-Methylnaphthalene & 330 & UG/KG & $u$ & NP & & 330 UG/KG & 1 \\
\hline Hexachlorocyclopentadiene & 330 & UG/KG & $u$ & NP & & 330 UG/KG & 1 \\
\hline 2, 4, 6-Trichlorophenol & 330 & UGKG & $u$ & NP & & 330 UG/KG & 1 \\
\hline 2, 4,5-Trichlorophenol & 1600 & UG/KG & $u$ & NP & & 1600 UG/KG & 1 \\
\hline 2-Chloronaphthalene & 330 & UG/KG & $u$ & NP & & 330 UG/KG & 1 \\
\hline 2-Nitroaniline & 1600 & UG/KG & $u$ & NP & & 1600 UG/KG & 1 \\
\hline Dimethyl Phthalate & 330 & UG/KG & $u$ & NP & & 330 UG/KG & 1 \\
\hline Acenaphthylene & 330 & UG/KG & $u$ & NP & & 330 UG/KG & 1 \\
\hline 2, 6-Dinitrotoluene & 330 & UG/KG & $u$ & NP & & 330 UG/KG & 1 \\
\hline 3-Nitroaniline & 1600 & UGKG & $u$ & NP & & 1600 UG/KG & 1 \\
\hline Acenaphthene & 330 & UG/KG & $u$ & NP & & 330 UG/KG & 1 \\
\hline 2, 4-Dinitrophenol & 1600 & UG/KG & $u$ & NP & & 1600 UG/KG & 1 \\
\hline 4-Nitrophenol & 1600 & UG/KG & $u$ & NP & & 1600 UG/KG & 1 \\
\hline Dibenzofuran & 330 & UG/KG & $u$ & NP & & 330 UG/KG & 1 \\
\hline 2, 4-Dinitrotoluene & 330 & UG/KG & $u$ & NP & & 330 UG/KG & 1 \\
\hline Diethylphthalate & 330 & UG/KG & $u$ & NP & & 330 UG/KG & 1 \\
\hline 4-Chlorophenyl phenyl ether & 330 & UGKG & $u$ & NP & & 330 UG/KG & 1 \\
\hline Fluorene & 330 & UG/KG & $u$ & NP & & 330 UG/KG & 1 \\
\hline 4-Nitroaniline & 1600 & UG/KG & $u$ & NP & & 1600 UGKG & 1 \\
\hline 4, 6-Dinitro-2-Methylphenol & 1600 & UG/KG & $u$ & NP & & 1600 UGKG & 1 \\
\hline N-Nitrosodiphenylamine & 330 & UG/KG & $u$ & NP & & 330 UG/KG & 1 \\
\hline 4-Bromophenyl phenyl ether & 330 & UG/KG & $u$ & NP & & 330 UG/Ka & 1 \\
\hline Hexachlorobenzene & 330 & UGKG & $u$ & NP & & 330 UG/KG & 1 \\
\hline Pentachlorophenol & 1600 & UG/KG & $u$ & NP & & 1600 UG/KG & 1 \\
\hline Phenanthrene & 330 & UG/KG & $u$ & NP & & 330 UG/KG & 1 \\
\hline
\end{tabular}


Appendix D: Results of Semivolatile Organic Compound Analyses. Salmon Site

\begin{tabular}{|c|c|c|c|c|c|c|c|c|}
\hline COMPOUND & RESULTS & UNITS & $\begin{array}{l}\text { DATA } \\
\text { QUAL }\end{array}$ & $\begin{array}{l}\text { DATA } \\
\text { VALID. }\end{array}$ & NOTES & CRDL & UNITS & DILUTION \\
\hline \multicolumn{9}{|c|}{ TR18-BS-90-150A-E (CON'T) } \\
\hline Anthracene & 330 & UG/KG & $u$ & NP & & & U UG/KG & 1 \\
\hline Di-N-Butylphthalate & 330 & UG/KG & $u$ & NP & & & O UG/KG & 1 \\
\hline Fluoranthene & 330 & UG/KG & $u$ & NP & & & O UG/KG & 1 \\
\hline Pyrene & 330 & UG/KG & $u$ & NP & & & O UG/KG & 1 \\
\hline Butyl Benzyl Phthalate & 330 & $U G / K G$ & $u$ & NP & & & O UG/KG & 1 \\
\hline 3, 3'-Dichlorobenzidine & 660 & UG/KG & $u$ & NP & & 66 & O UG/KG & 1 \\
\hline Benzo(a)Anthracene & 330 & UG/KG & $u$ & NP & & 33 & O UG/KG & 1 \\
\hline Chrysene & 330 & UG/KG & $u$ & NP & & 33 & O UG/KG & 1 \\
\hline bis(2-Ethylhexyl)phthalate & 330 & UG/KG & $u$ & NP & & 33 & 0 UG/KG & 1 \\
\hline di-N-Octyl Phthalate & 330 & UG/KG & $u$ & NP & & 33 & O UG/KG & 1 \\
\hline Benzo(b)Fluoranthene & 330 & UG/KG & $u$ & NP & & 33 & O UG/KG & 1 \\
\hline Benzo(k)Fluoranthene & 330 & UG/KG & $u$ & NP & & 33 & 0 UG/KG & 1 \\
\hline Benzo(a)Pyrene & 330 & UG/KG & $u$ & NP & & 33 & 0 UG/KG & 1 \\
\hline Indeno(1,2,3-CD)Pyrene & 330 & UG/KG & $u$ & NP & & & 0 UG/KG & 1 \\
\hline Dibenzo(a.h)Anthracene & 330 & UG/KG & $u$ & NP & & & O UG/KG & 1 \\
\hline Benzo(g,h,i)Peryiene & 330 & UG/KG & $u$ & NP & & & 0 UG/KG & 1 \\
\hline Unknown-1 & 94 & UG/KG & J & NP & & & UG/KG & 1 \\
\hline Unknown-2 & 67 & UG/KG & J & NP & & & UG/KG & 1 \\
\hline Nitrobenzene-d5 & 78 & $\%$ & & & & & $\%$ & 1 \\
\hline 2-Fluorobiphenyl & 77 & $\%$ & & & & & $\%$ & 1 \\
\hline Terphenyl-d14 & 65 & $\%$ & & & & & $\%$ & 1 \\
\hline Phenol-d5 & 80 & $\%$ & & & & & $\%$ & 1 \\
\hline 2-Fluorophenol & 74 & $\%$ & & & & & $\%$ & 1 \\
\hline 2, 4, 6-Tribromophenol & 79 & $\%$ & & & & & $\%$ & 1 \\
\hline
\end{tabular}




\begin{tabular}{|c|c|c|c|c|c|c|c|}
\hline COMPOUND & RESULTS & UNITS & $\begin{array}{l}\text { DATA } \\
\text { QUAL }\end{array}$ & $\begin{array}{l}\text { DATA } \\
\text { VALID. }\end{array}$ & NOTES & CRDL UNITS & DILUTION \\
\hline \multicolumn{8}{|l|}{ TA19-BS-0-150A-E } \\
\hline Phenol & 330 & UG/KG & $u$ & NP & & 330 UG/KG & 1 \\
\hline bis(2-Chloroethyl) ether & 330 & VaKG & $u$ & NP & & 330 UG/KG & 1 \\
\hline 2-Chlorophenol & 330 & UGKG & $u$ & NP & & 330 Ua/KG & 1 \\
\hline 1, 3-Dichlorobenzene & 330 & Va/Ka & $u$ & NP & & 330 UG/KG & 1 \\
\hline 1, 4-Dichlorobenzene & 330 & Va/Ka & $u$ & NP & & 330 UG/KG & 1 \\
\hline Benzyl Alcohol & 330 & VQKG & $u$ & NP & & 330 UG/KG & 1 \\
\hline 1, 2-Dichlorobenzene & 330 & UG/KG & $u$ & NP & & 330 UG/KG & 1 \\
\hline 2-Methylphenol & 330 & UG/KG & $u$ & NP & & 330 UG/KG & 1 \\
\hline bis(2-Chloroisopropyl) ether & 330 & UG/KG & $u$ & NP & & 330 Ua/KG & 1 \\
\hline 4-Methylphenol & 330 & UG/KG & $u$ & NP & & 330 UG/KG & 1 \\
\hline N-Nitroso-di-nPropylamine & 330 & UG/KG & $u$ & NP & & 330 UG/KG & 1 \\
\hline Hexachloroethane & 330 & Ua/Ko & $u$ & NP & & 330 UGKG & 1 \\
\hline Nitrobenzene & 330 & UGKG & $u$ & NP & & 330 Ua/KG & 1 \\
\hline Isophorone & 330 & UGKG & $u$ & NP & & 330 UG/KG & 1 \\
\hline 2-Nitrophenol & 330 & UG/KG & $u$ & NP & & 330 UG/KG & 1 \\
\hline 2, 4-Dimethyiphenol & 330 & UGKG & $u$ & NP & & 330 UG/KG & 1 \\
\hline Benzoic Acid & 1600 & UaKG & $u$ & NP & & 1600 Ua/KG & 1 \\
\hline bis(2Chloroethoxy)methane & 330 & UGKG & $u$ & NP & & 330 UG/KG & 1 \\
\hline 2, 4-Dichlorophenol & 330 & UGKG & $u$ & NP & & 330 Ua/KG & 1 \\
\hline 1, 2, 4-Trichlorobenzene & 330 & UaKG & $u$ & NP & & 330 UG/KG & 1 \\
\hline Naphthalene & 330 & UGKG & $u$ & NP & & 330 UG/KG & 1 \\
\hline 4-Chloroaniline & 330 & UG/KG & $u$ & NP & & 330 UG/KG & 1 \\
\hline Hexachlorobutadiene & 330 & UG/KG & u & NP & & 330 UG/KG & 1 \\
\hline 4-Chloro-3-Methylphenol & 330 & UGKG & $u$ & NP & & 330 UG/KG & 1 \\
\hline 2-Methylnaphthalene & 2300 & UGKG & & NP & & 330 UG/KG & 1 \\
\hline Hexachlorocyclopentadiene & 330 & UG/KG & $u$ & NP & & 330 UG/KG & 1 \\
\hline 2, 4, 6-Trichlorophenol & 330 & UGKG & $u$ & NP & & 330 Ua/KG & 1 \\
\hline 2, 4, 5-Trichlorophenol & 1600 & UGKG & $u$ & NP & & 1600 UG/KG & 1 \\
\hline 2-Chloronaphthalene & 330 & UG/KG & $u$ & NP & & 330 UG/KG & 1 \\
\hline 2-Nitroaniline & 1600 & UG/KG & $u$ & NP & & 1600 UG/KG & 1 \\
\hline Dimethyl Phthalate & 330 & UGKG & U & NP & & 330 UG/KG & 1 \\
\hline Acenaphthylene & 330 & UG/KG & $u$ & NP & & 330 UG/KG & 1 \\
\hline 2. 6-Dinitrotoluene & 330 & UG/KG & $u$ & NP & & 330 UG/KG & 1 \\
\hline 3-Nitroaniline & 1600 & UGKG & U & NP & & 1600 UG/KG & 1 \\
\hline Acenaphthene & 330 & UGKG & $u$ & NP & & 330 UG/KG & 1 \\
\hline 2, 4-Dinitrophenol & 1600 & UGKG & $u$ & NP & & 1600 UG/KG & 1 \\
\hline 4-Nitrophenol & 1600 & UG/KG & $u$ & NP & & $1600 \mathrm{UG} / \mathrm{KG}$ & 1 \\
\hline Dibenzofuran & 330 & $U G / K G$ & $u$ & NP & & 330 UG/KG & 1 \\
\hline 2, 4-Dinitrotoluene & 330 & UGKG & $u$ & NP & & 330 UG/KG & 1 \\
\hline Diethylphthalate & 330 & UG/KG & $u$ & NP & & 330 UG/KG & 1 \\
\hline 4-Chlorophenyl phenyl ether & 330 & UG/KG & $u$ & NP & & 330 UG/KG & 1 \\
\hline Fluorene & 650 & UGKG & & NP & & 330 UG/KG & 1 \\
\hline 4-Nitroaniline & 1600 & UG/KG & $u$ & NP & & 1600 UG/KG & 1 \\
\hline 4, 6-Dinitro-2-Methylphenol & 1600 & UG/KG & $u$ & NP & & 1600 UG/KG & 1 \\
\hline N-Nitrosodiphenylamine & 330 & UG/KG & $u$ & NP & & 330 UG/KG & 1 \\
\hline 4-Bromophenyl phenyl ether & 330 & UG/KG & $u$ & NP & & 330 UG/KG & 1 \\
\hline Hexachlorobenzene & 330 & UG/KG & $u$ & NP & & 330 UG/KG & 1 \\
\hline Pentachlorophenol & 1600 & UG/KG & $u$ & NP & & 1600 UaKG & 1 \\
\hline Phenanthrene & 420 & UQKKG & & NP & & 330 UG/KG & 1 \\
\hline
\end{tabular}


Appendix D: Results of Semivolatile Organic Compound Analyses. Salmon Site

\begin{tabular}{|c|c|c|c|c|c|c|c|}
\hline COMPOUND & RESULTS & UNITS & $\begin{array}{l}\text { DATA } \\
\text { QUAL }\end{array}$ & $\begin{array}{l}\text { DATA } \\
\text { VALID. }\end{array}$ & NOTES & CRDL UNITS & DILUTION \\
\hline \multicolumn{8}{|l|}{ TA19-8S-0-150A-E (CON'T) } \\
\hline Anthracene & 330 & va/ka & $u$ & NP & & 330 UG/KG & 1 \\
\hline DI-N-Butylphthalate & 330 & UG/KG & $u$ & NP & & 330 UG/KG & 1 \\
\hline Fluoranthene & 330 & va/Ka & $u$ & NP & & 330 UG/KG & 1 \\
\hline Pyrene & 330 & Ua/KO & $u$ & NP & & 330 UG/KG & 1 \\
\hline Butyl Benzyl Phthalate & 49 & UG/KG & J & NP & & 330 UG/KG & 1 \\
\hline 3, 3'-Dichlorobenzidine & 660 & UG/KG & $u$ & NP & & 660 Ua/KG & 1 \\
\hline Benzo(a)Anthracene & 330 & vaka & $u$ & NP & & 330 UaKKa & 1 \\
\hline Chrysene & 330 & UG/KG & $u$ & NP & & 330 UG/KG & 1 \\
\hline bis(2-Ethylhexyl)phthalate & 41 & UaKG & J & NP & & 330 UaKKo & 1 \\
\hline di-N-Octyl Phthalate & 330 & $U G / K O$ & $u$ & NP & & 330 UG/KG & 1 \\
\hline Benzo(b)Fluoranthene & 330 & UG/KG & $u$ & NP & & 330 UG/KG & 1 \\
\hline Benzo(k)Fluoranthene & 330 & $U G / K G$ & $u$ & NP & & 330 UG/KG & 1 \\
\hline Benzo(a)Pyrene & 330 & $U G / K G$ & u & NP & & 330 UG/KG & 1 \\
\hline Indeno(1,2,3-CD)Pyrene & 330 & UG/KG & $u$ & NP & & 330 UG/KG & 1 \\
\hline Dibenzo(a.h)Anthracene & 330 & UG/KG & $u$ & NP & & 330 UG/KG & 1 \\
\hline Benzo(g,h,i)Perylene & 330 & UG/KG & $u$ & NP & & 330 UG/KG & 1 \\
\hline Undecane & 740 & UG/KG & J & NP & & UG/KG & 1 \\
\hline Unknown-1 & 650 & UGKKO & J & NP & & va/Ko & 1 \\
\hline Dodecane & 2300 & UGKG & J & NP & & vake & 1 \\
\hline Unknown-2 & 1100 & Ua/KG & J & NP & & UaKa & 1 \\
\hline Unknown-3 & 1300 & UGKG & $\mathrm{J}$ & NP & & UaKG & 1 \\
\hline Unknown-4 & 2600 & VaKo & $\mathrm{J}$ & NP & & vane & 1 \\
\hline Unknown-5 & 780 & Ua/Ka & J & NP & & UGKG & 1 \\
\hline Unknown-6 & 760 & UG/KG & J & NP & & UG/KG & 1 \\
\hline Unknown-7 & 1400 & UG/KG & J & NP & & UG/KG & 1 \\
\hline Unknown-8 & 1400 & UG/KG & J & NP & & UG/KG & 1 \\
\hline Tetradecane & 6300 & UG/KG & $\mathrm{J}$ & NP & & UGKG & 1 \\
\hline Unknown-9 & 2900 & UG/KG & $\mathrm{J}$ & NP & & UG/KG & 1 \\
\hline Unknown-10 & 3300 & UG/KG & J & NP & & vaKk & 1 \\
\hline Unknown-11 & 1300 & UGKG & J & NP & & UG/KG & 1 \\
\hline Unknown-12 & 2900 & UG/KG & J & NP & & UG/KG & 1 \\
\hline Unknown-13 & 1600 & UG/KG & J & NP & & UG/KG & 1 \\
\hline Pentadecane & 4400 & UG/KG & J & NP & & UG/KG & 1 \\
\hline Unknown-14 & 1400 & UGKO & J & NP & & UGKG & 1 \\
\hline Unknown-15 & 1000 & UG/KG & J & NP & & UG/KG & 1 \\
\hline Unknown-16 & 1000 & UG/KG & J & NP & & UG/KG & 1 \\
\hline Unknown-17 & 1000 & UG/KG & J & NP & & Ua/KG & 1 \\
\hline Heptadecane & 3800 & UG/KG & $\mathrm{J}$ & NP & & UG/KG & 1 \\
\hline Nitrobenzene-d5 & 75 & $\%$ & & & & $\%$ & 1 \\
\hline 2-Fluorobiphenyl & 85 & $\%$ & & & & $\%$ & 1 \\
\hline Terphenyl-d14 & 65 & $\%$ & & & & $\%$ & 1 \\
\hline Phenol-d5 & 80 & $\%$ & & & & $\%$ & 1 \\
\hline 2-Fluorophenol & 75 & $\%$ & & & & $\%$ & 1 \\
\hline 2, 4, 6-Tribromophenol & 78 & $\%$ & & & & $\%$ & 1 \\
\hline
\end{tabular}




\section{Appendix E}

\section{Results of Gross Alpha and Beta Analyses}


Appendix E: Results of Gross Alpha and Beta Analyses, Salmon Site

\begin{tabular}{|c|c|c|c|c|c|c|c|c|}
\hline SAMPLE & & RESULT & UNITS & $\begin{array}{r}2 \text { SIGMA } \\
\text { ERROR }(+/ \cdot) \\
\end{array}$ & $\begin{array}{l}\text { DATA } \\
\text { VALID. } \\
\end{array}$ & UNITS & MDA & UNITS \\
\hline \multirow[t]{2}{*}{ TR1-ES-001 } & Alpha (total) & 6.36 & PCI/G & 1.43 & J & PCI/G & 1.44 & PCI/G \\
\hline & Beta (total) & 10.9 & PCI/G & 2.00 & $\cdots$ & PCI/G & 2.5 & PCI/G \\
\hline \multirow[t]{2}{*}{ TR2-ES-355N-175E-5Z } & Alpha (total) & 5.54 & PCl/G & 1.52 & J & $\mathrm{PCl} / \mathrm{G}$ & 1.85 & PCI/G \\
\hline & Bota (total) & 15.6 & PCI/G & 2.40 & $\cdots$ & $P C / / G$ & 2.7 & COUG \\
\hline \multirow[t]{2}{*}{ TR2-ES-356N-175E-8Z } & Alpha (total) & 7.62 & $\mathrm{PC} / \mathrm{G}$ & 1.55 & J & PCI/G & 1.36 & PCI/G \\
\hline & Beta (total) & 18.3 & $\mathrm{PCl} / \mathrm{O}$ & 2.60 & $\cdots$ & PCI/G & 2.7 & PCI/G \\
\hline \multirow[t]{2}{*}{ TR2A-ES-365N-200E-3.5Z } & Alpha (total) & 9.37 & $\mathrm{PCl} / \mathrm{G}$ & 1.82 & J & $\mathrm{PCl} / \mathrm{G}$ & 1.6 & PCI/G \\
\hline & Beta (total) & 14.6 & PCI/G & 2.60 & $\cdots$ & $\mathrm{PCl} / \mathrm{G}$ & 3.2 & PCI/G \\
\hline \multirow[t]{2}{*}{ TR2A-ES-360N-200E-8Z } & Alpha (total) & 10.3 & PCI/G & 2.00 & J & $\mathrm{PCl} / \mathrm{G}$ & 1.7 & PCI/G \\
\hline & Beta (total) & 18.1 & PCI/G & 2.70 & $\cdots$ & PCI/G & 2.9 & PCI/G \\
\hline \multirow[t]{2}{*}{ TR3-ES-45ON-400E-3Z } & Alpha (total) & 6.77 & PCI/G & 1.52 & J & PCI/G & 1.63 & PCI/G \\
\hline & Beta (total) & 13.4 & PCI/G & 2.20 & $\cdots$ & PCI/G & 2.5 & PCVG \\
\hline \multirow[t]{2}{*}{ TR3-ES-430N-400E-15Z } & Aipha (total) & 10.4 & $\mathrm{PC} / \mathrm{G}$ & 6.70 & $J$ & PCV/G & 8.7 & PCUO \\
\hline & Beta (total) & 12.6 & $\mathrm{PC} / \mathrm{G}$ & 3.90 & $\cdots$ & PCI/G & 5.2 & PCI/G \\
\hline \multirow[t]{2}{*}{ TR4-ES-260-490-8 } & Alpha (total) & 11.8 & PCI/G & 2.00 & J & PCI/G & 1.7 & PCVG \\
\hline & Bota (total) & 26.2 & PCIG & 3.30 & -- & PCI/G & 2.6 & PCUG \\
\hline \multirow[t]{2}{*}{ TR5-ES-590-570-12 } & Alpha (total) & 11.2 & $\mathrm{PCl} / \mathrm{G}$ & 2.20 & $J$ & $\mathrm{PCI} / \mathrm{G}$ & 2 & PCI/G \\
\hline & Beta (total) & 15.4 & PCUG & 2.60 & $\cdots$ & PCI/G & 3 & PCUG \\
\hline \multirow[t]{2}{*}{ TR6-ES-495-585-12 } & Alpha (total) & 16.8 & PCU/G & 3.4 & NP & PCI/G & 3.2 & PCVG \\
\hline & Beta (total) & 11.8 & $\mathrm{PCI/G}$ & 1.8 & NP & PCI/G & 2 & PCVO \\
\hline \multirow[t]{2}{*}{ TA6-ES-485-590-6 } & Alpha (total) & 11.7 & PCUG & 2.9 & NP & PCI/G & 3.1 & $\mathrm{PCI} / \mathrm{G}$ \\
\hline & Beta (total) & 7.21 & $\mathrm{PCI} / \mathrm{G}$ & 1.53 & NP & PCI/G & 2.04 & PCI/G \\
\hline \multirow[t]{2}{*}{ TA7-ES-558-650-5 } & Alpha (total) & 11 & PCVG & 2.20 & $J$ & PCVG & 2.1 & PCUG \\
\hline & Beta (total) & 17.8 & PCVG & 2.70 & - & PCVG & 3 & PCVG \\
\hline
\end{tabular}


Appendix E: Results of Gross Alpha and Beta Analyses. Salmon Site

\begin{tabular}{|c|c|c|c|c|c|c|c|c|}
\hline SAMPLE & & RESULT & UNITS & $\begin{array}{r}2 \text { SIGMA } \\
\text { ERROR }(+/ \cdot) \\
\end{array}$ & $\begin{array}{l}\text { DATA } \\
\text { VALID. } \\
\end{array}$ & UNITS & MDA & UNITS \\
\hline \multirow[t]{2}{*}{ TA7-ES-560-650-14 } & Alpha (total) & 10.3 & PCVIO & 2.10 & J & $\mathrm{PCI/G}$ & 2.2 & PCVOA \\
\hline & Beta (total) & 40.7 & PCI/G & 4.40 & -.. & PCI/G & 1.1 & PCVOa \\
\hline \multirow{2}{*}{ TRB-ES-780-600-3 } & Alpha (total) & 8.21 & PCI/G & 1.78 & J & PCl/G & 1.79 & PCI/O \\
\hline & Beta (total) & 13.6 & PCIO & 2.30 & $\cdots$ & $\mathrm{PCl} / \mathrm{G}$ & 2.7 & PCIV \\
\hline \multirow[t]{2}{*}{ TR8-ES-780-600-3-DUP } & Alpha (total) & 8 & PCI/G & 1.79 & NP & PCI/G & 1.86 & PCVO \\
\hline & Bota (total) & 14.1 & PCI/G & 2.40 & NP & PCI/G & 2.8 & PCIVG \\
\hline \multirow[t]{2}{*}{ TA10-ES-180-790-15 } & Alpha (total) & 2.43 & PCI/G & 0.93 & J & PCI/G & 1.22 & PCIIG \\
\hline & Beta (total) & 5.69 & PCI/G & 1.61 & $\cdots$ & PCI/G & 2.37 & PCI/G \\
\hline \multirow[t]{2}{*}{ TR10-ES-190-790-8 } & Alpha (total) & 7.91 & PCI/G & 1.77 & J & PCI/G & 1.82 & PCI/G \\
\hline & Beta (total) & 10.4 & PCI/G & 2.00 & $\cdots$ & $P C / / O$ & 2.6 & PCI/G \\
\hline \multirow[t]{2}{*}{ TR11-ES-180-930-3 } & Alpha (total) & 14.2 & PCUG & 3.7 & NP & PCVG & 4.2 & PCUG \\
\hline & Beta (total) & 7.8 & PCI/G & 1.65 & NP & PCI/G & 2.16 & PCVG \\
\hline \multirow[t]{2}{*}{ TR13-ES-210-1110-6 } & Alpha (total) & 4.57 & PCUG & 2.32 & NP & PCIVG & 3.4 & PCI/G \\
\hline & Beta (total) & 5.22 & PCI/G & 1.46 & NP & PCI/G & 2.13 & PCI/G \\
\hline \multirow[t]{2}{*}{ TR13-ES-212-1150-6 } & Alpha (total) & 11.1 & PCI/G & 2.8 & NP & PCUG & 2.7 & PCUQ \\
\hline & Bota (total) & 6.58 & PCI/G & 1.66 & NP & PCI/G & 2.36 & PCVG \\
\hline \multirow[t]{2}{*}{ TR14-ES-80-1189-5 } & Alpha (total) & 15.2 & PCI/G & 3.6 & NP & PCI/G & 3.6 & PCVG \\
\hline & Beta (total) & 13.2 & $\mathrm{PCI} / \mathrm{G}$ & 2.1 & NP & $\mathrm{PCI} / \mathrm{G}$ & 2.2 & PCVG \\
\hline \multirow[t]{2}{*}{ TR14-ES-90-1200-3 } & Alpha (total) & 5.7 & PCUG & 2.46 & NP & PCVG & 3.43 & PCVOG \\
\hline & Beta (total) & 3.66 & PCI/G & 1.47 & NP & PCI/G & 2.31 & PCI/G \\
\hline \multirow[t]{2}{*}{ TR-15-ES-20-1180-.5 } & Alpha (total) & 9.3 & $\mathrm{PCl} / \mathrm{G}$ & 3.34 & NP & $\mathrm{PCI/G}$ & 4.56 & PCUG \\
\hline & Beta (total) & 6.15 & $\mathrm{PCI} / \mathrm{G}$ & 1.81 & NP & PCVG & 2.7 & PCVG \\
\hline
\end{tabular}


Appendix E: Results of Gross Alpha and Beta Analyses. Salmon Site

\begin{tabular}{|c|c|c|c|c|c|c|c|c|}
\hline SAMPLE & & RESULT & UNITS & $\begin{array}{r}2 \text { SIGMA } \\
\text { ERROR }(+/-) \\
\end{array}$ & $\begin{array}{l}\text { DATA } \\
\text { VALID. } \\
\end{array}$ & UNITS & MDA & UNITS \\
\hline \multirow[t]{2}{*}{ TR-15-ES-20-1180-.5DUP } & Alpha (total) & 10.4 & PCI/G & 3.1 & NP & $\mathrm{PCI/G}$ & 3.8 & PCI/G \\
\hline & Beta (total) & 8.83 & PCI/G & 1.71 & NP & PCI/G & 2.16 & PCI/G \\
\hline \multirow[t]{2}{*}{ TR16-ES-200-80-1.5 } & Alpha (total) & 10.4 & $P C I / G$ & 4.4 & NP & $\mathrm{PCI} / \mathrm{G}$ & 6.5 & PCI/G \\
\hline & Beta (total) & 11.8 & PCI/G & 2 & NP & $\mathrm{PC} / / \mathrm{G}$ & 2.4 & PCI/G \\
\hline \multirow[t]{2}{*}{ TR17-ES-30-120-5 } & Alpha (total) & 10 & PCI/G & 2.4 & NP & $\mathrm{PCl/G}$ & 2.6 & PCUG \\
\hline & Beta (total) & 10.5 & PCI/G & 1.6 & NP & $P C / / G$ & 1.8 & PCI/G \\
\hline \multirow{2}{*}{ TR17-ES-30-125-8 } & Alpha (total) & 10.2 & PCI/G & 2.6 & NP & PCI/G & 3.1 & PCUG \\
\hline & Beta (total) & 13.1 & $\mathrm{PCl} / \mathrm{G}$ & 1.8 & NP & PCI/G & 1.8 & PCUG \\
\hline \multirow[t]{2}{*}{ TR18-BS-90-150A-E } & Alpha (total) & 18.4 & PCI/G & 3.3 & NP & PCI/G & 2.6 & PCI/G \\
\hline & Beta (total) & 12.2 & PCI/G & 1.8 & NP & PCI/G & 2 & $\mathrm{PCI} / \mathrm{G}$ \\
\hline \multirow[t]{2}{*}{ TR19-BS-0-150A-E } & Alpha (total) & 15.9 & PCI/G & 3.1 & NP & PCI/G & 2.9 & PCUG \\
\hline & Beta (total) & 9.44 & $\mathrm{PCl} / \mathrm{G}$ & 1.66 & NP & PCI/G & 2.04 & PCI/G \\
\hline
\end{tabular}


Appendix F

Results of Gamma Spectroscopy Analyses 
Appendix F: Results of Gamma Spectroscopy Analyses, Salmon Site

\begin{tabular}{|c|c|c|c|c|c|c|c|c|}
\hline SAMPLE & COMPOUND & RESULTS & UNITS & $\begin{array}{l}\text { DATA } \\
\text { VALID. }\end{array}$ & $\begin{array}{r}2 \text { SIGMA } \\
\text { ERROR }(+/-) \\
\end{array}$ & UNITS & MDA & URIIS \\
\hline \multirow[t]{2}{*}{ TR1-ES-001 } & Lead 212 & 0.36 & $\mathrm{PCl} / \mathrm{G}$ & $\ldots$ & 0.09 & $\mathrm{PCI} / \mathrm{G}$ & 0.15 & PCI/G \\
\hline & Radium-226 & 0.42 & PCI/G & ..- & 0.08 & $\mathrm{PCl} / \mathrm{G}$ & 0.17 & PCI/G \\
\hline \multirow[t]{3}{*}{ TR2-ES-355N-175E-5Z } & Potassium 40 & 3.49 & PCI/G & $\ldots$ & 1.00 & $\mathrm{PCI/G}$ & 1.75 & $P C / / G$ \\
\hline & Lead 212 & 0.45 & $\mathrm{PCl} / \mathrm{G}$ & $\ldots$ & 0.12 & $\mathrm{PCI} / \mathrm{G}$ & 0.20 & PCI/G \\
\hline & Radium-226 & 0.54 & $\mathrm{PCl} / \mathrm{G}$ & $\ldots$ & 0.14 & $P C I / G$ & 0.29 & PCIG \\
\hline \multirow[t]{4}{*}{ TR2-ES-356N-175E-8Z } & Cesium 137 & 0.34 & $\mathrm{PCI/G}$ & $\ldots$ & 0.08 & PCI/G & 0.11 & $\mathrm{PCl} / \mathrm{G}$ \\
\hline & Thallium 208 & 0.14 & PCI/G & $\ldots$ & 0.06 & $\mathrm{PCI} / \mathrm{G}$ & 0.13 & $\mathrm{PCl} / \mathrm{G}$ \\
\hline & Lead 210 & 1.39 & PCI/G & $\ldots$ & 1.05 & $P C I / G$ & 1.24 & PCI/G \\
\hline & Lead 212 & 0.43 & $\mathrm{PCl} / \mathrm{G}$ & $\ldots$ & 0.07 & $\mathrm{PCl} / \mathrm{G}$ & 0.16 & $\mathrm{PCl} / \mathrm{G}$ \\
\hline \multirow[t]{3}{*}{ TR2A-ES-365N-200E-3.5Z } & Cesium 137 & 0.36 & $\mathrm{PCl} / \mathrm{G}$ & $\ldots$ & 0.08 & $\mathrm{PCl} / \mathrm{G}$ & 0.11 & $\mathrm{PC} / / \mathrm{G}$ \\
\hline & Lead 212 & 0.51 & $\mathrm{PCl} / \mathrm{G}$ & $\cdots$ & 0.07 & $P C / / G$ & 0.20 & $\mathrm{PCI/G}$ \\
\hline & Radium-226 & 0.75 & PCI/G & $\cdots$ & 0.10 & $\mathrm{PCl} / \mathrm{G}$ & 0.22 & $\mathrm{PC} / \mathrm{G}$ \\
\hline \multirow[t]{3}{*}{ TR2A-ES-360N-200E-8Z } & Lead 212 & 0.59 & PCI/G & $\ldots$ & 0.08 & PCI/G & 0.18 & $\mathrm{PCI} / \mathrm{G}$ \\
\hline & Bismuth 214 & 0.24 & $\mathrm{PCl} / \mathrm{G}$ & $\ldots$ & 0.18 & $P C / / G$ & 0.22 & PCI/G \\
\hline & Radium-226 & 0.66 & $\mathrm{PCl} / \mathrm{G}$ & ... & 0.10 & $P C / / G$ & 0.20 & $\mathrm{PCl} / \mathrm{s}$ \\
\hline \multirow[t]{2}{*}{ TR3-ES-450N-400E-3Z } & Thallium 208 & 0.25 & PCl/G & -- & 0.04 & $P C I / G$ & 0.14 & PCI/G \\
\hline & Radium-226 & 0.44 & PCI/G & $\ldots$ & 0.13 & $\mathrm{PCI/G}$ & 0.30 & PCI/G \\
\hline \multirow[t]{3}{*}{ TR3-ES-430N-400E-15Z } & Thallium 208 & 0.25 & PCI/G & $\ldots$ & 0.08 & $P C I / G$ & 0.12 & $\mathrm{PCI} / \mathrm{G}$ \\
\hline & Lead 212 & 0.59 & PCI/G & ... & 0.09 & $P C / / G$ & 0.16 & PCI/G \\
\hline & Radium-226 & 0.36 & PCI/G & $\ldots$ & 0.05 & PCI/G & 0.25 & PCI/G \\
\hline \multirow[t]{6}{*}{ TR4-ES-260-490-8 } & Potassium 40 & 6.27 & $\mathrm{PCl} / \mathrm{G}$ & ... & 1.32 & PCI/G & 2.74 & PCI/G \\
\hline & Thallium 208 & 0.39 & PCI/G & $\ldots$ & 0.07 & PCI/G & 0.08 & PCI/G \\
\hline & Lead 210 & 2.65 & PCI/G & ... & 1.25 & PCI/G & 1.49 & PCI/G \\
\hline & Lead 212 & 1.39 & PCI/G & $\ldots$ & 0.15 & $P C I / G$ & 0.16 & PCI/G \\
\hline & Radium-226 & 1.38 & PCI/G & $\ldots$ & 0.13 & PCI/G & 0.26 & $\mathrm{PCl} / \mathrm{G}$ \\
\hline & Thorium 234 & 2.26 & PCl/G & $\ldots$ & 0.79 & $\mathrm{PCI} / \mathrm{G}$ & 1.34 & $\mathrm{PCl} / \mathrm{G}$ \\
\hline \multirow[t]{2}{*}{ TR5-ES-590-570-12 } & Thallium 208 & 0.31 & PCI/G & - & 0.08 & PCI/G & 0.16 & PCI/G \\
\hline & Lead 212 & 0.53 & PCI/G & $\ldots$ & 0.12 & PCI/G & 0.24 & PCIG \\
\hline
\end{tabular}


Appendix F: Results of Gamma Spectroscopy Analyses, Salmon Site

\begin{tabular}{|c|c|c|c|c|c|c|c|c|}
\hline SAMPLE & COMPOUND & RESULTS & UNITS & $\begin{array}{l}\text { DATA } \\
\text { VALID. } \\
\end{array}$ & $\begin{array}{r}2 \text { SIGMA } \\
\text { ERROR (+/-) } \\
\end{array}$ & UNITS & MDA & UNITS \\
\hline \multirow[t]{4}{*}{ TR6-ES-495-585-12 } & Thallium 208 & 0.44 & $P C / / G$ & NP & 0.05 & PCI/G & 0.11 & PCI/G \\
\hline & Lead 212 & 1.11 & $\mathrm{PCl/G}$ & $N P$ & 0.10 & PCl/G & 0.17 & PCI/G \\
\hline & Radium-226 & 0.60 & PCI/G & $\mathrm{NP}$ & 0.06 & $\mathrm{PCl} / \mathrm{G}$ & 0.19 & $P C I / G$ \\
\hline & Radium-228 & 1.28 & PCI/G & NP & 0.19 & $\mathrm{PCl} / \mathrm{G}$ & 0.37 & $\mathrm{PCl/G}$ \\
\hline TR6-ES-485-590-6 & Lead 212 & 0.46 & $\mathrm{PCl} / \mathrm{G}$ & NP & 0.12 & $\mathrm{PCI/G}$ & 0.22 & PCI/G \\
\hline \multirow[t]{3}{*}{ TR7-ES-558-650-5 } & Thallium 208 & 0.34 & $P C / / G$ & $\ldots$ & 0.09 & $\mathrm{PCl/G}$ & 0.14 & PCI/G \\
\hline & Lead 212 & 0.95 & PCI/G & $\ldots$ & 0.13 & PCI/G & 0.15 & $\mathrm{PCl} / \mathrm{G}$ \\
\hline & Radium-226 & 0.67 & $\mathrm{PCI} / \mathrm{G}$ & $\cdots$ & 0.11 & PCI/G & 0.20 & PCIVG \\
\hline \multirow[t]{4}{*}{ TR7-ES-560-650-14 } & Potassium 40 & 3.32 & PCI/G & $\ldots$ & 0.85 & PCI/G & 2.20 & PCI/G \\
\hline & Thallium 208 & 0.32 & $\mathrm{PCl} / \mathrm{G}$ & $\ldots$ & 0.07 & $\mathrm{PCl} / \mathrm{G}$ & 0.14 & PCI/G \\
\hline & Lead 212 & 1.19 & $\mathrm{PCl/G}$ & ... & 0.11 & $\mathrm{PCI/G}$ & 0.20 & PCI/G \\
\hline & Radium-226 & 0.68 & PCI/G & $\cdots$ & 0.11 & PCI/G & 0.20 & $\mathrm{PCI/G}$ \\
\hline TR8-ES-780-600-3 & Lead 212 & 0.40 & $\mathrm{PCI} / \mathrm{G}$ & ... & 0.14 & PCI/G & 0.18 & PCIVG \\
\hline \multirow[t]{2}{*}{ TR8-ES-780-600-3-DUP } & Lead 212 & 0.56 & $P C / / G$ & NP & 0.07 & $\mathrm{PCl} / \mathrm{G}$ & 0.18 & PCI/G \\
\hline & Radium-226 & 0.59 & $P C / / G$ & NP & 0.11 & $P C l / G$ & 0.22 & PCI/G \\
\hline TA10-ES-180-790-15 & Lead 212 & 0.26 & $P C / / G$ & $\ldots$ & 0.09 & $\mathrm{PCI/G}$ & 0.17 & PCI/G \\
\hline \multirow[t]{2}{*}{ TR10-ES-190-790-8 } & Thallium 208 & 0.22 & PCI/G & $\ldots$ & 0.08 & PCI/G & 0.12 & $P C / / G$ \\
\hline & Lead 212 & 0.61 & $P C I / G$ & $\cdots$ & 0.09 & $P C / / G$ & 0.13 & PCI/G \\
\hline \multirow[t]{2}{*}{ TR11-ES-180-930-3 } & Thallium 208 & 0.17 & $\mathrm{PCI/G}$ & NP & 0.08 & $\mathrm{PCl/G}$ & 0.10 & PCI/G \\
\hline & Lead 212 & 0.36 & $\mathrm{PCI} / \mathrm{G}$ & NP & 0.08 & $\mathrm{PCI/G}$ & 0.18 & PCVIG \\
\hline TR13-ES-210-1110-6 & Lead 212 & 0.59 & $\mathrm{PCI/G}$ & NP & 0.12 & PCI/G & 0.18 & PCI/G \\
\hline TR13-ES-212-1150-6 & Lead 212 & 0.52 & PCI/G & NP & 0.14 & $\mathrm{PCI/G}$ & 0.23 & PCl/a \\
\hline \multirow[t]{4}{*}{ TR14-ES-80-1189-5 } & Cerium 141 & 0.17 & $\mathrm{PCl/G}$ & NP & 0.07 & $\mathrm{PCI/G}$ & 0.07 & PCI/G \\
\hline & Thallium 208 & 0.13 & PCI/G & NP & 0.08 & $P C / / G$ & 0.11 & PCVO \\
\hline & Lead 212 & 0.48 & PCU/G & NP & 0.08 & $P C I / G$ & 0.14 & PCVG \\
\hline & Uranium 235 & 0.41 & PCI/G & NP & 0.18 & PCl/G & 0.33 & PCV/G \\
\hline \multirow[t]{2}{*}{ TR14-ES-90-1200-3 } & Thallium 208 & 0.22 & PCI/G & NP & 0.10 & $P C / / G$ & 0.14 & PCI/G \\
\hline & Lead 212 & 0.56 & PCI/G & NP & 0.12 & $P C / / G$ & 0.18 & PCI/G \\
\hline
\end{tabular}


Appendix F: Results of Gamma Spectroscopy Analyses, Salmon Site

\begin{tabular}{|c|c|c|c|c|c|c|c|c|}
\hline SAMPLE & COMPOUND & RESULTS & UNITS & $\begin{array}{l}\text { DATA } \\
\text { VALID. }\end{array}$ & $\begin{array}{r}2 \text { SIGMA } \\
\text { ERROR }(+/ \cdot) \\
\end{array}$ & UNITS & MDA & UNITS \\
\hline \multirow[t]{3}{*}{ TR-15-ES-20-1180-.5 } & Thallium 208 & 0.32 & PCl/G & NP & 0.07 & PCI/G & 0.12 & PCI/G \\
\hline & Lead 212 & 0.82 & PCI/G & $N P$ & 0.08 & PCI/G & 0.19 & PCI/G \\
\hline & Radium-226 & 0.50 & $P C / / G$ & NP & 0.07 & $\mathrm{PCl/G}$ & 0.21 & PCI/G \\
\hline TR-15-ES-20-1180-.5DUP & Lead 212 & 0.51 & $P C / / G$ & NP & 0.15 & $\mathrm{PCl/G}$ & 0.19 & PCI/G \\
\hline TR16-ES-200-80-1.5 & Lead 212 & 0.80 & $\mathrm{PCI/G}$ & NP & 0.05 & PCl/G & 0.15 & PCI/G \\
\hline \multirow[t]{3}{*}{ TR17-ES-30-120-5 } & Thallium 208 & 0.26 & PCI/G & $N P$ & 0.08 & PCI/G & 0.18 & $P C I / G$ \\
\hline & Lead 212 & 0.56 & $\mathrm{PCl/G}$ & NP & 0.16 & $P C / / G$ & 0.23 & $\mathrm{PCl} / \mathrm{G}$ \\
\hline & Radium-226 & 0.37 & PCI/G & NP & 0.13 & $P C I / G$ & 0.37 & PCI/G \\
\hline \multirow[t]{5}{*}{ TR17-ES-30-125-8 } & Potassium 40 & 4.54 & PCI/G & NP & 0.80 & $\mathrm{PCl} / \mathrm{G}$ & 2.46 & $P C / / G$ \\
\hline & Thallium 208 & 0.34 & PCI/G & NP & 0.06 & $P C / / G$ & 0.14 & $\mathrm{PCI} / \mathrm{G}$ \\
\hline & Lead 212 & 1.14 & PCI/G & NP & 0.15 & PCI/G & 0.17 & $\mathrm{PCl} / \mathrm{G}$ \\
\hline & Radium-226 & 0.48 & PCI/G & NP & 0.09 & PCI/G & 0.24 & $\mathrm{PC} / / \mathrm{G}$ \\
\hline & Thorium 234 & 1.59 & PCI/G & NP & 0.46 & PCl/G & 1.24 & PCI/G \\
\hline \multirow[t]{5}{*}{ TR18-BS-90-150A-E } & Potassium 40 & 5.45 & PCI/G & NP & 1.59 & PCI/G & 3.21 & PCI/G \\
\hline & Thallium 208 & 0.27 & PCI/G & NP & 0.07 & $\mathrm{PCl} / \mathrm{G}$ & 0.14 & $\mathrm{PCI} / \mathrm{G}$ \\
\hline & Lead 210 & 1.34 & PCl/G & NP & 0.57 & $P C I / G$ & 1.31 & ف 1. 1 \\
\hline & Lead 212 & 0.70 & $P C / / G$ & NP & 0.09 & PCI/G & 0.19 & $P C / / G$ \\
\hline & Radium-226 & 0.61 & PCI/G & NP & 0.11 & $\mathrm{PCl} / / \mathrm{G}$ & 0.22 & $P C l / G$ \\
\hline \multirow[t]{2}{*}{ TR19-BS-0-150A-E } & Lead 212 & 0.66 & $P C / / G$ & $\mathrm{NP}$ & 0.09 & PCI/G & 0.17 & $\mathrm{PCI} / \mathrm{G}$ \\
\hline & Radium-226 & 0.58 & PCI/G & NP & 0.11 & $\mathrm{PCl} / \mathrm{G}$ & 0.19 & PCI/G \\
\hline
\end{tabular}

NOTE: NON-DETECTS NOT REPORTED 
Appendix G

Results of Metals and Cyanide Analyses 
Appendix G: Results of Metals and Cyanide Analyses, Salmon Site

\begin{tabular}{|c|c|c|c|c|c|c|c|c|}
\hline SAMPLE & COMPOUND & RESULTS & UNITS & $\begin{array}{l}\text { DATA } \\
\text { QUAL } \\
\end{array}$ & $\begin{array}{l}\text { DATA } \\
\text { VALID. } \\
\end{array}$ & CRDL & UNITS & DILUTION \\
\hline \multirow[t]{24}{*}{ TR1-ES-001 } & Arsenic & 0.98 & $M G / K G$ & $B$ & $J$ & 2 & $M G / K G$ & 1 \\
\hline & Cyanide & 0.06 & $M G / K G$ & $B$ & J & 0.5 & $M G / K G$ & 1 \\
\hline & Mercury & 0.48 & $M G / K G$ & & - & 0.1 & $M G / K G$ & 1 \\
\hline & Aluminum & 814 & $M G / K G$ & & - & 40 & $M G / K G$ & 1 \\
\hline & Antimony & 7 & $M G / K G$ & U & UJ & 12 & $M G / K G$ & 1 \\
\hline & Barium & 13.8 & $M G / K G$ & $B$ & $\mathrm{~J}$ & 40 & $M G / K G$ & 1 \\
\hline & Beryllium & 0.48 & $M G / K G$ & U & $\ldots$ & 1 & $M G / K G$ & 1 \\
\hline & Cadmium & 0.72 & $M G / K G$ & $B$ & $J$ & 1 & $M G / K G$ & 1 \\
\hline & Calcium & 386 & $M G / K G$ & $B$ & J & 1000 & $M G / K G$ & 1 \\
\hline & Chromium & 5.1 & $M G / K G$ & & $\cdots$ & 2 & $M G / K G$ & 1 \\
\hline & Cobalt & 1.5 & $M G / K G$ & $B$ & $J$ & 10 & $M G / K G$ & 1 \\
\hline & Copper & 77.2 & $M G / K G$ & & - & 5 & $M G / K G$ & 1 \\
\hline & Iron & 1910 & $M G / K G$ & & - & 20 & $M G / K G$ & 1 \\
\hline & Magnesium & 54.4 & $M G / K G$ & B & $J$ & 1000 & $M G / K G$ & 1 \\
\hline & Manganese & 67.3 & MG/KG & & $\ldots$ & 3 & $M G / K G$ & 1 \\
\hline & Nickel & 6 & $M G / K G$ & $B$ & $J$ & 8 & $M G / K G$ & 1 \\
\hline & Potassium & 434 & $M G / K G$ & $U$ & .-- & 1000 & $M G / K G$ & 1 \\
\hline & Silver & 1.4 & $M G / K G$ & B & $J$ & 2 & $M G / K G$ & 1 \\
\hline & Sodium & 54.7 & $M G / K G$ & $B$ & $J$ & 1000 & $M G / K G$ & 1 \\
\hline & Vanadium & 4.9 & MG/KG & B & $U$ & 10 & $M G / K G$ & 1 \\
\hline & Zinc & 490 & MGKG & & - & 4 & MG/KG & 1 \\
\hline & Lead & 5.2 & MGKG & & - & 0.6 & $M G / K G$ & 1 \\
\hline & Selenium & 0.22 & $M G / K G$ & U & UJ & 1 & $M G / K G$ & 1 \\
\hline & Thallium & 0.2 & $M G / K G$ & U & $\ldots$ & 2 & $M G / K G$ & 1 \\
\hline
\end{tabular}


Appendix G: Results of Metals and Cyanide Analyses, Salmon Site

\begin{tabular}{|c|c|c|c|c|c|c|c|c|}
\hline SAMPLE & COMPOUND & RESULTS & UNITS & $\begin{array}{l}\text { DATA } \\
\text { QUAL }\end{array}$ & $\begin{array}{l}\text { DATA } \\
\text { VALID. }\end{array}$ & CRDL & UNITS & DILUTION \\
\hline \multirow[t]{24}{*}{ TR2-ES-355N-175E-5Z } & Arsenic & 3.3 & MG/KG & & $\mathrm{J}$ & 2 & $M G / K G$ & 1 \\
\hline & Cyanide & 0.05 & MG/KG & $U$ & $\ldots$ & 0.5 & $M G / K G$ & 1 \\
\hline & Mercury & 6.5 & MGKG & & - & 0.1 & $M G / K G$ & 1 \\
\hline & Aluminum & 4800 & $M G / K G$ & & - & 40 & $M G / K G$ & 1 \\
\hline & Antimony & 7 & $M G / K G$ & U & UJ & 12 & $M G / K G$ & 1 \\
\hline & Barium & 63.7 & Ma/KG & & $\cdots$ & 40 & $M G / K G$ & 1 \\
\hline & Beryllium & 0.48 & $M G / K G$ & $U$ & $\cdots$ & 1 & $M G / K G$ & 1 \\
\hline & Cadmium & 4 & MG/KG & & - & 1 & $\mathrm{MG} / \mathrm{KG}$ & 1 \\
\hline & Calcium & 1390 & MQ/KG & & - & 1000 & $M G / K G$ & 1 \\
\hline & Chromium & 12.2 & MG/KG & & $\cdots$ & 2 & $M G / K G$ & 1 \\
\hline & Cobalt & 1.8 & $M G / K G$ & $B$ & J & 10 & $M G / K G$ & 1 \\
\hline & Copper & 72.3 & MGKG & & - & 5 & $M G / K G$ & 1 \\
\hline & Iron & 11700 & MQRKG & & - & 20 & $M G / K G$ & 1 \\
\hline & Magnesium & 283 & $M G / K G$ & $B$ & J & 1000 & $M G / K G$ & 1 \\
\hline & Manganese & 386 & $M G / K G$ & & $\ldots$ & 3 & $M G / K G$ & 1 \\
\hline & Nickel & 23.6 & MG/KG & & $\cdots$ & 8 & $M G / K G$ & 1 \\
\hline & Potassium & 434 & $M G / K G$ & $U$ & $\ldots$ & 1000 & $M G / K G$ & 1 \\
\hline & Silver & 63.8 & $M G / K G$ & & -- & 2 & $M G / K G$ & 1 \\
\hline & Sodium & 127 & $M G / K G$ & B & $J$ & 1000 & $M G / K G$ & 1 \\
\hline & Vanadium & 11.9 & MG/KG & & - & 10 & MGKG & 1 \\
\hline & Zinc & 519 & Ma/KG & & - & 4 & MG/KG & 1 \\
\hline & Lead & 28.5 & MG/KG & & - & 6 & $M G / K G$ & 10 \\
\hline & Selenium & 0.22 & MG/KG & $U$ & UJ & 1 & $M G / K G$ & 1 \\
\hline & Thallium & 0.2 & $M G / K G$ & $U$ & - & 2 & $M G / K G$ & 1 \\
\hline
\end{tabular}


Appendix G: Results of Metals and Cyanide Analyses, Salmon Site

\begin{tabular}{|c|c|c|c|c|c|c|c|c|}
\hline SAMPLE & COMPOUND & RESULTS & UNITS & $\begin{array}{l}\text { DATA } \\
\text { QUAL }\end{array}$ & $\begin{array}{c}\text { DATA } \\
\text { VALID. }\end{array}$ & CRDL & UNITS & DILUTION \\
\hline \multirow[t]{24}{*}{ TR2-ES-356N-175E-8Z } & Arsenic & $\overline{6.1}$ & $M G / K G$ & & $J$ & 2 & $M G / K G$ & 1 \\
\hline & Cyanide & 0.05 & $M G / K G$ & $U$ & $\cdots$ & 0.49 & $M G / K G$ & 1 \\
\hline & Mercury & 5.4 & MG/KG & & $\ldots$ & 0.1 & $M G / K G$ & 1 \\
\hline & Aluminum & 8250 & MG/KG & & - & 40 & MG/KG & 1 \\
\hline & Antimony & 11.7 & $M G / K G$ & B & J & 12 & $M G / K G$ & 1 \\
\hline & Barium & 177 & MaKKG & & - & 40 & MG/KG & 1 \\
\hline & Beryllium & 0.48 & $M G / K G$ & U & $\ldots$ & 1 & $M G / K G$ & 1 \\
\hline & Cadmium & 10.5 & $M G / K G$ & & $\cdots$ & 1 & $M Q / K G$ & 1 \\
\hline & Calcium & 3770 & MG/KG & & $\ldots$ & 1000 & $M G / K G$ & 1 \\
\hline & Chromium & 22 & MG/KG & & $\cdots$ & 2 & $M G / K G$ & 1 \\
\hline & Cobalt & 57.8 & MG/KG & & $\ldots$ & 10 & $M G / K G$ & 1 \\
\hline & Copper & $159 n$ & Ma/KG & & - & 5 & MG/KG & 1 \\
\hline & Iron & 19300 & $M G / K G$ & & - & 20 & MG/KG & 1 \\
\hline & Magnesium & 499 & MG/KG & $B$ & J & 1000 & $\mathrm{MG} / \mathrm{KG}$ & 1 \\
\hline & Manganese & 3460 & $M G / K G$ & & $\ldots$ & 3 & MG/KG & 1 \\
\hline & Nickel & 19.4 & $M G / K G$ & & $\ldots$ & 8 & MG/KG & 1 \\
\hline & Potassium & 434 & $M G / K G$ & $U$ & $\cdots$ & 1000 & $M G / K G$ & 1 \\
\hline & Silver & 54.5 & $M G / K G$ & & $\ldots$ & 2 & $M G / K G$ & 1 \\
\hline & Sodium & 151 & $M G / K G$ & B & J & 1000 & $M G / K G$ & 1 \\
\hline & Vanadium & 15.7 & MG/KG & & - & 10 & $M G / K G$ & 1 \\
\hline & Zinc & 2710 & $M G / K G$ & & - & 4 & $M G / K G$ & 1 \\
\hline & Lead & 133 & $M G / K G$ & & $\ldots$ & 30 & $M G / K G$ & 50 \\
\hline & Selenium & 0.22 & $M G / K G$ & $U$ & UJ & 1 & $M G / K G$ & 1 \\
\hline & Thallium & 0.2 & $M G / K G$ & U & -.- & 2 & $M G / K G$ & 1 \\
\hline
\end{tabular}


Appendix G: Results of Metals and Cyanide Analyses, Salmon Site

\begin{tabular}{|c|c|c|c|c|c|c|c|c|}
\hline SAMPLE & COMPOUND & RESULTS & UNITS & $\begin{array}{l}\text { DATA } \\
\text { QUAL }\end{array}$ & $\begin{array}{r}\text { DATA } \\
\text { VALID. } \\
\end{array}$ & CROL & UNITS & DILUTION \\
\hline \multirow[t]{24}{*}{ TR2A-ES-365N-200E-3.5Z } & Arsenic & 2.9 & $M G / K G$ & & $J$ & 2 & MG/KG & 1 \\
\hline & Cyanide & 0.05 & MG/KG & $B$ & $J$ & 0.49 & $M G / K G$ & 1 \\
\hline & Mercury & 0.4 & MG/KG & & $\cdots$ & 0.1 & $M G / K G$ & 1 \\
\hline & Aluminum & 5720 & MGKG & & - & 40 & MG/KG & 1 \\
\hline & Antimony & 7 & $M G / K G$ & $U$ & UJ & 12 & $M G / K G$ & 1 \\
\hline & Barium & 197 & MGKG & & - & 40 & MG/KG & 1 \\
\hline & Beryllium & 0.48 & $M G / K G$ & $U$ & - & 1 & MG/KG & 1 \\
\hline & Cadmium & 2 & MG/KG & & $\cdots$ & 1 & MG/KG & 1 \\
\hline & Calcium & 7310 & MOKG & & $\cdots$ & 1000 & MGKG & 1 \\
\hline & Chromium & 179 & $M Q / K G$ & & $\cdots$ & 2 & MG/KG & 1 \\
\hline & Cobalt & 2.6 & $M Q / K G$ & B & $J$ & 10 & $M G / K G$ & 1 \\
\hline & Copper & 83.9 & MGKG & & $\cdots$ & 5 & $M G / K G$ & 1 \\
\hline & Iron & 9330 & MQ/KG & & - & 20 & $M G / K a$ & 1 \\
\hline & Magnesium & 404 & $M G / K G$ & B & $\mathrm{J}$ & 1000 & $M G / K G$ & 1 \\
\hline & Manganese & 143 & $M G / K G$ & & $\cdots$ & 3 & $M G / K G$ & 1 \\
\hline & Nickel & 8 & $M G / K G$ & B & $\mathrm{J}$ & 8 & $M G / K Q$ & 1 \\
\hline & Potassium & 434 & $M G / K G$ & $U$ & $\cdots$ & 1000 & MG/KG & 1 \\
\hline & Silver & 1.7 & $M G / K G$ & $B$ & $\mathrm{~J}$ & 2 & $M G / K G$ & 1 \\
\hline & Sodium & 206 & MG/KG & $B$ & $J$ & 1000 & $M G / K G$ & 1 \\
\hline & Vanadium & 14.8 & MGKG & & - & 10 & $M G / K G$ & 1 \\
\hline & Zinc & 333 & MG/KG & & $\cdots$ & 4 & MG/KG & 1 \\
\hline & Lead & 199 & $M G / K G$ & & $\cdots$ & 30 & MG/KG & 50 \\
\hline & Selenıum & 0.22 & $M G / K G$ & $U$ & US & 1 & $M G / K G$ & 1 \\
\hline & Thallium & 0.2 & $M G / K G$ & $U$ & $\ldots$ & 2 & $M G / K G$ & 1 \\
\hline
\end{tabular}


Appendix G: Results of Metals and Cyanide Analyses. Salmon Site

\begin{tabular}{|c|c|c|c|c|c|c|c|c|}
\hline SAMPLE & COMPOUND & RESULTS & UNITS & $\begin{array}{l}\text { DATA } \\
\text { QUAL }\end{array}$ & $\begin{array}{l}\text { DATA } \\
\text { VALID. }\end{array}$ & CRDL & UNITS & DILUTION \\
\hline \multirow[t]{24}{*}{ TR2A-ES-360N-200E-8Z } & Arsenic & 2.3 & MG/KG & & $J$ & 2 & MGKG & 1 \\
\hline & Cyanide & 0.05 & MQ/KG & $U$ & $\cdots$ & 0.5 & $M G / K G$ & 1 \\
\hline & Mercury & 0.16 & MaKa & & - & 0.1 & MGKG & 1 \\
\hline & Aluminum & 3840 & MGKG & & - & 40 & MGKG & 1 \\
\hline & Antimony & 7 & $M G / K G$ & $u$ & UJ & 12 & $M G / K G$ & 1 \\
\hline & Barium & 16.2 & MQ/KO & $B$ & $J$ & 40 & MQKG & 1 \\
\hline & Beryllium & 0.48 & Ma/KG & $u$ & $\ldots$ & 1 & MG/KG & 1 \\
\hline & Cadmium & 0.76 & $M G / K G$ & $B$ & J & 1 & $M G / K G$ & 1 \\
\hline & Calcium & 515 & MG/KG & B & J & 1000 & $M Q / K G$ & 1 \\
\hline & Chromium & 6.3 & MG/KG & & $\cdots$ & 2 & $M G / K G$ & 1 \\
\hline & Cobalt & 1.1 & MG/KG & $u$ & $\ldots$ & 10 & $M G / K G$ & 1 \\
\hline & Copper & 34.5 & Ma/KG & & - & 5 & MGKG & 1 \\
\hline & Iron & 3950 & Ma/KG & & - & 20 & Ma/KG & 1 \\
\hline & Magnesium & 149 & MG/KG & B & $J$ & 1000 & MG/KG & 1 \\
\hline & Manganese & 26.5 & MG/KG & & ..- & 3 & MQ/KG & 1 \\
\hline & Nickel & 4.9 & MG/KG & $B$ & J & 8 & MG/KG & 1 \\
\hline & Potassium & 434 & MG/KG & $U$ & $\ldots$ & 1000 & $M G / K G$ & 1 \\
\hline & Silver & 1.3 & MG/KG & B & $J$ & 2 & $M G / K G$ & 1 \\
\hline & Sodium & 42.2 & MG/KG & B & $U$ & 1000 & $M G / K G$ & 1 \\
\hline & Vanadium & 10.3 & MGKG & & - & 10 & MGKG & 1 \\
\hline & Zinc & 55 & MQ/KG & & - & 4 & MGKG & 1 \\
\hline & Lead & 21.5 & MQ/KG & & - & 2.4 & MGKG & 4 \\
\hline & Selenium & 0.22 & MEKG & U & UJ & 1 & MG/KG & 1 \\
\hline & Thallium & 0.2 & $M G / K G$ & $U$ & $\cdots$ & 2 & $M G / K G$ & 1 \\
\hline
\end{tabular}


Appendix G: Results of Metals and Cyanide Analyses, Salmon Site

\begin{tabular}{|c|c|c|c|c|c|c|c|c|}
\hline SAMPLE & COMPOUND & RESULTS & UNITS & $\begin{array}{l}\text { DATA } \\
\text { QUAL }\end{array}$ & $\begin{array}{l}\text { DATA } \\
\text { VALID. }\end{array}$ & CRDL & UNITS & DILUTION \\
\hline \multirow[t]{24}{*}{ TR3-ES-45ON-40OE-3Z } & Arsenic & 2.3 & MaKG & & $J$ & 2 & MG/KG & 1 \\
\hline & Cyanide & 0.05 & MaKG & $U$ & $\cdots$ & 0.48 & MG/KG & 1 \\
\hline & Mercury & 0.05 & MEKG & U & $\cdots$ & 0.1 & $M G / K G$ & 1 \\
\hline & Aluminum & 6400 & MaKo & & $\cdots$ & 40 & MG/KG & 1 \\
\hline & Antimony & 7 & MO/KG & U & UJ & 12 & $M G / K G$ & 1 \\
\hline & Barium & 30.7 & MEKG & B & $J$ & 40 & MGKG & 1 \\
\hline & Beryllium & 0.48 & MGKG & U & $\cdots$ & 1 & MG/KG & 1 \\
\hline & Cadmium & 0.58 & MGKG & U & $\cdots$ & 1 & MOKG & 1 \\
\hline & Calcium & 1940 & MQKG & & - & 1000 & MOKG & 1 \\
\hline & Chromium & 40.6 & MGKG & & - & 2 & MG/KO & 1 \\
\hline & Cobalt & 1.4 & MGKG & B & $J$ & 10 & $M G / K G$ & 1 \\
\hline & Copper & 4.3 & MG/KO & B & U & 5 & MG/KO & 1 \\
\hline & Iron & 6810 & MGKO & & - & 20 & MGKO & 1 \\
\hline & Magnesium & 323 & MG/KG & B & $J$ & 1000 & $M G / K G$ & 1 \\
\hline & Manganese & 28.3 & MaKG & & $\cdots$ & 3 & MQ/KG & 1 \\
\hline & Nickel & 4.7 & MaKa & B & $J$ & 8 & MG/KG & 1 \\
\hline & Potassium & 434 & MOKG & U & - & 1000 & MaKO & 1 \\
\hline & Silver & 0.82 & MaKa & $U$ & $\cdots$ & 2 & MGKG & 1 \\
\hline & Sodium & 595 & MGKG & B & $J$ & 1000 & MGKG & 1 \\
\hline & Vanadium & 14.8 & MGKG & & - & 10 & MGKG & 1 \\
\hline & Zinc & 16.1 & MGKO & & - & 4 & MGKG & 1 \\
\hline & Lead & 4 & MOKG & & $\cdots$ & 0.6 & $M G / K G$ & 1 \\
\hline & Selenium & 0.22 & MG/KG & $U$ & UJ & 1 & MG/KG & 1 \\
\hline & Thallium & 0.2 & MG/KG & $U$ & - & 2 & $M G / K G$ & 1 \\
\hline
\end{tabular}


Appendix G: Results of Metals and Cyanide Analyses, Salmon Site

\begin{tabular}{|c|c|c|c|c|c|c|c|c|}
\hline SAMPLE & COMPOUND & RESULTS & UNITS & $\begin{array}{l}\text { DATA } \\
\text { QUAL }\end{array}$ & $\begin{array}{l}\text { DATA } \\
\text { VALID. }\end{array}$ & CRDL & UNITS & DILUTION \\
\hline \multirow[t]{24}{*}{ TR3-ES-430N-400E-152 } & Arsenic & 2.2 & MGKO & & J & 2 & MEKG & 1 \\
\hline & Cyanide & 0.05 & MaKo & U & - & 0.48 & MG/KG & 1 \\
\hline & Mercury & 0.05 & MaKa & $U$ & - & 0.1 & MaKa & 1 \\
\hline & Aluminum & 5030 & MaKO & & - & 40 & MaKa & 1 \\
\hline & Antimony & 7 & MGKG & $u$ & UJ & 12 & MG/KG & 1 \\
\hline & Barium & 19.3 & Ma/KG & B & $J$ & 40 & MaKG & 1 \\
\hline & Beryllium & 0.48 & MaKa & U & - & 1 & MGKG & 1 \\
\hline & Cadmium & 0.58 & MOKG & $U$ & - & 1 & MOKG & 1 \\
\hline & Calcium & 851 & MEKG & B & J & 1000 & MaKG & 1 \\
\hline & Chromium & 9.8 & MGKG & & - & 2 & MGKG & 1 \\
\hline & Cobalt & 1.1 & MGKO & U & - & 10 & MGKG & 1 \\
\hline & Copper & 3.5 & MOKG & B & $U$ & 5 & MQKG & 1 \\
\hline & Iron & 6180 & MOKKO & & - & 20 & MOKG & 1 \\
\hline & Magnesium & 215 & MGKG & B & $J$ & 1000 & MaKG & 1 \\
\hline & Manganese & 24.8 & $M G / K G$ & & $\cdots$ & 3 & MG/KG & 1 \\
\hline & Nickel & 3.6 & MOKо & U & - & 8 & MGKG & 1 \\
\hline & Potassium & 434 & MGKG & $U$ & - & 1000 & MGKG & 1 \\
\hline & Silver & 0.82 & MEKG & $U$ & - & 2 & $M G / K G$ & 1 \\
\hline & Sodium & 129 & MaKK & B & $J$ & 1000 & MGKO & 1 \\
\hline & Vanadium & 11.6 & MOKG & & - & 10 & MOKG & 1 \\
\hline & Zinc & 12.8 & MaKo & & - & 4 & MEKG & 1 \\
\hline & Lead & 4 & MaKa & & - & 0.6 & MGKG & 1 \\
\hline & Solenium & 0.22 & MOKG & $U$ & US & 1 & MGKG & 1 \\
\hline & Thallium & 0.2 & MG/KG & $U$ & - & 2 & MG/KG & 1 \\
\hline
\end{tabular}


Appendix G: Results of Metals and Cyanide Analyses, Salmon Site

\begin{tabular}{|c|c|c|c|c|c|c|c|c|}
\hline SAMPLE & COMPOUND & RESULTS & UNITS & $\begin{array}{l}\text { DATA } \\
\text { QUAL }\end{array}$ & $\begin{array}{r}\text { DATA } \\
\text { VALID. }\end{array}$ & CADL & UNITS & DILUTION \\
\hline \multirow[t]{24}{*}{ TR4-ES-260-490-8 } & Arsenic & 1.9 & $M G K G$ & $B$ & $J$ & 2 & $M G / K G$ & 1 \\
\hline & Cyanide & 0.05 & MaKo & $U$ & - & 0.49 & $M G / K G$ & 1 \\
\hline & Mercury & 0.05 & MG/KG & $U$ & $\cdots$ & 0.1 & MGKG & 1 \\
\hline & Aluminum & 8800 & MGKG & & - & 40 & MaKa & 1 \\
\hline & Antimony & 7 & $M G / K G$ & U & UJ & 12 & $M G / K G$ & 1 \\
\hline & Barium & 75.3 & MGKG & & - & 40 & $M G / K G$ & 1 \\
\hline & Beryllium & 0.54 & MGKG & $B$ & $\mathrm{~J}$ & 1 & Ma/Ka & 1 \\
\hline & Cadmium & 0.66 & MGKG & $B$ & $J$ & 1 & MG/KG & 1 \\
\hline & Calcium & 1170 & MaKG & & $\rightarrow$ & 1000 & MQKG & 1 \\
\hline & Chromium & 24 & MGKG & & $\cdots$ & 2 & MGKG & 1 \\
\hline & Cobalt & 4 & MGKG & $B$ & J & 10 & MG/KG & 1 \\
\hline & Copper & 5.6 & MGKG & & $U$ & 5 & MGKO & 1 \\
\hline & Iron & 13000 & MGKG & & - & 20 & MQ/KG & 1 \\
\hline & Magnesium & 881 & MG/KG & B & $J$ & 1000 & $M G / K G$ & 1 \\
\hline & Manganese & 67.1 & MGKG & & $\cdots$ & 3 & $M G / K G$ & 1 \\
\hline & Nickel & 7.2 & MQKKG & B & J & 8 & MG/KG & 1 \\
\hline & Potassium & 697 & MaKG & B & $\mathrm{J}$ & 1000 & MG/KG & 1 \\
\hline & Silver & 0.82 & MGKG & $U$ & - & 2 & MGKG & 1 \\
\hline & Sodium & 315 & MaKG & B & J & 1000 & MEKG & 1 \\
\hline & Vanadium & 18.8 & MQKKG & & - & 10 & MOKKG & 1 \\
\hline & Zinc & 17.9 & MOKO & & - & 4 & MaKa & 1 \\
\hline & Load & 4.8 & MaKo & & - & 0.6 & MaKG & 1 \\
\hline & Selenium & 0.22 & MOKG & $U$ & US & 1 & MOKG & 1 \\
\hline & Thallium & 0.2 & MG/KG & $U$ & $\ldots$ & 2 & MG/KG & 1 \\
\hline
\end{tabular}


Appendix G: Results of Metals and Cyanide Analyses, Salmon Site

\begin{tabular}{|c|c|c|c|c|c|c|c|c|}
\hline SAMPLE & COMPOUND & RESULTS & UNITS & $\begin{array}{l}\text { DATA } \\
\text { QUAL }\end{array}$ & $\begin{array}{l}\text { DATA } \\
\text { VALID. }\end{array}$ & CRDL & UNITS & DILUTION \\
\hline \multirow[t]{24}{*}{ TR5-ES-590-570-12 } & Arsenic & 2.1 & MGKG & & $J$ & 2 & MGKG & 1 \\
\hline & Cyanide & 0.05 & MGKG & $U$ & - & 0.49 & MGKG & 1 \\
\hline & Mercury & 0.05 & MGKG & U & - & 0.1 & MGKG & 1 \\
\hline & Aluminum & 8570 & MGKG & & - & 40 & MGKG & 1 \\
\hline & Antimony & 7 & $M G / K G$ & U & J & 12 & $M G / K G$ & 1 \\
\hline & Barium & 36.7 & MGKG & B & J & 40 & $M G / K G$ & 1 \\
\hline & Beryllium & 0.48 & MGKG & $U$ & - & 1 & $M G / K G$ & 1 \\
\hline & Cadmium & 0.58 & MG/KG & $U$ & - & 1 & MG/KG & 1 \\
\hline & Calcium & 567 & MGKG & B & J & 1000 & MG/KG & 1 \\
\hline & Chromium & 8.4 & MGKG & & - & 2 & MGKG & 1 \\
\hline & Cobait & 1.7 & MGKG & B & $J$ & 10 & MGKG & 1 \\
\hline & Copper & 5 & MGKG & B & $U$ & 5 & $M G / K G$ & 1 \\
\hline & Iron & 7600 & MGKG & & - & 20 & MGKG & 1 \\
\hline & Magnesium & 328 & MGKG & B & $J$ & 1000 & $M G / K G$ & 1 \\
\hline & Manganese & 47.6 & $M G / K G$ & & $\ldots$ & 3 & $M G / K G$ & 1 \\
\hline & Nickel & 5.6 & MG/KG & B & J & 8 & MGKG & 1 \\
\hline & Potassium & 434 & MG/KG & $U$ & - & 1000 & MG/KG & 1 \\
\hline & Silver & 0.82 & MG/KG & U & - & 2 & MG/KG & 1 \\
\hline & Sodium & 481 & MGKG & B & $J$ & 1000 & MG/KG & 1 \\
\hline & Vanadium & 18.2 & MaKG & & - & 10 & MGKG & 1 \\
\hline & Zinc & 10.1 & MONKG & & - & 4 & MaKG & 1 \\
\hline & Lead & 4.8 & MaKG & & - & 0.6 & MG/KG & 1 \\
\hline & Selenium & 0.22 & MGKG & $U$ & $w$ & 1 & MaKG & 1 \\
\hline & Thallium & 0.2 & MG/KG & $U$ & - & 2 & $M G / K G$ & 1 \\
\hline
\end{tabular}


Appendix G: Results of Metals and Cyanide Analyses. Salmon Site

\begin{tabular}{|c|c|c|c|c|c|c|c|c|}
\hline SAMPLE & COMPOUND & RESULTS & UNITS & $\begin{array}{l}\text { DATA } \\
\text { QUAL }\end{array}$ & $\begin{array}{l}\text { DATA } \\
\text { VALID. }\end{array}$ & CRDL & UNITS & DILUTION \\
\hline \multirow[t]{24}{*}{ TR6-ES-495-585-12 } & Arsenic & 1.9 & $M G / K G$ & $B$ & NP & 2 & $M G / K G$ & 1 \\
\hline & Cyanide & 0.05 & $M G / K G$ & $U$ & NP & 0.5 & $M G / K G$ & 1 \\
\hline & Mercury & 0.05 & MG/KG & $U$ & NP & 0.1 & $M G / K G$ & 1 \\
\hline & Aluminum & 10200 & $M G / K G$ & & NP & 40 & $M G / K G$ & 1 \\
\hline & Antimony & 4.5 & $M G / K G$ & $U$ & NP & 12 & $M G / K G$ & 1 \\
\hline & Barium & 24.6 & $M G / K G$ & $B$ & NP & 40 & $M G / K G$ & 1 \\
\hline & Beryllium & 0.35 & $M G / K G$ & $B$ & NP & 1 & $M G / K G$ & 1 \\
\hline & Cadmium & 0.7 & $M G / K G$ & $B$ & NP & 1 & $M G / K G$ & 1 \\
\hline & Calcium & 7510 & MG/KG & & NP & 1000 & MG/KG & 1 \\
\hline & Chromium & 52.9 & MG/KG & & NP & 2 & $M G / K G$ & 1 \\
\hline & Cobalt & 1.1 & $M G / K G$ & $U$ & NP & 10 & $M G / K G$ & 1 \\
\hline & Copper & 6.5 & $M G / K G$ & & NP & 5 & MG/KG & 1 \\
\hline & Iron & 11200 & MG/KG & & NP & 20 & $M G / K G$ & 1 \\
\hline & Magnesium & 227 & MG/KG & B & NP & 1000 & $M G / K G$ & 1 \\
\hline & Manganese & 37.8 & $M G / K G$ & & NP & 3 & $M G / K G$ & 1 \\
\hline & Nickel & 5.2 & $M G / K G$ & $B$ & NP & 8 & $M G / K G$ & 1 \\
\hline & Potassium & 316 & MG/KG & $B$ & NP & 1000 & $M G / K G$ & 1 \\
\hline & Silver & 0.82 & $M G / K G$ & U & NP & 2 & $M G / K G$ & 1 \\
\hline & Sodium & 198 & MG/KG & B & NP & 1000 & $M G / K G$ & 1 \\
\hline & Vanadium & 27.3 & MG/KG & & NP & 10 & $M G / K G$ & 1 \\
\hline & Zinc & 9.1 & MG/KG & & NP & 4 & $M G / K G$ & 1 \\
\hline & Lead & 7.9 & $M G / K G$ & & NP & 1.2 & $M G / K G$ & 2 \\
\hline & Selenium & 0.4 & $M G / K G$ & B & NP & 1 & $M G / K G$ & 1 \\
\hline & Thallium & 0.18 & $M G / K G$ & $\mathrm{U}$ & NP & 2 & $M G / K G$ & 1 \\
\hline
\end{tabular}


Appendix G: Results of Metals and Cyanide Analyses, Salmon Site

\begin{tabular}{|c|c|c|c|c|c|c|c|c|}
\hline SAMPLE & COMPOUND & RESULTS & UNITS & $\begin{array}{l}\text { DATA } \\
\text { QUAL }\end{array}$ & $\begin{array}{l}\text { DATA } \\
\text { VALID. }\end{array}$ & CRDL & UNITS & DILUTION \\
\hline \multirow[t]{24}{*}{ TR6-ES-485-590-6 } & Arsenic & 1.3 & $M G / K G$ & $B$ & NP & 2 & $M G / K G$ & 1 \\
\hline & Cyanide & 0.05 & MG/KG & $U$ & NP & 0.49 & MG/KG & 1 \\
\hline & Mercury & 0.05 & MGKG & U & NP & 0.1 & MG/KG & 1 \\
\hline & Aluminum & 5030 & MGKG & & NP & 40 & MG/KG & 1 \\
\hline & Antimony & 4.5 & $M G / K G$ & U & NP & 12 & $M G / K G$ & 1 \\
\hline & Barium & 43.1 & MGKG & & NP & 40 & $M O / K G$ & 1 \\
\hline & Beryllium & 0.34 & MGKG & U & NP & 1 & $M G / K G$ & 1 \\
\hline & Cadmium & 0.58 & $M G / K G$ & U & NP & 1 & $M G / K G$ & 1 \\
\hline & Calcium & 900 & MG/KG & B & NP & 1000 & $M G / K G$ & 1 \\
\hline & Chromium & 28.2 & MGJKG & & NP & 2 & $M G / K G$ & 1 \\
\hline & Cobalt & 4.5 & $M G / K G$ & B & NP & 10 & MG/KG & 1 \\
\hline & Copper & 3.7 & MGKG & B & $N P$ & 5 & MG/KG & 1 \\
\hline & Iron & 4960 & MGKG & & NP & 20 & MEKG & 1 \\
\hline & Magnesium & 172 & $M G / K G$ & B & NP & 1000 & MG/KG & 1 \\
\hline & Manganese & 118 & $M G / K G$ & & NP & 3 & $M G / K G$ & 1 \\
\hline & Nickel & 4.4 & MGKG & B & NP & 8 & MG/KG & 1 \\
\hline & Potassium & 185 & MG/KG & B & NP & 1000 & $M G / K G$ & 1 \\
\hline & Silver & 0.82 & $M G / K G$ & $U$ & NP & 2 & $M G / K G$ & 1 \\
\hline & Sodium & 69.1 & $M G / K G$ & $B$ & NP & 1000 & MG/KG & 1 \\
\hline & Vanadium & 9.8 & MG/KG & B & NP & 10 & MaKG & 1 \\
\hline & Zinc & 11.1 & MGKG & & NP & 4 & MG/KG & 1 \\
\hline & Lead & 4.1 & Ma/Ka & & NP & 0.6 & MaKG & 1 \\
\hline & Selenium & 0.22 & MG/KG & U & NP & 1 & $M G / K G$ & 1 \\
\hline & Thallium & 0.18 & $M G / K G$ & $U$ & NP & 2 & $M G / K G$ & 1 \\
\hline
\end{tabular}


Appendix G: Results of Metals and Cyanide Analyses, Salmon Site

\begin{tabular}{|c|c|c|c|c|c|c|c|c|}
\hline SAMPLE & COMPOUND & RESULTS & UNITS & $\begin{array}{l}\text { DATA } \\
\text { QUAL }\end{array}$ & $\begin{array}{r}\text { DATA } \\
\text { VALID. }\end{array}$ & CRDL & UNITS & DILUTION \\
\hline \multirow[t]{24}{*}{ TR7-ES-560-650-14 } & Arsenic & 3 & $M G / K G$ & & $J$ & 2 & $\mathrm{MG} / \mathrm{KG}$ & 1 \\
\hline & Cyanide & 0.05 & MG/KG & $U$ & $\ldots$ & 0.49 & $M G / K G$ & 1 \\
\hline & Mercury & 0.07 & $M G / K G$ & $B$ & $J$ & 0.1 & $M G / K G$ & 1 \\
\hline & Aluminum & 6470 & $M G / K G$ & & $\ldots$ & 40 & $M G / K G$ & 1 \\
\hline & Antimony & 7 & MG/KG & $U$ & UJ & 12 & $M G / K G$ & 1 \\
\hline & Barium & 29.8 & $M G / K G$ & $B$ & $J$ & 40 & MG/KG & 1 \\
\hline & Beryllium & 0.48 & $M G / K G$ & $U$ & -- & 1 & $M G / K G$ & 1 \\
\hline & Cadmium & 0.58 & $M G / K G$ & $U$ & $\ldots$ & 1 & $M G / K G$ & 1 \\
\hline & Calcium & 3460 & MG/KG & & - & 1000 & MG/KG & 1 \\
\hline & Chromium & 26.4 & $M G / K G$ & & $\ldots$ & 2 & $M G / K G$ & 1 \\
\hline & Cobalt & 2 & $M G / K G$ & B & $J$ & 10 & $M G / K G$ & 1 \\
\hline & Copper & 5 & $M G / K G$ & & $\cdots$ & 5 & $M G / K G$ & 1 \\
\hline & Iron & 10800 & $M G / K G$ & & $\ldots$ & 20 & $M G / K G$ & 1 \\
\hline & Magnesium & 464 & $M G / K G$ & B & $J$ & 1000 & $M G / K G$ & 1 \\
\hline & Manganese & 53.9 & $M G / K G$ & & $\ldots$ & 3 & $M G / K G$ & 1 \\
\hline & Nickel & 5.2 & $M G / K G$ & $B$ & $J$ & 8 & $M G / K G$ & 1 \\
\hline & Potassium & 440 & $M G / K G$ & $B$ & $J$ & 1000 & $M G / K G$ & 1 \\
\hline & Silver & 0.82 & $M G / K G$ & $U$ & -. & 2 & $M G / K G$ & 1 \\
\hline & Sodium & 1210 & $M G / K G$ & & $\cdots$ & 1000 & $M G / K G$ & 1 \\
\hline & Vanadium & 22.4 & $M G / K G$ & & -- & 10 & $M G / K G$ & 1 \\
\hline & Zinc & 12.9 & $M G / K G$ & & - & 4 & $M G / K G$ & 1 \\
\hline & Lead & 7.3 & $M G / K G$ & & - & 1.2 & $M G / K G$ & 2 \\
\hline & Selenium & 0.22 & $M G / K G$ & $U$ & US & 1 & $M G / K G$ & 1 \\
\hline & Thallium & 0.2 & $M G / K G$ & $U$ & $\cdots$ & 2 & $M G / K G$ & 1 \\
\hline
\end{tabular}


Appendix G: Results of Metals and Cyanide Analyses. Salmon Site

\begin{tabular}{|c|c|c|c|c|c|c|c|c|}
\hline SAMPLE & COMPOUND & RESULTS & UNITS & $\begin{array}{l}\text { DATA } \\
\text { QUAL }\end{array}$ & $\begin{array}{l}\text { DATA } \\
\text { VALID. }\end{array}$ & CRDL & UNITS & DILUTION \\
\hline \multirow[t]{24}{*}{ TR7-ES-558-650-5 } & Arsenic & 3.6 & $M G / K G$ & & $\mathrm{~J}$ & 2 & $M G / K G$ & 1 \\
\hline & Cyanide & 0.05 & MG/KG & U & -- & 0.49 & MG/KG & 1 \\
\hline & Mercury & 0.05 & MG/KG & $U$ & - & 0.1 & $M G / K G$ & 1 \\
\hline & Aluminum & 10600 & MGKG & & - & 40 & $M G / K G$ & 1 \\
\hline & Antimony & 7 & $M G / K G$ & $U$ & UJ & 12 & $M G / K G$ & 1 \\
\hline & Barium & 30.8 & $M G / K G$ & B & J & 40 & $M G / K G$ & 1 \\
\hline & Beryllium & 0.48 & $M G / K G$ & U & $\ldots$ & 1 & $M G / K G$ & 1 \\
\hline & Cadmium & 0.58 & $M G / K G$ & $U$ & $\ldots$ & 1 & $M G / K G$ & 1 \\
\hline & Calcium & 554 & $M G / K G$ & B & $J$ & 1000 & $M G / K G$ & 1 \\
\hline & Chromium & 10.4 & $M G / K G$ & & $\ldots$ & 2 & $M G / K G$ & 1 \\
\hline & Cobalt & 1.3 & $M G / K G$ & B & J & 10 & $M G / K G$ & 1 \\
\hline & Copper & 5.1 & $M G / K G$ & & $U$ & 5 & $M G / K G$ & 1 \\
\hline & Iron & 12200 & $M G / K G$ & & - & 20 & $M G / K G$ & 1 \\
\hline & Magnesium & 324 & $M G / K G$ & B & J & 1000 & $M G / K G$ & 1 \\
\hline & Manganese & 101 & $M G / K G$ & & $\ldots$ & 3 & $M G / K G$ & 1 \\
\hline & Nickel & 5.9 & $M G / K G$ & $B$ & $J$ & 8 & $M G / K G$ & 1 \\
\hline & Potassium & 434 & $M G / K G$ & $U$ & -- & 1000 & $M G / K G$ & 1 \\
\hline & Silver & 0.82 & $M G / K G$ & U & $\ldots$ & 2 & $M G / K G$ & 1 \\
\hline & Sodium & 394 & $M G / K G$ & B & J & 1000 & $M G / K G$ & 1 \\
\hline & Vanadium & 26.8 & $M G / K G$ & & - & 10 & $M G / K G$ & 1 \\
\hline & Zinc & 9.5 & $M G / K G$ & & - & 4 & $M G / K G$ & 1 \\
\hline & Lead & 7 & $M G / K G$ & & - & 1.2 & $M G / K G$ & 2 \\
\hline & Selenium & 0.22 & $M G / K G$ & $U$ & UJ & 1 & $M G / K G$ & 1 \\
\hline & Thallium & 0.2 & $M G / K G$ & U & $\ldots$ & 2 & $M G / K G$ & 1 \\
\hline
\end{tabular}


Appendix G: Results of Metals and Cyanide Analyses, Salmon Site

\begin{tabular}{|c|c|c|c|c|c|c|c|c|}
\hline SAMPLE & COMPOUND & RESULTS & UNITS & $\begin{array}{l}\text { DATA } \\
\text { QUAL } \\
\end{array}$ & $\begin{array}{l}\text { DATA } \\
\text { VALID. } \\
\end{array}$ & CRDL & UNITS & DILUTION \\
\hline \multirow[t]{24}{*}{ TR8-ES-780-600-3 } & Arsenic & 3.1 & $M G / K G$ & & $J$ & 2 & $M G / K G$ & 1 \\
\hline & Cyanide & 0.05 & $M G / K G$ & U & $\ldots$ & 0.5 & $M G / K G$ & 1 \\
\hline & Mercury & 0.05 & MG/KG & $U$ & - & 0.1 & $M G / K G$ & 1 \\
\hline & Aluminum & 8670 & MG/KG & & - & 40 & $M G / K G$ & 1 \\
\hline & Antimony & 7 & $M G / K G$ & $U$ & UJ & 12 & $M G / K G$ & 1 \\
\hline & Barium & 18.5 & MG/KG & B & $J$ & 40 & $M G / K G$ & 1 \\
\hline & Beryllium & 0.48 & $M G / K G$ & $U$ & - & 1 & $M G / K G$ & 1 \\
\hline & Cadmium & 0.58 & $M G / K G$ & $u$ & - & 1 & $M G / K G$ & 1 \\
\hline & Calcium & 159 & $M G / K G$ & B & - & 1000 & $M G / K G$ & 1 \\
\hline & Chromium & 10.4 & $M G / K G$ & & - & 2 & $M G / K G$ & 1 \\
\hline & Cobalt & 1.6 & $M G / K G$ & $B$ & J & 10 & $M G / K G$ & 1 \\
\hline & Copper & 4.2 & $M G / K G$ & $\mathrm{~B}$ & $U$ & 5 & $M G / K G$ & 1 \\
\hline & Iron & 9250 & $M G / K G$ & & - & 20 & $M G / K G$ & 1 \\
\hline & Magnesium & 272 & $M G / K G$ & $B$ & J & 1000 & $M G / K G$ & 1 \\
\hline & Manganese & 13.9 & $M G / K G$ & & -- & 3 & $M G / K G$ & 1 \\
\hline & Nickel & 4.6 & $M G / K G$ & $B$ & $J$ & 8 & $M G / K G$ & 1 \\
\hline & Potassium & 434 & $M G / K G$ & $U$ & $\ldots$ & 1000 & $M G / K G$ & 1 \\
\hline & Silver & 0.82 & $M G / K G$ & $U$ & - & 2 & $M G / K G$ & 1 \\
\hline & Sodium & 36.5 & $M G / K G$ & $B$ & $U$ & 1000 & $M G / K G$ & 1 \\
\hline & Vanadium & 20.5 & $M G / K G$ & & - & 10 & $M G / K G$ & 1 \\
\hline & Zinc & 7.6 & $M G / K G$ & & - & 4 & $M G / K G$ & 1 \\
\hline & Lead & 4.3 & $M G / K G$ & & $\ldots$ & 0.6 & $M G / K G$ & 1 \\
\hline & Selenium & 0.26 & $M G / K G$ & $B$ & J & 1 & $M G / K G$ & 1 \\
\hline & Thallium & 0.2 & $M G / K G$ & U & $\ldots$ & 2 & $M G / K G$ & 1 \\
\hline
\end{tabular}


Appendix G: Results of Metals and Cyanide Analyses. Salmon Site

\begin{tabular}{|c|c|c|c|c|c|c|c|c|}
\hline SAMPLE & COMPOUND & RESULTS & UNITS & $\begin{array}{l}\text { DATA } \\
\text { QUAL } \\
\end{array}$ & $\begin{array}{l}\text { DATA } \\
\text { VALID. } \\
\end{array}$ & CRDL & UNITS & DILUTION \\
\hline \multirow[t]{24}{*}{ TR8-ES-780-600-3-DUP } & Arsenic & 3.3 & $M G / K G$ & & NP & 2 & $M G / K G$ & 1 \\
\hline & Cyanide & 0.05 & $M G / K G$ & U & NP & 0.49 & $M G / K G$ & 1 \\
\hline & Mercury & 0.05 & MG/KG & $U$ & NP & 0.1 & $M G / K G$ & 1 \\
\hline & Aluminum & 10400 & MG/KG & & NP & 40 & $M G / K G$ & 1 \\
\hline & Antimony & 7 & $M G / K G$ & U & NP & 12 & $M G / K G$ & 1 \\
\hline & Barium & 19.9 & $\mathrm{MG} / \mathrm{KG}$ & B & NP & 40 & $M G / K G$ & 1 \\
\hline & Beryllium & 0.48 & $M G / K G$ & $U$ & NP & 1 & MG/KG & 1 \\
\hline & Cadmium & 0.65 & $M G / K G$ & $B$ & NP & 1 & $M G / K G$ & 1 \\
\hline & Calcium & 149 & $M G / K G$ & B & NP & 1000 & MG/KG & 1 \\
\hline & Chromium & 11.7 & $M G / K G$ & & NP & 2 & MG/KG & 1 \\
\hline & Cobalt & 1.7 & $M G / K G$ & B & NP & 10 & MG/KG & 1 \\
\hline & Copper & 4.7 & $M G / K G$ & B & NP & 5 & $M G / K G$ & 1 \\
\hline & Iron & 9250 & $M G / K G$ & & NP & 20 & $M G / K G$ & 1 \\
\hline & Magnesium & 345 & $M G / K G$ & B & NP & 1000 & $M G / K G$ & 1 \\
\hline & Manganese & 16.4 & MG/KG & & $N P$ & 3 & $M G / K G$ & 1 \\
\hline & Nickel & 6.7 & MG/KG & B & NP & 8 & $M G / K G$ & 1 \\
\hline & Potassium & 434 & $M G / K G$ & $U$ & NP & 1000 & $M G / K G$ & 1 \\
\hline & Silver & 0.82 & $M G / K G$ & $U$ & NP & 2 & MG/KG & 1 \\
\hline & Sodium & 42.1 & $M G / K G$ & $B$ & NP & 1000 & $M G / K G$ & 1 \\
\hline & Vanadium & 21.5 & $M G / K G$ & & NP & 10 & MG/KG & 1 \\
\hline & Zinc & 10 & MG/KG & & NP & 4 & MG/KG & 1 \\
\hline & Lead & 3.8 & MG/KG & & NP & 0.6 & $M G / K G$ & 1 \\
\hline & Selenium & 0.22 & $M G / K G$ & $U$ & $N P$ & 1 & $M G / K G$ & 1 \\
\hline & Thallium & 0.2 & $M G / K G$ & $U$ & $N P$ & 2 & $M G / K G$ & 1 \\
\hline
\end{tabular}


Appendix G: Results of Metals and Cyanide Analyses, Salmon Site

\begin{tabular}{|c|c|c|c|c|c|c|c|c|}
\hline SAMPLE & COMPOUND & RESULTS & UNITS & $\begin{array}{l}\text { DATA } \\
\text { QUAL }\end{array}$ & $\begin{array}{l}\text { DATA } \\
\text { VALID. }\end{array}$ & CRDL & UNITS & DILUTION \\
\hline \multirow[t]{18}{*}{ TR8-ES-780-600-3-MS } & Arsenic & 66 & $\%$ & & $\mathrm{NP}$ & & $\%$ & 1 \\
\hline & Cyanide & 95 & $\%$ & & NP & & $\%$ & 1 \\
\hline & Mercury & 117 & $\%$ & & NP & & $\%$ & 1 \\
\hline & Antimony & 61 & $\%$ & & NP & & $\%$ & 1 \\
\hline & Barium & 104 & $\%$ & & NP & & $\%$ & 1 \\
\hline & Beryllium & 107 & $\%$ & & NP & & $\%$ & 1 \\
\hline & Cadmium & 112 & $\%$ & & NP & & $\%$ & 1 \\
\hline & Chromium & 108 & $\%$ & & NP & & $\%$ & 1 \\
\hline & Cobalt & 114 & $\%$ & & NP & & $\%$ & 1 \\
\hline & Copper & 105 & $\%$ & & NP & & $\%$ & 1 \\
\hline & Manganese & 114 & $\%$ & & NP & & $\%$ & 1 \\
\hline & Nickel & 110 & $\%$ & & NP & & $\%$ & 1 \\
\hline & Silver & 110 & $\%$ & & NP & & $\%$ & 1 \\
\hline & Vanadium & 106 & $\%$ & & NP & & $\%$ & 1 \\
\hline & Zinc & 111 & $\%$ & & NP & & $\%$ & 1 \\
\hline & Lead & 79 & $\%$ & & $N P$ & & $\%$ & 1 \\
\hline & Selenium & 51 & $\%$ & & NP & & $\%$ & 1 \\
\hline & Thallium & 97 & $\%$ & & NP & & $\%$ & 1 \\
\hline
\end{tabular}


Appendix G: Results of Metals and Cyanide Analyses, Salmon Site

\begin{tabular}{|c|c|c|c|c|c|c|c|c|}
\hline SAMPLE & COMPOUND & RESULTS & UNITS & $\begin{array}{l}\text { DATA } \\
\text { QUAL }\end{array}$ & $\begin{array}{r}\text { DATA } \\
\text { VALID. }\end{array}$ & CRDL & UNITS & DILUTION \\
\hline \multirow[t]{24}{*}{ TR10-ES-180-790-15 } & Arsenic & 0.85 & $M G / K G$ & $B$ & UJ & 2 & $M G / K G$ & 1 \\
\hline & Cyanide & 0.05 & $M G / K G$ & $U$ & $--\cdot$ & 0.49 & $M G / K G$ & 1 \\
\hline & Mercury & 0.05 & $M G / K G$ & $U$ & $\cdots$ & 0.1 & $M G / K G$ & 1 \\
\hline & Aluminum & 1180 & MG/KG & & - & 40 & MG/KG & 1 \\
\hline & Antimony & 7 & $M G / K G$ & U & UJ & 12 & $M G / K G$ & 1 \\
\hline & Barium & 4.4 & $M G / K G$ & $B$ & $J$ & 40 & $M G / K G$ & 1 \\
\hline & Beryllium & 0.48 & $M G / K G$ & U & $\cdots$ & 1 & $M G / K G$ & 1 \\
\hline & Cadmium & 0.58 & $M G / K G$ & $U$ & - & 1 & $M G / K G$ & 1 \\
\hline & Calcium & 255 & $M G / K G$ & $\mathrm{~B}$ & $J$ & 1000 & $M G / K G$ & 1 \\
\hline & Chromium & 2.4 & MG/KG & & -- & 2 & $M G / K G$ & 1 \\
\hline & Cobalt & 1.1 & $M G / K G$ & $U$ & $\cdots$ & 10 & $M G / K G$ & 1 \\
\hline & Copper & 0.88 & $M G / K G$ & $U$ & $\cdots$ & 5 & $M G / K G$ & 1 \\
\hline & Iron & 1260 & $M G / K G$ & & $\cdots$ & 20 & $M G / K G$ & 1 \\
\hline & Magnesium & 57.3 & $M G / K G$ & B & $\mathrm{J}$ & 1000 & $M G / K G$ & 1 \\
\hline & Manganese & 3.8 & $M G / K G$ & & $\cdots$ & 3 & $M G / K G$ & 1 \\
\hline & Nickel & 3.6 & $M G / K G$ & $U$ & $--\cdot$ & 8 & $M G / K G$ & 1 \\
\hline & Potassium & 434 & $M G / K G$ & $U$ & $\cdots$ & 1000 & $M G / K G$ & 1 \\
\hline & Silver & 0.82 & $M G / K G$ & $U$ & $\cdots$ & 2 & $M G / K G$ & 1 \\
\hline & Sodium & 53.2 & MG/KG & $B$ & $J$ & 1000 & $M G / K G$ & 1 \\
\hline & Vanadium & 4.5 & $M G / K G$ & B & U & 10 & $M G / K G$ & 1 \\
\hline & Zinc & 2.1 & $M G / K G$ & B & $J$ & 4 & $M G / K G$ & 1 \\
\hline & Lead & 1.2 & $M G / K G$ & & $\cdots$ & 0.6 & $M G / K G$ & 1 \\
\hline & Selenium & 0.22 & $M G / K G$ & $U$ & UJ & 1 & $M G / K G$ & 1 \\
\hline & Thallium & 0.2 & $M G / K G$ & $U$ & $\cdots$ & 2 & $M G / K G$ & 1 \\
\hline
\end{tabular}


Appendix G: Results of Metals and Cyanide Analyses, Salmon Site

\begin{tabular}{|c|c|c|c|c|c|c|c|c|}
\hline SAMPLE & COMPOUND & RESULTS & UNITS & $\begin{array}{l}\text { DATA } \\
\text { QUAL }\end{array}$ & $\begin{array}{r}\text { DATA } \\
\text { VALID. }\end{array}$ & CRDL & UNITS & DILUTION \\
\hline \multirow[t]{24}{*}{ TR10-ES-190-790-8 } & Arsenic & 3.5 & MG/KG & & $J$ & 2 & $M G / K G$ & 1 \\
\hline & Cyanide & 0.05 & $M G / K G$ & $U$ & $\cdots$ & 0.49 & $M G / K G$ & 1 \\
\hline & Mercury & 0.05 & $M G / K G$ & U & $\cdots$ & 0.1 & $M G / K G$ & 1 \\
\hline & Aluminum & 4400 & $M G / K G$ & & - & 40 & $M G / K G$ & 1 \\
\hline & Antimony & 7 & $M G / K G$ & $U$ & UJ & 12 & MG/KG & 1 \\
\hline & Barium & 33.1 & $M G / K G$ & $B$ & $\cdots$ & 40 & $M G / K G$ & 1 \\
\hline & Beryllium & 0.48 & MG/KG & U & - & 1 & $M G / K G$ & 1 \\
\hline & Cadmium & 0.71 & MG/KG & $B$ & $J$ & 1 & $M G / K G$ & 1 \\
\hline & Calcium & 3160 & $M G / K G$ & & $\cdots$ & 1000 & $M G / K G$ & 1 \\
\hline & Chromium & 248 & $M G / K G$ & & $\cdots$ & 2 & $M G / K G$ & 1 \\
\hline & Cobalt & 1.2 & $M G / K G$ & B & $J$ & 10 & $M G / K G$ & 1 \\
\hline & Copper & 3.3 & $M G / K G$ & B & U & 5 & $M G / K G$ & 1 \\
\hline & Iron & 6260 & $M G / K G$ & & - & 20 & $M G / K G$ & 1 \\
\hline & Magnesium & 327 & $M G / K G$ & B & $J$ & 1000 & $M G / K G$ & 1 \\
\hline & Manganese & 37.7 & $M G / K G$ & & $\ldots$ & 3 & $M G / K G$ & 1 \\
\hline & Nickel & 5.3 & $M G / K G$ & B & $J$ & 8 & $M G / K G$ & 1 \\
\hline & Potassium & 434 & $M G / K G$ & $U$ & -- & 1000 & $M Q / K G$ & 1 \\
\hline & Silver & 0.82 & $M G / K G$ & $U$ & - & 2 & $M G / K G$ & 1 \\
\hline & Sodium & 78.8 & $M G / K G$ & B & $J$ & 1000 & MG/KG & 1 \\
\hline & Vanadium & 12.4 & $M G / K G$ & & - & 10 & $M G / K G$ & 1 \\
\hline & Zinc & 57.8 & Ma/KG & & - & 4 & MQKKG & 1 \\
\hline & Lead & 10 & MGKG & & $\ldots$ & 1.2 & MG/KG & 2 \\
\hline & Selenium & 0.22 & MG/KG & $U$ & UJ & 1 & $M G / K G$ & 1 \\
\hline & Thallium & 0.2 & $M G / K G$ & $\mathrm{U}$ & $\cdots$ & 2 & $M G / K G$ & 1 \\
\hline
\end{tabular}


Appendix G: Results of Metals and Cyanide Analyses. Salmon Site

\begin{tabular}{|c|c|c|c|c|c|c|c|c|}
\hline SAMPLE & COMPOUND & RESULTS & UNITS & $\begin{array}{l}\text { DATA } \\
\text { QUAL } \\
\end{array}$ & $\begin{array}{l}\text { DATA } \\
\text { VALID. } \\
\end{array}$ & CRDL & UNITS & DILUTION \\
\hline \multirow[t]{24}{*}{ TR11-ES-180-930-3 } & Arsenic & 1.5 & $M G / K G$ & $B$ & $\mathrm{NP}$ & 2 & $M G / K G$ & 1 \\
\hline & Cyanide & 0.05 & $M G / K G$ & $U$ & NP & 0.49 & $M G / K G$ & 1 \\
\hline & Mercury & 0.05 & $M G / K G$ & U & NP & 0.1 & $M G / K G$ & 1 \\
\hline & Aluminum & 3610 & $M G / K G$ & & NP & 40 & $M G / K G$ & 1 \\
\hline & Antimony & 4.5 & $M G / K G$ & $U$ & NP & 12 & $M G / K G$ & 1 \\
\hline & Barium & 9.9 & $M G / K G$ & $B$ & NP & 40 & $M G / K G$ & 1 \\
\hline & Beryllium & 0.34 & $M G / K G$ & $U$ & NP & 1 & $M G / K G$ & 1 \\
\hline & Cadmium & 0.58 & $M G / K G$ & $U$ & NP & 1 & $M G / K G$ & 1 \\
\hline & Calcium & 197 & $M G / K G$ & B & NP & 1000 & $M G / K G$ & 1 \\
\hline & Chromium & 5.4 & MG/KG & & NP & 2 & $M G / K G$ & 1 \\
\hline & Cobalt & 1.1 & $M G / K G$ & $U$ & NP & 10 & $M G / K G$ & 1 \\
\hline & Copper & 2.1 & $M G / K G$ & B & NP & 5 & $M G / K G$ & 1 \\
\hline & Iron & 4340 & $M G / K G$ & & NP & 20 & $M G / K G$ & 1 \\
\hline & Magnesium & 114 & $M G / K G$ & B & NP & 1000 & $M G / K G$ & 1 \\
\hline & Manganese & 12.6 & $M Q / K G$ & & NP & 3 & $M G / K G$ & 1 \\
\hline & Nickel & 2.7 & $M G / K G$ & $B$ & NP & 8 & $M G / K G$ & 1 \\
\hline & Potassium & 165 & $M G / K G$ & $B$ & NP & 1000 & $M G / K G$ & 1 \\
\hline & Silver & 0.82 & $M G / K G$ & $U$ & $N P$ & 2 & $M G / K G$ & 1 \\
\hline & Sodium & 28.9 & $M O / K G$ & $B$ & NP & 1000 & $M G / K G$ & 1 \\
\hline & Vanadium & 11.1 & $M G / K G$ & & NP & 10 & $M G / K G$ & 1 \\
\hline & Zinc & 4.7 & MG/KG & & NP & 4 & $M G / K G$ & 1 \\
\hline & Lead & 2.8 & $M G / K G$ & & NP & 0.6 & $M G / K G$ & 1 \\
\hline & Selenium & 0.22 & $M G / K G$ & $U$ & NP & 1 & MG/KG & 1 \\
\hline & Thallium & 0.19 & $M G / K G$ & $B$ & NP & 2 & $M G / K G$ & 1 \\
\hline
\end{tabular}


Appendix G: Results of Metals and Cyanide Analyses. Salmon Site

\begin{tabular}{|c|c|c|c|c|c|c|c|c|}
\hline SAMPLE & COMPOUND & RESULTS & UNITS & $\begin{array}{l}\text { DATA } \\
\text { QUAL }\end{array}$ & $\begin{array}{l}\text { DATA } \\
\text { VALID. }\end{array}$ & CADL & UNITS & DILUTION \\
\hline \multirow[t]{24}{*}{ TR13-ES-210-1110-6 } & Arsenic & 2 & MG/KG & $B$ & NP & 2 & $\mathrm{MG/KG}$ & 1 \\
\hline & Cyanide & 0.05 & MG/KG & $U$ & NP & 0.49 & Ma/KG & 1 \\
\hline & Mercury & 0.05 & MG/KG & $U$ & NP & 0.1 & Ma/Ka & 1 \\
\hline & Aluminum & 4360 & MGKG & & NP & 40 & MaNG & 1 \\
\hline & Antimony & 4.5 & $M Q / K G$ & $U$ & NP & 12 & $M Q / K G$ & 1 \\
\hline & Barium & 17.7 & $M Q / K G$ & B & NP & 40 & MG/KG & 1 \\
\hline & Beryllium & 0.34 & MG/KG & U & $N P$ & 1 & MG/KG & 1 \\
\hline & Cadmium & 0.58 & MG/KG & $U$ & NP & 1 & $M G / K G$ & 1 \\
\hline & Calcium & 4970 & MaKG & & NP & 1000 & MG/KG & 1 \\
\hline & Chromium & 29.1 & $M G / K G$ & & NP & 2 & $M G / K G$ & 1 \\
\hline & Cobalt & 1.1 & $M G / K G$ & U & NP & 10 & MOKKG & 1 \\
\hline & Copper & 3.5 & Ma/KG & $B$ & NP & 5 & $M G / K G$ & 1 \\
\hline & Iron & 6400 & MaKa & & NP & 20 & $M G / K G$ & 1 \\
\hline & Magnesium & 194 & MG/KG & B & NP & 1000 & MOKKO & 1 \\
\hline & Manganes $\theta$ & 37.2 & Ma/KG & & NP & 3 & Ma/KG & 1 \\
\hline & Nickel & 2.9 & Ma/KO & B & NP & 8 & MaKG & 1 \\
\hline & Potassium & 258 & MG/KG & $B$ & NP & 10 & MaKG & 1 \\
\hline & Silver & 0.82 & MG/KG & U & NP & 2 & Ma/KG & 1 \\
\hline & Sodium & 203 & $M G / K G$ & B & NP & 1000 & MGKG & 1 \\
\hline & Vanadium & 15.7 & MGKO & & NP & 10 & MaKG & 1 \\
\hline & Zinc & 13.8 & MaKG & & NP & 4 & MaKa & 1 \\
\hline & Lead & 5 & MGKO & & NP & 0.6 & MG/KG & 1 \\
\hline & Selenium & 0.22 & $M G / K G$ & $U$ & $N P$ & 1 & MG/KG & 1 \\
\hline & Thallium & 0.18 & $M G / K G$ & $U$ & $N P$ & 2 & $M G / K G$ & 1 \\
\hline
\end{tabular}


Appendix G: Results of Metals and Cyanide Analyses. Salmon Site

\begin{tabular}{|c|c|c|c|c|c|c|c|c|}
\hline SAMPLE & COMPOUND & RESULTS & UNITS & $\begin{array}{l}\text { DATA } \\
\text { QUAL } \\
\end{array}$ & $\begin{array}{l}\text { DATA } \\
\text { VALID. }\end{array}$ & CRDL & UNITS & DILUTION \\
\hline \multirow[t]{24}{*}{ TA13-ES-212-1150-6 } & Arsenic & 2.2 & MQ/KG & & NP & 2 & $M G / K G$ & 1 \\
\hline & Cyanide & 0.05 & no/Ko & $u$ & NP & 0.49 & $M G / K G$ & 1 \\
\hline & Mercury & 0.05 & MaKKG & $u$ & NP & 0.1 & MOKKG & 1 \\
\hline & Aluminum & 3920 & MaKKa & & NP & 40 & MOKKG & 1 \\
\hline & Antimony & 4.5 & MG/KO & $U$ & NP & 12 & $M O / K G$ & 1 \\
\hline & Barium & 46.4 & MOKKG & & NP & 40 & Ma/Ka & 1 \\
\hline & Beryllium & 0.34 & MOKKO & $u$ & NP & 1 & MQ/KG & 1 \\
\hline & Cadmium & 0.58 & MO/KO & $u$ & NP & 1 & MaKKG & 1 \\
\hline & Calcium & 14700 & MG/KG & & NP & 5000 & MG/KG & 5 \\
\hline & Chromium & 181 & MG/KG & & NP & 2 & $M G / K G$ & 1 \\
\hline & Cobalt & 1.1 & $M Q / K G$ & $U$ & NP & 10 & $M G / K G$ & 1 \\
\hline & Copper & 3.4 & MG/KO & B & NP & 5 & $M G / K G$ & 1 \\
\hline & Iron & 4360 & MQ/KG & & NP & 20 & MG/KG & 1 \\
\hline & Magnesium & 311 & $M Q / K G$ & B & NP & 1000 & $M G / K G$ & 1 \\
\hline & Manganes $\theta$ & 80 & $M G / K G$ & & $N P$ & 3 & $M G / K G$ & 1 \\
\hline & Nickel & 3.8 & Ma/KG & B & NP & 8 & $M Q / K G$ & 1 \\
\hline & Potassium & 338 & MG/KG & $B$ & NP & 1000 & $M G / K G$ & 1 \\
\hline & Silver & 0.82 & MG/KG & U & NP & 2 & $M G / K G$ & 1 \\
\hline & Sodium & 1350 & MOKKG & & NP & 1000 & MG/KG & 1 \\
\hline & Vanadium & 10.1 & MQKKG & & NP & 10 & MENG & 1 \\
\hline & Zinc & 61.2 & MaKG & & NP & 4 & MaKG & 1 \\
\hline & Lead & 14.2 & MGKG & & NP & 1.2 & $M G / K G$ & 2 \\
\hline & Selenium & 0.28 & MG/KG & B & NP & 1 & $M G / K G$ & 1 \\
\hline & Thallium & 0.18 & MG/KG & U & NP & 2 & $M G / K G$ & 1 \\
\hline
\end{tabular}


Appendix G: Results of Metals and Cyanide Analyses, Salmon Site

\begin{tabular}{|c|c|c|c|c|c|c|c|c|}
\hline SAMPLE & COMPOUND & RESULTS & UNITS & $\begin{array}{l}\text { DATA } \\
\text { QUAL } \\
\end{array}$ & $\begin{array}{r}\text { DATA } \\
\text { VALID. } \\
\end{array}$ & CRDL & UNITS & DILUTION \\
\hline \multirow[t]{24}{*}{ TR14-ES-80-1189-5 } & Arsenic & 1.5 & $M G / K G$ & $B$ & NP & 2 & $M G / K G$ & 1 \\
\hline & Cyanide & 0.05 & $M G / K G$ & $U$ & NP & 0.5 & $M G / K G$ & 1 \\
\hline & Mercury & 0.05 & Ma/KO & $U$ & NP & 0.1 & $M G / K G$ & 1 \\
\hline & Aluminum & 3450 & MaKG & & NP & 40 & $M a / K G$ & 1 \\
\hline & Antimony & 4.5 & MG/KG & $U$ & NP & 12 & $M G / K G$ & 1 \\
\hline & Barium & 9.3 & MG/KG & $B$ & NP & 40 & MG/KG & 1 \\
\hline & Beryllium & 0.34 & MGKG & $U$ & NP & 1 & MG/KO & 1 \\
\hline & Cadmium & 0.58 & MaKG & $U$ & NP & 1 & $M G / K G$ & 1 \\
\hline & Calcium & 218 & MaKG & $B$ & NP & 1000 & MG/KG & 1 \\
\hline & Chromium & 4.6 & MG/KG & & NP & 2 & $M G / K G$ & 1 \\
\hline & Cobalt & 1.1 & Ma/KG & $U$ & NP & 10 & $M G / K G$ & 1 \\
\hline & Copper & 1.9 & $M G / K G$ & $B$ & NP & 5 & $M G / K G$ & 1 \\
\hline & Iron & 4720 & $M Q / K G$ & & NP & 20 & $M G / K G$ & 1 \\
\hline & Magnesium & 68.5 & $M G / K G$ & $B$ & NP & 1000 & $M G / K a$ & 1 \\
\hline & Manganese & 25.8 & $M G / K G$ & & NP & 3 & $M G / K G$ & 1 \\
\hline & Nickel & 2.4 & $M G / K G$ & $B$ & NP & 8 & $M G / K G$ & 1 \\
\hline & Potassium & 181 & MQ/KG & $B$ & NP & 1000 & $M G / K G$ & 1 \\
\hline & Silver & 0.82 & $M G / K G$ & $U$ & NP & 2 & MQKKG & 1 \\
\hline & Sodium & 372 & MEKG & $B$ & NP & 1000 & MG/KG & 1 \\
\hline & Vanadium & 12.5 & MaKo & & NP & 10 & MGKG & 1 \\
\hline & Zinc & 3.3 & MaKG & B & NP & 4 & MGKG & 1 \\
\hline & Lead & 3.4 & MEKG & & NP & 0.6 & $M G K G$ & 1 \\
\hline & Selenium & 0.22 & MQ/KO & $U$ & NP & 1 & $M G / K G$ & 1 \\
\hline & Thallium & 0.18 & $M G / K G$ & $U$ & NP & 2 & $M G / K G$ & 1 \\
\hline
\end{tabular}


Appendix G: Results of Metals and Cyanide Analyses, Salmon Site

\begin{tabular}{|c|c|c|c|c|c|c|c|c|}
\hline SAMPLE & COMPOUND & RESULTS & UNITS & $\begin{array}{l}\text { DATA } \\
\text { QUAL }\end{array}$ & $\begin{array}{l}\text { DATA } \\
\text { VALID. }\end{array}$ & CRDL & UNITS & DILUTION \\
\hline \multirow[t]{24}{*}{ TR14-ES-90-1200-3 } & Arsenic & 2.1 & $M G / K G$ & & $\mathrm{NP}$ & 2 & $M G / K G$ & 1 \\
\hline & Cyanide & 0.05 & $M G / K G$ & $U$ & NP & 0.49 & $M G / K G$ & 1 \\
\hline & Mercury & 0.05 & $M G / K G$ & U & NP & 0.1 & $M G / K G$ & 1 \\
\hline & Aluminum & 5770 & $M G / K G$ & & NP & 40 & $M G / K G$ & 1 \\
\hline & Antimony & 4.5 & $M G / K G$ & U & NP & 12 & $M G / K G$ & 1 \\
\hline & Barium & 12 & $M G / K G$ & $B$ & NP & 40 & $M G / K G$ & 1 \\
\hline & Beryllium & 0.34 & $M G / K G$ & $U$ & NP & 1 & $M G / K G$ & 1 \\
\hline & Cadmium & 0.58 & $M G / K G$ & $U$ & NP & 1 & $M G / K G$ & 1 \\
\hline & Calcium & 683 & $M G / K G$ & $B$ & NP & 1000 & $M G / K G$ & 1 \\
\hline & Chromium & 5.5 & $M G / K G$ & & NP & 2 & $M G / K G$ & 1 \\
\hline & Cobalt & 1.1 & $M G / K G$ & $U$ & NP & 10 & $M G / K G$ & 1 \\
\hline & Copper & 3.1 & $M G / K G$ & B & NP & 5 & $M G / K G$ & 1 \\
\hline & Iron & 6880 & $M G / K G$ & & NP & 20 & $M G / K G$ & 1 \\
\hline & Magnesium & 147 & $M G / K G$ & B & NP & 1000 & $M G / K G$ & 1 \\
\hline & Manganese & 25.6 & $M G / K G$ & & NP & 3 & $M G / K G$ & 1 \\
\hline & Nickel & 3.7 & $M G / K G$ & $B$ & NP & 8 & $M G / K G$ & 1 \\
\hline & Potassium & 258 & $M G / K G$ & $B$ & NP & 1000 & $M G / K G$ & 1 \\
\hline & Silver & 0.82 & $M G / K G$ & $U$ & NP & 2 & $M G / K G$ & 1 \\
\hline & Sodium & 48.2 & $M G / K G$ & $B$ & NP & 1000 & $M G / K G$ & 1 \\
\hline & Vanadium & 17.4 & $M G / K G$ & & NP & 10 & $M G / K G$ & 1 \\
\hline & Zinc & 7.5 & $M G / K G$ & & NP & 4 & $M G / K G$ & 1 \\
\hline & Lead & 4.1 & $M G / K G$ & & NP & 0.6 & $M G / K G$ & 1 \\
\hline & Selenium & 0.22 & $M G / K G$ & $U$ & NP & 1 & $M G / K G$ & 1 \\
\hline & Thallium & 0.18 & $M G / K G$ & U & NP & 2 & $M G / K G$ & 1 \\
\hline
\end{tabular}


Appendix G: Results of Metals and Cyanide Analyses, Salmon Site

\begin{tabular}{|c|c|c|c|c|c|c|c|c|}
\hline SAMPLE & COMPOUND & RESULTS & UNITS & $\begin{array}{l}\text { DATA } \\
\text { QUAL }\end{array}$ & $\begin{array}{l}\text { DATA } \\
\text { VALID. }\end{array}$ & CRDL & UNITS & DILUTION \\
\hline \multirow[t]{24}{*}{ TR-15-ES-20-1180-.5 } & Arsenic & 2.6 & $M G / K G$ & & NP & 2 & $M G / K G$ & 1 \\
\hline & Cyanide & 0.05 & $M G / K G$ & U & NP & 0.5 & $M G / K G$ & 1 \\
\hline & Mercury & 0.05 & $M G / K G$ & U & NP & 0.1 & $M G / K G$ & 1 \\
\hline & Aluminum & 7710 & $M G / K G$ & & NP & 40 & $M G / K G$ & 1 \\
\hline & Antimony & 4.5 & $M G / K G$ & U & NP & 12 & $M G / K G$ & 1 \\
\hline & Barium & 52 & $M G / K G$ & & NP & 40 & $M G / K G$ & 1 \\
\hline & Beryllium & 0.35 & $M G / K G$ & B & NP & 1 & $M G / K G$ & 1 \\
\hline & Cadmium & 0.58 & $M G / K G$ & $U$ & NP & 1 & $M G / K G$ & 1 \\
\hline & Calcium & 3950 & $M G / K G$ & & NP & 1000 & $M G / K G$ & 1 \\
\hline & Chromium & 77 & $M G / K G$ & & NP & 2 & MG/KG & 1 \\
\hline & Cobalt & 1.7 & $M G / K G$ & $B$ & NP & 10 & $M G / K G$ & 1 \\
\hline & Copper & 5.2 & MG/KG & & NP & 5 & $M G / K G$ & 1 \\
\hline & Iron & 7880 & $M G / K G$ & & NP & 20 & $M G / K G$ & 1 \\
\hline & Magnesium & 390 & $M G / K G$ & B & NP & 1000 & $M G / K G$ & 1 \\
\hline & Manganese & 164 & $M G / K G$ & & NP & 3 & $M G / K G$ & 1 \\
\hline & Nickel & 6.5 & $M G / K G$ & B & NP & 8 & $M G / K G$ & 1 \\
\hline & Potassium & 360 & $M G / K G$ & B & NP & 1000 & $M G / K G$ & 1 \\
\hline & Silver & 0.82 & $M G / K G$ & $U$ & NP & 2 & $M G / K G$ & 1 \\
\hline & Sodium & 69.4 & MG/KG & B & NP & 1000 & MG/KG & 1 \\
\hline & Vanadium & 17.9 & $M G / K G$ & & NP & 10 & $M G / K G$ & 1 \\
\hline & Zinc & 28.6 & $M G / K G$ & & NP & 4 & $M G / K G$ & 1 \\
\hline & Lead & 8.1 & MQ/KG & & NP & 0.6 & MG/KG & 1 \\
\hline & Selenium & 0.22 & $M G / K G$ & $U$ & NP & 1 & $M G / K G$ & 1 \\
\hline & Thallium & 0.18 & $M G / K G$ & $U$ & NP & 2 & $M G / K G$ & 1 \\
\hline
\end{tabular}


Appendix G: Results of Metals and Cyanide Analyses, Salmon Site

\begin{tabular}{|c|c|c|c|c|c|c|c|c|}
\hline SAMPLE & COMPOUND & RESULTS & UNITS & $\begin{array}{l}\text { DATA } \\
\text { QUAL }\end{array}$ & $\begin{array}{l}\text { DATA } \\
\text { VALID. }\end{array}$ & CRDL & UNITS & DILUTION \\
\hline \multirow[t]{24}{*}{ TR-15-ES-20-1180-.5 DUP } & Arsenic & 2.5 & $M G / K G$ & & $\mathrm{NP}$ & 2 & $M G / K G$ & 1 \\
\hline & Cyanide & 0.05 & $M G / K G$ & $U$ & NP & 0.49 & MG/KG & 1 \\
\hline & Mercury & 0.05 & $M G / K G$ & $U$ & NP & 0.1 & $M G / K G$ & 1 \\
\hline & Aluminum & 6860 & $M G / K G$ & & NP & 40 & $M G / K G$ & 1 \\
\hline & Antimony & 4.5 & $M G / K G$ & $U$ & NP & 12 & $M G / K G$ & 1 \\
\hline & Barium & 31.6 & $M G / K G$ & B & NP & 40 & MG/KG & 1 \\
\hline & Beryllium & 0.34 & $M G / K G$ & $U$ & NP & 1 & $M G / K G$ & 1 \\
\hline & Cadmium & 0.58 & $M G / K G$ & $u$ & NP & 1 & $M G / K G$ & 1 \\
\hline & Calcium & 2090 & $M G / K G$ & & NP & 1000 & $M G / K G$ & 1 \\
\hline & Chromium & 40.8 & $M G / K G$ & & NP & 2 & $M G / K G$ & 1 \\
\hline & Cobalt & 1.8 & $M G / K G$ & $B$ & NP & 10 & $M G / K G$ & 1 \\
\hline & Copper & 4.4 & $M G / K G$ & B & NP & 5 & $M G / K G$ & 1 \\
\hline & Iron & 7430 & $M Q / K G$ & & NP & 20 & $M G / K G$ & 1 \\
\hline & Magnesium & 275 & $M G / K G$ & B & NP & 1000 & $M G / K G$ & 1 \\
\hline & Manganese & 78.5 & $M G / K G$ & & $N P$ & 3 & $M G / K G$ & 1 \\
\hline & Nickel & 4.3 & $M G / K G$ & B & NP & 8 & $M G / K G$ & 1 \\
\hline & Potassium & 287 & $M G / K G$ & $B$ & $N P$ & 1000 & MGiKG & 1 \\
\hline & Silver & 0.82 & $M G / K G$ & $U$ & $N P$ & 2 & $M G / K G$ & 1 \\
\hline & Sodium & 51.2 & $M G / K G$ & $B$ & NP & 1000 & $M G / K G$ & 1 \\
\hline & Vanadium & 17.8 & $M G / K G$ & . & NP & 10 & $M G / K G$ & 1 \\
\hline & Zinc & 17.3 & $M G / K G$ & & NP & 4 & $M G / K G$ & 1 \\
\hline & Lead & 9.1 & $M G / K G$ & & NP & 0.6 & $M G / K G$ & 1 \\
\hline & Selenium & 0.22 & $M G / K G$ & $U$ & NP & 1 & $M G / K G$ & 1 \\
\hline & Thallium & 0.18 & $M G / K G$ & U & $N P$ & 2 & $M G / K G$ & 1 \\
\hline
\end{tabular}


Appendix G: Results of Metals and Cyanide Analyses, Salmon Site

\begin{tabular}{|c|c|c|c|c|c|c|c|c|}
\hline SAMPLE & COMPOUND & RESULTS & UNITS & $\begin{array}{l}\text { DATA } \\
\text { QUAL } \\
\end{array}$ & $\begin{array}{l}\text { DATA } \\
\text { VALID. } \\
\end{array}$ & CRDL & UNITS & DILUTION \\
\hline \multirow[t]{18}{*}{ TR-15-ES-20-1180-.5 MS } & Arsenic & 90 & $\%$ & & NP & & $\%$ & 1 \\
\hline & Cyanide & 96 & $\%$ & & NP & & $\%$ & 1 \\
\hline & Mercury & 100 & $\%$ & & NP & & $\%$ & 1 \\
\hline & Antimony & 52 & $\%$ & & NP & & $\%$ & 1 \\
\hline & Barium & 95 & $\%$ & & NP & & $\%$ & 1 \\
\hline & Beryllium & 99 & $\%$ & & NP & & $\%$ & 1 \\
\hline & Cadmium & 109 & $\%$ & & NP & & $\%$ & 1 \\
\hline & Chromium & 78 & $\%$ & & NP & & $\%$ & 1 \\
\hline & Cobalt & 110 & $\%$ & & NP & & $\%$ & 1 \\
\hline & Copper & 98 & $\%$ & & NP & & $\%$ & 1 \\
\hline & Manganese & 55 & $\%$ & & NP & & $\%$ & 1 \\
\hline & Nickel & 101 & $\%$ & & NP & & $\%$ & 1 \\
\hline & Silver & 99 & $\%$ & & NP & & $\%$ & 1 \\
\hline & Vanadium & 102 & $\%$ & & NP & & $\%$ & 1 \\
\hline & Zinc & 100 & $\%$ & & NP & & $\%$ & 1 \\
\hline & Lead & 143 & $\%$ & & NP & & $\%$ & 1 \\
\hline & Selenium & 55 & $\%$ & & NP & & $\%$ & 1 \\
\hline & Thallium & 91 & $\%$ & & NP & & $\%$ & 1 \\
\hline
\end{tabular}


Appendix G: Results of Metals and Cyanide Analyses, Salmon Site

\begin{tabular}{|c|c|c|c|c|c|c|c|c|}
\hline SAMPLE & COMPOUND & RESULTS & UNITS & $\begin{array}{l}\text { DATA } \\
\text { QUAL }\end{array}$ & $\begin{array}{l}\text { DATA } \\
\text { VALID. }\end{array}$ & CRDL & UNITS & DILUTION \\
\hline \multirow[t]{24}{*}{ TR16-ES-200-80-1.5 } & Arsenic & 1.7 & $M G / K G$ & B & NP & 2 & $M G / K G$ & 1 \\
\hline & Cyanide & 0.05 & $M G / K G$ & $U$ & NP & 0.5 & $M G / K G$ & 1 \\
\hline & Mercury & 0.05 & $M G / K G$ & U & NP & 0.1 & $M G / K G$ & 1 \\
\hline & Aluminum & 8430 & $M G / K G$ & & NP & 40 & $M G / K G$ & 1 \\
\hline & Antimony & 4.5 & $M G / K G$ & $U$ & NP & 12 & $M G / K G$ & 1 \\
\hline & Barium & 17.4 & $M G / K G$ & $B$ & NP & 40 & $M G / K G$ & 1 \\
\hline & Beryllium & 0.34 & $M G / K G$ & $U$ & NP & 1 & $M G / K G$ & 1 \\
\hline & Cadmium & 0.58 & $M G / K G$ & $U$ & NP & 1 & $M G / K G$ & 1 \\
\hline & Calcium & 153 & $M G / K G$ & $B$ & NP & 1000 & $M G / K G$ & 1 \\
\hline & Chromium & 6.8 & $M G / K G$ & & NP & 2 & $M G / K G$ & 1 \\
\hline & Cobalt & 1.6 & $M G / K G$ & $B$ & NP & 10 & $M G / K G$ & 1 \\
\hline & Copper & 3.6 & $M G / K G$ & $B$ & NP & 5 & $M G / K G$ & 1 \\
\hline & Iron & 6320 & $M G / K G$ & & NP & 20 & $M G / K G$ & 1 \\
\hline & Magnesium & 273 & $M G / K G$ & $B$ & NP & 1000 & $M G / K G$ & 1 \\
\hline & Manganese & 17.1 & $M G / K G$ & & NP & 3 & $M G / K G$ & 1 \\
\hline & Nickel & 5.4 & $M G / K G$ & $\mathrm{~B}$ & NP & 8 & $M G / K G$ & 1 \\
\hline & Potassium & 263 & $M G / K G$ & $\mathrm{~B}$ & NP & 1000 & $M G / K G$ & 1 \\
\hline & Silver & 0.82 & $M G / K G$ & $U$ & NP & 2 & $M G / K G$ & 1 \\
\hline & Sodium & 35.4 & $M G / K G$ & $B$ & NP & 1000 & $M G / K G$ & 1 \\
\hline & Vanadium & 16.8 & $M G / K G$ & & NP & 10 & $M G / K G$ & 1 \\
\hline & Zinc & 9.3 & $M G / K G$ & & NP & 4 & $M G / K G$ & 1 \\
\hline & Lead & 4.3 & $M G / K G$ & & NP & 0.6 & $M G / K G$ & 1 \\
\hline & Selenium & 0.22 & $M G / K G$ & $U$ & NP & 1 & $M G / K G$ & 1 \\
\hline & Thallium & 0.34 & $M G / K G$ & $B$ & NP & 2 & $M G / K G$ & 1 \\
\hline
\end{tabular}


Appendix G: Results of Metals and Cyanide Analyses, Salmon Site

\begin{tabular}{|c|c|c|c|c|c|c|c|c|}
\hline SAMPLE & COMPOUND & RESULTS & UNITS & $\begin{array}{l}\text { DATA } \\
\text { QUAL }\end{array}$ & $\begin{array}{r}\text { DATA } \\
\text { VALID. }\end{array}$ & CRDL & UNITS & DILUTION \\
\hline \multirow[t]{24}{*}{ TR17-ES-30-120-5 } & Arsenic & 2.7 & MG/KG & & NP & 2 & $M G / K G$ & 1 \\
\hline & Cyanide & 0.05 & $M G / K G$ & U & NP & 0.5 & $M G / K G$ & 1 \\
\hline & Mercury & 0.05 & $M G / K G$ & U & NP & 0.1 & $M G / K G$ & 1 \\
\hline & Aluminum & 6660 & $M G / K G$ & & NP & 40 & $M G / K G$ & 1 \\
\hline & Antimony & 4.5 & $M G / K G$ & U & NP & 12 & $M G / K G$ & 1 \\
\hline & Barium & 33.9 & $M G / K G$ & $B$ & NP & 40 & $M G / K G$ & 1 \\
\hline & Beryllium & 0.41 & $M G / K G$ & $B$ & NP & 1 & $M G / K G$ & 1 \\
\hline & Cadmium & 0.58 & $M G / K G$ & $U$ & NP & 1 & $M G / K G$ & 1 \\
\hline & Calcium & 785 & $M G / K G$ & $B$ & NP & 1000 & $M G / K G$ & 1 \\
\hline & Chromium & 6.8 & $M G / K G$ & & NP & 2 & $M G / K G$ & 1 \\
\hline & Cobalt & 2.2 & $M G / K G$ & $B$ & NP & 10 & $M G / K G$ & 1 \\
\hline & Copper & 5.6 & $M G / K G$ & & NP & 5 & $M G / K G$ & 1 \\
\hline & Iron & 7140 & $M G / K G$ & & NP & 20 & $M G / K G$ & 1 \\
\hline & Magnesium & 829 & $M G / K G$ & $B$ & NP & 1000 & $M G / K G$ & 1 \\
\hline & Manganese & 77.3 & $M G / K G$ & & NP & 3 & $M G / K G$ & 1 \\
\hline & Nickel & 5.1 & $M G / K G$ & $B$ & NP & 8 & $M G / K G$ & 1 \\
\hline & Potassium & 533 & $M G / K G$ & $B$ & NP & 1000 & $M G / K G$ & 1 \\
\hline & Silver & 0.82 & $M G / K G$ & $U$ & NP & 2 & $M G / K G$ & 1 \\
\hline & Sodium & 47.9 & $M G / K G$ & $B$ & NP & 1000 & $M G / K G$ & 1 \\
\hline & Vanadium & 11.4 & $M G / K G$ & & NP & 10 & $M G / K G$ & 1 \\
\hline & Zinc & 11.5 & $M G / K G$ & & NP & 4 & $M G / K G$ & 1 \\
\hline & Lead & 5.2 & $M G / K G$ & & NP & 0.6 & $M G / K G$ & 1 \\
\hline & Selenium & 0.25 & $M G / K G$ & $B$ & NP & 1 & $M G / K G$ & 1 \\
\hline & Thallium & 0.31 & $M G / K G$ & $B$ & NP & 2 & $M G / K G$ & 1 \\
\hline
\end{tabular}


Appendix G: Results of Metals and Cyanide Analyses, Salmon Site

\begin{tabular}{|c|c|c|c|c|c|c|c|c|}
\hline SAMPLE & COMPOUND & RESULTS & UNITS & $\begin{array}{l}\text { DATA } \\
\text { QUAL }\end{array}$ & $\begin{array}{l}\text { DATA } \\
\text { VALID. }\end{array}$ & CRDL & UNITS & DILUTION \\
\hline \multirow[t]{24}{*}{ TR17-ES-30-125-8 } & Arsenic & 1.4 & $M G / K G$ & $B$ & NP & 2 & $M G / K G$ & 1 \\
\hline & Cyanide & 0.05 & $M G / K G$ & $U$ & NP & 0.49 & $M G / K G$ & 1 \\
\hline & Mercury & 0.05 & $M G / K G$ & $u$ & NP & 0.1 & $M G / K G$ & 1 \\
\hline & Aluminum & 5330 & $M G / K G$ & & NP & 40 & $M G / K G$ & 1 \\
\hline & Antimony & 4.5 & $M G / K G$ & $U$ & NP & 12 & $M G / K G$ & 1 \\
\hline & Barium & 33.7 & $M G / K G$ & B & NP & 40 & $M G / K G$ & 1 \\
\hline & Beryllium & 0.35 & $M G / K G$ & $B$ & NP & 1 & $M G / K G$ & 1 \\
\hline & Cadmium & 0.58 & $M G / K G$ & U & NP & 1 & $M G / K G$ & 1 \\
\hline & Calcium & 748 & $M G / K G$ & B & NP & 1000 & $M G / K G$ & 1 \\
\hline & Chromium & 5.9 & $M G / K G$ & & NP & 2 & $M G / K G$ & 1 \\
\hline & Cobait & 2.4 & $M G / K G$ & B & NP & 10 & $M G / K G$ & 1 \\
\hline & Copper & 3.3 & $M G / K G$ & B & NP & 5 & $M G / K G$ & 1 \\
\hline & Iron & 7350 & $M G / K G$ & & NP & 20 & $M G / K G$ & 1 \\
\hline & Magnesium & 651 & $M G / K G$ & $B$ & $\mathrm{NP}$ & 1000 & $M G / K G$ & 1 \\
\hline & Manganese & 142 & $M G / K G$ & & NP & 3 & $M G / K G$ & 1 \\
\hline & Nickel & 4.5 & $M G / K G$ & $B$ & NP & 8 & $M G / K G$ & 1 \\
\hline & Potassium & 360 & $M G / K G$ & B & NP & 1000 & $M G / K G$ & 1 \\
\hline & Silver & 0.82 & $M G / K G$ & $U$ & NP & 2 & $M G / K G$ & 1 \\
\hline & Sodium & 52.8 & $M G / K G$ & B & $N P$ & 1000 & $M G / K G$ & 1 \\
\hline & Vanadium & 10.9 & $M G / K G$ & & NP & 10 & MG/KG & 1 \\
\hline & Zinc & 13.9 & $M G / K G$ & & NP & 4 & $M G / K G$ & 1 \\
\hline & Lead & 18.6 & $M G / K G$ & & NP & 2.4 & $M G / K G$ & 4 \\
\hline & Selenium & 0.22 & $M G / K G$ & $U$ & $N P$ & 1 & $M G / K G$ & 1 \\
\hline & Thallium & 0.18 & $M G / K G$ & $U$ & NP & 2 & $M G / K G$ & 1 \\
\hline
\end{tabular}


Appendix G: Results of Metals and Cyanide Analyses, Salmon Site

\begin{tabular}{|c|c|c|c|c|c|c|c|c|}
\hline SAMPLE & COMPOUND & RESULTS & UNITS & $\begin{array}{l}\text { DATA } \\
\text { QUAL }\end{array}$ & $\begin{array}{l}\text { DATA } \\
\text { VALID. }\end{array}$ & CRDL & UNITS & DILUTION \\
\hline \multirow[t]{24}{*}{ TR18-BS-90-150A-E } & Arsenic & 1.7 & $M G / K G$ & $B$ & $\mathrm{NP}$ & 2 & $M G / K G$ & 1 \\
\hline & Cyanide & 0.05 & MG/KG & $U$ & NP & 0.49 & $M G / K G$ & 1 \\
\hline & Mercury & 0.05 & $M G / K G$ & $U$ & NP & 0.1 & $M G / K G$ & 1 \\
\hline & Aluminum & 5560 & $M G / K G$ & & NP & 40 & $M G / K G$ & 1 \\
\hline & Antimony & 4.5 & $M G / K G$ & $U$ & NP & 12 & MG/KG & 1 \\
\hline & Barium & 21.6 & $M G / K G$ & $B$ & NP & 40 & $M G / K G$ & 1 \\
\hline & Beryllium & 0.34 & $M G / K G$ & $U$ & NP & 1 & $M G / K G$ & 1 \\
\hline & Cadmium & 0.58 & $M G / K G$ & $U$ & NP & 1 & $M G / K G$ & 1 \\
\hline & Calcium & 244 & $M G / K G$ & B & NP & 1000 & $M G / K G$ & 1 \\
\hline & Chromium & 6.3 & $M G / K G$ & & NP & 2 & $M G / K G$ & 1 \\
\hline & Cobalt & 1.3 & $M G / K G$ & B & NP & 10 & $M G / K G$ & 1 \\
\hline & Copper & 3.2 & MG/KG & $B$ & NP & 5 & $M G / K G$ & 1 \\
\hline & Iron & 6170 & $M G / K G$ & & NP & 20 & $M G / K Q$ & 1 \\
\hline & Magnesium & 322 & $M G / K G$ & $B$ & NP & 1000 & $M G / K G$ & 1 \\
\hline & Manganese & 13.7 & $M G / K G$ & & NP & 3 & $M G / K G$ & 1 \\
\hline & Nicitiel & 4.4 & $M G / K G$ & $B$ & NP & 8 & $M G / K G$ & 1 \\
\hline & Potassium & 329 & $M G / K G$ & B & $N P$ & 1000 & $M G / K G$ & 1 \\
\hline & Silver & 0.82 & $M G / K G$ & $U$ & $N P$ & 2 & $M G / K G$ & 1 \\
\hline & Sodium & 36.7 & $M Q / K G$ & $B$ & NP & 1000 & $M G / K G$ & 1 \\
\hline & Vanadium & 13.4 & MG/KG & & $N P$ & 10 & $M G / K G$ & 1 \\
\hline & Zinc & 7.3 & $M G / K G$ & & NP & 4 & $M G / K G$ & 1 \\
\hline & Lead & 4.6 & $M G / K G$ & & NP & 0.6 & MG/KG & 1 \\
\hline & Selenium & 0.22 & $M G / K G$ & $U$ & NP & 1 & $M G / K G$ & 1 \\
\hline & Thallium & 0.18 & $M G / K G$ & $U$ & NP & 2 & $M G / K G$ & 1 \\
\hline
\end{tabular}


Appendix G: Results of Metals and Cyanide Analyses, Salmon Site

\begin{tabular}{|c|c|c|c|c|c|c|c|c|}
\hline SAMPLE & COMPOUND & RESULTS & UNITS & $\begin{array}{l}\text { DATA } \\
\text { QUAL }\end{array}$ & $\begin{array}{r}\text { DATA } \\
\text { VALID. } \\
\end{array}$ & CRDL & UNITS & DILUTION \\
\hline \multirow[t]{24}{*}{ TR19-BS-0-150A-E } & Arsenic & 3.1 & $M G / K G$ & & NP & 2 & $M G / K G$ & 1 \\
\hline & Cyanide & 0.05 & MG/KG & $U$ & NP & 0.49 & $M G / K G$ & 1 \\
\hline & Mercury & 0.05 & $M G / K G$ & $U$ & NP & 0.1 & MG/KG & 1 \\
\hline & Aluminum & 9180 & MG/KG & & NP & 40 & $M G / K G$ & 1 \\
\hline & Antimony & 4.5 & $M G / K G$ & $U$ & NP & 12 & $M G / K G$ & 1 \\
\hline & Barium & 22.4 & $M G / K G$ & B & NP & 40 & $M G / K G$ & 1 \\
\hline & Beryllium & 0.34 & $M G / K G$ & $U$ & NP & 1 & $M G / K G$ & 1 \\
\hline & Cadmium & 0.58 & $M G / K G$ & $U$ & NP & 1 & $M G / K G$ & 1 \\
\hline & Calcium & 479 & $M G / K G$ & $B$ & NP & 1000 & $M G / K G$ & 1 \\
\hline & Chromium & 24.5 & $M G / K G$ & & NP & 2 & $M G / K G$ & 1 \\
\hline & Cobalt & 1.5 & $M G / K G$ & B & NP & 10 & $M G / K G$ & 1 \\
\hline & Copper & 4.6 & $M G / K G$ & B & NP & 5 & $M G / K G$ & 1 \\
\hline & Iron & 7970 & $M G / K G$ & & NP & 20 & $M G / K G$ & 1 \\
\hline & Magnesium & 372 & $M G / K G$ & $B$ & NP & 1000 & $M G / K G$ & 1 \\
\hline & Manganese & 43.4 & $M G / K G$ & & NP & 3 & $M G / K G$ & 1 \\
\hline & Nickel & 6.7 & $M G / K G$ & B & NP & 8 & MG/KG & 1 \\
\hline & Potassium & 384 & $M G / K G$ & $B$ & NP & 1000 & $M G / K G$ & 1 \\
\hline & Silver & 0.82 & $M G / K G$ & $U$ & NP & 2 & $M G / K G$ & 1 \\
\hline & Sodium & 97.6 & $M G / K G$ & B & NP & 1000 & $M G / K G$ & 1 \\
\hline & Vanadium & 18.7 & $M G / K G$ & & NP & 10 & $M G / K G$ & 1 \\
\hline & Zinc & 15.8 & $M G / K G$ & & NP & 4 & $M G / K G$ & 1 \\
\hline & Lead & 4.9 & $M G / K G$ & & NP & 0.6 & $M G / K G$ & 1 \\
\hline & Selenium & 0.22 & MG/KG & $U$ & NP & 1 & $M G / K G$ & 1 \\
\hline & Thallium & 0.2 & $M G / K G$ & $B$ & NP & 2 & $M G / K G$ & 1 \\
\hline
\end{tabular}




\section{Appendix $\mathrm{H}$}

Results of Dioxin/Furan Compound Analyses 
Appendix H: Results of Dioxin/Furan Compound Analyses, Salmon Site

\begin{tabular}{|c|c|c|c|c|c|c|c|c|}
\hline SAMPLE & ANALYTE & RESULTS & $\begin{array}{l}\text { DATA } \\
\text { QUAL }\end{array}$ & $\begin{array}{l}\text { DATA } \\
\text { VALID }\end{array}$ & UNITS & CRDL & UNITS & DILUTION \\
\hline \multirow[t]{13}{*}{ TR2-ES-355N-175E-5Z } & TCDD & 1.0 & $\bar{U}$ & $\overline{-\cdots}$ & UG/KG & 1.0 & UG/KG & 1 \\
\hline & TCDF & 1.0 & U & $\cdots$ & UG/KG & 1.0 & UG/KG & 1 \\
\hline & PeCDD & 2.4 & $U$ & $\cdots$ & UG/KG & 2.4 & $U G / K G$ & 1 \\
\hline & PeCDF & 2.4 & $U$ & $\cdots$ & UG/KG & 2.4 & $U G / K G$ & 1 \\
\hline & $\mathrm{HXCDD}$ & 2.4 & $U$ & $\ldots$ & UG/KG & 2.4 & UG/KG & 1 \\
\hline & $\mathrm{H} \times \mathrm{CDF}$ & 2.4 & $U$ & $\cdots$ & UG/KG & 2.4 & $U G / K G$ & 1 \\
\hline & HPCDD & 2.4 & $U$ & $\cdots$ & $U G / K G$ & 2.4 & $U G / K G$ & 1 \\
\hline & $\mathrm{HpCDF}$ & 2.4 & U & $\cdots$ & UG/KG & 2.4 & $U G / K G$ & 1 \\
\hline & OCDD & 4.8 & $U$ & $\ldots$ & UG/KG & 4.8 & $U G / K G$ & 1 \\
\hline & OCDF & 4.8 & U & $\cdots$ & UG/KG & 4.8 & UG/KG & 1 \\
\hline & C13-2378-TCDD & 86.0 & & & $\%$ & & $\%$ & 1 \\
\hline & C13-2378-TCDF & 90.0 & & & $\%$ & & $\%$ & 1 \\
\hline & C13.OCDD & 74.0 & & & $\%$ & & $\%$ & 1 \\
\hline \multirow[t]{13}{*}{ TR2-ES-356N-175E-8Z } & TCDD & 1.0 & $U$ & $\cdots$ & UG/KG & 1.0 & $U G / K G$ & 1 \\
\hline & TCDF & 2.2 & & - & UG/KG & 1.0 & UG/KG & 1 \\
\hline & PeCDD & 2.4 & $U$ & $\cdots$ & UG/KG & 2.4 & $U G / K G$ & 1 \\
\hline & PeCDF & 2.4 & $U$ & $\cdots$ & UG/KG & 2.4 & UG/KG & 1 \\
\hline & $\mathrm{H} \times \mathrm{CDD}$ & 2.4 & $U$ & $\cdots$ & UG/KG & 2.4 & UG/KG & 1 \\
\hline & $H \times C D F$ & 2.7 & & $\ldots$ & UG/KG & 2.4 & UG/KG & 1 \\
\hline & $\mathrm{HPCDD}$ & 2.4 & U & $\cdots$ & UG/KG & 2.4 & $U G / K G$ & 1 \\
\hline & $\mathrm{HPCDF}$ & 2.6 & & $-\cdot$ & UG/KG & 2.4 & $U G / K G$ & 1 \\
\hline & OCDD & 4.8 & $U$ & $\cdots$ & $U G / K G$ & 4.8 & $U G / K G$ & 1 \\
\hline & OCDF & 4.8 & $u$ & $\ldots$ & UG/KG & 4.8 & UG/KG & 1 \\
\hline & C13-2378-TCDD & 84.0 & & & $\%$ & & $\%$ & 1 \\
\hline & C13-2378-TCDF & 88.0 & & & $\%$ & & $\%$ & 1 \\
\hline & $C 13-O C D D$ & 71.0 & & & $\%$ & & $\%$ & 1 \\
\hline \multirow[t]{13}{*}{ TR2A-ES-365N-200E-3.5Z } & TCDD & 1.0 & $U$ & $\cdots$ & UG/KG & 1.0 & UG/KG & 1 \\
\hline & TCDF & 1.0 & U & $\cdots$ & UG/KG & 1.0 & UG/KG & 1 \\
\hline & PeCDD & 2.5 & $U$ & $\cdots$ & UG/KG & 2.5 & IIG/KG & 1 \\
\hline & PeCDF & 2.5 & U & $\ldots$ & UG/KG & 2.5 & UG/KG & 1 \\
\hline & $H \times C D D$ & 2.5 & U & $\ldots$ & UG/KG & 2.5 & UG/KG & 1 \\
\hline & $H \times C D F$ & 2.5 & $u$ & $\cdots$ & $U G / K G$ & 2.5 & UG/KG & 1 \\
\hline & $\mathrm{HpCDD}$ & 2.5 & $u$ & $\cdots$ & UG/KG & 2.5 & UG/KG & 1 \\
\hline & $\mathrm{HpCDF}$ & 2.5 & $U$ & $\ldots$ & UG/KG & 2.5 & UG/KG & 1 \\
\hline & OCDD & 4.9 & $U$ & $\ldots$ & UG/KG & 4.9 & UG/KG & 1 \\
\hline & OCDF & 4.9 & $U$ & $\cdots$ & UG/KG & 4.9 & UG/KG & 1 \\
\hline & C13-2378-TCDD & 82.0 & & & $\%$ & & $\%$ & 1 \\
\hline & C13-2378-TCDF & 84.0 & & & $\%$ & & $\%$ & 1 \\
\hline & C13-OCDD & 71.0 & & & $\%$ & & $\%$ & 1 \\
\hline
\end{tabular}


Appendix H: Results of Dioxin/Furan Compound Analyses, Salmon Site

\begin{tabular}{|c|c|c|c|c|c|c|c|c|}
\hline SAMPLE & ANALYTE & RESULTS & $\begin{array}{l}\text { DATA } \\
\text { QUAL }\end{array}$ & $\begin{array}{l}\text { DATA } \\
\text { VALID }\end{array}$ & UNITS & CADL & UNITS & DILUTION \\
\hline \multirow[t]{13}{*}{ TR2A-ES-360N-200E-8Z } & TCDD & 1.0 & $U$ & $\cdots$ & UG/KG & 1.0 & $U G / K G$ & 1 \\
\hline & TCDF & 1.0 & $U$ & $\cdots$ & $U G / K G$ & 1.0 & UG/KG & 1 \\
\hline & PECDD & 2.4 & $U$ & $\cdots$ & UG/KG & 2.4 & UG/KG & 1 \\
\hline & $P_{\Theta C D F}$ & 2.4 & $U$ & $\cdots$ & UG/KG & 2.4 & UG/KG & 1 \\
\hline & $\mathrm{H} \times C D D$ & 2.4 & $U$ & $\cdots$ & $U G / K G$ & 2.4 & UG/KG & 1 \\
\hline & HxCDF & 2.4 & U & $\cdots$ & UG/KG & 2.4 & UG/KG & 1 \\
\hline & HpCDD & 2.4 & U & $\cdots$ & UG/KG & 2.4 & UG/KG & 1 \\
\hline & HpCDF & 2.4 & U & $\cdots$ & UG/KG & 2.4 & UG/KG & 1 \\
\hline & OCDD & 4.8 & $U$ & $\cdots$ & UG/KG & 4.8 & $U G / K G$ & 1 \\
\hline & OCDF & 4.8 & $U$ & $\cdots$ & UQ/KG & 4.8 & $U G / K G$ & 1 \\
\hline & C13-2378-TCDD & 87.0 & & & $\%$ & & $\%$ & 1 \\
\hline & C13-2378-TCDF & 92.0 & & & $\%$ & & $\%$ & 1 \\
\hline & C13-OCDD & 71.0 & & & $\%$ & & $\%$ & 1 \\
\hline
\end{tabular}


Appendix I
Results of Total Petroleum Hydrocarbon Analyses 
Appendix I: Results of Total Petroleum Hydrocarbons Analyses, Salmon Site

\begin{tabular}{|c|c|c|c|c|c|c|c|}
\hline SAMPLE & RESULTS & UNITS & $\begin{array}{l}\text { DATA } \\
\text { QUAL. }\end{array}$ & $\begin{array}{l}\text { DATA } \\
\text { VALID. }\end{array}$ & CRDL & UNITS & DILUTION \\
\hline TR3-ES-450N-400E-3Z & 3380.0 & MGKG & & - & 250 & MaKo & 50 \\
\hline TR3-ES-430N-400E-15Z & 1110.0 & MGKG & & - & 125 & MQKO & 25 \\
\hline TR4-ES-260-490-8 & 114.0 & MaKa & & - & 5 & $M G / K G$ & 1 \\
\hline TR5-ES-590-570-12 & 57.6 & MaKa & & - & 5 & MaKO & 1 \\
\hline TR6-ES-495-585-12 & 117.0 & MaKG & & NP & 4.95 & MQNG & 1 \\
\hline TR6-ES-485-590-6 & 21.3 & MQKO & & NP & 4.96 & MG/KG & 1 \\
\hline TR7-ES-558-650-5 & 4940.0 & Maka & & - & 1000 & MGKG & 200 \\
\hline TR7-ES-560-650-14 & 2780.0 & MaKO & & - & 1000 & MQKKG & 200 \\
\hline TR8-ES-780-600-3 & 5.0 & MGKG & $U$ & $\cdots$ & 5 & MaKe & 1 \\
\hline TR8-ES-780-600-3-MS & 102.0 & $\%$ & & NP & & $\%$ & 1 \\
\hline TA8-ES-780-600-3-MSD & 98.0 & $\%$ & & NP & & $\%$ & 1 \\
\hline TR10-ES-180-790-15 & 18.8 & MaKG & & - & 5 & MaKO & 1 \\
\hline TA10-ES-190-790-8 & 346.0 & MGKG & & - & 25 & MGKG & 5 \\
\hline TR11-ES-180-930-3 & 9.3 & MEKG & & NP & 4.98 & MGKG & 1 \\
\hline TR13-ES-210-1110-6 & 2240.0 & MaKa & & NP & 124 & MQKO & 25 \\
\hline TR13-ES-212-1150-6 & 9120.0 & MGKG & & NP & 500 & MGKG & 100 \\
\hline TR14-ES-80-1189-5 & 5.0 & MG/KG & $U$ & NP & 4.98 & MG/KG & 1 \\
\hline TR14-ES-90-1200-3 & 5.0 & MG/KG & $U$ & NP & 4.95 & MG/KG & 1 \\
\hline TR-15-ES-20-1180-.5 & 40.2 & MaKG & & NP & 4.98 & MGKG & 1 \\
\hline TR-15-ES-20-1180-.5MS & 97.0 & $\%$ & & NP & & $\%$ & 1 \\
\hline TR-15-ES-20-1180-.5MSD & 146.0 & $\%$ & • & NP & & $\%$ & 1 \\
\hline TR16-ES-200-80-1.5 & 5.0 & MG/KG & $u$ & NP & 4.97 & MGKG & 1 \\
\hline TR17-ES-30-120-5 & 5.0 & MGKG & u & NP & 4.97 & MGKG & 1 \\
\hline TR17-ES-30-125-8 & 5.0 & MGKG & $U$ & NP & 4.95 & MGKG & 1 \\
\hline TR19-BS-0-150A-E & 252.0 & MG/KG & & $N P$ & 24.9 & MGKG & 5 \\
\hline
\end{tabular}




\section{Appendix $\mathrm{J}$ \\ Results of Tritium Analyses}

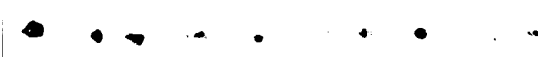


Appendix J: Results of Tritium Analyses, Salmon Site

\begin{tabular}{|c|c|c|c|c|c|c|c|c|}
\hline SAMPLE & RESULTS & UNITS & $\begin{array}{l}\text { DATA } \\
\text { VALID } \\
\end{array}$ & $\begin{array}{r}2 \text { SIGMA } \\
\text { ERROR }(+/-) \\
\end{array}$ & UNITS & MDA & UNITS & DILUTION \\
\hline TR18-BS-90-150A-E & 0.01 & PCI/G & NP & 0.02 & PCI/G & 0.04 & $\mathrm{PC} / / \mathrm{G}$ & 1 \\
\hline TR19-BS-0-150A-E & 0.03 & PCl/G & NP & 0.03 & PCI/G & 0.04 & PCI/G & 1 \\
\hline
\end{tabular}




\section{Appendix K Sample Collection Logs}


SAMPLE COLLECTION LOG

FoE OD23032262

INTERNATIONAL

CORPORATION

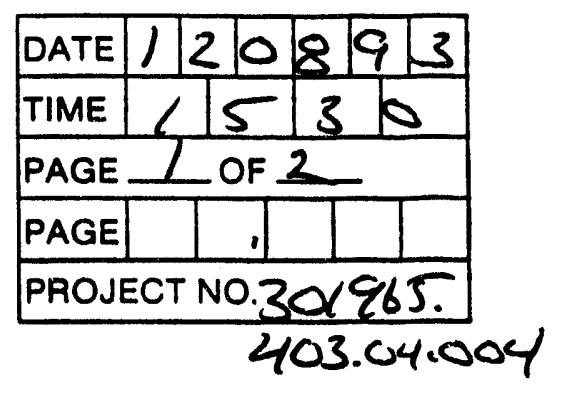

project name Salmon test Site

SAMPLE NO. IR/-ES-OOOI

sample location TRENCH - REel Pits

SAMPLE TYPE SCI GRAB

COMPOSITE — Yes $\angle$ No

COMPOSITE TYPE $\sim A$

DEPTH OF SAMPLE 3 feet

weather Clear $50^{\circ} \mathrm{f}$

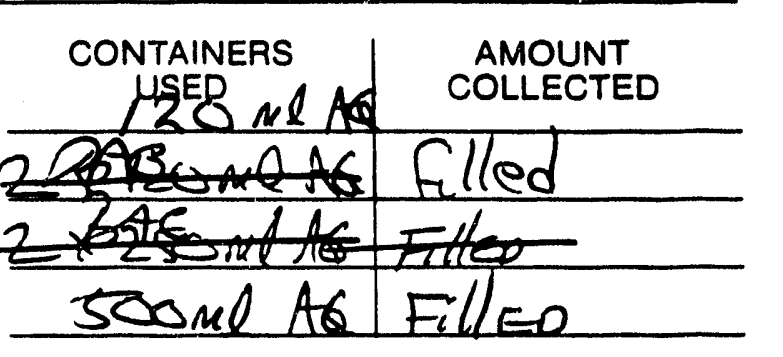

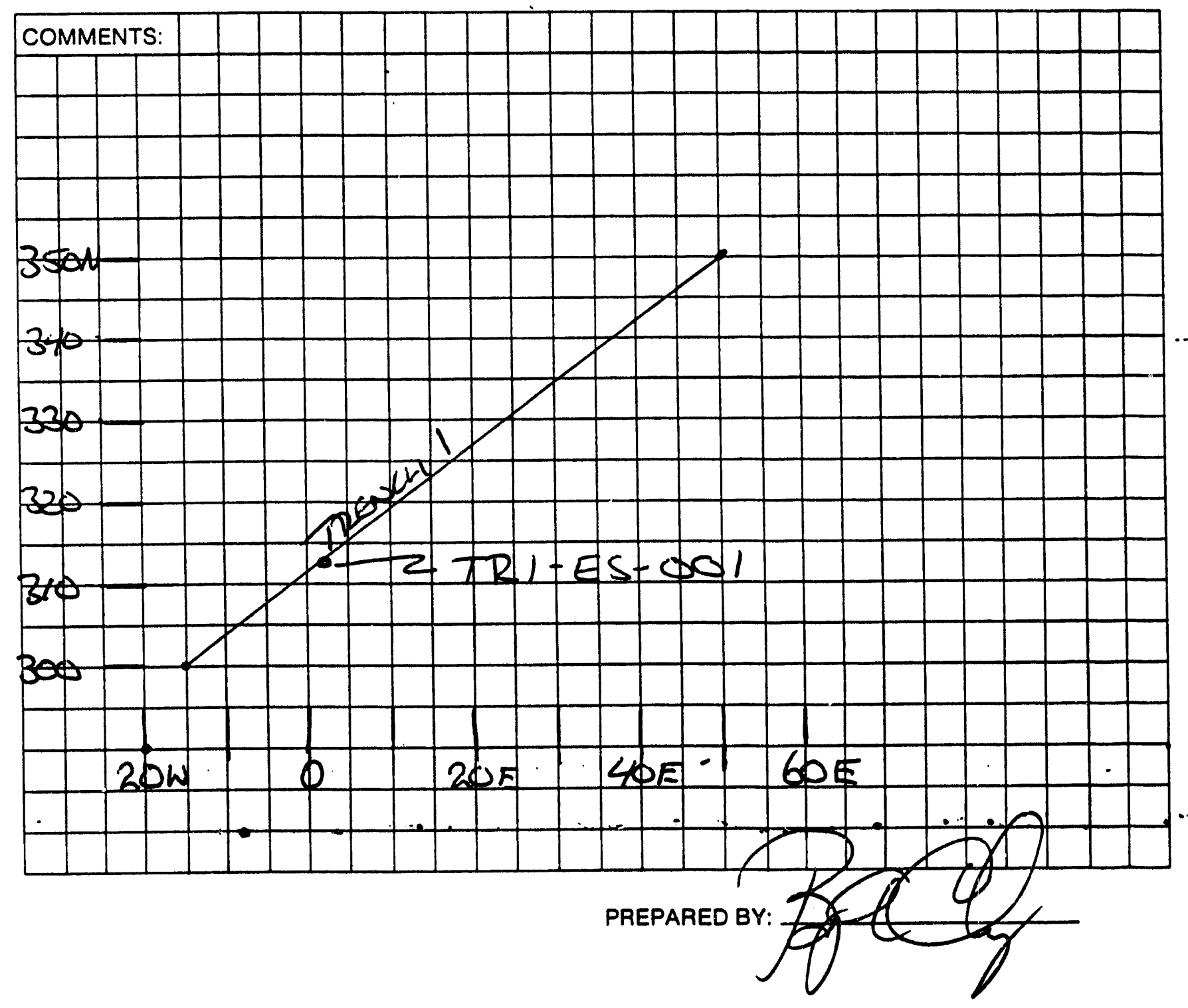




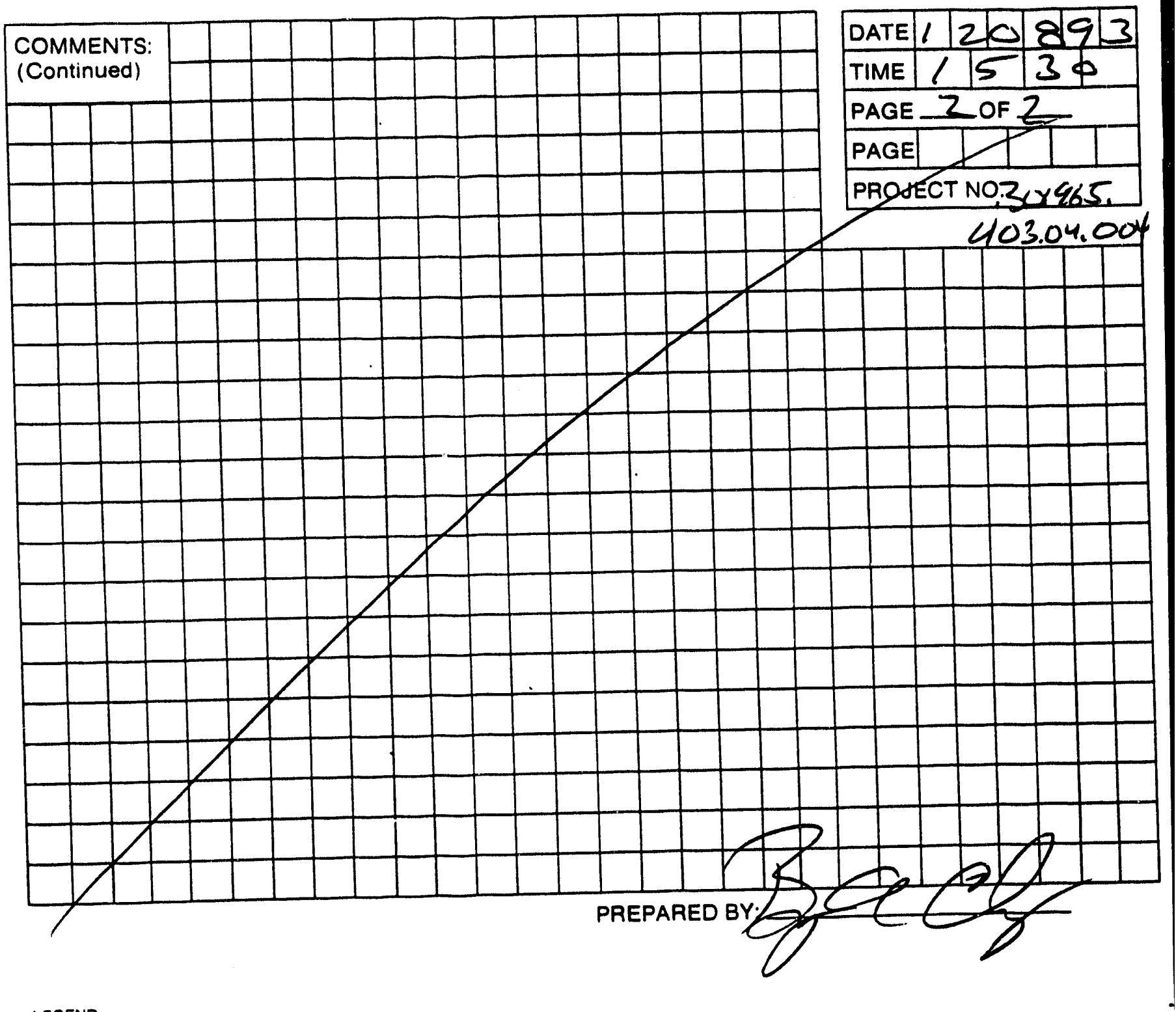

LEGEND

1. A SAMPLE COLLECTION LOG IS TO BE COMPLETED FOR EACH SAMPLE.

2. ALWAYS COMPLETE BOTH SIDES. IF SECOND SIDE IS NOT USED. DRAW A LINE THROUGH IT AND MARK N/A. FILL IN CONTROL BLOCK AND PREPARED BY.

3. ALL ENTRIES ON LOG ARE TO BE COMPLETED, IF NOT APPLICABLE MARK N/A.

4. DATE: USE MONTH/DAY/YEAR: I.E.. 10/30/85

5. TIME: USE 24-HOUR CLOCK: I.E., 1835 FOR 6:35 P.M

6. PAGE: EACH SAMPLE TEAM SHOULD NUMBER PAGE - OF SINGLE DAY, I.E., IF THEAE AAE A TOTAL OF 24 PAGES (INCLUDING FRONTAND BACK) NUMBER

7. SAMPLE LOCATION: USE BORING OR MONITORING WELL NUMBER, GRIOLOCATION TTRANSECT). SAMPLING STATION SETE IN COMMENT SECTION IF NECESSARY. COORDINATE TO PHYSICAL FEATURES WITH DISTANCES. INCLUDE SKETCH IN COMMENT SECTIONIF NECESSARY. 8. SAMPLE TYPE: USE THE FOLLOWING - SOIL: WATER ISUAF
DRUM CONTENTS: OIL: VEGETATION; WIPE: SEDIMENT.

9. COMPOSITE TYPE: I.E., 24-HOUR, LIST SAMPLE NUMBERS IN COMPOSITE. SPATIAL COMPOSITE.

10. DEPTH OF SAMPLE: GIVE UNITS, WRITE OUT UNITS SUCH AS INCHES, FEET. DON'T USE ' OR "

11. WEATHER: APPROXIMATE TEMPERATURE, SUN AND MOISTURE CONDITIONS.

12. CONTAINERS USED: LIST EACH CONTAINER TYPE AS NUN PLASTIC: 1 - 3 INCH STEEL TUBE: 1 - 8 OZ. GLASS JAR)

13. AMOUNT COLLECTED: VOLUME IN CONTAINEAS (E.G. 1/2 FULL). 
Project name SArmon

SAMPLE NO. JR2-ES-355N-135-5Z

SAMPLE LOCATION IREN $\angle 42$

SAMPLE TYPE Sol/ Graza

COMPOSITE —YES «NO

COMPOSITE TYPE N N/A

DEPTH OF SAMPLE 5 feec

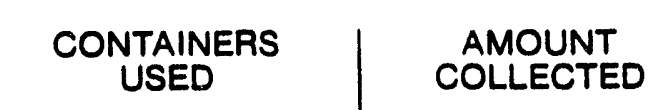

$130 \mathrm{~m} / \mathrm{AG} \quad \mathrm{Fill}$ $500 \mathrm{ml}$ AG Fill $\mathrm{EP}_{0}$

weather Clearnig; V. humla

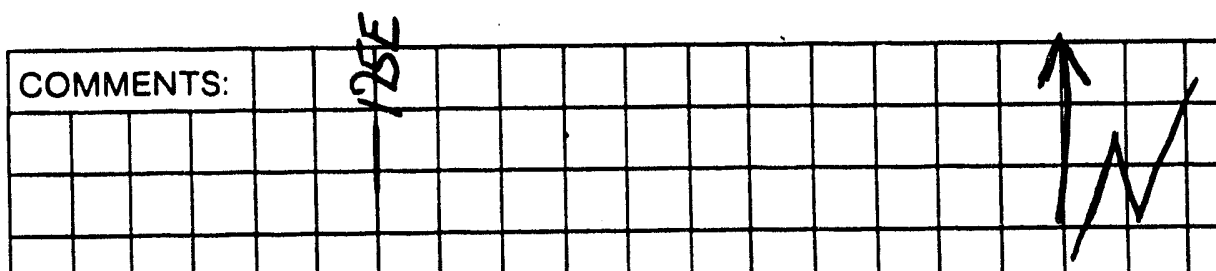

380

30

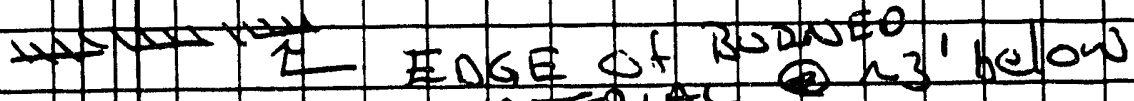
MAjERLC

360

27R2ES-3.55-125-52

\section{5}

EQGe of Burnen Mationar

(1) nz' below suffece.

340

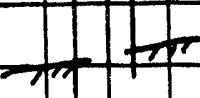

7

PREPARED BY:

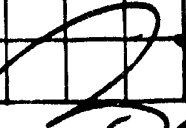




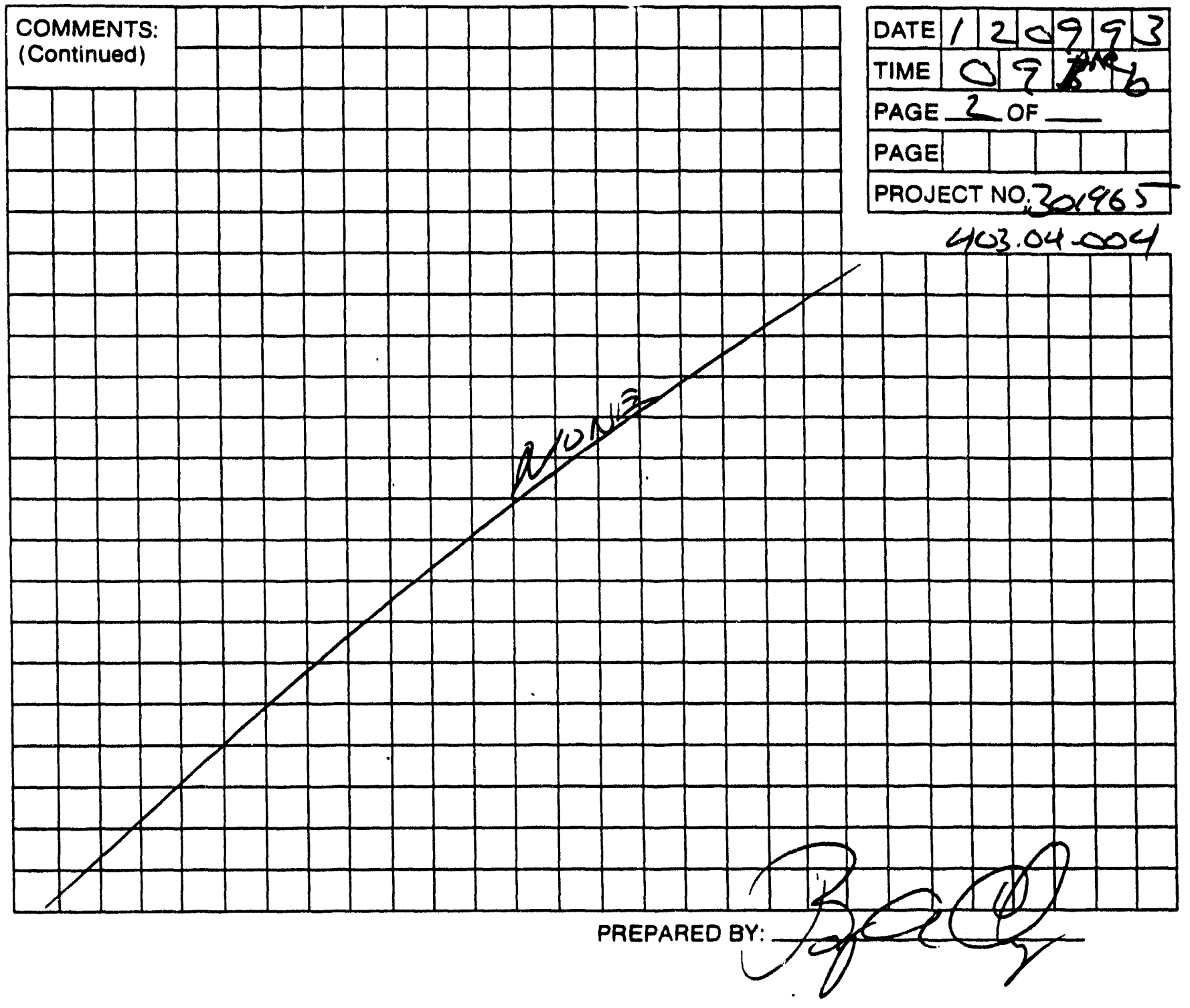

LEGEND

1. A SAMPLE COLLECTION LOG IS TO BE COMPLETED FOR EACH SAMPLE.

2. ALWAYS COMPLETE BOTH SIDES. IF SECOND SIDE IS NOT USED, DRAW A LINE THROUGH IT AND MARK N/A. FILL IN CONTROL BLOCK AND PREPARED $\mathrm{QY}$.

3. ALL ENTAIES ON LOQ ARE TO BE COMPLETED, IF NOT APPLICABLE MARK N/A.

4. DATE: USE MONTH/DAY/YEAR: 1.E., 10/30/85

5. TIME: USE 24-HOUR CLOCK: I.E., 1835 FOR 6:35 P.M.

6. PAGE: EACH SAMPLE TEAM SHOULD NUMBER PAGE __ OF__ FOR THE DAY'S ACTIV. TIES FOR ALL SHEETS PREPARED ON A SINGLE DAY, I.E., IF THERE ARE A TOTAL OF 24 PAGES (INCLUDING FRONT AND BACK) NUMBt.A 1 OF 24, 2 OF 24, ETC.

7. SAMPLE LOCATION: USE BORING OR MONITORING WELL NUMBER, GRIO LOCATION (TRANSECT). SAMPLING STATICN I.D.. OR COORDINATE TO PHYSICAL FEATURES WITH DISTANCES. INCLUDE SKETCH IN COMMENT SECTION IF NECESSARY.

8. SAMPLE TYPE: USE THE FOLLOWING - SOIL: WATER (SURFACE OR GROUND): AIR (FILTERS. TUBES. AMBIENT. PERSONNEL): SLUDGE: DRUM CONTENTS: OIL: VEGETATION; WIPE: SEDIMENT.

9. COMPOSITE TYPE: I.E., 24-MOUR, LIST SAMPLE NUMBERS IN COMPOSITE, SPATIAL COMPOSITE.

10. DEPTH OF SAMPLE: GIVE UNITS. WRITE OUT UNITS SUCH AS INCHES. FEET. DON'T USE ' OR "

11. WEATHER: APPROXIMATE TEMPERATURE, SUN AND MOISTURE CONDITIONS.

12. CONTAINERS USED: LIST EACH CONTAINER TYPE AS NUMBER, VOLUME, MATERIAL (E.G., 2 - IL GLASS; 1 - 40 ML GLASS VIAL: 1 - 400 ML PLASTIC: 1 - 3 INCH STEEL TUBE: 8 OZ. GLASG JAR)

13. AMOUNT COLLECTED: VOLUME IN CONTAINERS (E.G. 1/2 FULL). 


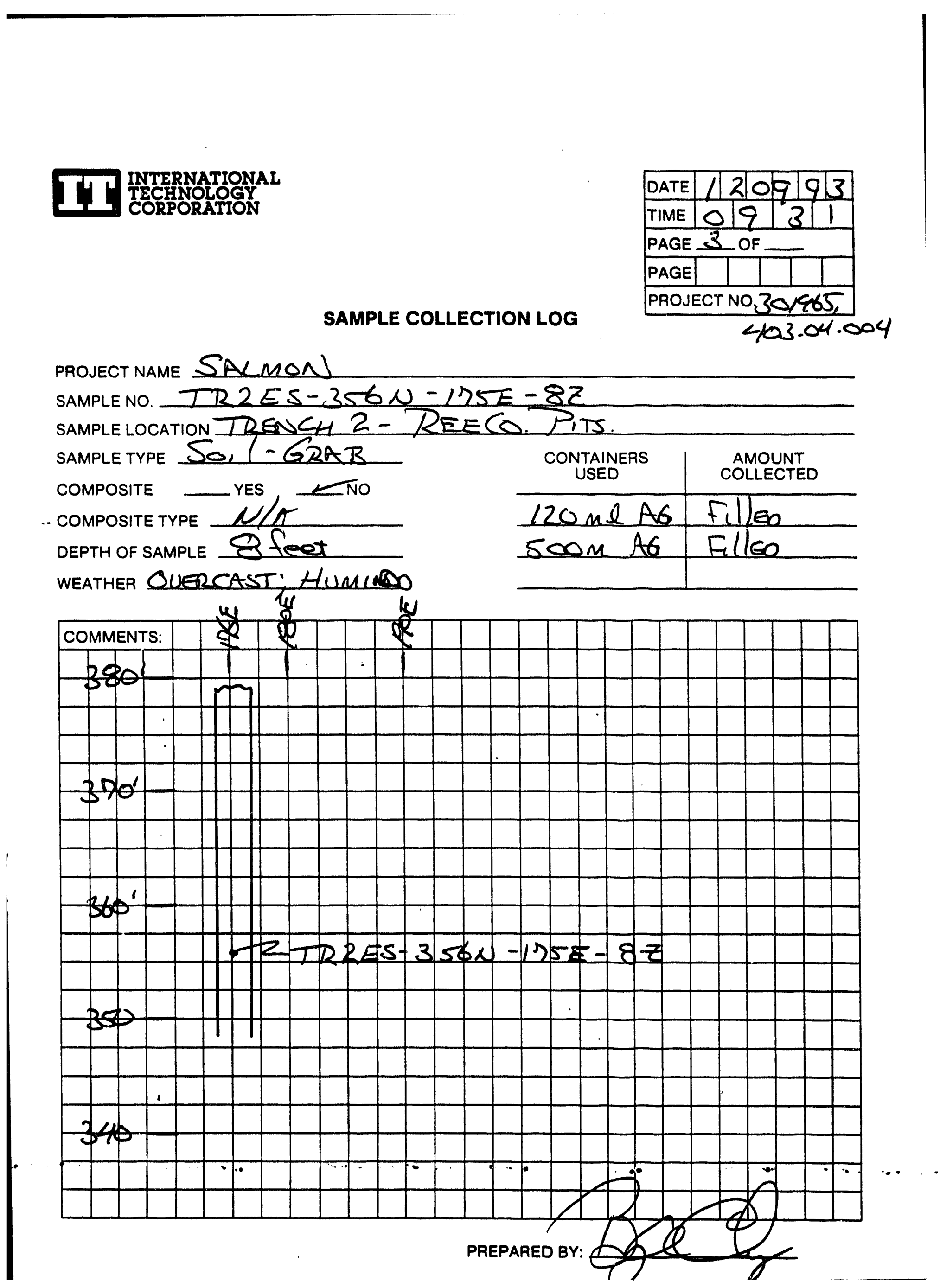




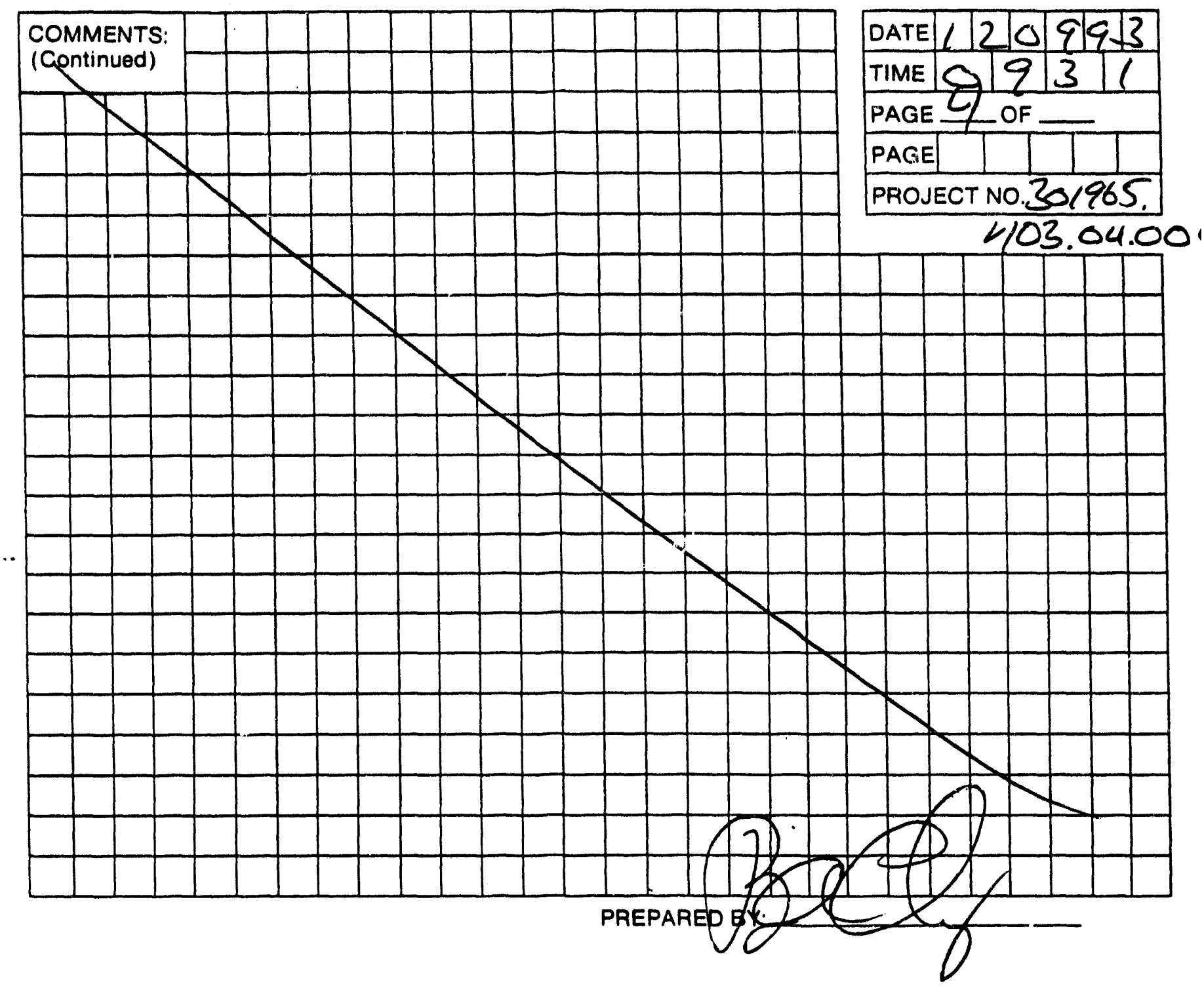

\section{LEGEND}

1. A SAMPLE COLLECTION LOG IS TO Be COMPLETED For EACH SAMPLE.

2. ALWAYS COMPLETE BOTH SIDES. IF SECOND SIDE IS NOT USED, DRA W A LINE THROUGH IT AND MARK N/A. FILL IN CONTROL BLOCK AND PREPARED BY.

3. All entaies on log ARE TO BE COMPLETEd, if nOt APPLiCABle MA RK N/A.

4. DATE: USE MONTH/DAYMEAR: I.E., 10/30/85

S. TIME: USE 24-HOUR CLOCK: I.E., 1835 FOR 6:35 P.M.

6. PAGE: EACH SAMPLE TEAM SHOULD NUMBER PAGE __ OF___ FOR THE DAY'S ACTIVITIES FOR ALL SHEETS PREPARED ON A SINGLE DAY, I.E., IF THERE ARE A TOTAL OF 24 PAGES (INCLUDING FRONT AND BACK) NUMBER 1 OF 24, 2 OF 24. ETC.

7. SAMPLE LOCATION: USE BORING OR MONITORING WELL NUMEER, GRID LOCATION (TRANSECT), SAMPLING STATION I.D., OR COORDINATE TO PHYSICAL FEATURES WITH DISTANCES. INCLUDE SKETCH IN COMMENT SECTION IF NECESSARY.

8. SAMPLE TYPE: USE THE FOLLOWING - SOIL: WATER (SUPFACE OR GROUND): AIR (FILTERS. TUBES, AMBIENT, PERSONNEL): SLUDGE; DRUM CONTENTS: OIL: VEGETATION: WIPE: SEDIMENT.

9. COMPOSITE TYPE: I.E., 24-HOUR, LIST SAMPLE NUMBERS IN COMPOSITE, SPATIAL COMPOSITE.

10. DEPTH OF SAMPLE: GIVE UNITS. WRITE OUT UNITS SUCH AS INCHES. FEET. DON'T USE ' OR ".

11. WEATHER: APPROXIMATE TEMPERATURE. SUN AND MOISTURE CONDITIONS.

12. CLNTANERS USED: LIST EACH CONTAINER TYPE AS NUMBER, VOLUME, MATEAIAL (E.G., 2 - IL GLASS: 4 - 40 ML GLASS VIAL: 1 - 400 ML PLASTIC: 1 - 3 INCH STEEL TUBE: 1 - 8 OZ. GLASS JAR)

13. AMOUNT COLLECTED: VOLUME IN CONTAINERS (E.O. 1/2 FULLL). 
Project name SAkmon

SAMPLENO. TR2A ES-360N-200E-8Z

sample location IREUCH 2A-REe Go PITS.

SAMPLE TYPE SOil GRAB

COMPOSITE YYES NNO

.. COMPOSITE TYPE $\propto / A$

DEPTH OF SAMPLE \& foer

WEATHER Cldy 50

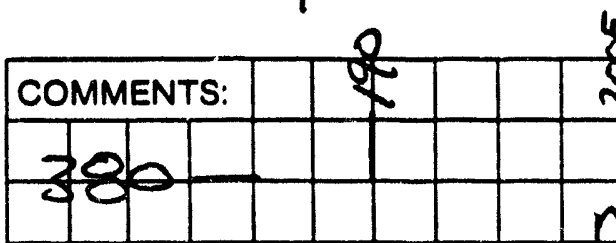

$y$

N

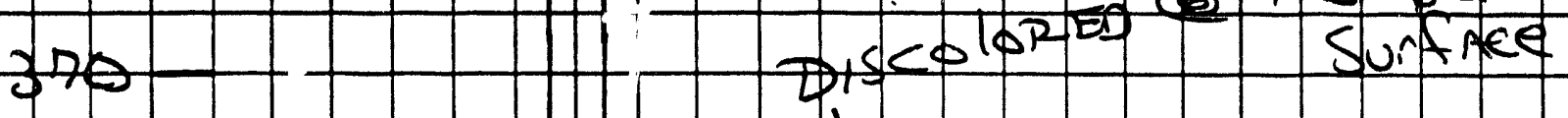

14

360 T2 T2A-ES-360N-200E.8R

30

340

$-$.

$-\cdot \cdot+\cdot \cdot+\cdot \cdot \cdot$

AMOUNT

COLLECTED

$120 \mathrm{ml}$ AG $190 \%$ fill

soo me As filleo.

b) 13 ' zelon 


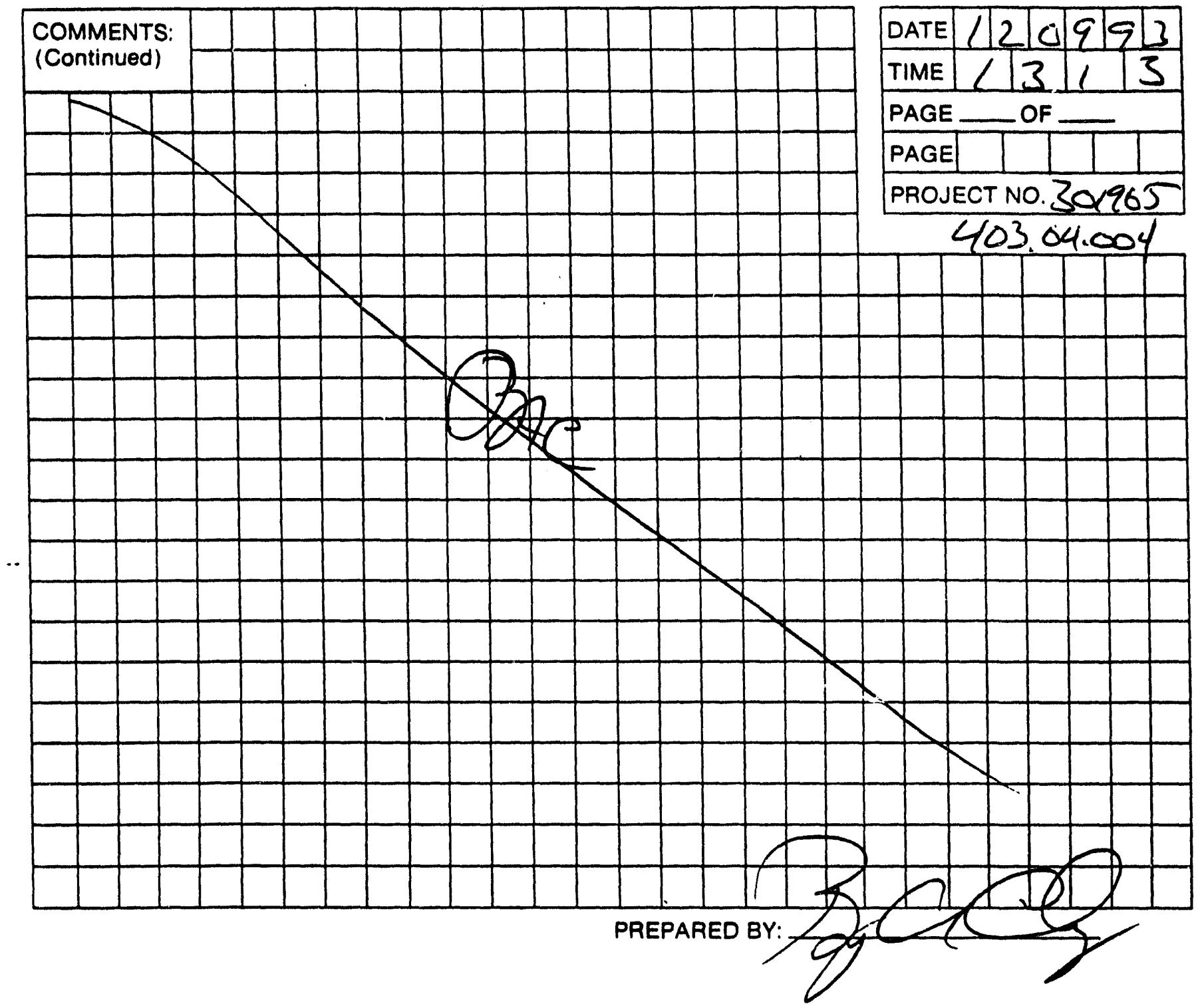

LEGEND

1. A SAMPLE COLLECTION LOG IS TO BE COMPLETED FOR EACH SAMPLE.

2. ALWAYS COMPLETE BOTH SIDES. IF SECOND SIDE IS NOT USED. DRAW A LINE THROUGH IT AND MARK N/A. FILL IN CONTROL BLOCK AND PAEPAAED BY.

3. ALL ENTRIES ON LOG ARE TO BE COMPLETED. If NOT APPLICABLE MARK N/A.

4. DATE: USE MONTH/DAY/YEAR: I.E., 10/30/85

5. TIME: USE 24-HOUA CLOCK: I.E., 1835 FOR 6:35 P.M.

6. PAGE: EACH SAMPLE TEAM SHOULD NUMBER PAGE__ OF__ FOR THE DAY'S ACTIVITIES FOR ALL SHEETS PREPARED ON A SINGLE DAY, I.E., IF THERE ARE A TOTAL OF 24 PAGES (INCLUDING FRONT AND BACK) NUMBER 1 OF 24, 2 OF 24, ETC.

7. SAMPLE LOCATION: USE BORING OR MONITORING WELL NUMBER, GRIO LOCATION (TRANSECT). SAMPLING STATION I.D.. OR COORDINATE TO PHYSICAL FEATURES WITH DISTANCES. INCLUDE SKETCH IN COMMENT SECTION IF NECESSARY.

8. SAMPLE TYPE: USE THE FOLLOWING - SOIL: WATER (SUPFACE OR GROUND): AIR (FILTERS, TUBES. AMBIENT, PERSONNEL): SLUDGE; DRUM CONTENTS: OIL: VEGETATION: WIPE: SEDIMENT.

9. COMPOSITE TYPE: I.E., 24-HOUR, LIST SAMPLE NUMBERS IN COMPOSITE, SPATIAL COMPOSITE.

10. DEPTH OF SAMPLE: GIVE UNITS. WRITE OUT UNITS SUCH AS INCHES, FEET. DON'T USE ' OR ".

11. WEATHER: APPROXIMATE TEMPERATURE. SUN ANO MOISTURE CONDITIONS.

12. CONTAINERS USED: LIST EACH CONTAINER TYPE AS NUMBER, VOLUME, MATERIAL (E.G., 2 - IL GLASS: 4 - 40 ML GLASS VIAL: 1 - 400 ML PLASTIC: 1 - 3 INCH STEEL TUBE: 1 - 8 OZ. GLASS JAP).

13. AMOUNT COLLECTED: VOLÜME IN CONTAINERS (E.G. I/2 FULLL). 
PROJECT NAME SARmonj

SAMPLE NO. TR2A-ES-365N-2OCVE-3.5Z

SAMPLE LOCATION IDENCH $2 A$ - ReECO. Pits.

SAMPLE TYPE SOML GRAB

COMPOSITE YYES -NO

.. COMPOSITE TYPE $N / A$

DEPTH OF SAMPLE 3.5 feec

WEATHER P.cidy $50^{\circ}$

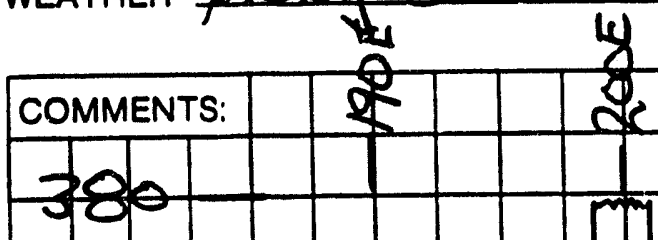

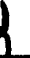

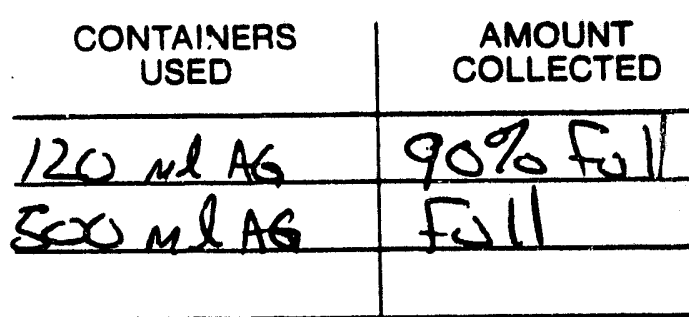

$120 \mathrm{mlAG} 90 \%$ full

t) TIR $2 A-E S-365 y-200 E-3.5 z$

360

$3=0$

850

$2 / 6$ 


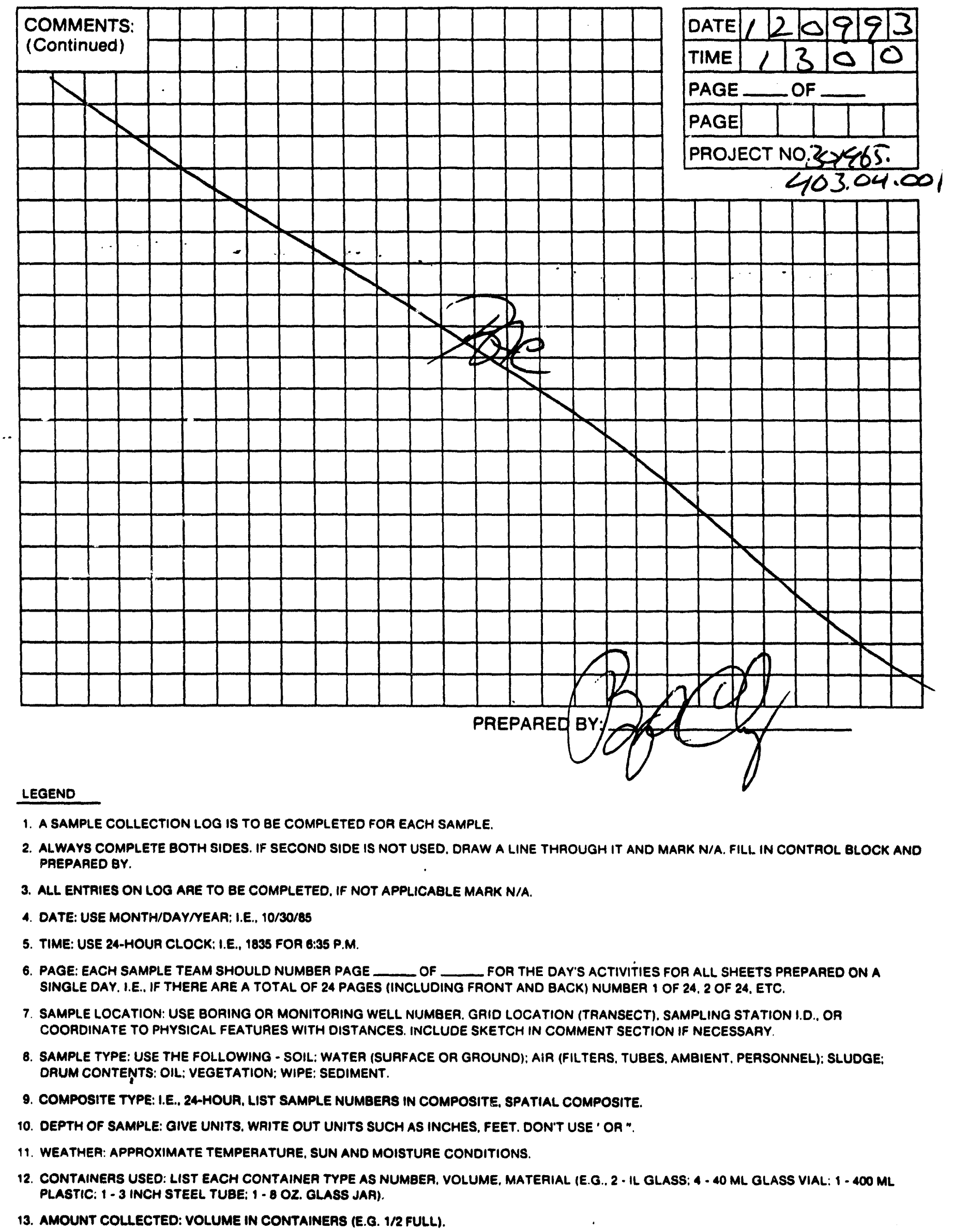


PRoJect NAME SACMON

SAMPLE NO IR3 - ES - L50N-400E-3Z

SAMPLE LOCATION IRENEH 2 -REECQ PITS

SAMPLE TYPE Soul GDAB

COMPOSITE —YES کNO

.. COMPOSITE TYPE N/A

CONTAINERS USED

DEPTH OF SAMPLE 3 feet.

WEATHER cldy $-245^{\circ}$

$120 \mathrm{ml}$ A6. $90 \%$ foll

soomlt6 Foll.

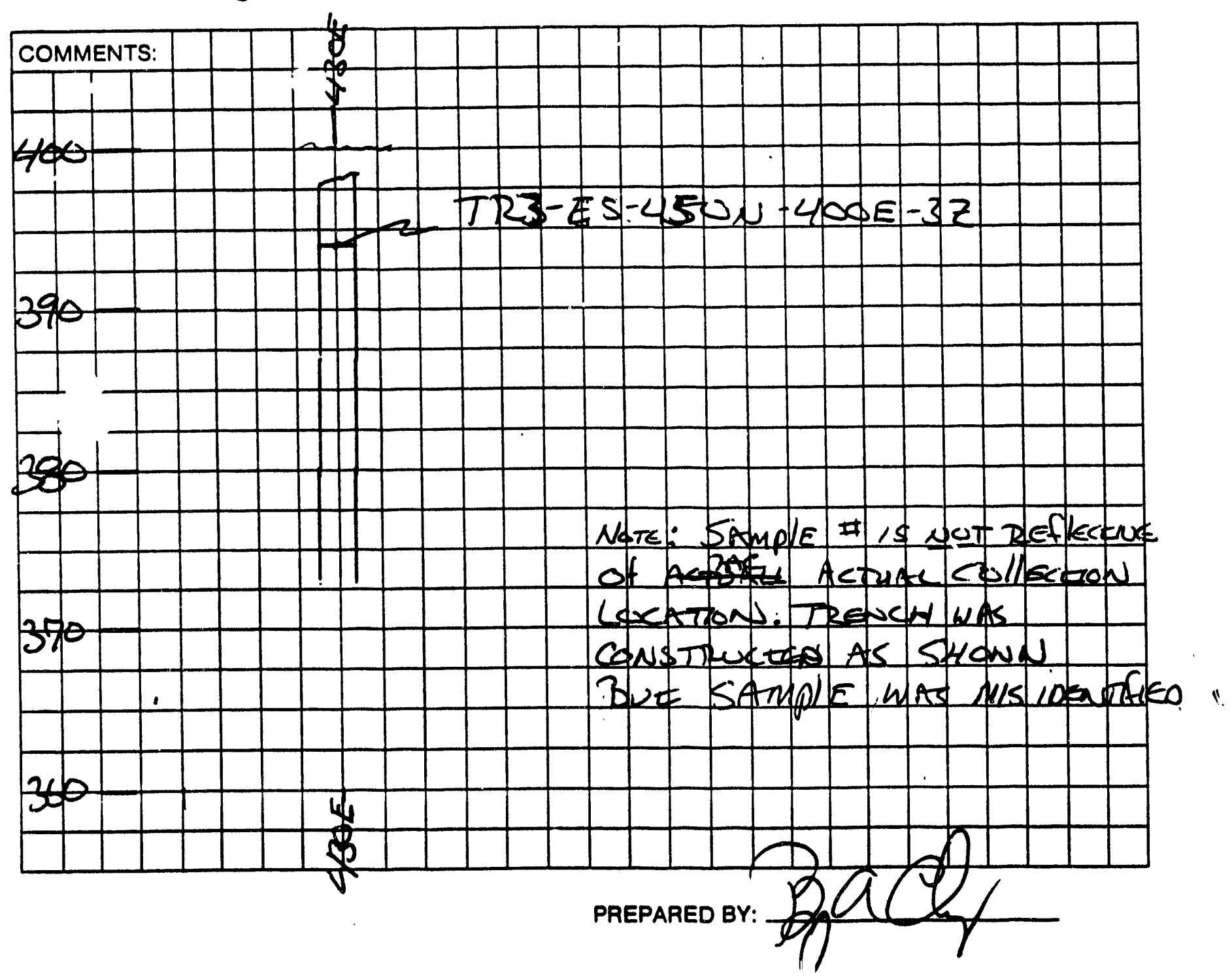




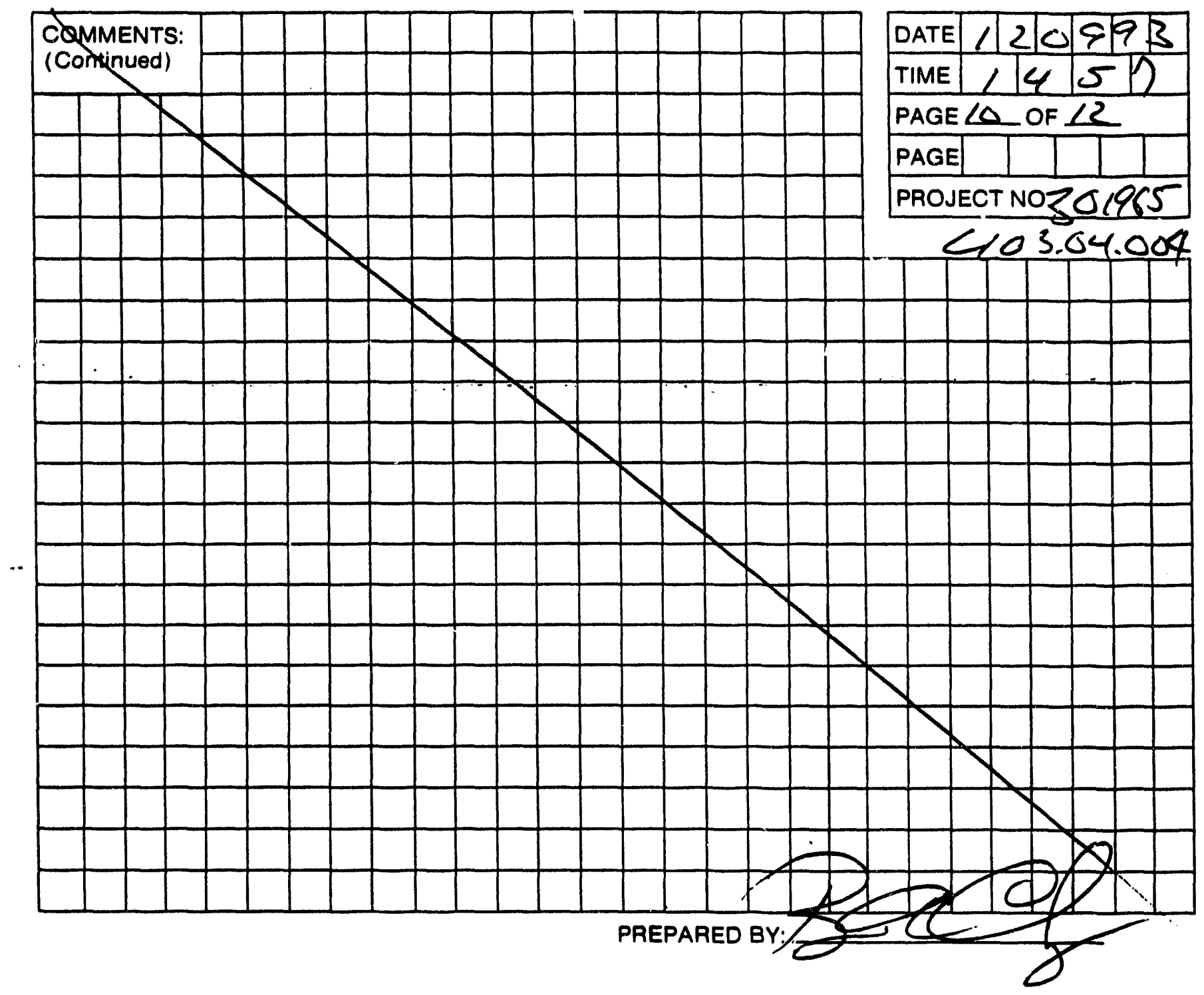

LEGENO

1. A SAMPLE COLLECTION LOG IS TO BE COMPLETED FOR EACH SAMPLE.

2. ALWAYS COMPLETE BOTH SIDES, IF SECOND SIDE IS NOT USED. ORAW A LINE THROUGH IT AND MARK N/A. FILL IN CONTROL BLOCK AND PREPARED BY.

3. ALL ENTRIES ON LOQ ARE TO BE COMPLETED, IF NOT APPLICABLE MARK N/A.

4. DATE: USE MONTH/DAYTYEAP; I.E., 10/30/85

5. TIME: USE 24-HOUR CLOCK: I.E., 1835 FOR 6:35 P.M.

6. PAGE: EACH SAMPLE TEAM SHOULD NUMBER PAGE __ OF _ FOR THE DAY'S ACTIVITIES FOR ALL SHEETS PREPARED ON A SINGLE DAY, I.E., IF THERE ARE A TOTAL OF 24 PAGES (INCLUDING FRONT AND BACK) NUMBER 1 OF 24, 2 OF 24. ETC.

7. SAMPLE LOCATION: USE BORING OF MONITORING WELL NUMBER. GRID LOCATION (TRANSECT). SAMPLING STATION I.D. OR COORDINATE TO PHYSICAL FEATURES WITH DISTANCES. INCLUDE SKETCH IN COMMENT SECTION IF NECESSARY.

8. SAMPLE TYPE: USE THE FOLLOWING - SOIL: WATER (SUAFACE OR GROUND): AIR (FILTERS. TUBES. AMBIENT, PEASONNEL): SLUDGE; DAUM CONTENTS: OIL: VEGETATION: WIPE: SEDIMENT.

9. COMPOSITE TYPE: I.E., 24-HOUR, LIST SAMPLE NUMBERS IN COMPOSITE. SPATIAL COMPOSITE.

10. DEPTH OF SAMPLE: GIVE UNITS. WAITE OUT UNITS SUCH AS INCHES, FEET. DON'T USE ' OR ".

11 WEATHER: APPROXIMATE TEMPERATURE. SUN AND MOISTURE CONOITIONS.

12. CONTAINERS USED: LIST EACH CONTAINEA TYPE AS NUMBER, VOLUME, MATEAIAL (E.G., 2 - IL GLASS: 4 - 40 ML GLASS VIAL: 1 - 400 ML PLASTIC: 1 - 3 INCH STEEL TUBE: 1 - 8 OZ. GLASS JAR).

13. AMOUNT COLLECTED: VOLUME IN CONTAINERS (E.G. 1/2 FULL). 
PROJeCt NAME SALMOA:

SAMPLE COLLECTION LOG

SAMPLENO. TR 3 -ES - $4 / 30 N-400 E-15 Z$

SAMPLE LOCATION IRENCH 3

SAMPLE TYPE SO.1 GRAB

COMPOSITE —YES —NO

.. COMPOSITE TYPE N N/A.

DEPTH OF SAMPLE 15 feer

\begin{tabular}{c|c}
$\begin{array}{c}\text { CONTAINERS } \\
\text { USED }\end{array}$ & $\begin{array}{c}\text { AMOUNT } \\
\text { COLLECTED }\end{array}$ \\
\hline $120 \mathrm{~m}$ \& AG & full \\
\hline $500 \mathrm{ml} A G$ & full
\end{tabular}

weather PTly Cloudy

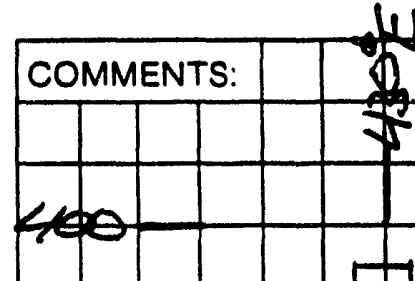

30

20

$2+R 3-E S-4130 x-400 E-152$

Note: Sample is votreffectule of Abua colleconon Losamol.

300 Irone hat constroceson as sícun But stanple cuas mis sontifion 


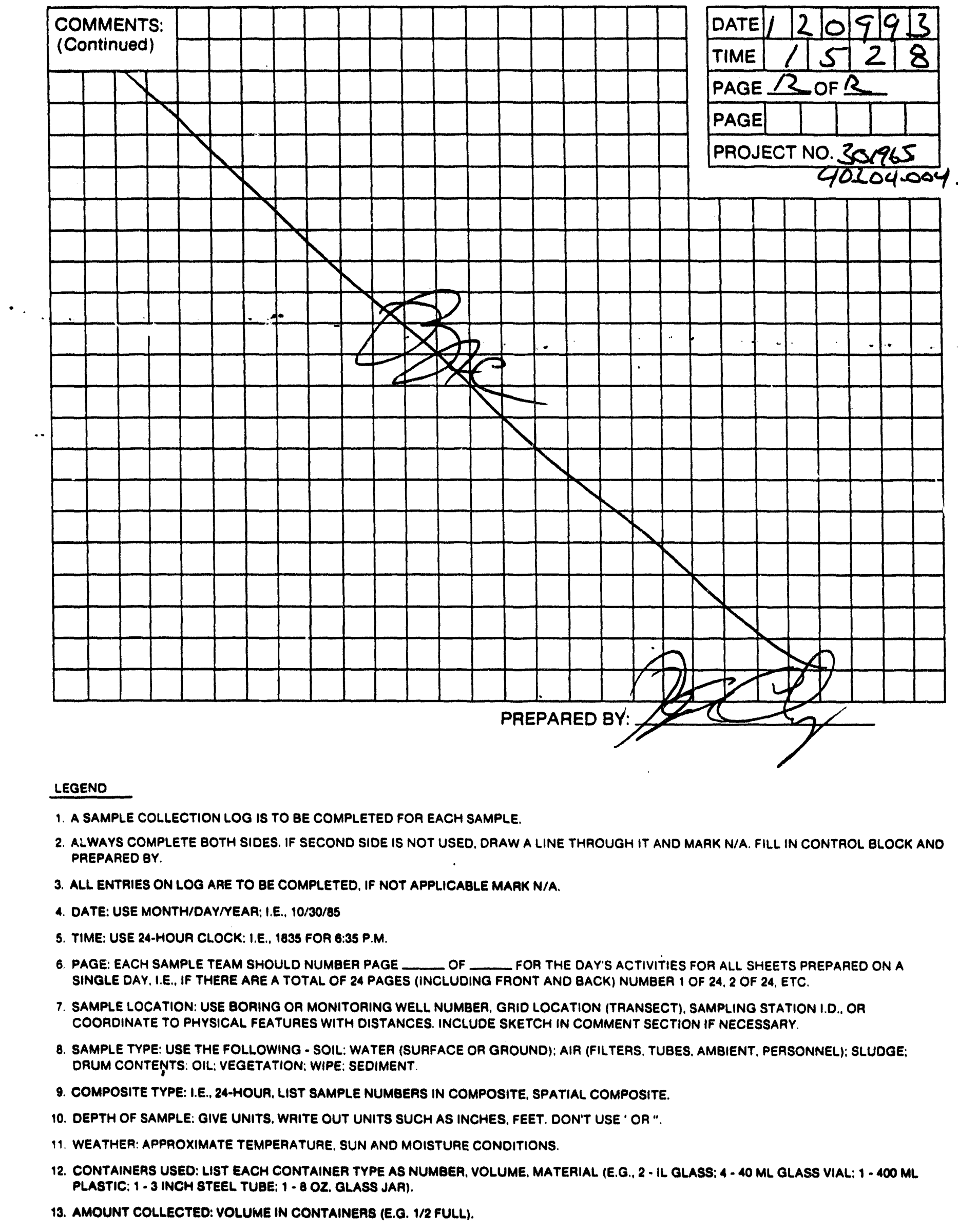


PROJECT NAME SALMOAl

SAMPLE NO. JR LI-ES - 260-490-8

SAMPLE LOCATION JRENU4 4 - REE LO PITS

SAMPLE TYPE SOLl GRAB

COMPOSITE —YES —NO

COMPOSITE TYPE

DEPTH OF SAMPLE \& $\&$ EeT

WEATHER is, EHCOAh, Clen

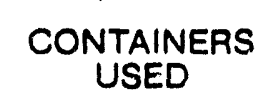
USED

$120 \mathrm{ml}$ AG $120 \mathrm{ml}$ Sooml AG $500 \mathrm{ml}$.

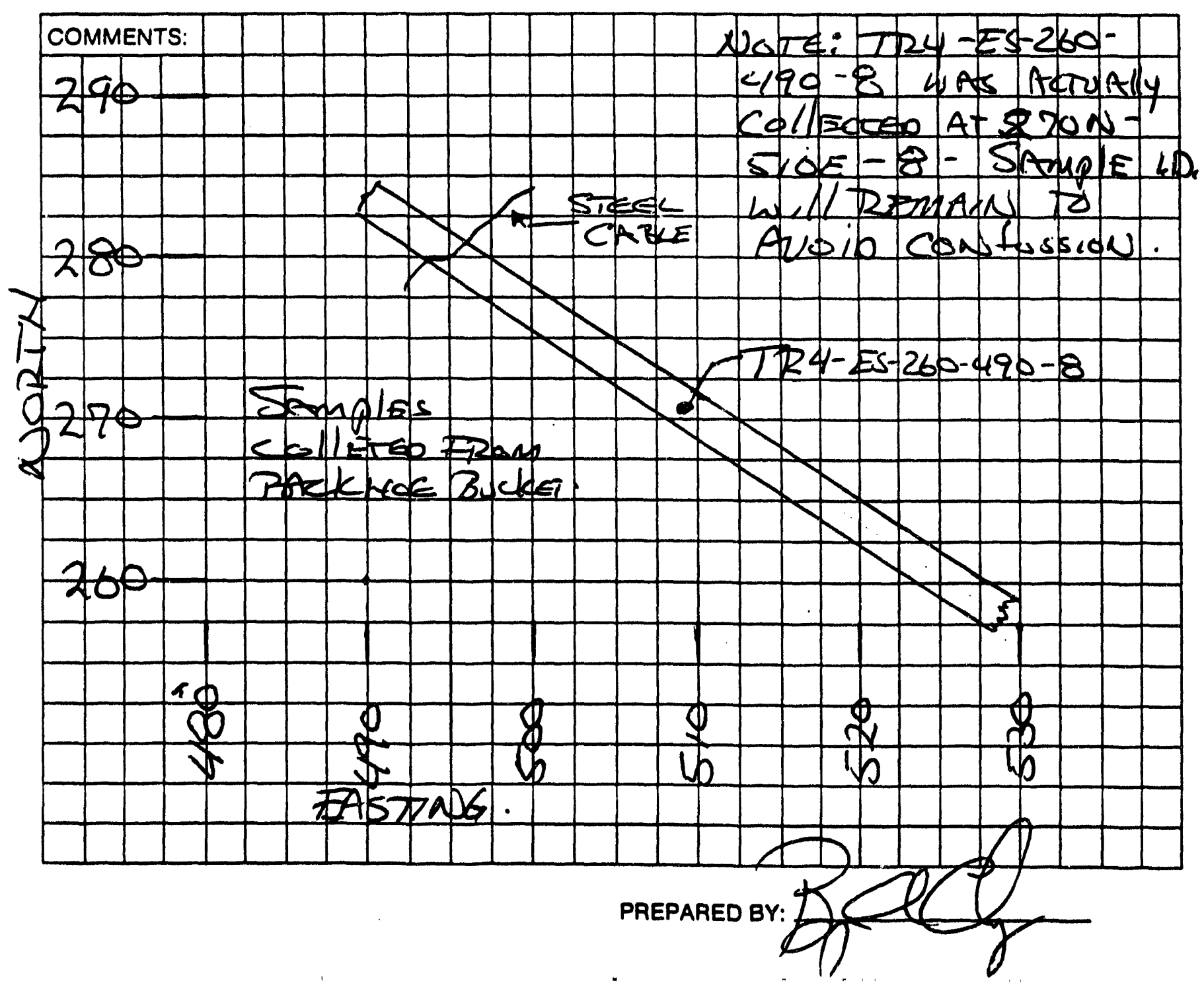




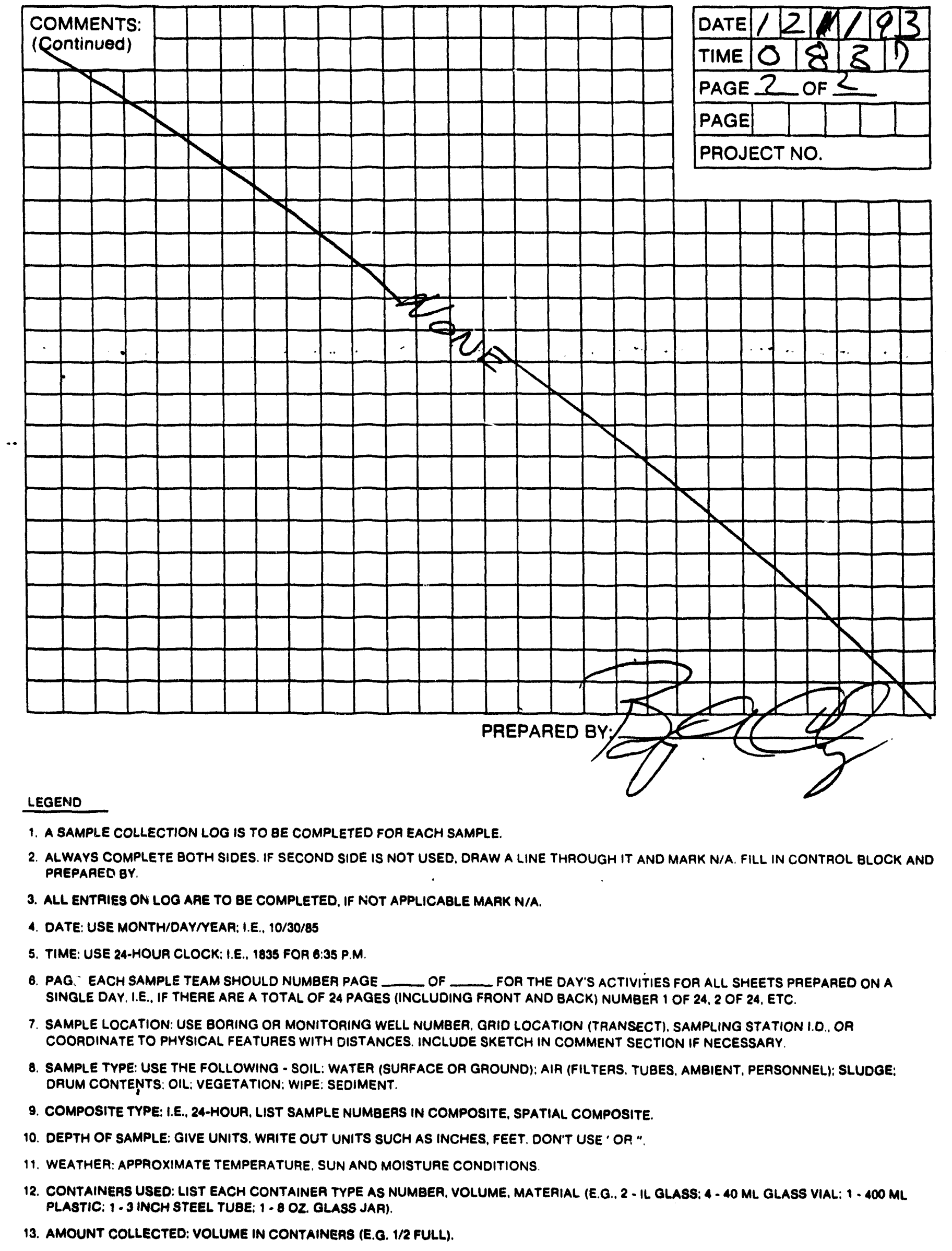


INTERNATIONAL

TECHNOLOGY
SAMPLE COLLECTION LOG

$$
\begin{aligned}
& \begin{array}{|l|l|l|l|l|l|l|}
\hline \text { DATE } & 1 & 2 & 1 & 1 & 9 & 3 \\
\hline
\end{array} \\
& \begin{array}{|l|l|l|l|l|}
\hline \text { TIME } & 1 & 1 & 4 & 0 \\
\hline
\end{array} \\
& \text { PAGE L OF \& } \\
& \text { PAGE } \\
& \text { PROJECT NO.ZOC } 965 \\
& 403.04 .004
\end{aligned}
$$

PRoject NAME SAlmon SAMPLE NO. TR/O-ES-180-1990-E

SAMPLE LOCATION IRANHAP- REELS. PITS

SAMPLE TYPE SO II GRAB

COMPOSITE — YES $=$ NO

COMPOSITE TYPE

DEPTH OF SAMPLE WeATHER clear CoOl.
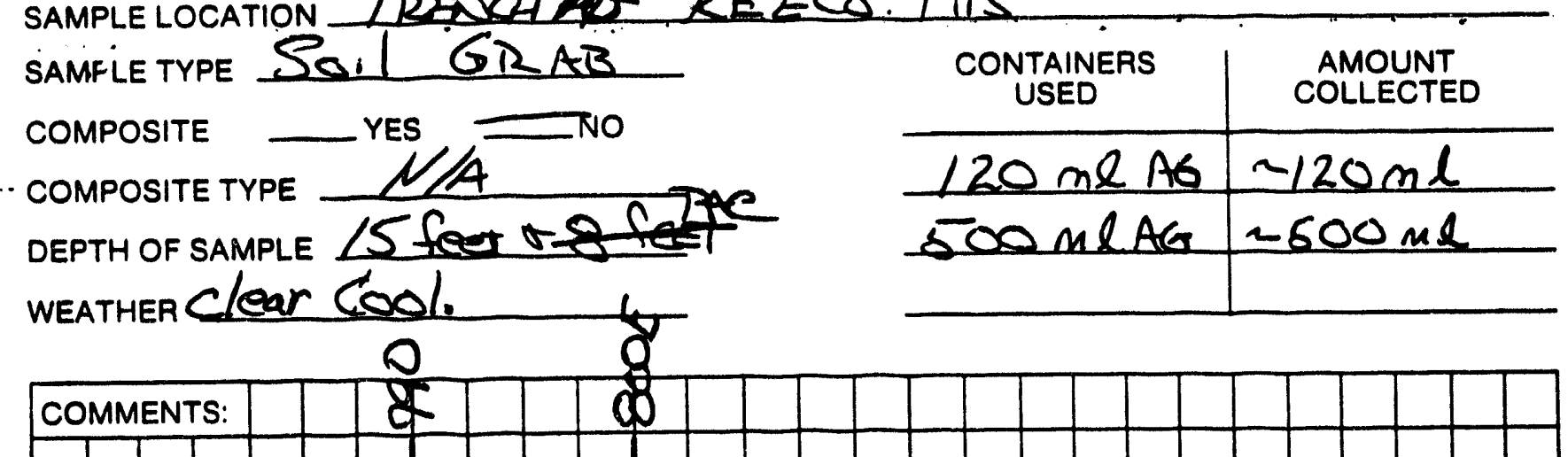

200

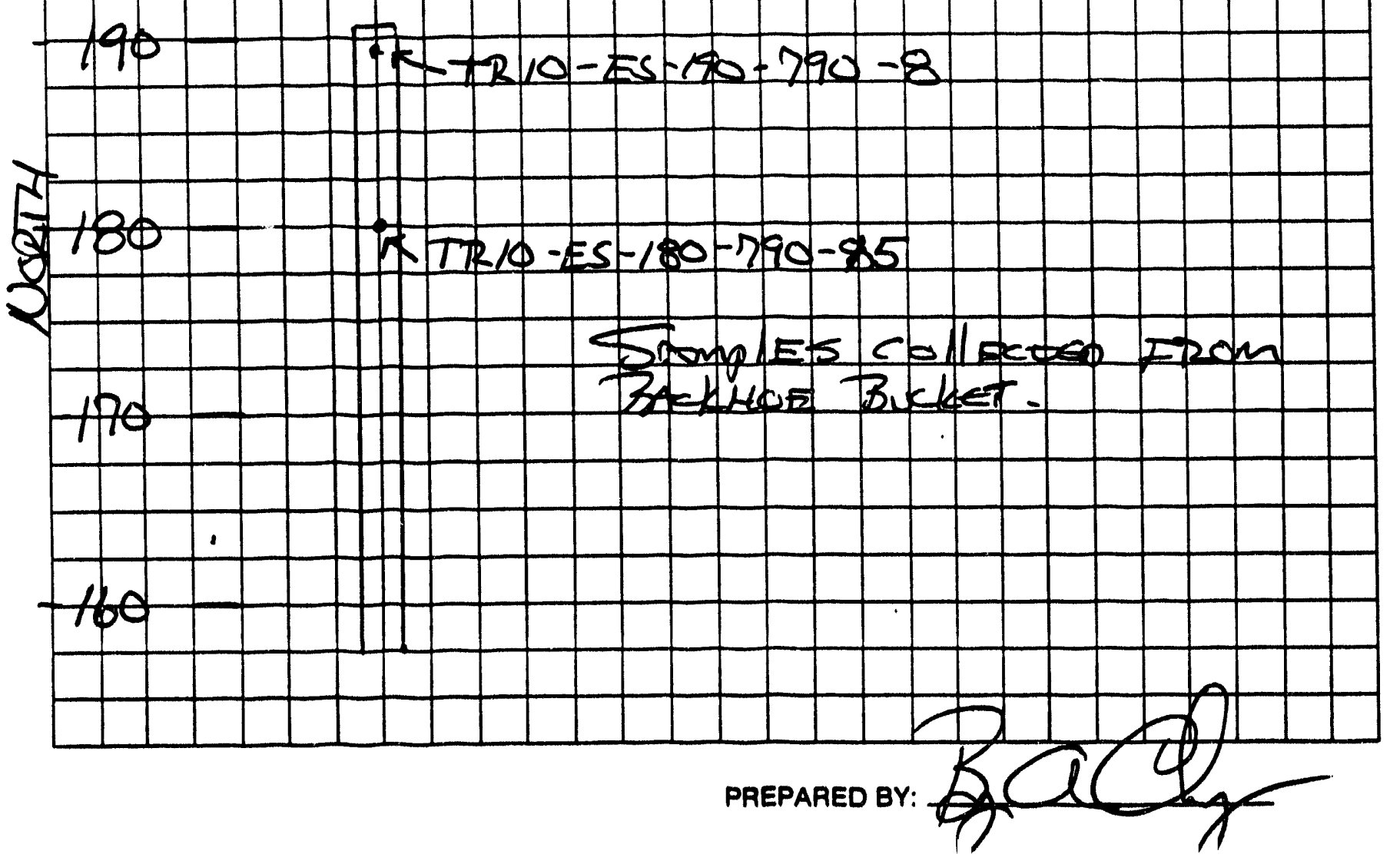




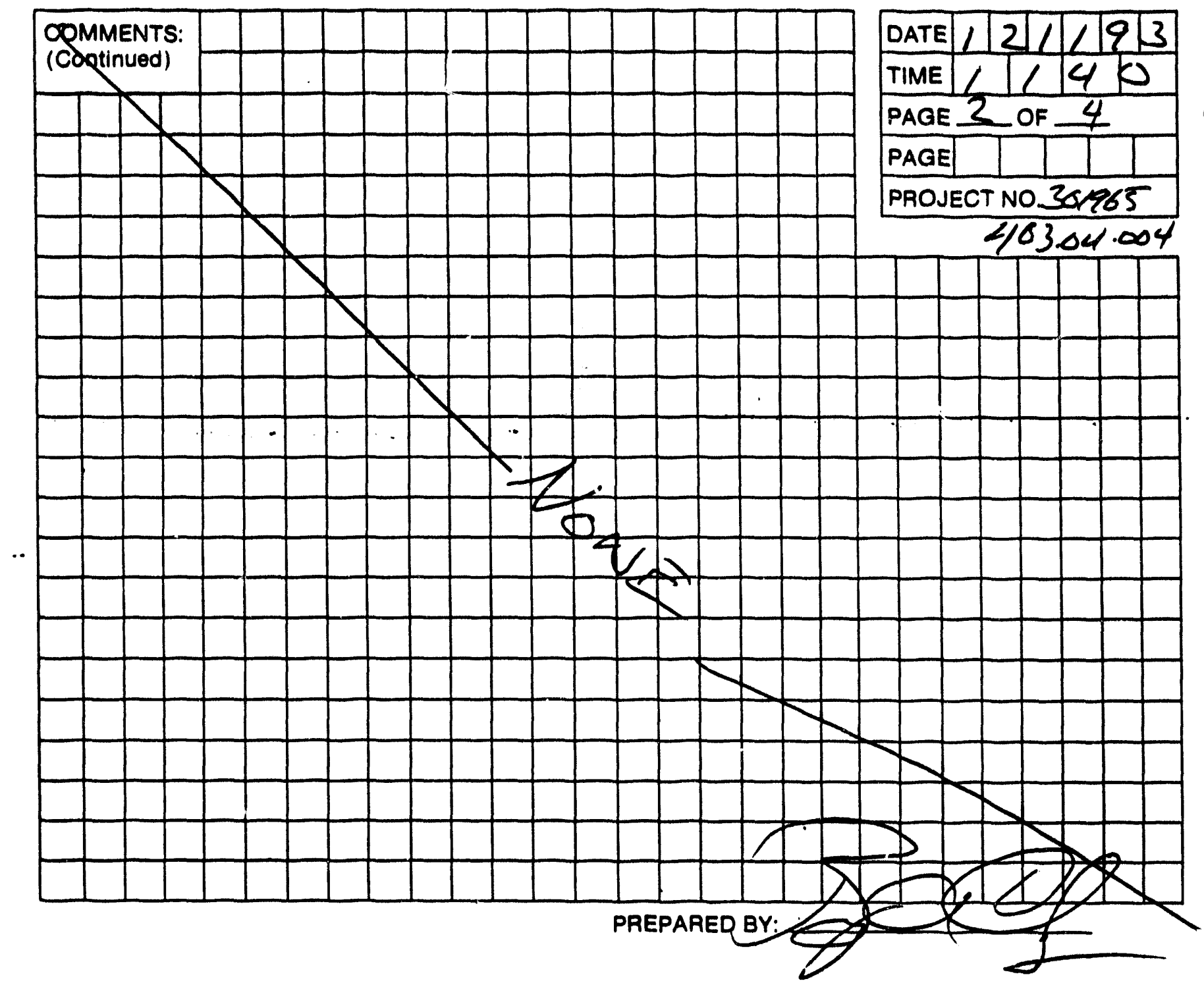

LEGEND

1. A SAMPLE COLLECTION LOG IS TO BE COMPLETED FOR EACH SAMPLE.

2. ALWAYS COMPLETE BOTH SIDES. IF SECOND SIDE IS NOT USEO. DRAW A LINE THROUGH IT AND MARK N/A. FILL IN CONTROL BLOCK AND PREPARED BY.

3. ALL ENTRIES ON LOQ ARE TO BE COMPLETED, IF NOT APPLICABLE MARK N/A.

4. DATE: USE MONTH/DAYMEAR: 1.E., 10/30/85

5. TIME: USE 24-HOUR CLOCK: I.E., 1835 FOR 8:35 P.M.

6. PAGE: EACH SAMPLE TEAM SHOULO NUMBER PAGE ___ OF___ FOR THE DAY'S ACTIVITIES FOR ALL SHEETS PAEPARED ON A SINGLE DAY. I.E.. IF THERE ARE A TOTAL OF 24 PAGES (INCLUDING FRONT AND BACK) NUMBEA 1 OF 24, 2 OF 24. ETC.

7. SAMPLE LOCATION: USE BORING OR MONITORING WELL NUMBER, GRID LOCATION (TRANSECT). SAMPLING STATION I.D., OR COOADINATE TO PHYSICAL FEATURES WITH DISTANCES. INCLUDE SKETCH IN COMMENT SECTION IF NECESSARY.

8. SAMPLE TYPE: USE THE FOLLOWINO - SOIL: WATER (SURFACE OR GROUND): AIR (FILTERS. TUBES. AMBIENT, PERSONNEL): SLUDGE: DRUM CONTENTS: OIL: VEGETATION: WIPE: SEDIMENT.

9. COMPOSITE TYPE: I.E., 24-HOUR, LIST SAMPLE NUMBERS IN COMPOSITE, SPATIAL COMPOSITE.

10. DEPTH OF SAMPLE: GIVE UNITS. WAITE OUT UNITS SUCH AS INCHES, FEET. DON'T USE ' OR ".

11. WEATHER: APPROXIMATE TEMPERATURE, SUN AND MOISTURE CONOITIONS.

12. CONTAINEAS USED: LIST EACH CONTAINER TYPE AS NUMBEA, VOLUME, MATERIAL, (E.G., 2 - IL GLASS; 4 - 40 ML GLASS VIAL: 1 - 400 ML PLASTIC: 1 - 3 INCH STEEL TUBE: 1 - 8 OZ. GLASS JA 9).

13. AMOUNT COLLECTED: VOLUME IN CONTAINERS (E.Q. 1/2 FULL). 
SAMPLE COLLECTION LOG

\begin{tabular}{|c|c|c|}
\hline DATE & 11211 & $1 / 9 \sqrt{3}$ \\
\hline TIME & 11 & 153 \\
\hline PAGE & 3 of & \\
\hline PAGE & & \\
\hline PROJE & ETT NO. & $\alpha 965_{1}$ \\
\hline
\end{tabular}

PROJeCt NAME SALmonj

SAMPLENO. TR/O-ES - 190.790-8

SAMPLE LCCATION TREUCH 10 - REECO.

SAMPLE TYPE SOII GRAB

COMPOSITE —YES —NO

COMPOSITE TYPE weather Clear Cocil. $500 \mathrm{ml}$ AS $1500 \mathrm{ml}$

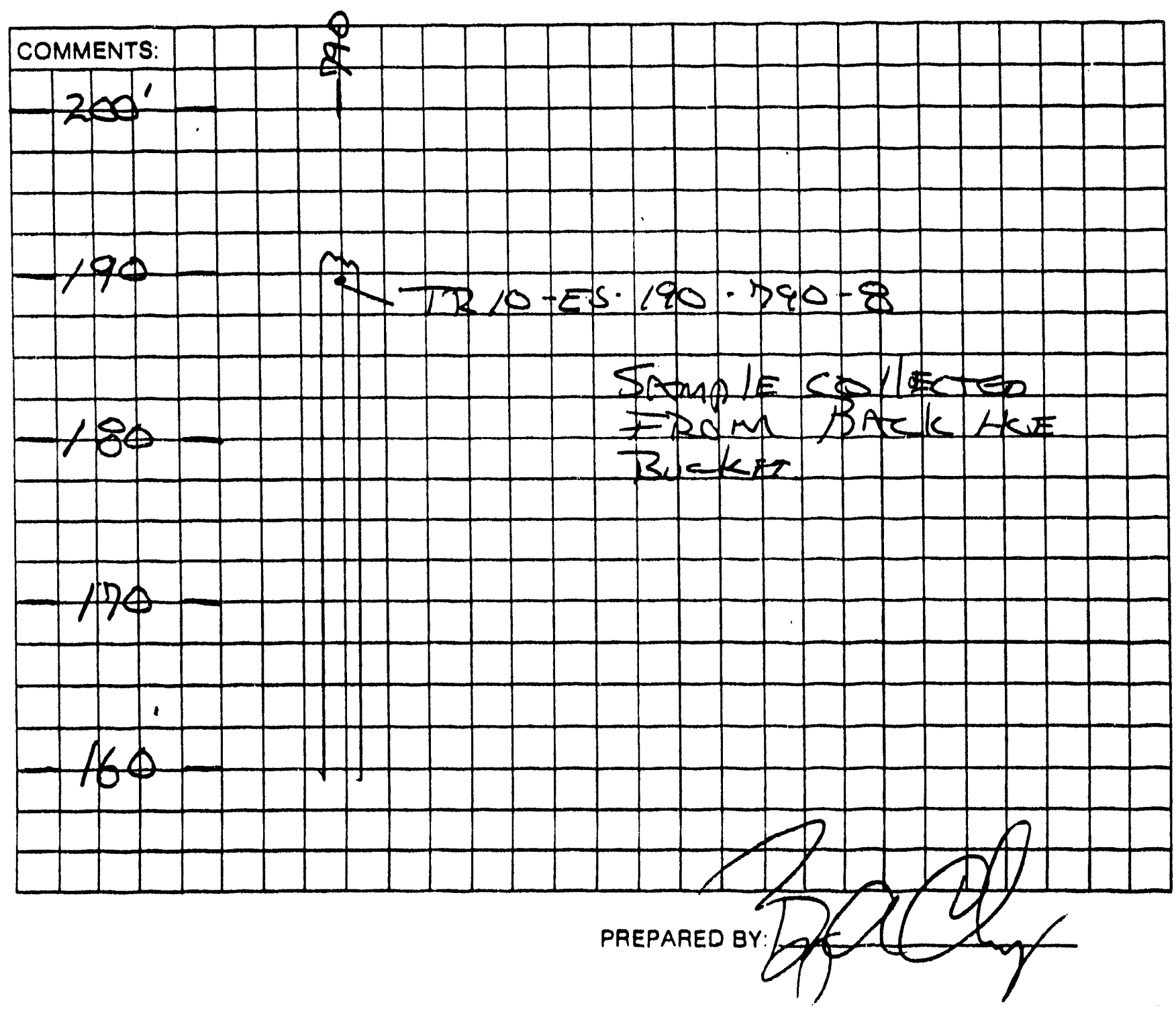




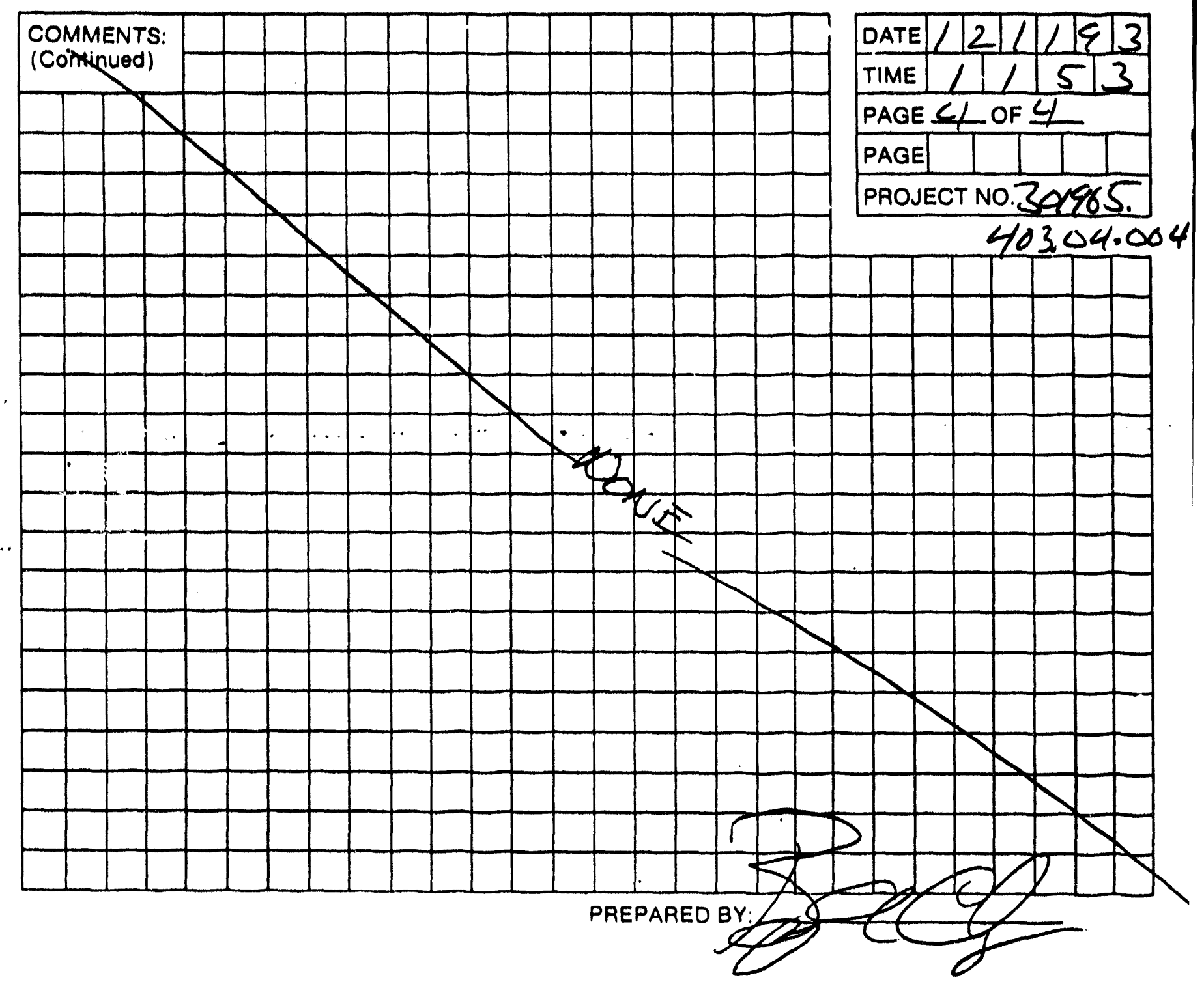

LEGEND

1. A SAMPLE COLLECTION LOO IS TO BE COMPLETED FOR EACH SAMPLE.

2. ALWAYS COMPLETE BOTH SIDES. IF SECOND SIDE IS NOT USEO. DAAW A LINE THROUGH IT ANO MARK N/A. FILL. IN CONTROL BLOCK AND PAEPAAED BY.

3. ALL ENTAIES ON LOQ ARE TO BE COMPLETED. IF NOT APPLICABLE MAAK N/A.

4. DATE: USE MONTH/OAY/YEAR: I.E., 10/30/85

3. TIME: USE 24.HOUA CLOCK: 1.E., 1835 FOR 6:35 P.M.

6. Page: each sample team should numbea page __ of __ for the day's activities for all sheets Paepared on a SINGLE DAY, I.E. IF THERE ARE A TOTAL OF 24 PAGES (INCLUDING FAONT AND BACK) NUMBER I OF 24. 2 OF 24. ETC

7 SAMPLE LOCATION USE BORING OR MONITOAING WELL NUMDER. GAIO LOCATION (TRANSECTI. SAMPLING STATIONI.D OR COOROINATE TO PHYSICAL FEATURES WITH DISTANCES. INCLUDE SKETCH IN COMMENT SECTION IF NECESSAAY

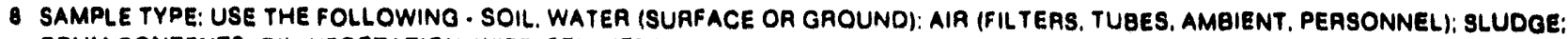
ORUM CONTENTS: OIL: VEGETATION, WIPE: SEDIMENT

9. COMPOSITE TYPE: I.E., 24-HOUA, LIST SAMPLE NUMBEAS IN COMPOSITE, SPATIAL COMPOSITE.

10. OEPTH OF SAMPLE: OIVE UNITS. WAITE OUT UNITS SUCH AS INCHES, FEET. DON'T USE ' OR "

11. WEATHER: APPAOXIMATE TEMPERATUAE, SUN AND MOISTUAE CONOITIONS

12. CONTAINERS USED: LIST EACH CONTAINER TYPE AS NUMBER. VOLUME, MATEAIAL IE. $0.2 \cdot 12$ GLASS: 4 - 40 ML GLASS VIAL: 1 - 400 ML PLASTIC: 1 - 3 INCH STEEL TUBE: 1 - 8 OZ. GLASS JAP).

13. AMOUNT COLLECTED: VOLUME IN CONTAINEAS (E.O. 1/2 FULLL). 


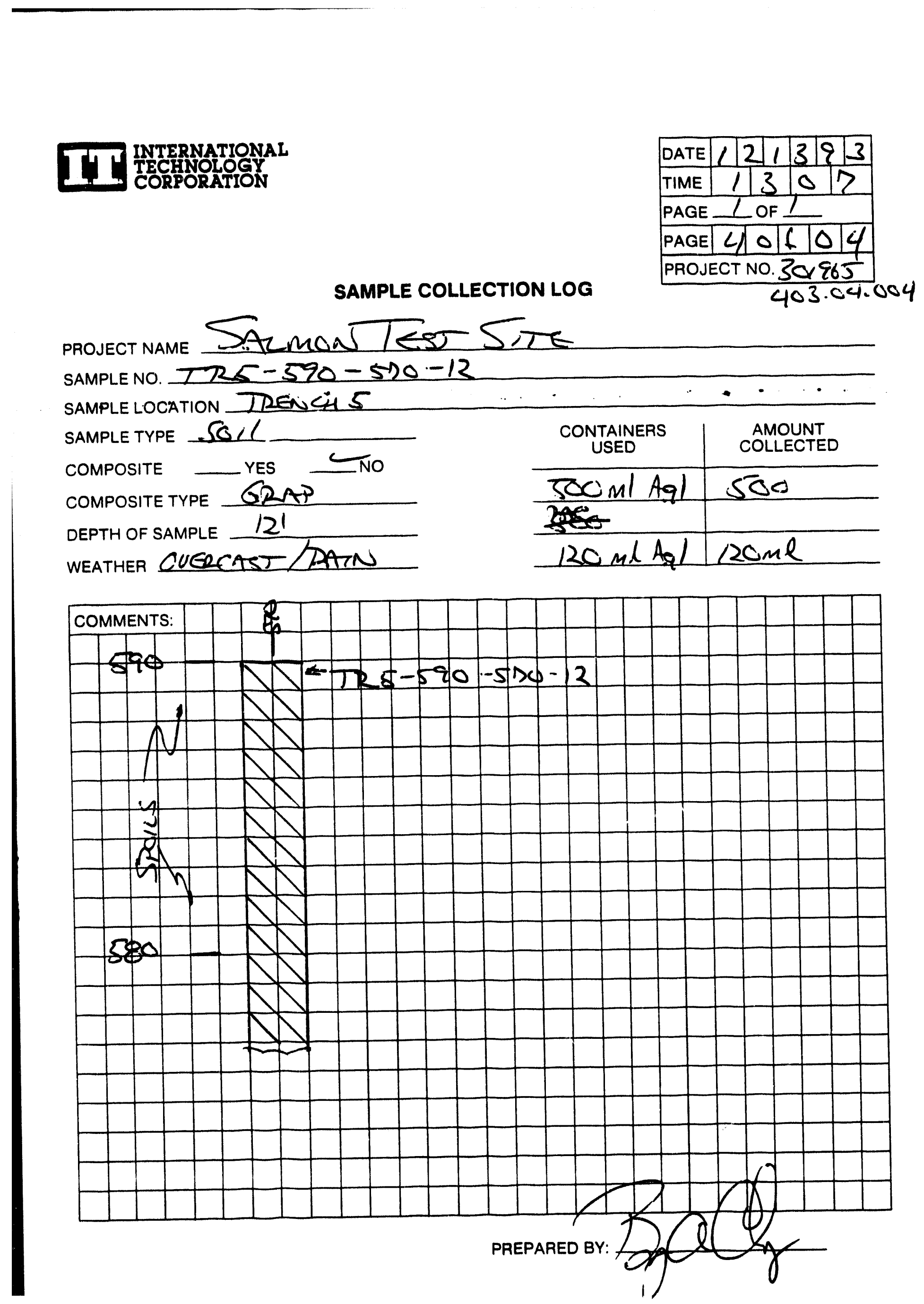




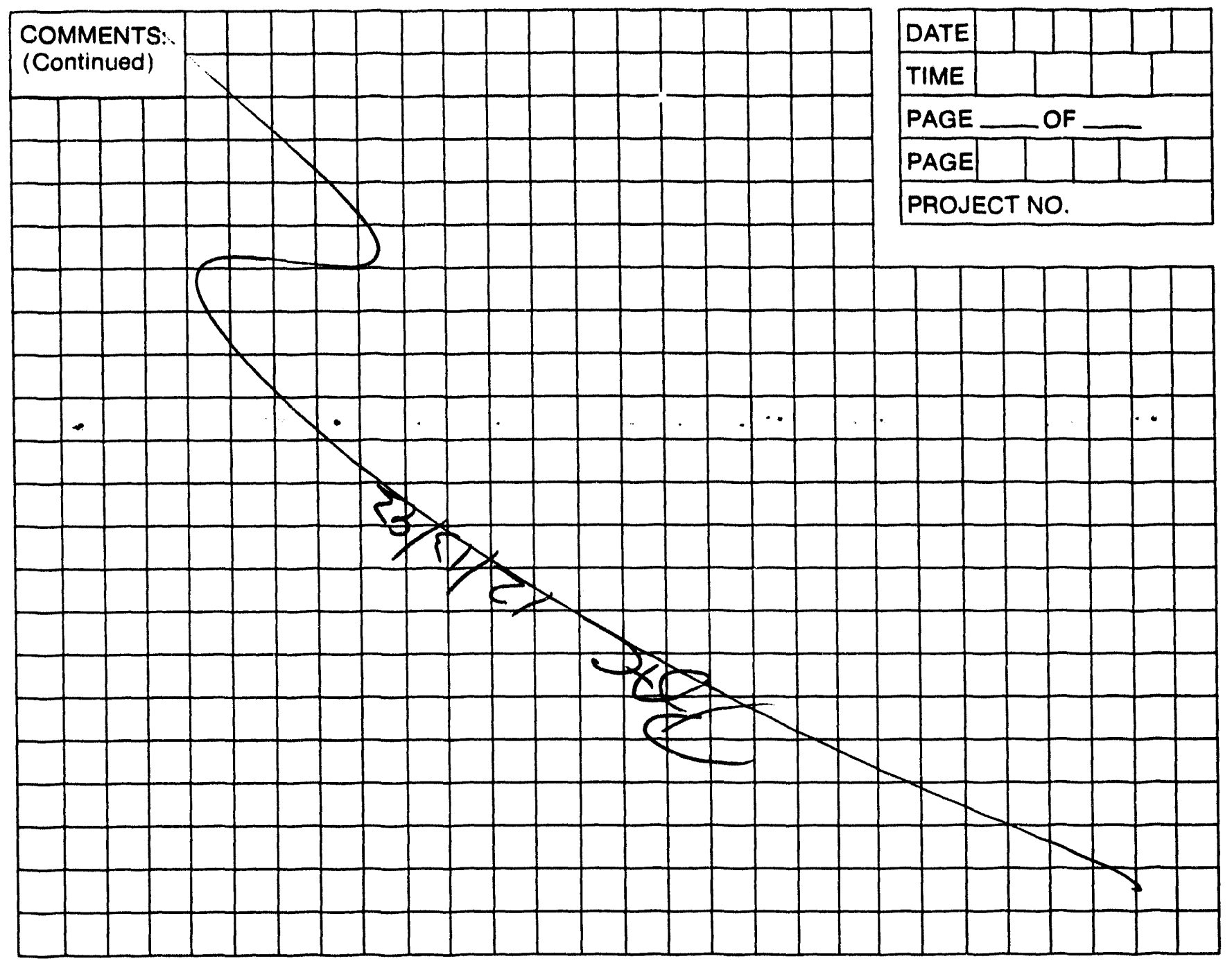

PREPARED BY:

\section{LEGEND}

1. A SAMPLE COLLECTION LOG IS TO BE COMPLETED FOR EACH SAMPLE.

2. ALWAYS COMPLETE BOTH SIDES. IF SECOND SIDE IS NOT USED. DRAW A LINE THROUGH IT AND MARK N/A. FILL IN CONTROL BLOCK AND PREPARED BY.

3. ALL ENTRIES ON LOG ARE TO BE COMPLETED. IF NOT APPLICABLE MARK N/A.

4. DATE: USE MONTH/DAY/YEAR: I.E., 10/30/85

5. TIME: USE 24-HOUR CLOCK: I.E., 1835 FOR 6:35 P.M.

6. PAGE: EACH SAMPLE TEAM SHOULO NUMBER PAGE ___ OF___ FOR THE DAY'S ACTIVITIES FOR ALL SHEETS PREPARED ON A SINGLE DAY. I.E.. IF THERE ARE A TOTAL OF 24 PAGES (INCLUDING FRONT AND BACK) NUMBER 1 OF 24, 2 OF 24. ETC

7. SAMPLE LOCATION: USE BORING OR MONITORING WELL NUMBER, GRID LOCATION (TRANSECT). SAMPLING STATION I.D., OR COORDINATE TO PHYSICAL FEATURES WITH DISTANCES. INCLUDE SKETCH IN COMMENT SECTION IF NECESSARY.

8. SAMPLE TYPE: USE THE FOLLOWING - SOIL: WATER (SURFACE OR GROUND): AIR (FILTERS. TUBES. AMBIENT. PERSONNEL): SLUDGE; DRUM CONTENTS: OIL: VEGETATION: WIPE: SEDIMENT.

9. COMPOSITE TYPE: I.E., 24-HOUR, LIST SAMPLE NUMBEAS IN COMPOSITE. SPATIAL COMPOSITE.

10. DEPTH OF SAMPLE: GIVE UNITS. WRITE OUT UNITS SUCH AS INCHES, FEET. DON'T USE ' OR ".

11. WEATHER: APPROXIMATE TEMPERATURE. SUN AND MOISTURE CONDITIONS.

12. CONTAINERS USED: LIST EACH CONTAINER TYPE AS NUMBER. VOLUME. MATERIAL (E.G., 2 - IL GLASS; 4 - 40 ML GLASS VIAL: 1 - 400 ML PLASTIC: 1 - 3 INCH STEEL TUBE; 1 - 8 OZ. GLASS JAP).

13. AMOUNT COLLECTED: VOLUME IN CONTAINERS (E.G. 1/2 FULL). 
SAMPLE COLLECTION LOG

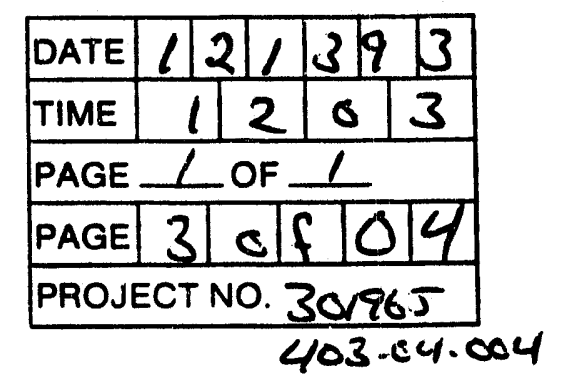

project name Salmon Test Site

SAMPLE NO. IRD-ES- $560-650-14$

sample LOCATION TRENCA 2 5A-2

SAMPLE TYPE Sod

COMPOSITE — YES

COMPOSITE TYPE $\frac{14-\text { GRAB }}{14^{\prime}}$

DEPTH OF SAMPLE 14

WEATHER OVERCAST

\begin{tabular}{c|c}
$\begin{array}{c}\text { CONTAINERS } \\
\text { USED }\end{array}$ & $\begin{array}{c}\text { AMOUNT } \\
\text { COLLECTED }\end{array}$ \\
\hline $1500 \mathrm{ml}$ A. gl & $500 \mathrm{ml}$ \\
\hline $120 \mathrm{ml}$ A gl & $120 \mathrm{ml}$ \\
\hline
\end{tabular}

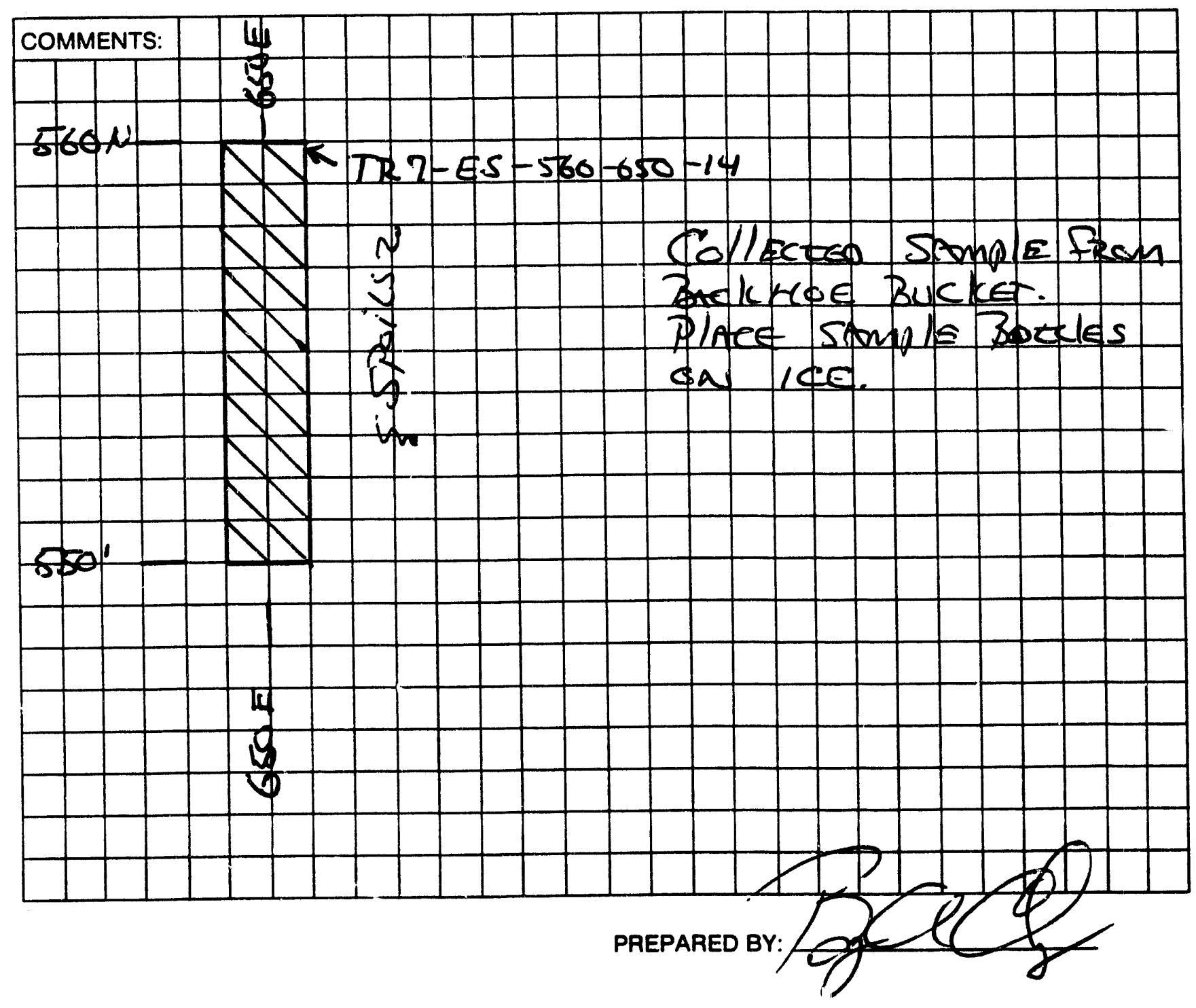




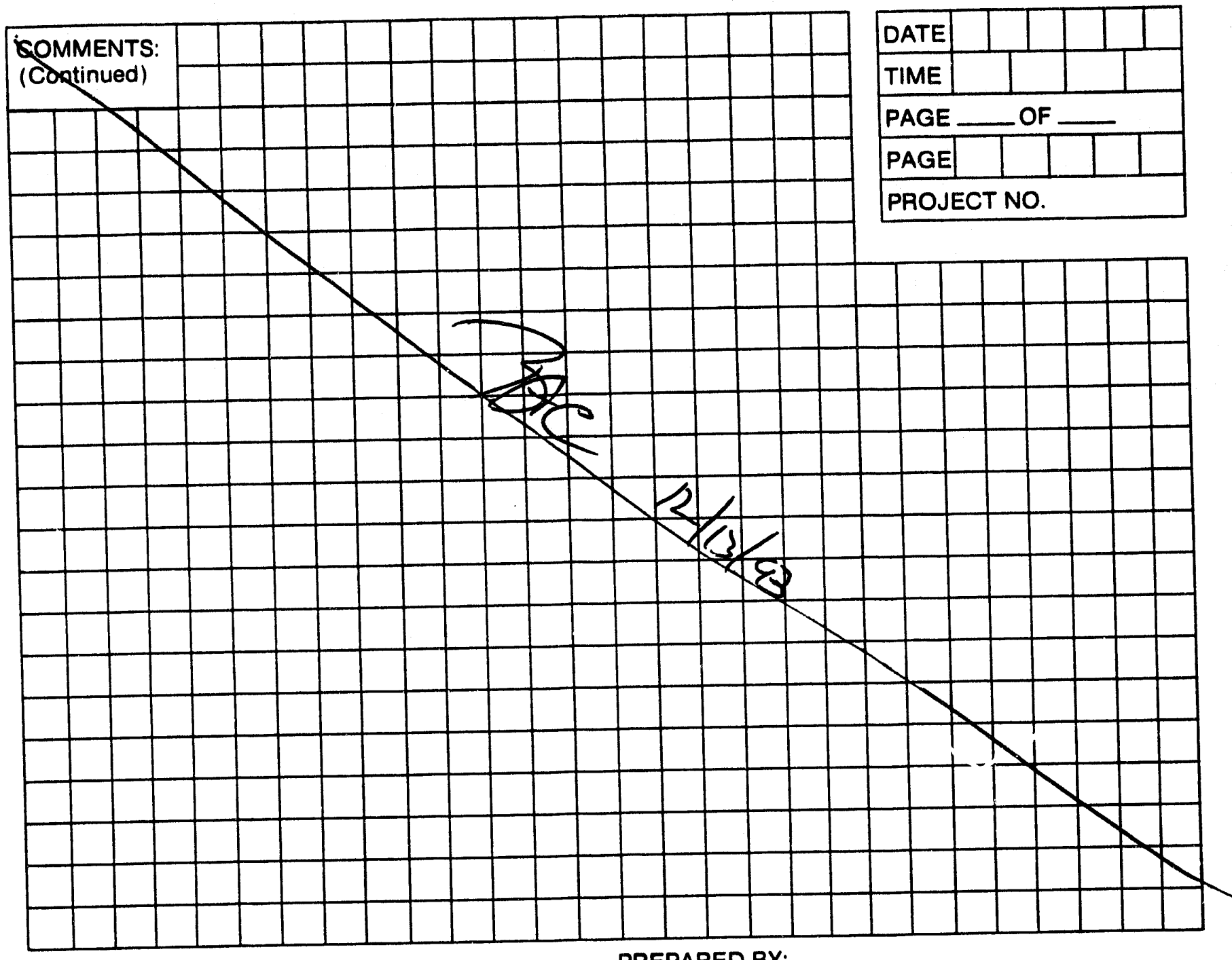

PREPARED BY:

LEGEND

1. A SAMPLE COLLECTION LOG IS TO BE COMPLETED FOR EACH SAMPLE.

2. ALWAYS COMPLETE BOTH SIDES. IF SECOND SIDE IS NOT USED. DRAW A LINE THROUGH IT AND MARK N/A. FILL IN CONTROL BLOCK AND PREPARED BY.

3. ALL ENTRIES ON LOG ARE TO BE COMPLETED. IF NOT APPLICABLE MARK N/A.

4 DATE: USE MONTH/DAY/YEAR: I.E., 10/30/85

5. TIME: USE 24-HOUR CLOCK: I.E., 1835 FOR 6:35 P.M.

6. PAGE: EACH SAMPLE TEAM SHOULD NUMBER PAGE — OF
SINGLE DAY. I.E.. IF THERE ARE A TOTAL OF 24 PAGES (INCLUDING FRONT AND BACK) NUMBER 1 OF 24. 2 OF 24. ETC.

SAMPLE LOCATION: USE BORING OR MONITORING WELL NUMBER. GRID LOCATION (TRANSECT). SAMPLING STATION I.D.. OR COORDINATE TO PHYSICAL FEATURES WITH DISTANCES. INCLUDE SKETCH IN COMMENT SECTION IF NECESSARY.

8. SAMPLE TYPE: USE THE FOLLOWING - SOIL: WATER (SURFACE OR GROUND): AIR (FILTERS. TUBES, AMBIENT. PERSONNEL): SLUDGE: DRUM CONTENTS: OIL: VEGETATION: WIPE: SEDIMENT

9. COMPOSITE TYPE: I.E., 24-HOUR. LIST SAMPLE NUMBERS IN COMPOSITE. SPATIAL COMPOSITE.

10. DEPTH OF SAMPLE: GIVE UNITS. WAITE OUT UNITS SUCH AS INCHES. FEET. DON'T USE ' OR ".

11. WEATHER: APPROXIMATE TEMPERATURE. SUN AND MOISTURE CONDITIONS.

12. CONTAINERS USED: LIST EACH CONTAINER TYPE AS NUMBER. VOLUME. MATERIAL (E.G., 2 - IL GLASS: 4 - 40 ML GLASS VIAL: 1 - 400 ML PLASTIC: 1 - 3 INCH STEEL TUBE: 1 - 8 OZ. GLASS JAR).

13. AMOUNT COLLECTED: VOLUME IN CONTAINERS (E.G. 1/2 FULLL). 
SAMPLE COLLECTION LOG

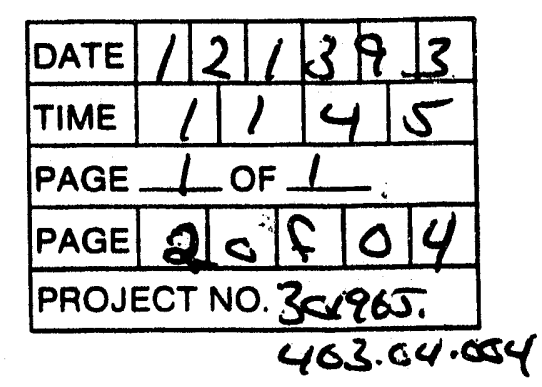

project name Salmon Test Site

SAMPLE NO. IR?- $E S-.558-650-5$

SAMPLE LOCATION TRENCH? - SAT

SAMPLE TYPE SE ,l

COMPOSITE Y YES

COMPOSITE TYPE GRAB

DEPTH OF SAMPLE 5 "

WeATHER QUERCAST

\begin{tabular}{c|c}
$\begin{array}{c}\text { CONTAINERS } \\
\text { USED }\end{array}$ & $\begin{array}{c}\text { AMOUNT } \\
\text { COLLECTED }\end{array}$ \\
\hline $150 \mathrm{~m} / \mathrm{m} / \mathrm{a} .91$ & $500 \mathrm{ml}$ \\
\hline $120 \mathrm{ml} / \mathrm{ag} /$ & $120 \mathrm{ml}$ \\
\hline
\end{tabular}

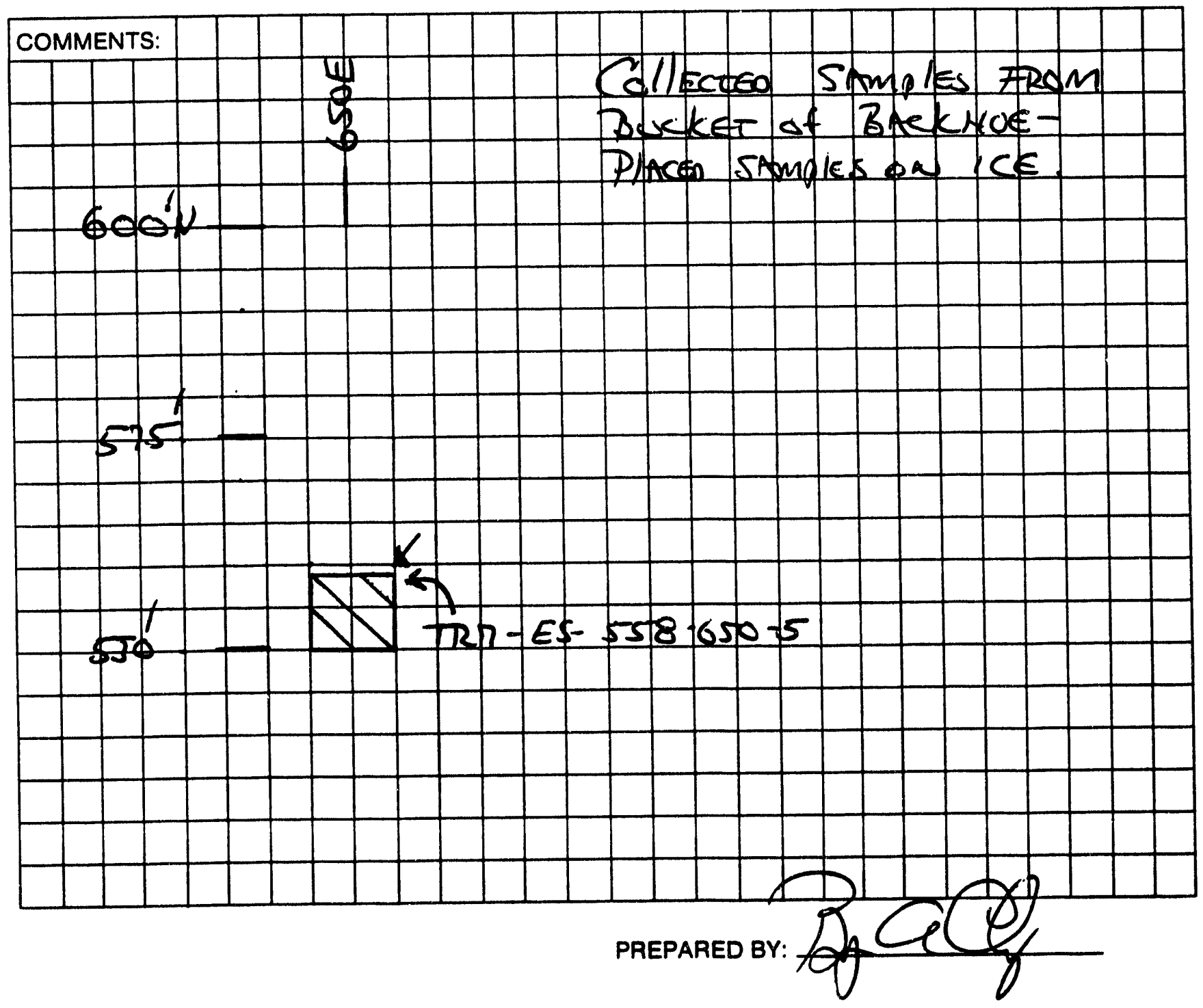




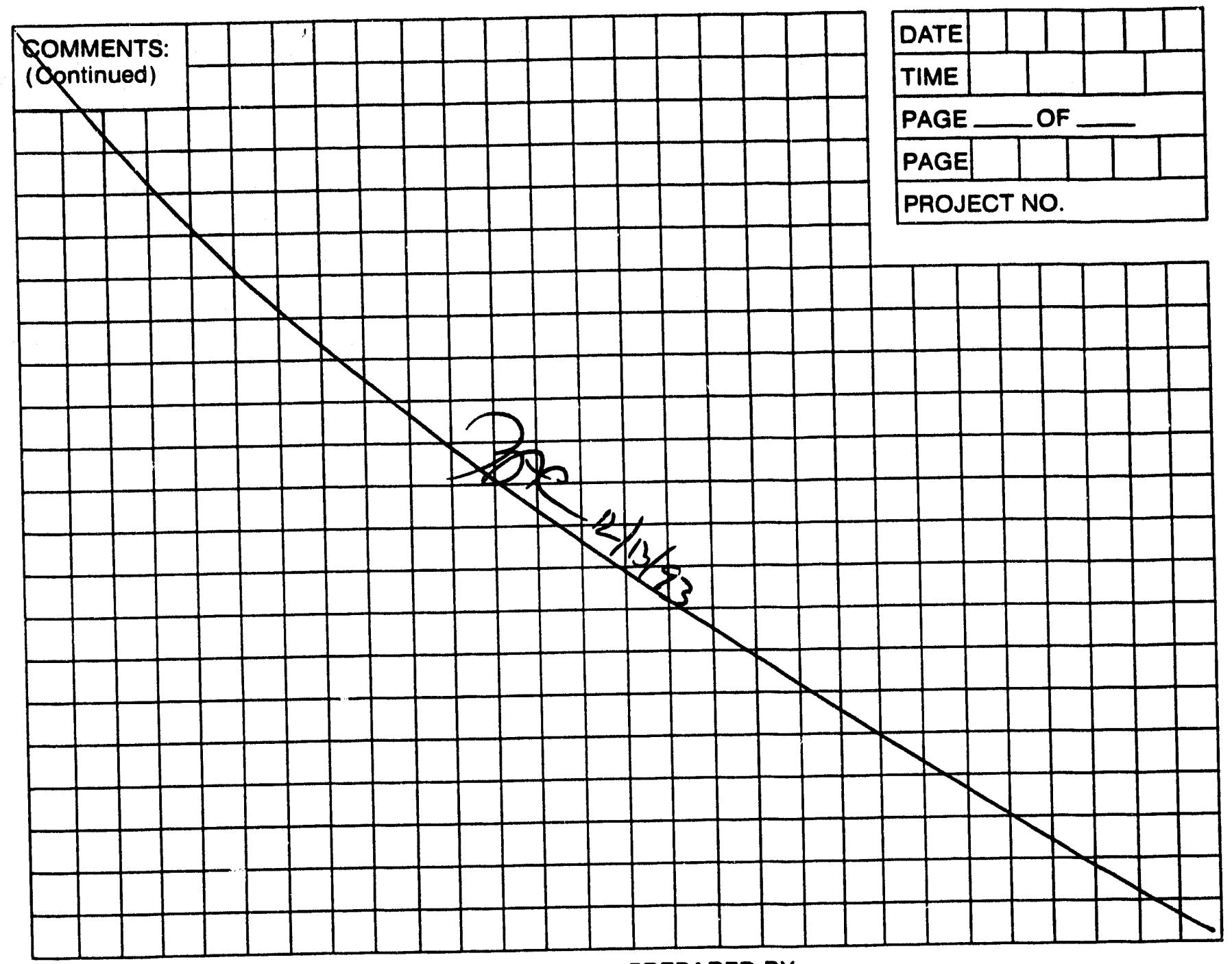

PREPARED BY:

LEGEND

1 A SAMPLE COLLECTION LOG IS TO BE COMPLETED FOR EACH SAMPLE.

2. ALWAYS COMPLETE BOTH SIDES. IF SECOND SIDE IS NOT USED. DRAW A LINE THROUGH IT AND MARK N/A. FILL IN CONTROL BLOCK AND PREPARED BY.

3. ALL ENTRIES ON LOG ARE TO BE COMPLETED. IF NOT APPLICABLE MARK N/A.

4 DATE: USE MONTH/DAY/YEAR: I.E., 10/30/85

5. TIME: USE 24-HOUR CLOCK: I.E., 1835 FOR 6:35 P.M.

PAGE: EACH SAMPLE TEAM SHOULD NUMBER PAGE __ OF__ FOR THE DAY'S ACTIVITIES FOR ALL SHEETS PREPARED ON A SINGLE DAY. I.E., IF THERE ARE A TOTAL OF 24 PAGES (INCLUDING FRONT AND BACK) NUMBER 1 OF 24. 2 OF 24. ETC.

7. SAMPLE LOCATION: USE BORING OR MONITORING WELL NUMBER. GRID LOCATION (TRANSECT), SAMPLING STATION I.D.. OR COORDINATE TO PHYSICAL FEATURES WITH DISTANCES. INCLUDE SKETCH IN COMMENT SECTION IF NECESSARY

8. SAMPLE TYPE: USE THE FOLLOWING - SOIL: WATER (SURFACE OR GROUND); AIR (FILTERS. TUBES. AMBIENT, PERSONNEL): SLUDGE: DRUM CONTENTS: OIL: VEGETATION: WIPE: SEDIMENT

9. COMPOSITE TYPE: I.E., 24-HOUR, LIST SAMPLE NUMBERS IN COMPOSITE, SPATIAL COMPOSITE.

10. DEPTH OF SAMPLE: GIVE UNITS. WRITE OUT UNITS SUCH AS INCHES. FEET. DON'T USE ' OR "

11. WEATHER: APPROXIMATE TEMPERATURE. SUN AND MOISTURE CONDITIONS.

12. CONTAINERS USED: LIST EACH CONTAINER TYPE AS NUMBER. VOLUME. MATERIAL (E.G., 2 - IL GLASS; 4 - 40 ML GLASS VIAL: 1 - 400 ML PLASTIC: 1 - 3 INCH STEEL TUBE: 1 - 8 OZ. GLASS JAR)

13. AMOUNT COLLECTED: VOLUME IN CONTAINERS (E.G. 1/2 FULL). 
576006

SAMPLE COLLECTION LOG

\begin{tabular}{|l|l|l|l|l|l|l|}
\hline DATE & 1 & 2 & 1 & 3 & 9 & 3 \\
\hline TIME & 1 & 0 & 1 & 0 \\
\hline PAGE & 1 & OF & 1 \\
\hline PAGE & 1 & 0 & $\mathrm{f}$ & 0 & $\mathbf{4}$ \\
\hline PROJECT NO. & $0.965 .4 \mathrm{~s} 3$ \\
\hline
\end{tabular}

Project name SAlmoN test, Site

SAMPLE NO. IRE - ES - $280-600-3$

SAMPLE LOCATION DRENCH \&

SAMPLE TYPE SOIl

COMPOSITE — YES -NO

gonpostat TYPE GTL GRAB

DEPTH OF SAMPLE $3^{\prime}$

WEATHER OVERCAST

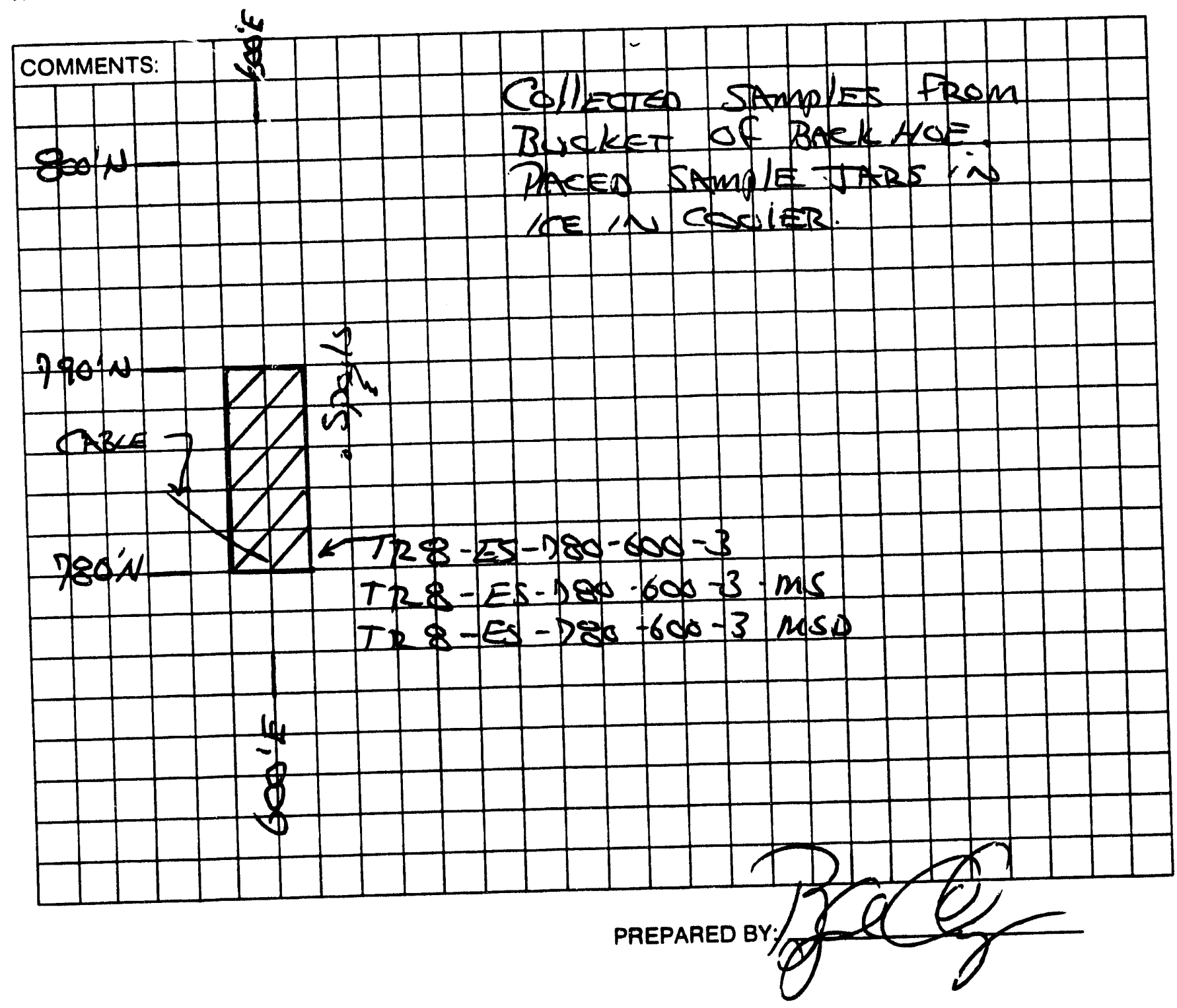




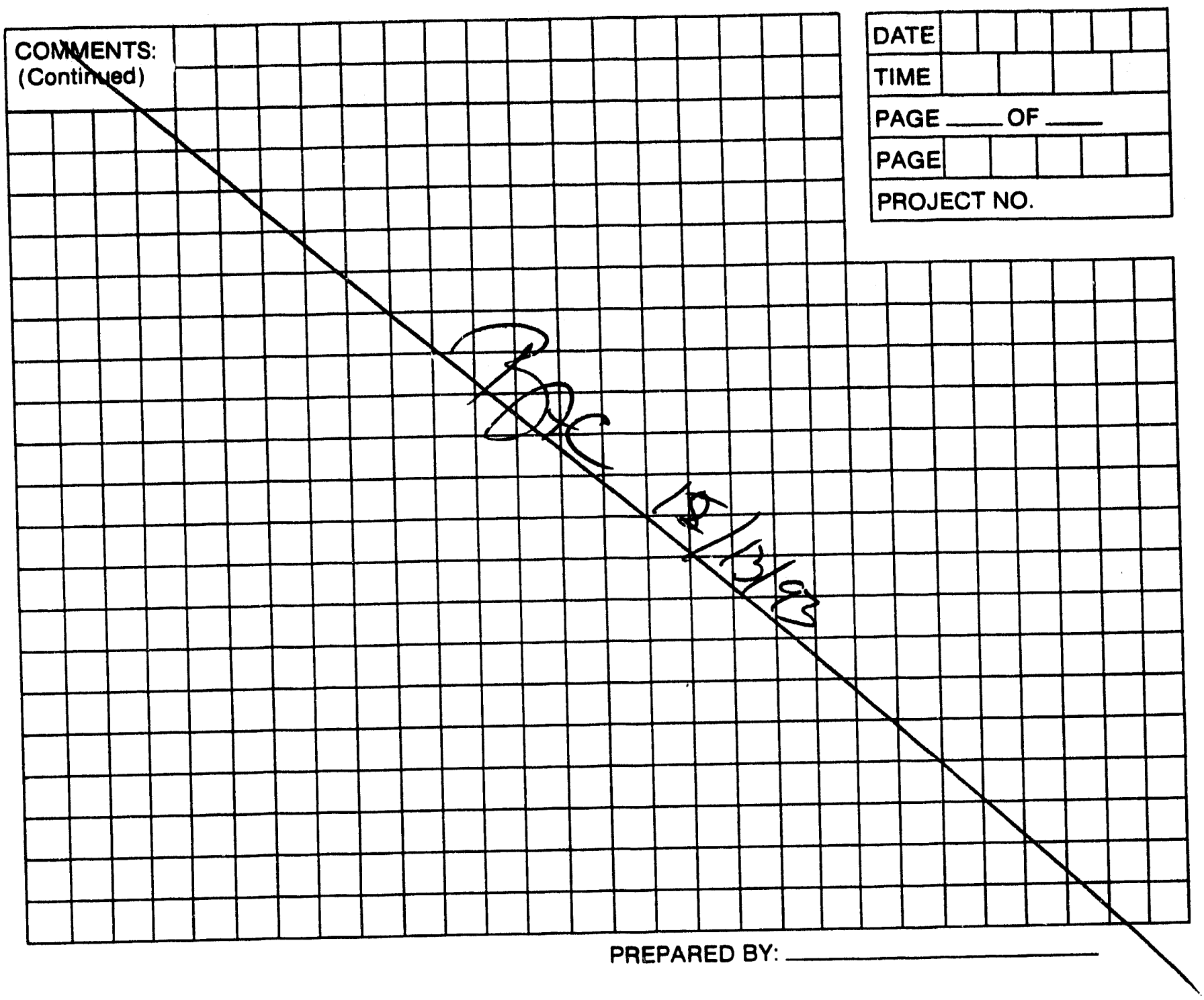

LEGEND

I A SAMPLE COLLECTION LOG IS TO BE COMPLETED FOR EACH SAMPLE.

2. ALWAYS COMPLETE BOTH SIDES. IF SECOND SIDE IS NOT USED. DRAW A LINE THROUGH IT AND MARK N/A. FILL IN CONTROL BLOCK AND PREPARED BY

3. ALL ENTRIES ON LOG ARE TO BE COMPLETED. IF NOT APPLICABLE MARK N/A

4. DATE: USE MONTH/DAY/YEAR: I.E., 10/30/85

5. TIME: USE 24-HOUR CLOCK: 1.E., 1835 FOR 6:35 P.M.

6. PAGE: EACH SAMPLE TEAM SHOULD NUMBER PAGE OF _ FOR THE DAY'S ACTIVITIES FOR ALL SHEETS PREPARED ON A THERE ARE A TOTAL OF 24 PAGES (INCLUDING FRONT AND BACK) NUMBER 1 OF 24. 2 OF 24. ETC

T. SAMPLE LOCATION: USE BORING OR MONITORING WELL NUMBER. GRID LOCATION (TRANSECT), SAMPLING STATION I.D.. OR PHYSICAL FEATURES WITH DISTANCES. INCLUDE SKETCH IN COMMENT SECTION IF NECESSARY.

SAMPLE TYPE: USE THE FOLLOWING - SOIL: WATER (SURFACE OR GROUND): AIR (FILTERS. TUBES, AMBIENT, PERSONNEL): SLUDGE: DRUM CONTENTS: OIL: VEGETATION: WIPE: SEDIMENT

9. COMPOSITE TYPE: I.E., 24-HOUP. LIST SAMPLE NUMBERS IN COMPOSITE. SPATIAL COMPOSITE.

10. DEPTH OF SAMPLE: GIVE UNITS. WRITE OUT UNITS SUCH AS INCHES, FEET. DON'T USE ' OR "

11. WEATHER: APPROXIMATE TEMPERATURE. SUN AND MOISTURE CONDITIONS

12. CONTAINERS USED: LIST EACH CONTAINER TYPE AS NUMBER, VOLUME. MATERIAL (E.G., 2 - IL GLASS: 4 - 40 ML GLASS VIAL: 1 - 400 ML PLASTIC: 1 - 3 INCH STEEL TUBE; 1 - 8 OZ. GLASS JAR).

13. AMOUNT COLLECTED: VOLUME IN CONTAINERS (E.G. 1/2 FULL). 
SD 007

FEDEX 0723032923

$12 / 17 / 93$

TH T INTR NATIONAL TECHNOLOGY

SAMPLE COLLECTION LOG

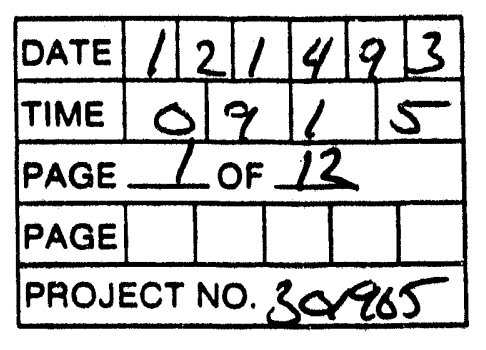

project name Salmon Test Site SAMPLE NO. IR $6-E S-495-585-12$

sample location Re geo Pits -

SAMPLE TYPE GRAB FrOM BACkHoE COMPOSITE SOII Yes $\chi_{\text {NO }}$

COMPOSITE TYPE $\overline{12 \text { Fear }}$ WEATHER QUERCAST

\begin{tabular}{c|c}
$\begin{array}{c}\text { CONTAINERS } \\
\text { USED }\end{array}$ & $\begin{array}{c}\text { AMOUNT } \\
\text { COLLECTED }\end{array}$ \\
\hline$A G, 120 \mathrm{ml}$ & $120 \mathrm{ml}$ \\
\hline AG $500 \mathrm{ml}$ & $500 \mathrm{~m}$ \\
\hline & \\
\hline
\end{tabular}

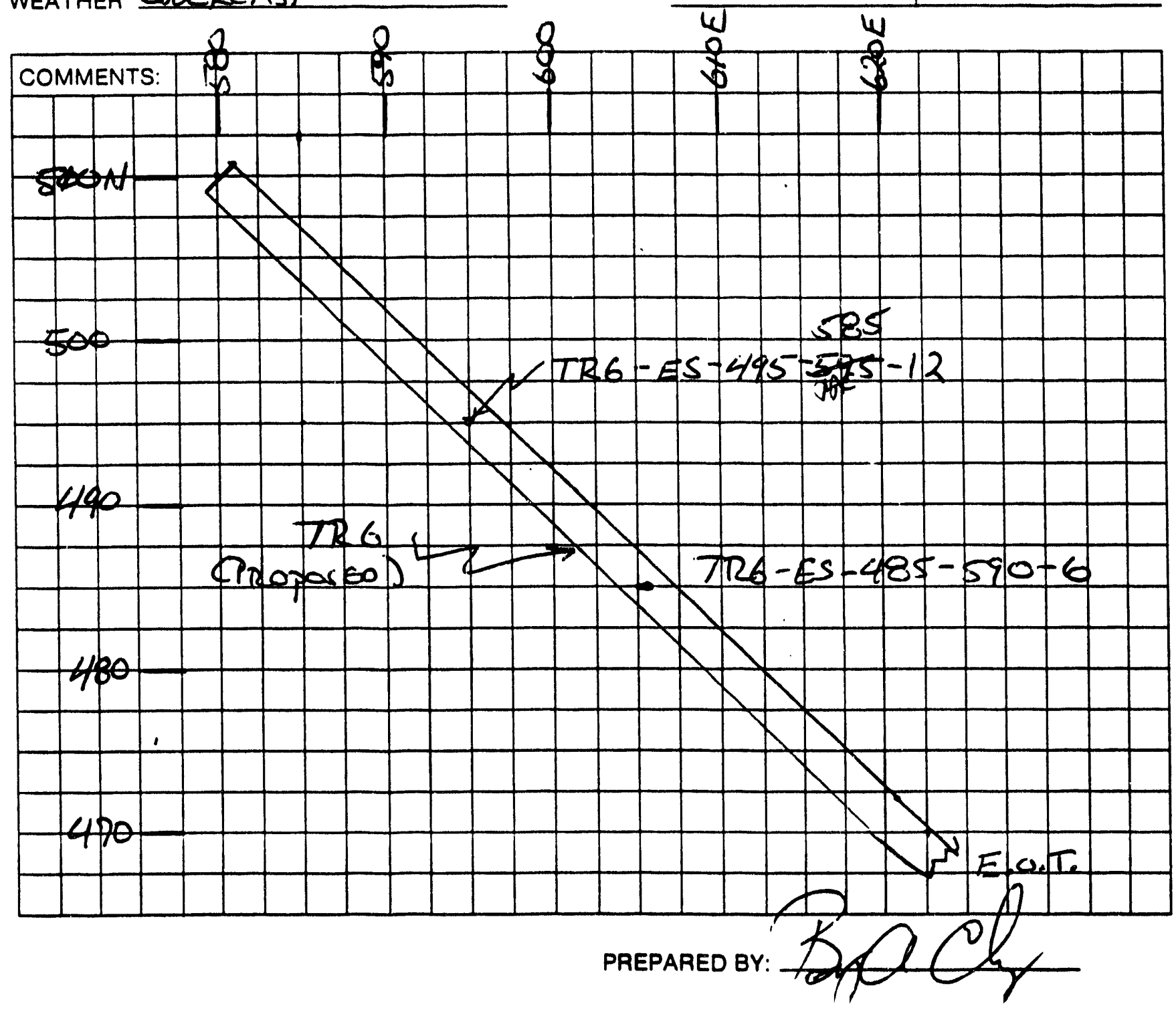




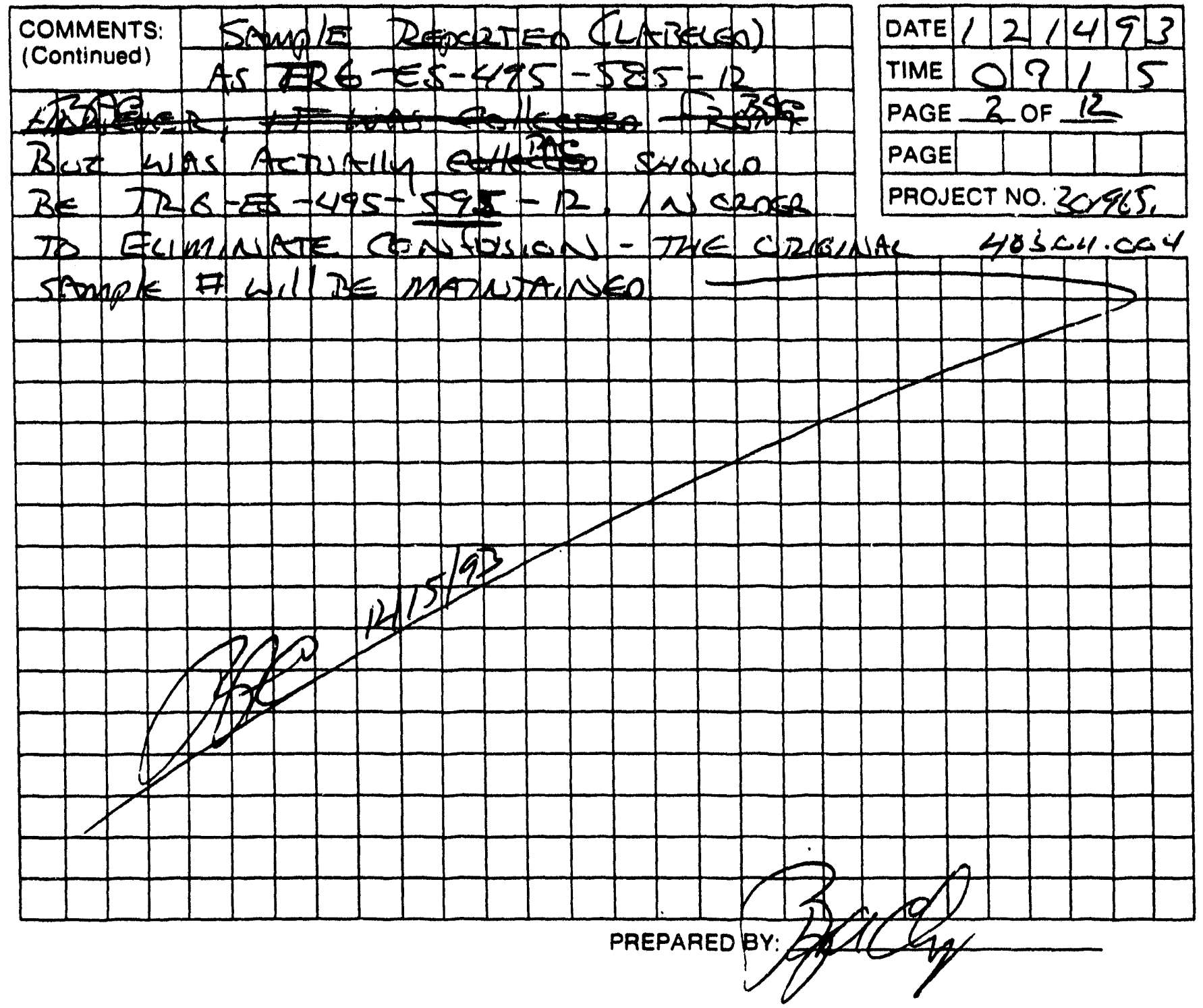

LEGEND

1 A SAMPLE COLLECTION LOG IS TO BE COMPLETED FOR EACH SAMPLE.

2. ALWAYS COMPLETE BOTH SIDES. If SECOND SIDE IS NOT USED. DRAW A LINE THROUGH IT AND MARK N/A. FILL IN CONTROL BLOCK AND PREPARED BY.

3. ALL ENTRIES ON LOG ARE TO BE COMPLETED. IF NOT APPLICABLE MARK N/A.

4. DATE: USE MONTH/OAY/YEAR: I.E., 10/30/85

5. TIME: USE 24-HOUA CLOCK: I.E., 1835 FOR 6:35 P.M.

6 PAGE: EACH SAMPLE TEAM SHOULD NUMBER PAGE __ OF FOR THE DAY'S ACTIVITIES FOR ALL SHEETS PREPARED ON A SINGLE DAY. I.E., IF THERE ARE A TOTAL OF 24 PAGES (INCLUDING FRONT AND BACKI NUMBER IOF 24. 2 OF 24. ETC

7 SAMPLE LOCATION: USE BORING OR MONITORING WELL NUMBER. GRID LOCATION (TRANSECT), SAMPLING STATION ID. OR COORDINATE TO PHYSICAL. FEATURES WITH DISTANCES. INCLUDE SKETCH IN COMMENT SECTION IF NECESSAAY

8. SAMPLE TYPE. USE THE FOLLOWING - SOIL: WATER (SURFACE OR GROUND): AIR (FILTERS. TUBES. AMBIENT. PERSONNEL): SLUDGE: DRUM CONTENTS: OIL: VEGETATION: WIPE: SEDIMENT

9. COMPOSITE TYPE: I.E., 24-HOUR, LIST SAMPLE NUMBERS IN COMPOSITE, SPATIAL COMPOSITE.

10. DEPTH OF SAMPLE. GIVE UNITS. WAITE OUT UNITS SUCH AS INCHES. FEET DON'T USE ' OR "

11 WEATHER APPROXIMATE TEMPERATURE. SUN AND MOISTURE CONDITIONS.

12. CONTAINERS USED: LIST EACH CONTAINER TYPE AS NUMBER, VOLUME, MATERIAL (E. O. 2 - $1 \mathrm{~L}$ GLASS, 4 - 40 ML GLASS VIAL: 1 . 400 ML PLASTIC. 1 - 3 INCH STEEL TUBE: 1 - 8 OZ. GLASS JAR)

13. AMOUNT COLLECTED: VOLUME IN CONTAINERS (E.G. 1/2 FULLL) 
project name SALmoal TEST Site

SAMPLENO. IR6-ES-485-590-6

SAMPLE LOCATION REECO.

SAMPLE TYPE SOII

COMPOSITE _YES LNO

COMPOSITE TYPE

DEPTH OF SAMPLE 6

WEATHER OUECAST.

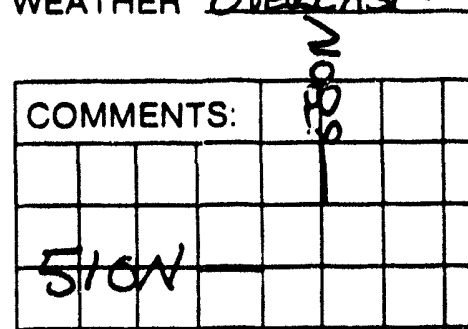

\begin{tabular}{c|c}
$\begin{array}{c}\text { CONTAINERS } \\
\text { USED }\end{array}$ & $\begin{array}{c}\text { AMOUNT } \\
\text { COLLECTED }\end{array}$ \\
\hline $120 \mathrm{~m} A 6$ & $120 \mathrm{ml}$ \\
\hline $500 \mathrm{~m} A 6$ & $500 \mathrm{ml}$ \\
\hline
\end{tabular}

के

500

490

TRENCH6

2

TR6-Es-485-590-6

\begin{tabular}{l} 
\\
\hline 400
\end{tabular}

NotE - Suple WAS

Collecreo or AT $485 \%$, 605 5 Bure 


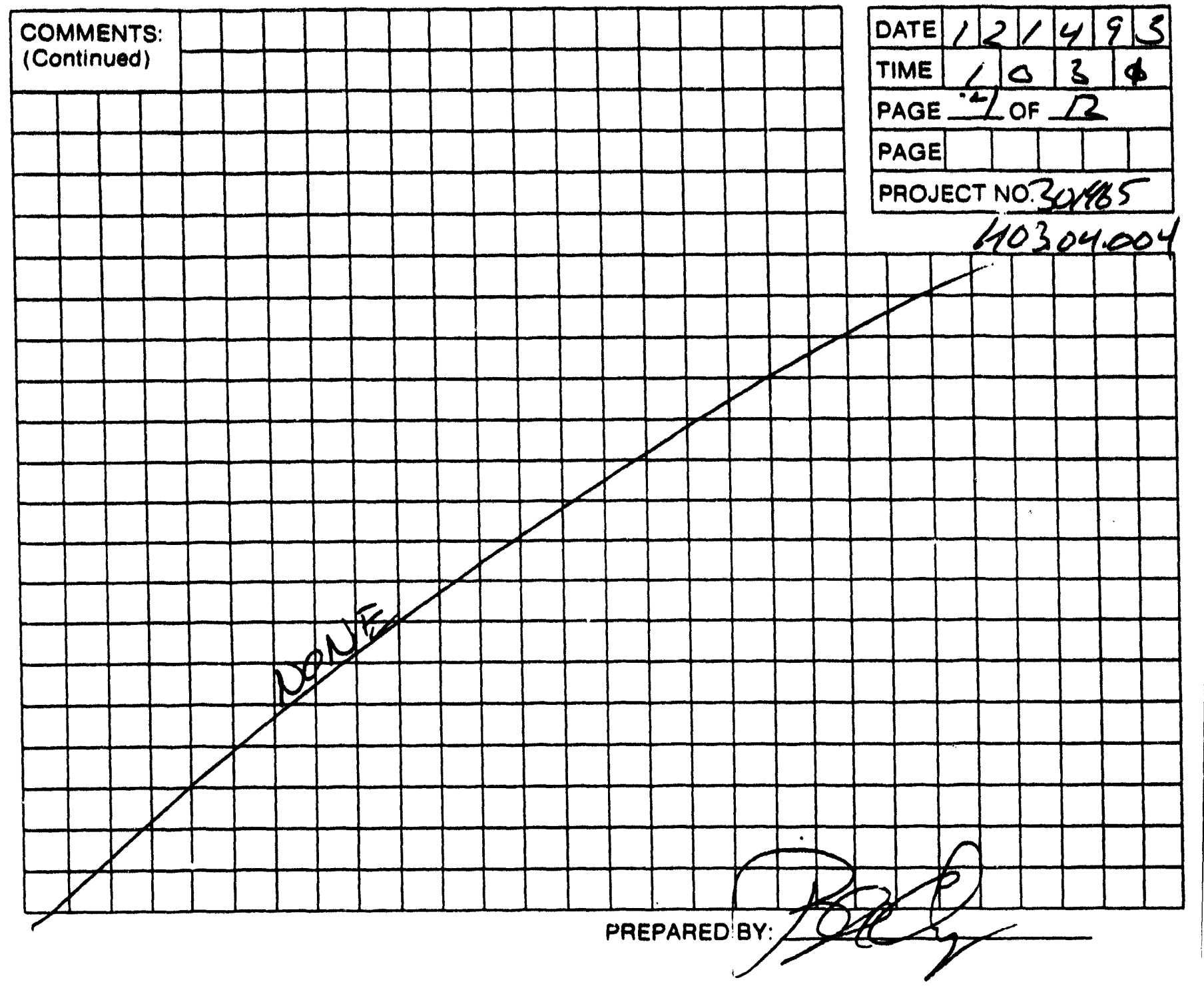

LEGEND

1 a SAMPLE COLLECTION LOG IS TO Be COMPLETED FoR EACH SAMPLE.

2. ALWAYS COMPLETE BOTH SIDES. IF SECOND SIDE IS NOT USED. DRAW A LINE THROUGH IT AND MARK N/A. FILL IN CONTROL BLOCK AND PREPARED BY.

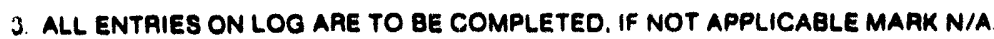

4 DATE: USE MONTH/DAYTYEAR: I.E., 10/30/05

5. TIME: USE 24.HOUR CLOCK: I.E., 1835 FOR 6:35 PM.

6. PAGE: EACH SAMPLE TEAM SHOULD NUMBER PAGE ___ OF___ FOR THE DAY'S ACTIVITIES FOR ALL SHEETS PREPARED ON A SINGLE DAY. I.E.. IF THERE ARE A TOTAL OF 24 PAGES (INCLUDING FRONT AND BACK) NUMBER 1 OF 24. 2 OF 24. ETC.

7 SAMPLE LOCATION. USE BORING OR MONITORING WELL NUMBER. GRID LOCATION ITRANSECTI. SAMPLING STATION IO.. OR COORDINATE TO PHYSICAL FEATURES WITH DISTANCES. INCLUDE SKETCH IN COMMENT SECTION IF NECESSARY

8 SAMPLE TYPE: USE THE FOLLOWING - SOIL: WATEA (SUAFACE OR GROUND): AIR (FILTERS. TUBES. AMBIENT. PERSONNEL): SLUDGE: DAUM CONTENTS: OIL: VEGETATION: WIPE: SEDIMENT

9. COMPOSITE TYPE: I.E., 24-HOUA, LIST SAMPLE NUMBERS IN COMPOSITE. SPATIAL COMPOSITE.

10. DEPTH OF SAMPLE: GIVE UNITS. WRITE OUT UNITS SUCH AS INCHES. FEET DON'T USE ' OR ".

11 WEATHER APPROXIMATE TEMPERATURE, SUN ANO MOISTURE CONDITIONS

12. CONTAINEAS USEO: LIST EACH CONTAINER TYPE AS NUMBER, VOLUME. MATERIAL IE G. 2 - IL GLASS: 4 - 40 ML GLASS VIAL; 1 - 400 ML PLASTIC: 1 - 3 INCH STEEL TUBE: 1 - 8 OZ. GLASS JAA).

13. AMOUNT COLLECTED: VOLUME IN CONTAINEAS (E.O. 1/2 FULL). 
$\sin 000$

SAMPLE COLLECTION LOG

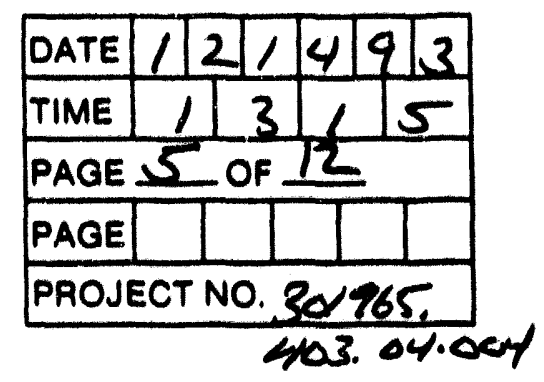

project name Salmon Test Site SAMPLE NO. IR B-ES - 210- $1110-6$.

SAMPLE LOCATION

SAMPLE TYPE SOT)

COMPOSITE

YES C NO

COMPOSITE TYPE

DEPTH OF SAMPLE 6

Weather Queacass, $\mathrm{COO} /$

\begin{tabular}{c|c}
$\begin{array}{c}\text { CONTAINERS } \\
\text { USED }\end{array}$ & $\begin{array}{c}\text { AMOUNT } \\
\text { COLLECTED }\end{array}$ \\
\hline $120 \mathrm{ml} A 6$ & $120 \mathrm{ml}$ \\
\hline $500 \mathrm{ml} A 6$ & $120 \mathrm{ml}$ \\
\hline & \\
\hline
\end{tabular}

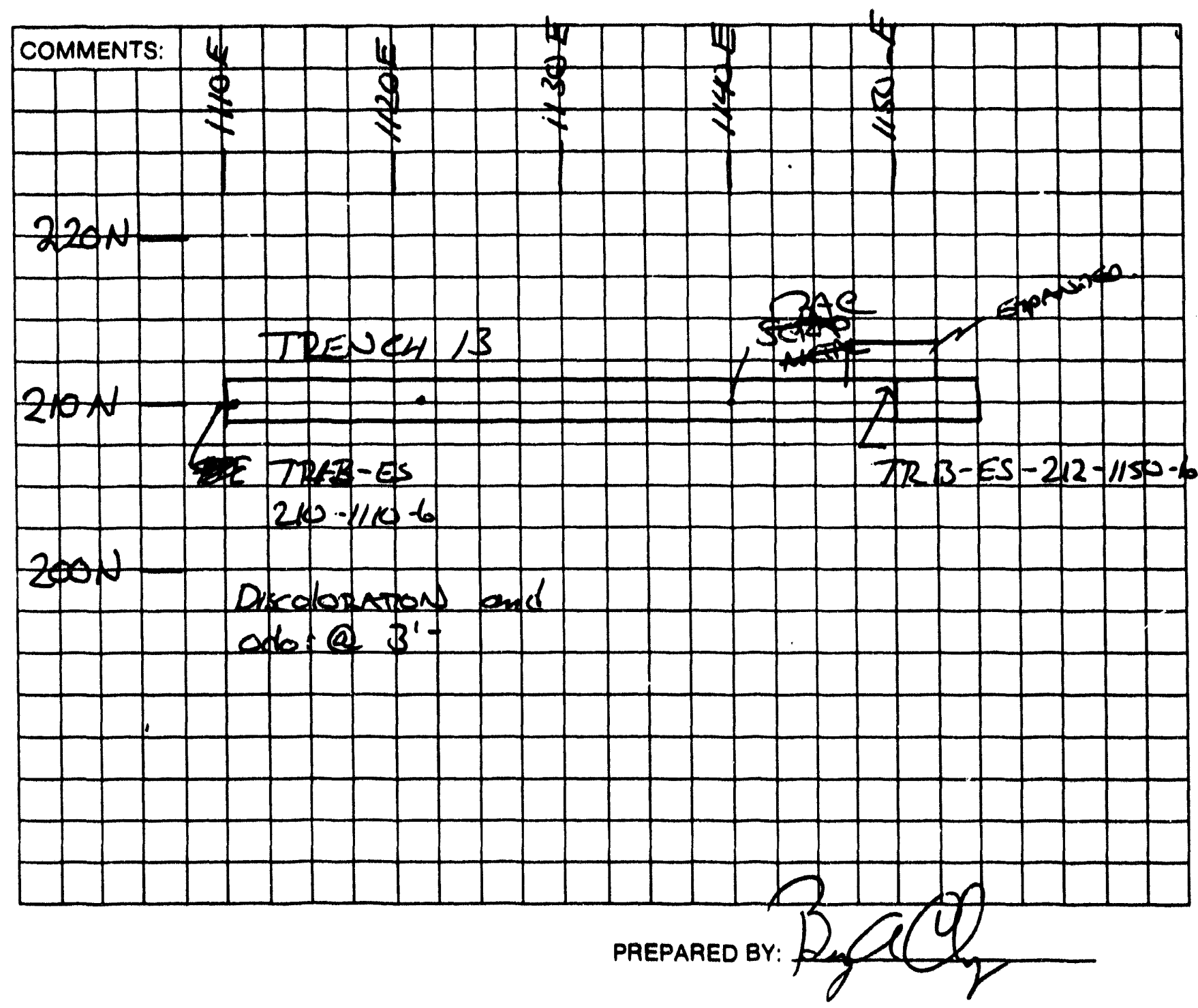




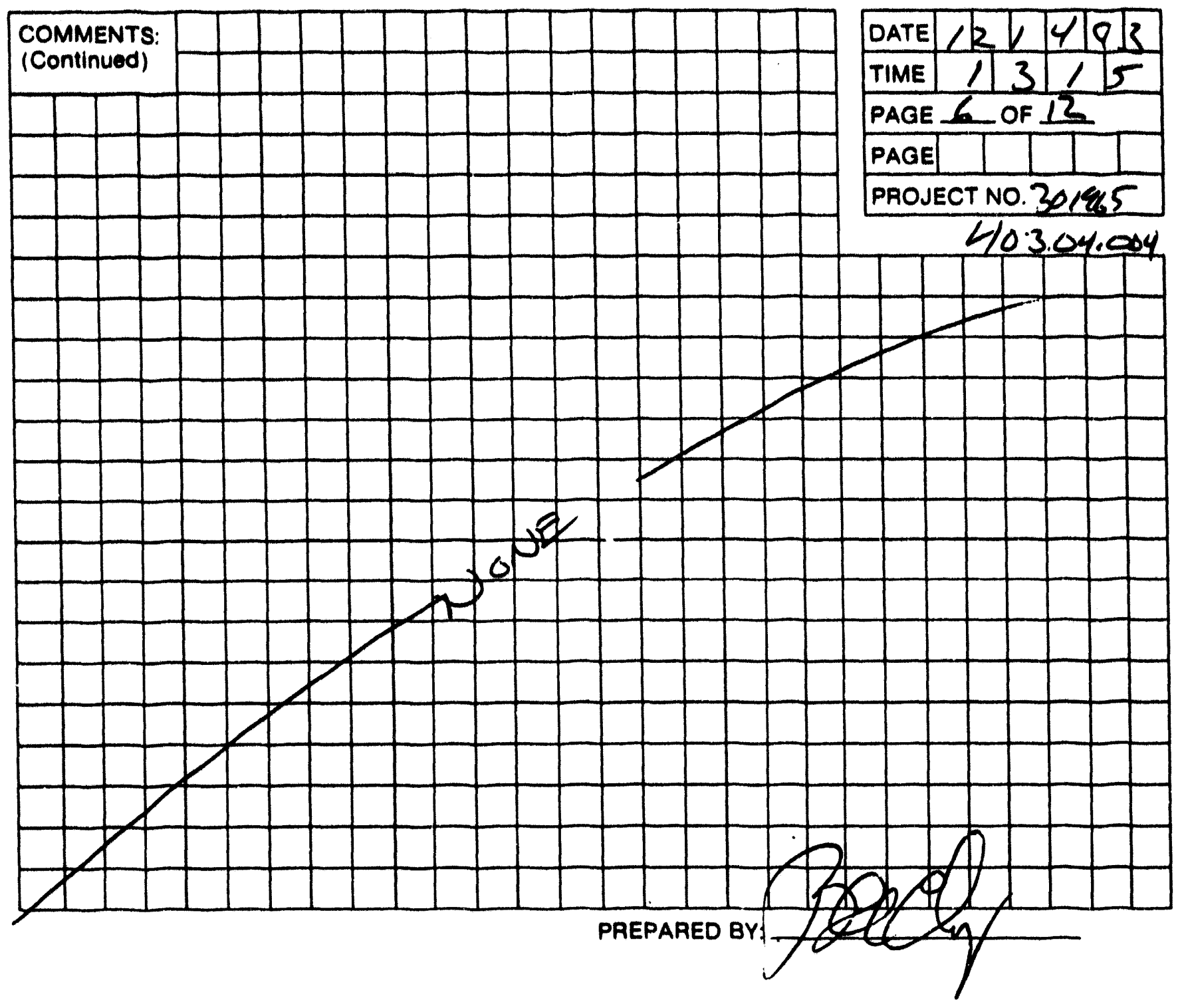

\section{LEQEND}

1 A SAMPLE COLLECTION LOQ IS TO BE COMPLETED FOA EACH SAMPLE.

2. ALWAYS COMPLETE BOTH SIDES. IF SECONO SIDE IS NOT USED. DAAW A LINE THROUGH IT AND MAPK N/A. FILL IN CONTROL BLOCK AND PAEPARED BY.

3. ALL ENTAIES ON LOQ AAE TO BE COMPLETED, IF NOT APPLICABLE MAAK N/A.

4 DATE USE MONTH/DAYTYEAR: I.E. 10/30/85

3. TIME: USE 24-HOUA CLOCK: I.E., 1835 FOR 6.35 PM

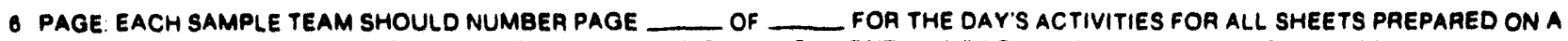
SINGLE DAY IE. IF THERE ARE A TOTAL OF 24 PAGES (INCLUDING FAONT AND BACKI NUMBER 1 OF 24. 2 OF 24. ETC.

7 SAMPLE LOCATION USE BORING OR MONITOAING WELL NUMBER. GRIO LOCATION ITRANSECTI. SAMPLING STATION I.D. OR COORDINATE TO PHYSICAL FEATUAES WITH DISTANCES INCLUDE SKETCH IN COMMENT SECTION IF NECESSARY

- SAMPLE TYPE: USE THE FOLLOWINO - SOIL. WATER (SURFACE OR GROUND). AIR (FILTERS. TUBES. AMBIENT. PERSONNEL): SLUDGE: DRUM CONTENTS OIL. VEGETATION. WIPE SEDIMENT

9. COMPOSITE TYPE: I.E., 24-HOUA, LIST SAMPLE NUMEEAS IN COMPOSITE. SPATIAL COMPOSITE

10 DEPTH OF SAMPLE GIVE UNITS. WRITE OUT UNITS SUCH AS INCHES. FEET DON'T USE ' OR "

11 Weathea appaoximate temperature. sun and moisture conditions

12. CONTAINEAS USEO. LIST EACH CONTAINEA TYPE AS NUMEER. VOLUME. MATERIAL IE G . 2 - IL GLASS: 4 - 40 ML GLASS VIAL: 1 - 400 ML PLASTIC: 1 - 3 INCH STEEL TUEE: 1 - 8 OZ. QLASS JAR)

13. AMOUNT COLLECTED: VOLUME IN CONTAINEAS (E.G. 1/2 FULL). 


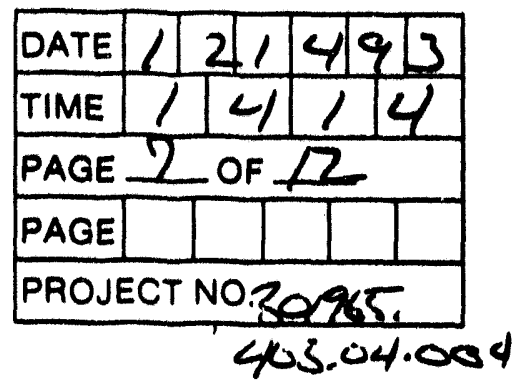

PROJECT NAME SAzmoal

SAMPLE NO. IR/3-ZES-212-1150-6

sample location DzZEC PIts - 5A-2

SAMPLE TYPE Soul

COMPOSITE —YES —NO

COMPOSITE TYPE

DEPTH OF SAMPLE la

WeATHER Ovecant - cool.

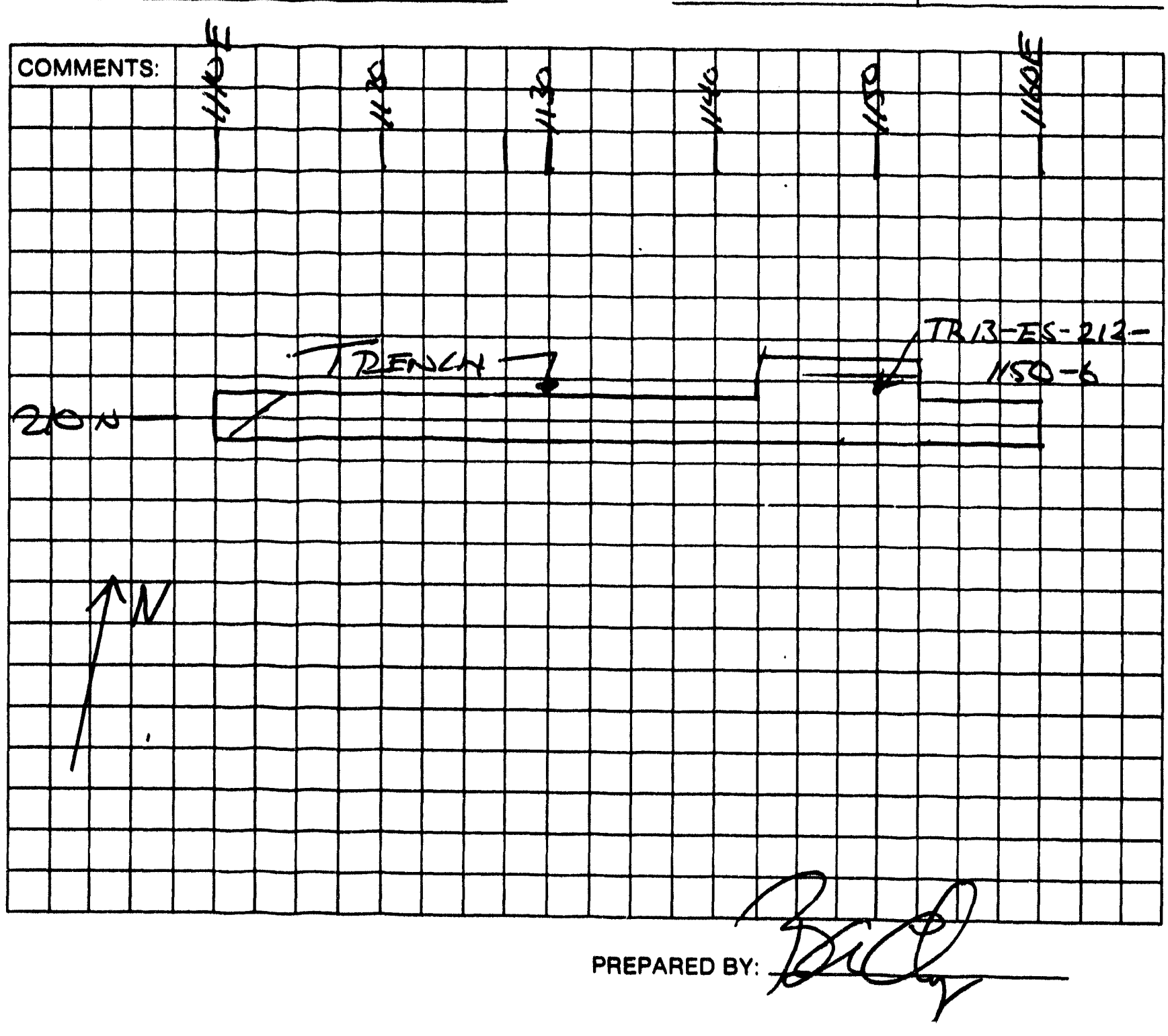

\begin{tabular}{c|c}
$\substack{\text { CONTAINERS } \\
\text { USED }}$ & $\begin{array}{c}\text { AMOUNT } \\
\text { COLLECTED }\end{array}$
\end{tabular}

$120 \mathrm{md} \mathrm{AS} 120 \mathrm{ml}$ $500 \mathrm{ml}$ A6 $500 \mathrm{ml}$ 


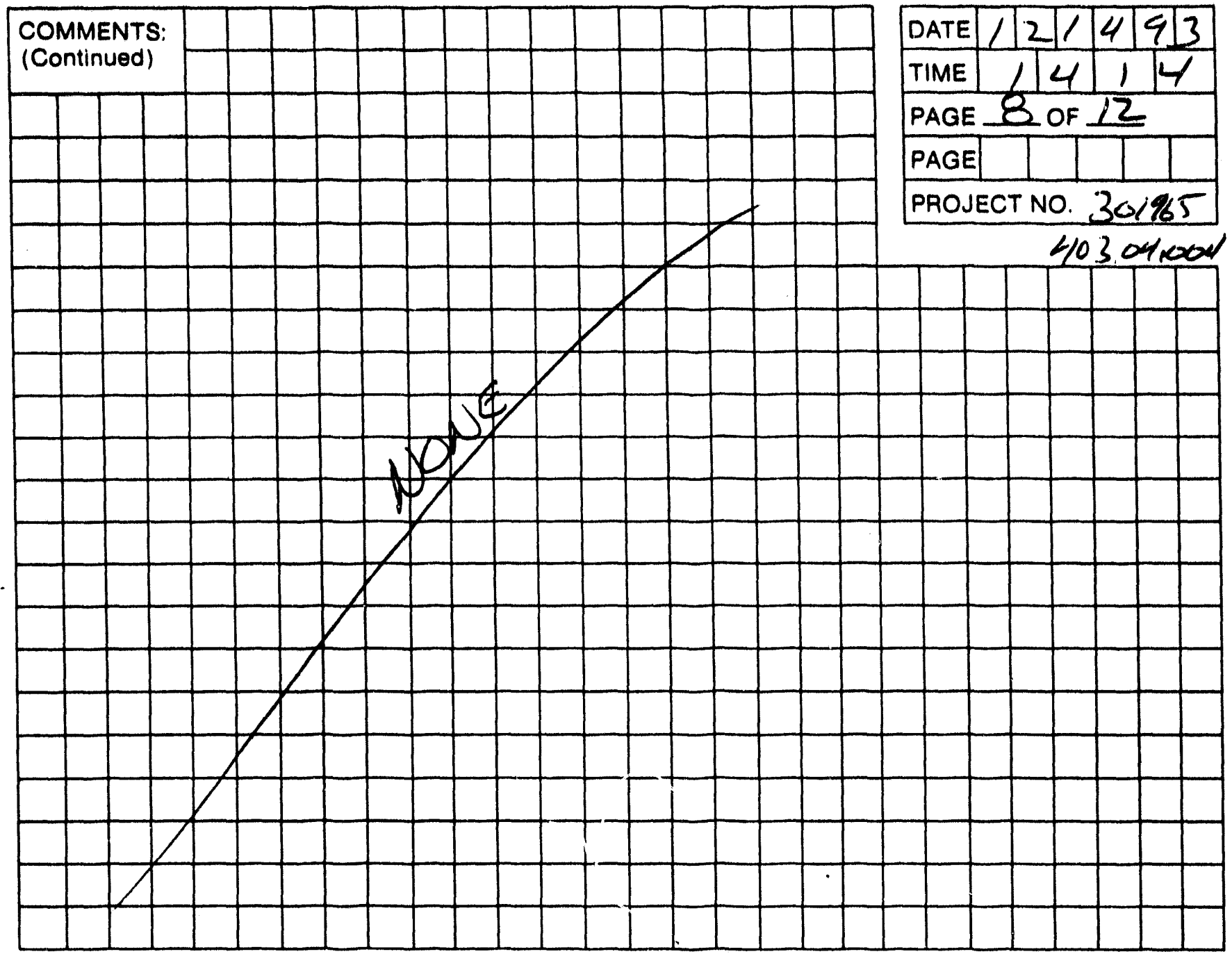

PREPARED BY:

\section{LEGEND}

1. A SAMPLE COLLECTION LOg IS TO BE COMPLETED FOA EACH SAMPLE

2. ALWAYS COMPLETE BOTH SIDES. IF SECOND SIDE IS NOT USED. DRAW A LINE THROUGH IT AND MARK N/A. FILL IN CONTROL BLCICK AND PAEPARED BY.

3. ALL ENTAIES ON LOG ARE TO BE COMPLETED. IF NOT APPLICABLE MARK N/A.

4. DATE: USE MONTH/DAY/YEAR: I.E., 10/30/85

5. TIME: USE 24-HOUR CLOCK: I.E., 1835 FOR 6:35 P.M.

6 PAGE: EACH SAMPLE TEAM SHOULO NUMBER PAGE ___ OF___ FOR THE DAYS ACTIVITIES FOR ALL SHEETS PREPARED ON A SINGLE DAY, I.E., IF THERE ARE A TOTAL OF 24 PAGES (INČLLUDING FRONT AND BACK) NUMBEA 1 OF 24. 2 OF 24. ETC.

7 SAMPLE LOCATION USE BORING OR MONITORING WELL NUMBER. GRIO LOCATION ITRANSECTI. SAMPLING STATION ID. OR COORDINATE TO PHYSICAL FEATURES WITH DISTANCES INCLUDE SKETCH IN COMMENT SECTION IF NECESSARY

8. SAMPLE TYPE USE THE FOLLOWING - SOIL: WATER (SURFACE OR GROUND). AIA (FILTERS. TUBES. AMBIENT. PERSONNEL): SLUDGE: DRUM CONTENTS OIL: VEGETATION: WIPE: SEDIMENT

9 COMPOSITE TYPE: I.E., 24-HOUA, LIST SAMPLE NUMBERS IN COMPOSITE, SPATIAL COMPOSITE.

10. DEPTH OF SAMPLE GIVE UNITS. WAITE OUT UNITS SUCH AS INCHES. FEET DON'T USE ' OR"

11 WEATHER APPROXIMATE TEMPERATURE. SUN AND MOISTURE CONDITIONS

12 CONTAINEAS USED. LIST EACH CONTAINER TYPE AS NUMBER. VOLUME. MATERIAL IE G . 2 - IL GLASS. 4 - $40 \mathrm{ML}$ GLASS VIAL, 1 - $400 \mathrm{ML}$ PLASTIC. 1 - 3 INCH STEEL TUBE: 1 - 8 OZ. GLASS JAR ?

13. AMOUNT COLLECTED: VOLUME IN CONTAINEAS (E.G. 1/2 FULL) 


\section{SAMPLE COLLECTION LOG}

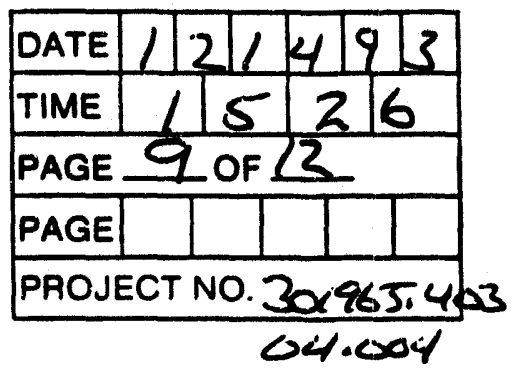

Project name SAzmronl TEST Sitz-

SAMPLE NO. IR/4-ES - $90-1200-3$

sample location ReeCo Pris.

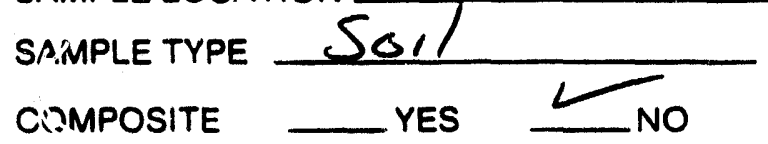

COMPOSITE TYPE

DEPTH OF SAMPLE 3 EEET

WEATHER Guecant ad

\begin{tabular}{c|c}
$\begin{array}{c}\text { CONTAINERS } \\
\text { USED }\end{array}$ & \multicolumn{1}{|c}{$\begin{array}{c}\text { AMOUNT } \\
\text { COLLECTED }\end{array}$} \\
\hline $120 \mathrm{ml} A G$ & 120 \\
\hline $500 \mathrm{ml} A G$ & $500 \mathrm{ml}$ \\
\hline & \\
\hline
\end{tabular}

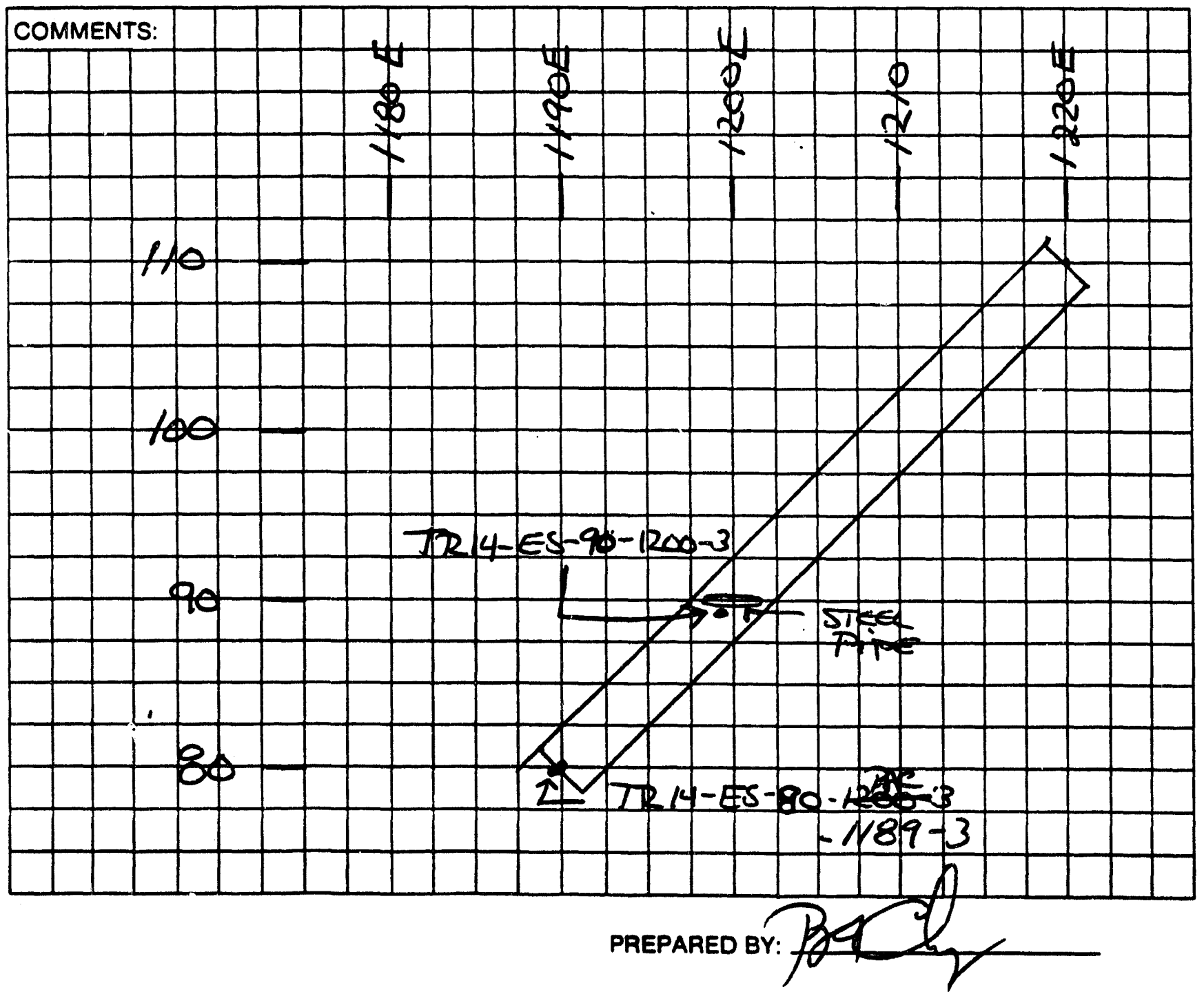




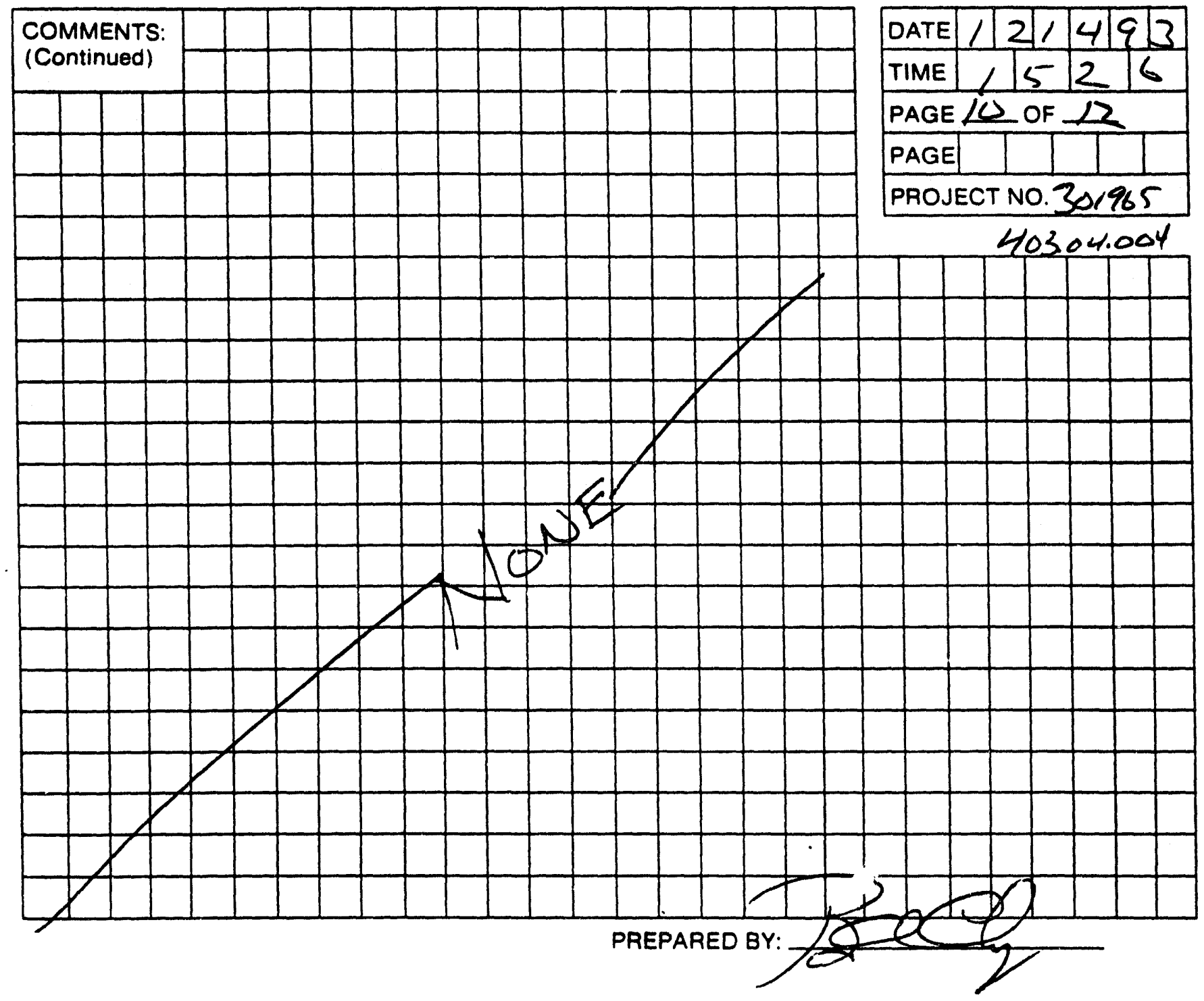

LEGEND

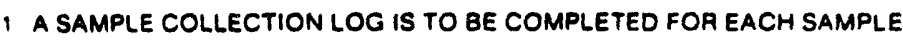

2. ALWAYS COMPLETE BOTH SIDES IF SECOND SIDE IS NOT USED. DRAW A LINE THROUGH IT ANO MARK N/A FILL. IN CONTROL BLOCK AND PAEPARED BY

3. AlL ENTAIES ON LOG ARE TO BE COMPLETED. IF NOT APPLICABLE MARK N/A.

4 DATE: USE MONTH/DAY/YEAR: I.E.. 10/30/85

5. TIME: USE 24-HOUR CLOCK, I.E., 1835 FOR 6:35 P M

6 PAGE: EACH SAMPLE TEAM SHOULO NUMBER PAGE ___ OF___ FOR THE DAY'S ACTIVITIES FOR ALL SHEETS PREPAREO ON A SINGLE DAY, I.E. IF THERE ARE A TOTAL OF 24 PAGES (INCLUDING FRONT AND BACKI NUMBER 1 OF 24.2 OF 24 ETC

7 SAMPLE LOCATION USE BORING OR MONITORING WELL NUMBEA. GRIO LOCATION (TRANSECT). SAMPLING STATION I D. OR COORDINATE TO PHYSICAL FEATURES WITH DISTANCES. INCLUDE SKETCH IN COMMENT SECTION IF NECESSARY

8 SAMPLE TYPE USE THE FOLLOWING - SOIL: WATER (SURFACE OR GROUND): AIR (FILTERS. TUBES. AMBIENT. PERSONNEL). SLUDGE: DRUM CONTENTS OIL. VEGETATION: WIPE. SEDIMENT

9. COMPOSITE TYPE: I.E., 24-HOUR. LIST SAMPLE NUMBEAS IN COMPOSITE. SPATIAL COMPOSITE.

10. DEPTH OF SAMPLE: GIVE UNITS. WAITE OUT UNITS SUCH AS INCHES. FEET DON'T USE ' OR "

11 WEATHER APPROXIMATE TEMPERATURE. SUN AND MOISTURE CONDITIONS.

12. CONTAINERS USED: LIST EACH CONTAINER TYPE AS NUMBER, VOLUME, MATERIAL IE G. 2 - IL GLASS: 4 - 40 ML GLASS VIAL: 1 - 400 ML PLASTIC: 1 - 3 INCH STEEL TUBE: 1 - 8 OZ. GLASS JAR)

13. AMOUNT COLLECTED: VOLUME IN CONTAINERS (E.G. 1/2 FULL). 
SOS $\infty$ ?

inTERNATIONAL TECANATOGX
CORPORATION

SAMPLE COLLECTION LOG

Project Name Salmon

SAMPLE NO. TRU4-ES-80-1189=3

sample location Deco Pits

SAMPLE TYPE So,

COMPOSITE — YES LNO

COMPOSITE TYPE

DEPTH OF SAMPLE 3 FEeT

WEATHER QUECOAT-COOI

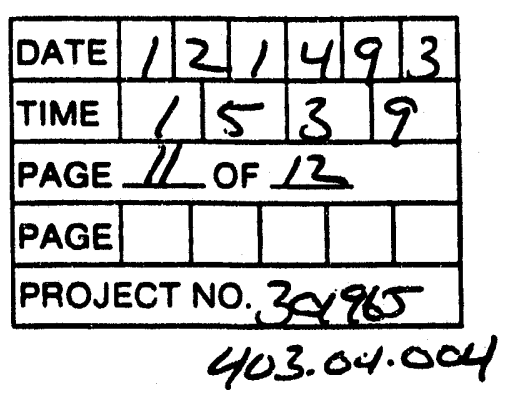

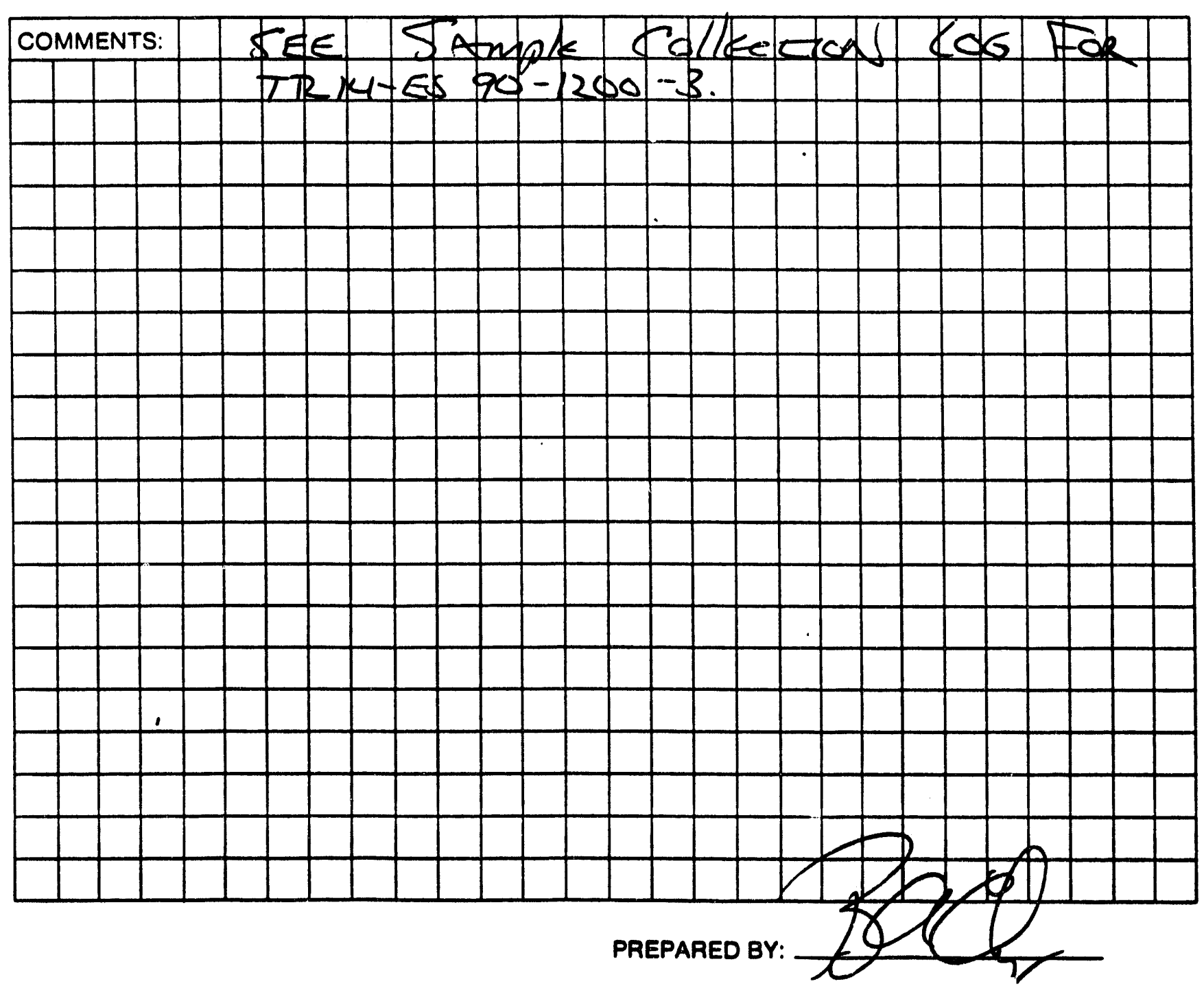




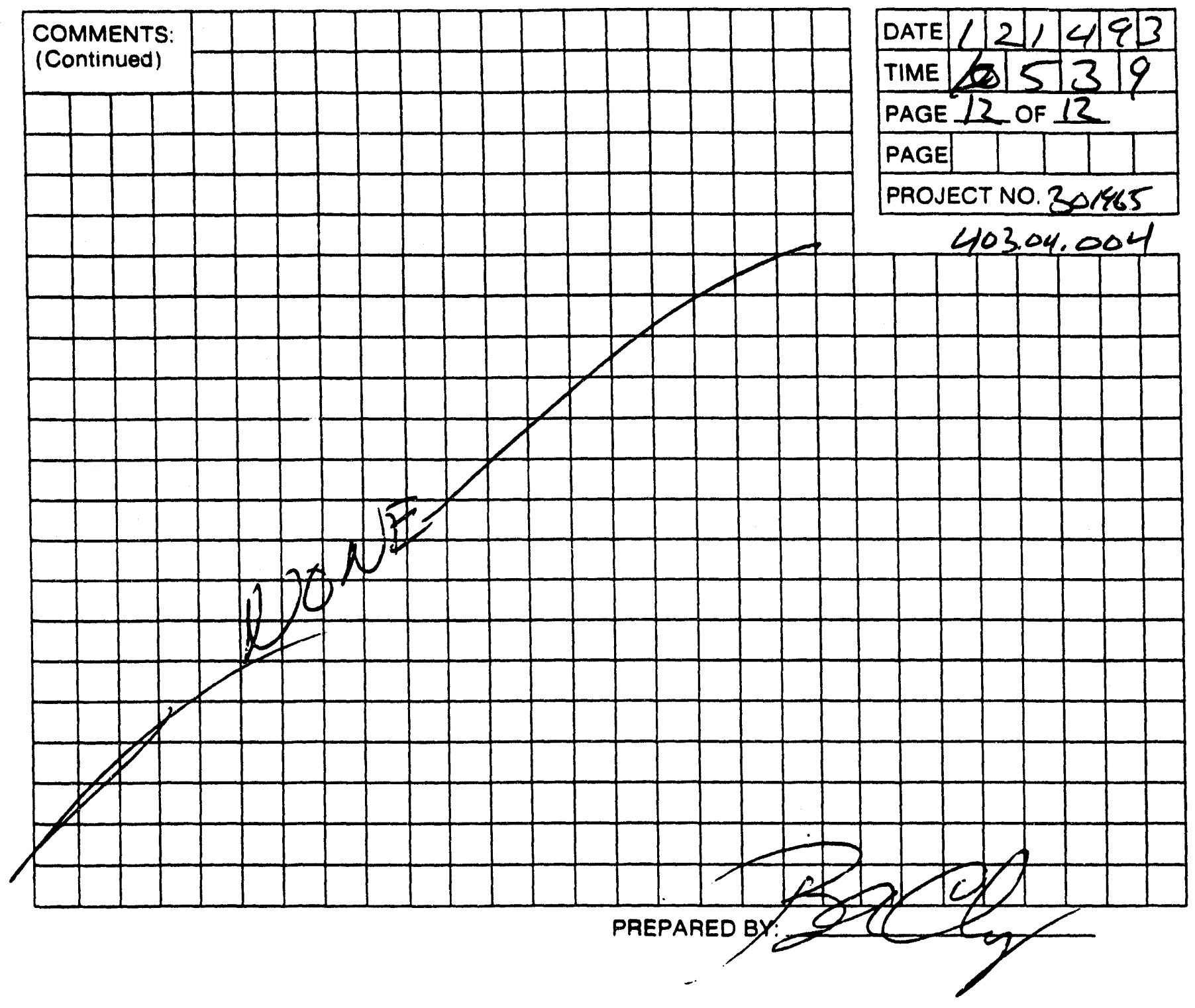

LEGEND

1 a sample collection log is to ge CoMpleted for eACH SAMPLE.

2. ALWAYS COMPLETE BOTH SIDES. IF SECOND SIDE IS NOT USED. DRAW A LINE THROUGH IT AND MARK N/A FILL IN CONTAOL BLOCK AND PREPARED BY.

3. ALL ENTAIES ONLOG ARE TO BE COMPLETED. If NOT APPLICABLE MARK N/A.

4. DATE: USE MONTH/OAY/YEAR: I.E., 10/30/85

5. TIME: USE 24-HOUR CLOCK: I.E., 1835 FOR 6:35 P M

6 PAGE: EACH SAMPLE TEAM SHOULD NUMBER PAGE___ OF___ FOR THE DAYS ACTIVITIES FOR ALL SHEETS PREPARED ON A SINGLE DAY. 1.E., IF THERE ARE A TOTAL OF 24 PAGES (INCLUDING FRONT AND BACK) NUMBER 1. OF 24. 2 OF 24. ETC

7 SAMPLE LOCATION: USE BORING OR MONITORING WELL NUMBEA. GRID LOCATION (TRANSECT), SAMPLING STATION I D. OA COORDINATE TO PHYSICAL FEATURES WITH DISTANCES. INCLUDE SKETCH IN COMMENT SECTION IF NECESSARY

8. SAMPLE TYPE: USE THE FOLLOWING - SOIL. WATER (SURFACE OR GROUNDI. AIR (FILTERS. TUBES. AMBIENT. PERSONNEL): SLUDGE: DRUM CONTENTS: OIL: VEGETATION: WIPE SEDIMENT

9. COMPOSITE TYPE: I.E.. 24-HOUA, LIST SAMPLE NUMEERS IN COMPOSITE, SPATIAL COMPOSITE.

10. DEPTH OF SAMPLE: GIVE UNITS. WRITE OUT UNITS SUCH AS INCHES. FEET. DON'T USE ' OR ".

: WEATHER. APPROXIMATE TEMPERATURE. SUN ANO MOISTURE CONDITIONS

12. CONTAINERS USED: LIST EACH CONTAINER TYPE AS NUMBER, VOLUME. MATERIAL IE.G . 2 - IL GLASS. 4 - 40 ML GLASS VIAL: 1 - 400 ML PLASTIC: 1 - 3 INCH STEEL TUBE; 1 - 8 OZ. GLASS JAR)

13. AMOUNT COLLECTED: VOLUME IN CONTAINERS (E.G. 1/2 FULL) 
PRoject name SAlmoN)

SAMPLENO. IR/I-ES-180-930-2

sample location TRenCH $\|$ - ReElo PITS AREA

SAMPLE TYPE SOI/ GRAB

COMPOSITE —YES LNO

COMPOSITE TYPE $N / N$

DEPTH OF SAMPLE 2 FEET

weather Claung Cool $240^{\circ} \mathrm{F}$

\begin{tabular}{c|c}
$\begin{array}{c}\text { CONTAINERS } \\
\text { USED }\end{array}$ & \multicolumn{1}{c}{$\begin{array}{c}\text { AMOUNT } \\
\text { COLLECTED }\end{array}$} \\
\hline $120 \mathrm{~m} / \mathrm{A} 6$ & 120 \\
\hline $2 \times 250 \mathrm{~m} / \mathrm{AG}$ & 500 \\
\hline
\end{tabular}

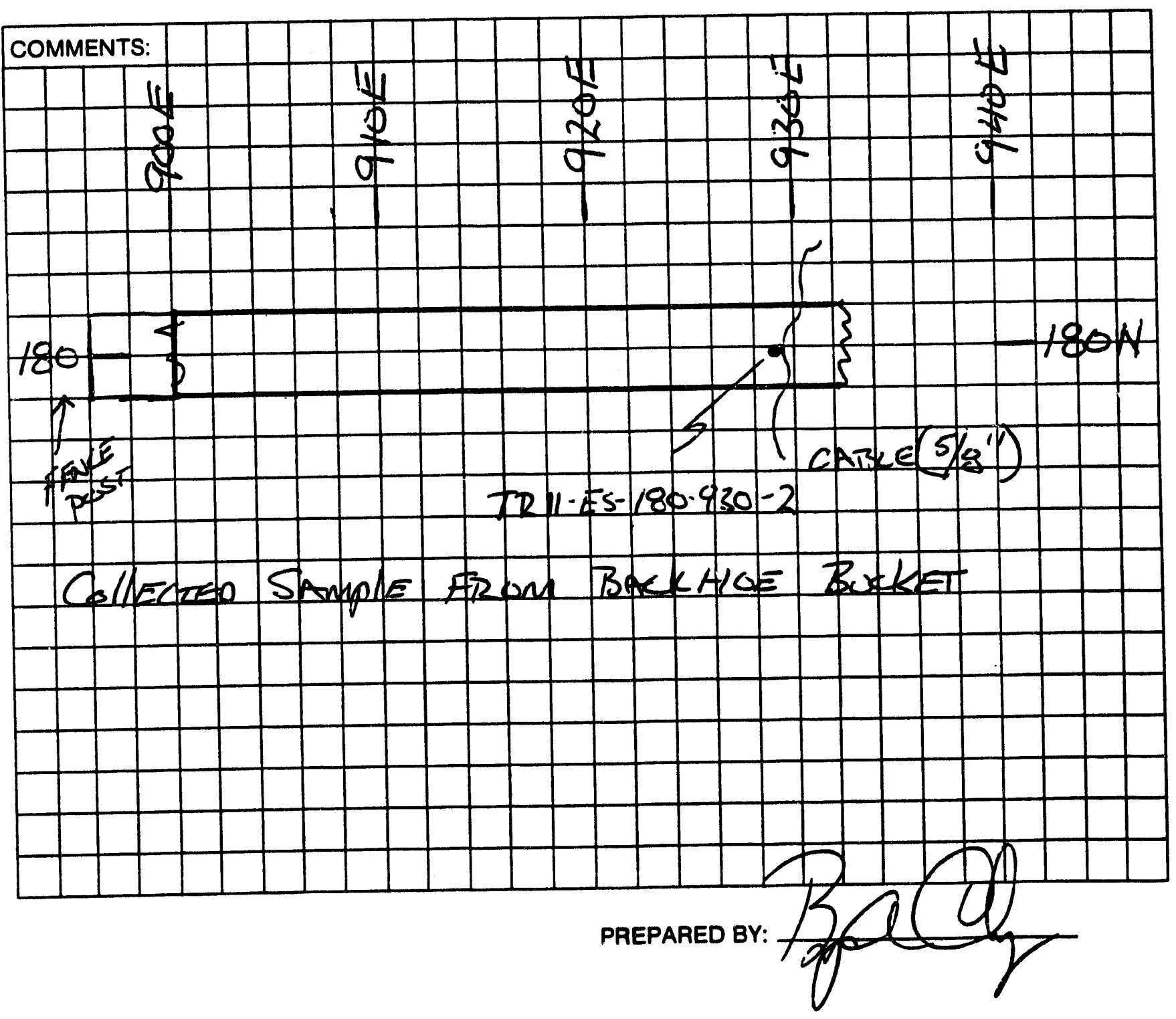




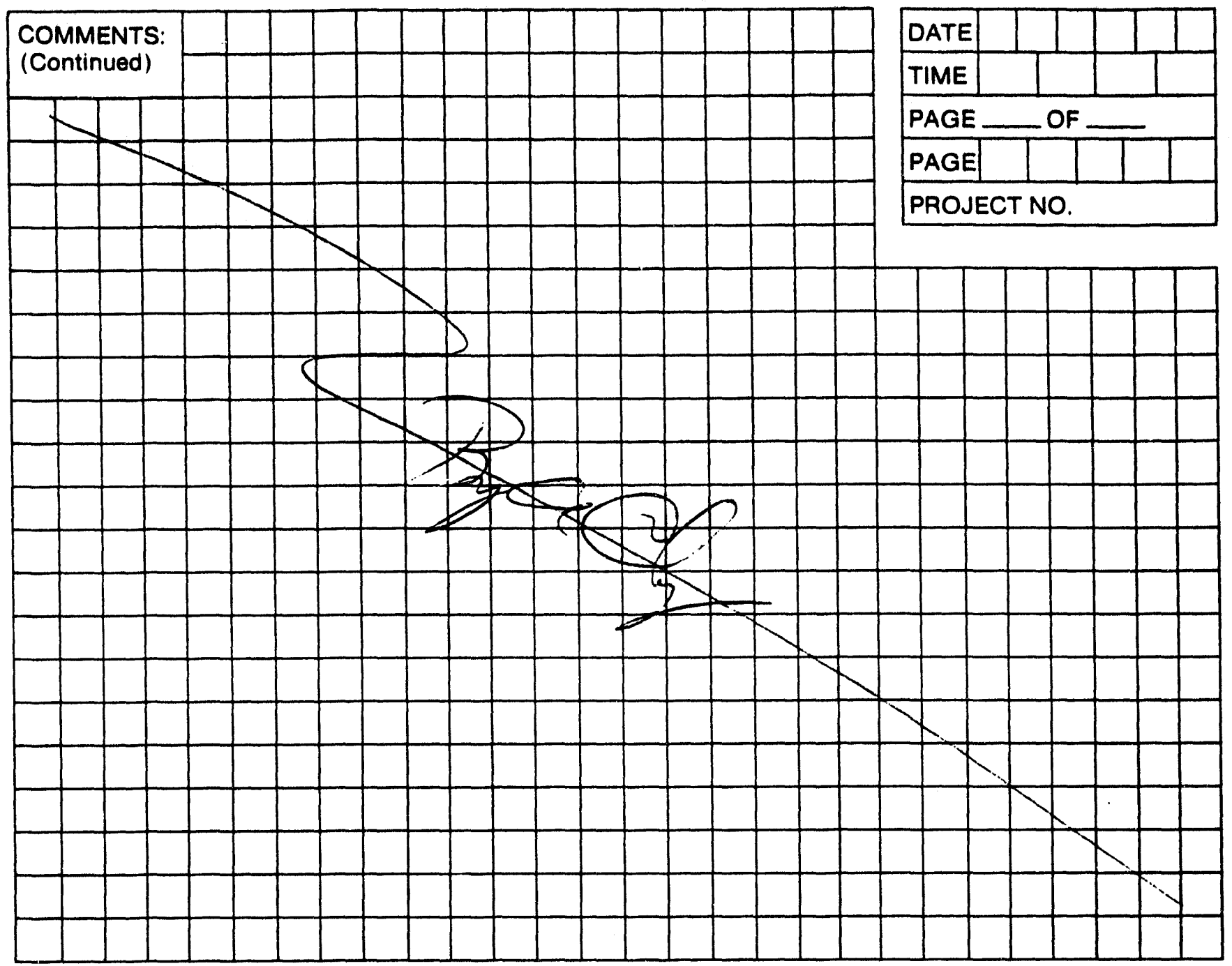

PREPARED BY:

\section{LEGEND}

1 A SAMPLE COLLECTION LOG IS TO BE COMPLETED FOR EACH SAMPLE

2. ALWAYS COMPLETE BOTH SIDES. IF SECOND SIDE IS NOT USED. DRAW A LINE THROUGH IT AND MARK N/A. FILL IN CONTROL BLOCK AND PREPARED BY.

3. ALL ENTRIES ON LOG ARE TO BE COMPLETED. IF NOT APPLICABLE MARK N/A.

4. DATE: USE MONTH/DAY/YEAR: I.E.. 10/30/85

5. TIME: USE 24-HOUR CLOCK: I.E. 1835 FOR 6:35 P.M

6. PAGE: EACH SAMPLE TEAM SHOULD NUMBER PAGE OF FOR THE DAY'S ACTIVITIES FOR ALL SHEETS PREPARED ON A SINGLE DAY. I.E.. IF THERE ARE A TOTAL OF 24 PAGES (INCLUDING FRONT AND BACK) NUMBER 1 OF 24,2 OF 24. ETC

7 SAMPLE LOCATION USE BORING OR MONITORING WELL NUMBER. GRID LOCATION (TRANSECTI. SAMPLING STATION I.D., OR COORDINATE TO PHYSICAL FEATURES WITH DISTANCES. INCLUDE SKETCH IN COMMENT SECTION IF NECESSARY.

8. SAMPLE TYPE: USE THE FOLLOWING - SOIL: WATER (SURFACE OR GROUND): AIR (FILTERS. TUBES. AMBIENT. PERSONNEL); SLUDGE: DRUM CONTENTS: OIL: VEGETATION: WIPE: SEDIMENT

9 COMPOSITE TYPE: I.E., 24-HOUR. LIST SAMPLE NUMBERS IN COMPOSITE. SPATIAL COMPOSITE.

10 DEPTH OF SAMPLE: GIVE UNITS. WRITE OUT UNITS SUCH AS INCHES. FEET. DON'T USE ' OR ".

11. WEATHER: APPROXIMATE TEMPERATURE. SUN AND MOISTURE CONDITIONS.

12. CONTAINERS USED: LIST EACH CONTAINER TYPE AS NUMBER. VOL ' JME. MATERIAL (E.G., 2 - IL GLASS; 4 - 40 ML GLASS VIAL: 1 - 400 ML PLASTIC 1 - 3 INCH STEEL TUBE: 1 - 8 OZ. GLASS JARI

13. AMOUNT COLLECTED: VOLUME IN CONTAINERS (E.G. 1/2 FULL). 
SAMPLE COLLECTION LOG

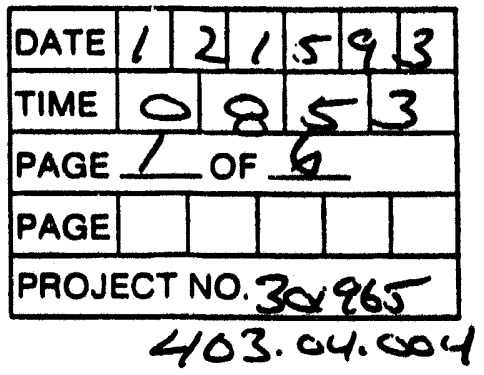

PRoject Name SACmonl

SAMPLE NO. TR/S - ES - 20-1180-0.5MS

SAMPLE LOCATION IDENCH IS REECO PITS.

SAMPLE TYPE So,

COMPOSITE YYES

COMPOSITE TYPE

DEPTH OF SAMPLE - 5 feet USED

AMOUNT

COLLECTED

WeATHER Cleagjcool

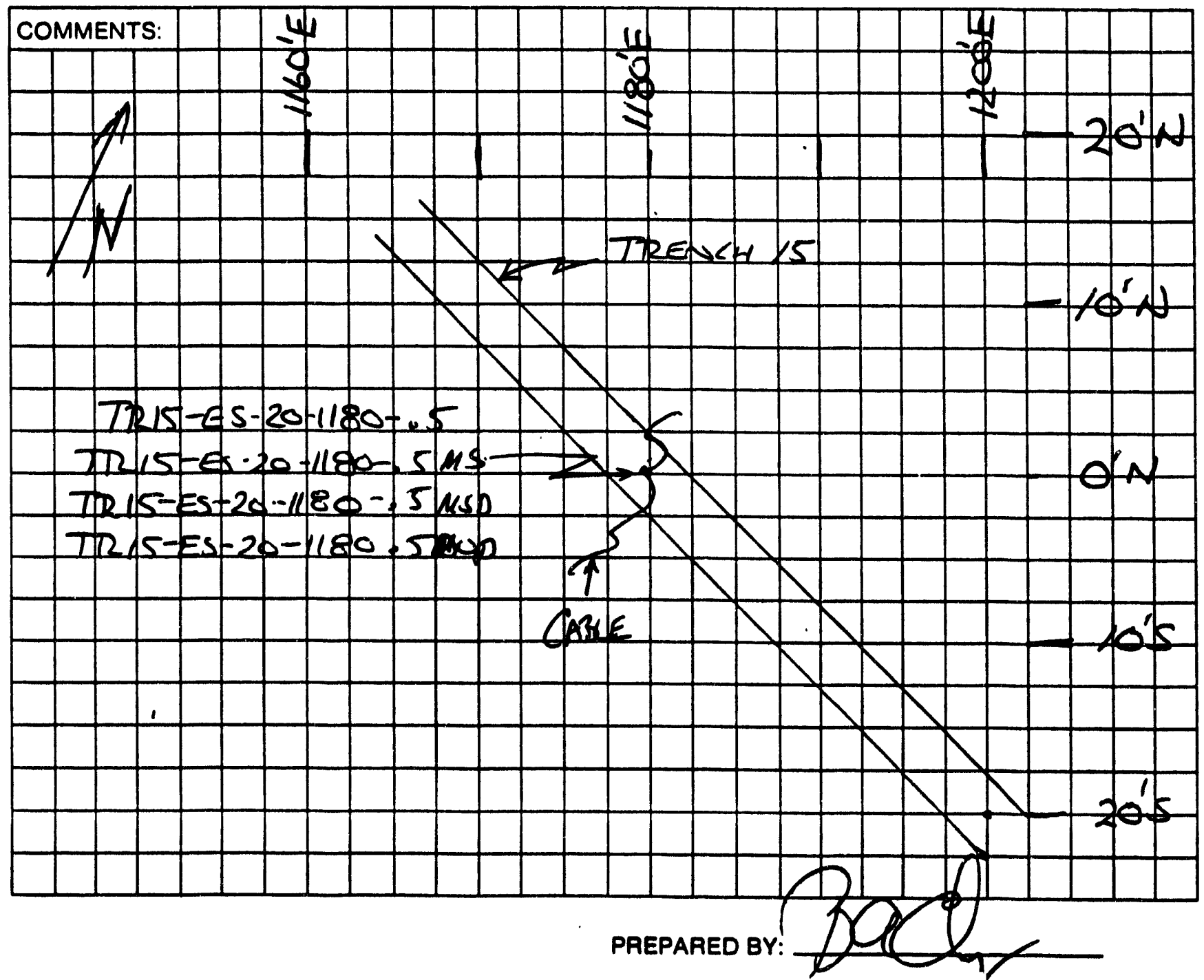




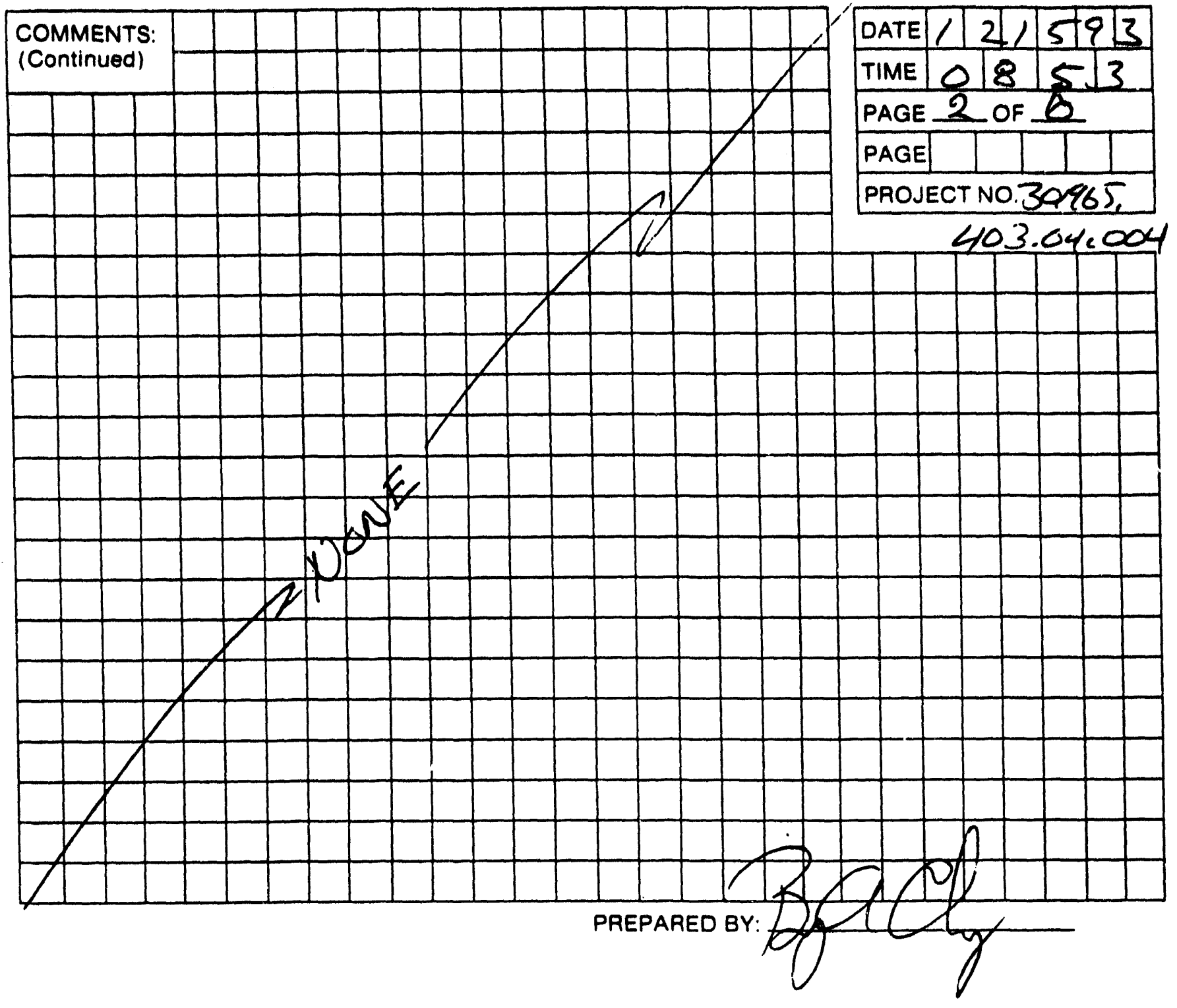

LEGEND

I A SAMPLE COLLECTION LOG IS TO Be COMPLETEO For EACH SAMPLE.

2. ALWAYS COMPLETE BOTH SIDES. IF SECOND SIDE IS NOT USED. DRAW A LINE THROUGH IT AND MARK N/A FILL IN CONTROL BLOCK AND PREPARED BY.

3. ALL ENTRIES ON LOG ARE TO BE COMPLETED. IF NOT APPLICABLE MARK N/A.

4 DATE: USE MONTH/DAY/YEAR: 1.E., 10/30/85

5 TIME: USE 24-HOUR CLOCK: I.E., 1835 FOR B:35 PM.

6 PAGE: EACH SAMPLE TEAM SHOULD NUMBER PAGE __ OF__ FOR THE DAYS ACTIVITIES TOR ALL SHEETS PREPARED ON A SINGLE DAY IE.. IF THERE ARE A TOTAL OF 24 PAGES IINCLUDING FAONT AND BACKI NUMBER 1 OF 24. 2 OF 24. ETC

7 SAMPLE LOCATION USE BORING OR MONITORING WELL NUMBER. GRID LOCATION ITRAI SECTI. SAMPLING STATION ID. OR COORDINATE TO PHYSICAL FEATURES WITH DISTANCES INCLUDE SKETCH IN COMMENT SECTION IF NECESSARY

8 SAMPLE TYPE: USE THE FOLLOWING - SOIL. WATER (SURFACE OR GROUNDI. AIR (FILTERS. TUBES. AMBIENT. PERSONNELI. SLUDGE: DAUM CONTENTS. OIL. VEGETATION: WIPE. SEDIMENT

9 COMPOSITE TYPE: I.E., 24-HOUR, LIST SAMPLE NUMBERS IN COMPOSITE. SPATIAL COMPOSITE.

10 OEPTH OF SAMPLE: GIVE UNITS. WRITE OUT UNITS SUCH AS INCHES. FEET. DON'T USE ' OR "

"1 WEATHER APPROXIMATE TEMPERATURE. SUN ANO MOISTURE CONOITIONS

12 CONTAINERS USED: LIST EACH CONTAINER TYPE AS NUMBER. VOLUME. MATERIAL IE. G., 2 - IL GLASS: 4 - 40 ML GLASS VIAL: 1 - 400 ML PLASTIC: 1 - 3 INCH STEEL TUBE: 1 - 8 OZ. GLASS JARI.

13. AMOUNT COLLECTED: VOLUME IN CONTAINEAS (E.G. 1/2 FULLL). 
MTERMTTOANaL ThCopotion

SAMPLE COLLECTION LOG

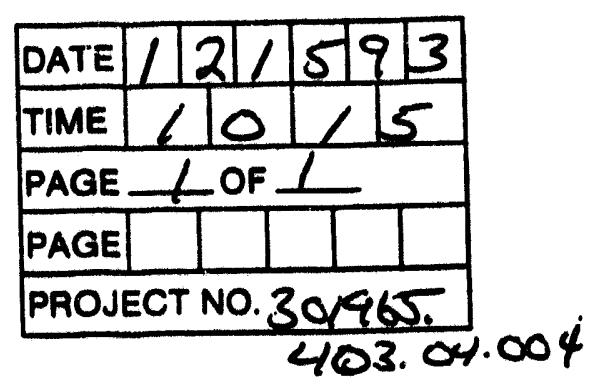

project name Salmon

SAMPLE NO. TR /6-ES-200-80-15

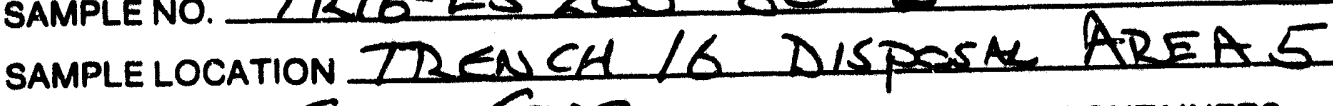

SAMPLE TYPE SOM GRAB

COMPOSITE - YES oNO

COMPOSITE TYPE NA

DEPTH OF SAMPLE 1.5 FEET

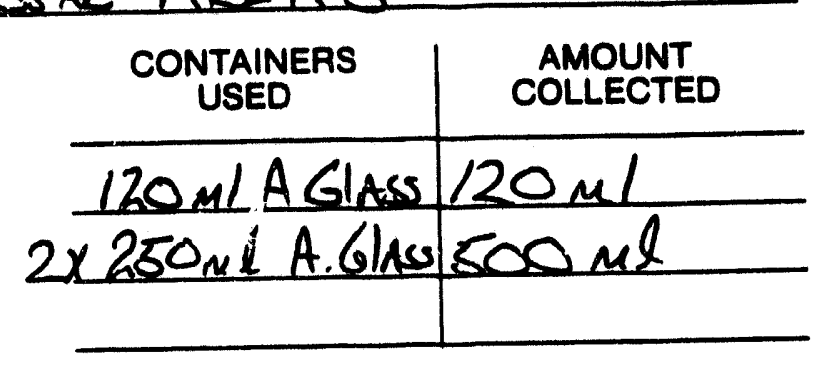

WEATHER $47^{\circ} \mathrm{F} / \mathrm{ClONDY}$

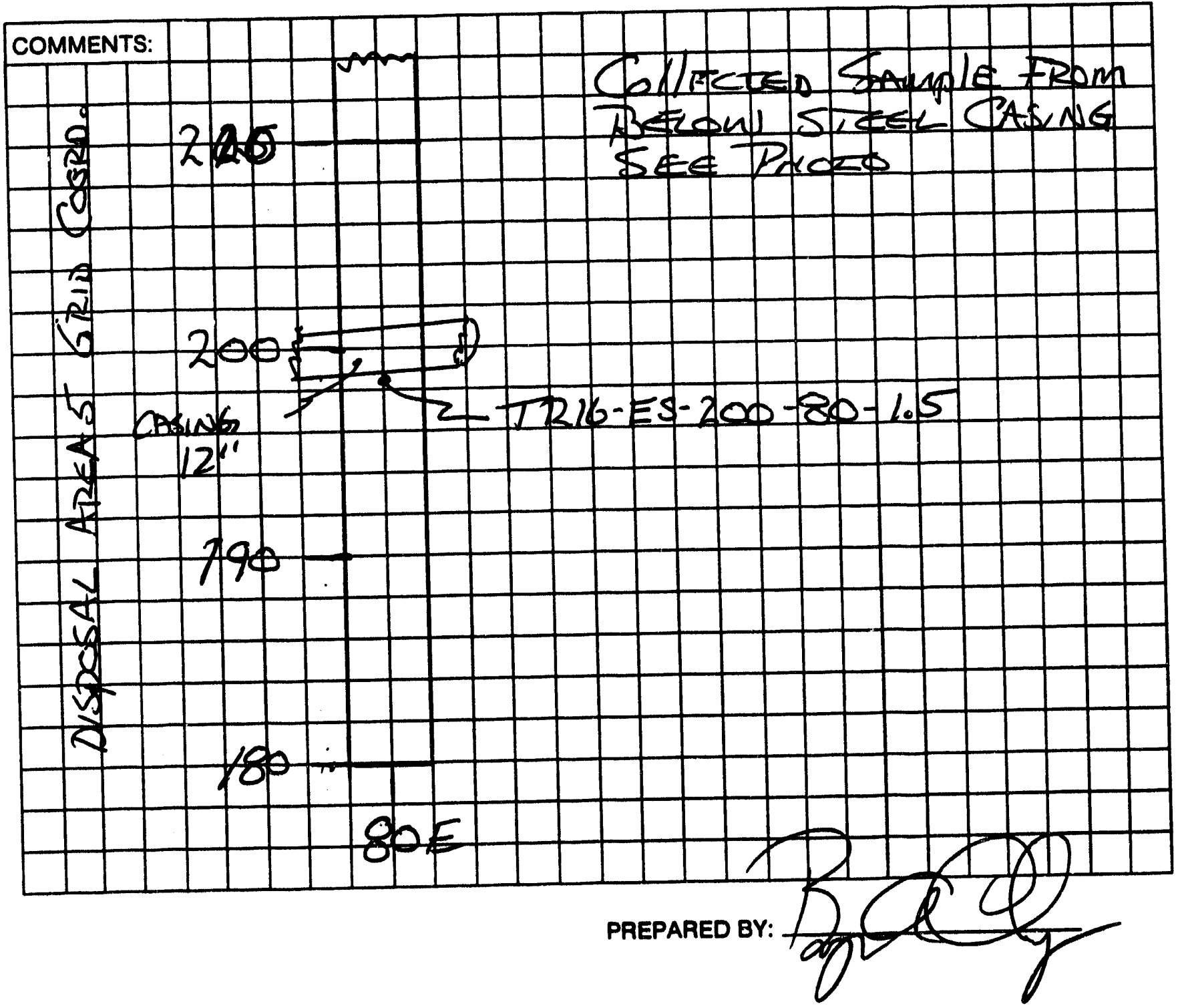




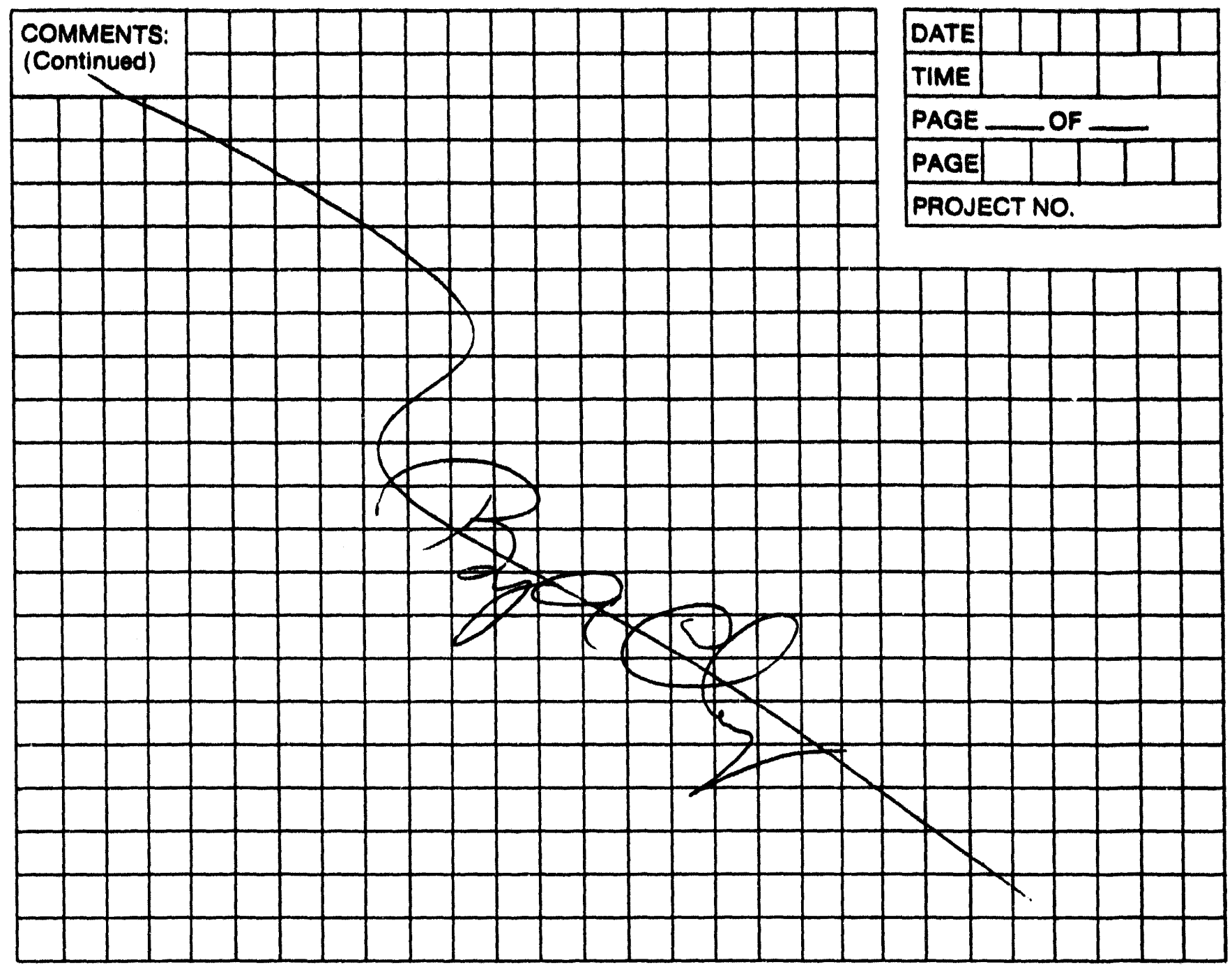

PREPARED BY:

\section{LEGEND}

1 A SAMPLE COLLECTION LOG IS TO BE COMPLETED FOR EACH SAMPLE.

2. ALWAYS COMPLETE BOTH SIDES. IF SECOND SIDE IS NOT USED. DRAW A LINE THROUGH IT AND MARK N/A. FILL IN CONTROL BLOCK AND PAEPARED BY.

3. ALL. ENTAIES ON LOG ARE TO BE COMPLETED, IF NOT APPLICABLE MAAK N/A.

4. DATE: USE MONTH/DAY/YEAA: I.E.. 10/30/85

5. TIME: USE 24-HOUR CLOCK: 1.E., 1835 FOR 6:35 P.M.

6. PAGE: EACH SAMPLE TEAM SHOULD NUMBER PAGE ___ OF __ FOR THE DAY'S ACTIVITIES FOR ALL SHEETS PREPARED ON A SINGLE DAY, I.E., IF THERE ARE A TOTAL OF 24 PAGES (INCLUDING FAONT AND BACK) NUMBER 1 OF 24, 2 OF 24, ETC.

7. SAMPLE LOCATION: USE BORING OR MONITORING WELL NUMBER, GRID LOCATION (TRANSECTI. SAMPLING STATION I.D., OR COORDINATE TO PHYSICAL FEATURES WITH DISTANCES. INCLUDE SKETCH IN COMMENT SECTION IF NECESSARY

8. SAMPLE TYPE: USE THE FOLLOWING - SOIL: WATER (SURFACE OR GROUND): AIR (FILTERS. TUBES. AMBIENT, PEASONNEL): SLUDGE: DRUM CONTENTS: OIL: VEGETATION: WIPE: SEDIMENT.

9. COMPOSITE TYPE: I.E., 24-HOUA, LIST SAMPLE NUMBERS IN COMPOSITE. SPATIAL COMPOSITE.

10. DEPTH OF SAMPLE: GIVE UNITS. WRITE OUT UNITS SUCH AS INCHES, FEET. DON'T USE ' OR ".

11. WEATHER: APPAOXIMATE TEMPERATURE, SUN AND MOISTURE CONDITIONS.

12. CONTAINERS USED: LIST EACH CONTAINER TYPE AS NUMBER. VOLUME, MATERIAL (E.G. 2 - IL GLASS: 4 - 40 ML GLASS VIAL: 1 - 400 ML PLASTIC: 1 - 3 INCH STEEL TUBE: 1 - 8 OZ. GLASS JARI

13. AMOUNT COLLECTED: VOLUME IN CONTAINEAS (E.G. 1/2 FULLL). 


\section{SAMPLE COLLECTION LOQ}

\begin{tabular}{|l|l|l|l|l|l|l|}
\hline DATE & 1 & 2 & 1 & 6 & 9 & 3 \\
\hline TIME & 0 & 9 & 0 & 0 \\
\hline PAGE & L & OF & 4 & \\
\hline PAGE & & & & & \\
\hline PROJECT NO. & 309650 \\
\hline
\end{tabular}

Project Name Salmon

SAMPLE NO. IR/7-ES-30-120-5

sample location "Gas STATton"

SAMPLE TYPE

COMPOSITE —YES $\longrightarrow$ NO

COMPOSITE TYPE LKA

DEPTH OF SAMPLE 5 feet

WEATHER COOL OUErcont

\begin{tabular}{c|c}
$\begin{array}{c}\text { CONTAINERS } \\
\text { USED }\end{array}$ & $\begin{array}{c}\text { AMOUNT } \\
\text { COLLECTED }\end{array}$ \\
\hline $120 \mathrm{~m} / \mathrm{AG}$ & $120 \mathrm{ml}$ \\
\hline $2 \times 250 \mathrm{ml}$ AS & $500 \mathrm{ml}$
\end{tabular}

COMMENTS:

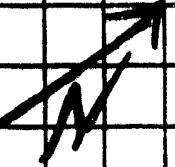

$7+k$
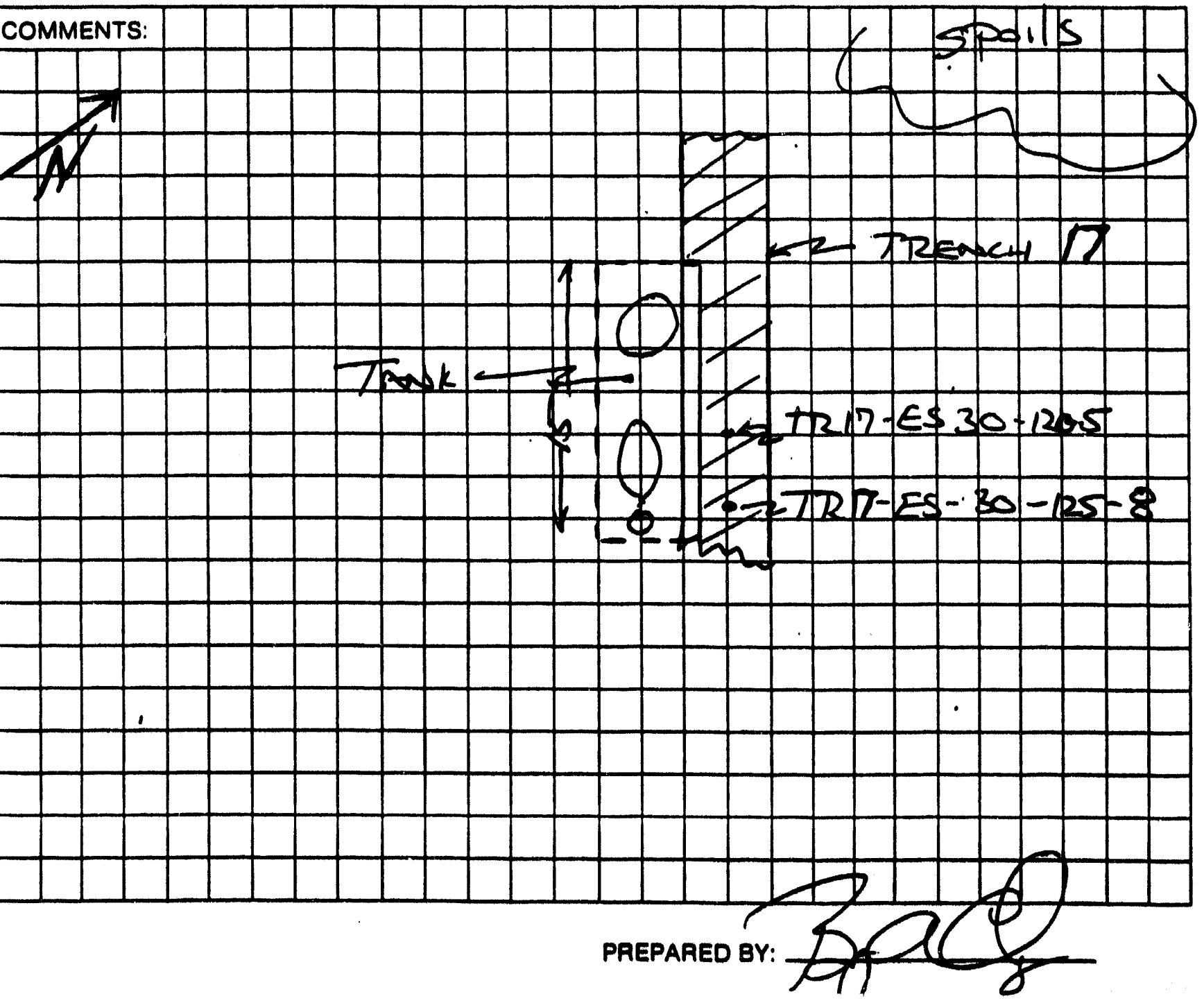


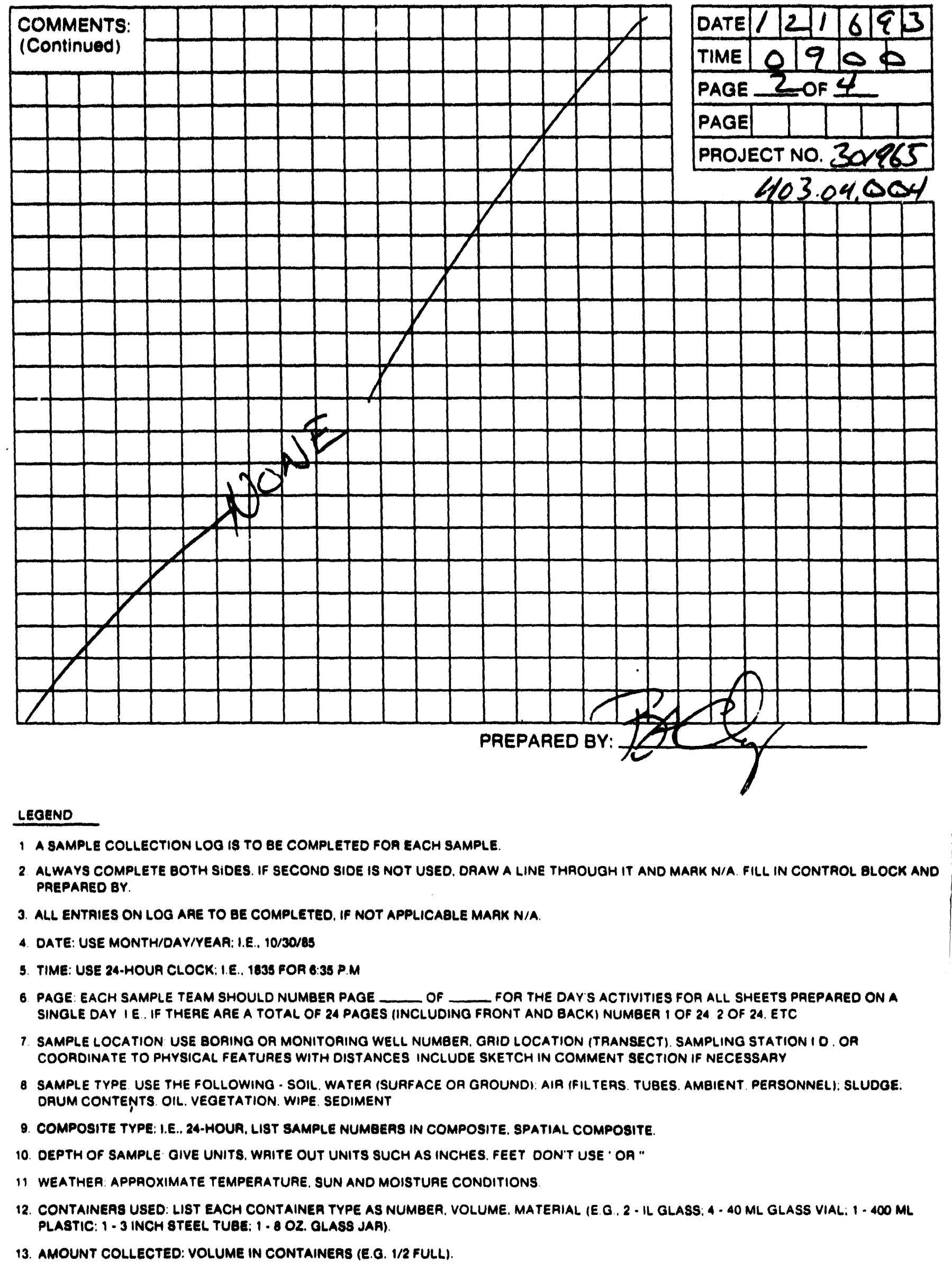


SAG $00 ?$

IT MTrpatrosaL conn

SAMPLE COLLECTION LOG

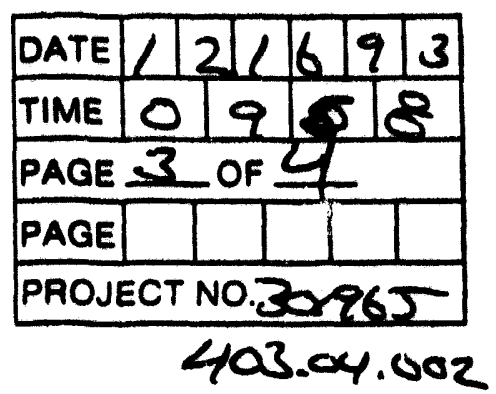

PRoject name Salmon SAMPLE NO. IR 12-ES-30-125-8

SAMPLE LOCATION "GAS STANTON:"

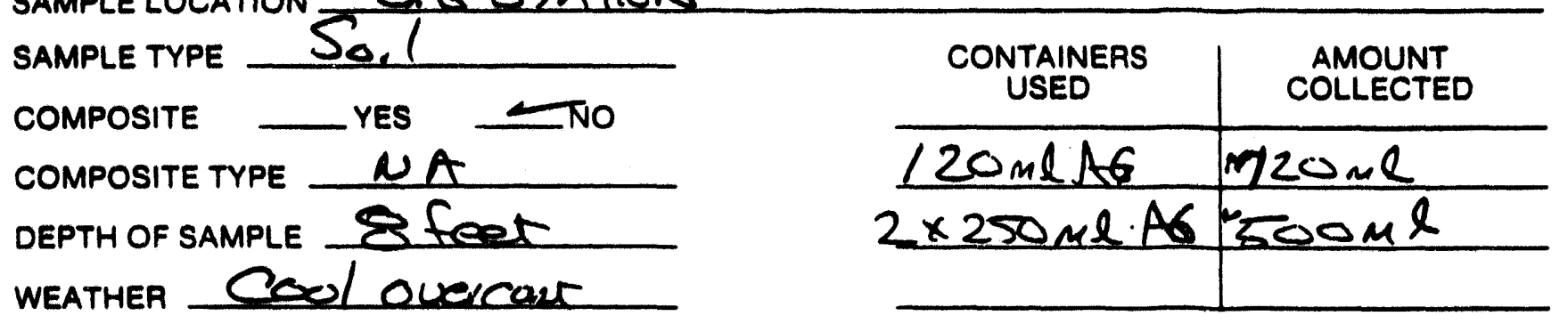

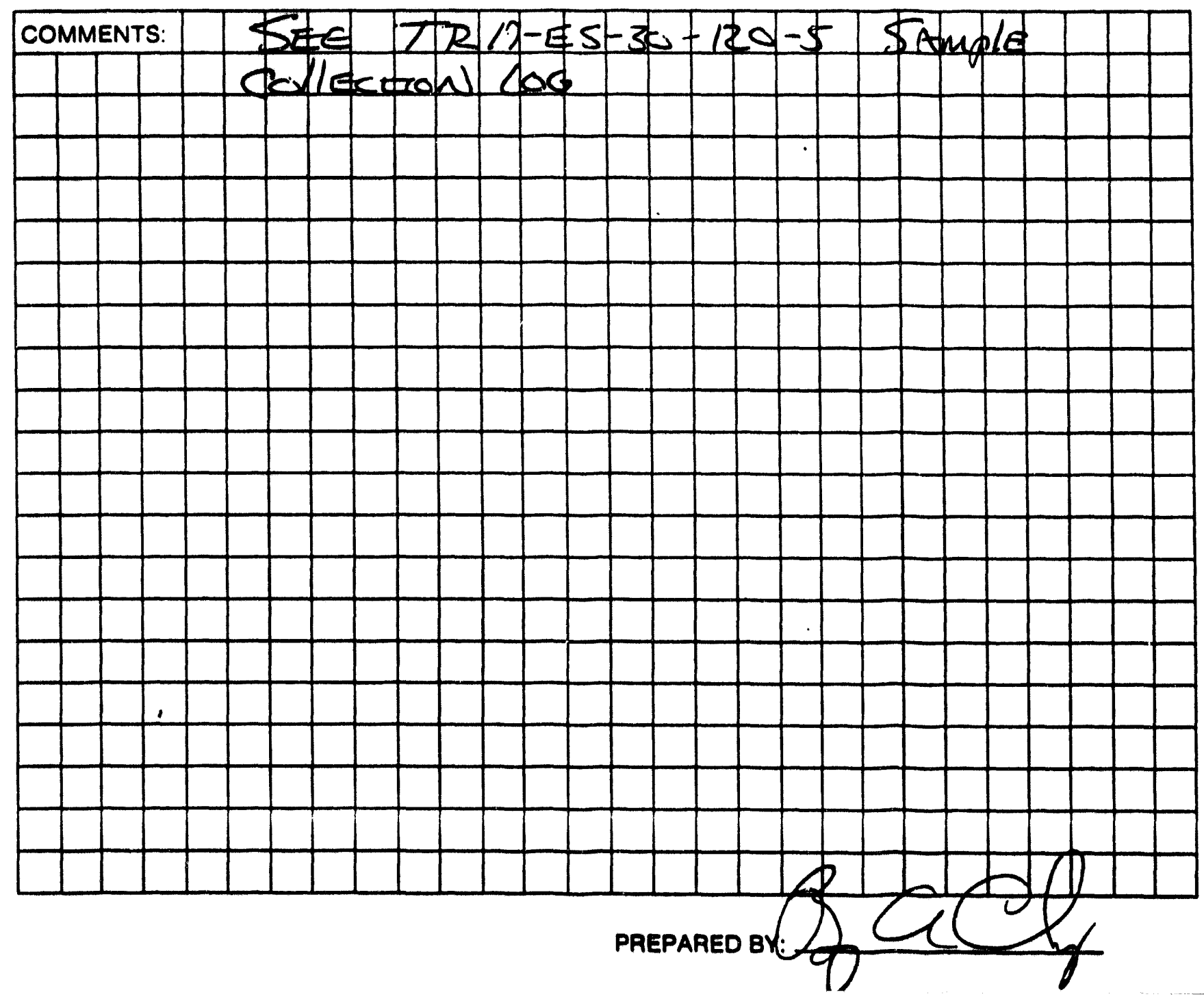




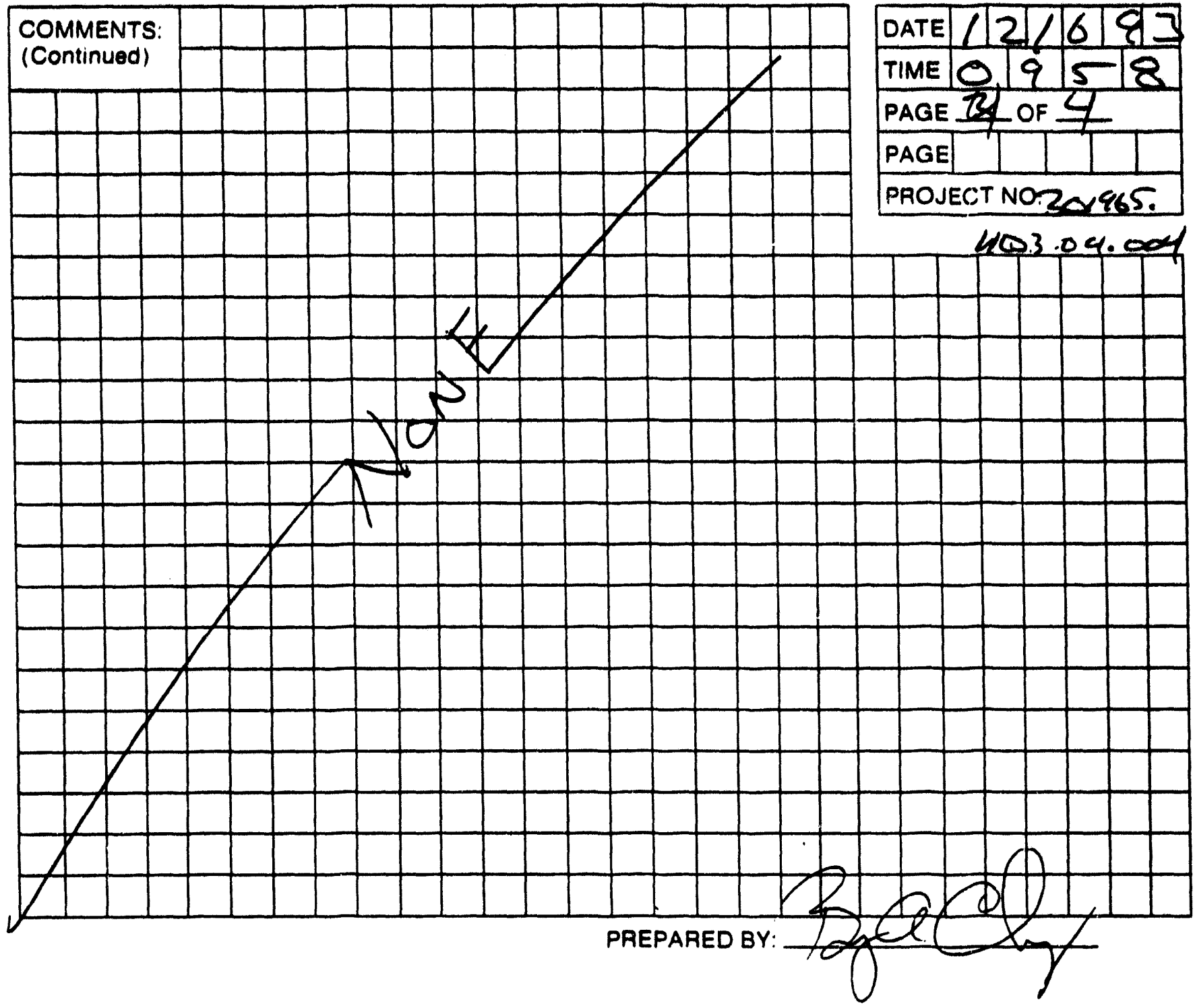

LEGEND

1 a sAMPLE COLLECTION LOG IS TO BE COMPLETED For EACH SAMPLE

2 ALWAYS COMPLETE BOTH SIDES. IF SECOND SIDE IS NOT USED. ORAW A LINE THROUGH IT ANO MARK N/A FILL IN CONTROL BLOCK ANO PAEPARED BY

3. all entaies on log afe to be COMPLeted. if nOt APPLicable maAk N/A.

4 DATE USE MONTH/DAY/YEAP: IE.. 10/30/85

5. TIME: USE 24-HOUR CLOCK: IE.. 1835 FOR 6.35 P M

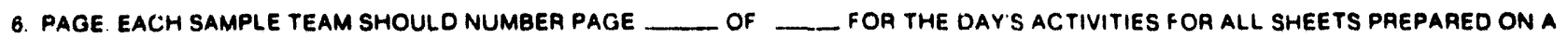
SINGLE DAY IE. IF THERE ARE A TOTAL OF 24 PAGES (INCLUDING FRONT AND BACK) NUMBER 1 OF 24.2 OF 24 ETC

7 SAMPLE LOCATION USE BORING OR MONITORING WELL NUMBER. GRID LOCATION ITRANSECTI. SAMPLING STATION ID. OR COORDINATE TO PHYSICAL FEATURES WITH DISTANCES INCLUDE SKETCH IN COMMENT SECTION IF NECESSARY

a SAMPLE TYPE USE THE FOLLOWING . SOIL. WATER (SURFACE OR GROUND). AIP (FILTERS. TUBES. AMBIENT. PERSONNELI. SLUDGe. DRUM CONTENTS OIL. VEGETATION. WIPE. SEOIMENT

9. COMPOSITE TYPE: I.E., 24-HOUP. LIST SAMPLE NUMBEAS IN COMPOSITE. SPATIAL COMPOSITE.

10 DEPTH OF SAMPLE gIVE UNITS. WRITE OUT UNITS SUCH AS INCHES. FEET DON'T USE ' OR "

11 WEATHER APPROXIMATE TEMPERATURE. SUN ANO MOISTURE CONDITIONS

12. CONTAINEAS USEO LIST EACH CONTAINER TYPE AS NUMBER, VOLUME. MATERIAL IE O. 2 - IL GLASS. 4 - 40 ML GLASS VIAL: 1 - 400 ML PLASTIC: 1 - 3 INCH STEEL TUBE: 1 - 8 OZ. GLASS JARI

13. AMOUNT COLLECTED: VOLUME IN CONTAINEAS (E.G. 1/2 FULL). 
SDGOS?

INTERNATIONAL TECHNOLOGY

SAMPLE COLLECTION LOG

project name Salmon test Site

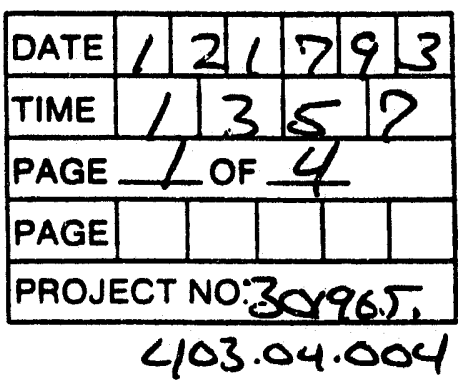

SAMPLE NO. IR/8-BS-90-150A-E

sample location Disposal Area 5

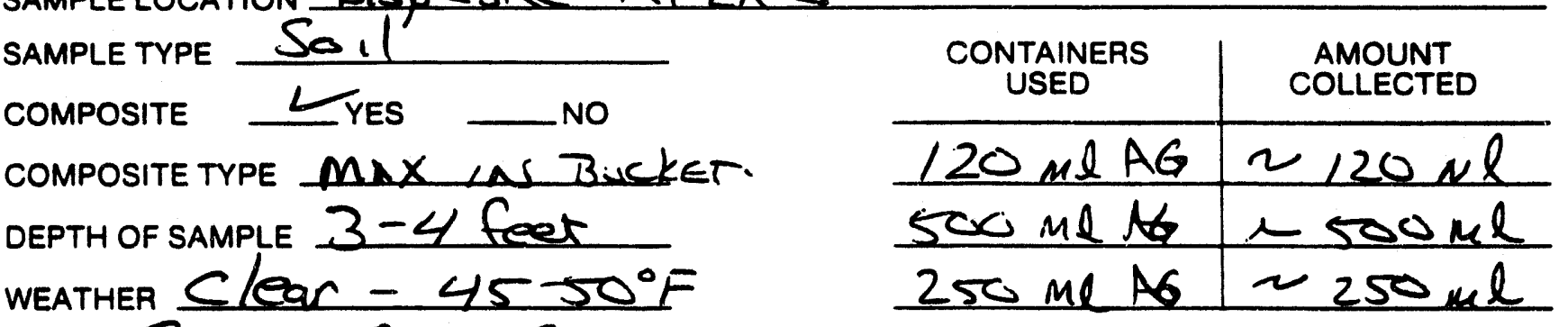

Small gRID COORDNATÉS

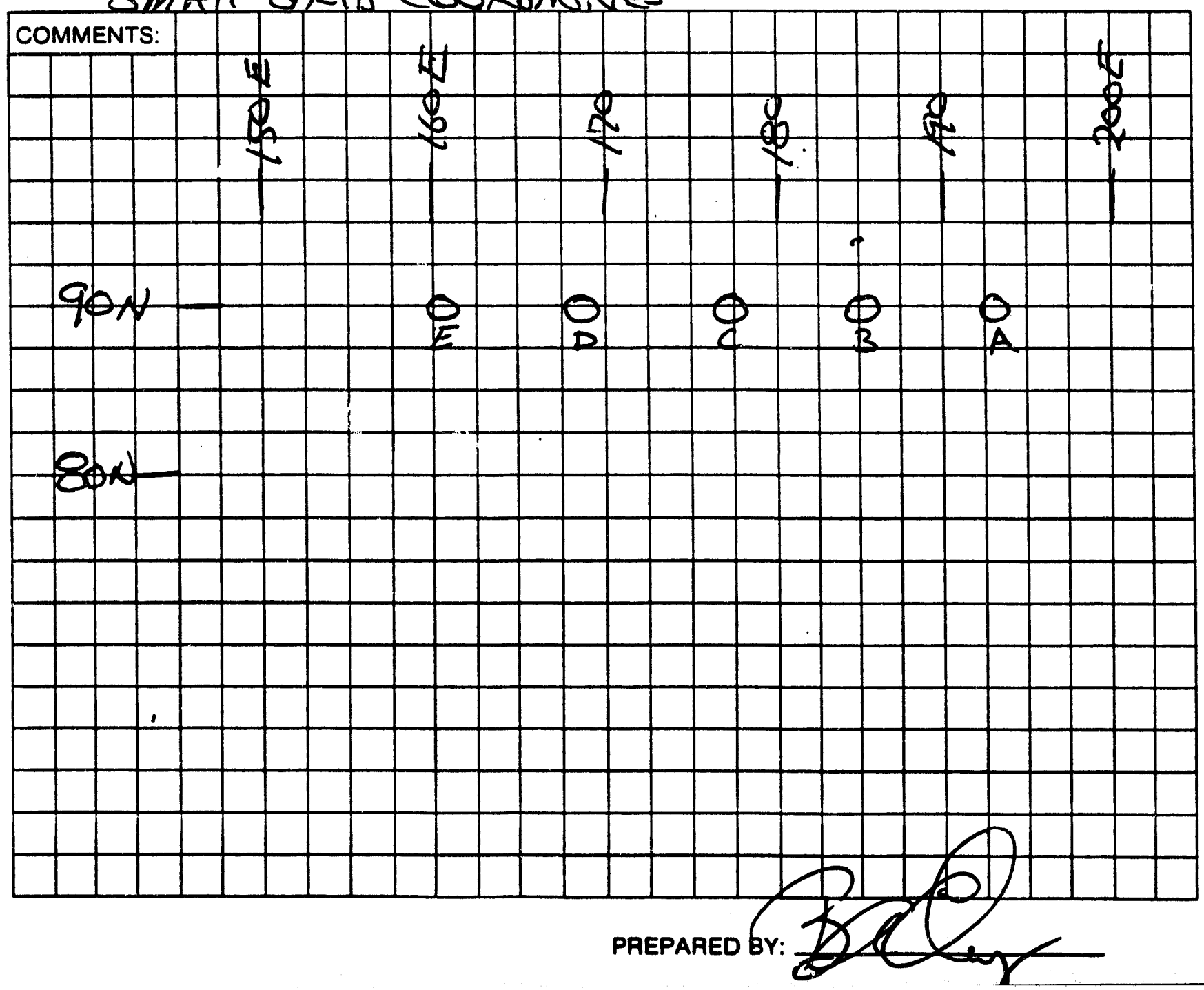




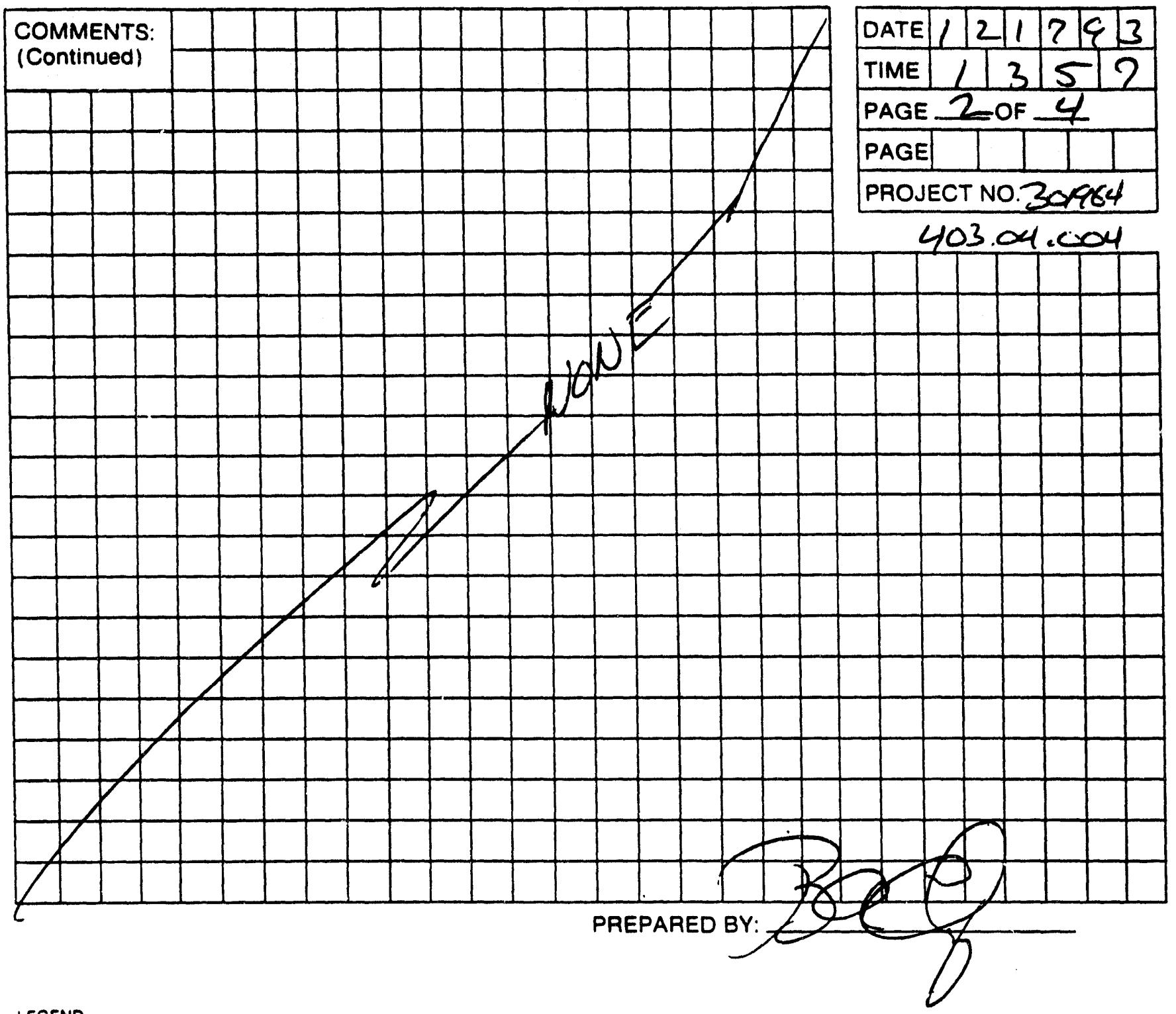

LEGEND

1 A SAMPLE COLLECTION LOG IS TO BE COMPLETED FOR EACH SAMPLE

2. ALWAYS COMPLETE BOTH SIDES. IF SECOND SIOE IS NOT USED. DRAW A LINE THROUGH IT AND MARK N/A FILL IN CONTROL BLOCK AND PAEPARED BY

3. ALL ENTRIES ON LOG ARE TO BE COMPLETED, IF NOT APPLICABLE MARK N/A

4 DATE: USE MONTH/DAYTYEAR: I.E. 10/30/85

5. TIME: USE 24-HOUR CLOCK: 1.E., 1835 FOR 6:35 PM

6 PAGE: EACH SAMPLE TEAM SHOULD NUMBER PAGE __ OF__ FOR THE DAYS ACTIVITIES FOR ALL SHEETS PREPARED ON A SINGLE DAY IE.. IF THERE ARE A TOTAL OF 24 PAGES (INCLUDING FRONT AND BACK) NUMBER 1 OF 24. 2 OF 24. ETC

7 SAMPLE LOCATION: USE BORING OR MONITORING WELL NUMBER. GRIO LOCATION (TRANSECTI. SAMPLING STATIONID. OR COORDINATE TO PHYSICAL FEATURES WITH DISTANCES. INCLUDE SKETCH IN COMMENT SECTION IF NECESSARY

8 SAMPLE TYPE: USE THE FOLLOWING - SOIL. WATER (SURFACE OR GROUNOI AIR (FILTERS. TUBES. AMBIENT. PERSONNEL): SLUDGE: DRUM CONTENTS. OIL. VEGETATION: WIPE. SEDIMENT

9. COMPOSITE TYPE: I.E., 24-HOUR. LIST SAMPLE NUMBEAS IN COMPOSITE. SPATIAL COMPOSITE

10 DEPTH OF SAMPLE: GIVE UNITS. WAITE OUT UNITS SUCH AS INCHES. FEET. DON'T USE ' OR "

11 WEATHER APPROXIMATE TEMPERATURE. SUN ANO MOISTURE CONDITIONS

12. CONTAINERS USED: LIST EACH CONTAINEA TYPE AS NUMBER. VOLUME. MATERIAL IE.G., 2 - IL GLASS: 4 - 40 ML GLASS VIAL: 1 - 400 ML PLASTIC: 1 - 3 INCH STEEL : UBE: 1 - 8 OZ. GLASS JARI

13. AMOUNT COLLECTED: VOLUME IN CONTAINERS (E.G. 1/2 FULL). 
SAMPLE COLLECTION LOG

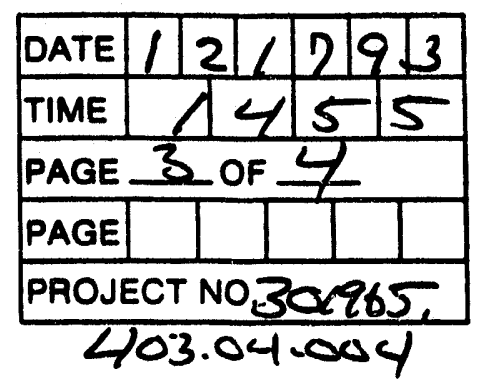

PROJECTNAME SACMOAN TEST SITE

SAMPLE NO. IR/9-BS-O-\% AE

SAMPLE LOCATION SOUTH GNO DMPASAR ARsa 5

SAMPLE TYPE SOII BORING

COMPOSITE UTES -NO

COMPOSITE TYPE GN P/ACTR SHEST

DEPTH OF SAMPLE $3-4$ feet

weather Clear $40-45^{\circ} \mathrm{F}$

\begin{tabular}{c|c}
$\begin{array}{c}\text { CONTAINERS } \\
\text { USED }\end{array}$ & $\begin{array}{c}\text { AMOUNT } \\
\text { COLLECTED }\end{array}$ \\
\hline $120 \mathrm{ml} A 6$ & $120 \mathrm{ml}$ \\
\hline $500 \mathrm{ml} \mathrm{Ab}$ & $500 \mathrm{ml}$ \\
\hline $250 \mathrm{ml} A 6$ & $250 \mathrm{ml}$ \\
\hline
\end{tabular}

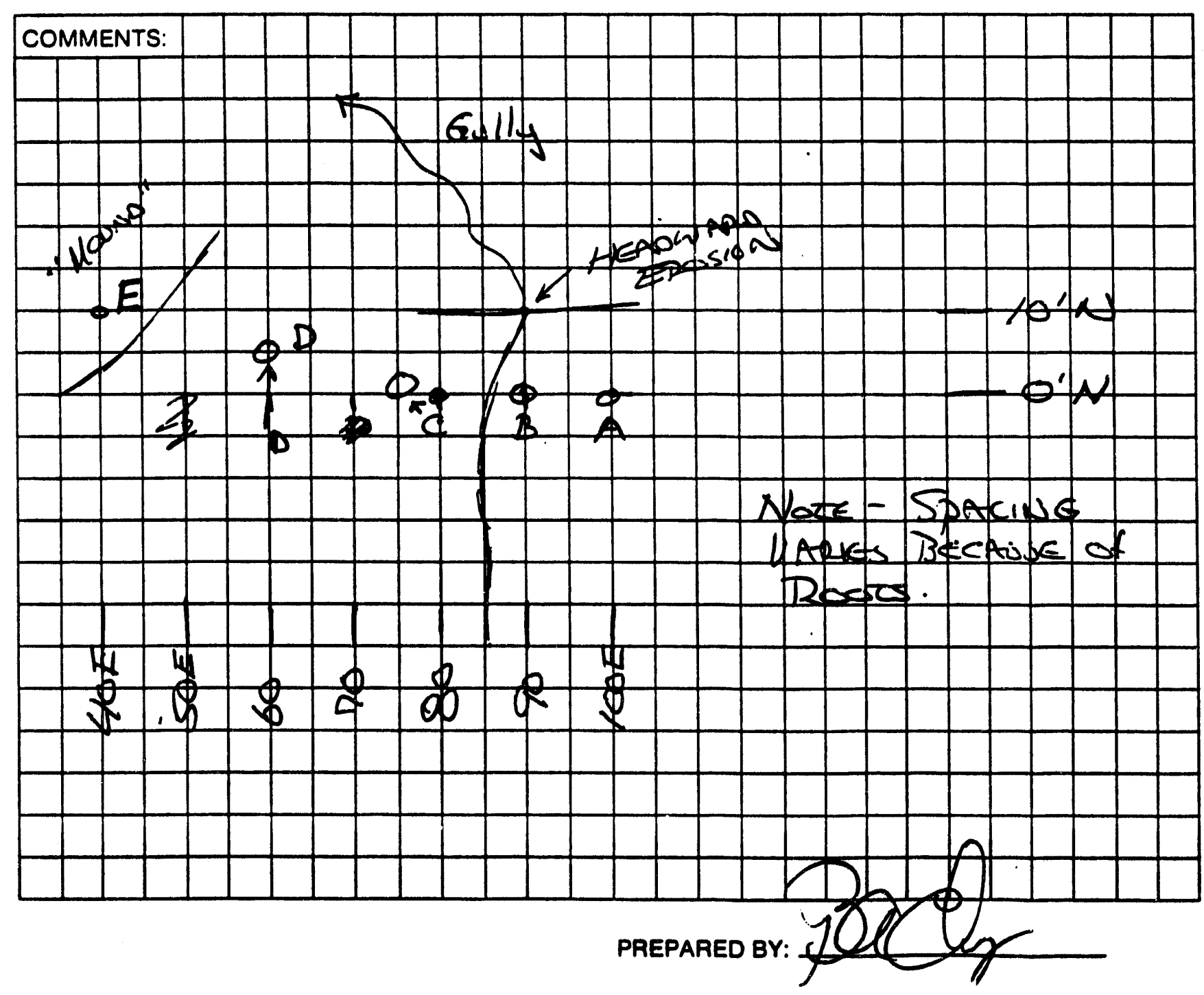




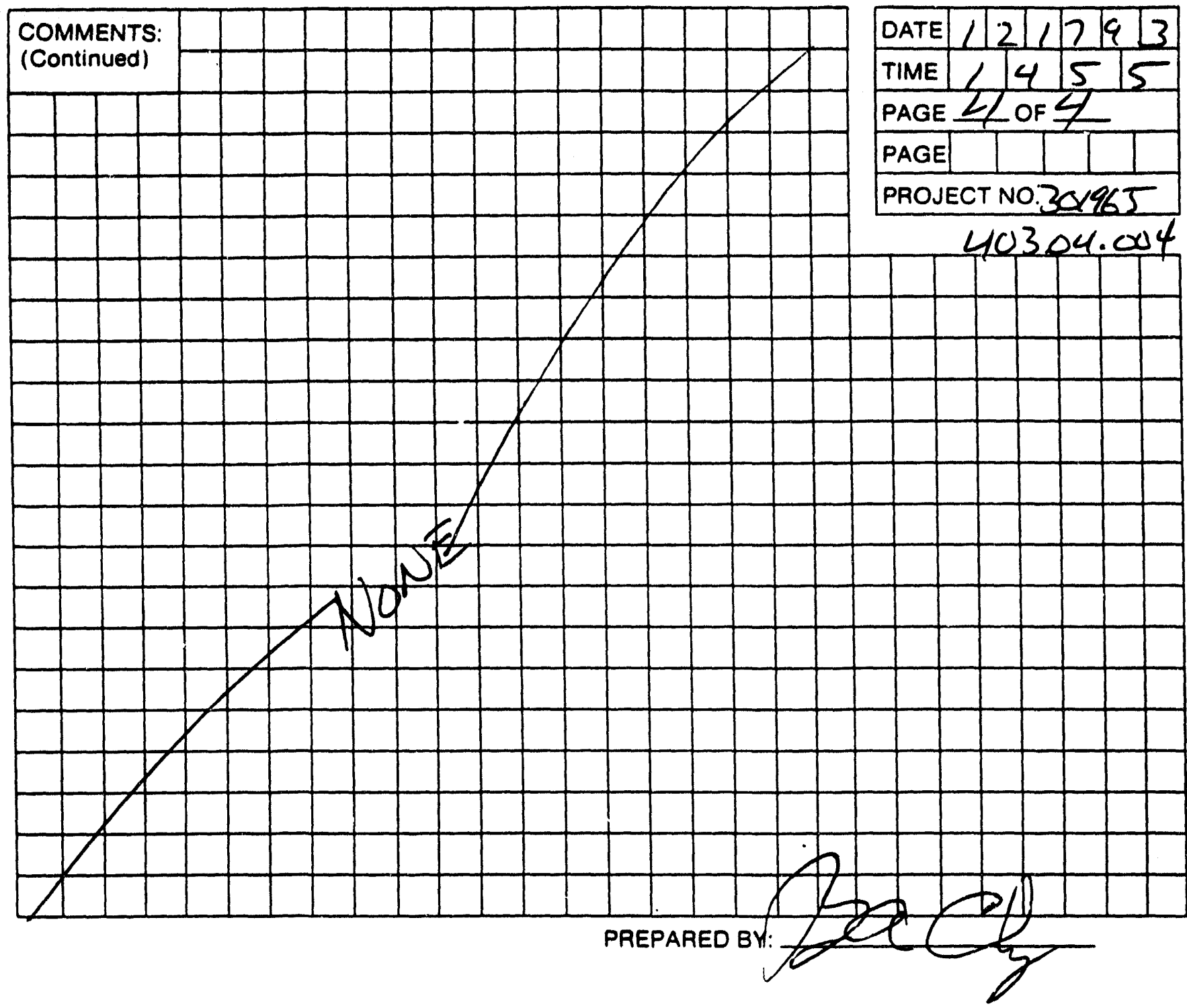

LEGEND

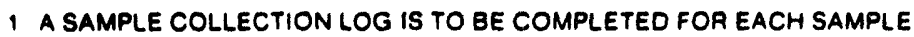

2 ALWAYS COMPLETE BOTH SIDES IF SECOND SIDE IS NOT USED. DRAW A LINE THROUGH IT AND MARK N/A. FILL IN CONTROL BLOCK AND PAEPAREO BY

3. ALL ENTRIES ON LOG ARE TO BE COMPLETED. If NOT APPLICABle MARK N/A.

4 DATE: USE MONTH/DAY/YEAR: 1.E., 10/30/85

5. TIME: USE 24-HOUR CLOCK. I.E.. 1835 FOR 6.35 P M

6 PAgE: EACH SAMPLE TEAM SHOULD NUMBER PAGE __ OF FOR THE DAYS ACTIVITIES FOR ALL SHEETS PREPARED ON A SINGLE DAY. I.E. IF THERE ARE A TOTAL OF 24 PAGES IINCLUDING FRONT AND BACKI NUMBER 1OF 24. 2 OF 24. ETC

7 SAMPLE LOCATION USE BORING OR MONITORING WELL NUMBER, GRIO LOCATION (TRANSECT). SAMPLING STATIONIO. OR COORDINATE TO PHYSICAL FEATURES WITH DISTANCES. INCLUDE SKETCH IN COMMENT SECTION IF NECESSARY

8. SAMPLE TYPE: USE THE FOLLOWING - SOIL: WATER (SUAFACE OR GROUNDI. AIR (FILTERS. TUBES. AMBIENT. PERSONNELI: SLUDGE. DRUM CONTENTS. OIL: VEGETATION: WIPE: SEDIMENT

9. COMPOSITE TYPE: I.E., 24-HOUR. LIST SAMPLE NUMBEAS IN COMPOSITE. SPATIAL COMPOSITE.

10. DEPTH OF SAMPLE. GIVE UNITS. WRITE OUT UNITS SUCH AS INCHES. FEET. DON'T USE ' OR"

11 WEATHER: APPROXIMATE TEMPERATURE. SUN ANO MOISTURE CONOITIONS

12. CONTAINERS USED: LIST EACH CONTAINER TYPE AS NUMBER. VOLUME. MATERIAL IE.G.. 2 - IL GLASS: 4 - 40 IAL GLASS VIAL: 1 - 400 ML PLASTIC: 1 - 3 INCH STEEL TUBE: 1 - 8 OZ. GLASS JAR).

13. AMOUNT COLLECTED: VOLUME IN CONTAINEAS (E.G. 1/2 FULL). 
Copies

Robert Bell

Mississippi Department of Health

Division of Radiological Health

P.O. Box 1700

Jackson, Mississippi 39215-1700

Roxanne Danz

DOE Nevada Operations Office

P.O. Box 98518

Las Vegas, Nevada 89193-8518

Phillip Weathersby

Mississippi Department of Environmental Quality

Office of Pollution Control

P.O. Box 10385

Jackson, Mississippi 39289-0385

IT Corporation

4330 S. Valley View, Suite 114

Las Vegas, Nevada 89023-0345

Ethan Merril

U.S. Department of Energy Headquarters

12800 Littlebrook Road

Germantown, Maryland 20874

Anita Mullen

U.S. Environmental Protection Agency

Environmental Monitoring Systems Laboratory

P.O. Box 93478

Las Vegas, Nevada 89193-3478

Purvis Library

Purvis, Mississippi 39475

Paul Tatum

Tatum Lumbar Company

P.O. Box 15547

Hattiesburg, Mississippi 39404

U.S. Department of Energy

Office of Scientific and Technical Information

175 Oak Ridge Turnpike

P.O. Box 62

Oak Ridge, Tennessee 37831

U.S. Department of Energy

Nevada Operations Office

Technical Library

P.O. Box 98518

Las Vegas, Nevada $89193-8518$ 